\title{
Application Of Advanced Diagonalization Methods To Quantum Spin Systems
}

\author{
By
}

Jieyu Wang

A thesis submitted in partial fulfillment of the requirements for the degree of Master of Science (MSc) in Computational Sciences

The School of Graduate Studies

Laurentian University

Sudbury, Ontario, Canada

(C) Jieyu Wang, 2014 


\section{Thesis Defence Committee/COMITÉ DE SOUTENANCE DE THÈSE \\ Laurentian Université/Université Laurentienne \\ School of Graduate Studies/École des études supérieures}

Title of Thesis

Titre de la thèse

Application Of Advanced Diagonalization Methods To

Quantum Spin Systems

Name of Candidate

Nom du candidat

Wang, Jieyu

Degree

Diplôme

Master of Science

Department/Program

Computational Sciences

Date of Defence

Département/Programme

Date de la soutenance

March 28, 2014

\section{APPROVED/APPROUVÉ}

Thesis Examiners/Examinateurs de thèse:

Dr. Ralf Meyer

(Supervisor/Directeur de thèse)

Dr. Gennady Chitov

(Committee member/Membre du comité)

Dr. Abdellatif Serghini

(Committee member/Membre du comité)

Dr. William Atkinson

(External Examiner/Examinateur externe)

Approved for the School of Graduate Studies Approuvé pour l'École des études supérieures

Dr. David Lesbarrères

M. David Lesbarrères

Director, School of Graduate Studies

Directeur, École des études supérieures

\section{ACCESSIBILITY CLAUSE AND PERMISSION TO USE}

I, Jieyu Wang, hereby grant to Laurentian University and/or its agents the non-exclusive license to archive and make accessible my thesis, dissertation, or project report in whole or in part in all forms of media, now or for the duration of my copyright ownership. I retain all other ownership rights to the copyright of the thesis, dissertation or project report. I also reserve the right to use in future works (such as articles or books) all or part of this thesis, dissertation, or project report. I further agree that permission for copying of this thesis in any manner, in whole or in part, for scholarly purposes may be granted by the professor or professors who supervised my thesis work or, in their absence, by the Head of the Department in which my thesis work was done. It is understood that any copying or publication or use of this thesis or parts thereof for financial gain shall not be allowed without my written permission. It is also understood that this copy is being made available in this form by the authority of the copyright owner solely for the purpose of private study and research and may not be copied or reproduced except as permitted by the copyright laws without written authority from the copyright owner. 


\section{Abstract}

Quantum spin models play an important role in theoretical condensed matter physics and quantum information theory. One numerical technique that is frequently used in studies of quantum spin systems is exact diagonalization. In this approach, numerical methods are used to find the lowest eigenvalues and associated eigenvectors of the Hamilton matrix of the quantum system. The computational problem is thus to determine the lowest eigenpairs of an extremely large, sparse matrix.

Although many sophisticated iterative techniques for the determination of a small number of lowest eigenpairs can be found in the literature, most exact diagonalization studies of quantum spin systems have employed the Lanczos algorithm. In contrast to this, other methods have been applied very successfully to the similar problem of electronic structure calculations. The well known VASP code for example uses a Block Davidson method as well as the residualminimization - direct inversion of the iterative subspace algorithm (RMM-DIIS).

The Davidson algorithm is closely related to the Lanczos method but usually needs less iterations. The RMM-DIIS method was originally proposed by Pulay and later modified by Wood and Zunger. The RMM-DIIS method is particularly interesting if more than one eigenpair is sought since it does not require orthogonalization of the trial vectors at each step.

In this work I study the efficiency of the Lanczos, Block Davidson and RMM-DIIS method when applied to basic quantum spin models like the spin-1/2 Heisenberg chain, ladder and dimerized ladder. I have implemented all three methods and are currently applying the methods to the different models. In our presentation I will compare the three algorithms based on the number of 
iterations to achieve convergence, the required computational time.

An Intel's Many-Integrated Core architecture with Intel Xeon Phi coprocessor 5110P integrates 60 cores with 4 hardware threads per core was used for RMM-DIIS method, the achieved parallel speedups were compared with those obtained on a conventional multi-core system. 


\section{Acknowledgements}

At first, I would like to acknowledge my supervisor Dr. Ralf Meyer, with whom I worked together on this research. He led me to this scientific field, and helped me to improve program. The algorithms became more efficient through his guidance.

This work has been financially supported by Laurentian University and the Natural Science and Engineering Research Council of Canada (NSERC). I gratefully acknowledge generous allocation of computer time on the facilities of the Shared Hierarchical Academic Research Network (SHARCNET) and Compute/Calcul Canada (https://computecanada.ca/) 


\section{Table of Contents}

Thesis Defence Committee .................................................................................................... ii

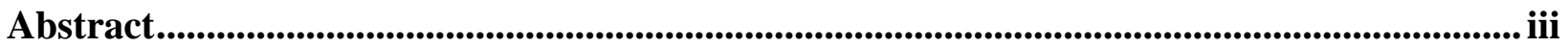

Acknowledgements ............................................................................................................................................. V

Table of Contents ......................................................................................................................................... vi

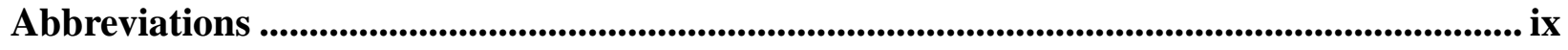

List of Tables..................................................................................................................................................... $x$

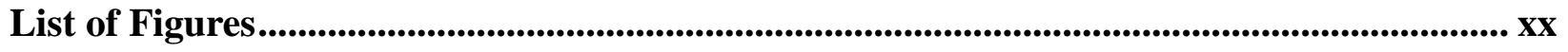

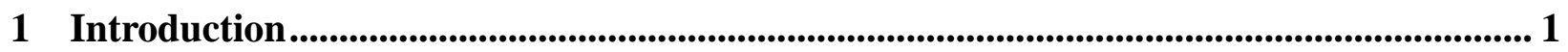

2 Backgrounds ......................................................................................................................................... 5

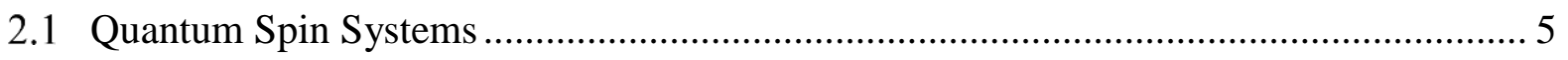

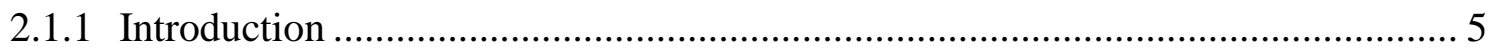

2.1.2 Numerical Methods for Quantum Models............................................................. 6

2.1.3 Quantum Spin Systems Considered in this Work ………………....................... 9

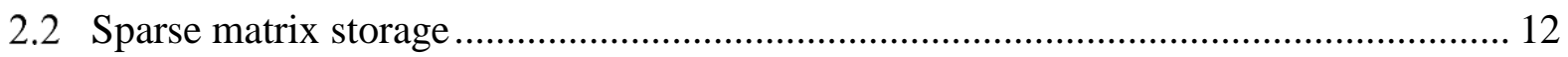

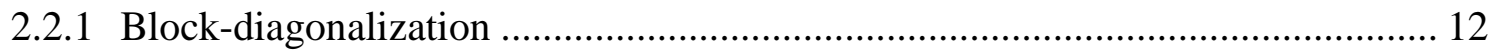

2.2.2 Sparse matrix storage (CSR) ……................................................................ 14

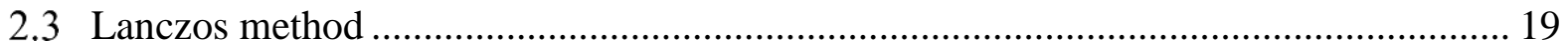

2.4 Davidson and Block Davidson method.................................................................... 22

2.4.1 Davidson method......................................................................................... 22

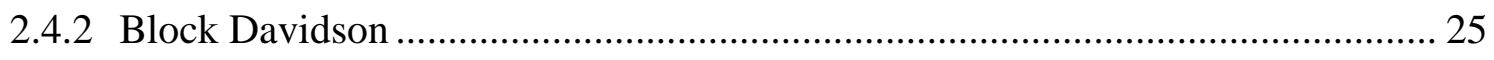

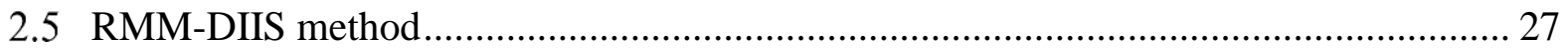


3 Implementations.................................................................................................................................. 32

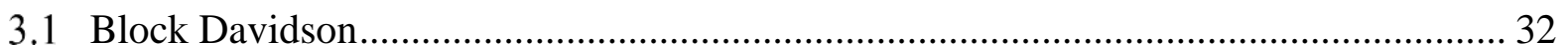

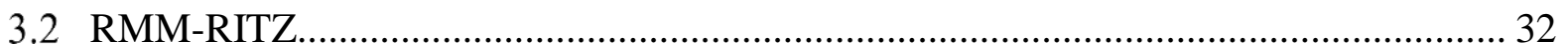

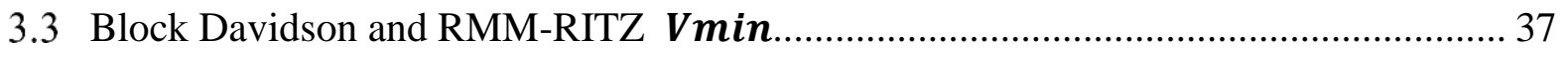

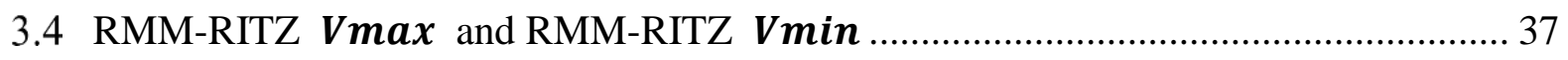

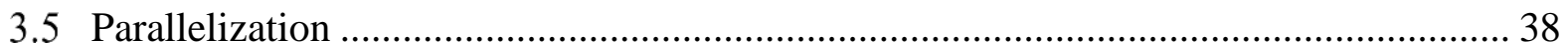

4 Results ............................................................................................................................................... 39

4.1 Comparison of different block size ............................................................................. 39

4.2 Results of Block Davidson and RMM-DIIS ................................................................ 54

4.2.1 Heisenberg Chain ............................................................................................ 55

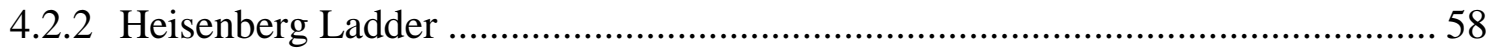

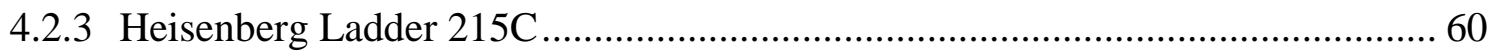

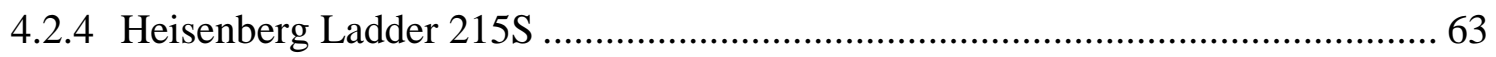

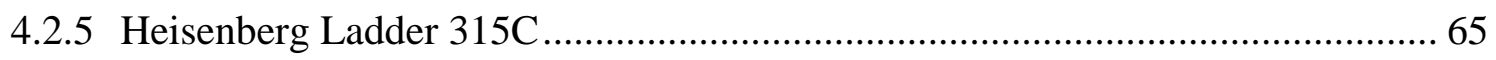

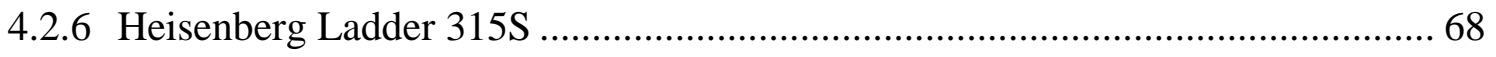

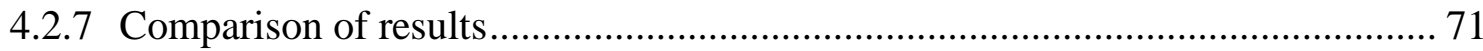

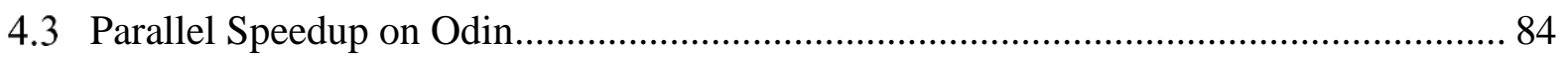

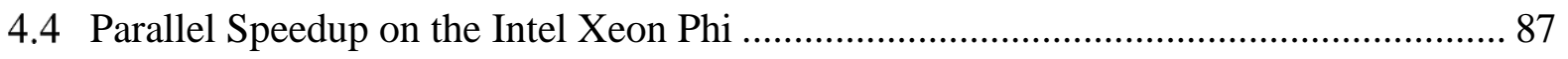

4.4.1 Heisenberg Chain ............................................................................................ 90

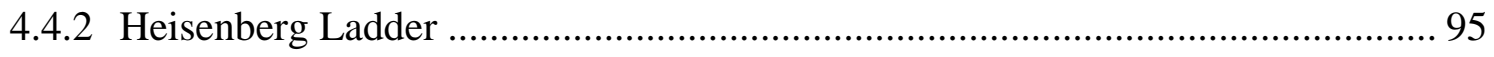

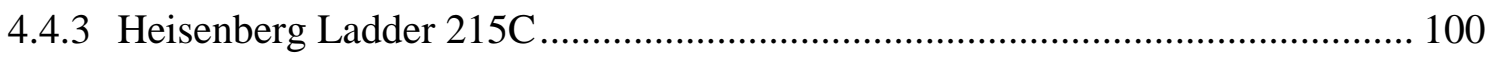

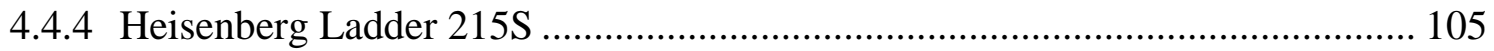

4.4.5 Heisenberg Ladder 315C ................................................................................ 111

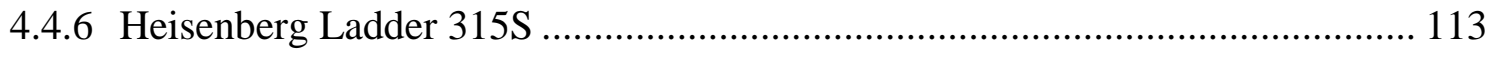

4.4.7 Time and speedup of different models on Xeon Phi ........................................ 116

4.4.8 Comparison of Speedup between Xeon Phi and Odin ..................................... 132 


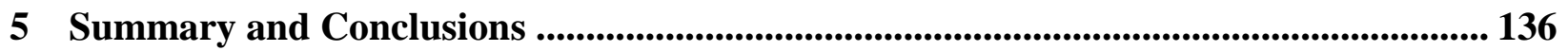

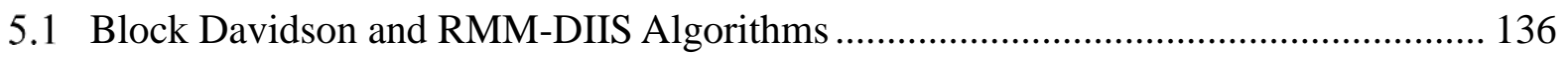

5.2 Parallel Speedup on Intel Xeon Phi ....................................................................... 138

5.3 Comparison with other exact diagonalization studies .............................................. 139

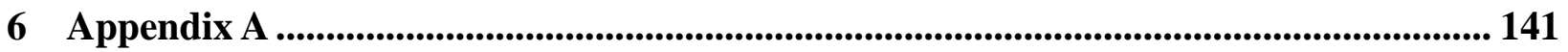

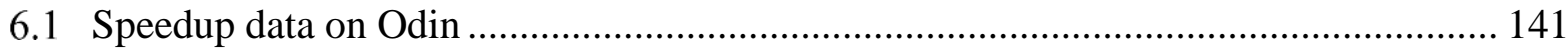

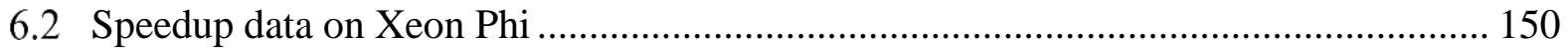

6.2.1 Heisenberg Chain on Xeon Phi .................................................................. 150

6.2.2 Heisenberg Ladder on Xeon Phi ..................................................................... 168

6.2.3 Heisenberg Ladder 215C on Xeon Phi ............................................................ 186

6.2.4 Heisenberg Ladder 215S on Xeon Phi .............................................................. 204

6.2.5 Heisenberg Ladder 315C on Xeon Phi ............................................................ 222

6.2.6 Heisenberg Ladder 315S on Xeon Phi ....................................................... 228

7 Reference 


\section{Abbreviations}

\begin{tabular}{|c|c|}
\hline $\mathrm{COO}$ & Coordinate format \\
\hline CSR & Compressed Sparse Row format \\
\hline NNZ & Number of Non-Zero element(s) \\
\hline MGS & Modified Gram-Schmidt orthogonalization procedure \\
\hline BKDV & Block Davidson \\
\hline RMM-DIIS/ & Residual Minimization Method - Direct Inversion in the \\
\hline RMM & Iterative Subspace \\
\hline Chain $(N)$ & Heisenberg Chain model (with $N$ spins) \\
\hline Ladder $2 /$ Ladder $(N)$ & Two-leg Heisenberg ladder model (with $N$ spins) \\
\hline Ladder $215 \mathrm{C}(N)$ & $\begin{array}{l}\text { Two-leg Dimerized Heisenberg ladder COLUMNAR model } \\
\text { with dimerization parameter }=0.5 \text { (with } N \text { spins) }\end{array}$ \\
\hline Ladder $215 \mathrm{~S}(N)$ & $\begin{array}{l}\text { Two-leg Dimerized Heisenberg ladder STAGGERED } \\
\text { model with dimerization parameter }=0.5 \text { (with } N \text { spins) }\end{array}$ \\
\hline Ladder $315 \mathrm{C}(N)$ & $\begin{array}{l}\text { Three-leg Dimerized Heisenberg ladder COLUMNAR } \\
\text { model with dimerization parameter }=0.5 \text { (with } N \text { spins) }\end{array}$ \\
\hline Ladder $315 \mathrm{~S}(N)$ & $\begin{array}{l}\text { Three-leg Dimerized Heisenberg ladder STAGGERED } \\
\text { model with dimerization parameter }=0.5 \text { (with } N \text { spins) }\end{array}$ \\
\hline Xeon Phi & $\begin{array}{l}\text { An Intel's Many-Integrated Core architecture with Intel } \\
\text { Xeon Phi coprocessor } 5110 \mathrm{P} \text { integrates } 60 \text { cores with } 4 \\
\text { hardware threads per core. }\end{array}$ \\
\hline Odin & $\begin{array}{l}\text { A machine with } 2 \times \text { Intel X5650 Xeon processor }(2.67 \mathrm{GHz}) \\
\text { and } 12 \mathrm{~GB} \text { of DDR3-1333 RAM, }\end{array}$ \\
\hline
\end{tabular}




\section{List of Tables}

Table 2.1 Full size dimension and reduced dimension in different size....................................................... 13

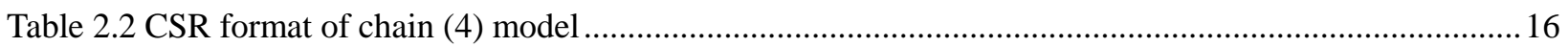

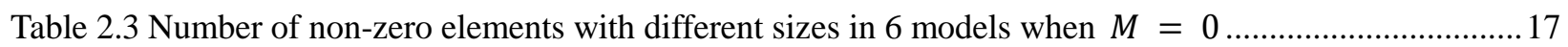

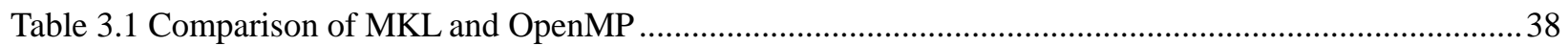

Table 4.1 Report 1 Convergence of Ladder 215S(24) by Block Davidson..................................................... 41

Table 4.2 Report 1 Convergence of Ladder 215S(24) by Block Davidson and RMM-RITZ Vmin.................41

Table 4.3 Report 1 Convergence of Ladder 215S(24) by RMM-RITZ Vmax ............................................. 42

Table 4.4 Report 1 Convergence of Ladder 215S(24) by RMM-RITZ Vmax and Vmin .............................42

Table 4.5 Report 2 Convergence of Ladder 215S(24) by Block Davidson......................................................43

Table 4.6 Report 2 Convergence of Ladder 215S(24) by Block Davidson and RMM-RITZ Vmin..................43

Table 4.7 Report 2 Convergence of Ladder 215S(24) by RMM-RITZ Vmax ..............................................44

Table 4.8 Report 2 Convergence of Ladder 215S(24) by RMM-RITZ Vmax and Vmin .............................44

Table 4.9 Report 3 Convergence of Ladder 215S(24) by Block Davidson.......................................................45

Table 4.10 Report 3 Convergence of Ladder 215S(24) by Block Davidson and RMM-RITZ Vmin............... 45

Table 4.11 Report 3 Convergence of Ladder 215S(24) by RMM-RITZ Vmax ...............................................46

Table 4.12 Report 3 Convergence of Ladder 215S(24) by RMM-RITZ Vmax and Vmin ...........................46

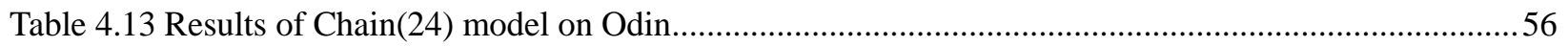

Table 4.14 Results of Chain(18) model on Odin...........................................................................................57

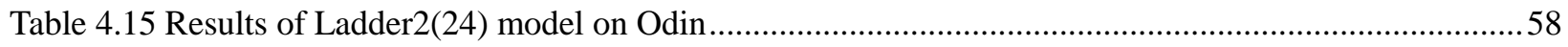

Table 4.16 Results of Ladder2(18) model on Odin......................................................................................5

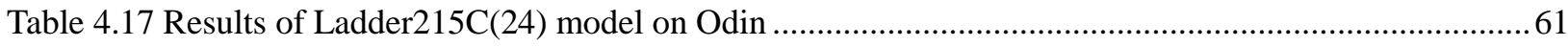

Table 4.18 Results of Ladder215C(18) model on Odin .............................................................................62

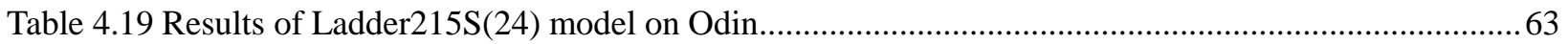


Table 4.20 Results of Ladder215S(18) model on Odin.

Table 4.21 Comparison of results of Ladder315C(24) model on Odin .66

Table 4.22 Comparison of results of Ladder315C(18) model on Odin 67

Table 4.23 Comparison of results of Ladder315S(24) model on Odin 68

Table 4.24 Comparison of results of Ladder315S(18) model on Odin 69

Table 4.25 Comparison of the results of two-leg model with $N=24$ on Odin .74

Table 4.26 Comparison of the results of three-leg model with $N=24$ on Odin .75

Table 4.27 Calculations of Chain(24) model by 4 different methods .76

Table 4.28 Calculations of Ladder2(24) model by 4 different methods .77

Table 4.29 Calculations of Ladder215C(24) model by 4 different methods 78

Table 4.30 Calculations of Ladder215S(24) model by 4 different methods 79

Table 4.31 Calculations of Ladder315C(24) model by 4 different methods .80

Table 4.32 Calculations of Ladder315S(24) model by 4 different methods 81

Table 4.33 Comparison of the results of two-leg models with $N=18$ on Odin. .82

Table 4.34 Comparison of the results of three-leg models with $N=18$ on Odin. .83

Table 4.35 Speedup of two-leg models with different size $N=26,24$, and 22 on Odin .84

Table 4.36 Speedup of two-leg models with different size $N=20,18$ and 16 on Odin. .85

Table 4.37 Speedup of three-leg models with different size $N=24$ and 18 on an Intel Xeon Phi .86

Table 4.38 Speedup of Chain $(N)$ with $N=26,24$ and 22 on an Intel Xeon Phi .90

Table 4.39 Speedup of Chain $(N)$ with $N=20,18$ and 16 on an Intel Xeon Phi 91

Table 4.40 Speedup of Ladde2( $N)$ with $N=26,24$ and 22 on an Intel Xeon Phi. .95

Table 4.41 Speedup of Ladder2( $N)$ with $N=20,18$ and 16 on an Intel Xeon Phi .96

Table 4.42 Speedup of Ladder 215C $(N)$ with $N=26,24$ and 22 on Xeon Phi 100

Table 4.43 Speedup of Ladder 215C $(N)$ with $N=20,18$ and 16 on an Intel Xeon Phi. 101

Table 4.44 Speedup of Ladder 215S $(N)$ with $N=26,24$ and 22 on an Intel Xeon Phi 106

Table 4.45 Speedup of Ladder 215S $(N)$ with $N=20,18$ and 16 on an Intel Xeon Phi 107 
Table 4.46 Speedup of Ladder 315C $(N)$ with $N=24$ and 18 on an Intel Xeon Phi

Table 4.47 Speedup of Ladder 315S $(N)$ with $N=24$ and 18 on an Intel Xeon Phi

Table 4.48 Comparison of time and speedup of Chain, Ladder and Ladder 215C with $N=24$ on an Intel Xeon

Phi with KMP_THREAD_AFFINITY set to scatter. 120

Table 4.49 Comparison of time and speedup of Ladder215S, Ladder315C and Ladder315S with $N=24$ on an Intel Xeon Phi coprocessor with KMP_THREAD_AFFINITY set to scatter ..... 121

Table 4.50 Comparison of time and speedup of Chain, Ladder and Ladder 215C with $N=24$ on an Intel Xeon Phi coprocessor with KMP_THREAD_AFFINITY set to compact 122

Table 4.51 Comparison of time and speedup of Ladder215S, Ladder315C and Ladder315S with $N=24$ on an Intel Xeon Phi coprocessor with KMP_THREAD_AFFINITY set to compact 123

Table 4.52 Comparison of time and speedup of Chain, Ladder and Ladder 215C with $N=24$ on an Intel Xeon

Phi coprocessor with KMP_THREAD_AFFINITY set to balanced 124

Table 4.53 Comparison of time and speedup of Ladder215S, Ladder315C and Ladder315S with $N=24$ on an Intel Xeon Phi coprocessor with KMP_THREAD_AFFINITY set to balanced. 125

Table 4.54 Comparison of time and speedup of Chain, Ladder and Ladder 215C with $N=18$ on an Intel Xeon Phi coprocessor with KMP_THREAD_AFFINITY set to scatter 126

Table 4.55 Comparison of time and speedup of Ladder215S, Ladder315C and Ladder315S with $N=18$ on an Intel Xeon Phi coprocessor with KMP_THREAD_AFFINITY set to scatter .... 127

Table 4.56 Comparison of time and speedup of Chain, Ladder and Ladder 215C with $N=18$ on an Intel Xeon Phi coprocessor with KMP_THREAD_AFFINITY set to compact 128

Table 4.57 Comparison of time and speedup of Ladder215S, Ladder315C and Ladder315S with $N=18$ on an Intel Xeon Phi coprocessor with KMP_THREAD_AFFINITY set to compact 129

Table 4.58 Comparison of time and speedup of Chain, Ladder and Ladder 215C with $N=18$ on an Intel Xeon Phi coprocessor with KMP_THREAD_AFFINITY set to balanced 130

Table 4.59 Comparison of time and speedup of Ladder215S, Ladder315C and Ladder315S with $N=18$ on an Intel Xeon Phi coprocessor with KMP_THREAD_AFFINITY set to balanced. 131 
Table 4.60 Comparison of time and speedup between Xeon Phi and Odin with $N=24$

Table 4.61 Comparison of time and speedup between Xeon Phi and Odin with $N=18$.

Table 4.62 Comparison Ladder 215C $(N)$ with $N=16,18,20,22,24,26$ on two machines 134

Table 6.1 Time and speedup of Chain $(N)$ model with $N=26,24$ and 22 on Odin...... 141

Table 6.2 Time and speedup of Chain $(N)$ model with $N=20,18$ and16 on Odin. 142

Table 6.3 Time and speedup of Ladder2( $N)$ model with $N=26,24$ and 22, on Odin 143

Table 6.4 Time and speedup of Ladder2( $N)$ model with $N=20,18$ and16 on Odin 144

Table 6.5 Time and speedup of Ladder $215 \mathrm{C}(N)$ with $N=26,24$ and 22 on Odin 145

Table 6.6 Time and speedup of Ladder $215 \mathrm{C}(N)$ with $N=20,18$ and16 on Odin. 146

Table 6.7 Time and speedup of Ladder $215 \mathrm{~S}(N)$ with $N=26,24$ and 22 on Odin 147

Table 6.8 Time and speedup of Ladder $215 \mathrm{~S}(N)$ with $N=20,18$ and 16 on Odin 148

Table 6.9 Time and speedup of Ladder $315 \mathrm{C}(N)$ and $315 \mathrm{~S}(N)$ model with $N=24$ and 18 on Odin

Table 6.10 Time and speedup of Chain $(N)$ model with $N=26$ on an Intel Xeon Phi coprocessor with

KMP_THREAD_AFFINITY set to scatter..

Table 6.11 Time and speedup of Chain $(N)$ model with $N=24$ on an Intel Xeon Phi coprocessor with KMP_THREAD_AFFINITY set to scatter.. 151

Table 6.12 Time and speedup of Chain $(N)$ model with $N=22$ on an Intel Xeon Phi coprocessor with KMP_THREAD_AFFINITY set to scatter 152

Table 6.13 Time and speedup of Chain $(N)$ model with $N=20$ on an Intel Xeon Phi coprocessor with KMP_THREAD_AFFINITY set to scatter. 153

Table 6.14 Time and speedup of Chain $(N)$ model with $N=18$ on an Intel Xeon Phi coprocessor with KMP_THREAD_AFFINITY set to scatter.. 154

Table 6.15 Time and speedup of Chain $(N)$ model with $N=16$ on an Intel Xeon Phi coprocessor with KMP_THREAD_AFFINITY set to scatter....... 155

Table 6.16 Time and speedup of Chain $(N)$ model with $N=26$ on an Intel Xeon Phi coprocessor with KMP_THREAD_AFFINITY set to compact. 156 
Table 6.17 Time and speedup of Chain $(N)$ model with $N=24$ on an Intel Xeon Phi coprocessor with

KMP_THREAD_AFFINITY set to compact.

Table 6.18 Time and speedup of Chain $(N)$ model with $N=22$ on an Intel Xeon Phi coprocessor with

KMP_THREAD_AFFINITY set to compact. 158

Table 6.19 Time and speedup of Chain $(N)$ model with $N=20$ on an Intel Xeon Phi coprocessor with

KMP_THREAD_AFFINITY set to compact.

Table 6.20 Time and speedup of Chain $(N)$ model with $N=18$ on an Intel Xeon Phi coprocessor with

KMP_THREAD_AFFINITY set to compact.

Table 6.21 Time and speedup of Chain $(N)$ model with $N=16$ on an Intel Xeon Phi coprocessor with

KMP_THREAD_AFFINITY set to compact.

Table 6.22 Time and speedup of Chain $(N)$ model with $N=26$ on an Intel Xeon Phi coprocessor with

KMP_THREAD_AFFINITY set to balanced.

Table 6.23 Time and speedup of Chain $(N)$ model with $N=24$ on an Intel Xeon Phi coprocessor with

KMP_THREAD_AFFINITY set to balanced.

Table 6.24 Time and speedup of Chain $(N)$ model with $N=22$ on an Intel Xeon Phi coprocessor with

KMP_THREAD_AFFINITY set to balanced.

Table 6.25 Time and speedup of Chain $(N)$ model with $N=20$ on an Intel Xeon Phi coprocessor with

KMP_THREAD_AFFINITY set to balanced.

Table 6.26 Time and speedup of Chain $(N)$ model with $N=18$ on an Intel Xeon Phi coprocessor with

KMP_THREAD_AFFINITY set to balanced. 166

Table 6.27 Time and speedup of Chain $(N)$ model with $N=16$ on an Intel Xeon Phi coprocessor with

KMP_THREAD_AFFINITY set to balanced.

Table 6.28 Time and speedup of Ladder2( $N)$ model with $N=26$ on an Intel Xeon Phi coprocessor with

KMP_THREAD_AFFINITY set to scatter...

Table 6.29 Time and speedup of Ladder2( $N)$ model with $N=24$ on an Intel Xeon Phi coprocessor with

KMP_THREAD_AFFINITY set to scatter.. 169 
Table 6.30 Time and speedup of Ladder2( $N)$ model with $N=22$ on an Intel Xeon Phi coprocessor with

KMP_THREAD_AFFINITY set to scatter.

Table 6.31 Time and speedup of Ladder2( $N)$ model with $N=20$ on an Intel Xeon Phi coprocessor with

KMP_THREAD_AFFINITY set to scatter...

Table 6.32 Time and speedup of Ladder2( $N)$ model with $N=18$ on an Intel Xeon Phi coprocessor with

KMP_THREAD_AFFINITY set to scatter

Table 6.33 Time and speedup of Ladder2( $N)$ model with $N=16$ on an Intel Xeon Phi coprocessor with

KMP_THREAD_AFFINITY set to scatter.

Table 6.34 Time and speedup of Ladder2( $N)$ model with $N=26$ on an Intel Xeon Phi coprocessor with

KMP_THREAD_AFFINITY set to compact.

Table 6.35 Time and speedup of Ladder2( $N)$ model with $N=24$ on an Intel Xeon Phi coprocessor with

KMP_THREAD_AFFINITY set to compact.

Table 6.36 Time and speedup of Ladder2( $N)$ model with $N=22$ on an Intel Xeon Phi coprocessor with

KMP_THREAD_AFFINITY set to compact.

Table 6.37 Time and speedup of Ladder2(N) model with $N=20$ on an Intel Xeon Phi coprocessor with

KMP_THREAD_AFFINITY set to compact.

Table 6.38 Time and speedup of Ladder2(N) model with $N=18$ on an Intel Xeon Phi coprocessor with

KMP_THREAD_AFFINITY set to compact.

Table 6.39 Time and speedup of Ladder2( $N)$ model with $N=16$ on an Intel Xeon Phi coprocessor with

KMP_THREAD_AFFINITY set to compact.

Table 6.40 Time and speedup of Ladder2( $N)$ model with $N=26$ on an Intel Xeon Phi coprocessor with

KMP_THREAD_AFFINITY set to balanced.

Table 6.41 Time and speedup of Ladder2( $N)$ model with $N=24$ on an Intel Xeon Phi coprocessor with

KMP_THREAD_AFFINITY set to balanced.

Table 6.42 Time and speedup of Ladder2( $N)$ model with $N=22$ on an Intel Xeon Phi coprocessor with

KMP_THREAD_AFFINITY set to balanced... 
Table 6.43 Time and speedup of Ladder2( $N)$ model with $N=20$ on an Intel Xeon Phi coprocessor with

KMP_THREAD_AFFINITY set to balanced.

Table 6.44 Time and speedup of Ladder2( $N)$ model with $N=18$ on an Intel Xeon Phi coprocessor with

KMP_THREAD_AFFINITY set to balanced.

Table 6.45 Time and speedup of Ladder2( $N)$ model with $N=16$ on an Intel Xeon Phi coprocessor with

KMP_THREAD_AFFINITY set to balanced.

Table 6.46 Time and speedup of Ladder $215 \mathrm{C}(N)$ model with $N=26$ on an Intel Xeon Phi coprocessor with

KMP_THREAD_AFFINITY set to scatter.. 186

Table 6.47 Time and speedup of Ladder $215 \mathrm{C}(N)$ model with $N=24$ on an Intel Xeon Phi coprocessor with

KMP_THREAD_AFFINITY set to scatter...

Table 6.48 Time and speedup of Ladder $215 \mathrm{C}(N)$ model with $N=22$ on an Intel Xeon Phi coprocessor with

KMP_THREAD_AFFINITY set to scatter.

Table 6.49 Time and speedup of Ladder 215C $(N)$ model with $N=20$ on an Intel Xeon Phi coprocessor with

KMP_THREAD_AFFINITY set to scatter......

Table 6.50 Time and speedup of Ladder $215 \mathrm{C}(N)$ model with $N=18$ on an Intel Xeon Phi coprocessor with

KMP_THREAD_AFFINITY set to scatter.

Table 6.51 Time and speedup of Ladder $215 \mathrm{C}(N)$ model with $N=16$ on an Intel Xeon Phi coprocessor with

KMP_THREAD_AFFINITY set to scatter.

Table 6.52 Time and speedup of Ladder $215 \mathrm{C}(N)$ model with $N=26$ on an Intel Xeon Phi coprocessor with

KMP_THREAD_AFFINITY set to compact.

Table 6.53 Time and speedup of Ladder $215 \mathrm{C}(N)$ model with $N=24$ on an Intel Xeon Phi coprocessor with

KMP_THREAD_AFFINITY set to compact.

Table 6.54 Time and speedup of Ladder $215 \mathrm{C}(N)$ model with $N=22$ on an Intel Xeon Phi coprocessor with

KMP_THREAD_AFFINITY set to compact.

Table 6.55 Time and speedup of Ladder 215C $(N)$ model with $N=20$ on an Intel Xeon Phi coprocessor with

KMP_THREAD_AFFINITY set to compact. 
Table 6.56 Time and speedup of Ladder $215 \mathrm{C}(N)$ model with $N=18$ on an Intel Xeon Phi coprocessor with KMP_THREAD_AFFINITY set to compact.

Table 6.57 Time and speedup of Ladder $215 \mathrm{C}(N)$ model with $N=16$ on an Intel Xeon Phi coprocessor with KMP_THREAD_AFFINITY set to compact.

Table 6.58 Time and speedup of Ladder $215 \mathrm{C}(N)$ model with $N=26$ on an Intel Xeon Phi coprocessor with KMP_THREAD_AFFINITY set to balanced.... 198

Table 6.59 Time and speedup of Ladder $215 \mathrm{C}(N)$ model with $N=24$ on an Intel Xeon Phi coprocessor with KMP_THREAD_AFFINITY set to balanced.

Table 6.60 Time and speedup of Ladder $215 \mathrm{C}(N)$ model with $N=22$ on an Intel Xeon Phi coprocessor with KMP_THREAD_AFFINITY set to balanced. 200

Table 6.61 Time and speedup of Ladder $215 \mathrm{C}(N)$ model with $N=20$ on an Intel Xeon Phi coprocessor with KMP_THREAD_AFFINITY set to balanced. 201

Table 6.62 Time and speedup of Ladder $215 \mathrm{C}(N)$ model with $N=18$ on an Intel Xeon Phi coprocessor with KMP_THREAD_AFFINITY set to balanced.

Table 6.63 Time and speedup of Ladder $215 \mathrm{C}(N)$ model with $N=16$ on an Intel Xeon Phi coprocessor with KMP_THREAD_AFFINITY set to balanced.

Table 6.64 Time and speedup of Ladder 215S $(N)$ model with $N=26$ on an Intel Xeon Phi coprocessor with KMP_THREAD_AFFINITY set to scatter..

Table 6.65 Time and speedup of Ladder 215S $(N)$ model with $N=24$ on an Intel Xeon Phi coprocessor with KMP_THREAD_AFFINITY set to scatter...

Table 6.66 Time and speedup of Ladder 215S $(N)$ model with $N=22$ on an Intel Xeon Phi coprocessor with

KMP_THREAD_AFFINITY set to scatter.

Table 6.67 Time and speedup of Ladder 215S $(N)$ model with $N=20$ on an Intel Xeon Phi coprocessor with KMP_THREAD_AFFINITY set to scatter.......

Table 6.68 Time and speedup of Ladder 215S $(N)$ model with $N=18$ on an Intel Xeon Phi coprocessor with KMP_THREAD_AFFINITY set to scatter... 
Table 6.69 Time and speedup of Ladder 215S $(N)$ model with $N=16$ on an Intel Xeon Phi coprocessor with KMP_THREAD_AFFINITY set to scatter

Table 6.70 Time and speedup of Ladder $215 \mathrm{~S}(N)$ model with $N=26$ on an Intel Xeon Phi coprocessor with KMP_THREAD_AFFINITY set to compact.

Table 6.71 Time and speedup of Ladder 215S $(N)$ model with $N=24$ on an Intel Xeon Phi coprocessor with KMP_THREAD_AFFINITY set to compact.

Table 6.72 Time and speedup of Ladder 215S $(N)$ model with $N=22$ on an Intel Xeon Phi coprocessor with KMP_THREAD_AFFINITY set to compact.

Table 6.73 Time and speedup of Ladder 215S $(N)$ model with $N=20$ on an Intel Xeon Phi coprocessor with

KMP_THREAD_AFFINITY set to compact.

Table 6.74 Time and speedup of Ladder 215S $(N)$ model with $N=18$ on an Intel Xeon Phi coprocessor with

KMP_THREAD_AFFINITY set to compact.

Table 6.75 Time and speedup of Ladder 215S $(N)$ model with $N=16$ on an Intel Xeon Phi coprocessor with

KMP_THREAD_AFFINITY set to compact.

Table 6.76 Time and speedup of Ladder 215S $(N)$ model with $N=26$ on an Intel Xeon Phi coprocessor with

KMP_THREAD_AFFINITY set to balanced.

Table 6.77 Time and speedup of Ladder 215S $(N)$ model with $N=24$ on an Intel Xeon Phi coprocessor with

KMP_THREAD_AFFINITY set to balanced.

Table 6.78 Time and speedup of Ladder 215S $(N)$ model with $N=22$ on an Intel Xeon Phi coprocessor with

KMP_THREAD_AFFINITY set to balanced.

Table 6.79 Time and speedup of Ladder 215S $(N)$ model with $N=20$ on an Intel Xeon Phi coprocessor with

KMP_THREAD_AFFINITY set to balanced.

Table 6.80 Time and speedup of Ladder 215S $(N)$ model with $N=18$ on an Intel Xeon Phi coprocessor with

KMP_THREAD_AFFINITY set to balanced.

Table 6.81 Time and speedup of Ladder 215S $(N)$ model with $N=16$ on an Intel Xeon Phi coprocessor with

KMP_THREAD_AFFINITY set to balanced. 
Table 6.82 Time and speedup of Ladder $315 \mathrm{C}(N)$ model with $N=24$ on an Intel Xeon Phi coprocessor with KMP_THREAD_AFFINITY set to scatter.

Table 6.83 Time and speedup of Ladder $315 \mathrm{C}(N)$ model with $N=18$ on an Intel Xeon Phi coprocessor with KMP_THREAD_AFFINITY set to scatter......

Table 6.84 Time and speedup of Ladder $315 \mathrm{C}(N)$ model with $N=24$ on an Intel Xeon Phi coprocessor with KMP_THREAD_AFFINITY set to compact..

Table 6.85 Time and speedup of Ladder $315 \mathrm{C}(N)$ model with $N=18$ on an Intel Xeon Phi coprocessor with KMP_THREAD_AFFINITY set to compact.

Table 6.86 Time and speedup of Ladder $315 \mathrm{C}(N)$ model with $N=24$ on an Intel Xeon Phi coprocessor with

KMP_THREAD_AFFINITY set to balanced.

Table 6.87 Time and speedup of Ladder $315 \mathrm{C}(N)$ model with $N=18$ on an Intel Xeon Phi coprocessor with

KMP_THREAD_AFFINITY set to balanced.

Table 6.88 Time and speedup of Ladder 315S $(N)$ model with $N=24$ on an Intel Xeon Phi coprocessor with

KMP_THREAD_AFFINITY set to scatter......

Table 6.89 Time and speedup of Ladder $315 \mathrm{~S}(N)$ model with $N=18$ on an Intel Xeon Phi coprocessor with

KMP_THREAD_AFFINITY set to scatter...

Table 6.90 Time and speedup of Ladder 315S $(N)$ model with $N=24$ on an Intel Xeon Phi coprocessor with KMP_THREAD_AFFINITY set to compact.

Table 6.91 Time and speedup of Ladder 315S $(N)$ model with $N=18$ on an Intel Xeon Phi coprocessor with

KMP_THREAD_AFFINITY set to compact.

Table 6.92 Time and speedup of Ladder 315S $(N)$ model with $N=24$ on an Intel Xeon Phi coprocessor with

KMP_THREAD_AFFINITY set to balanced.

Table 6.93 Time and speedup of Ladder 315S $(N)$ model with $N=18$ on an Intel Xeon Phi coprocessor with KMP_THREAD_AFFINITY set to balanced. 


\section{List of Figures}

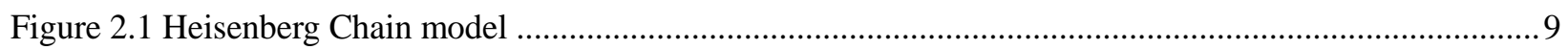

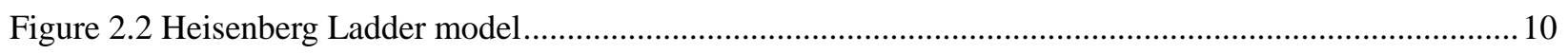

Figure 2.3 Dimerized Heisenberg ladder columnar model …................................................................... 11

Figure 2.4 Dimerized Heisenberg ladder staggered model ...................................................................... 11

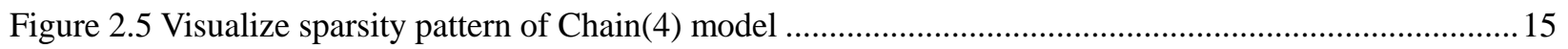

Figure 2.6 Visualize sparsity pattern of Chain(24) model ............................................................................. 18

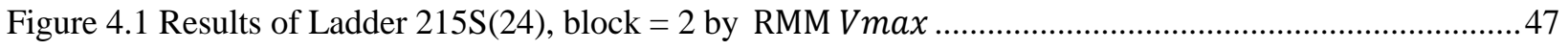

Figure 4.2 Results of Ladder 215S(24), block = 2/4 by RMM Vmax\& Vmin ..............................................47

Figure 4.3 Results of Ladder 215S(24), block = 3 by RMM Vmax .......................................................... 47

Figure 4.4 Results of Ladder 215S(24), block = 3/4 by RMM Vmax\& Vmin .............................................. 47

Figure 4.5 Results of Ladder 215S(24), block = 4 by RMM Vmax ...........................................................48

Figure 4.6 Results of Ladder 215S(24), block = 4/4 by RMM Vmax\& Vmin ............................................. 48

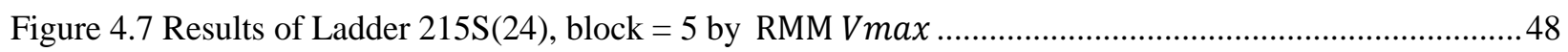

Figure 4.8 Results of Ladder 215S(24), block = 5/4 by RMM Vmax \& Vmin ........................................... 48

Figure 4.9 Results of Ladder 215S(24), block = 6 by RMM Vmax ......................................................... 49

Figure 4.10 Results of Ladder 215S(24), block = 6/4 by RMM Vmax\& Vmin ............................................. 49

Figure 4.11 Results of Ladder 215S(24), block = 2 by BKDV ..............................................................50

Figure 4.12 Results of Ladder 215S(24), block = 2/4 by BKDV and RMM Vmin .......................................50

Figure 4.13 Results of Ladder 215S(24), block = 3 by BKDV .................................................................51

Figure 4.14 Results of Ladder 215S(24), block = 3/4 by BKDV and RMM Vmin .......................................51

Figure 4.15 Results of Ladder 215S(24), block = 4 by BKDV ...................................................................51

Figure 4.16 Results of Ladder 215S(24), block = 4/4 by BKDV and RMM Vmin ......................................51

Figure 4.17 Results of Ladder 215S(24), block = 5 by BKDV ...................................................................52 
Figure 4.18 Results of Ladder 215S(24), block = 5/4 by BKDV and RMM Vmin......

Figure 4.19 Results of Ladder 215S(24), block = 6 by BKDV ...............................................................52

Figure 4.20 Results of Ladder 215S(24), block = 6/4 by BKDV and RMM Vmin .........................................52

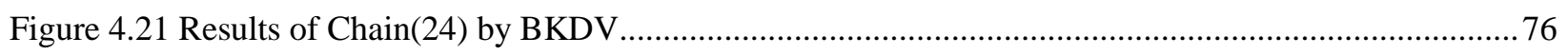

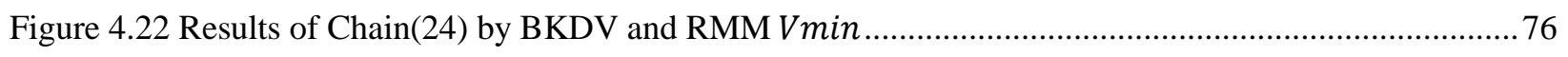

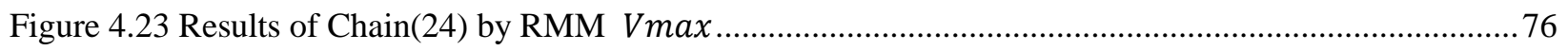

Figure 4.24 Results of Chain(24) by RMM Vmax and RMM Vmin ........................................................ 76

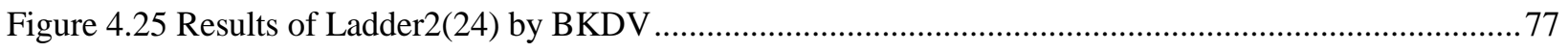

Figure 4.26 Results of Ladder2(24) by BKDV and RMM Vmin ............................................................. 77

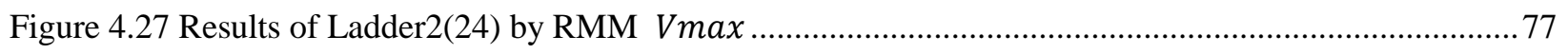

Figure 4.28 Results of Ladder2(24) by RMM V $\max$ and RMM Vmin .....................................................77

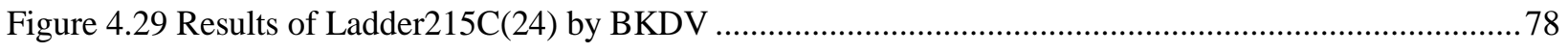

Figure 4.30 Results of Ladder215C(24) model by BKDV and RMM Vmin ............................................ 78

Figure 4.31 Results of Ladder215C(24) by RMM Vmax ...................................................................... 78

Figure 4.32 Results of Ladder215C(24) by RMM Vmax and RMM Vmin ............................................78

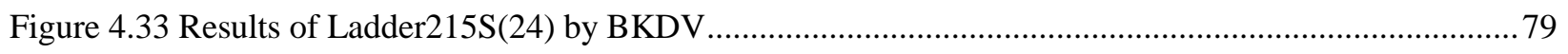

Figure 4.34 Results of Ladder215S(24) by BKDV and RMM Vmin ......................................................... 79

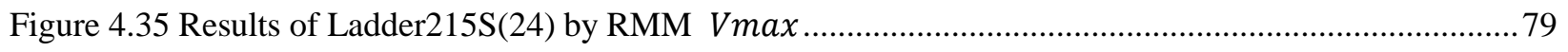

Figure 4.36 Results of Ladder215S(24) by RMM Vmax and RMM Vmin ................................................ 79

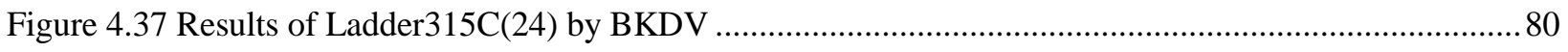

Figure 4.38 Results of Ladder315C(24) by BKDV and RMM V min ...................................................... 80

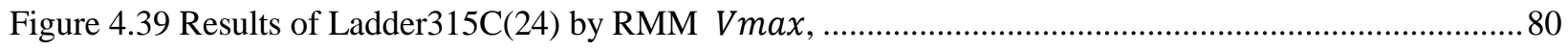

Figure 4.40 Results of Ladder315C(24) by RMM Vmax and RMM Vmin..............................................8 80

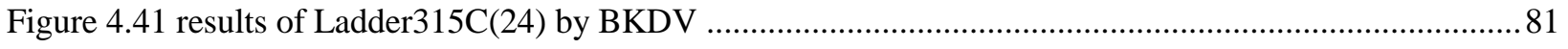

Figure 4.42 results of Ladder315S(24) by BKDV and RMM Vmin ....................................................... 81

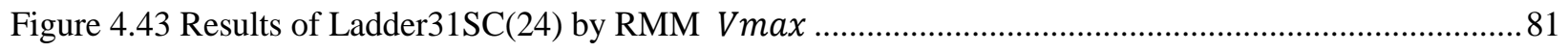


Figure 4.44 Results of Ladder315S(24) by RMM Vmax and RMM Vmin.....

Figure 4.45 Allocation with scatter affinity type .89

Figure 4.46 Allocation with compact affinity type .89

Figure 4.47 Allocation with balanced affinity type

Figure 4.48 Parallel speedup of Chain(24) model on an Intel Xeon Phi with KMP_THREAD_AFFINITY set to scatter, compact and balanced.

Figure 4.49 Parallel speedup of Chain(22) model on an Intel Xeon Phi with KMP_THREAD_AFFINITY set to scatter, compact and balanced.

Figure 4.50 Parallel speedup of Chain(24) model on an Intel Xeon Phi with KMP_THREAD_AFFINITY set to scatter, compact and balanced. .93

Figure 4.51 Parallel speedup of Chain(18) model on an Intel Xeon Phi with KMP_THREAD_AFFINITY set to scatter, compact and balanced. .93

Figure 4.52 Parallel speedup of Chain(16) model on an Intel Xeon Phi with KMP_THREAD_AFFINITY set to scatter, compact and balanced. 94

Figure 4.53 Parallel speedup of Ladder2(24) model on an Intel Xeon Phi with KMP_THREAD_AFFINITY set to scatter, compact and balanced 97

Figure 4.54 Parallel speedup of Ladder2(22) model on an Intel Xeon Phi with KMP_THREAD_AFFINITY set to scatter, compact and balanced .97

Figure 4.55 Parallel speedup of Ladder2(20) model on an Intel Xeon Phi with KMP_THREAD_AFFINITY set to scatter, compact and balanced ... 98

Figure 4.56 Parallel speedup of Ladder2(18) mode on an Intel Xeon Phi with KMP_THREAD_AFFINITY set to scatter, compact and balanced .98

Figure 4.57 Parallel speedup of Ladder2(16) model on an Intel Xeon Phi with KMP_THREAD_AFFINITY set to scatter, compact and balanced .99

Figure 4.58 Parallel speedup of Ladder215C(26) model on an Intel Xeon Phi with KMP_THREAD_AFFINITY set to scatter, compact and balanced 102 
Figure 4.59 Parallel speedup of Ladder215C(24) model on an Intel Xeon Phi with

KMP_THREAD_AFFINITY set to scatter, compact and balanced

Figure 4.60 Parallel speedup of Ladder215C(22) model on an Intel Xeon Phi with

KMP_THREAD_AFFINITY set to scatter, compact and balanced

Figure 4.61 Parallel speedup of Ladder215C(20) model on an Intel Xeon Phi with

KMP_THREAD_AFFINITY set to scatter, compact and balanced 103

Figure 4.62 Parallel speedup of Ladder215C(18) model on an Intel Xeon Phi with

KMP_THREAD_AFFINITY set to scatter, compact and balanced 104

Figure 4.63 Parallel speedup of Ladder215C(16) model on an Intel Xeon Phi with

KMP_THREAD_AFFINITY set to scatter, compact and balanced 104

Figure 4.64 Parallel speedup of Ladder215S(24) model on an Intel Xeon Phi with

KMP_THREAD_AFFINITY set to scatter, compact and balanced 108

Figure 4.65 Parallel speedup of Ladder215S(22) model on an Intel Xeon Phi with

KMP_THREAD_AFFINITY set to scatter, compact and balanced 108

Figure 4.66 Parallel speedup of Ladder215S(20) model on an Intel Xeon Phi with

KMP_THREAD_AFFINITY set to scatter, compact and balanced

Figure 4.67 Parallel speedup of Ladder215S(18) model on an Intel Xeon Phi with

KMP_THREAD_AFFINITY set to scatter, compact and balanced

Figure 4.68 Parallel speedup of Ladder215S(16) model on an Intel Xeon Phi with

KMP_THREAD_AFFINITY set to scatter, compact and balanced 110

Figure 4.69 Parallel speedup of Ladder315C(24) model on an Intel Xeon Phi with

KMP_THREAD_AFFINITY set to scatter, compact and balanced

Figure 4.70 Parallel speedup of Ladder315C(18) model on an Intel Xeon Phi with

KMP_THREAD_AFFINITY set to scatter, compact and balanced

Figure 4.71 Parallel speedup of Ladder315S(24) model on an Intel Xeon Phi with

KMP_THREAD_AFFINITY set to scatter, compact and balanced 
Figure 4.72 Parallel speedup of Ladder315S(24) model on an Intel Xeon Phi with

KMP_THREAD_AFFINITY set to scatter, compact and balanced

Figure 4.73 Parallel speedup of Ladder215C $(N)$ with $N=16,18,20,22,24$ and 26 on an Intel Xeon Phi

coprocessor with KMP_THREAD_AFFINITY is Scatter...

Figure 4.74 Parallel speedup of Ladder215C $(N)$ with $N=16,18,20,22,24$ and 26 on an Intel Xeon Phi

coprocessor with KMP_THREAD_AFFINITY is compact

Figure 4.75 Parallel speedup of Ladder215C $(N)$ with $N=16,18,20,22,24$ and 26 on an Intel Xeon Phi

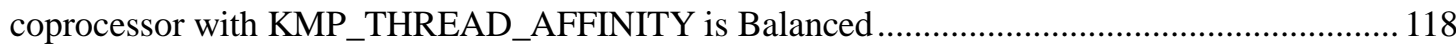

Figure 4.76 Comparison of time between Odin and Xeon Phi on Ladder215C(24) ...................................... 134

xxiv 


\section{Introduction}

The invention of electronic computers has revolutionized all areas of sciences. The application of computational techniques has opened the door to new approaches and made it possible to study systems of unprecedented complexity. Many important discoveries in biology, chemistry, physics, materials science, etc. have been made with the help of modern computers. This development is ongoing and the invention of new computing technology and better computational techniques will fuel scientific discoveries for the foreseeable future.

One area of science that has immensely benefited from computational techniques is quantum mechanics. Very few quantum problems can be solved exactly with analytical methods. Other models can only be studied with the help of approximations or with computational techniques.

Quantum spin models are a large class of quantum models that play an important role in theoretical condensed matter physics and quantum information theory. These models can be used to study certain magnetic materials as well as general properties of quantum systems. Improved computational techniques might make it possible to study larger systems or systems that currently cannot be studied computationally and could lead to new insights in this fascinating branch of physics.

One computational technique that is often used to study quantum spin models is called exact diagonalization. In this approach, numerical methods are used to determine the 
lowest eigenvalues and associated eigenvectors of the Hamilton matrix describing the model Ref. [30]. What makes this approach computationally very challenging is the fact that the Hamilton matrix of quantum spin models growth exponentially with the size of the system. The main computational problem is thus to find a few of the lowest eigenvalues and associated eigenvectors (eigenpairs). Eigenpairs rather than eigenvalues are required in many cases since they allow the calculation of a wider spectrum of physical properties.

The exact diagonalization method has been used to study the properties of a variety of different quantum spin models. Examples of studies of one-dimensional models can be found in Ref. [11][14][18][21][24][25]. Two-dimensional quantum models have also been studied with this method (see e.g. Ref. [1][2][13][15]).

In earlier studies (e.g. Ref. [11][14]) the size of the systems that could be studied was extremely low (less than 20 spins) since technical limitations prohibited the studying of systems with larger Hamilton matrices. More recently, system with 28 - 40 spins have been studied Ref. [1][2][13][15].

Many exact diagonalization studies of quantum spin systems use the Lanczos method Ref. [10] for the determination of the lowest eigenvalues and eigenvectors. Many other methods for this problem can however be found in the literature (see e.g. Ref. [10]). Some of these methods might be advantageous, in particular if one needs to determine not only the lowest eigenvalue but several of the low-lying eigenpairs.

The first goal of this work is to test whether the Block-Davidson algorithm Ref. [6] and the Residual Minimization — Direct Inversion of the Iterative Subspace (RMM-DIIS) 
method Ref. [23] can be employed successfully in exact diagonalization studies of quantum spin systems. Both methods have been used successfully in electronic structure calculations, which require the determination of the lowest eigenvalues of large matrices. The Block-Davidson method and the RMM-DIIS algorithm are both implemented in the well-known VASP code Ref. [16].

The Block-Davidson algorithm Ref. [6] is closely related to the Lanczos method but it usually requires less iterations. Conceptually, both of these methods search for the eigenvector with the lowest eigenvalue. If several of the lowest eigenvalues are sought, the trial states must be reorthogonalized at every step. Otherwise, the different trial vectors may converge towards each other. For large matrices, this reorthogonalization procedure is computationally rather intensive and may dominate the total computing time.

The RMM-DIIS method does not attempt to minimize the eigenvalue but the norm of the residual vector. For this reason, trial vectors will not converge towards each other if the initial states are sufficiently separated in the state space. The RMM-DIIS method does not require a reorthogonalization at every step. Even if this method requires more iterations, this method may take substantially less computing time Ref. [16]. For quantum spin systems this effect may even be larger than for electronic structure calculations due to the larger matrix sizes in this case.

In order to test the Block-Davidson method and the RMM-DIIS algorithm in the context of quantum spin systems, computer programs for these two methods have been developed and applied to a number of spin-1/2 Heisenberg systems. The aim of these calculations was to find whether these methods do work properly for such typical 
problems and to assess their potential. The goal was not immediately to perform calculations of larger systems than was possible previously.

As pointed out above, scientific progress is fueled by advances in computational methods as well as in computing technology. In recent times, increases in computing power were mainly achieved through the introduction of multi-core processors that integrate several processor cores on a single chip. In order to take advantage of modern computers, programs must be able to run efficiently in parallel on such processors. This can be problematic if a problem uses large amounts of memory like it is the case for the quantum spin systems studied in this thesis. In such cases, the computing time may be limited by memory access speed and the usage of more CPU core might not result in substantial time savings.

The next generation of processors for computing problems will be so-called many-core processors that integrate hundreds or even thousands of CPU cores on a single chip. An early example of this forthcoming technology is the Intel Xeon Phi coprocessor Ref. [3]. Current Xeon Phi processors provide $60 \mathrm{CPU}$ cores with 4 hardware threads per core and a high memory band width on a single chip. The second goal of this thesis is to test whether this new architecture provides a suitable basis for exact diagonalization studies of quantum spin systems. To this end, the program for RMM-DIIS method has been employed on an Xeon Phi coprocessor and the achieved parallel speedups were compared with those obtained on a conventional multi-core system. 


\section{Backgrounds}

\subsection{Quantum Spin Systems}

\subsubsection{Introduction}

The study of quantum spin systems or quantum spin models is an important branch of theoretical physics. The origins of these systems lies in the field of magnetism. The first quantum spin systems where invented as models to study the properties of insulating magnetic materials Ref. [22].

Today, quantum spin models are still used to study the behavior of magnetic materials. (see e.g Ref. [1][2][13][11][15][14][18][21][24][25])In addition to this, they are also used as basic models to understand fundamental phenomena of quantum mechanics, like quantum phase transitions. Finally, quantum spin systems play a role in the field of quantum computing since interacting spin systems can be used to realize quantum computing devices.

In physics, the spin is an intrinsic property of elementary particles, for example electrons. The spin of a particle behaves like a tiny angular momentum and it has an associated magnetic moment. An important property of spins is that it is quantized. Each spin has an associated quantum number $S$. which is a positive integer or half-integer number. A spin can only be in one of $2 S+1$ states that are often characterized by $S^{z}=$ $-S,-S+1, \ldots, S-1, S$. An important case is $S=1 / 2$ when the spin can only take one 
of two states: $S^{z}=+1 / 2,-1 / 2$.

A quantum spin system is a system that contains an ensemble of $N$ spins that interact with each other through some kind of interaction. An example for a class of quantum spin models that I use in this work is the antiferromagnetic Heisenberg model with spin $S=1 / 2$. There are however many more types of quantum spin models.

In the Heisenberg model, the spins of the system are immobile and they interact through an exchange interaction whose strength is determined by an interaction constant $J$. In principle each spin in a Heisenberg model might interact with all other spins. In practice, however, one often uses the so-called nearest neighbor approximation. This approximation restricts the interaction so that each spin interacts only with its closest neighbours. If the interaction between two spins is not zero, one says that there is a bond between the two spins.

\subsubsection{Numerical Methods for Quantum Models}

Theoretical physicists use many different methods to study quantum spin systems. In some cases, it is possible to obtain exact information about the properties of a model through analytical calculations. If this is not possible, approximative analytical methods or numerical methods can be employed.

Three important numerical techniques for the studying of quantum spin models are Density Matrix Renormalization Group Ref. [27], Quantum Monte Carlo Simulation (see, e.g., Ref. [9]), and Exact Diagonalization (see, e.g., Ref. [30]). This work focuses 
on the latter method which is described in the following paragraphs.

In quantum mechanics, a system is characterized by a linear operator (Hamilton operator) which acts on elements of a Hilbert space. The elements of the Hilbert space correspond to the possible states of the system. For a spin system with a finite number of spins $N$, the Hilbert space becomes a finite-dimensional vector space and the Hamilton operator a quadratic matrix.

The exact diagonalization method uses numerical techniques to find the eigenvalues and eigenvectors of the Hamilton matrix of a quantum system. The reason for this is that the physical properties of the model described by the Hamilton matrix can be understood from the eigenvalues and eigenvectors of the matrix. For many applications a sufficient understanding of the systems can be obtained from the lowest eigenvalues and their eigenvectors.

While conceptually easy to understand, the practical use of the exact diagonalization method faces enormous technical problems. The primary reason for these difficulties is the large size of the Hamilton matrices even for relatively small systems. For a spin $-1 / 2$ Heisenberg model, i.e. a Heisenberg model with $S=1 / 2$, with $N$ spins, the dimension of the state vector space is $2^{N}$. This means that even for a modest system with 20 spins the vector space is of dimension 1,048,576 and the Hamilton matrix has more than a trillion elements.

A numerical treatment of the Hamilton matrices of quantum spin systems is only possible since only a small fraction of the matrix elements of these matrices are nonzero. It is therefore possible to use so-called sparse-matrix techniques that store only the 
non-zero elements of the matrix.

A spin- $1 / 2$ Heisenberg model is determined by the number of bonds. Each bond, i.e. each pair of spins with a non-zero interaction constant $J$, adds $2^{N-1}$ off-diagonal elements to the matrix. In addition to this, most of the diagonal elements are non-zero. If one neglects the small number of diagonal elements that might be zero, the fraction of matrix elements that are non-zero in a spin-1/2 Heisenberg model with $b$ bonds is given by $(b+2) 2^{-(N+1)}$.

The size of eigenvalue problem to be handled in the exact diagonalization method can be further reduced if one makes use of the symmetries of the problem. Heisenberg models contain a number of symmetries that can be used to block diagonalize the Hamilton matrix. Each of the blocks can be treated separately which leads to a substantial reduction of the computational effort.

In this work the only symmetry that is used to reduce the problem size is the conservation of the total spin. This symmetry is related to the fact that when the Hamilton matrix is applied, the number of spins $N_{+}$that are in the $+1 / 2$ state and the number of spins $N_{\text {- that }}$ are in the $-1 / 2$ state does not change. This symmetry makes it possible to classify the eigenstates of the Hamilton matrix by the difference $M=N_{+}-$ $N_{-}$. In this work, only the subspace of states with $M=0$ has been considered. The dimension of this this subspace is given by equation $\left(\frac{N !}{(N / 2) !(N / 2) !}\right)$ 


\subsubsection{Quantum Spin Systems Considered in this Work}

Three different types of antiferromagnetic Heisenberg models with $S=1 / 2$ are used in this work. The term antiferromagnetic means that the interaction constant between two spins favors the antiparallel alignment: one spin is in the $+1 / 2$ state while the other is in the $-1 / 2$ state. The four models are called the Heisenberg chain, the Heisenberg ladder and the dimerized Heisenberg ladder.

The Heisenberg chain is a simple one-dimensional arrangement of the spins. Each spin interacts only with its immediate neighbours to the left and right.

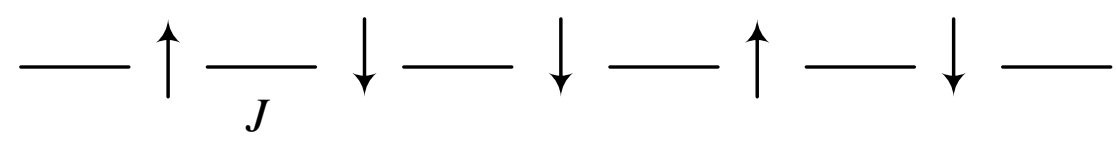

Figure 2.1 Heisenberg Chain model

Example for exact diagonalization studies of spin chains can be found in Ref. [11][14]. In this work, periodic boundary conditions are applied to all models. This means that the spin at the left end of the chain interacts with the spin at the right end of the chain so that the chain effectively has no end. All bonds in the chain use the same interaction strength $J=1$.

The Heisenberg ladder is a generalization of the Heisenberg chain [2]. A $k$-leg ladder consists of $k$ parallel Heisenberg chains. These chains are called the legs of the ladder. In addition to the interaction along the legs, each spin interacts with its neighbours in the adjacent chains. 
As in the case of the chain, periodic boundary conditions are applied. The Heisenberg ladder, is characterized by two interaction constants. Interactions among neighbours along the legs use $J_{\|}$whereas the bonds along the rungs of the ladder use $J_{\perp}$. In this work $J_{\|}=1$ and $J_{\perp}=\kappa$ is used.

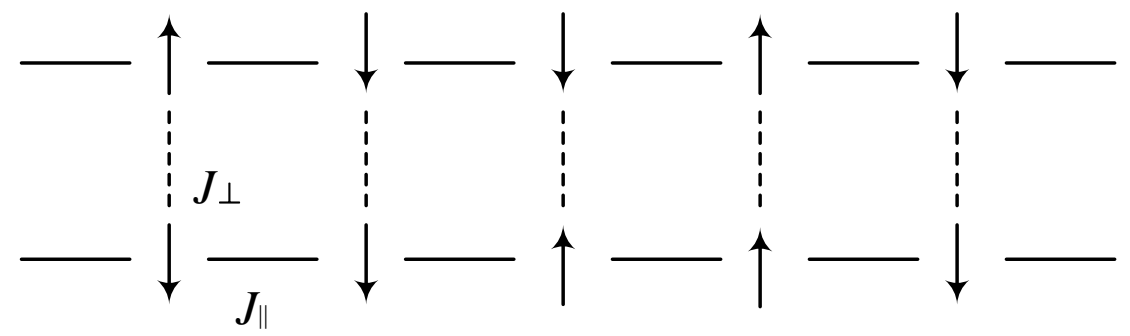

Figure 2.2 Heisenberg Ladder model

The dimerized Heisenberg ladder model Ref.[19][29] uses the same geometric arrangement of the spins as the ladder model. The difference is that the interaction strength of the bonds along the ladder legs is modulated. Two different values of the interaction constant are used in an alternating pattern. The two values used in this work are $J_{\|, 1}=3 / 2$ and $J_{\|, 2}=1 / 2$.

The modulation of the bond strength along the ladder legs allows for two different subtypes of the dimerized ladder. In the columnar model, the strength of the corresponding bonds in all legs is the same. In the staggered model, the pattern is shifted by one bond between the legs. 


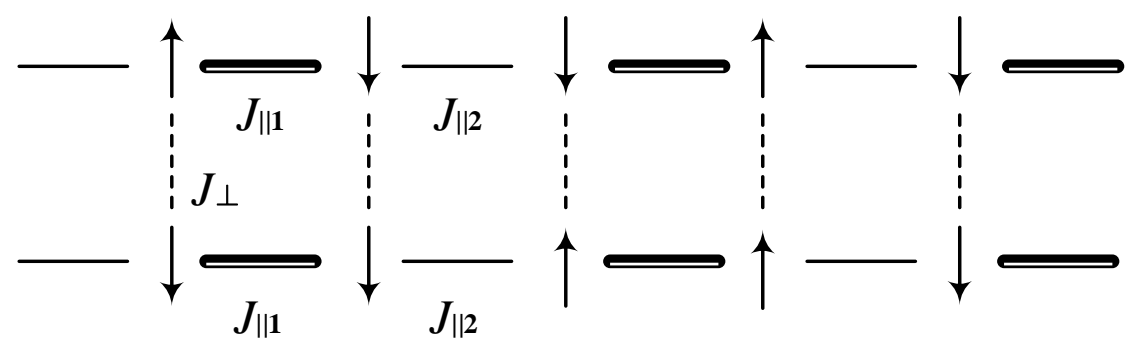

Figure 2.3 Dimerized Heisenberg ladder columnar model

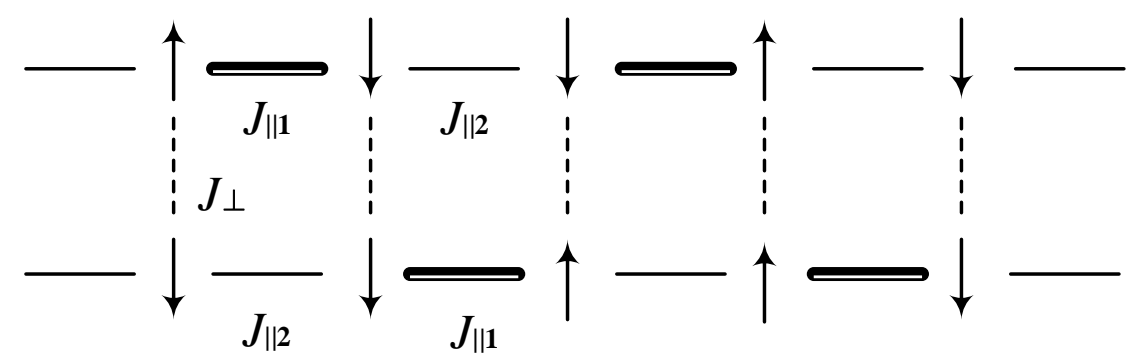

Figure 2.4 Dimerized Heisenberg ladder staggered model 


\subsection{Sparse matrix storage}

\subsubsection{Block-diagonalization}

Fortunately, however, it is possible to reduce the size of the matrices. The Heisenberg models possess a number of symmetries that can be exploited to perform a block diagonalization of the Hamilton matrix. The block diagonalization divides the Hilbert space into a number of subspaces that do not interact with each other. It is then possible to diagonalize each of these subspaces independently from the others. This reduces the size of the computational problem effectively to the size of the largest subspace.

Depending on the details of the Heisenberg model, multiple symmetries can be exploited to reduce the system size. Some of these symmetries are more complicated than others. For simplicity, in this work only the conservation of the $z$-component of the total spin $S^{z}$ has been exploited. After the block diagonalization under this symmetry, the dimension of the largest subspace $\left(S^{z}=0\right)$ is given by $\frac{N !}{(N / 2) !(N / 2) !}$. The calculations in this work have always been done in the subspace $S^{z}=0$.

In Table 2.1 the full systems size and the size of the $S^{z}=0$ subspace are compared. The third column (percentage) shows the fraction of the reduced system size compared to the total size. For example, in the case of $N=26$, the dimension of the $S^{z}=0$ subspace is less than $16 \%$ of the dimension of the full Hamilton matrix. 
Table 2.1 Full size dimension and reduced dimension in different size

\begin{tabular}{|r|r|r|r|}
\hline \multicolumn{1}{r|}{$N$} & Full size dimension $=N^{2}$ & Reduced dimension $=\frac{N !}{(N / 2) !(N / 2) !}$ & Percentage \\
\hline 2 & 4 & 2 & $50.0000 \%$ \\
\hline 4 & 16 & 6 & $37.5000 \%$ \\
\hline 6 & 64 & 20 & $31.2500 \%$ \\
\hline 8 & 256 & 70 & $27.3438 \%$ \\
\hline 10 & 1024 & 252 & $24.6094 \%$ \\
\hline 12 & 4096 & 924 & $22.5586 \%$ \\
\hline 14 & 16384 & 3432 & $20.9473 \%$ \\
\hline 16 & 65536 & 12870 & $19.6381 \%$ \\
\hline 18 & 262144 & 48620 & $18.5471 \%$ \\
\hline 20 & 1048576 & 184756 & $17.6197 \%$ \\
\hline 22 & 4194304 & 705432 & $16.8188 \%$ \\
\hline 24 & 16777216 & 2704156 & $16.1180 \%$ \\
\hline 26 & 67108864 & 10400600 & $15.4981 \%$ \\
\hline
\end{tabular}




\subsubsection{Sparse matrix storage (CSR)}

The huge size of the Hamilton matrices describing quantum spin systems makes it impossible to directly store the complete matrices except for very small system sizes $N$. Fortunately, these matrices are very sparse, i.e. the vast majority of elements is zero.

For very sparse matrices, different storage techniques can be used that store only the non-zero elements of the matrix. These techniques require that in addition to the value of the matrix elements, the position of the element in the matrix (row and column index) is stored.

In this work, I use the Compressed Sparse Row (CSR) format Ref. [26]. This is a common scheme that is supported by many libraries. The CSR format stores all elements that belong to the same row in subsequent addresses. This makes is unnecessary to store the row index for each individual element.

The CSR format uses three arrays: value, column and row. The value array stores the values of all non-zero elements of the matrix. The column array stores for each elements its column index. Column and value have clearly the same size. Finally, the row array stores for each row the index of its first non-zero element in the column and value arrays. This array needs only one entry per row. 


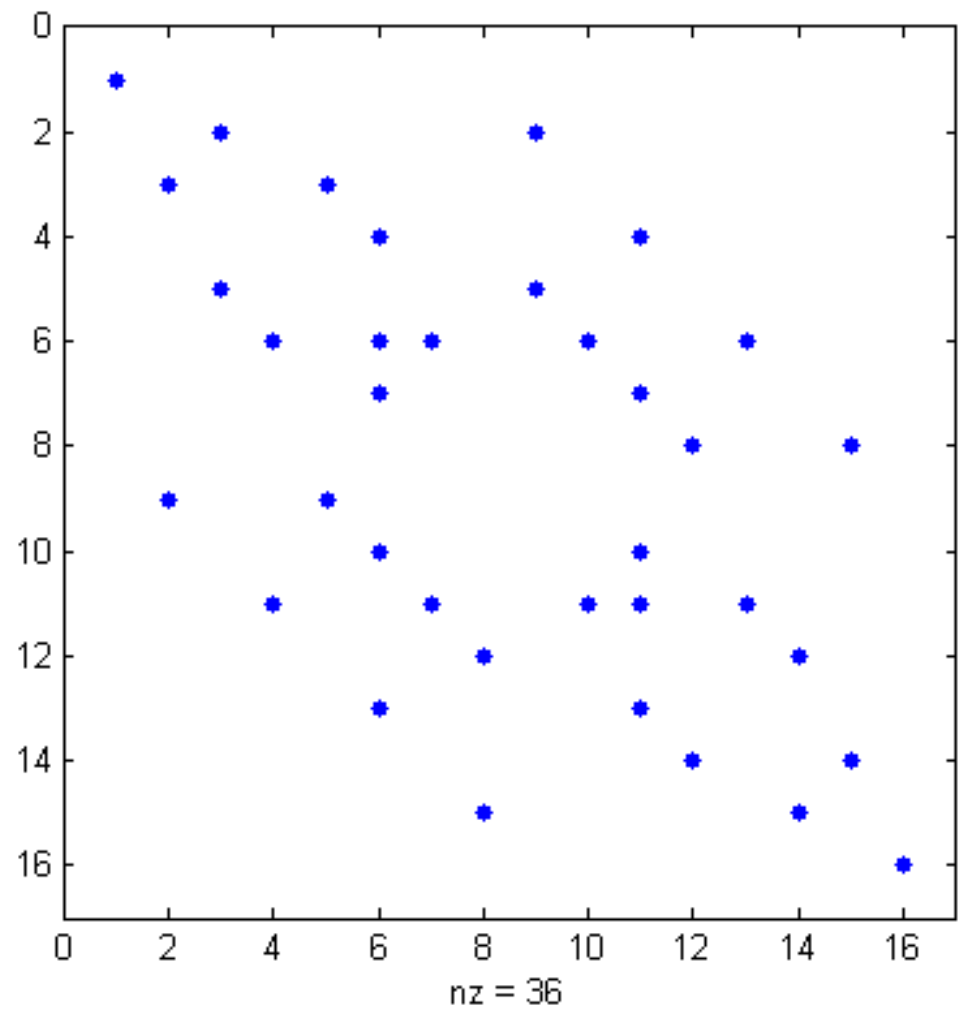

Figure 2.5 Visualize sparsity pattern of Chain(4) model

For example, Chain(4) is a $16 \times 16$ matrix, the expression is shown in Eq.(2.1). Figure 2.5 shows how sparse the matrix it is. There are 36 non-zero elements in Chain (4) model, if use full size, it needs store $16 \times 16=196$ values and their positions. According to Table 2.2 , it needs $36 \times 2+16=88$ values (include indices) to store Chain(4) model, save rate $=88 /(196 \times 3) \times 100 \% \approx 14.97 \%$. 
Chain $(4)=$

$\left(\begin{array}{llllllllllllllll}1 & 0 & 0 & 0 & 0 & 0 & 0 & 0 & 0 & 0 & 0 & 0 & 0 & 0 & 0 & 0 \\ 0 & 0 & 0.5 & 0 & 0 & 0 & 0 & 0 & 0.5 & 0 & 0 & 0 & 0 & 0 & 0 & 0 \\ 0 & 0.5 & 0 & 0 & 0.5 & 0 & 0 & 0 & 0 & 0 & 0 & 0 & 0 & 0 & 0 & 0 \\ 0 & 0 & 0 & 0 & 0 & 0.5 & 0 & 0 & 0 & 0 & 0.5 & 0 & 0 & 0 & 0 & 0 \\ 0 & 0 & 0.5 & 0 & 0 & 0 & 0 & 0 & 0.5 & 0 & 0 & 0 & 0 & 0 & 0 & 0 \\ 0 & 0 & 0 & 0.5 & 0 & -1 & 0.5 & 0 & 0 & 0.5 & 0 & 0 & 0.5 & 0 & 0 & 0 \\ 0 & 0 & 0 & 0 & 0 & 0.5 & 0 & 0 & 0 & 0 & 0.5 & 0 & 0 & 0 & 0 & 0 \\ 0 & 0 & 0 & 0 & 0 & 0 & 0 & 0 & 0 & 0 & 0 & 0.5 & 0 & 0 & 0.5 & 0 \\ 0 & 0.5 & 0 & 0 & 0.5 & 0 & 0 & 0 & 0 & 0 & 0 & 0 & 0 & 0 & 0 & 0 \\ 0 & 0 & 0 & 0 & 0 & 0.5 & 0 & 0 & 0 & 0 & 0.5 & 0 & 0 & 0 & 0 & 0 \\ 0 & 0 & 0 & 0.5 & 0 & 0 & 0.5 & 0 & 0 & 0.5 & -1 & 0 & 0.5 & 0 & 0 & 0 \\ 0 & 0 & 0 & 0 & 0 & 0 & 0 & 0.5 & 0 & 0 & 0 & 0 & 0 & 0.5 & 0 & 0 \\ 0 & 0 & 0 & 0 & 0 & 0.5 & 0 & 0 & 0 & 0 & 0.5 & 0 & 0 & 0 & 0 & 0 \\ 0 & 0 & 0 & 0 & 0 & 0 & 0 & 0 & 0 & 0 & 0 & 0.5 & 0 & 0 & 0.5 & 0 \\ 0 & 0 & 0 & 0 & 0 & 0 & 0 & 0.5 & 0 & 0 & 0 & 0 & 0 & 0.5 & 0 & 0 \\ 0 & 0 & 0 & 0 & 0 & 0 & 0 & 0 & 0 & 0 & 0 & 0 & 0 & 0 & 0 & 1\end{array}\right)$

Table 2.2 CSR format of chain (4) model

\begin{tabular}{|l|r|r|r|r|r|r|r|r|r|}
\hline Value & 1 & 0.5 & 0.5 & 0.5 & 0.5 & 0.5 & 0.5 & 0.5 & 0.5 \\
\hline Row & 1 & 2 & & 4 & & 6 & & 8 & \\
\hline Column & 1 & 3 & 9 & 2 & 5 & 6 & 11 & 3 & 9 \\
\hline \hline Value & 0.5 & -1 & 0.5 & 0.5 & 0.5 & 0.5 & 0.5 & 0.5 & 0.5 \\
\hline Row & 10 & & & & & 15 & & 17 & \\
\hline Column & 4 & 6 & 7 & 10 & 13 & 6 & 11 & 12 & 15 \\
\hline \hline Value & 0.5 & 0.5 & 0.5 & 0.5 & 0.5 & 0.5 & 0.5 & -1 & 0.5 \\
\hline Row & 19 & & 21 & & 23 & & & & \\
\hline Column & 2 & 5 & 6 & 11 & 4 & 7 & 10 & 11 & 13 \\
\hline \hline Value & 0.5 & 0.5 & 0.5 & 0.5 & 0.5 & 0.5 & 0.5 & 0.5 & 1 \\
\hline Row & 28 & & 30 & & 32 & & 34 & & 36 \\
\hline Column & 8 & 14 & 6 & 11 & 12 & 15 & 8 & 14 & 16 \\
\hline
\end{tabular}


Table 2.3 Number of non-zero elements with different sizes in Chain, Ladder2, Ladder215C, Ladder 215S, Ladder315C and Ladder315S when $M=0$

\begin{tabular}{|c|c|c|c|}
\hline $\operatorname{Model}(N)$ & $M=0$ & & \\
\hline $\operatorname{size} N$ & Chain & $\begin{array}{l}\text { Two-leg Ladder: } \\
\text { Ladder2 } \\
\text { Ladder } 215 \mathrm{C} \\
\text { Ladder } 215 \mathrm{~S}\end{array}$ & $\begin{array}{l}\text { Three-leg Ladder: } \\
\text { Ladder315C } \\
\text { Ladder315S }\end{array}$ \\
\hline 2 & 4 & 4 & - \\
\hline 4 & 22 & 22 & - \\
\hline 6 & 92 & 128 & 104 \\
\hline 8 & 390 & 550 & - \\
\hline 10 & 1652 & 2352 & - \\
\hline 12 & 6972 & 9996 & 11004 \\
\hline 14 & 29304 & 42240 & - \\
\hline 16 & 122694 & 177606 & - \\
\hline 18 & 511940 & 743600 & 820820 \\
\hline 20 & 2129556 & 3101956 & - \\
\hline 22 & 8834696 & 12899328 & - \\
\hline 24 & 36564892 & 53495260 & 59138716 \\
\hline 26 & 151016712 & 221324768 & - \\
\hline
\end{tabular}

Table 2.3 shows how many non-zero elements in different models with different size when $M=0$. Number of non-zero elements of Chain model is the least, two-leg Heisenberg Ladder (Ladder2, Ladder215C and Ladder215S) models have same Number of non-zero elements, and three-leg Heisenberg (Ladder315C and Ladder315S) models have the most Number of non-zero elements in these six models.

Form Figure 2.6, we can get there are 212695496 non-zero elements in this model, $\frac{\text { number of non-zero elements }}{\text { number of all elements }}=\frac{212695496}{2^{24} \times 2^{24}} \approx 7.6 \times 10^{-7}$, this ratio shows how sparse the Chain(24) model is, hence using Compressed Sparse Row (CSR) format to compress matrix and store it that is necessary, because CSR can save so much storage. 


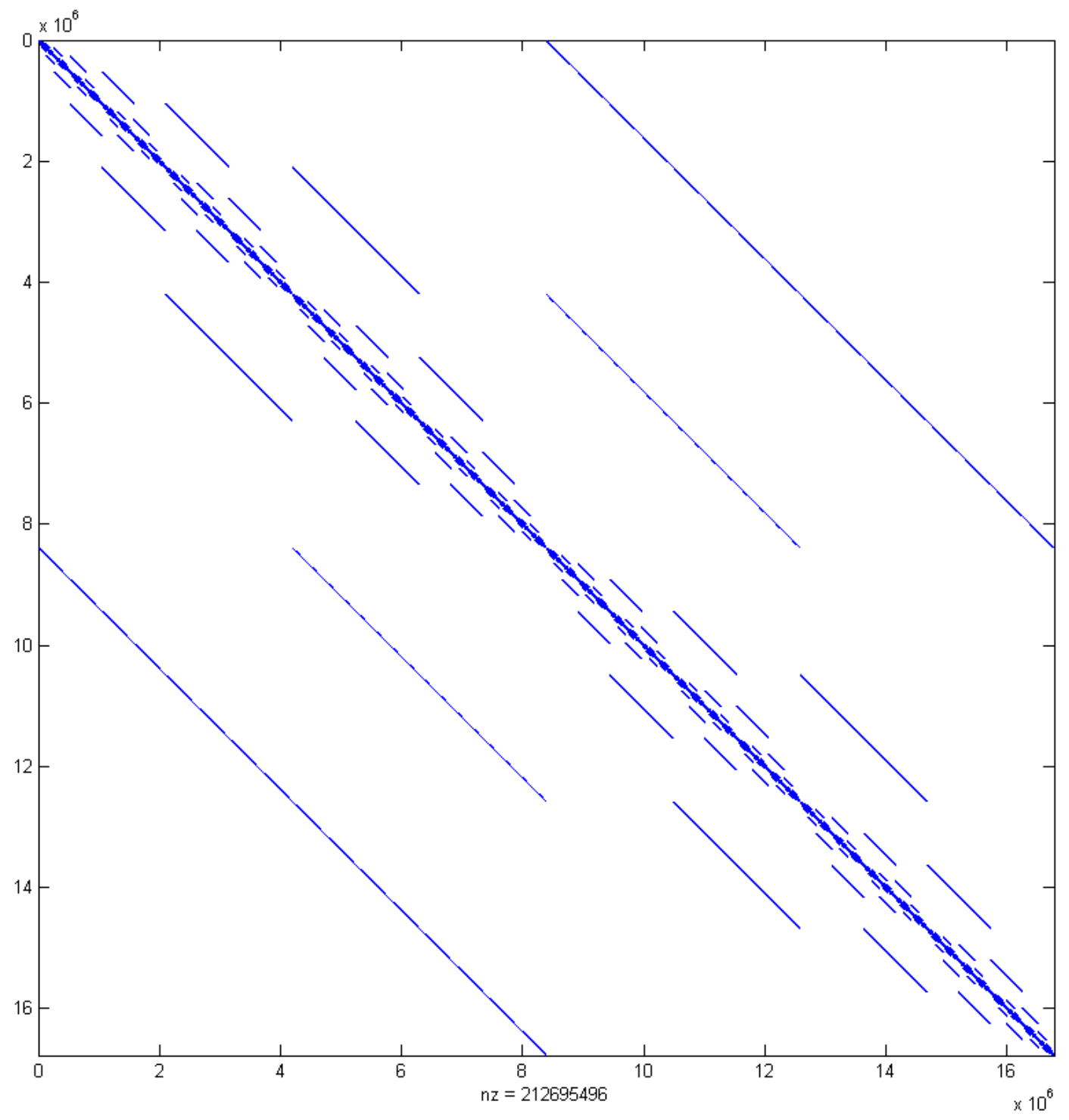

Figure 2.6 Visualize sparsity pattern of Chain(24) model 


\subsection{Lanczos method}

Lanczos developed the Lanczos theory in the 1950s Ref. [10], it is suitable for solving the eigenvalues problem of large symmetrical sparse matrices.

The Lanczos algorithm is an iterative method which transforms the matrix A into a series of symmetric (Hermitian) tridiagonal matrices

$$
T^{k}=\left[\begin{array}{lllll}
\alpha_{1} & \beta_{1} & 0 & \cdots & 0 \\
\beta_{1} & \alpha_{2} & \ddots & \ddots & \vdots \\
0 & \ddots & \ddots & \ddots & 0 \\
\vdots & \ddots & \ddots & \ddots & \beta_{k-1} \\
0 & \cdots & 0 & \beta_{k-1} & \alpha_{k}
\end{array}\right]
$$

with the help of the orthogonal transformations

$$
T^{k}=Q^{k^{T}} A Q^{k}
$$

where $=Q^{k}=\left[q_{1}, q_{2}, \ldots q_{k}\right]$ is an orthogonal matrix. As $k$ increases, the eigenvalues of $T^{k}$ approximate the eigenvalues of $A$. In the limiting case $k=N, T^{N}$ is similar to $A$ so that the eigenvalue spectrums of the two matrices are identical (apart from numerical errors).

The algorithm starts with a randomly chosen normalized vector $q_{1}$. At the $k^{\text {th }}$ iteration, $q_{k+1}$ is found by applying the matrix $A$ to the vector $q_{k}$ and orthogonalization of the result with respect to the previous vectors. This orthogonalization procedure would normally be a computationally expensive procedure and it would require the storage of all previous vectors. The reason for the success of 
the Lanczos method is, however, that it can be shown that for symmetric (Hermitian) matrices it is sufficient to orthogonalize with respect to the last two vectors. An effective method to do this is shown in Algorithm 1 Ref. [10]. It should be mentioned however, that numerical errors lead to a degradation of the orthogonality of the vectors.

In practice, the number of iterations performed is much smaller than the dimension of the matrix A. When the algorithm stops, at iteration $k$ the lowest eigenvalues of the matrix $T^{k+1}$ are used as approximations for the lowest eigenvalues of $A$.

Since the vectors $q_{k}$ are obtained by successive application of the matrix $A$ and subsequent orthogonalization, the space spanned by the vectors $\left[q_{1}, q_{2}, \ldots q_{k}\right]$ is the Krylov subspace

$$
K\left(A, q_{1}, k\right)=\operatorname{span}\left\{q_{1}, A q_{1}, A^{2} q_{1}, \ldots, A^{k-1} q_{1}\right\}
$$

The eigenvalues found by the Lanczos algorithm are therefore the lowest eigenvalues of the matrix A projected into the subspace $K\left(A, q_{1}, k\right)$. 


\section{Algorithm 1 Lanczos method}

Input: matrix $A$, where $A$ is a $n \times n$ symmetrical sparse matrix. vector $w$, where $w$ has $n$ elements with rand $[-1,1]$.

Output: The lowest $k$ eigenvalues $\lambda_{m}$ (for $m=1: k$ )

1. $v_{1: n}=0 ; \beta_{1}=1 ; k=1 ; \mid w>=\frac{\mid w>}{\|\mid w>\|_{2}}$

2. while $\beta_{k} \neq 1$

3. if $\quad k \neq 1$

4. for $i=1: n$

5. $\quad t=w_{i}$;

6.

$$
w_{i}=\frac{v_{i}}{\beta_{k}}
$$

7.

$$
v_{i}=-\beta_{k} t ;
$$

8. end for

9. end if

10.

$|v>=| v>+A \mid w>$

11. $k=k+1$;

12.

$$
\alpha_{k}=\langle w| v>\text {; }
$$

13.

$|v>=| v>-\alpha_{k} \mid w>$;

14.

$$
\beta_{k}=\|\mid v>\|_{2}
$$

15. end while

16. Make $T=\left[\begin{array}{lllll}\alpha_{1} & \beta_{1} & 0 & \cdots & 0 \\ \beta_{1} & \alpha_{2} & \ddots & \ddots & \vdots \\ 0 & \ddots & \ddots & \ddots & 0 \\ \vdots & \ddots & \ddots & \ddots & \beta_{k-1} \\ 0 & \cdots & 0 & \beta_{k-1} & \alpha_{k}\end{array}\right]$

17. Compute eigenvalues of $T$; 


\subsection{Davidson and Block Davidson method}

The Davidson method is closely related to the Lanczos algorithm. Like the latter method, they are iterative methods that project the matrix A onto a sequence of subspaces of increasing dimensions Ref. [23]. The Davidson method is particularly suited for diagonally dominant matrices. Contrary to the Lanczos algorithm, the Davidson method requires at each step an explicit orthogonalization of the new vector with respect to the previous iterative subspace.

The Block Davidson method is a generalization of the Davidson method that allows the simultaneous search for the $l$ lowest eigenpairs.

\subsubsection{Davidson method}

Davidson originally published his method in 1975 as a method to find the lowest eigenstates in quantum chemistry problems Ref. [8]. Later, the method has been improved by Liu Ref. [17] and Murray et al. Ref. [20].

At each iteration, the Davidson method performs a Rayleigh-Ritz step that determines the lowest eigenpair of the projected matrix in the current iterative subspace (Step 3-7 in Algorithm 2). The algorithm than calculates the residual vector $\left|r^{k}\right\rangle=$ $\left(A-\lambda^{k} I\right) \mid z>$ for this eigenpair. If the norm of this vector is below the given convergence criterion "epsilon", the algorithm stops. 
If the residual vector is above the convergence criterion, a pre-conditioner $C^{k}$ is applied to the residual vector. The resulting vector is than orthogonalized with respect to all previous vectors and added to the iteration space $V^{k+1}$. It is the preconditioning step which separates the Davidson method from the Lanczos algorithm Ref.[7]. In this work, I use the diagonal pre-conditioner proposed in Ref. [8] (step 11 in Algorithm 2). Other pre-conditioners could be used here and might give better results. The application of the pre-conditioner destroys the orthogonality properties of Lanczos algorithm. This is the reason at each step the new search vector must be orthogonalized with respect to the complete search space. 


\section{Algorithm 2 Davidson method}

Input: matrix $A$, where $A$ is a $n \times n$ symmetrical sparse matrix. matrix $D$, where $D$ is main diagonal of $A$, same size as $A$. integer $m$, where $m$ is maximum number of iteration. vector $\left|v^{1}\right\rangle$, an initial eigenvector with rand $[-1,1]$. tolerance $\varepsilon$, where is a threshold, if $\|\mid r>\|_{2}<\varepsilon$ then break.

Output: The lowest eigenpair $\lambda^{k}, \mid a^{k}>$

1. Pick up an initial unit vector $\mid v^{1}>$

2. $\left|v^{1}>=\frac{\mid v^{1}>}{\left\|\mid v^{1}>\right\|_{2}} ; V^{1}=\right| v^{1}>$;

3. for $k=1: m$

4. Compute the Rayleigh matrix $H^{k}=V^{k^{T}} A V^{k}$;

5. Compute the lowest eigenpairs $\left(\lambda^{k}, \mid a^{k}>\right)$ of $H^{k}$;

6. Compute the Ritz vector $|z\rangle=V^{k} \mid a^{k}>$;

7. $\quad$ Compute the residual vector $\left|r^{k}>=\left(A-\lambda^{k} I\right)\right| z>$;

8. if $\left\|\mid r^{k}>\right\|_{2}<\varepsilon$

9. convergence $=1$ and exit

10. end if

11. Compute $C^{k}=\left(D-\lambda^{k} I\right)^{-1}$;

12. Compute the new directions $\left|t^{k}\right\rangle=C^{k}\left|r^{k}\right\rangle$

13. $V^{k+1}=\operatorname{MGS}\left(\left[V^{k}, \mid t^{k}>\right]\right)$

14. end for

15. Set $\left|v^{1}>=\right| z>$, go to 2 


\subsubsection{Block Davidson}

The Block Davidson method is a generalized formulation of Davidson's method. This version of the algorithm attempts to determine simultaneously the lowest $l$ eigenpairs of $A$.

In this work, I follow the formulation of the Block Davidson algorithm described by Crouzeix et al. in Ref. [6] (see algorithm 3). The principle difference between algorithm 2 and algorithm 3 is that the blocked version calculates in each iteration the lowest $l$ eigenpairs and residual vectors of the matrix projected onto the search space. After the convergence check, if the norm of residual vector is below the given convergence criterion $\varepsilon$, the algorithm stops, else all $l$ residual vectors are preconditioned and added to the subspace.

Another difference to the original Davidson method is a limitation of the dimension of the subspace. Since it is too costly to store all previous vectors, the algorithm enforces a maximum dimension $b$ for the iterative subspace. If adding of the new vectors exceeds this size, the algorithm restarts with the lowest $l$ Ritz vectors and the preconditioned residual vectors. 


\section{Algorithm 3 Block Davidson}

Input: matrix $A$, where $A$ is a $n \times n$ symmetrical sparse matrix. matrix $D$, where $D$ is main diagonal of $A$, same size as $A$. integer $l$, where $l$ is number of the lowest eigenpairs need to be calculated.

matrix $V^{1}$, where $V^{1}$ is a random $n \times l$ matrix, all elements of $V^{1}$ within range [-1,1], make $V^{1}=M G S\left(V^{1}\right)$ as initial eigenvectors of $A$. integer $m$, where $m$ is maximum number of iteration. integer $b$, which $b$ limits the dimension of the basis. tolerance $\varepsilon$, where is a threshold, if $\|\mid r>\|_{2}<\varepsilon$ then break.

Output: $\quad$ the lowest $l$ eigenpairs $\lambda_{j}, \mid a_{j}>(j=1,2, \ldots, l)$

1. Pick up the initial orthonormal basis $V^{1}$;

2. for $k=1: m$

3. Compute the matrix $W^{k}=A V^{k}$;

4. Compute the Rayleigh matrix $H^{k}=V^{k^{T}} W^{k}$;

5. Compute the lowest $l$ eigenpairs $\left(\lambda_{i}^{k}, a_{i}^{k}\right)$ of $H^{k} ; \quad$ for $i=1: l$

6. Compute the Ritz vector $\left|z_{i}^{k}>=V^{k}\right| a_{i}^{k}>$; for $i=1: l$

7. The residual vector $\left|r_{i}^{k}>=\lambda_{i}^{k}\right| a_{i}^{k}>-W^{k}\left|a_{i}^{k}\right\rangle ; \quad$ for $i=1: l$

8. If $\max _{i=1: l}\left\|\mid r_{i}^{k}>\right\|_{2}<\varepsilon$

9. $\quad$ convergence $=1$ and exit

10. end if

11. $\quad$ Compute $C_{i}^{k}=\left(D-\lambda_{i}^{k} I\right)^{-1}$;

for $i=1: l$

12. Compute the new directions $\left|t_{i}^{k}>=C_{i}^{k}\right| r_{i}^{k}>$ for $i=1: l$

13. If $\operatorname{dim}\left(V^{k}\right)<b$

14. $\quad V^{k+1}=\operatorname{MGS}\left(V^{k},\left|t_{1}^{k}>,\right| t_{2}^{k}>\cdots \mid t_{l}^{k}>\right)$

15. else

16. $\quad V^{k+1}=\operatorname{MGS}\left(\left|z_{1}^{k}>,\right| z_{2}^{k}>\cdots\left|z_{l}^{k}>,\right| t_{1}^{k}>,\left|t_{2}^{k}>\cdots\right| t_{l}^{k}>\right)$

17. end if

18. end for 


\subsection{RMM-DIIS method}

The Residual Minimization Method - Direct Inversion in the Iterative Subspace method (RMM-DIIS method) was proposed by Péter Pulay in 1980 Ref. [23], Wood and Zunger were the first to apply the method to the eigenvalue problem in electronic structure calculations Ref. [31].

The residual minimization scheme is based on the idea to minimize the norm of the residual vector in the iterative subspace generated by the previous iterations. The advantage of this is that if the trial vectors are sufficiently separated, they will converge to different eigenstates independently. This is in contrast to methods which attempt to minimize the Rayleigh quotient (like the Davidson algorithms) where the trial states must be explicitly orthogonalized in order to avoid that all trial vectors converge towards the eigenvector of the lowest eigenvalue. This is an important difference since the GramSchmidt orthonorrmalization procedure which requires $C(n)=\Theta\left(n^{3}\right)$ operations, dominates the execution time of the Block Davidson algorithm for larger matrices. Since the residual minimization method does not require explicit orthogonalization at every step it has the potential to be faster than the Block Davidson method even if the method itself is more complex.

In the RMM-DIIS method, the residual vector is used:

$$
\left|r^{k}>=\left(A-\lambda^{k} I\right)\right| a^{k}>
$$

With each iteration, the residual vector $\left|r^{k}\right\rangle$ will converge towards zero. The $\lambda^{k}$ is

the eigenvalue of $A$ when the residual vector $\left\|\mid r^{k}>\right\|_{2}=0$, or when $\left\|\mid r^{k}>\right\| \|_{2}$ is less than a threshold $\varepsilon(\varepsilon=0$ ideally). 
$\lambda^{k}$ is an approximate eigenvalue, it can be calculated directly by the Rayleigh quotient Eq.(2.6), using Hamiltonian matrix $A$ and approximate eigenvector $\left|a^{k}\right\rangle$.

$$
\lambda^{k}=\frac{<a^{k}|A| a^{k}>}{<a^{k} \mid a^{k}>}
$$

Most iterative methods use an increment vector $\left|\delta x^{k}\right\rangle$ to obtain the approximate eigenvector at the next iteration.

$$
\left|a^{k+1}>=\right| a^{k}>+\mid \delta x^{k}>
$$

Ideally, the Eq. (2.8)would bring the residual vector to zero, getting $\mid r^{k+1}>$ to zero yields.

$$
\begin{aligned}
\mid r\left(\lambda, \mid a^{k}+\delta x>\right)> & =(A-\lambda I) \mid a^{k}+\delta x^{k}> \\
& =(A-\lambda I)\left|a^{k}>+(A-\lambda I)\right| \delta x^{k}> \\
& =0
\end{aligned}
$$

The formal solution of this equation is:

$$
\mid \delta x^{k}>=\frac{-(A-\lambda I) \mid a^{k}>}{(A-\lambda I)}
$$

Unfortunately, however there is no straightforward way solve Eq.(2.9), because $(A-\lambda I)^{-1}$ needs a matrix inversion operating, which may consume more time than traditional way to solve eigenproblem and $\lambda$ is not necessarily the exact eigenvalue. Instead, the RMM-DIIS method sets the increment vector $\mid \delta x^{k}>$ along the direction of the residual vector $\mid r^{k-1}>\left(\left|\delta x^{0}>=\right| a^{0}>\right)$.

$$
\mid \delta x^{k}>=\left\{\begin{array}{l}
\mid r^{k-1}>, k>0 \\
\mid a^{k}>, k=0
\end{array}\right.
$$


The vector at the next iteration is written as a linear combination of increment vector at the current iterations:

$$
\left|a^{k+1}>=\sum_{j=0}^{k} \alpha_{j}\right| \delta x^{j}>
$$

The coefficient $\alpha_{j}$ are obtained from the condition that the square of the norm of the residual vector $\rho^{2}=\left\|\mid r^{k+1}>\right\|_{2}^{2}$ is minimal. This leads to the condition Ref.[31].

$$
\begin{aligned}
\frac{\delta \rho^{2}}{\delta \alpha_{j}^{*}} & =\frac{\delta}{\delta \alpha_{j}^{*}} \frac{\sum_{g, h=0}^{k} \alpha_{g}^{*} \alpha_{h}<r^{g} \mid r^{h}>}{\sum_{g, h=0}^{k} \alpha_{g}^{*} \alpha_{h}<\delta x^{g}|I| \delta x^{h}>} \\
& =\frac{\delta}{\delta \alpha_{j}^{*}} \frac{\sum_{g, h=0}^{k} \alpha_{g}^{*} \alpha_{h}<\delta x^{g}\left|\left(A-\lambda^{o l d} I\right)\right|\left(A-\lambda^{o l d} I\right) \mid \delta x^{h}>}{\sum_{g, h=0}^{k} \alpha_{g}^{*} \alpha_{h}<\delta x^{g}|I| \delta x^{h}>} \\
& =0
\end{aligned}
$$

$\alpha_{j}$ (for $j=0,1,2, \ldots, k$ ) can be regarded as the components of a $(k+1)$ dimensional vector $|\alpha\rangle$. The problem of finding $\alpha_{j}$ is equivalent to finding the eigenvector corresponding to the lowest eigenvalue $\rho^{2}$ of the generalized Hermitian eigenproblem:

$$
P\left|\alpha>=\rho^{2} Q\right| \alpha>
$$

where

$$
P_{(g, h)}=<\delta x_{i}^{g}\left|\left(A-\lambda_{i}^{k} I\right)\right|\left(A-\lambda_{i}^{k} I\right) \mid \delta x_{i}^{h}>
$$

and

$$
Q_{(g, h)}=<\delta x_{i}^{g}|I| \delta x_{i}^{h}>
$$

The lowest eigenvalue of this problem corresponds to the minim value of $\rho^{2}$. Since 
$P$ and $Q$ are small $(k+1) \times(k+1)$ symmetric matrices the solution of this generalized eigenproblem requires only a negligible amount of time.

The lowest eigenvalue $\rho^{2}$ corresponds to the minimum value of the square of the norm of the residual vector. The components of the corresponding eigenvector $|\alpha\rangle$ can then be used to obtain the next trial eigenvector $\left|a^{k+1}\right\rangle$ according to Eq.(2.11). Finally, the new approximate eigenvalue is obtained as in Eq.(2.6):

$$
\lambda_{\mathrm{i}}^{k+1}=\frac{<a_{i}^{k+1}|A| a_{i}^{k+1}>}{<a_{i}^{k+1} \mid a_{i}^{k+1}>}
$$

If the new residual vector $\left|r^{k+1}\right\rangle$ fulfills the convergence criterion, in other word, if $\left\|\mid r_{i}^{k+1}>\right\|_{2}<\varepsilon$ (for $i=1: l$ ) then the program is stopped. If one of the residual vector $\mid r_{i}^{k+1}>$ does not satisfy the criterion, $k$ is increased by one and the procedure is repeated starting with Eq.(2.10). 


\section{Algorithm 4 RMM-DIIS method}

Input: matrix $A$, where $A$ is a $n \times n$ symmetrical sparse matrix.

integer $l$, where $l$ is number of the lowest eigenpairs need to be calculated.

matrix $a^{0}$, where $a^{0}=\left[\left|a_{1}^{0}>,\right| a_{2}^{0}>, \ldots, \mid a_{l}^{0}>\right]$, is a random $n \times l$

matrix, and contains $l$ initial eigenvectors of $A$.

vector $\lambda^{0}$, where $\lambda^{0}=\left[\lambda_{1}^{0}, \lambda_{2}^{0}, \ldots \lambda_{l}^{0}\right], \lambda$ is the eigenvalues corresponding to the eigenvectors in $V$.

integer $m$, where $m$ is maximum number of iterations.

tolerance $\varepsilon$, where is a threshold, if $\|\mid r>\|_{2}<\varepsilon$ then break.

Output: the lowest $l$ eigenpairs $\lambda_{i}^{j}, \mid a_{i}^{j}>(j=1,2, \ldots, l)$

1. pick input eigenpairs $\lambda_{i}^{0}, \mid a_{i}^{0}>$

for $i=1: l$

2. let $\left|\delta x_{i}^{0}\right\rangle=\left|a_{i}^{0}\right\rangle$

3. Compute the $A \mid a_{i}^{0}>$ and store it;

4. for $k=0: m$

5. Compute residual vector $\left|\delta x_{i}^{k+1}>=A\right| a_{i}^{k}>-\lambda_{i}^{k} \mid a_{i}^{k}>$

6. if $\left\|\mid r_{i}^{k}>\right\|_{2}<\varepsilon$

7. convergence $=1$ and exit

8. end if

9. $\quad$ Compute $P_{g . h}=<\delta x_{i}^{g}\left|\left(A-\lambda_{i}^{k} I\right)\right|\left(A-\lambda_{i}^{k} I\right) \mid \delta x_{i}^{h}>\quad \begin{aligned} & \text { for } g=0: k+1 \\ & \text { for } h=0: k+1\end{aligned}$

10. Compute $Q_{g, h}=<\delta x_{i}^{g}|I| \delta x_{i}^{h}>\quad$ for $g=0: k+1$

11. Compute the lowest eigenvector $|\alpha\rangle$ in generalized eigenproblem of $P$ and $Q$;

12. Compute new eigenvector $\left|a_{i}^{k+1}>=\sum_{j=0}^{k+1} \alpha_{j}\right| \delta x_{i}^{j}>$;

13. $\quad$ Compute new eigenvalue $\quad \lambda_{i}^{k+1}=\frac{\left\langle a_{i}^{k+1}|A| a_{i}^{k+1}\right\rangle}{\left\langle a_{i}^{k+1} \mid a_{i}^{k+1}\right\rangle}$;

14. $\quad$ store $A \mid a_{i}^{k+1}>$

15. end for 


\section{Implementations}

In this section, there are four algorithms listed (Block Davidson, RMM-RITZ, and two combination methods: BK-RMM and RMM-RMM) which are implemented. All implementations are achieved by $\mathrm{C}++$, in order to get results more quickly, Intel's Math Kernel Library (MKL) is used in this work frequently, and OpenMP is used to parallelize some vector operations. There are also some calculations and graphs by Matlab.

\subsection{Block Davidson}

I implemented the Block Davidson algorithm same as Algorithm 3 in page 25.

\subsection{RMM-RITZ}

The dimension of $P$ and $Q$ are not limited in the original RMM-DIIS method, when the number of iteration grows up, size of $P$ and $Q$ becomes larger, therefore I set a block number $b$ to limit the dimension in my implementation. On the hand setting a block number can save calculations, execution time and reduce memory requirement, on the other hand using block RMM-DIIS may lost some information and obtain a degenerated eigenvector, but normally, it also can reach convergent at final.

Similar to the Block Davidson method, implementation of the RMM-DIIS method require a blocking scheme for practical reasons. Although in theory the dimensions of the matrices $P$ and $Q$ are not limited, it is impractical to store more than a few of 
vectors $\left|\delta x_{i}\right\rangle$.

Since the trial vectors are iterated independently (without the need for an orthogonalization step), the RMM-DIIS blocking scheme works a bit different. The algorithm picks one trial vectors and iterates it until the size of the subspace for this vector has reached the block size. Then the procedure is repeated with a different trial vector. After all states have been iterated, a Ritz projection is performed that finds the $l$ lowest eigenvectors in the combined iterative subspace.

In addition to a reduction of the memory size, the Ritz projection has a number of other advantages. First, the Ritz projection drives the trial states towards the lowest eigenvalues and it makes sure that it is not possible that by accident two trial vectors converge towards the same eigenvector. In addition to this, it separates and orthogonalizes trial states converging towards eigenstates with the same eigenvalue (degenerate eigenvectors).

About the Ritz projection: I made two versions of the Ritz projection: One includes all intermediate vectors in the Ritz matrix $=V^{T} A V$ where $A$ is the matrix whose eigenvalues are sought, and $V=\left|r_{1}^{0}>\cdots\right| r_{1}^{\text {block }}>,\left|r_{2}^{0}>\cdots\right| r_{2}^{\text {block }}>, \ldots \mid r_{l}^{1}>$ $\cdots \mid r_{l}^{\text {block }}>$ (I call this variant of the algorithm maximum $V$, because $V$ include all Ritz information this version, it makes $V$ in a maximum size). This enlarges the search space of the Ritz projection and leads in all cases to a convergence towards the lowest eigenvalues. In RMM-Ritz maximum $V$ version, convergence is guaranteed for random initial eigenvectors, where I set the elements in initial eigenvectors with a range from 1 to -1 . The other version of the Ritz projection, uses only the latest eigenvectors. (I call this variant of the algorithm minimum $V$.) $V=\left|a_{1}^{\text {block }}>,\right| a_{2}^{\text {block }}>\cdots \mid a_{l}^{\text {block }}>$. The 
minimum $V$ version uses less memory and less calculations steps than maximum $V$ version, but this version needs good enough initial eigenvectors to start.

Set $\quad M_{g, h}=<V^{g} \mid A V^{h}>\quad$ and $\quad N_{g, h}=<V^{g} \mid V^{h}>$ for $g, h=0: l \times$ block -1. $M$ and $N$ are $(l \times$ block $) \times(l \times$ block $)$ symmetric matrices with small size, the generalized eigenproblem in a short time. After solving the generalized eigenproblem between $M$ and $N$, the eigenvectors $\beta$ which are result in generalized eigenproblem of $M$ and $N$ can be put into Eq.(3.1) to new eigenvalue Eq.(2.6):

$$
\mid a_{i}^{0}>_{s}=\sum_{j=0}^{d-1} \beta_{j, s} V_{i, j}
$$

The new residual vector $\mid r^{0}>$ fulfills the convergence criterion, if $\left\|\mid r_{i}^{0}>\right\| \|_{2}<\varepsilon$, for $i=1: l$, then stop the program is stop, if the residual vector $\left|r_{i}^{0}\right\rangle$ does not satisfy the criterion, let $k=k+1$ and go back to RMM step. 


\section{Algorithm 5 RMM- RITZ}

Input: matrix $A$, where $A$ is a $n \times n$ symmetrical sparse matrix.

integer $l$, where $l$ is number of the lowest eigenpairs need to be calculated.

matrix $a$, where $a=\left[\left|a_{1}^{0}>,\right| a_{2}^{0}>, \ldots, \mid a_{l}^{0}>\right]$, is a random $n \times l$

matrix, and contains $l$ initial eigenvectors of $A$.

integer $m$, where $m$ is maximum number of iterations.

integer $k$, where $k=1$ as initial value.

integer block, which limits the dimension of the block.

tolerance $\varepsilon$, where is a threshold, if norm of residual vector smaller than

$\varepsilon$ then break.

maximum $V$ or minimum $V$

Output: the lowest $l$ eigenpairs $\lambda_{i}^{j}, \mid a_{i}^{j}>(j=1,2, \ldots, l)$

\section{RMM - DIIS step}

\section{1. for $i=1: l$}

2. pick an input eigenvector $\left|a_{i}^{0}\right\rangle$, let $\left|r_{i}^{0}\right\rangle=\left|a_{i}^{0}\right\rangle$

3. Compute $\lambda_{i}^{0}=\frac{<a_{i}^{0}|A| a_{i}^{0}>}{<a_{i}^{0} \mid a_{i}^{0}>}$

4. for $b=0:$ block

5. $\quad$ Compute the $A \mid r_{i}^{b}>$ and store it;

6. Compute the $\left|r_{i}^{b+1}>=A\right| a_{i}^{b}>-\lambda_{i}^{b} \mid a_{i}^{b}>$

7. Let $P_{g . h}=<r_{i}^{g}\left|\left(A-\lambda_{i}^{b} I\right)\right|\left(A-\lambda_{i}^{b} I\right) \mid r_{i}^{h}>$

8. $\quad$ Let $Q_{g, h}=<r_{i}^{g}|I| r_{i}^{h}>$ for $g=0: b+1$ for $h=0: b+1$ for $g=0: b+1$ for $h=0: b+1$

Compute the lowest eigenvector $\mid \alpha>$ in generalized eigenproblem of $P$ and $Q$;

10. Compute new eigenvector $\left|a_{i}^{b+1}>=\sum_{j=0}^{b+1} \alpha_{j}\right| r_{i}^{j}>$;

11. $\quad$ Compute new eigenvalue $\frac{<a_{i}^{b+1}|A| a_{i}^{b+1}>}{<a_{i}^{b+1} \mid a_{i}^{b+1}>}$; Store $|A| a_{i}^{b+1}>$;

\section{end for}

\section{2. end for}

\section{3. end RMM-DIIS step}




\section{RITZ step}

14. if Using maximum $\mathrm{V}$

15. $d=l \times$ block;

16. $\quad V=\left|r_{1}^{0}>\cdots\right| r_{1}^{\text {block }}>,\left|r_{2}^{0}>\cdots\right| r_{2}^{\text {block }}>, \ldots\left|r_{l}^{1}>\cdots\right| r_{l}^{\text {block }}>$

17. $A V=A\left|r_{1}^{0}>\cdots A\right| r_{1}^{\text {block }}>A\left|r_{2}^{0}>\cdots A\right| r_{2}^{\text {block }}>\cdots A\left|r_{l}^{1}>\cdots A\right| r_{l}^{\text {block }}>$

18. else Using minimum size $\mathrm{V}$

19. $d=l$

20. $\quad V=\left|a_{1}^{\text {block }}>, \quad\right| a_{2}^{\text {block }}>\cdots \mid a_{l}^{\text {block }}>$;

21. $A V=A\left|a_{1}^{\text {block }}>, A\right| a_{2}^{\text {block }}>\cdots A \mid a_{l}^{\text {block }}>$;

22. end if

23. Compute $M_{g, h}=<V^{g} \mid A V^{h}>$

for $g=0: d-1$

for $h=0: d-1$

24. Compute $N_{g, h}=<V^{g} \mid V^{h}>$ for $g=0: d-1$ for $h=0: d-1$

25. Compute eigenvector $\beta$ in generalized eigenproblem of $M$ and $N$;

26. Compute new eigenvectors(s)

$$
\mid a_{i}^{0}>_{s}=\sum_{j=0}^{d-1} \beta_{j, s} V_{i, j} \quad \begin{array}{ll}
\text { for } i=1: l \\
\text { for } s=1: \operatorname{dim}(A)
\end{array}
$$

27. Compute new eigenvalue(s) $\quad \lambda_{i}^{0}=\frac{\left.<a_{i}^{0}|A| a_{i}^{0}\right\rangle}{<a_{i}^{0}\left|a_{i}^{0}\right\rangle} \quad$ for $i=1: l$

28. Compute residual vector $\left.\left|r_{i}^{0}\right\rangle=A\left|a_{i}^{0}>-\lambda_{i}^{0}\right| a_{i}^{0}\right\rangle \quad$ for $i=1: l$

29. if $\max _{i=1: l-2}\left\|\mid r_{i}^{0}>\right\|_{2}<\varepsilon$

30. convergence $=1$ and exit

31. else if $k<m$

32. $k=k+1$ and back to RMM-DIIS step 1

33. end if

34. end RITZ step 


\subsection{Block Davidson and RMM-RITZ $\boldsymbol{V}_{\min }$}

1. Set an integer $m$ which limits the number of iteration of the Block Davidson algorithm, and a tolerance $\varepsilon_{1}$;

2. Set an integer $r$ which limits the number of iteration of the RMM-RITZ $V_{\min }$ algorithm, and a tolerance $\varepsilon_{2}$;

3. Operate the Block Davidson algorithm;

4. Store the eigenvectors which are the results of the Block Davidson;

5. Operate the RMM-RITZ $V_{\min }$ algorithm using the eigenvector obtained with the Block Davidson algorithm;

\subsection{RMM-RITZ $\boldsymbol{V}_{\max }$ and RMM-RITZ $\boldsymbol{V}_{\min }$}

1. Set an integer $m$ which limits number of iteration of the RMM-RITZ $V_{\max }$ algorithm, and a tolerance $\varepsilon_{1}$;

2. Set an integer $r$ which limits number of iteration of the RMM-RITZ $V_{\min }$ algorithm, and a tolerance $\varepsilon_{2}$;

3. Operate the RMM-RITZ $V_{\max }$ algorithm;

4. Store the eigenvectors;

5. Operate the RMM-RITZ $V_{\min }$ algorithm using the eigenvector obtained with the RMM-RITZ $V_{\max }$ algorithm; 


\subsection{Parallelization}

In my Block Davidson and RMM-DIIS algorithms can be run in parallel on sharedmemory computers. To this end the programs make heavy use of Intel's Math Kernel Library (MKL). In particular, multiplications of the sparse Hamilton matrix with a vector are performed with the help of MKL. Additionally, OpenMP is used to parallelize some vector operations. Two computers were used for the calculations in this program: one is a dual hex-core machine with Intel $2 \times$ Intel X5650 Xeon processor $(2.67 \mathrm{GHz})$ and 12GB of DDR3-1333 RAM, 12 cores with 12 threads. The second machine is an Intel's Many-Integrated Core architecture with Intel Xeon Phi coprocessor 5110P integrates 60 cores with 4 hardware threads per core. Table 3.1 compares MKL and OpenMP on several operations, in same operation have different elapsed times.

Table 3.1 Comparison of MKL and OpenMP

\begin{tabular}{|c|c|c|c|}
\hline MKL & Faster? & OpenMP & Faster? \\
\hline dnrm2_ & $\mathbf{x}$ & $\begin{array}{l}\text { \#pragma omp parallel for } \\
\text { reduction(+:norm2) }\end{array}$ & $\checkmark$ \\
\hline ddot_ & X & $\begin{array}{l}\text { \#pragma omp parallel for } \\
\text { reduction(+:dot) }\end{array}$ & $\checkmark$ \\
\hline daxpy_ & X & \#pragma omp parallel for & $\checkmark$ \\
\hline dscal_ & $x$ & \#pragma omp parallel for & $\checkmark$ \\
\hline dgemv_ & x & \#pragma omp parallel for & $V$ \\
\hline
\end{tabular}

According to Table 3.1, obviously, OpenMP has a better than MKL on some operations;

hence I used OpenMP instead of MKL to make these programs obtain acceleration on elapsed time, and improve speedup. 


\section{Results}

In this chapter, I will show my results, include comparison of different block, comparison of different algorithms, and parallel speedup on two different machines.

I find out the top 6 eigenvalues can all reach to convergence easily in all 6 models in this project, but when I tried to calculate out top 8 eigenpairs, there were some different result among the different models and different size, I want to compare them so that's the reason why I determined to calculated top 8 eigenvalues.

\subsection{Comparison of different block size}

In this section I describe result of calculation to study the influence of the block size on the convergence speed. There are 3 reports to compare different among 5 block sizes in 4 methods on two-leg Heisenberg Ladder staggered model, I abbreviated it Ladder 215S, where $N=24, M=0$ (All results in section 4.1 are calculated by an Intel Xeon Phi coprocessor 5110P.)

In total I have implement four approaches: the first one is the Block Davidson method only; the second one is the RMM-DIIS $V_{\max }$ method only, the third one is started the calculation with the Block Davidson method and switched to the RMM-DIIS $V_{\min }$ after 60 iterations or when the norms of all residual vectors was below $5 \times 10^{-2}$; the last one is the RMM-DIIS $V_{\max }$ and RMM-DIIS $V_{\min }$ combination method, the switch conditions is same as the third method: reach to 60 iterations or when the norms of all 
residual vector s was below $5 \times 10^{-2}$; For all of 4 methods, exit program if number of iterations exceed 240 or when the norms of all residual vectors was below $5 \times 10^{-4}$. The number $5 \times 10^{-2}$ and $5 \times 10^{-4}$ were chosen so that convergence could be achieved is a reasonable time in most cases for all system sizes. Practical applications might require more stringent criterions.

The minimum size of block is 2 , hence I started from 2 , and increased the size of the block until 6 . There are 20 items in each report, they used same vectors as initial eigenvectors. It can compare the result objectively. In order to proof the result doesn't happen by accident, I tested three different initial eigenvectors in each report, and they have revealed convergence.

In the following reports, I have recorded 5 attributions; The total number of iterations and the number of blocks the in RMM-RITZ algorithm if applicable (in brackets); The convergence status for the lowest eight eigenvalues (if the norm of the residual is vector is below the tolerance); 'count system' shows how many $A \mid v>$ operation were performed where $A$ is the Hamilton matrix, $\mid v>$ is a vector with same column size as $A$; 'clock time' is the execution time in seconds; 'Memory' recorded the memory usage, I checked memory with the help of the 'top' command of Ubuntu. 
Table 4.1 Report 1 Convergence of Ladder 215S(24) by Block Davidson

\begin{tabular}{|l|l|l|l|r|l|}
\hline Block size & Iteration & $\begin{array}{l}\text { conve } \\
\text { rgent }\end{array}$ & $\begin{array}{l}\text { Count } \\
\text { Sys }\end{array}$ & Clock time (sec.) & Memory \\
\hline BKDV 2 & 240 & no & 5752 & 956.5 & $18062 \mathrm{MB}$ \\
\hline BKDV 3 & 240 & no & 6424 & 956.2 & $18227 \mathrm{MB}$ \\
\hline BKDV 4 & 125 & yes & 3976 & 521.5 & $18392 \mathrm{MB}$ \\
\hline BKDV 5 & 94 & yes & 3352 & 395.5 & $18557 \mathrm{MB}$ \\
\hline BKDV 6 & 75 & yes & 2960 & 321.7 & $18723 \mathrm{MB}$ \\
\hline
\end{tabular}

Table 4.2 Report 1 Convergence of Ladder 215S(24) by Block Davidson and RMMRITZ $V_{\min }$

\begin{tabular}{|c|c|c|c|c|c|c|}
\hline Block size & Iteration & $\begin{array}{l}\text { conve } \\
\text { rgent }\end{array}$ & $\begin{array}{l}\text { Count } \\
\text { Sys }\end{array}$ & \multicolumn{2}{|c|}{ Clock time (sec.) } & Memory \\
\hline \multirow{2}{*}{$\begin{array}{l}\text { BKDV } 2 \\
\text { RMM } 4 V_{\text {min }}\end{array}$} & 60 & no & 1432 & 239.6 & \multirow{2}{*}{261.3} & $18161 \mathrm{MB}$ \\
\hline & $78(26)$ & yes & 865 & 21.7 & & $18141 \mathrm{MB}$ \\
\hline \multirow{2}{*}{$\begin{array}{l}\text { RMM } 3 \\
\text { RMM } 4 V_{\text {min }}\end{array}$} & 60 & no & 1664 & 250.5 & \multirow{2}{*}{300.4} & $18498 \mathrm{MB}$ \\
\hline & $180(60)$ & no & 1987 & 50.0 & & $18133 \mathrm{MB}$ \\
\hline \multirow{2}{*}{$\begin{array}{l}\text { BKDV } 4 \\
\text { RMM } 4 V_{\text {min }}\end{array}$} & 39 & yes & 1224 & 160.3 & \multirow{2}{*}{183.7} & $18814 \mathrm{MB}$ \\
\hline & $84(28)$ & yes & 931 & 23.4 & & $18133 \mathrm{MB}$ \\
\hline \multirow{2}{*}{$\begin{array}{l}\text { BKDV } 5 \\
\text { RMM } 4 V_{\text {min }}\end{array}$} & 38 & yes & 1336 & 159.8 & \multirow{2}{*}{179.8} & $19160 \mathrm{MB}$ \\
\hline & $72(24)$ & yes & 799 & 20.1 & & $18149 \mathrm{MB}$ \\
\hline \multirow{2}{*}{$\begin{array}{l}\text { BKDV } 6 \\
\text { RMM } 4 V_{\text {min }}\end{array}$} & 32 & yes & 1240 & 138.6 & \multirow{2}{*}{157.0} & $19491 \mathrm{MB}$ \\
\hline & $66(22)$ & yes & 733 & 18.42 & & $18149 \mathrm{MB}$ \\
\hline
\end{tabular}


Table 4.3 Report 1 Convergence of Ladder 215S(24) by RMM-RITZ $V_{\max }$

\begin{tabular}{|l|l|l|l|r|l|}
\hline Block size & Iteration & conve & \multicolumn{1}{l|}{$\begin{array}{l}\text { Count } \\
\text { rgent }\end{array}$} & \multicolumn{1}{l|}{ Clock time (sec.) } & Memory \\
\hline RMM 2 $\boldsymbol{V}_{\max }$ & $240(240)$ & no & 4087 & 122.0 & $18161 \mathrm{MB}$ \\
\hline RMM 3 $\boldsymbol{V}_{\max }$ & $240(120)$ & no & 3007 & 103.8 & $18514 \mathrm{MB}$ \\
\hline RMM 4 $\boldsymbol{V}_{\max }$ & $201(67)$ & yes & 2218 & 86.1 & $18846 \mathrm{MB}$ \\
\hline RMM 5 $\boldsymbol{V}_{\max }$ & $160(40)$ & yes & 1647 & 71.7 & $19176 \mathrm{MB}$ \\
\hline RMM 6 $\boldsymbol{V}_{\max }$ & $125(25)$ & yes & 1134 & 60.9 & $19516 \mathrm{MB}$ \\
\hline
\end{tabular}

Table 4.4 Report 1 Convergence of Ladder 215S(24) by RMM-RITZ $V_{\max }$ and $V_{\min }$

\begin{tabular}{|c|c|c|c|c|c|c|}
\hline Block size & iteration & $\begin{array}{l}\text { conve } \\
\text { rgent }\end{array}$ & $\begin{array}{l}\text { Count } \\
\text { Sys }\end{array}$ & \multicolumn{2}{|c|}{ Clock time (sec.) } & Memory \\
\hline \multirow{2}{*}{$\begin{array}{l}\text { RMM } 2 V_{\max } \\
\text { RMM } 4 V_{\min }\end{array}$} & $60(60)$ & no & 1027 & 30.5 & \multirow{2}{*}{80.4} & $18145 \mathrm{MB}$ \\
\hline & $180(60)$ & no & 1987 & 50.0 & & $18125 \mathrm{MB}$ \\
\hline \multirow{2}{*}{$\begin{array}{l}\mathbf{R M M} 3 V_{\max } \\
\mathbf{R M M} 4 V_{\min }\end{array}$} & $60(30)$ & no & 757 & 26.0 & \multirow{2}{*}{76.1} & $18498 \mathrm{MB}$ \\
\hline & $180(60)$ & no & 1987 & 50.0 & & $18133 \mathrm{MB}$ \\
\hline \multirow{2}{*}{$\begin{array}{l}\mathrm{RMM} 4 V_{\max } \\
\mathrm{RMM} 4 V_{\min }\end{array}$} & $60(20)$ & no & 667 & 25.8 & \multirow{2}{*}{43.4} & $18814 \mathrm{MB}$ \\
\hline & $63(21)$ & yes & 700 & 17.6 & & $18133 \mathrm{MB}$ \\
\hline \multirow{2}{*}{$\begin{array}{l}\text { RMM } 5 V_{\max } \\
\mathbf{R M M} 4 V_{\min }\end{array}$} & $60(15)$ & no & 622 & 26.7 & \multirow{2}{*}{54.3} & $19160 \mathrm{MB}$ \\
\hline & $99(33)$ & yes & 1096 & 27.6 & & $18149 \mathrm{MB}$ \\
\hline \multirow{2}{*}{$\begin{array}{l}\text { RMM } 6 V_{\max } \\
\text { RMM } 4 V_{\min }\end{array}$} & $60(12)$ & no & 595 & 28.1 & \multirow{2}{*}{44.9} & $19491 \mathrm{MB}$ \\
\hline & $60(20)$ & yes & 667 & 16.8 & & $18149 \mathrm{MB}$ \\
\hline
\end{tabular}


Table 4.5 Report 2 Convergence of Ladder 215S(24) by Block Davidson

\begin{tabular}{|l|l|l|l|r|l|}
\hline Block size & Iteration & $\begin{array}{l}\text { conve } \\
\text { rgent }\end{array}$ & $\begin{array}{l}\text { Count } \\
\text { Sys }\end{array}$ & Clock time (sec.) & Memory \\
\hline BKDV 2 & 240 & no & 4087 & 954.9 & $18161 \mathrm{MB}$ \\
\hline BKDV 3 & 220 & yes & 2757 & 564.2 & $18514 \mathrm{MB}$ \\
\hline BKDV 4 & 157 & yes & 1723 & 504.1 & $18846 \mathrm{MB}$ \\
\hline BKDV 5 & 124 & yes & 1278 & 417.9 & $19176 \mathrm{MB}$ \\
\hline BKDV 6 & 105 & yes & 1036 & 318.9 & $19516 \mathrm{MB}$ \\
\hline
\end{tabular}

Table 4.6 Report 2 Convergence of Ladder 215S(24) by Block Davidson and RMMRITZ $V_{\min }$

\begin{tabular}{|c|c|c|c|c|c|c|}
\hline Block size & iteration & $\begin{array}{l}\text { conve } \\
\text { rgent }\end{array}$ & $\begin{array}{l}\text { Count } \\
\text { Sys }\end{array}$ & \multicolumn{2}{|c|}{ Clock time (sec.) } & Memory \\
\hline \multirow{2}{*}{$\begin{array}{l}\text { BKDV } 2 \\
\text { RMM } 4 V_{\text {min }}\end{array}$} & 60 & no & 1432 & 239.1 & \multirow{2}{*}{263.3} & $18161 \mathrm{MB}$ \\
\hline & $87(29)$ & yes & 964 & 24.2 & & $18141 \mathrm{MB}$ \\
\hline \multirow{2}{*}{$\begin{array}{l}\text { RMM } 3 \\
\text { RMM } 4 V_{\text {min }}\end{array}$} & 44 & yes & 1216 & 180.5 & \multirow{2}{*}{198.1} & $18498 \mathrm{MB}$ \\
\hline & $63(21)$ & yes & 700 & 17.6 & & $18133 \mathrm{MB}$ \\
\hline \multirow{2}{*}{$\begin{array}{l}\text { BKDV } 4 \\
\text { RMM } 4 V_{\text {min }}\end{array}$} & 47 & yes & 1480 & 194.2 & \multirow{2}{*}{214.3} & $18814 \mathrm{MB}$ \\
\hline & $72(24)$ & yes & 799 & 20.1 & & $18133 \mathrm{MB}$ \\
\hline \multirow{2}{*}{$\begin{array}{l}\text { BKDV } 5 \\
\text { RMM } 4 V_{\text {min }}\end{array}$} & 38 & yes & 1336 & 159.9 & \multirow{2}{*}{183.3} & $19160 \mathrm{MB}$ \\
\hline & $84(28)$ & yes & 931 & 23.4 & & $18149 \mathrm{MB}$ \\
\hline \multirow{2}{*}{$\begin{array}{l}\text { BKDV } 6 \\
\text { RMM } 4 V_{\text {min }}\end{array}$} & 30 & yes & 1160 & 126.2 & \multirow{2}{*}{147.1} & $19491 \mathrm{MB}$ \\
\hline & $75(25)$ & yes & 832 & 20.9 & & $18149 \mathrm{MB}$ \\
\hline
\end{tabular}


Table 4.7 Report 2 Convergence of Ladder 215S(24) by RMM-RITZ $V_{\max }$

\begin{tabular}{|l|l|l|l|r|l|}
\hline Block size & Iteration & conve & \multicolumn{1}{l|}{$\begin{array}{l}\text { Count } \\
\text { rgent }\end{array}$} & \multicolumn{1}{l|}{ Clock time (sec.) } & Memory \\
\hline RMM 2 $\boldsymbol{V}_{\max }$ & $240(240)$ & no & 4087 & 122.1 & $18161 \mathrm{MB}$ \\
\hline RMM 3 $\boldsymbol{V}_{\max }$ & $240(120)$ & no & 3007 & 103.7 & $18514 \mathrm{MB}$ \\
\hline RMM 4 $\boldsymbol{V}_{\max }$ & $195(65)$ & yes & 2152 & 83.2 & $18846 \mathrm{MB}$ \\
\hline RMM 5 $\boldsymbol{V}_{\max }$ & $148(37)$ & yes & 1524 & 65.7 & $19176 \mathrm{MB}$ \\
\hline RMM 6 $\boldsymbol{V}_{\max }$ & $115(23)$ & yes & 1134 & 53.8 & $19516 \mathrm{MB}$ \\
\hline
\end{tabular}

Table 4.8 Report 2 Convergence of Ladder 215S(24) by RMM-RITZ $V_{\max }$ and $V_{\min }$

\begin{tabular}{|c|c|c|c|c|c|c|}
\hline Block size & iteration & $\begin{array}{l}\text { conve } \\
\text { rgent }\end{array}$ & $\begin{array}{l}\text { Count } \\
\text { Sys }\end{array}$ & \multicolumn{2}{|c|}{ Clock time (sec.) } & Memory \\
\hline \multirow{2}{*}{$\begin{array}{l}\text { RMM } 2 V_{\max } \\
\text { RMM } 4 V_{\min }\end{array}$} & $60(60)$ & no & 1027 & 30.6 & \multirow{2}{*}{80.7} & $18145 \mathrm{MB}$ \\
\hline & $180(60)$ & no & 1987 & 50.1 & & $18125 \mathrm{MB}$ \\
\hline \multirow{2}{*}{$\begin{array}{l}\text { RMM } 3 V_{\max } \\
\text { RMM } 4 V_{\min }\end{array}$} & $60(30)$ & no & 757 & 26.0 & \multirow{2}{*}{75.9} & $18498 \mathrm{MB}$ \\
\hline & $180(60)$ & no & 1987 & 49.9 & & $18133 \mathrm{MB}$ \\
\hline \multirow{2}{*}{$\begin{array}{l}\text { RMM } 4 V_{\max } \\
\text { RMM } 4 V_{\min }\end{array}$} & $60(20)$ & no & 667 & 25.7 & \multirow{2}{*}{49.9} & $18814 \mathrm{MB}$ \\
\hline & $87(29)$ & yes & 964 & 24.2 & & $18133 \mathrm{MB}$ \\
\hline \multirow{2}{*}{$\begin{array}{l}\text { RMM } 5 V_{\max } \\
\mathbf{R M M} 4 V_{\min }\end{array}$} & $60(15)$ & no & 662 & 26.7 & \multirow{2}{*}{51.7} & $19160 \mathrm{MB}$ \\
\hline & $90(30)$ & yes & 997 & 25.0 & & $18149 \mathrm{MB}$ \\
\hline \multirow{2}{*}{$\begin{array}{l}\text { RMM } 6 V_{\text {max }} \\
\text { RMM } 4 V_{\text {min }}\end{array}$} & $55(11)$ & yes & 546 & 25.7 & \multirow{2}{*}{41.6} & $19491 \mathrm{MB}$ \\
\hline & $57(19)$ & yes & 634 & 15.9 & & $18149 \mathrm{MB}$ \\
\hline
\end{tabular}


Table 4.9 Report 3 Convergence of Ladder 215S(24) by Block Davidson

\begin{tabular}{|l|l|l|l|r|l|}
\hline Block size & Iteration & $\begin{array}{l}\text { conve } \\
\text { rgent }\end{array}$ & $\begin{array}{l}\text { Count } \\
\text { Sys }\end{array}$ & Clock time (sec.) & Memory \\
\hline BKDV 2 & 232 & yes & 5560 & 920.5 & $18161 \mathrm{MB}$ \\
\hline BKDV 3 & 164 & yes & 4576 & 681.8 & $18514 \mathrm{MB}$ \\
\hline BKDV 4 & 107 & yes & 3400 & 416.1 & $18846 \mathrm{MB}$ \\
\hline BKDV 5 & 86 & yes & 3064 & 366.3 & $19176 \mathrm{MB}$ \\
\hline BKDV 6 & 67 & yes & 2640 & 276.8 & $19516 \mathrm{MB}$ \\
\hline
\end{tabular}

Table 4.10 Report 3 Convergence of Ladder 215S(24) by Block Davidson and RMMRITZ $V_{\min }$

\begin{tabular}{|c|c|c|c|c|c|c|}
\hline Block size & iteration & $\begin{array}{l}\text { conve } \\
\text { rgent }\end{array}$ & $\begin{array}{l}\text { Count } \\
\text { Sys }\end{array}$ & \multicolumn{2}{|c|}{ Clock time (sec.) } & Memory \\
\hline \multirow{2}{*}{$\begin{array}{l}\text { BKDV } 2 \\
\text { RMM } 4 V_{\text {min }}\end{array}$} & 60 & no & 1432 & 238.9 & \multirow{2}{*}{256.5} & $18161 \mathrm{MB}$ \\
\hline & $63(21)$ & yes & 700 & 17.6 & & $18141 \mathrm{MB}$ \\
\hline \multirow{2}{*}{$\begin{array}{l}\text { RMM } 3 \\
\text { RMM } 4 V_{\text {min }}\end{array}$} & 44 & yes & 1216 & 179.9 & \multirow{2}{*}{201.6} & $18498 \mathrm{MB}$ \\
\hline & $78(26)$ & yes & 865 & 21.7 & & $18133 \mathrm{MB}$ \\
\hline \multirow{2}{*}{$\begin{array}{l}\text { BKDV } 4 \\
\text { RMM } 4 V_{\text {min }}\end{array}$} & 38 & yes & 1192 & 145.8 & \multirow{2}{*}{162.5} & $18814 \mathrm{MB}$ \\
\hline & $60(20)$ & yes & 667 & 16.7 & & $18133 \mathrm{MB}$ \\
\hline \multirow{2}{*}{$\begin{array}{l}\text { BKDV } 5 \\
\text { RMM } 4 V_{\text {min }}\end{array}$} & 30 & yes & 1048 & 125.8 & \multirow{2}{*}{146.7} & $19160 \mathrm{MB}$ \\
\hline & $75(25)$ & yes & 832 & 20.9 & & $18149 \mathrm{MB}$ \\
\hline \multirow{2}{*}{$\begin{array}{l}\text { BKDV } 6 \\
\text { RMM } 4 V_{\text {min }}\end{array}$} & 28 & yes & 1072 & 117.9 & \multirow{2}{*}{133.8} & $19491 \mathrm{MB}$ \\
\hline & $57(19)$ & yes & 634 & 15.9 & & $18149 \mathrm{MB}$ \\
\hline
\end{tabular}


Table 4.11 Report 3 Convergence of Ladder 215S(24) by RMM-RITZ $V_{\max }$

\begin{tabular}{|l|l|l|l|r|l|}
\hline Block size & Iteration & conve & \multicolumn{1}{l|}{$\begin{array}{l}\text { Count } \\
\text { rgent }\end{array}$} & \multicolumn{1}{l|}{ Clock time (sec.) } & Memory \\
\hline RMM 2 $\boldsymbol{V}_{\max }$ & $240(240)$ & no & 4087 & 122.3 & $18161 \mathrm{MB}$ \\
\hline RMM 3 $\boldsymbol{V}_{\max }$ & $220(110)$ & yes & 2757 & 95.1 & $18514 \mathrm{MB}$ \\
\hline RMM 4 $\boldsymbol{V}_{\max }$ & $157(52)$ & yes & 1723 & 66.7 & $18846 \mathrm{MB}$ \\
\hline RMM 5 $\boldsymbol{V}_{\max }$ & $124(31)$ & yes & 1278 & 55.2 & $19176 \mathrm{MB}$ \\
\hline RMM 6 $\boldsymbol{V}_{\max }$ & $105(21)$ & yes & 1036 & 49.2 & $19516 \mathrm{MB}$ \\
\hline
\end{tabular}

Table 4.12 Report 3 Convergence of Ladder $215 \mathrm{~S}(24)$ by RMM-RITZ $V_{\max }$ and $V_{\text {min }}$

\begin{tabular}{|c|c|c|c|c|c|c|}
\hline Block size & iteration & $\begin{array}{l}\text { conve } \\
\text { rgent }\end{array}$ & $\begin{array}{l}\text { Count } \\
\text { Sys }\end{array}$ & \multicolumn{2}{|c|}{ Clock time (sec.) } & Memory \\
\hline \multirow{2}{*}{$\begin{array}{l}\text { RMM } 2 V_{\max } \\
\text { RMM } 4 V_{\min }\end{array}$} & $60(60)$ & no & 1027 & 30.5 & \multirow{2}{*}{42.3} & $18145 \mathrm{MB}$ \\
\hline & $42(14)$ & yes & 469 & 11.8 & & $18125 \mathrm{MB}$ \\
\hline \multirow{2}{*}{$\begin{array}{l}\text { RMM } 3 V_{\max } \\
\mathbf{R M M} 4 V_{\min }\end{array}$} & $60(30)$ & no & 757 & 26.0 & \multirow{2}{*}{42.7} & $18498 \mathrm{MB}$ \\
\hline & $60(20)$ & yes & 667 & 16.7 & & $18133 \mathrm{MB}$ \\
\hline \multirow{2}{*}{$\begin{array}{l}\text { RMM } 4 V_{\max } \\
\text { RMM } 4 V_{\min }\end{array}$} & $51(17)$ & yes & 568 & 21.9 & \multirow{2}{*}{32.0} & $18814 \mathrm{MB}$ \\
\hline & $36(12)$ & yes & 403 & 10.1 & & $18133 \mathrm{MB}$ \\
\hline \multirow{2}{*}{$\begin{array}{l}\text { RMM } 5 V_{\max } \\
\text { RMM } 4 V_{\min }\end{array}$} & $44(11)$ & yes & 458 & 19.6 & \multirow{2}{*}{41.3} & $19160 \mathrm{MB}$ \\
\hline & $78(26)$ & yes & 865 & 21.7 & & $18149 \mathrm{MB}$ \\
\hline \multirow{2}{*}{$\begin{array}{l}\text { RMM } 6 V_{\text {max }} \\
\text { RMM } 4 V_{\text {min }}\end{array}$} & $35(7)$ & yes & 350 & 16.4 & \multirow{2}{*}{36.5} & $19491 \mathrm{MB}$ \\
\hline & $72(24)$ & yes & 799 & 20.1 & & $18149 \mathrm{MB}$ \\
\hline
\end{tabular}




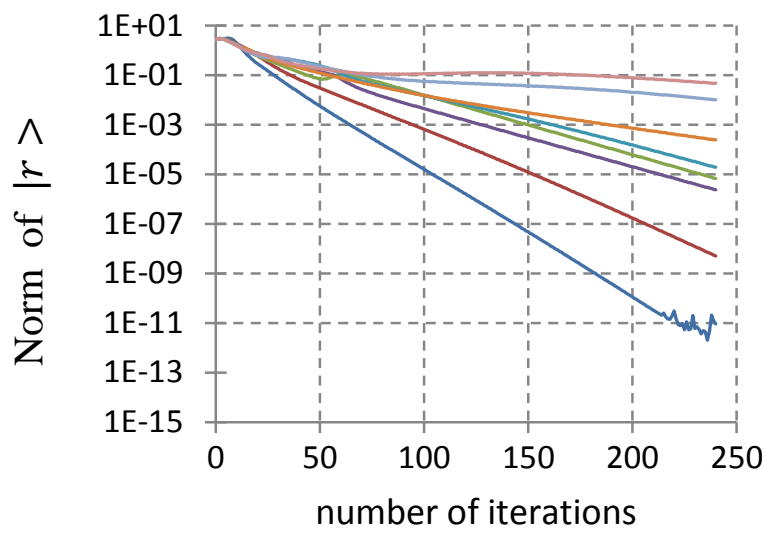

Figure 4.1 Results of Ladder 215S(24),

block $=\mathbf{2}$ by $\mathrm{RMM} \boldsymbol{V}_{\max }$

iteration $=240$,

time $=122.0 \mathrm{~s}$

on an Xeon Phi in report 1

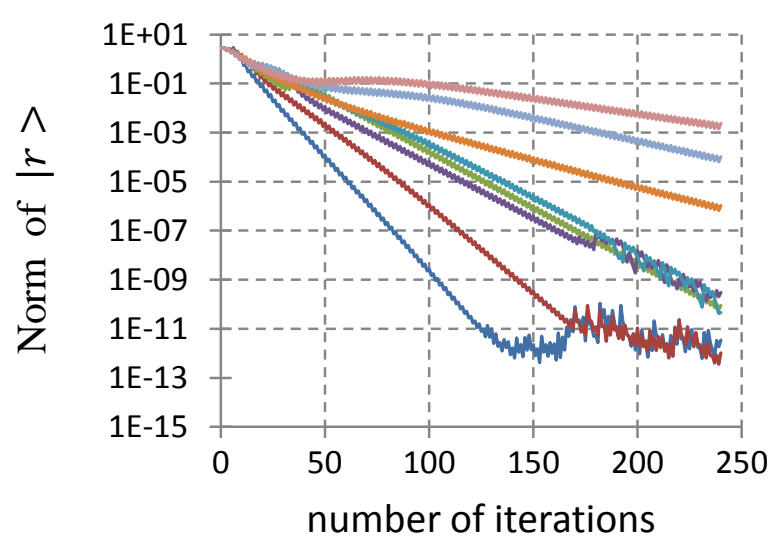

Figure 4.3 Results of Ladder 215S(24),

block $=\mathbf{3}$ by $\mathrm{RMM} \boldsymbol{V}_{\max }$

iteration $=240(120)$,

time $=103.8 \mathrm{~s}$

on an Xeon Phi in report 1

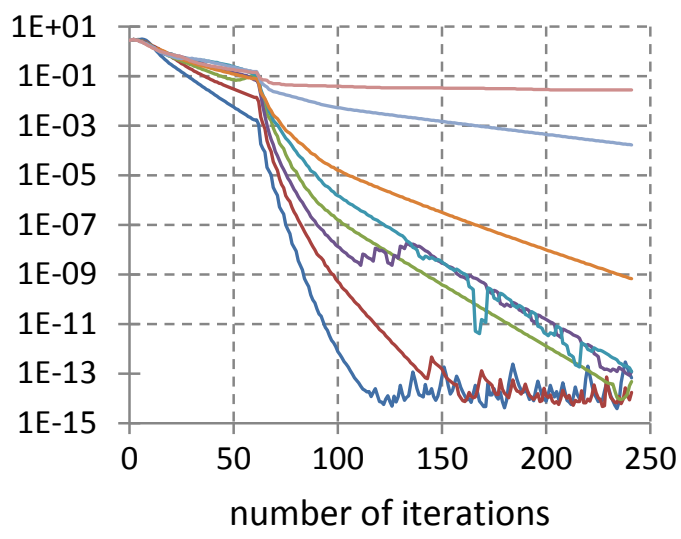

Figure 4.2 Results of Ladder 215S(24),

block $=2 / 4$ by $R M M V_{\max } \& V_{\min }$

iteration $=60(60) / 180(60)$,

time $=30.5 / 50.0(80.5) \mathrm{s}$

on an Xon Phi in report 1

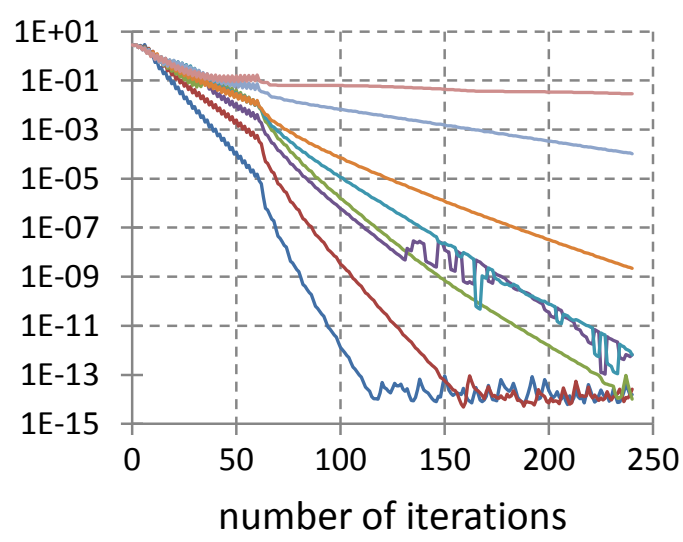

Figure 4.4 Results of Ladder 215S(24),

block $=3 / 4$ by $R M M V_{\max } \& V_{\min }$

iteration $=60(30) / 180(60)$,

time $=26.0 / 50.01(76.1) \mathrm{s}$

on an Xeon Phi in report 1 


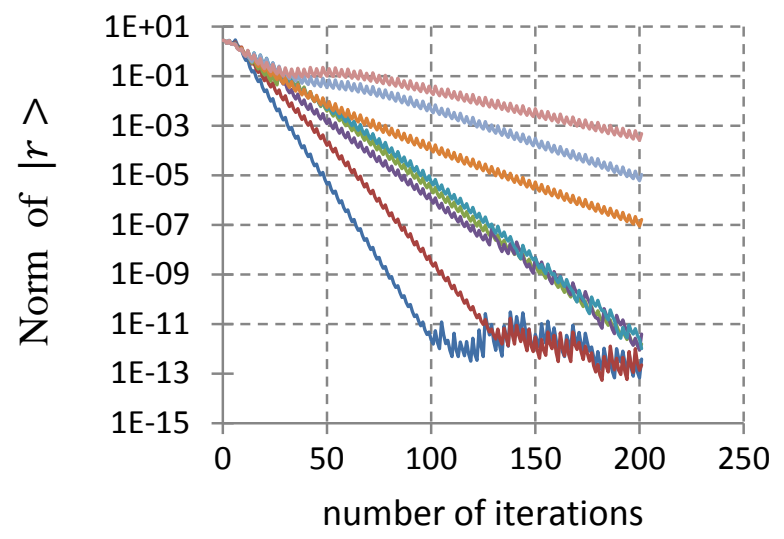

Figure 4.5 Results of Ladder 215S(24),

block $=4$ by $\mathrm{RMM} \boldsymbol{V}_{\max }$

iteration $=201(67)$,

time $=86.1 \mathrm{~s}$

on an Xeon Phi in report 1

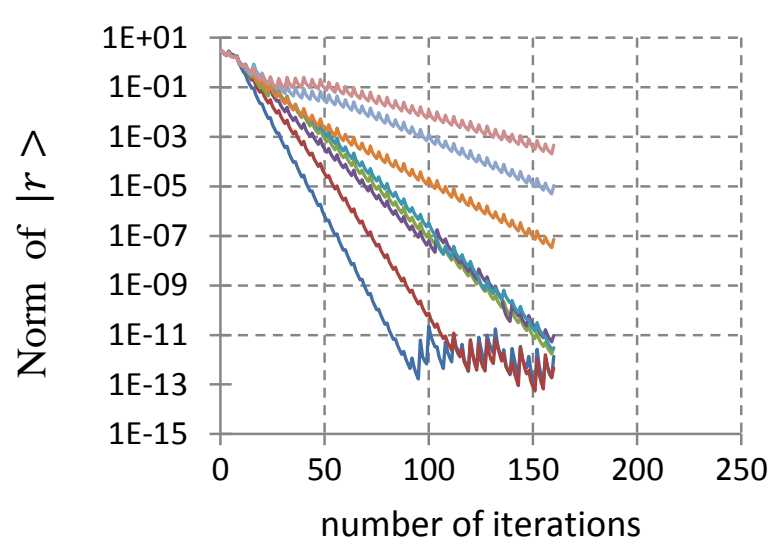

Figure 4.7 Results of Ladder 215S(24), block $=\mathbf{5}$ by $\mathbf{R M M} \boldsymbol{V}_{\max }$

iteration $=160(40)$,

time $=71.7 \mathrm{~s}$

on an Xeon Phi in report 1

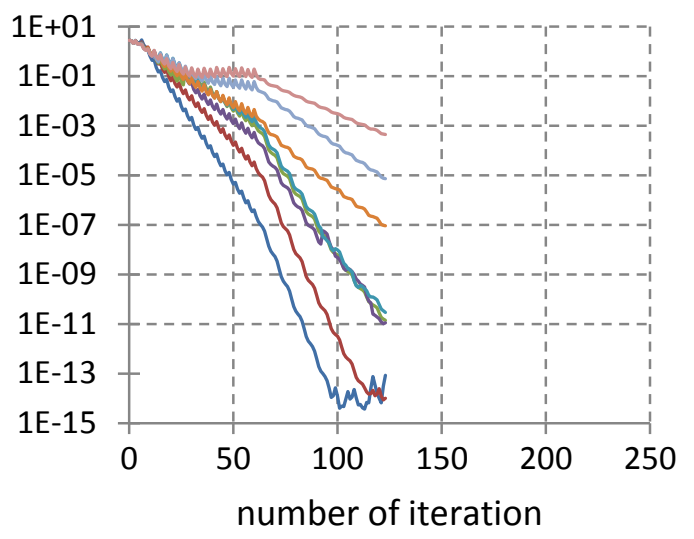

Figure 4.6 Results of Ladder 215S(24),

block $=4 / 4$ by $R M M V_{\max } \& V_{\min }$

iteration $=60(20) / 63(21)$,

time $=25.8 / 17.6(43.4) \mathrm{s}$

on an Xeon Phi in report 1

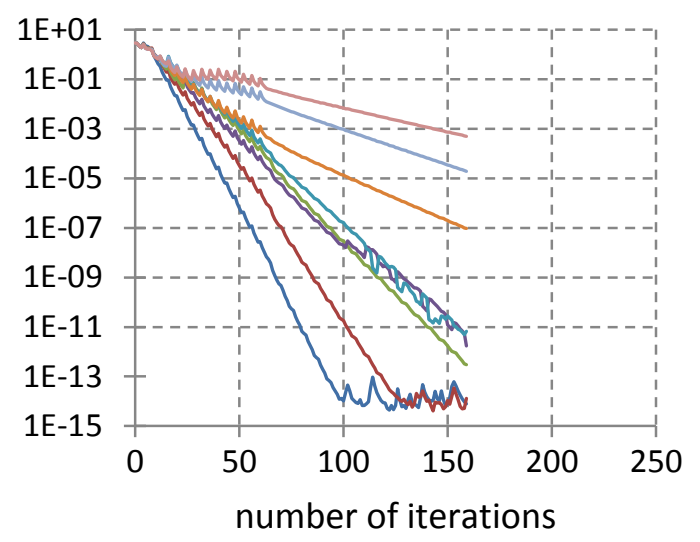

Figure 4.8 Results of Ladder 215S(24),

block $=5 / 4$ by $\mathrm{RMM} V_{\max } \& V_{\min }$

iteration $=60(15) / 99(33)$,

time $=26.7 / 27.6(54.3) \mathrm{s}$

on an Xeon Phi in report 1 


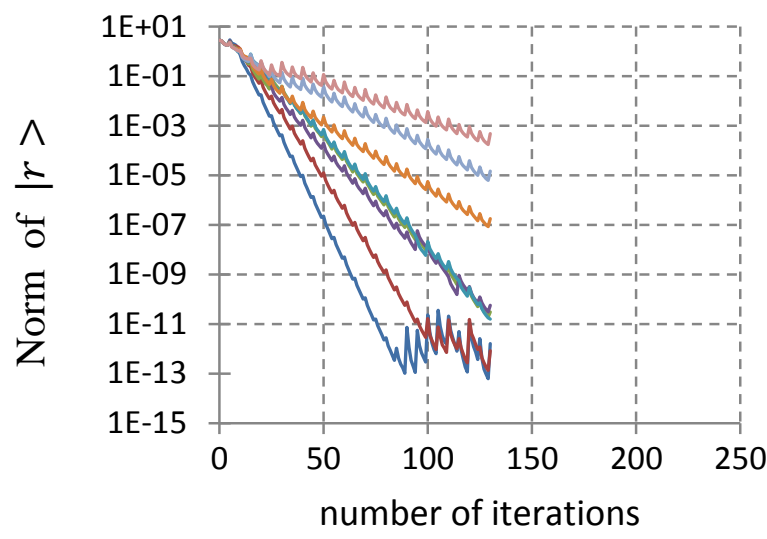

Figure 4.9 Results of Ladder 215S(24), block $=6$ by $\mathrm{RMM} V_{\max }$

iteration $=125(25)$,

time $=60.9 \mathrm{~s}$

on an Xeon Phi in report 1

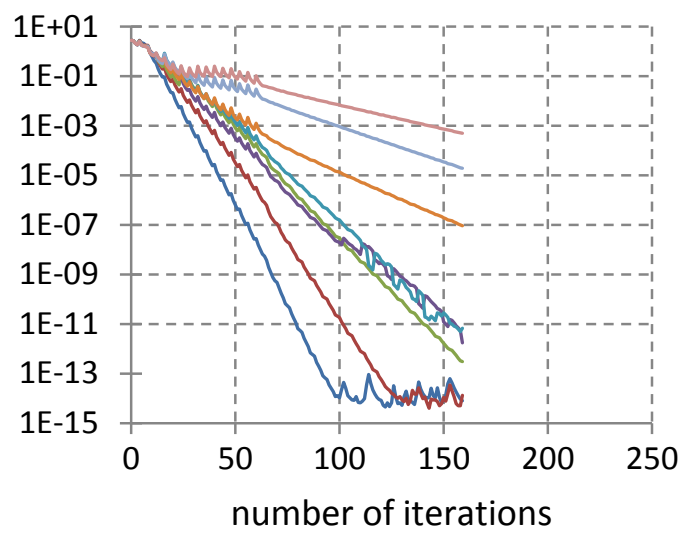

Figure 4.10 Results of Ladder 215S(24), block $=6 / 4$ by $R M M V_{\max } \& V_{\min }$ iteration $=60(12) / 60(20)$, time $=28.1 / 16.8(44.9) \mathrm{s}$ on an Xeon Phi in report 1

The error lines shown in the figures are the norm of residual vectors, because there are 8 eigenvalues were calculated, so there are 8 curves plotted in each figure.

Figure 4.1, Figure 4.3, Figure 4.5, Figure 4.7 and Figure 4.9 show results of RMM $V_{\max }$ method with different block size from 2 to 6 . As a measure for the error of the trial vectors, these figures show the norms of the residual vector as a function of the iteration number. When the block size increases, the time to reaching convergence shorter, but it required more memory. Just several error of eigenvalues can reach $5 \times 10^{-4}$ with block $=2$ and 3 , after block size larger than 3 , all eight eigenvalues can convergence at $5 \times 10^{-4}$. 
Figure 4.2, Figure 4.4, Figure 4.6, Figure 4.8 and Figure 4.10 show results of RMM $V_{\text {max }}$ and RMM $V_{\min }$ combination method with different block size from 2 to 6 in $\mathrm{RMM} V_{\max }$, and block size $=4$ in $\mathrm{RMM} V_{\min }$. They use the same initial eigenvectors as the ones in RMM $V_{\max }$ method. For this reason the first part of the graphs in the RMM combination methods are the same as RMM $V_{\max }$ when they have same block size. After the block size increases to 4 or bigger in RMM $V_{\max }$ and RMM $V_{\min }$ combination method, all eigenvalues finally reach the tolerance level.
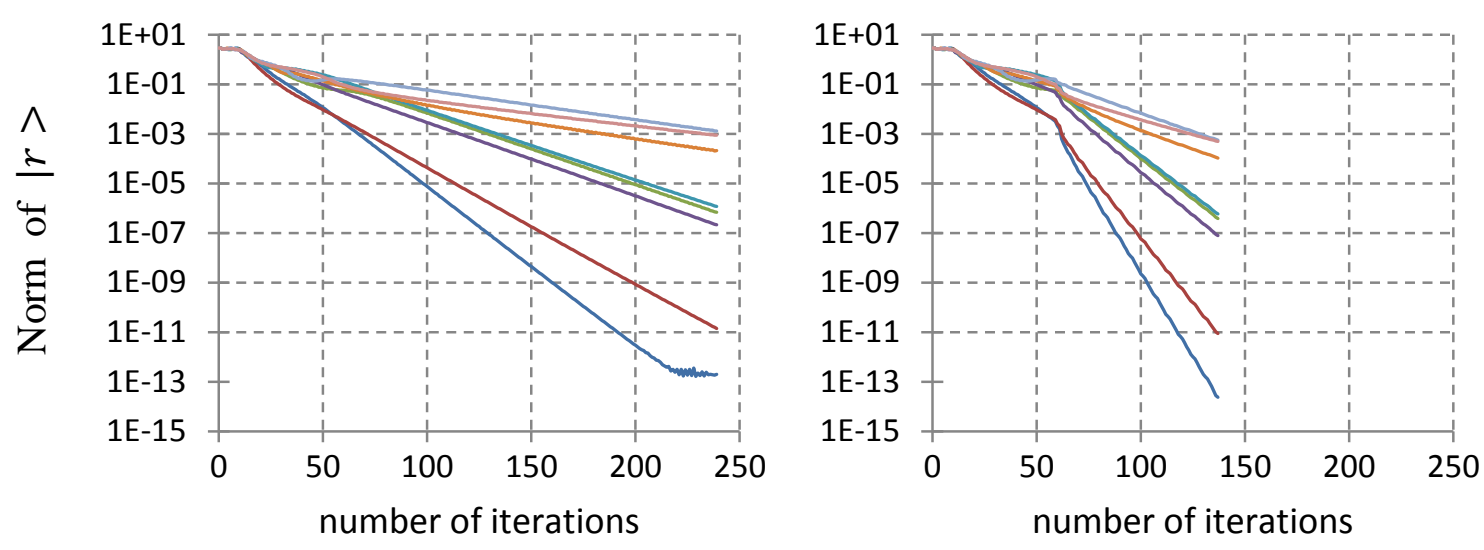

Figure 4.11 Results of Ladder 215S(24), Figure 4.12 Results of Ladder 215S(24), block $=2$ by BKDV block $=2 / 4$ by BKDV and RMM $V_{\min }$ iteration $=240$, iteration $=60(60) / 78(26)$, time $=956.5 \mathrm{~s}$ time $=239 \cdot 6 / 21 \cdot 7(261,3) \mathrm{s}$ on an Xeon Phi in report 1 on an Xeon Phi in report 1 


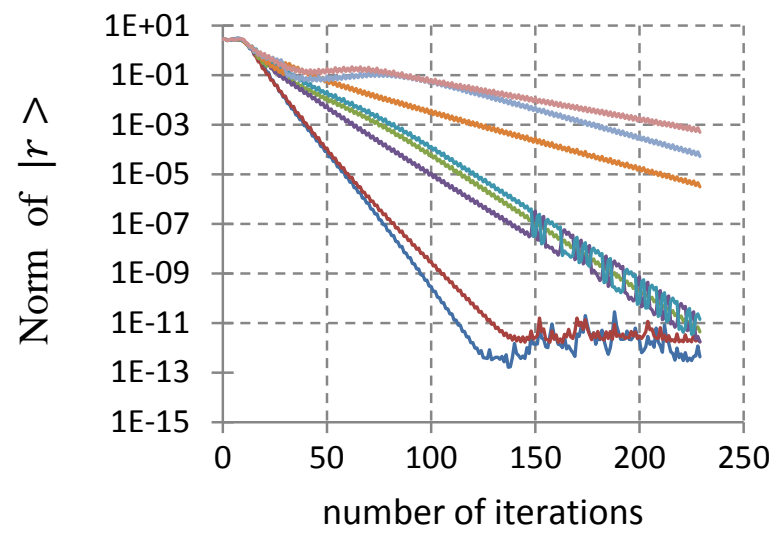

Figure 4.13 Results of Ladder 215S(24),

$$
\text { block }=3 \text { by BKDV }
$$

iteration $=240$,

time $=956.2 \mathrm{~s}$

on an Xeon Phi in report 1

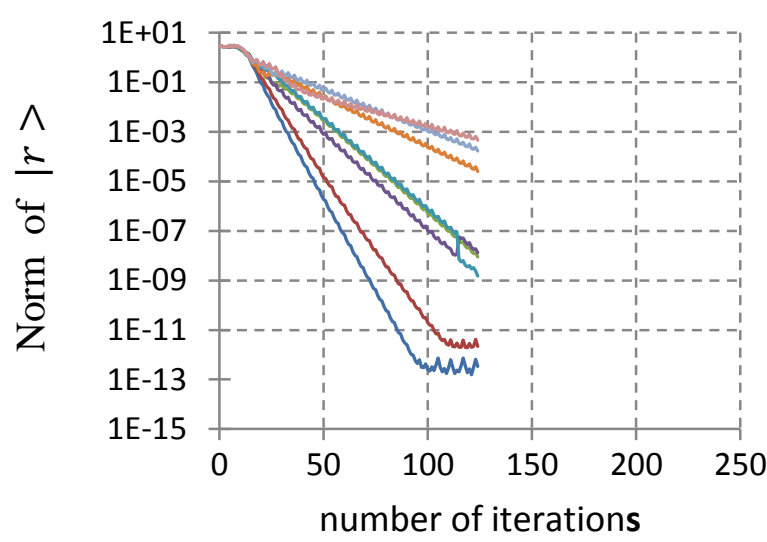

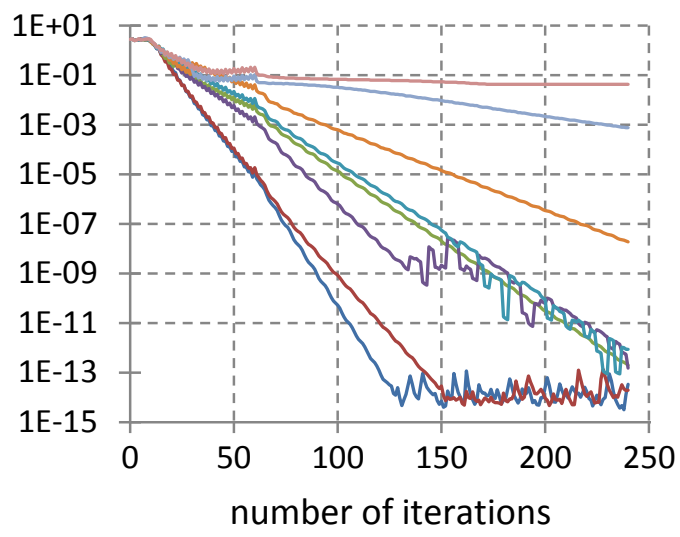

Figure 4.14 Results of Ladder 215S(24), block $=3 / 4$ by BKDV and RMM $V_{\min }$ iteration $=60 / 180(60)$, time $=250.5 / 50.0(300.5) \mathrm{s}$ on an Xeon Phi in report 1

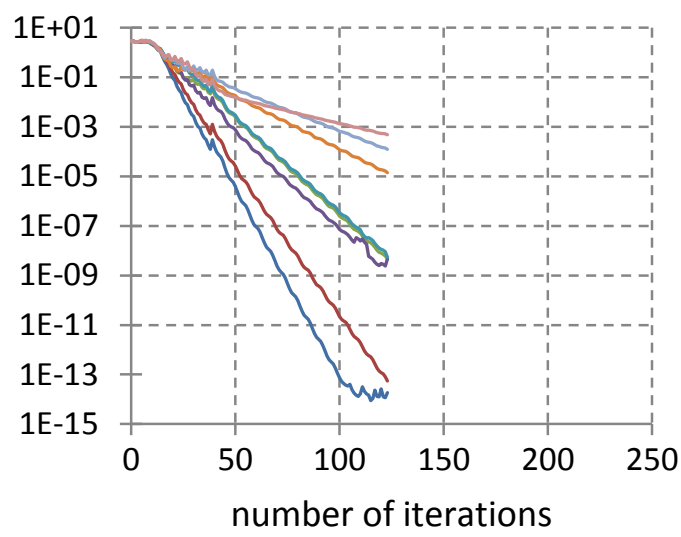

Figure 4.15 Results of Ladder 215S(24), Figure 4.16 Results of Ladder 215S(24), block $=4$ by BKDV block $=4 / 4$ by BKDV and RMM $V_{\min }$ iteration $=125$, iteration $=39 / 84(28)$, time $=521.5 \mathrm{~s}$ time $=160.3 / 23.4(183.7) \mathrm{s}$ on an Xeon Phi in report 1 on an Xeon Phi in report 1 


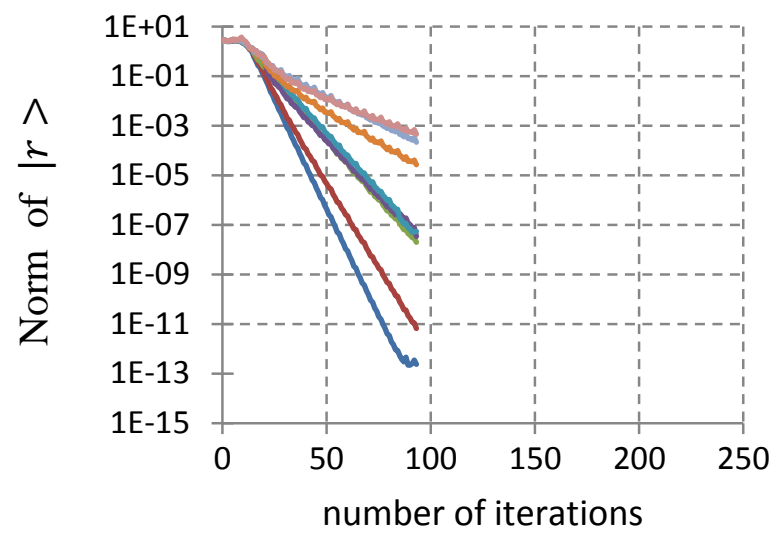

Figure 4.17 Results of Ladder 215S(24),

block = 5 by BKDV

iteration $=94$,

time $=395.5 \mathrm{~s}$

on an Xeon Phi in report 1

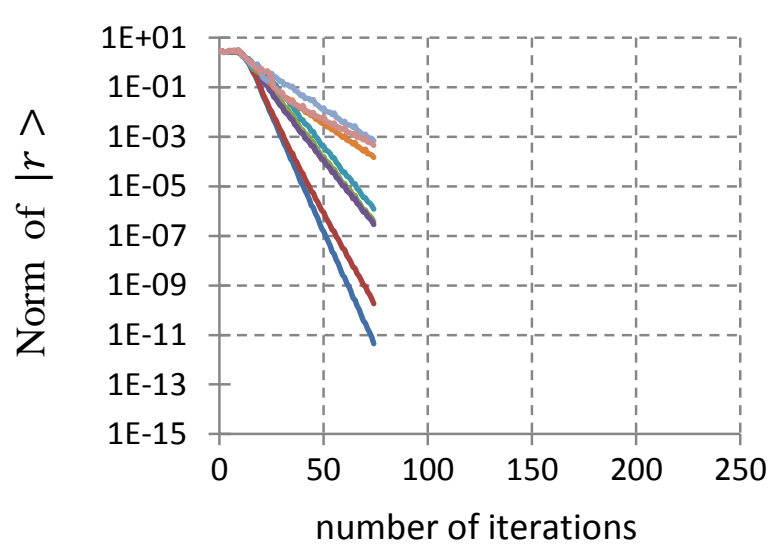

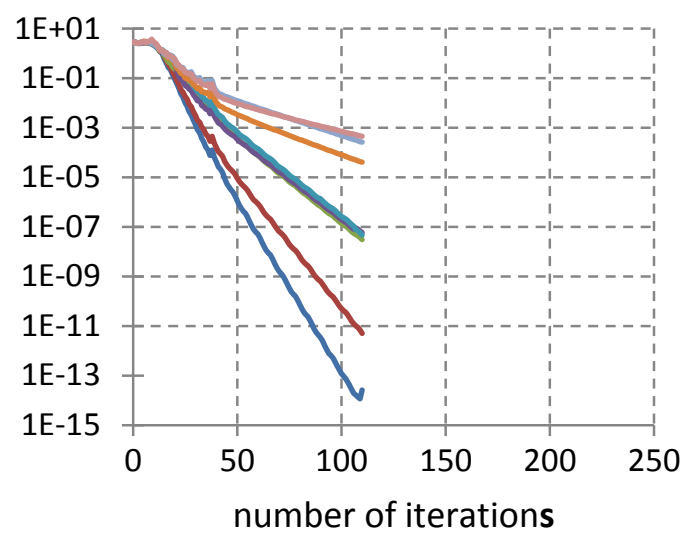

Figure 4.18 Results of Ladder 215S(24), block $=5 / 4$ by BKDV and RMM $V_{\min }$ iteration $=38 / 72(24)$, time $=159.8 / 20.1(179.8) \mathrm{s}$ on an Xeon Phi in report 1

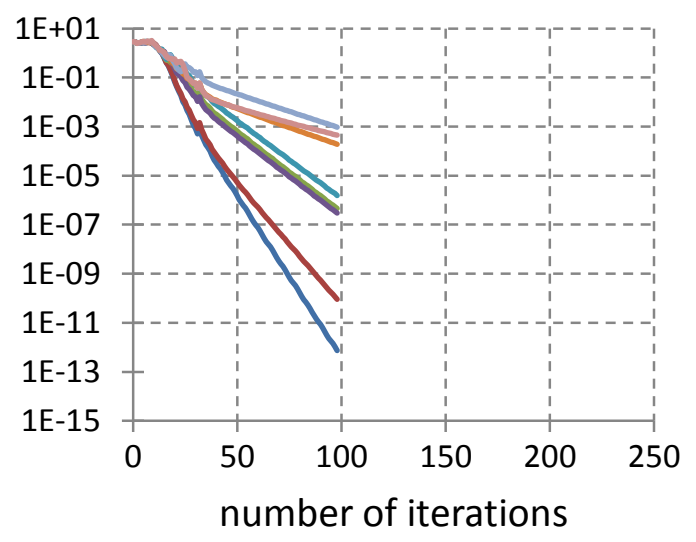

Figure 4.19 Results of Ladder 215S(24), Figure 4.20 Results of Ladder 215S(24), block $=6$ by BKDV block $=6 / 4$ by BKDV and RMM $V_{\min }$ iteration $=75$, iteration $=32 / 66(22)$, time $=321.7 \mathrm{~s}$ time $=138.6 / 156.977(295.530 .9) \mathrm{s}$ on an Xeon Phi in report 1 on an Xeon Phi in report 1 
Figure 4.11, Figure 4.13, Figure 4.15, Figure 4.17 and Figure 4.19 show the results of the Block Davidson method with block sizes of 2, $3,4,5$ and 6 . When the block size = 4, 8 all eigenvalues can reach convergence.

Figure 4.12, Figure 4.14, Figure 4.16, Figure 4.18 and Figure 4.20 show results of the Block Davidson and RMM $V_{\min }$ combination method with different block size from 2 to 6 , when the block size $=4,5$ and 6, the eigenvectors calculated by the Block Davidson are good enough, after calculation switch to RMM $V_{\min }$, all eigenvalues can convergence at final.

According to the reports, we observed that Block Davidson needs much more sys operations $(A \mid v>)$ than RMM-DIIS, this operation needs a certain time to perform. When number of block size was increasing, greater memory was required and less iterations were needed to reach convergent. In most cases, when the block size $=4$ or is over 4 , it could obtain appropriate eigenvectors and the corresponding eigenvalues were going to convergent. Therefore, in following programs, default block size is set by 4 . 


\subsection{Results of Block Davidson and RMM-DIIS}

In this section I tried to calculate the lowest 8 eigenvalues via 4 different algorithms on these six different models with size $N=18$ and 24 on Odin with setting MPI and OMP using 8 threads. (As before four algorithms are 1. RMM-DIIS algorithm, 2. RMM-DIIS $V_{\max }$ and $V_{\min }$ combination method, 3. Block Davidson algorithm and 4. Block Davidson and RMM-DIIS $V_{\min }$ combination method) I find out the top 6 eigenvalues can all reach to convergence easily in all 6 models in this project, reason for why try to calculate the lowest 8 eigenvalues is that not all lowest 8 eigenvalues can convergence at final in all models, therefore, I calculated the lowest 8 eigenpairs to compare which model can easy achieve required tolerance for all top 8 eigenvalues. All calculations block size $=4$, iterations were stopped when the norms of all residual vectors were blow $5 \times 10^{-4}$. In order to make sure that the result do not depend on the initial vectors, the calculations were repeated five times with a different set of random staring vectors (Report 1-5). For each report the four algorithms were run with the same starting vectors and the execution time and the number of iterations were measured.

In following results table in this section, I recorded 4 attributions, included total number of iterations and iterations number of block in RMM-RITZ algorithm if applicable (in the brackets); convergence status for the lowest eight eigenvalues (if norm of residual vector below the tolerance); 'clock time' is the execution time in seconds; 'Memory' recorded the memory usage, I checked memory by 'top' command in terminal of Ubuntu. 


\subsubsection{Heisenberg Chain}

Table 4.13 shows the result about the number of iterations and the execution time of Chain(24) model by 4 different algorithms. Not all eight errors of eigenvalues can reach to $5 \times 10^{-4}$ in this model. In most reports, top seven eigenvalues can be convergence.

Table 4.14 shows the result about Chain(18) model by 4 different algorithms. There are two reports show all errors below $5 \times 10^{-4}$ in RMM-DIIS $V_{\text {max }}$ and $V_{\text {min }}$ combination method. The lowest $8^{\text {th }}$ eigenvalue did not satisfy required convergent another methods. 
Table 4.13 Results of Chain(24) model on Odin

\begin{tabular}{|c|c|c|c|c|c|c|}
\hline 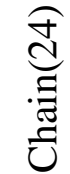 & Method name & $\begin{array}{l}\text { Number of } \\
\text { iteration } \\
\text { (block) }\end{array}$ & $\begin{array}{l}\text { Conv } \\
\text { ergent }\end{array}$ & \multicolumn{2}{|c|}{$\begin{array}{c}\text { Clock } \\
\text { time(second) }\end{array}$} & Memory \\
\hline \multirow{6}{*}{$\begin{array}{l}\vec{E} \\
\text { 这 } \\
\text { \&ै }\end{array}$} & BKDV 4 & 240 & $7 / 8$ & & 810.50 & $2327 \mathrm{MB}$ \\
\hline & \multirow{2}{*}{$\begin{array}{l}\text { BKDV } 4 \\
\text { RMM } V_{\text {min }} 4\end{array}$} & 38 & $8 / 8$ & 125.97 & \multirow{2}{*}{253.23} & $2327 \mathrm{MB}$ \\
\hline & & $180(60)$ & $7 / 8$ & 127.26 & & $2100 \mathrm{MB}$ \\
\hline & RMM $V_{\max } 4$ & $240(80)$ & $7 / 8$ & & 396.91 & $2760 \mathrm{MB}$ \\
\hline & RMM $V_{\max } 4$ & $60(20)$ & $6 / 8$ & 99.60 & \multirow{2}{*}{247.14} & $2780 \mathrm{MB}$ \\
\hline & $R M M V_{\min } 4$ & $180(60)$ & $7 / 8$ & 147.54 & & $2100 \mathrm{MB}$ \\
\hline \multirow{6}{*}{$\begin{array}{l}\stackrel{1}{0} \\
\stackrel{0}{2}\end{array}$} & BKDV 4 & 240 & $7 / 8$ & & 813.23 & $2327 \mathrm{MB}$ \\
\hline & BKDV 4 & 57 & $8 / 8$ & 189.12 & \multirow{2}{*}{334.55} & $2327 \mathrm{MB}$ \\
\hline & $\mathbf{R M M} V_{\min } 4$ & $180(60)$ & $7 / 8$ & 145.43 & & $2100 \mathrm{MB}$ \\
\hline & $R M M V_{\max } 4$ & $240(80)$ & $7 / 8$ & & 395.94 & $2760 \mathrm{MB}$ \\
\hline & RMM $V_{\max } 4$ & $57(19)$ & $8 / 8$ & 94.47 & \multirow{2}{*}{239.35} & $2780 \mathrm{MB}$ \\
\hline & $\mathbf{R M M} V_{\min } 4$ & $180(60)$ & $7 / 8$ & 144.88 & & $2100 \mathrm{MB}$ \\
\hline \multirow{6}{*}{$\begin{array}{l}\frac{1}{0} \\
\frac{0}{2} \\
\stackrel{2}{1}\end{array}$} & BKDV 4 & 240 & $7 / 8$ & & 820.20 & $2327 \mathrm{MB}$ \\
\hline & BKDV 4 & 35 & $8 / 8$ & 116.64 & \multirow{2}{*}{265.58} & $2327 \mathrm{MB}$ \\
\hline & $\mathrm{RMM} V_{\min } 4$ & $180(60)$ & $7 / 8$ & 148.94 & & $2100 \mathrm{MB}$ \\
\hline & $R M M V_{\max } 4$ & $240(80)$ & $7 / 8$ & & 401.13 & $2760 \mathrm{MB}$ \\
\hline & $\mathbf{R M M} V_{\max } 4$ & $45(15)$ & $8 / 8$ & 75.63 & \multirow{2}{*}{224.99} & $2780 \mathrm{MB}$ \\
\hline & $R M M V_{\min } 4$ & $180(60)$ & $7 / 8$ & 149.36 & & $2100 \mathrm{MB}$ \\
\hline \multirow{6}{*}{ 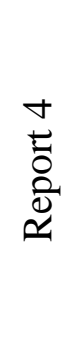 } & BKDV 4 & 240 & $7 / 8$ & & 825.19 & $2327 \mathrm{MB}$ \\
\hline & BKDV 4 & 50 & $8 / 8$ & 168.45 & \multirow{2}{*}{317.34} & $2327 \mathrm{MB}$ \\
\hline & $\mathrm{RMM} V_{\min } 4$ & $180(60)$ & $7 / 8$ & 148.89 & & $2100 \mathrm{MB}$ \\
\hline & RMM $V_{\max } 4$ & $240(80)$ & $7 / 8$ & & 402.33 & $2760 \mathrm{MB}$ \\
\hline & RMM $V_{\max } 4$ & $39(13)$ & $8 / 8$ & 65.55 & \multirow{2}{*}{214.91} & $2780 \mathrm{MB}$ \\
\hline & $\mathrm{RMM} V_{\min } 4$ & $180(60)$ & $7 / 8$ & 149.36 & & $2100 \mathrm{MB}$ \\
\hline \multirow{6}{*}{ 晜 } & BKDV 4 & 240 & $7 / 8$ & & 813.00 & $2327 \mathrm{MB}$ \\
\hline & BKDV 4 & 57 & $8 / 8$ & 191.21 & \multirow{2}{*}{338.52} & $2327 \mathrm{MB}$ \\
\hline & RMM $V_{\min } 4$ & $180(60)$ & $7 / 8$ & 147.31 & & $2100 \mathrm{MB}$ \\
\hline & RMM $V_{\max } 4$ & $192(64)$ & $8 / 8$ & & 318.60 & $2760 \mathrm{MB}$ \\
\hline & \multirow{2}{*}{ 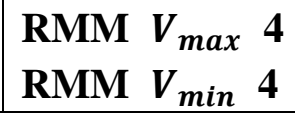 } & $36(12)$ & $8 / 8$ & 95.11 & \multirow{2}{*}{124.83} & $2780 \mathrm{MB}$ \\
\hline & & $180(60)$ & $7 / 8$ & 29.72 & & $2100 \mathrm{MB}$ \\
\hline
\end{tabular}


Table 4.14 Results of Chain(18) model on Odin

\begin{tabular}{|c|c|c|c|c|c|c|}
\hline 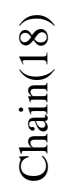 & Method name & $\begin{array}{l}\text { Number of } \\
\text { iteration } \\
\text { (block) }\end{array}$ & $\begin{array}{l}\text { Conve } \\
\text { rgent }\end{array}$ & \multicolumn{2}{|c|}{ Clock time(second) } & Memory \\
\hline \multirow{6}{*}{ 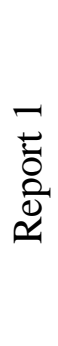 } & BKDV 4 & 240 & $7 / 8$ & & 4.29 & $689 \mathrm{MB}$ \\
\hline & \multirow{2}{*}{$\begin{array}{l}\text { BKDV } 4 \\
\text { RMM } V_{\min } 4\end{array}$} & 29 & $8 / 8$ & 0.54 & \multirow{2}{*}{1.14} & $689 \mathrm{MB}$ \\
\hline & & $180(60)$ & $7 / 8$ & 0.60 & & $689 \mathrm{MB}$ \\
\hline & RMM $V_{\max } 4$ & $240(80)$ & $7 / 8$ & \multirow{3}{*}{.34} & 1.93 & $694 \mathrm{MB}$ \\
\hline & \multirow{2}{*}{$\begin{array}{ll}R M M & V_{\max } 4 \\
R M M & V_{\min } 4\end{array}$} & $48(16)$ & $8 / 8$ & & \multirow{2}{*}{0.93} & $694 \mathrm{MB}$ \\
\hline & & $180(60)$ & $7 / 8$ & & & $689 \mathrm{MB}$ \\
\hline \multirow{6}{*}{ 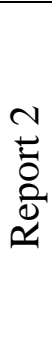 } & BKDV 4 & 240 & $7 / 8$ & \multicolumn{2}{|r|}{4.63} & $689 \mathrm{MB}$ \\
\hline & \multirow{2}{*}{$\begin{array}{l}\text { BKDV } 4 \\
\text { RMM } V_{\min } 4\end{array}$} & 47 & $8 / 8$ & 0.89 & \multirow{2}{*}{1.50} & $689 \mathrm{MB}$ \\
\hline & & $180(60)$ & $7 / 8$ & 0.61 & & $689 \mathrm{MB}$ \\
\hline & RMM $V_{\max } 4$ & $240(80)$ & $7 / 8$ & & 1.93 & $694 \mathrm{MB}$ \\
\hline & RMM $V_{\max } 4$ & $48(16)$ & $8 / 8$ & 0.37 & \multirow{2}{*}{0.75} & $694 \mathrm{MB}$ \\
\hline & $\mathbf{R M M} V_{\min } 4$ & $114(38)$ & $8 / 8$ & 0.38 & & $689 \mathrm{MB}$ \\
\hline \multirow{6}{*}{ 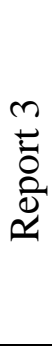 } & BKDV 4 & 240 & $7 / 8$ & & 4.67 & $689 \mathrm{MB}$ \\
\hline & BKDV 4 & 32 & $8 / 8$ & 0.61 & \multirow{2}{*}{1.21} & $689 \mathrm{MB}$ \\
\hline & $\mathrm{RMM} V_{\min } 4$ & $180(60)$ & $7 / 8$ & 0.60 & & $689 \mathrm{MB}$ \\
\hline & $R M M V_{\max } 4$ & $240(80)$ & $7 / 8$ & & 1.93 & $694 \mathrm{MB}$ \\
\hline & $\mathbf{R M M} V_{\max } 4$ & $45(15)$ & $8 / 8$ & 0.33 & \multirow{2}{*}{0.94} & $689 \mathrm{MB}$ \\
\hline & $\mathrm{RMM} V_{\min } 4$ & $114(38)$ & $8 / 8$ & 0.60 & & $689 \mathrm{MB}$ \\
\hline \multirow{6}{*}{$\begin{array}{l}\stackrel{0}{0} \\
\frac{0}{2}\end{array}$} & BKDV 4 & 240 & $7 / 8$ & & 4.53 & $689 \mathrm{MB}$ \\
\hline & BKDV 4 & 30 & $8 / 8$ & 0.55 & \multirow{2}{*}{1.13} & $689 \mathrm{MB}$ \\
\hline & $\mathrm{RMM} V_{\min } 4$ & $180(60)$ & $7 / 8$ & 0.58 & & $689 \mathrm{MB}$ \\
\hline & RMM $V_{\max } 4$ & $240(80)$ & $7 / 8$ & & 1.93 & 694MB \\
\hline & RMM $V_{\max } 4$ & $39(13)$ & $8 / 8$ & 0.29 & \multirow{2}{*}{0.87} & $689 \mathrm{MB}$ \\
\hline & $\mathrm{RMM} V_{\min } 4$ & $180(60)$ & $7 / 8$ & 0.58 & & $689 \mathrm{MB}$ \\
\hline \multirow{6}{*}{$\begin{array}{l}n \\
\stackrel{0}{0} \\
\stackrel{0}{2} \\
\approx\end{array}$} & BKDV 4 & 240 & $7 / 8$ & & 4.69 & $689 \mathrm{MB}$ \\
\hline & \multirow{2}{*}{$\begin{array}{l}\text { BKDV } 4 \\
\text { RMM } V_{\min } 4\end{array}$} & 28 & $8 / 8$ & 0.55 & \multirow{2}{*}{1.16} & $689 \mathrm{MB}$ \\
\hline & & $180(60)$ & $7 / 8$ & 0.61 & & $689 \mathrm{MB}$ \\
\hline & RMM $V_{\max } 4$ & $240(80)$ & $7 / 8$ & & .96 & $694 \mathrm{MB}$ \\
\hline & \multirow{2}{*}{ 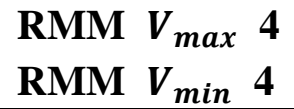 } & $36(12)$ & $8 / 8$ & 0.27 & 087 & $694 \mathrm{MB}$ \\
\hline & & $180(60)$ & $7 / 8$ & 0.61 & & $689 \mathrm{MB}$ \\
\hline
\end{tabular}




\subsubsection{Heisenberg Ladder}

Table 4.15 Results of Ladder2(24) model on Odin

\begin{tabular}{|c|c|c|c|c|c|c|}
\hline 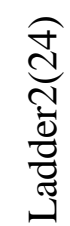 & Method name & $\begin{array}{l}\text { Number of } \\
\text { iteration } \\
\text { (block) }\end{array}$ & $\begin{array}{l}\text { Conve } \\
\text { rgent }\end{array}$ & \multicolumn{2}{|c|}{ Clock time(second) } & Memory \\
\hline \multirow{6}{*}{$\begin{array}{l}\bar{\partial} \\
\frac{0}{2} \\
\simeq\end{array}$} & BKDV 4 & 127 & $8 / 8$ & & 485.35 & $2520 \mathrm{MB}$ \\
\hline & \multirow{2}{*}{$\begin{array}{l}\text { BKDV } 4 \\
\text { RMM } V_{\min } 4 \\
\end{array}$} & 60 & $7 / 8$ & 228.20 & \multirow{2}{*}{348.47} & $2520 \mathrm{MB}$ \\
\hline & & $120(40)$ & $8 / 8$ & 120.26 & & $2293 \mathrm{MB}$ \\
\hline & RMM $V_{\max } 4$ & $165(55)$ & $8 / 8$ & & 300.04 & $2954 \mathrm{MB}$ \\
\hline & $\mathrm{RMM} V_{\max } 4$ & $60(20)$ & $8 / 8$ & 109.29 & \multirow{2}{*}{144.65} & $2974 \mathrm{MB}$ \\
\hline & $\mathbf{R M M} V_{\min } 4$ & $36(12)$ & $8 / 8$ & 35.36 & & $2293 \mathrm{MB}$ \\
\hline \multirow{6}{*}{ 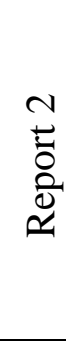 } & BKDV 4 & 143 & $8 / 8$ & & 549.74 & $2520 \mathrm{MB}$ \\
\hline & BKDV 4 & 53 & $8 / 8$ & 200.87 & \multirow{2}{*}{274.38} & $2520 \mathrm{MB}$ \\
\hline & $R M M V_{\min } 4$ & $75(25)$ & $8 / 8$ & 73.51 & & $2293 \mathrm{MB}$ \\
\hline & RMM $V_{\max } 4$ & 153(51) & $8 / 8$ & \multicolumn{2}{|r|}{284.16} & $2954 \mathrm{MB}$ \\
\hline & \multirow{2}{*}{$\begin{array}{ll}\text { RMM } V_{\max } & 4 \\
\text { RMM } V_{\text {min }} 4 \\
\end{array}$} & $60(20)$ & $7 / 8$ & 109.85 & \multirow{2}{*}{142.35} & $2974 \mathrm{MB}$ \\
\hline & & $33(11)$ & $8 / 8$ & 32.50 & & $2293 \mathrm{MB}$ \\
\hline \multirow{6}{*}{ 莒 } & BKDV 4 & 149 & $8 / 8$ & \multicolumn{2}{|r|}{568.20} & $2520 \mathrm{MB}$ \\
\hline & \multirow{2}{*}{$\begin{array}{l}\text { BKDV } 4 \\
\text { RMM } V_{\text {min }} 4\end{array}$} & 60 & $8 / 8$ & 226.18 & \multirow{2}{*}{304.82} & $2520 \mathrm{MB}$ \\
\hline & & $81(27)$ & $8 / 8$ & 78.64 & & $2293 \mathrm{MB}$ \\
\hline & RMM $V_{\max } 4$ & 201(67) & $8 / 8$ & & 363.33 & $2954 \mathrm{MB}$ \\
\hline & \multirow{2}{*}{$\begin{array}{lll}\mathrm{RMM} & V_{\max } & 4 \\
\mathrm{RMM} & V_{\min } & 4 \\
\end{array}$} & $60(20)$ & $7 / 8$ & 108.88 & \multirow{2}{*}{172.93} & 2974MB \\
\hline & & $66(22)$ & $8 / 8$ & 64.05 & & $2293 \mathrm{MB}$ \\
\hline \multirow{6}{*}{ 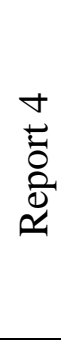 } & BKDV 4 & 152 & $8 / 8$ & \multicolumn{2}{|c|}{586.33} & $2520 \mathrm{MB}$ \\
\hline & \multirow{2}{*}{$\begin{array}{l}\text { BKDV } 4 \\
\text { RMM } V_{\text {min }} 4 \\
\end{array}$} & 60 & $8 / 8$ & 230.41 & \multirow{2}{*}{312.76} & $2520 \mathrm{MB}$ \\
\hline & & $84(28)$ & $8 / 8$ & 82.35 & & $2293 \mathrm{MB}$ \\
\hline & RMM $V_{\max } 4$ & $159(53)$ & $8 / 8$ & & 0.39 & $2954 \mathrm{MB}$ \\
\hline & RMM $V_{\max } 4$ & $60(20)$ & $7 / 8$ & 109.97 & \multirow{2}{*}{148.30} & 2974MB \\
\hline & $\mathrm{RMM} V_{\min } 4$ & $39(13)$ & $8 / 8$ & 38.33 & & $2293 \mathrm{MB}$ \\
\hline \multirow{6}{*}{ 范 } & BKDV 4 & 164 & $8 / 8$ & & 629.16 & $2520 \mathrm{MB}$ \\
\hline & \multirow{2}{*}{$\begin{array}{l}\text { BKDV } 4 \\
\text { RMM } V_{\text {min }} 4\end{array}$} & 60 & $7 / 8$ & 229.66 & \multirow{2}{*}{324.15} & $2520 \mathrm{MB}$ \\
\hline & & $96(32)$ & $8 / 8$ & 94.50 & & $2293 \mathrm{MB}$ \\
\hline & RMM $V_{\max } 4$ & $159(53)$ & $8 / 8$ & & 285.49 & $2954 \mathrm{MB}$ \\
\hline & RMM $V_{\max } 4$ & $57(19)$ & $8 / 8$ & 100.99 & 13371 & $2974 \mathrm{MB}$ \\
\hline & $\mathbf{R M M} V_{\min } 4$ & $33(11)$ & $8 / 8$ & 32.72 & 153.11 & $2293 \mathrm{MB}$ \\
\hline
\end{tabular}


Table 4.16 Results of Ladder2(18) model on Odin

\begin{tabular}{|c|c|c|c|c|c|c|}
\hline 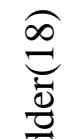 & Method name & $\begin{array}{l}\text { Number of } \\
\text { iteration }\end{array}$ & $\begin{array}{l}\text { Conve } \\
\text { rgent }\end{array}$ & \multicolumn{2}{|c|}{ Clock time(second) } & Memory \\
\hline \multirow{6}{*}{ 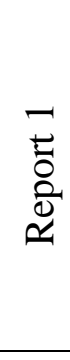 } & RMM $V_{\max } 4$ & $240(80)$ & $6 / 8$ & \multicolumn{2}{|r|}{2.05} & $699 \mathrm{MB}$ \\
\hline & \multirow{2}{*}{ 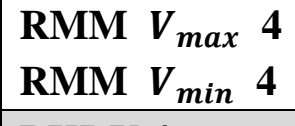 } & $39(13)$ & $8 / 8$ & 0.32 & \multirow{2}{*}{1.01} & $699 \mathrm{MB}$ \\
\hline & & $180(60)$ & $6 / 8$ & 0.69 & & $692 \mathrm{MB}$ \\
\hline & BKDV 4 & 240 & $6 / 8$ & & 5.32 & $692 \mathrm{MB}$ \\
\hline & BKDV 4 & 29 & $8 / 8$ & 0.62 & \multirow{2}{*}{1.31} & $692 \mathrm{MB}$ \\
\hline & $\mathbf{R M M} V_{\min } \mathbf{4}$ & $180(60)$ & $6 / 8$ & 0.69 & & $692 \mathrm{MB}$ \\
\hline \multirow{6}{*}{ 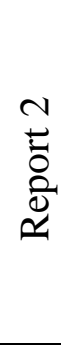 } & RMM $V_{\max } 4$ & $240(80)$ & $6 / 8$ & & 2.06 & $699 \mathrm{MB}$ \\
\hline & RMM $V_{\max } 4$ & $39(13)$ & $8 / 8$ & 0.31 & \multirow{2}{*}{0.99} & $699 \mathrm{MB}$ \\
\hline & RMM $V_{\min } 4$ & $180(60)$ & $6 / 8$ & 0.69 & & $692 \mathrm{MB}$ \\
\hline & BKDV 4 & 240 & $6 / 8$ & & 5.28 & $692 \mathrm{MB}$ \\
\hline & \multirow{2}{*}{$\begin{array}{l}\text { BKDV } 4 \\
\text { RMM } V_{\text {min }} 4 \\
\end{array}$} & 29 & $8 / 8$ & 0.62 & \multirow{2}{*}{1.31} & $692 \mathrm{MB}$ \\
\hline & & $180(60)$ & $6 / 8$ & 0.69 & & $692 \mathrm{MB}$ \\
\hline \multirow{6}{*}{$\begin{array}{l}\text { m } \\
\stackrel{0}{0} \\
\text { के }\end{array}$} & $\mathbf{R M M} V_{\max } 4$ & $240(80)$ & $6 / 8$ & & 2.04 & $699 \mathrm{MB}$ \\
\hline & \multirow{2}{*}{$\begin{array}{lll}R M M & V_{\max } & 4 \\
R M M & V_{\min } & 4 \\
\end{array}$} & $33(11)$ & $8 / 8$ & 0.25 & \multirow{2}{*}{0.92} & $699 \mathrm{MB}$ \\
\hline & & $180(60)$ & $6 / 8$ & 0.67 & & $692 \mathrm{MB}$ \\
\hline & BKDV 4 & 240 & $6 / 8$ & \multirow{3}{*}{$\begin{array}{l}.53 \\
.68 \\
\end{array}$} & 5.11 & $692 \mathrm{MB}$ \\
\hline & \multirow{2}{*}{$\begin{array}{l}\text { BKDV } 4 \\
\text { RMM } V_{\min } 4 \\
\end{array}$} & 26 & $8 / 8$ & & \multirow{2}{*}{1.21} & $692 \mathrm{MB}$ \\
\hline & & $180(60)$ & $6 / 8$ & & & $692 \mathrm{MB}$ \\
\hline \multirow{6}{*}{$\begin{array}{l}+ \\
\stackrel{0}{0} \\
\text { ¿े }\end{array}$} & $\mathbf{R M M} V_{\max } 4$ & $240(80)$ & $6 / 8$ & \multicolumn{2}{|r|}{2.01} & $699 \mathrm{MB}$ \\
\hline & \multirow{2}{*}{$\begin{array}{l}R M M V_{\max } 4 \\
R M M V_{\min } 4 \\
\end{array}$} & $39(13)$ & $8 / 8$ & 0.31 & \multirow{2}{*}{0.99} & $699 \mathrm{MB}$ \\
\hline & & $180(60)$ & $6 / 8$ & 0.69 & & $692 \mathrm{MB}$ \\
\hline & BKDV 4 & 240 & $6 / 8$ & & 5.17 & $692 \mathrm{MB}$ \\
\hline & BKDV 4 & 26 & $8 / 8$ & 0.54 & 123 & $692 \mathrm{MB}$ \\
\hline & RMM $V_{\min } 4$ & $180(60)$ & $6 / 8$ & 0.69 & & $692 \mathrm{MB}$ \\
\hline & RMM $V_{\max } 4$ & $240(80)$ & $6 / 8$ & & 2.00 & $699 \mathrm{MB}$ \\
\hline & RMM $V_{\max } 4$ & $39(13)$ & $8 / 8$ & 0.30 & 0.97 & $699 \mathrm{MB}$ \\
\hline 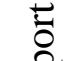 & $R M M V_{\min } 4$ & $180(60)$ & $6 / 8$ & 0.67 & ות. & $692 \mathrm{MB}$ \\
\hline$\stackrel{\bar{Q}}{q}$ & BKDV 4 & 240 & $6 / 8$ & & 5.11 & $692 \mathrm{MB}$ \\
\hline & BKDV 4 & 26 & $8 / 8$ & 0.53 & 1.21 & $692 \mathrm{MB}$ \\
\hline & RMM $V_{\min } 4$ & $180(60)$ & $6 / 8$ & 0.67 & & $692 \mathrm{MB}$ \\
\hline
\end{tabular}


Table 4.15 shows the result about the number of iterations and the execution time of the Ladder2(24) model by 4 different algorithms. All five reports show Ladder2(24) model can easy reach convergence form random star vectors. The variants RMM-DIIS (RMM-

DIIS $V_{\max }$ and $V_{\max }$ ) method is the fastest of all, the number of iterations of it is also the least one. The execution time of the RMM-DIIS only and the Block Davidson and the RMM-DIIS combination method are not much different, they are much faster than the Block Davidson.

From Table 4.16 , we can find that all reports show the lowest six eigenvalues of Ladder2(18) can be convergence by assort algorithms, and all eight eigenvalues are convergence to $5 \times 10^{-2}$ in combination method.

\subsubsection{Heisenberg Ladder 215C}

Table 4.17 shows the result about the number of iterations and the execution time of the Ladder215C(18) model by 4 different algorithms. All reports show the lowest seven eigenvalues can be convergence.

Table 4.18 shows the results about the number of iterations and the execution time of the Ladder215C(18) model by 4 different algorithms. In four out of the five reports at least one of the RMM-DIIS variants (RMM-DIIS $V_{\max }$ and $V_{\max }$ ) reached convergence for all eight eigenvalues. I think that the initial eight vectors in the last report were badly chosen, so that the $8^{\text {th }}$ eigenvalue converged only slowly. 
Table 4.17 Results of Ladder215C(24) model on Odin

\begin{tabular}{|c|c|c|c|c|c|c|}
\hline 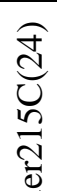 & Method name & $\begin{array}{l}\text { Number of } \\
\text { iteration }\end{array}$ & $\begin{array}{l}\text { Conve } \\
\text { rgent }\end{array}$ & \multicolumn{2}{|c|}{ Clock time(second) } & Memory \\
\hline \multirow{6}{*}{ 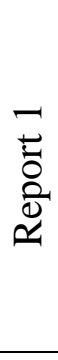 } & BKDV 4 & 240 & $7 / 8$ & & 932.50 & $2520 \mathrm{MB}$ \\
\hline & \multirow{2}{*}{$\begin{array}{l}\text { BKDV } 4 \\
\text { RMM } V_{\text {min }} 4\end{array}$} & 47 & $8 / 8$ & 177.77 & \multirow{2}{*}{354.25} & $2520 \mathrm{MB}$ \\
\hline & & $180(60)$ & $7 / 8$ & 176.48 & & $2293 \mathrm{MB}$ \\
\hline & RMM $V_{\max } 4$ & $240(80)$ & $7 / 8$ & & 438.45 & $2954 \mathrm{MB}$ \\
\hline & $\mathrm{RMM} V_{\max } 4$ & $54(18)$ & $8 / 8$ & 99.38 & \multirow{2}{*}{275.03} & $2974 \mathrm{MB}$ \\
\hline & $\mathrm{RMM} V_{\min } 4$ & $180(60)$ & $7 / 8$ & 175.65 & & $2293 \mathrm{MB}$ \\
\hline \multirow{6}{*}{ 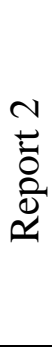 } & BKDV 4 & 240 & $7 / 8$ & & 927.53 & $2520 \mathrm{MB}$ \\
\hline & BKDV 4 & 47 & $8 / 8$ & 96.74 & \multirow{2}{*}{272.49} & $2520 \mathrm{MB}$ \\
\hline & $\mathrm{RMM} V_{\min } 4$ & $180(60)$ & $7 / 8$ & 175.76 & & $2293 \mathrm{MB}$ \\
\hline & RMM $V_{\max } 4$ & $240(80)$ & $7 / 8$ & \multicolumn{2}{|r|}{435.83} & $2954 \mathrm{MB}$ \\
\hline & \multirow{2}{*}{ 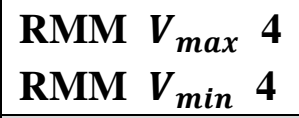 } & $54(18)$ & $8 / 8$ & 104.50 & \multirow{2}{*}{280.20} & 2974MB \\
\hline & & $180(60)$ & $7 / 8$ & 175.70 & & $2293 \mathrm{MB}$ \\
\hline \multirow{6}{*}{$\simeq$} & BKDV 4 & 240 & $7 / 8$ & & 919.23 & $2520 \mathrm{MB}$ \\
\hline & BKDV 4 & 27 & $8 / 8$ & 99.82 & \multirow{2}{*}{274.78} & $2520 \mathrm{MB}$ \\
\hline & $\mathrm{RMM} V_{\min } 4$ & $180(60)$ & $7 / 8$ & 174.96 & & $2293 \mathrm{MB}$ \\
\hline & RMM $V_{\max } 4$ & $240(80)$ & $7 / 8$ & & 436.77 & $2954 \mathrm{MB}$ \\
\hline & $\mathrm{RMM} V_{\max } 4$ & $57(19)$ & $8 / 8$ & 103.81 & \multirow{2}{*}{279.04} & 2974MB \\
\hline & RMM $V_{\min } 4$ & $180(60)$ & $7 / 8$ & 175.23 & & $2293 \mathrm{MB}$ \\
\hline \multirow{6}{*}{ 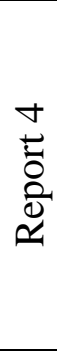 } & BKDV 4 & 240 & $7 / 8$ & & 921.35 & $2520 \mathrm{MB}$ \\
\hline & BKDV 4 & 30 & $8 / 8$ & 111.26 & \multirow{2}{*}{286.21} & $2520 \mathrm{MB}$ \\
\hline & $\mathrm{RMM} V_{\min } 4$ & $180(60)$ & $7 / 8$ & 174.94 & & $2293 \mathrm{MB}$ \\
\hline & RMM $V_{\max } 4$ & $240(80)$ & $7 / 8$ & & 435.58 & $2954 \mathrm{MB}$ \\
\hline & RMM $V_{\max } 4$ & $60(20)$ & $7 / 8$ & 109.23 & \multirow{2}{*}{284.23} & $2974 \mathrm{MB}$ \\
\hline & RMM $V_{\min } 4$ & $180(60)$ & $7 / 8$ & 175.00 & & $2293 \mathrm{MB}$ \\
\hline \multirow{6}{*}{ 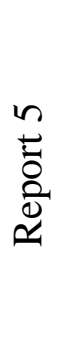 } & BKDV 4 & 240 & $7 / 8$ & & 858.31 & $2520 \mathrm{MB}$ \\
\hline & BKDV 4 & 40 & $8 / 8$ & 144.18 & \multirow{2}{*}{304.01} & $2520 \mathrm{MB}$ \\
\hline & $\mathrm{RMM} V_{\min } 4$ & $180(60)$ & $7 / 8$ & 159.83 & & $2293 \mathrm{MB}$ \\
\hline & RMM $V_{\max } 4$ & $240(80)$ & $7 / 8$ & & 397.89 & 2954MB \\
\hline & $\mathrm{RMM} V_{\max } 4$ & $51(17)$ & $8 / 8$ & 89.30 & \multirow{2}{*}{249.48} & 2974MB \\
\hline & RMM $V_{\min } 4$ & $180(60)$ & $7 / 8$ & 160.19 & & $2293 \mathrm{MB}$ \\
\hline
\end{tabular}


Table 4.18 Results of Ladder215C(18) model on Odin

\begin{tabular}{|c|c|c|c|c|c|c|}
\hline 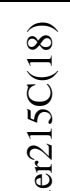 & Method name & $\begin{array}{l}\text { Number of } \\
\text { iteration }\end{array}$ & $\begin{array}{l}\text { Conv } \\
\text { ergent }\end{array}$ & \multicolumn{2}{|c|}{ Clock time(second) } & Memory \\
\hline \multirow{6}{*}{ 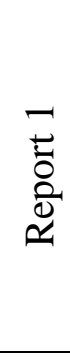 } & BKDV 4 & 240 & $7 / 8$ & \multicolumn{2}{|r|}{5.15} & $692 \mathrm{MB}$ \\
\hline & \multirow{2}{*}{$\begin{array}{l}\text { BKDV } 4 \\
\text { RMM } V_{\text {min }} 4\end{array}$} & 60 & $7 / 8$ & 1.28 & \multirow{2}{*}{1.96} & $692 \mathrm{MB}$ \\
\hline & & $180(60)$ & $7 / 8$ & 0.68 & & $692 \mathrm{MB}$ \\
\hline & $R M M V_{\max } 4$ & $240(60)$ & $7 / 8$ & & 2.02 & 699MB \\
\hline & $R M M V_{\max } 4$ & $60(20)$ & $7 / 8$ & 0.46 & \multirow{2}{*}{0.76} & $699 \mathrm{MB}$ \\
\hline & $R M M V_{\min } 4$ & $78(26)$ & $8 / 8$ & 0.30 & & $692 \mathrm{MB}$ \\
\hline \multirow{6}{*}{ 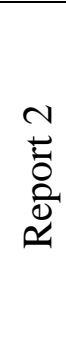 } & BKDV 4 & 160 & $8 / 8$ & & 3.45 & $692 \mathrm{MB}$ \\
\hline & BKDV 4 & 41 & $8 / 8$ & 0.86 & \multirow{2}{*}{1.34} & $692 \mathrm{MB}$ \\
\hline & $R M M V_{\min } 4$ & $123(41)$ & $8 / 8$ & 0.47 & & $692 \mathrm{MB}$ \\
\hline & $R M M V_{\max } 4$ & $213(70)$ & $8 / 8$ & & 1.87 & 699MB \\
\hline & RMM $V_{\max } 4$ & $60(20)$ & $7 / 8$ & 0.47 & \multirow{2}{*}{0.65} & $699 \mathrm{MB}$ \\
\hline & RMM $V_{\min } 4$ & $48(16)$ & $8 / 8$ & 0.18 & & $692 \mathrm{MB}$ \\
\hline \multirow{6}{*}{ 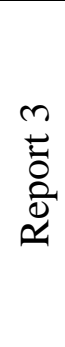 } & BKDV 4 & 143 & $8 / 8$ & & 3.06 & $692 \mathrm{MB}$ \\
\hline & BKDV 4 & 59 & $8 / 8$ & 1.25 & \multirow{2}{*}{1.53} & $692 \mathrm{MB}$ \\
\hline & $R M M V_{\min } 4$ & $75(25)$ & $8 / 8$ & 0.28 & & $692 \mathrm{MB}$ \\
\hline & RMM $V_{\max } 4$ & $240(80)$ & $7 / 8$ & & 1.99 & 699MB \\
\hline & RMM $V_{\max } 4$ & $60(20)$ & $8 / 8$ & 0.46 & \multirow{2}{*}{0.83} & 699MB \\
\hline & $\mathrm{RMM} V_{\min } 4$ & 99(33) & $8 / 8$ & 0.37 & & $692 \mathrm{MB}$ \\
\hline \multirow{6}{*}{ 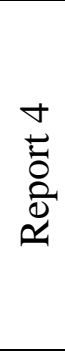 } & \begin{tabular}{|l|} 
BKDV 4 \\
\end{tabular} & 240 & $7 / 8$ & & 5.27 & $692 \mathrm{MB}$ \\
\hline & \multirow{2}{*}{\begin{tabular}{|l|} 
BKDV 4 \\
RMM $V_{\text {min }} 4$ \\
\end{tabular}} & 60 & $7 / 8$ & 1.30 & \multirow{2}{*}{2.00} & $692 \mathrm{MB}$ \\
\hline & & $180(60)$ & $7 / 8$ & 0.70 & & $692 \mathrm{MB}$ \\
\hline & $R M M V_{\max } 4$ & $135(45)$ & $8 / 8$ & & 1.25 & $699 \mathrm{MB}$ \\
\hline & RMM $V_{\max } 4$ & $48(16)$ & $8 / 8$ & 0.37 & \multirow{2}{*}{0.47} & $699 \mathrm{MB}$ \\
\hline & RMM $V_{\min } 4$ & $27(9)$ & $8 / 8$ & 0.10 & & $692 \mathrm{MB}$ \\
\hline \multirow{6}{*}{$\begin{array}{l}n \\
\stackrel{n}{0} \\
\stackrel{0}{2}\end{array}$} & BKDV 4 & 240 & $7 / 8$ & & 5.20 & $692 \mathrm{MB}$ \\
\hline & BKDV 4 & 60 & $7 / 8$ & 1.29 & \multirow{2}{*}{1.98} & $692 \mathrm{MB}$ \\
\hline & $R M M V_{\min } 4$ & $180(60)$ & $7 / 8$ & 0.69 & & $692 \mathrm{MB}$ \\
\hline & RMM $V_{\max } 4$ & $240(80)$ & $7 / 8$ & & 2.07 & $699 \mathrm{MB}$ \\
\hline & $R M M V_{\max } 4$ & $60(20)$ & $7 / 8$ & 0.47 & \multirow{2}{*}{1.15} & $699 \mathrm{MB}$ \\
\hline & RMM $V_{\min } 4$ & $180(60)$ & $7 / 8$ & 0.68 & & $692 \mathrm{MB}$ \\
\hline
\end{tabular}




\subsubsection{Heisenberg Ladder 215S}

Table 4.19 Results of Ladder215S(24) model on Odin

\begin{tabular}{|c|c|c|c|c|c|c|}
\hline 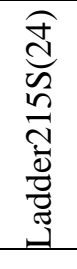 & \multirow[t]{2}{*}{ Method name } & \multirow{2}{*}{$\begin{array}{l}\text { Number of } \\
\text { iteration } \\
\text { (block) }\end{array}$} & \multirow{2}{*}{$\begin{array}{r}\text { Conv } \\
\text { ergent } \\
\mathbf{8 / 8}\end{array}$} & \multicolumn{2}{|c|}{ Clock time(second) } & \multirow{2}{*}{$\begin{array}{l}\text { Memory } \\
2520 \mathrm{MB}\end{array}$} \\
\hline \multirow{6}{*}{ 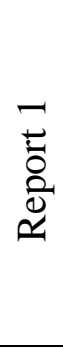 } & & & & & 538.96 & \\
\hline & \multirow{2}{*}{$\begin{array}{l}\text { BKDV } 4 \\
\text { RMM } V_{\min } 4\end{array}$} & $159(53)$ & $8 / 8$ & 200.11 & \multirow{2}{*}{276.40} & $2520 \mathrm{MB}$ \\
\hline & & $78(26)$ & $8 / 8$ & 76.28 & & $2293 \mathrm{MB}$ \\
\hline & RMM $V_{\max } 4$ & $162(54)$ & $8 / 8$ & \multicolumn{2}{|r|}{287.18} & $2954 \mathrm{MB}$ \\
\hline & \multirow{2}{*}{$\begin{array}{|lll|}\mathrm{RMM} & V_{\max } & 4 \\
\mathrm{RMM} & V_{\min } & 4 \\
\end{array}$} & $60(20)$ & $7 / 8$ & 106.37 & \multirow{2}{*}{141.59} & $2974 \mathrm{MB}$ \\
\hline & & $36(12)$ & $8 / 8$ & 35.22 & & $2293 \mathrm{MB}$ \\
\hline \multirow{6}{*}{$\overbrace{\substack{0 \\
0}}^{N}$} & BKDV 4 & 98 & $8 / 8$ & & 372.53 & $2520 \mathrm{MB}$ \\
\hline & \multirow{2}{*}{$\begin{array}{l}\text { BKDV } 4 \\
\text { RMM } V_{\min } 4 \\
\end{array}$} & $105(35)$ & $8 / 8$ & 130.89 & \multirow{2}{*}{186.61} & $2520 \mathrm{MB}$ \\
\hline & & $57(19)$ & $8 / 8$ & 55.73 & & $2293 \mathrm{MB}$ \\
\hline & RMM $V_{\max } 4$ & $153(51)$ & $8 / 8$ & \multicolumn{2}{|r|}{272.27} & $2954 \mathrm{MB}$ \\
\hline & \multirow{2}{*}{$\begin{array}{lll}\mathrm{RMM} & V_{\max } & 4 \\
\mathrm{RMM} & V_{\min } & 4 \\
\end{array}$} & $60(20)$ & $8 / 8$ & 106.31 & \multirow{2}{*}{135.85} & $2974 \mathrm{MB}$ \\
\hline & & $30(10)$ & $8 / 8$ & 29.54 & & $2293 \mathrm{MB}$ \\
\hline \multirow{6}{*}{$\stackrel{n}{0}_{\substack{0 \\
0}}^{0}$} & BKDV 4 & 140 & $8 / 8$ & \multicolumn{2}{|r|}{538.37} & $2520 \mathrm{MB}$ \\
\hline & \multirow{2}{*}{$\begin{array}{l}\text { BKDV } 4 \\
\text { RMM } V_{\min } 4\end{array}$} & $159(53)$ & $8 / 8$ & 201.74 & \multirow{2}{*}{278.52} & $2520 \mathrm{MB}$ \\
\hline & & $78(26)$ & $8 / 8$ & 76.77 & & $2293 \mathrm{MB}$ \\
\hline & \multirow{3}{*}{$\begin{array}{lll}\mathrm{RM} & V_{\max } & 4 \\
\mathrm{RM} & V_{\max } & 4 \\
\mathrm{RMM} & V_{\min } & 4 \\
\end{array}$} & $147(49)$ & $8 / 8$ & & 262.30 & $2954 \mathrm{MB}$ \\
\hline & & $51(17)$ & $8 / 8$ & 91.03 & \multirow{2}{*}{126.78} & $2974 \mathrm{MB}$ \\
\hline & & $36(12)$ & $8 / 8$ & 35.75 & & $2293 \mathrm{MB}$ \\
\hline \multirow{6}{*}{$\begin{array}{l}+ \\
\stackrel{ \pm}{0} \\
\stackrel{2}{2} \\
\stackrel{2}{2}\end{array}$} & BKDV 4 & 180 & $8 / 8$ & & 463.73 & $2520 \mathrm{MB}$ \\
\hline & \multirow{2}{*}{$\begin{array}{l}\text { BKDV } 4 \\
\text { RMM } V_{\min } 4 \\
\end{array}$} & $150(50)$ & $8 / 8$ & 126.47 & \multirow{2}{*}{223.69} & $2520 \mathrm{MB}$ \\
\hline & & $132(44)$ & $8 / 8$ & 97.22 & & $2293 \mathrm{MB}$ \\
\hline & RMM $V_{\max } 4$ & $240(80)$ & $7 / 8$ & & 290.50 & $2954 \mathrm{MB}$ \\
\hline & RMM $V_{\max } 4$ & $60(20)$ & $7 / 8$ & 73.16 & & $2974 \mathrm{MB}$ \\
\hline & $\mathrm{RMM} V_{\min } 4$ & $84(28)$ & $8 / 8$ & 61.63 & 19 & $2293 \mathrm{MB}$ \\
\hline & BKDV 4 & 128 & $8 / 8$ & & 490.24 & $2520 \mathrm{MB}$ \\
\hline & BKDV 4 & $132(44)$ & $8 / 8$ & 165.76 & & $2520 \mathrm{MB}$ \\
\hline 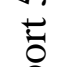 & $\mathrm{RMM} V_{\min } 4$ & $81(27)$ & $8 / 8$ & 78.91 & & $2293 \mathrm{MB}$ \\
\hline वे & RMM $V_{\max } 4$ & $210(70)$ & $8 / 8$ & & 373.92 & $2954 \mathrm{MB}$ \\
\hline & RMM $V_{\max } 4$ & $60(20)$ & $7 / 8$ & 105.33 & & $2974 \mathrm{MB}$ \\
\hline & $\mathrm{RMM} V_{\min } 4$ & $114(38)$ & $8 / 8$ & 110.89 & 210.22 & $2293 \mathrm{MB}$ \\
\hline
\end{tabular}


Table 4.20 Results of Ladder215S(18) model on Odin

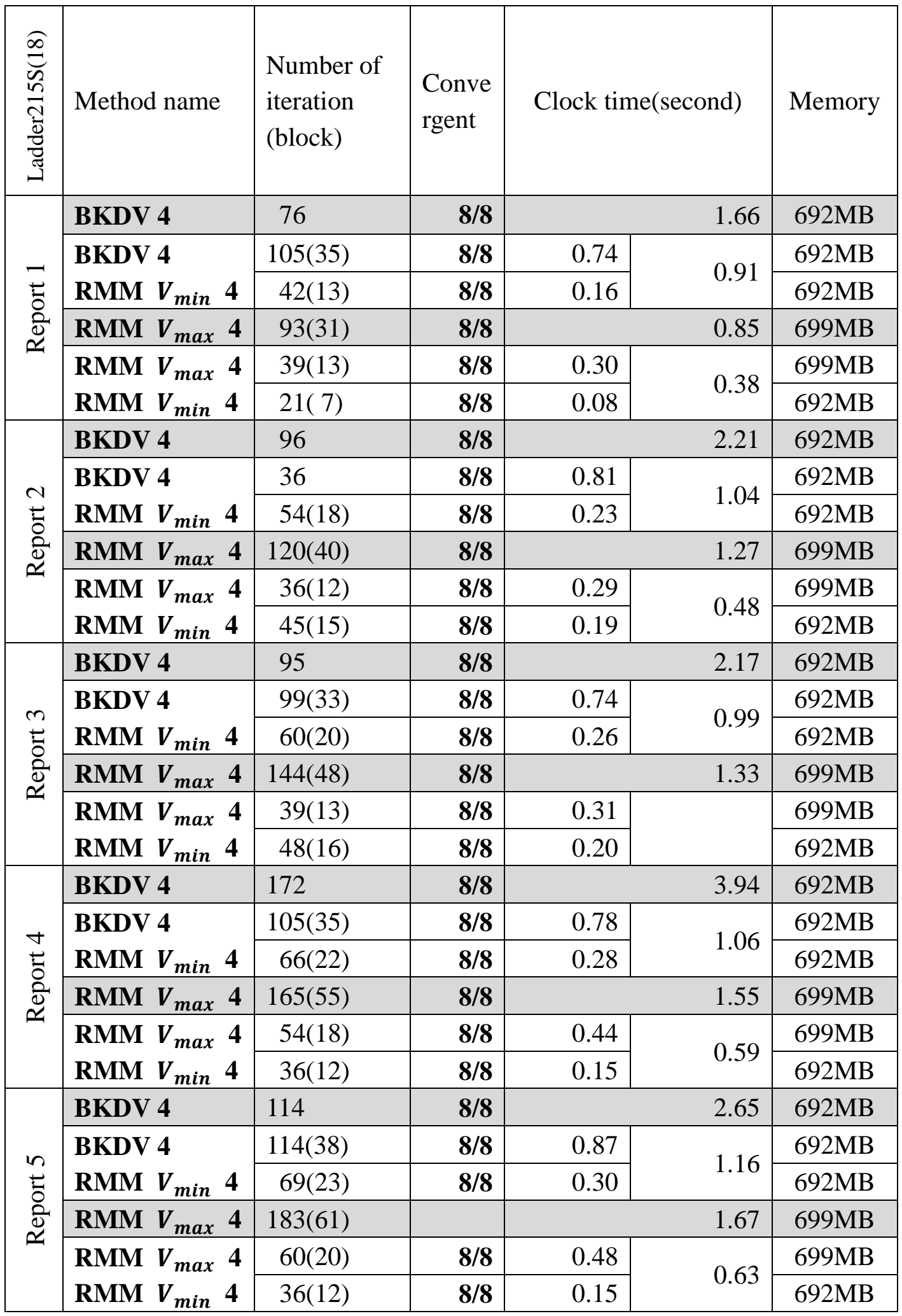


According to Table 4.19, we can get results about the number of iterations and the execution time of the Ladder215S(24) model by 4 different algorithms. Almost all result can reach convergence, the variants RMM-DIIS (RMM-DIIS $V_{\max }$ and $V_{\text {max }}$ ) method is the fastest of all, Block Davidson and RMM-DIIS combination method is the second fastest in most reports, the slowest one always Block Davidson method.

Table 4.20 shows the result about the number of iterations and the execution time of the Ladder215S(18) model. Results of all reports without exception can reach convergence at final Almost without exception, the variants RMM-DIIS (RMM-DIIS $V_{\max }$ and $\left.V_{\text {max }}\right)$ method is the fastest of all.

\subsubsection{Heisenberg Ladder 315C}

Table 4.21 shows the result about the number of iterations and the execution time of the Ladder315C(24) model, most results in these five report show Ladder315C(24) the lowest seven eigenvalues can reach convergence. Three of the all five reports show all eight eigenvalues can reach convergence via the combination RMM-DIIS.method (RMM-DIIS $V_{\max }$ and $\left.V_{\max }\right)$.

Table 4.42 shows the result about the number of iterations and the execution time of the Ladder315C(18) model, all results in five reports can reach convergence, the

combination RMM-DIIS (RMM $V_{\max }$ and $V_{\text {min }}$ ) is the fastest one of all, in the report5 of Table 4.22 the combination RMM-DIIS method is much faster than Block Davidson, even more than 6 times faster in this case. 
Table 4.21 Comparison of results of Ladder315C(24) model on Odin

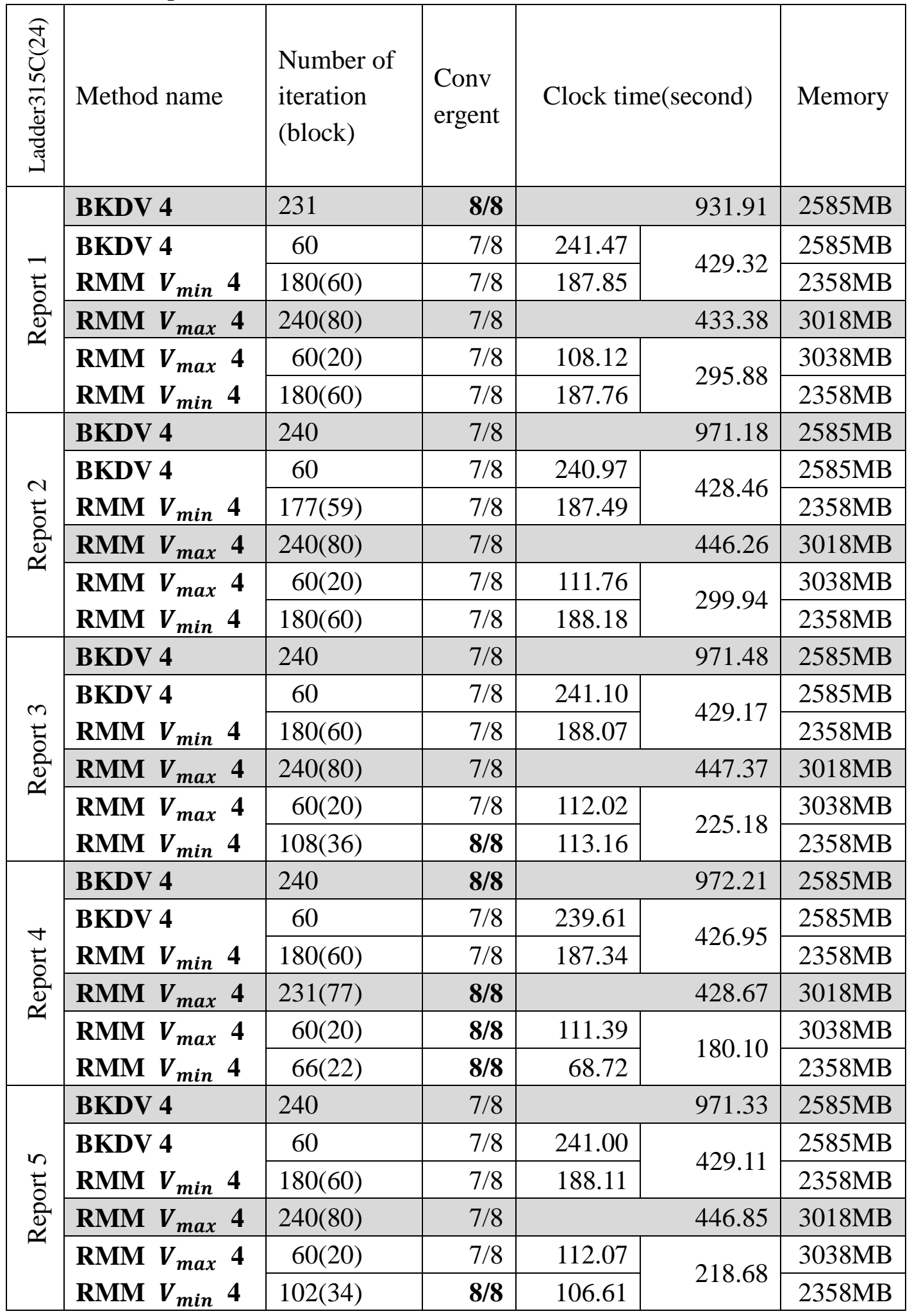


Table 4.22 Comparison of results of Ladder315C(18) model on Odin

\begin{tabular}{|c|c|c|c|c|c|c|}
\hline 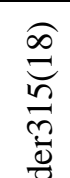 & & \multirow{2}{*}{$\begin{array}{l}\begin{array}{l}\text { Number of } \\
\text { iteration } \\
\text { (block) }\end{array} \\
129\end{array}$} & \multirow{2}{*}{\begin{tabular}{|r}
$\begin{array}{l}\text { Conv } \\
\text { ergent }\end{array}$ \\
$\mathbf{8 / 8}$ \\
\end{tabular}} & \multicolumn{2}{|c|}{ Clock time(second) } & \multirow[t]{2}{*}{ Memory } \\
\hline \multirow{6}{*}{ 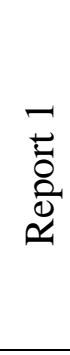 } & Method name & & & \multirow{3}{*}{$\begin{array}{l}0.72 \\
0.38 \\
\end{array}$} & 2.99 & \\
\hline & \multirow{2}{*}{$\begin{array}{l}\text { BKDV } 4 \\
\text { RMM } V_{\text {min }} 4\end{array}$} & $66(22)$ & $\mathbf{8 / 8}$ & & \multirow{2}{*}{1.10} & $692 \mathrm{MB}$ \\
\hline & & $90(30)$ & $8 / 8$ & & & $692 \mathrm{MB}$ \\
\hline & $\mathbf{R M M} V_{\max } 4$ & $153(51)$ & $8 / 8$ & & 1.36 & $699 \mathrm{MB}$ \\
\hline & $\mathrm{RMM} V_{\max } 4$ & $45(15)$ & $8 / 8$ & 0.36 & 054 & 699MB \\
\hline & $R M M V_{\min } 4$ & $42(14)$ & $8 / 8$ & 0.18 & 0.34 & $692 \mathrm{MB}$ \\
\hline & BKDV 4 & 116 & $8 / 8$ & & 2.73 & $692 \mathrm{MB}$ \\
\hline & BKDV 4 & $114(38)$ & $8 / 8$ & 0.89 & 1.20 & $692 \mathrm{MB}$ \\
\hline \pm & $\mathbf{R M M} V_{\min } 4$ & $72(24)$ & $8 / 8$ & 0.31 & & $692 \mathrm{MB}$ \\
\hline Qे & $\mathbf{R M M} V_{\max } 4$ & $165(55)$ & $8 / 8$ & & 1.47 & $699 \mathrm{MB}$ \\
\hline & $\mathbf{R M M} V_{\max } 4$ & $57(19)$ & $8 / 8$ & 0.46 & 061 & $699 \mathrm{MB}$ \\
\hline & $R M M V_{\min } 4$ & $36(12)$ & 8/8 & 0.15 & 0.01 & $692 \mathrm{MB}$ \\
\hline & BKDV 4 & 174 & $8 / 8$ & & 4.08 & $692 \mathrm{MB}$ \\
\hline & BKDV 4 & 60 & $7 / 8$ & 1.40 & 180 & $692 \mathrm{MB}$ \\
\hline \pm & RMM $V_{\min } 4$ & $114(38)$ & $8 / 8$ & 0.50 & 1.09 & $692 \mathrm{MB}$ \\
\hline ये & RMM $V_{\max } 4$ & 171(57) & $8 / 8$ & & 1.53 & $699 \mathrm{MB}$ \\
\hline & RMM $V_{\max } 4$ & $57(19)$ & $8 / 8$ & 0.46 & 067 & $699 \mathrm{MB}$ \\
\hline & $\mathbf{R M M} V_{\min } 4$ & $39(13)$ & $8 / 8$ & 0.17 & 0.02 & $692 \mathrm{MB}$ \\
\hline & BKDV 4 & 129 & $8 / 8$ & & 2.99 & $692 \mathrm{MB}$ \\
\hline & BKDV 4 & $114(38)$ & $8 / 8$ & 0.87 & 122 & $692 \mathrm{MB}$ \\
\hline 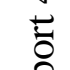 & $\mathrm{RMM} V_{\min } 4$ & $84(28)$ & $8 / 8$ & 0.36 & 1.22 & $692 \mathrm{MB}$ \\
\hline ये & $R M M V_{\max } 4$ & $168(66)$ & $8 / 8$ & & 1.52 & $699 \mathrm{MB}$ \\
\hline & $\mathbf{R M M} V_{\max } 4$ & $57(19)$ & $8 / 8$ & 0.46 & 0.64 & $699 \mathrm{MB}$ \\
\hline & RMM $V_{\min } 4$ & $42(14)$ & $8 / 8$ & 0.18 & 0.07 & $692 \mathrm{MB}$ \\
\hline & BKDV 4 & 141 & $8 / 8$ & & 3.24 & $692 \mathrm{MB}$ \\
\hline & BKDV 4 & 41 & $8 / 8$ & 0.93 & 1.31 & $692 \mathrm{MB}$ \\
\hline Ё & RMM $V_{\min } 4$ & $90(30)$ & $8 / 8$ & 0.38 & & $692 \mathrm{MB}$ \\
\hline ये & $\mathrm{RMM} V_{\max } 4$ & $147(49)$ & $8 / 8$ & & 1.37 & $699 \mathrm{MB}$ \\
\hline & RMM $V_{\max } 4$ & $39(13)$ & $8 / 8$ & 0.31 & 0.53 & $699 \mathrm{MB}$ \\
\hline & $\mathrm{RMM} V_{\min } 4$ & $51(17)$ & $8 / 8$ & 0.22 & 0.53 & $692 \mathrm{MB}$ \\
\hline
\end{tabular}




\subsubsection{Heisenberg Ladder 315S}

Table 4.23 Comparison of results of Ladder315S(24) model on Odin

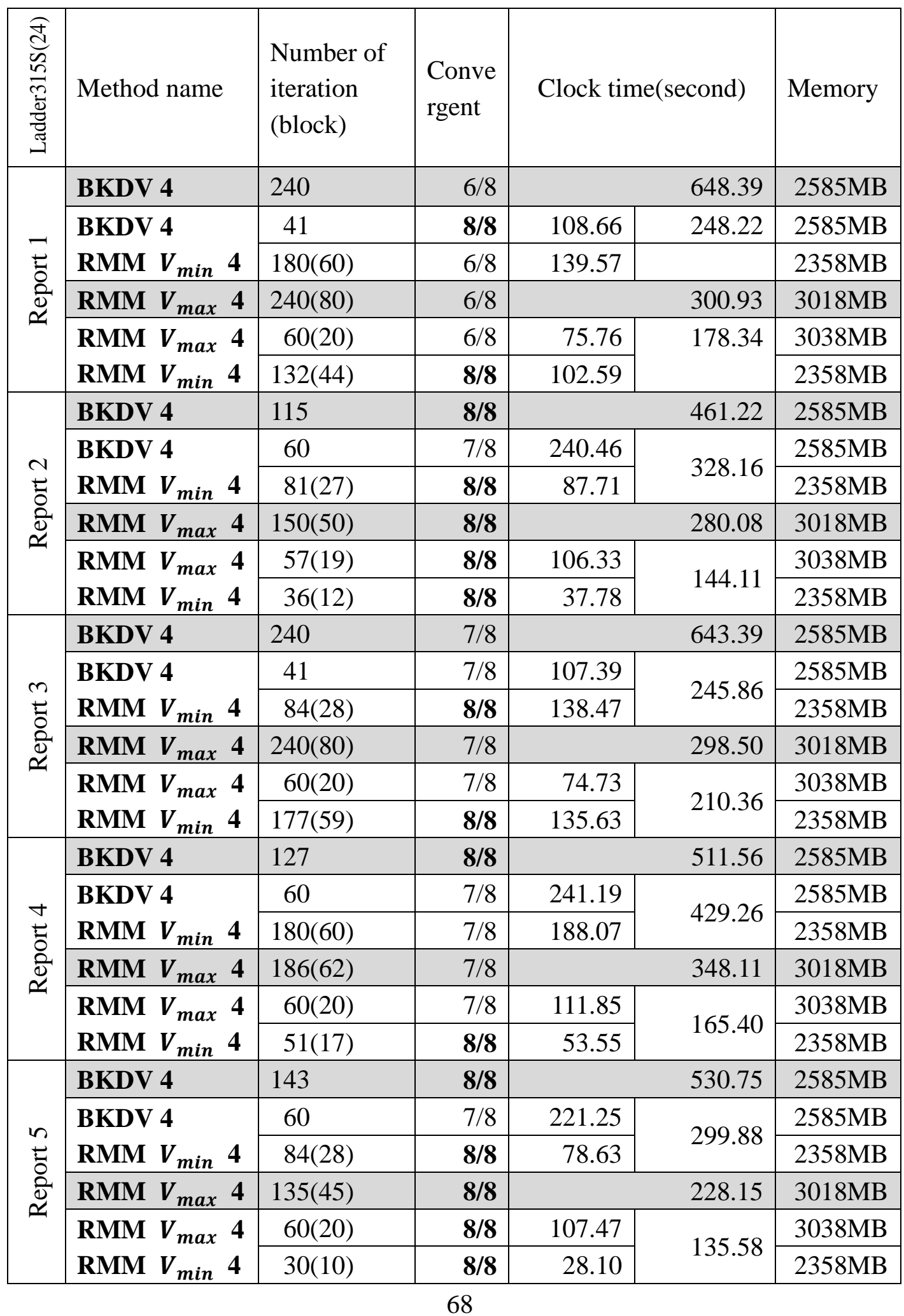


Table 4.24 Comparison of results of Ladder315S(18) model on Odin

\begin{tabular}{|c|c|c|c|c|c|c|}
\hline $\begin{array}{l}\stackrel{\infty}{=} \\
\stackrel{n}{n} \\
\stackrel{n}{n}\end{array}$ & Method name & $\begin{array}{l}\text { Number of } \\
\text { iteration }\end{array}$ & $\begin{array}{l}\text { Conv } \\
\text { ergent }\end{array}$ & \multicolumn{2}{|c|}{ Clock time (second) } & Memory \\
\hline \multirow{6}{*}{ 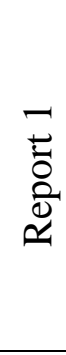 } & BKDV 4 & 89 & $8 / 8$ & & 2.08 & $692 \mathrm{MB}$ \\
\hline & \multirow{2}{*}{$\begin{array}{l}\text { BKDV } 4 \\
\text { RMM } V_{\text {min }} 4\end{array}$} & $87(29)$ & $8 / 8$ & 0.66 & \multirow{2}{*}{0.90} & $692 \mathrm{MB}$ \\
\hline & & $57(19)$ & $8 / 8$ & 0.24 & & $692 \mathrm{MB}$ \\
\hline & $\mathbf{R M M} V_{\max } 4$ & $123(41)$ & $8 / 8$ & & 1.18 & $700 \mathrm{MB}$ \\
\hline & RMM $V_{\max } 4$ & $45(15)$ & $8 / 8$ & 0.37 & \multirow{2}{*}{0.51} & $700 \mathrm{MB}$ \\
\hline & RMM $V_{\min } 4$ & $33(11)$ & $8 / 8$ & 0.14 & & $692 \mathrm{MB}$ \\
\hline \multirow{6}{*}{ 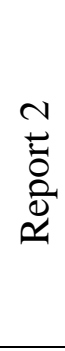 } & BKDV 4 & 96 & $8 / 8$ & & 2.21 & $692 \mathrm{MB}$ \\
\hline & \multirow{2}{*}{$\begin{array}{l}\text { BKDV } 4 \\
\text { RMM } V_{\text {min }} 4\end{array}$} & $108(35)$ & $8 / 8$ & 0.81 & \multirow{2}{*}{1.04} & $692 \mathrm{MB}$ \\
\hline & & $54(18)$ & $8 / 8$ & 0.23 & & $692 \mathrm{MB}$ \\
\hline & $R M M V_{\max } 4$ & $129(43)$ & $8 / 8$ & & 1.27 & $700 \mathrm{MB}$ \\
\hline & RMM $V_{\max } 4$ & $36(12)$ & $8 / 8$ & 0.29 & \multirow{2}{*}{0.48} & $700 \mathrm{MB}$ \\
\hline & $\mathbf{R M M} V_{\min } \mathbf{4}$ & $45(15)$ & $8 / 8$ & 0.19 & & $692 \mathrm{MB}$ \\
\hline \multirow{6}{*}{ 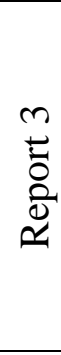 } & BKDV 4 & 95 & $8 / 8$ & & 2.17 & $692 \mathrm{MB}$ \\
\hline & \multirow{2}{*}{$\begin{array}{l}\text { BKDV } 4 \\
\text { RMM } V_{\text {min }} 4\end{array}$} & $99(33)$ & $8 / 8$ & 0.74 & \multirow{2}{*}{0.99} & $692 \mathrm{MB}$ \\
\hline & & $60(20)$ & $8 / 8$ & 0.26 & & $692 \mathrm{MB}$ \\
\hline & RMM $V_{\max } 4$ & $144(48)$ & $8 / 8$ & & 1.33 & $700 \mathrm{MB}$ \\
\hline & RMM $V_{\max } 4$ & $39(13)$ & $8 / 8$ & 0.31 & \multirow{2}{*}{0.52} & $700 \mathrm{MB}$ \\
\hline & $R M M V_{\min } 4$ & $48(16)$ & $8 / 8$ & 0.20 & & $692 \mathrm{MB}$ \\
\hline \multirow{6}{*}{ 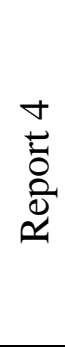 } & BKDV 4 & 172 & $8 / 8$ & & 3.94 & $692 \mathrm{MB}$ \\
\hline & BKDV 4 & $105(35)$ & $8 / 8$ & 0.78 & \multirow{2}{*}{1.06} & $692 \mathrm{MB}$ \\
\hline & RMM $V_{\min } 4$ & $66(22)$ & $8 / 8$ & 0.28 & & $692 \mathrm{MB}$ \\
\hline & RMM $V_{\max } 4$ & $165(55)$ & $8 / 8$ & & 1.55 & $700 \mathrm{MB}$ \\
\hline & RMM $V_{\max } 4$ & $54(18)$ & $8 / 8$ & 0.44 & \multirow{2}{*}{0.59} & $700 \mathrm{MB}$ \\
\hline & $R M M V_{\min } 4$ & $36(12)$ & $8 / 8$ & 0.15 & & $692 \mathrm{MB}$ \\
\hline \multirow{6}{*}{ 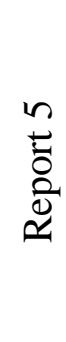 } & BKDV 4 & 114 & $8 / 8$ & & 2.65 & $692 \mathrm{MB}$ \\
\hline & \multirow{2}{*}{$\begin{array}{l}\text { BKDV } 4 \\
\text { RMM } V_{\text {min }} 4\end{array}$} & $114(38)$ & $8 / 8$ & 0.87 & \multirow{2}{*}{1.16} & $692 \mathrm{MB}$ \\
\hline & & $69(23)$ & $8 / 8$ & 0.30 & & 692MB \\
\hline & RMM $V_{\max } 4$ & $183(61)$ & $8 / 8$ & & 1.67 & $700 \mathrm{MB}$ \\
\hline & \multirow{2}{*}{$\begin{array}{ll}R M M & V_{\max } \\
R M M & V_{\min } 4\end{array}$} & $60(20)$ & $8 / 8$ & 0.48 & \multirow{2}{*}{0.63} & $700 \mathrm{MB}$ \\
\hline & & $36(12)$ & $8 / 8$ & 0.15 & & $692 \mathrm{MB}$ \\
\hline
\end{tabular}


Table 4.23 shows the result about the number of iterations and the execution time of the Ladder315S(24) model, the lowest 8 eigenvalues can reach convergence in most cases, the results are all convergence via the variants RMM-DIIS can all reach convergence.

Table 4.24 shows the result about the number of iterations and the execution time of the Ladder315S(18) model, all results in 5 reports can reach convergence, the variants RMM-DIIS is the fastest one of all, in report4 of Table 4.24, the variants RMMDIIS(RMM-DIIS $V_{\max }$ and $V_{\max }$ ) is much faster than Block Davidson, even more than 6 times faster in this case. 


\subsubsection{Comparison of results}

Table 4.25 and Table 4.26 show the result about the number of iterations and the execution time of all six models (Chain, Ladder2, Ladder215C, Ladder215S, Ladder315C and Ladder315S) with $N=24$ on Odin. Table 4.27, Table 4.28, Table 4.29, Table 4.30, Table 4.31 and Table 4.32 are figures tables, show result of all 6 different models result, how did they reach convergence. Table 4.33 and Table 4.34 show the result about the number of iterations and the execution time of models with size $N=18$ on Odin. According these tables and figures, we can find out some interesting things about the different models and different algorithms.

\section{Comparison of different models}

Convergent: For calculating the lowest eight eigenvalues, the Ladder2, Ladder215S and Ladder315S models, errors easily reach convergence at $5 \times 10^{-4}$, top 7 eigenvalues of Chain, Ladder215C and Ladder315C models can reach convergence when size $N=$ 24. The lowest 8 eigenvalues of all models can reach convergence with error blow $5 \times$ $10^{-4}$, except for the Chain model and Ladder2 when size $N=18$. The models with smaller size $N$ reach convergence faster than the models with larger size $N$, STAGGERED models are easier reach convergence faster than the COLUMNAR models.

Clock time: Calculations by RMM $V_{\max }$ did not reach convergence at maximum iteration in Chain, Ladder215C and Ladder315C models with $N=24$, we can get the execution times of these 3 models are 395.94s, 438.45s, 447.37s form Table 4.25 and 
Table 4.26, that means execution time of Chain $<$ Ladder215C $<$ Ladder315C when their number of iteration are all 240.

Memory: The Chain model uses less memory than the other 5 models; The required memory are same when two-leg Heisenberg Ladder models (Ladder2, Ladder215C and Ladder215S) were calculated, and their required memory are more than Chain models, but less than three-leg Heisenberg Ladder models (Ladder315C and Ladder315S). Three-leg Heisenberg Ladder models need same memory when they were calculated, their required memory is the largest among the all models. We can review Table 2.3 and find out Chain model has 36564892 non-zero elements, two-leg Heisenberg Ladder models have 53495260 non-zero elements, three-leg Heisenberg Ladder models have 59138716 non-zero elements when size $N=24$. The reason for why do three-leg Heisenberg Ladder models need more memory is there are more non-zero elements in these two models.

\section{Comparison of different algorithms}

Convergent: Each of Table 4.27, Table 4.28, Table 4.29, Table 4.30, Table 4.31 and Table 4.32 has 4 figures which show results of 4 different algorithms respectively. In most case, the error of top several eigenvalues can reach to $1 \times 10^{-11}$ via Block Davidson; RMM-DIIS $\boldsymbol{V}_{\boldsymbol{m a x}}$ algorithm can calculate results whose error can reach convergence around $1 \times 10^{-13}$; the errors are convergence to $1 \times 10^{-14}$ via the variants RMM-DIIS and Block Davidson RMM $V_{\min }$ combination method. Hence these two combination methods can calculate out eigenvalues more precise than single algorithm. 
Clock time: By checking all results, we can find out that the two variants RMM-DIIS $\left(\mathrm{RMM} V_{\max }\right.$ and RMM $\left.V_{\min }\right)$ are the fastest of all, no matter which model. It is much faster than another 3 algorithms, particularly compare with the Block Davidson method. The combination method of RMM $V_{\max }$ and RMM $V_{\min }$ are often 3-4 times faster than the Block Davidson method, even in some cases is more than 4 times faster, like the results of Ladder315C(24) in Table 4.26.

We can find out that RMM $V_{\max }$ is around 2 times faster than the Block Davidson, although the RMM $V_{\max }$ algorithm need more iterations to reach convergence. The reason why RMM-DIIS is faster than Block Davidson is that RMM-DIIS does not require a computationally expensive explicit orthogonalization step at each iteration. Since the method is based on the minimization of the residual vector, the trial vectors converge to the eigenvectors with eigenvalues closets to the current trial eigenvalues.

Memory: As an algorithm which can find out good enough initial eigenvectors in a combination method, RMM-DIIS $V_{\max }$ demands around $450 \mathrm{MB}$ more memory than Block Davidson method in all models, compare the required memory of Block Davidson (approximate $2300 \mathrm{MB}-2500 \mathrm{MB}), 450 \mathrm{MB}$ is not too much. RMM $V_{\max }$ is much faster than Block Davidson method, although it need more memory than Block Davidson method.

The order of required memory is RMM $V_{\max }>$ Block Davidson $>\mathrm{RMM} V_{\min }$, when used with the same block number. 
Table 4.25 Comparison of the results of Chain, Ladder2, Ladder215C and Ladder215S models with $N=24$ on Odin

\begin{tabular}{|c|c|c|c|c|c|}
\hline Methods name & $\begin{array}{l}\text { Number of } \\
\text { iteration } \\
\text { (block) }\end{array}$ & $\begin{array}{l}\text { Conv } \\
\text { ergent }\end{array}$ & \multicolumn{2}{|c|}{ Clock time (second) } & Memory \\
\hline \multicolumn{6}{|c|}{ Heisenberg Chain (24) } \\
\hline BKDV 4 & 240 & $7 / 8$ & \multicolumn{2}{|r|}{813.23} & $2327 \mathrm{MB}$ \\
\hline \multirow{2}{*}{$\begin{array}{l}\text { BKDV } 4 \\
\text { RMM } V_{\min } 4 \\
\end{array}$} & 57 & $8 / 8$ & 189.12 & \multirow{2}{*}{334.55} & $2327 \mathrm{MB}$ \\
\hline & $180(60)$ & $7 / 8$ & 145.43 & & $2100 \mathrm{MB}$ \\
\hline RMM $V_{\max } 4$ & $240(80)$ & $7 / 8$ & \multicolumn{2}{|r|}{395.94} & 2760MB \\
\hline \multirow{2}{*}{$\begin{array}{lll}\mathrm{RMM} & V_{\max } 4 \\
\mathrm{RMM} & V_{\min } 4 \\
\end{array}$} & $57(19)$ & $8 / 8$ & 94.47 & \multirow{2}{*}{239.35} & $2780 \mathrm{MB}$ \\
\hline & $180(60)$ & $7 / 8$ & 144.88 & & $2100 \mathrm{MB}$ \\
\hline \multicolumn{6}{|c|}{ Heisenberg Ladder2(24); } \\
\hline BKDV 4 & 143 & $8 / 8$ & & 549.74 & $2520 \mathrm{MB}$ \\
\hline \multirow{2}{*}{$\begin{array}{l}\text { BKDV } 4 \\
\text { RMM } V_{\min } 4\end{array}$} & 53 & $8 / 8$ & 200.87 & \multirow{2}{*}{274.38} & $2520 \mathrm{MB}$ \\
\hline & $75(25)$ & $8 / 8$ & 73.51 & & $2293 \mathrm{MB}$ \\
\hline $\mathrm{RMM} V_{\max } 4$ & $153(51)$ & $8 / 8$ & & 284.16 & $2954 \mathrm{MB}$ \\
\hline \multirow{2}{*}{$\begin{array}{lll}\mathrm{RMM} & V_{\max } & 4 \\
\mathrm{RMM} & V_{\min } & 4 \\
\end{array}$} & $60(20)$ & $7 / 8$ & 109.85 & \multirow{2}{*}{142.35} & $2974 \mathrm{MB}$ \\
\hline & $33(11)$ & $8 / 8$ & 32.50 & & $2293 \mathrm{MB}$ \\
\hline \multicolumn{6}{|c|}{ Heisenberg Ladderr215C(24) } \\
\hline BKDV 4 & 240 & $7 / 8$ & & 932.50 & $2520 \mathrm{MB}$ \\
\hline \multirow{2}{*}{$\begin{array}{l}\text { BKDV } 4 \\
\text { RMM } V_{\min } 4 \\
\end{array}$} & 47 & $8 / 8$ & 177.77 & \multirow{2}{*}{354.25} & $2520 \mathrm{MB}$ \\
\hline & $180(60)$ & $7 / 8$ & 176.48 & & $2293 \mathrm{MB}$ \\
\hline RMM $V_{\max } 4$ & $240(80)$ & $7 / 8$ & & 438.45 & $2954 \mathrm{MB}$ \\
\hline \multirow{2}{*}{$\begin{array}{lll}\mathrm{RMM} & V_{\max } & 4 \\
\mathrm{RMM} & V_{\min } & 4 \\
\end{array}$} & $54(18)$ & $8 / 8$ & 99.38 & \multirow{2}{*}{275.03} & $2974 \mathrm{MB}$ \\
\hline & $180(60)$ & $7 / 8$ & 175.65 & & $2293 \mathrm{MB}$ \\
\hline \multicolumn{6}{|c|}{ Heisenberg Ladderr215S(24) } \\
\hline BKDV 4 & 98 & $8 / 8$ & & 372.53 & $2520 \mathrm{MB}$ \\
\hline \multirow{2}{*}{$\begin{array}{l}\text { BKDV } 4 \\
\text { RMM } V_{\min } 4 \\
\end{array}$} & 35 & $8 / 8$ & 130.89 & \multirow{2}{*}{186.61} & $2520 \mathrm{MB}$ \\
\hline & $57(19)$ & $8 / 8$ & 55.73 & & $2293 \mathrm{MB}$ \\
\hline RMM $V_{\max } 4$ & $153(51)$ & $8 / 8$ & & 272.27 & $2954 \mathrm{MB}$ \\
\hline \multirow{2}{*}{$\begin{array}{lll}\mathrm{RMM} & V_{\max } & 4 \\
\mathrm{RMM} & V_{\min } & 4\end{array}$} & $60(20)$ & $8 / 8$ & 106.31 & \multirow{2}{*}{135.85} & $2974 \mathrm{MB}$ \\
\hline & $30(10)$ & $8 / 8$ & 29.54 & & $2293 \mathrm{MB}$ \\
\hline \multicolumn{6}{|c|}{$\begin{array}{l}\text { maximum iteration } m=240, \\
\begin{aligned} \text { combination method: } & \text { the } 1^{\text {st }} \text { part max iteration }=60, \text { tolerance }=5 \times 10^{-2} \\
& \text { the } 2^{\text {nd }} \text { part max iteration }=180, \text { tolerance }=5 \times 10^{-4}\end{aligned}\end{array}$} \\
\hline
\end{tabular}


Table 4.26 Comparison of the results of Ladder315C and Ladder315S models with $N=$ 24 on Odin

\begin{tabular}{|c|c|c|c|c|c|}
\hline Methods name & $\begin{array}{l}\text { Number of } \\
\text { iteration } \\
\text { (block) }\end{array}$ & $\begin{array}{r}\text { Conve } \\
\text { rgent }\end{array}$ & \multicolumn{2}{|c|}{ Clock time (second) } & Memory \\
\hline \multicolumn{6}{|c|}{ Heisenberg Ladderr315C(24) } \\
\hline BKDV 4 & 240 & $7 / 8$ & & 971.48 & $2585 \mathrm{MB}$ \\
\hline \multirow{2}{*}{$\begin{array}{l}\text { BKDV } 4 \\
\text { RMM } V_{\text {min }} 4 \\
\end{array}$} & 40 & $7 / 8$ & 241.10 & \multirow{2}{*}{429.17} & $2585 \mathrm{MB}$ \\
\hline & $180(60)$ & $7 / 8$ & 188.07 & & $2358 \mathrm{MB}$ \\
\hline$R M M V_{\max } 4$ & $240(80)$ & $7 / 8$ & & 447.37 & $3018 \mathrm{MB}$ \\
\hline \multirow{2}{*}{$\begin{array}{lll}R M M & V_{\text {max }} 4 \\
\text { RMM } & V_{\text {min }} 4\end{array}$} & $60(20)$ & $7 / 8$ & 112.02 & \multirow{2}{*}{225.18} & 3038MB \\
\hline & 108(36) & $8 / 8$ & 113.16 & & $2358 \mathrm{MB}$ \\
\hline \multicolumn{6}{|c|}{ Heisenberg Ladderr315S(24) } \\
\hline BKDV 4 & 115 & $8 / 8$ & & 461.22 & $2585 \mathrm{MB}$ \\
\hline \multirow{2}{*}{$\begin{array}{l}\text { BKDV } 4 \\
\text { RMM } V_{\text {min }} 4\end{array}$} & 60 & $7 / 8$ & 240.46 & \multirow{2}{*}{328.16} & $2585 \mathrm{MB}$ \\
\hline & $81(27)$ & $8 / 8$ & 87.71 & & $2358 \mathrm{MB}$ \\
\hline$R M M V_{\max } 4$ & $150(50)$ & $8 / 8$ & & 280.08 & 3018MB \\
\hline \multirow{2}{*}{$\begin{array}{ll}\text { RMM } V_{\text {max }} 4 \\
\text { RMM } V_{\text {min }} 4 \\
\end{array}$} & $57(19)$ & $8 / 8$ & 106.33 & \multirow{2}{*}{144.11} & 3038MB \\
\hline & $36(12)$ & $8 / 8$ & 37.78 & & $2358 \mathrm{MB}$ \\
\hline \multicolumn{6}{|c|}{ 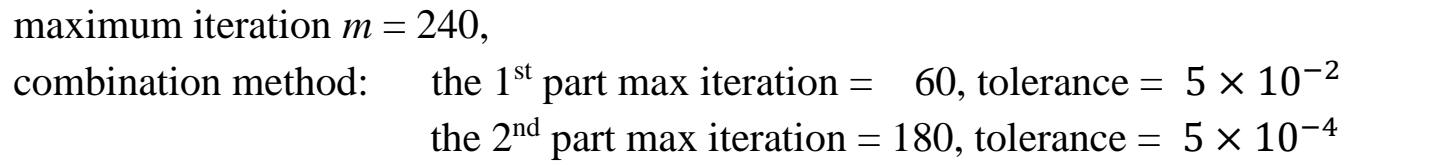 } \\
\hline
\end{tabular}


Table 4.27 Calculations of Chain(24) model by 4 different methods

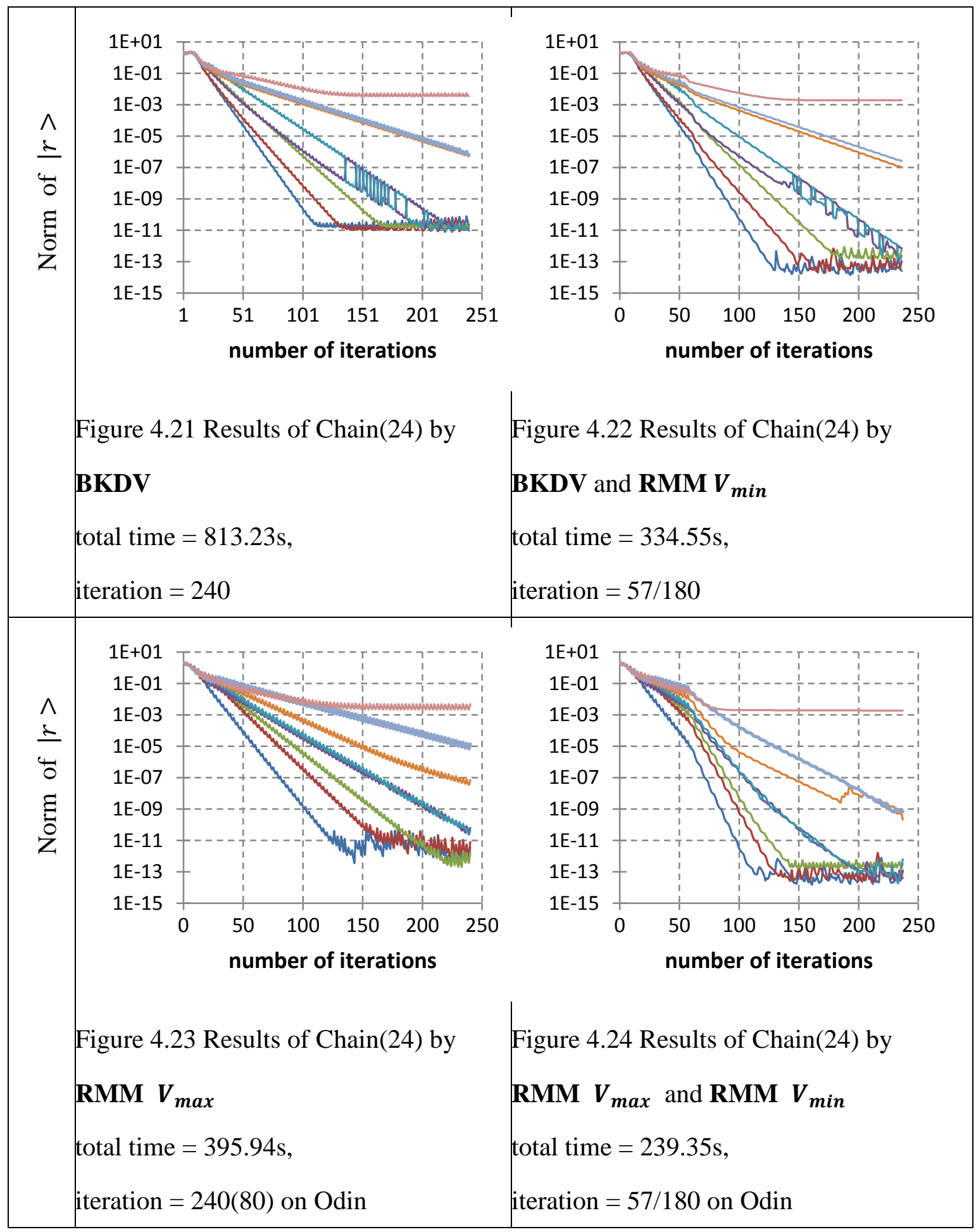


Table 4.28 Calculations of Ladder2(24) model by 4 different methods

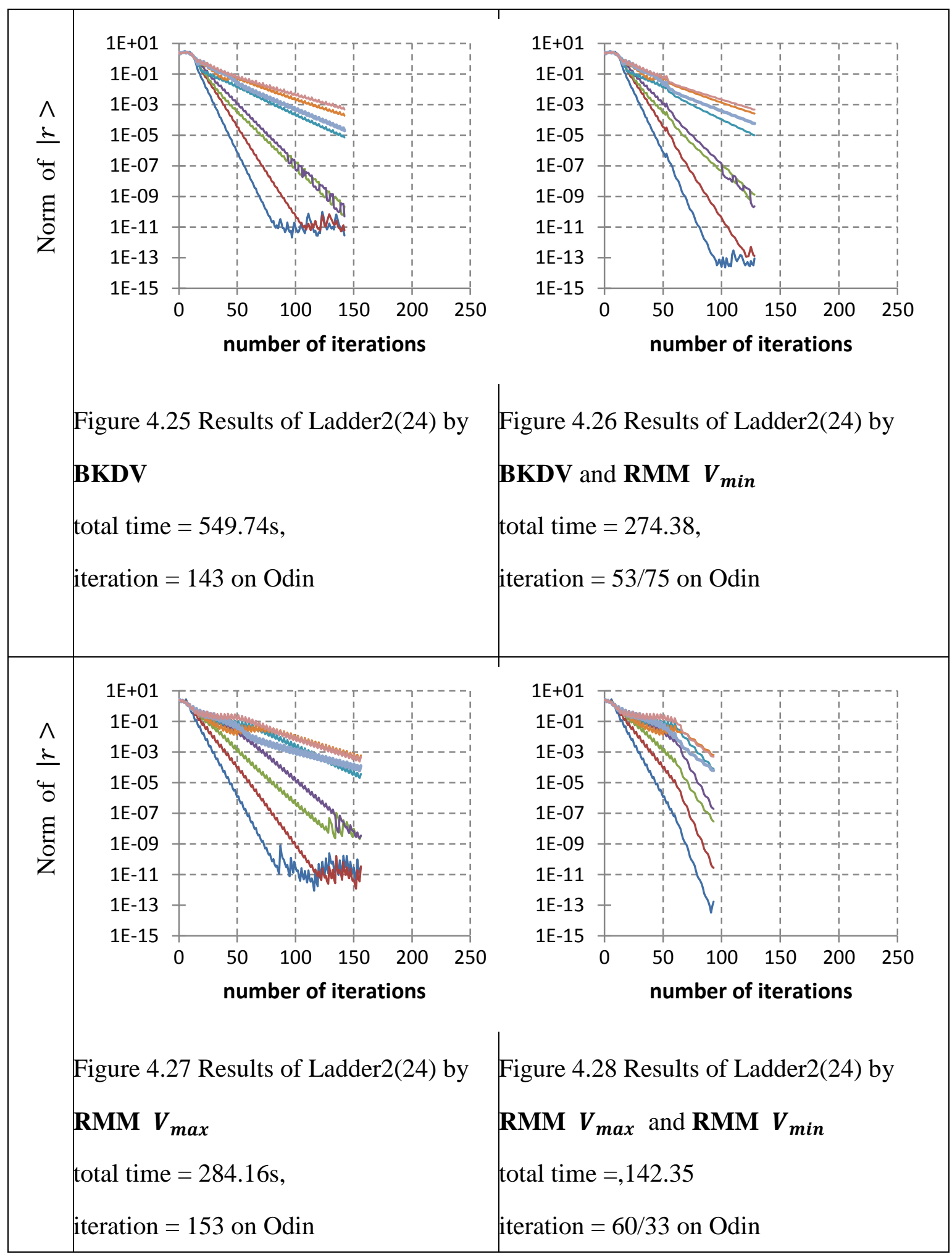


Table 4.29 Calculations of Ladder215C(24) model by 4 different methods

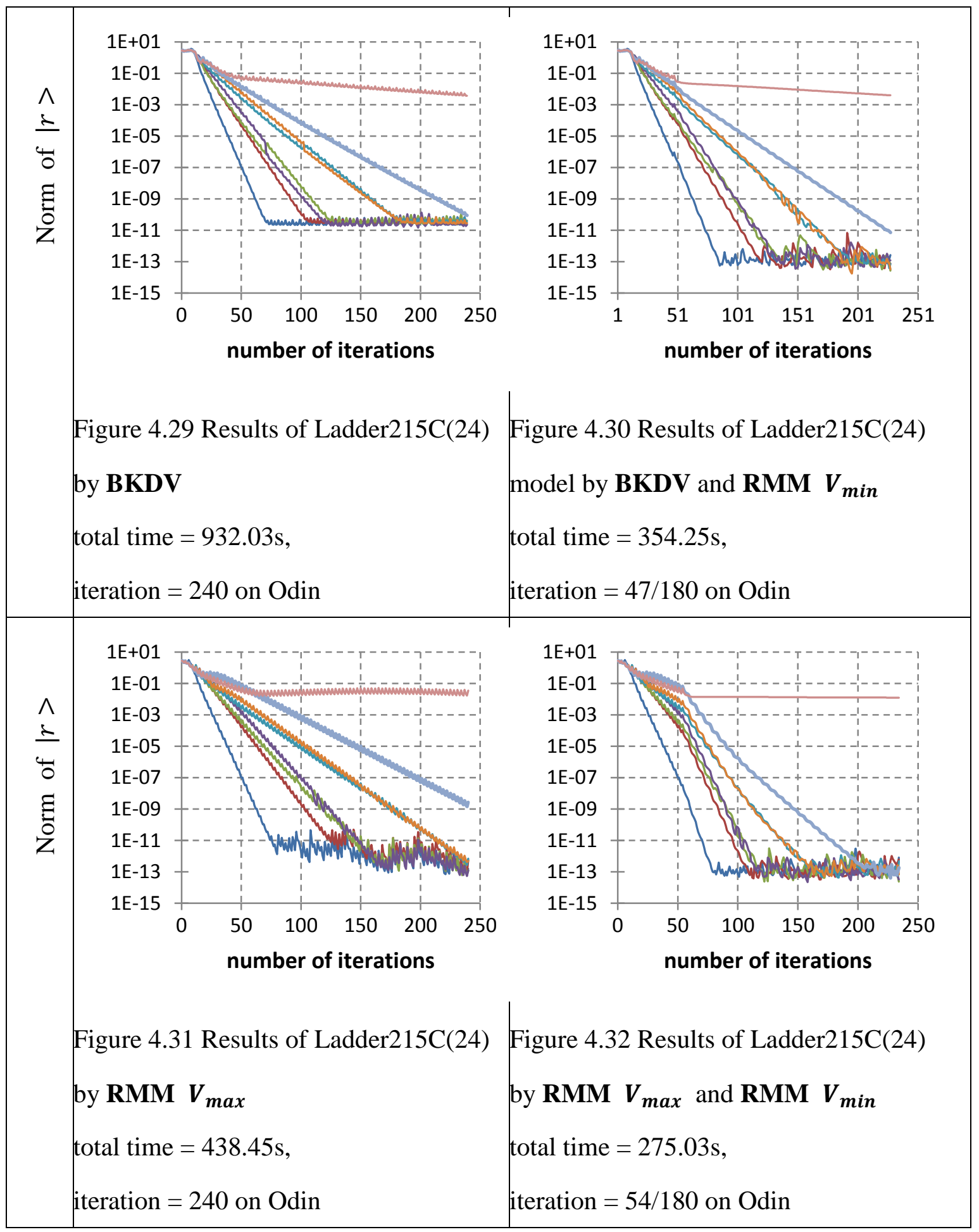


Table 4.30 Calculations of Ladder215S(24) model by 4 different methods

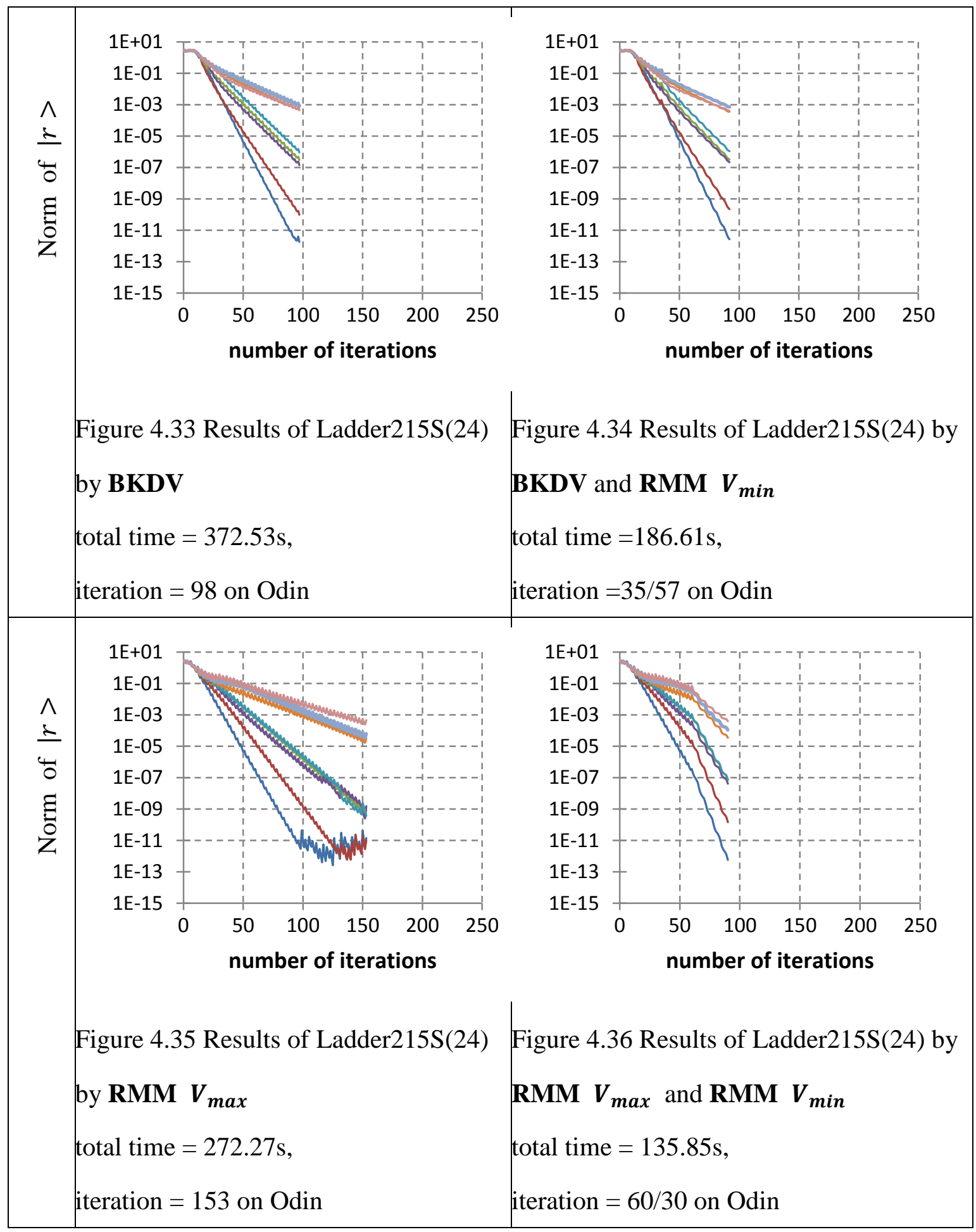


Table 4.31 Calculations of Ladder315C(24) model by 4 different methods

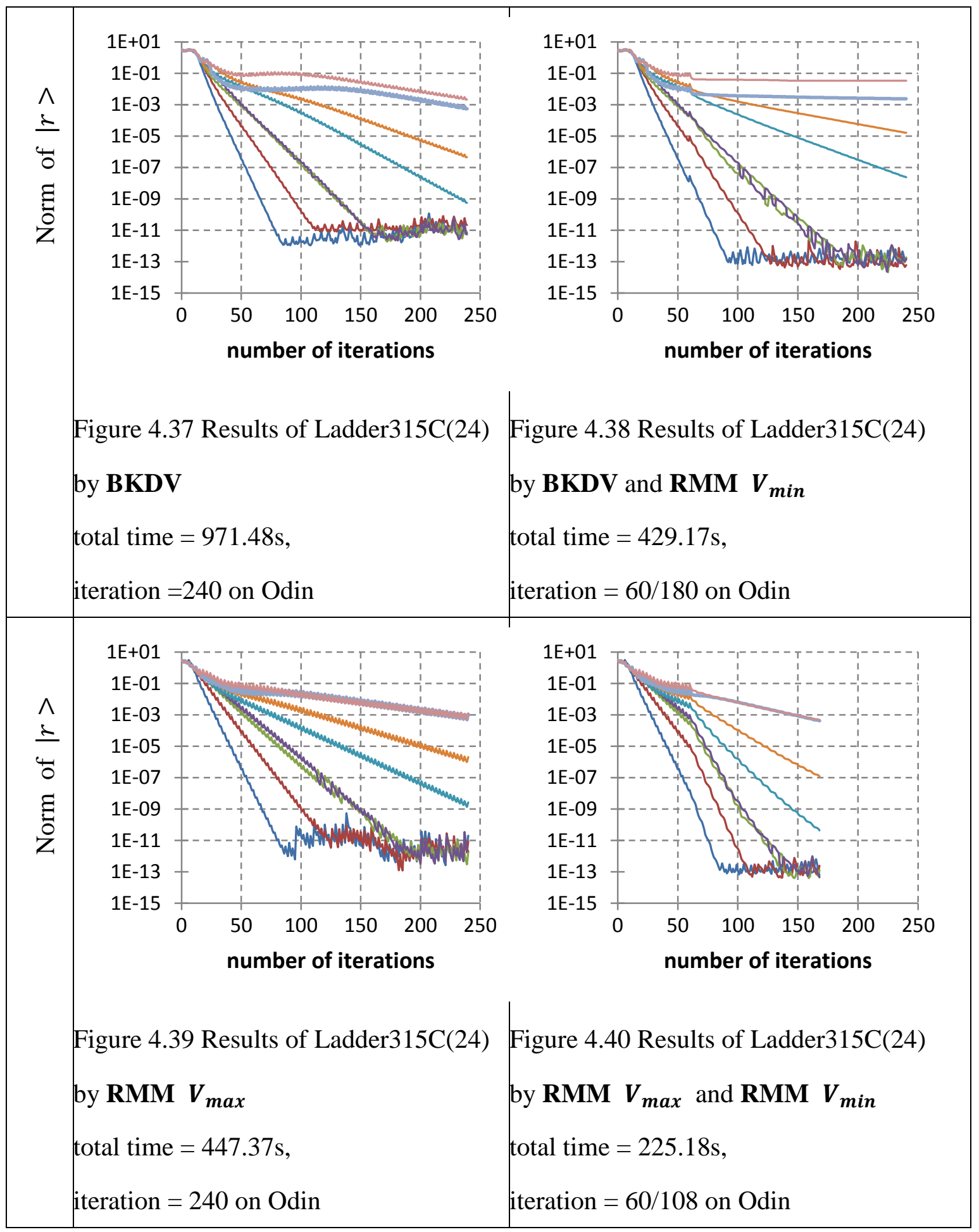


Table 4.32 Calculations of Ladder315S(24) model by 4 different methods

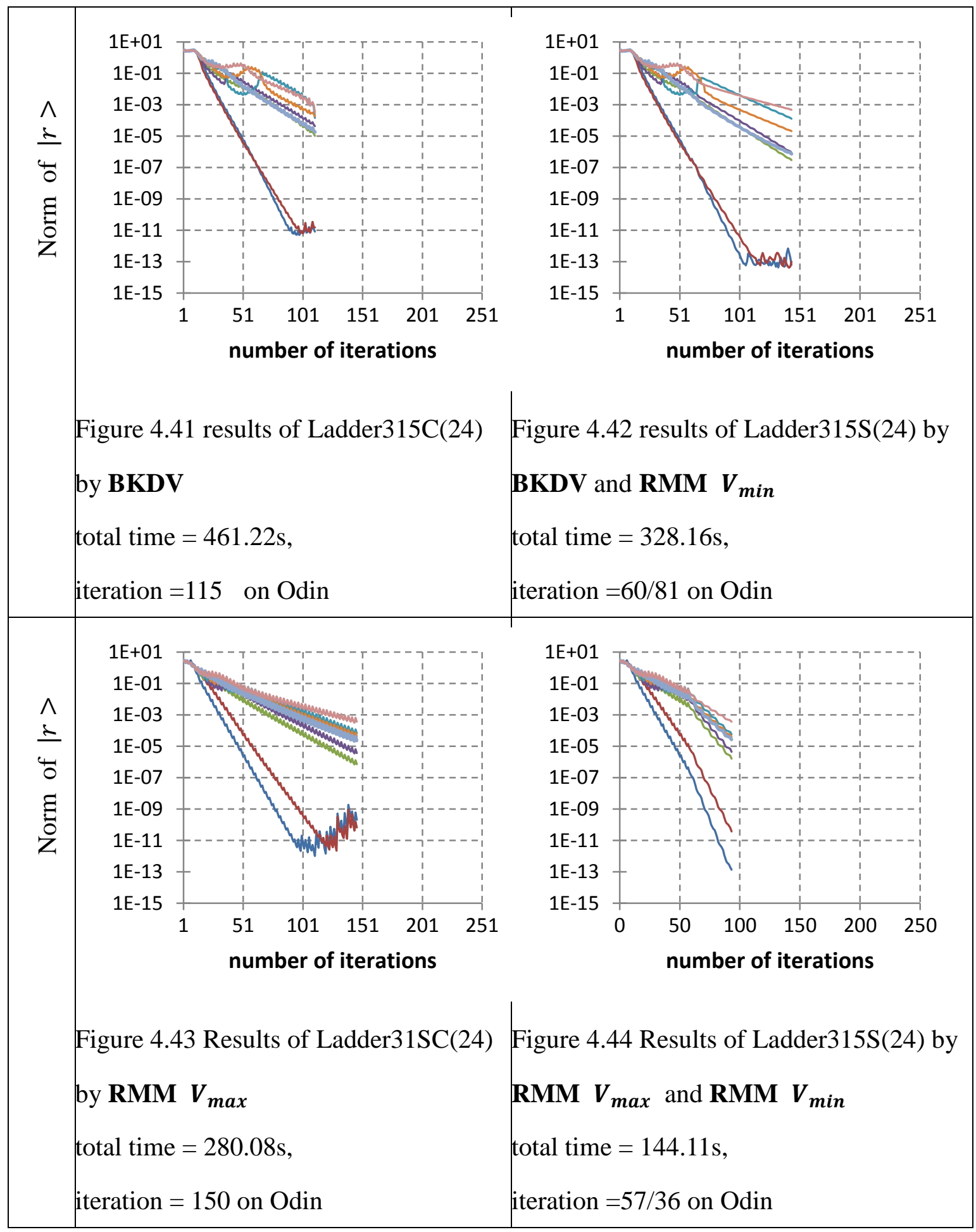


Table 4.33 Comparison of the results of Chain, Ladder2, Ladder215C and Ladder215S models with $N=18$ on Odin

\begin{tabular}{|c|c|c|c|c|c|}
\hline Methods & $\begin{array}{l}\text { Number of } \\
\text { iteration } \\
\text { (block) }\end{array}$ & $\begin{array}{l}\text { Conv } \\
\text { ergent }\end{array}$ & \multicolumn{2}{|c|}{ Clock time (second) } & Memory \\
\hline \multicolumn{6}{|c|}{ Heisenberg Chain (18) } \\
\hline BKDV 4 & 240 & $7 / 8$ & \multicolumn{2}{|r|}{4.29} & $689 \mathrm{MB}$ \\
\hline \multirow{2}{*}{$\begin{array}{l}\text { BKDV } 4 \\
\text { RMM } V_{\min } 4 \\
\end{array}$} & 29 & $8 / 8$ & 0.54 & \multirow{2}{*}{1.14} & $689 \mathrm{MB}$ \\
\hline & $180(60)$ & $7 / 8$ & 0.60 & & $689 \mathrm{MB}$ \\
\hline \multirow{3}{*}{$\begin{array}{ll}\mathbf{R M M} & V_{\max } 4 \\
\mathbf{R M M} & V_{\max } 4 \\
\mathbf{R M M} & V_{\min } 4 \\
\end{array}$} & $240(80)$ & $7 / 8$ & & 1.93 & $694 \mathrm{MB}$ \\
\hline & $48(16)$ & $8 / 8$ & 0.34 & \multirow{2}{*}{0.93} & $694 \mathrm{MB}$ \\
\hline & $180(60)$ & $7 / 8$ & 0.59 & & $689 \mathrm{MB}$ \\
\hline \multicolumn{6}{|c|}{ Heisenberg Ladderr2(18) } \\
\hline BKDV 4 & 240 & $6 / 8$ & & 5.32 & 692MB \\
\hline \multirow{2}{*}{$\begin{array}{l}\text { BKDV } 4 \\
\text { RMM } V_{\min } 4\end{array}$} & 29 & $8 / 8$ & 0.62 & \multirow{2}{*}{1.31} & 692MB \\
\hline & $180(60)$ & $6 / 8$ & 0.69 & & $692 \mathrm{MB}$ \\
\hline $\mathrm{RMM} V_{\max } 4$ & $240(80)$ & $6 / 8$ & & 2.05 & $699 \mathrm{MB}$ \\
\hline \multirow{2}{*}{$\begin{array}{lll}\mathrm{RMM} & V_{\max } & 4 \\
\mathrm{RMM} & V_{\min } & 4 \\
\end{array}$} & $39(13)$ & $8 / 8$ & 0.32 & \multirow{2}{*}{1.01} & 699MB \\
\hline & $180(60)$ & $6 / 8$ & 0.69 & & 692MB \\
\hline \multicolumn{6}{|c|}{ Heisenberg Ladderr215C(18) } \\
\hline \multirow{3}{*}{$\begin{array}{l}\text { BKDV } 4 \\
\text { RMM } V_{\min } 4\end{array}$} & 240 & $7 / 8$ & & 5.15 & 692MB \\
\hline & 60 & $7 / 8$ & 1.28 & \multirow{2}{*}{1.96} & 692MB \\
\hline & $180(60)$ & $7 / 8$ & 0.68 & & 692MB \\
\hline RMM $V_{\max } 4$ & $240(60)$ & $7 / 8$ & & 2.02 & 699MB \\
\hline \multirow{2}{*}{$\begin{array}{lll}\mathrm{RMM} & V_{\max } & 4 \\
\mathrm{RMM} & V_{\min } & 4 \\
\end{array}$} & $60(20)$ & $7 / 8$ & 0.46 & \multirow{2}{*}{0.76} & $699 \mathrm{MB}$ \\
\hline & $78(26)$ & $8 / 8$ & 0.30 & & 692MB \\
\hline \multicolumn{6}{|c|}{ Heisenberg Ladderr215S(18) } \\
\hline BKDV 4 & 76 & $8 / 8$ & & 1.66 & $692 \mathrm{MB}$ \\
\hline \multirow{2}{*}{$\begin{array}{l}\text { BKDV } 4 \\
\text { RMM } V_{\min } 4 \\
\end{array}$} & $105(35)$ & $8 / 8$ & 0.74 & \multirow{2}{*}{0.91} & 692MB \\
\hline & $42(13)$ & $8 / 8$ & 0.16 & & 692MB \\
\hline RMM $V_{\max } 4$ & $93(31)$ & $8 / 8$ & & 0.85 & 699MB \\
\hline \multirow{2}{*}{$\begin{array}{lll}\mathrm{RMM} & V_{\max } & 4 \\
\mathrm{RMM} & V_{\min } & 4 \\
\end{array}$} & $39(13)$ & $8 / 8$ & 0.30 & \multirow{2}{*}{0.38} & 699MB \\
\hline & $21(7)$ & $8 / 8$ & 0.08 & & $692 \mathrm{MB}$ \\
\hline \multicolumn{6}{|c|}{ 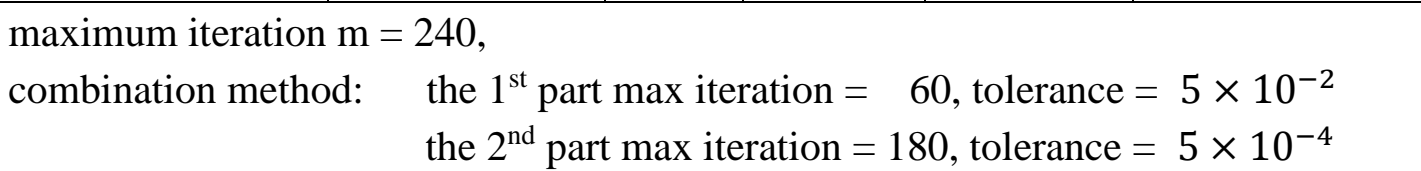 } \\
\hline
\end{tabular}


Table 4.34 Comparison of the results of Ladder315C and Ladder315S models with $N=$ 18 on Odin

\begin{tabular}{|c|c|c|c|c|c|}
\hline Methods & $\begin{array}{l}\text { Number of } \\
\text { iteration } \\
\text { (block) }\end{array}$ & $\begin{array}{l}\text { Conv } \\
\text { ergent }\end{array}$ & \multicolumn{2}{|c|}{ Clock time (second) } & Memory \\
\hline \multicolumn{6}{|c|}{ Heisenberg Ladderr315C(18) } \\
\hline BKDV 4 & 129 & $8 / 8$ & & 2.99 & $692 \mathrm{MB}$ \\
\hline \multirow{2}{*}{$\begin{array}{l}\text { BKDV } 4 \\
\text { RMM } V_{\min } 4\end{array}$} & $66(22)$ & $8 / 8$ & 0.72 & \multirow{2}{*}{1.10} & $692 \mathrm{MB}$ \\
\hline & $90(30)$ & $8 / 8$ & 0.38 & & $692 \mathrm{MB}$ \\
\hline & $153(51)$ & $8 / 8$ & & 1.36 & $700 \mathrm{MB}$ \\
\hline \multirow{2}{*}{$\begin{array}{lll}\mathbf{R M M} & V_{\max } & 4 \\
\mathbf{R M M} & V_{\min } & 4 \\
\end{array}$} & $45(15)$ & $8 / 8$ & 0.36 & \multirow{2}{*}{0.54} & $700 \mathrm{MB}$ \\
\hline & $42(14)$ & $8 / 8$ & 0.18 & & $692 \mathrm{MB}$ \\
\hline \multicolumn{6}{|c|}{ Heisenberg Ladderr315S(18) } \\
\hline \multirow{3}{*}{$\begin{array}{l}\text { BKDV } 4 \\
\text { BKDV } 4 \\
\text { RMM } V_{\min } 4\end{array}$} & 89 & $8 / 8$ & & 2.08 & $692 \mathrm{MB}$ \\
\hline & $87(29)$ & $8 / 8$ & 0.66 & \multirow{2}{*}{0.90} & $692 \mathrm{MB}$ \\
\hline & $57(19)$ & $8 / 8$ & 0.24 & & $692 \mathrm{MB}$ \\
\hline RMM $V_{\max } 4$ & $123(41)$ & $8 / 8$ & & 1.18 & $700 \mathrm{MB}$ \\
\hline \multirow{2}{*}{$\begin{array}{lll}\mathrm{RMM} & V_{\max } & 4 \\
\mathrm{RMM} & V_{\min } & 4 \\
\end{array}$} & $45(15)$ & $8 / 8$ & 0.37 & \multirow{2}{*}{0.51} & $700 \mathrm{MB}$ \\
\hline & $33(11)$ & $8 / 8$ & 0.14 & & $692 \mathrm{MB}$ \\
\hline \multicolumn{6}{|c|}{ 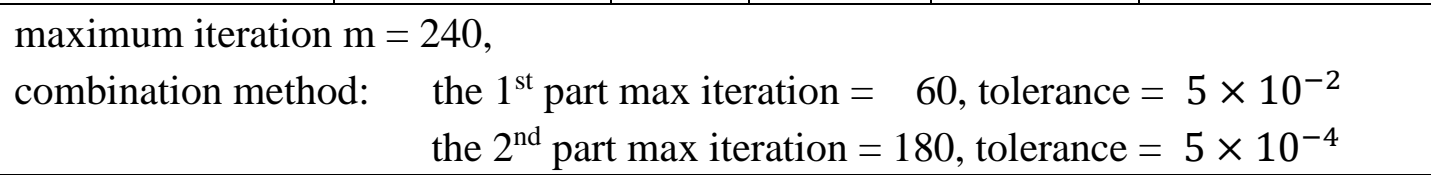 } \\
\hline
\end{tabular}




\subsection{Parallel Speedup on Odin}

All results in section 4.3 are calculated with the RMM-DIIS $V_{\max }$ and $V_{\min }$ method on a dual hex-core machine with $2 \times$ Intel X5650 Xeon processor $(2.67 \mathrm{GHz})$ and 12 GB of DDR3-1333 RAM, we called this machine Odin. The processor of Odin has 12 cores. I tested 6 different models with size $N=26,24,22,20,18$ and 16 .

Table 4.35 Speedup of Chain Ladder2 Ladder215C and Ladder215S models with different size $N=26,24$, and 22 on Odin

\begin{tabular}{|c|c|c|c|c|c|c|c|c|c|}
\hline \multicolumn{2}{|c|}{ Model } & \multicolumn{2}{|c|}{$\operatorname{Chain}(N)$} & \multicolumn{2}{|c|}{$\operatorname{Ladder} 2(N)$} & \multicolumn{2}{|c|}{ Ladder $215 \mathrm{C}(N)$} & \multicolumn{2}{|c|}{ Ladder $215 \mathrm{~S}(N)$} \\
\hline Size & Thread & time & speedup & time & speedup & time & speedup & time & speedup \\
\hline \multirow{7}{*}{$\begin{array}{l}\stackrel{0}{ } \\
\text { II } \\
Z\end{array}$} & 1 & 1169.72 & .00 & 1488.73 & 1.00 & 1450.58 & 1.00 & 1486.58 & 1.00 \\
\hline & 2 & 701.79 & 1.67 & 848.05 & 1.76 & 860.30 & 1.69 & 866.39 & 1.72 \\
\hline & 4 & 580.98 & 2.01 & 673.45 & 2.21 & 677.05 & 2.14 & 692.59 & 2.15 \\
\hline & 6 & 541.21 & 2.16 & 628.30 & 2.37 & 624.19 & 2.32 & 626.17 & 2.37 \\
\hline & 8 & 540.50 & 2.16 & 613.73 & 2.43 & 601.43 & 2.41 & 604.53 & 2.46 \\
\hline & 10 & 541.37 & 2.16 & 605.72 & 2.46 & 605.47 & 2.40 & 603.95 & 2.46 \\
\hline & 12 & 536.10 & 2.18 & 618.36 & 2.41 & 60 & 2.39 & 32 & 2.38 \\
\hline \multirow{7}{*}{\begin{tabular}{c}
\multirow{2}{*}{} \\
$I I$ \\
$Z$
\end{tabular}} & 1 & 256.06 & 1.00 & 307.08 & 1.00 & 314.33 & 1.00 & .40 & 1.00 \\
\hline & 2 & 181.14 & 1.41 & 218.76 & 1.40 & 217.28 & 1.45 & 213.90 & 1.48 \\
\hline & 4 & 152.09 & 1.68 & 181.19 & 1.69 & 180.05 & 1.75 & 175.99 & 1.80 \\
\hline & 6 & 147.76 & 1.73 & 170.64 & 1.80 & 169.13 & 1.86 & 169.10 & 1.88 \\
\hline & 8 & 146.09 & 1.75 & 169.46 & 1.81 & 166.61 & 1.89 & 170.62 & 1.86 \\
\hline & 10 & 150.71 & 1.70 & 174.97 & 1.76 & 167.96 & 1.87 & 176.56 & 1.80 \\
\hline & 12 & 153.24 & 1.67 & 177.76 & 1.73 & 170.44 & 1.84 & 176.25 & 1.80 \\
\hline \multirow{7}{*}{$\begin{array}{l}\text { N } \\
\text { II } \\
Z\end{array}$} & 1 & 60.15 & 1.00 & 70.89 & 1.00 & 70.92 & 1.00 & 70.19 & 1.00 \\
\hline & 2 & 38.33 & 1.57 & 43.87 & 1.62 & 45.10 & 1.57 & 44.89 & 1.56 \\
\hline & 4 & 29.97 & 2.01 & 34.85 & 2.03 & 36.80 & 1.93 & 35.91 & 1.95 \\
\hline & 6 & 26.81 & 2.24 & 32.65 & 2.17 & 34.20 & 2.07 & 33.15 & 2.12 \\
\hline & 8 & 26.38 & 2.28 & 32.20 & 2.20 & 33.75 & 2.10 & 33.29 & 2.11 \\
\hline & 10 & 27.14 & 2.22 & 32.19 & 2.20 & 34.22 & 2.07 & 34.11 & 2.06 \\
\hline & 12 & 26.55 & 2.27 & 32.43 & 2.19 & 34.54 & 2.05 & 34.58 & 2.03 \\
\hline
\end{tabular}

(time in seconds) 
Table 4.36 Speedup of Chain Ladder2 Ladder215C and Ladder215S models with different size $N=20,18$ and 16 on Odin

\begin{tabular}{|c|c|c|c|c|c|c|c|c|c|}
\hline \multicolumn{2}{|c|}{ Model } & \multicolumn{2}{|c|}{ Chain $(N)$} & \multicolumn{2}{|c|}{ Ladder $2(N)$} & \multicolumn{2}{|c|}{ Ladder $215 \mathrm{C}(N)$} & \multicolumn{2}{|c|}{ Ladder215S $(N)$} \\
\hline Size & Thread & time & speedup & time & speedup & time & speedup & time & speedup \\
\hline \multirow{7}{*}{$\begin{array}{l}\text { ते } \\
\text { ॥ } \\
\text { Z }\end{array}$} & 1 & 13.75 & 1.00 & 15.49 & 1.00 & 15.88 & 1.00 & 15.40 & 1.00 \\
\hline & 2 & 8.13 & 1.69 & 9.63 & 1.61 & 9.53 & 1.67 & 9.66 & 1.59 \\
\hline & 4 & 6.09 & 2.26 & 7.49 & 2.07 & 7.37 & 2.16 & 7.53 & 2.05 \\
\hline & 6 & 5.54 & 2.48 & 6.64 & 2.33 & 6.53 & 2.43 & 6.80 & 2.27 \\
\hline & 8 & 5.55 & 2.48 & 6.54 & 2.37 & 6.42 & 2.47 & 6.73 & 2.29 \\
\hline & 10 & 5.48 & 2.51 & 6.66 & 2.33 & 6.42 & 2.47 & 6.86 & 2.25 \\
\hline & 12 & 5.62 & 2.45 & 6.91 & 2.24 & 6.58 & 2.41 & 6.98 & 2.21 \\
\hline \multirow{7}{*}{$\begin{array}{l}\frac{\infty}{\|} \\
\mathrm{Z}\end{array}$} & 1 & 2.98 & 1.00 & 3.53 & 1.00 & 3.52 & 1.00 & 3.41 & 1.00 \\
\hline & 2 & 1.65 & 1.81 & 1.85 & 1.91 & 1.96 & 1.80 & 1.95 & 1.75 \\
\hline & 4 & 0.95 & 3.13 & 1.15 & 3.07 & 1.15 & 3.07 & 1.05 & 3.27 \\
\hline & 6 & 0.77 & 3.89 & 0.87 & 4.08 & 1.01 & 3.47 & 0.84 & 4.08 \\
\hline & 8 & 0.74 & 4.04 & 0.79 & 4.48 & 0.79 & 4.44 & 0.73 & 4.67 \\
\hline & 10 & 0.77 & 3.87 & 0.86 & 4.09 & 0.87 & 4.06 & 0.76 & 4.48 \\
\hline & 12 & 0.95 & 3.14 & 1.02 & 3.47 & 1.01 & 3.47 & 0.96 & 3.54 \\
\hline \multirow{7}{*}{$\begin{array}{c}0 \\
7 \\
\text { II } \\
Z\end{array}$} & 1 & 0.70 & 1.00 & 0.82 & 1.00 & 0.81 & 1.00 & 0.69 & 1.00 \\
\hline & 2 & 0.41 & 1.70 & 0.47 & 1.74 & 0.47 & 1.72 & 0.43 & 1.60 \\
\hline & 4 & 0.30 & 2.33 & 0.33 & 2.46 & 0.32 & 2.51 & 0.30 & 2.25 \\
\hline & 6 & 0.31 & 2.25 & 0.33 & 2.46 & 0.33 & 2.49 & 0.31 & 2.23 \\
\hline & 8 & 0.31 & 2.24 & 0.32 & 2.53 & 0.32 & 2.54 & 0.30 & 2.29 \\
\hline & 10 & 0.40 & 1.73 & 0.38 & 2.16 & 0.40 & 2.04 & 0.39 & 1.74 \\
\hline & 12 & 0.45 & 1.56 & 0.47 & 1.75 & 0.46 & 1.76 & 0.45 & 1.52 \\
\hline
\end{tabular}

(time in seconds) 
Table 4.37 Speedup of Ladder 315C and Ladder 315S models with different size $N=24$ and 18 on an Intel Xeon Phi

\begin{tabular}{|r|c|r|c|r|r|r|r|r|}
\hline \multicolumn{1}{|c|}{ Size } & \multicolumn{4}{|c|}{$N=24$} & \multicolumn{4}{c|}{$N=18$} \\
\hline Model & Ladder315C(N) & \multicolumn{2}{c|}{ Ladder315S(N) } & \multicolumn{1}{c|}{ Ladder315C(N) } & \multicolumn{2}{c|}{ Ladder315S(N) } \\
\hline Threads & time & speedup & time & speedup & time & speedup & time & speedup \\
\hline 1 & 351.83 & 1.00 & 361.07 & 1.00 & 3.92 & 1.00 & 3.92 & 1.00 \\
\hline 2 & 249.30 & 1.41 & 247.25 & 1.46 & 2.20 & 1.78 & 2.39 & 1.64 \\
\hline 4 & 198.73 & 1.77 & 191.09 & 1.89 & 1.23 & 3.17 & 1.29 & 3.04 \\
\hline 6 & 187.16 & 1.88 & 180.32 & 2.00 & 0.98 & 3.99 & 1.03 & 3.80 \\
\hline 8 & 180.08 & 1.95 & 177.01 & 2.04 & 0.91 & 4.31 & 1.00 & 3.93 \\
\hline 10 & 183.46 & 1.92 & 181.74 & 1.99 & 0.98 & 4.01 & 0.96 & 4.10 \\
\hline 12 & 187.74 & 1.87 & 186.23 & 1.94 & 1.02 & 3.84 & 1.07 & 3.65 \\
\hline
\end{tabular}

(time in seconds)

According the Table 4.35, Table 4.36 and Table 4.37, the speedup coefficient are very similar for 8, 10 and 12 threads, In most case, the speedup can reach peak when 8 cores were used in calculations. The number of speedup is larger than 4 in most modes with $N=18$; the maximum number of speedup of 4.67 occurred in the Ladder215S (18) model with 8 threads. Compare using single thread, each core assumes 4.67/8 $=0.58375$ workload. Except when $N=18$, the best speedup for each model and size is around 2 . As before, best speedup were obtained when using 8 threads. The speedup of the Ladder models is a little better than for the Chain model's on Odin. 


\subsection{Parallel Speedup on the Intel Xeon Phi}

In this section, all calculations were made with the RMM-DIIS $V_{\max }$ and $V_{\min }$ method on an Intel Xeon Phi coprocessor 5110P. The Intel Xeon Phi co-processor is a many-core processor based on Intel's Many-integrated Core architecture. It integrates 60 cores with 4 hardware threads per core on one chip. On this machine, the speedup depends on the placement of the threads on the cores, the so-called thread affinity type. The thread affinity can be selected by setting the environment variable KMP_THREAD_AFFINITY.

There are 3 affinity types supported on Intel Many Integrated Core Architecture Ref.[3]: scatter, compact and balanced. The threads are placed on separate cores until all cores have at least one thread under scatter allocation type. The threads are placed to one core until this core is full, and go to next one under compact allocation type. The thread allocation under balanced type is balanced over the cores and the threads allocated to a core are neighbors of each other.

Figure 4.45, Figure 4.46 and Figure 4.47 are simulations of threads allocation under scatter, compact and balanced type. The graphs illustrate the allocation in a 3cores system, in each core allows 4 threads across.

Figure 4.45 shows scatter type, when the threads are placed on separate cores. After

each core has a thread, the following threads will be placed to the core which has the least threads. Scatter type uses as many cores as possible. 
In Figure 4.46, the threads allocate in one core, after allocating 4 threads, HTO is full, and then the threads were placed to next core HT1, compact type lets threads across to one core, and allocates maximum number threads of this core, this method of allocation makes workload of each core is unbalanced when there is no enough workload, it uses cores as less as possible.

Figure 4.47 diagrams the bal anced type, every two consecutive threads are placed to each core when there are 6 threads need to be placed. Each core will have 3 threads if there are 9 threads need to be placed. The threads allocated to a core are neighbors of each other. Hence cache utilization should be efficient if the threads access data that is near in store. The balanced type always gives an average number of threads to each core.

In this section, each result based on the average values of 5 tests, all tests table are in section 6.2 Speedup data on the Intel Xeon Phi.

I planned to record 6 different models with size 26, 24, 22, 20, 18, 16 by 3 different affinity types. There were some problems with Xeon Phi machine at the end of tests, hence, the reports with size $N=26$ were not tested, except Ladder215C models. That's the reason why some data are missing. The first column of each table shows the number of threads from 1 to 240 (1, 10 to 240 in steps of 10$)$. 


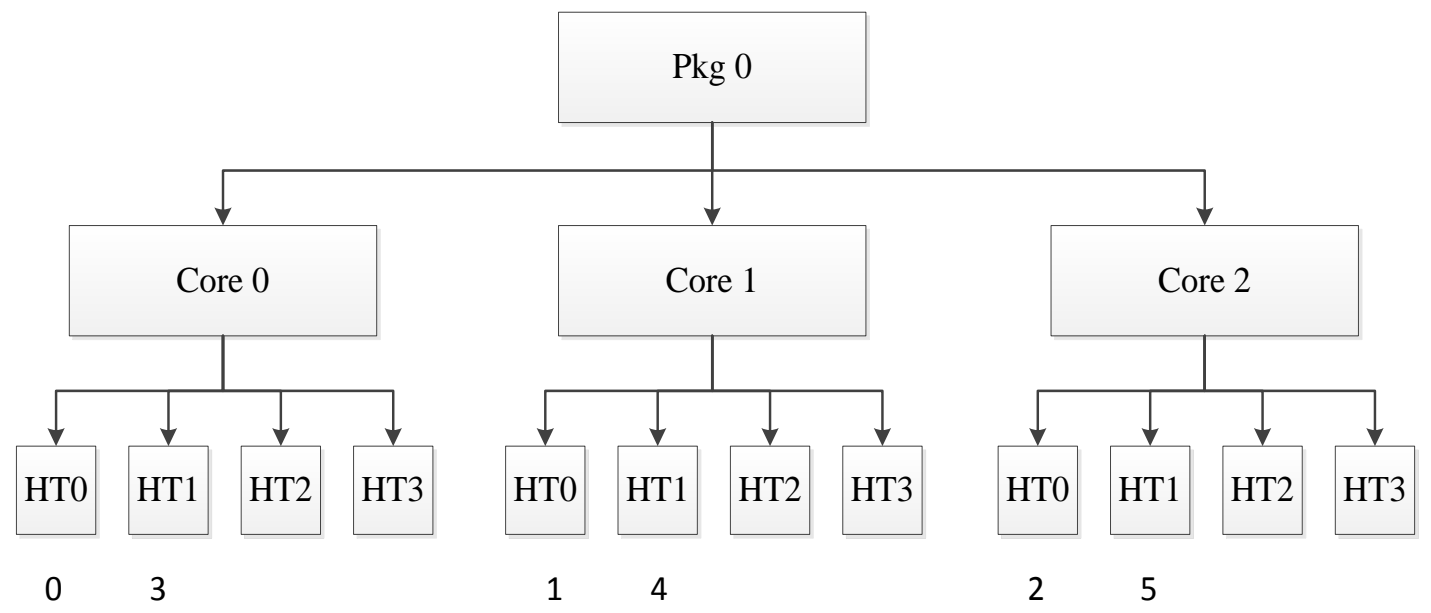

Figure 4.45 Allocation with scatter affinity type

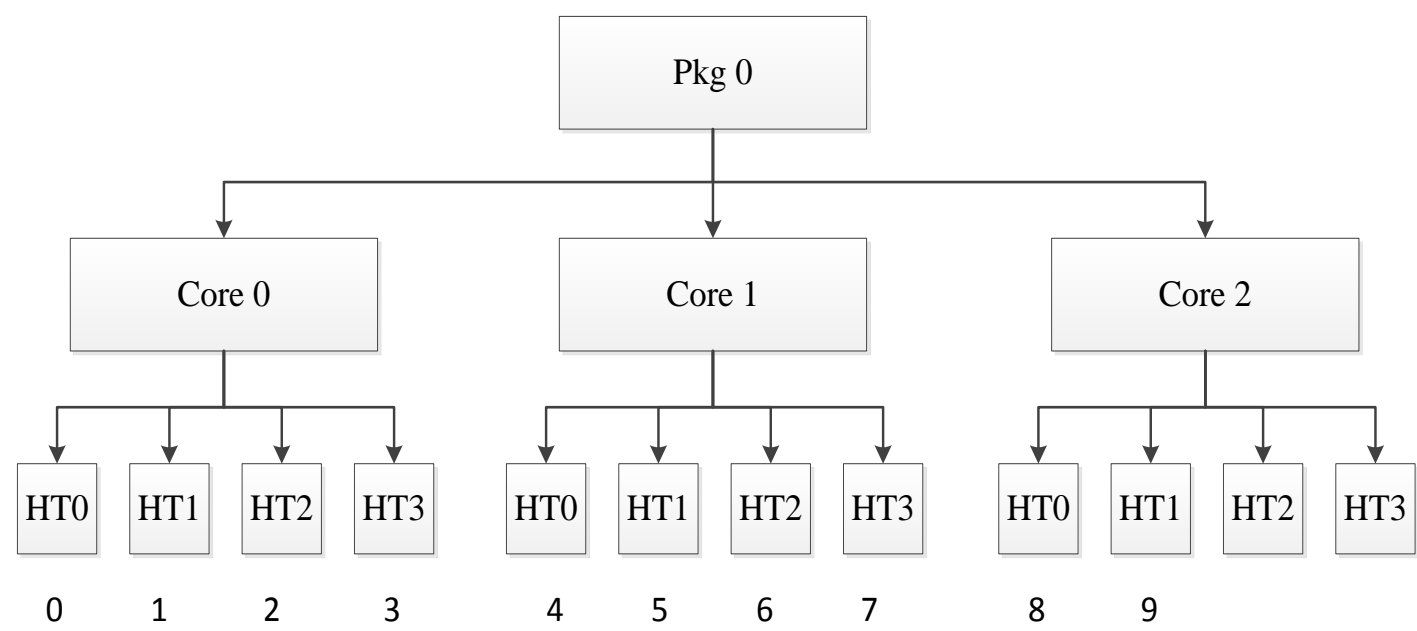

Figure 4.46 Allocation with compact affinity type

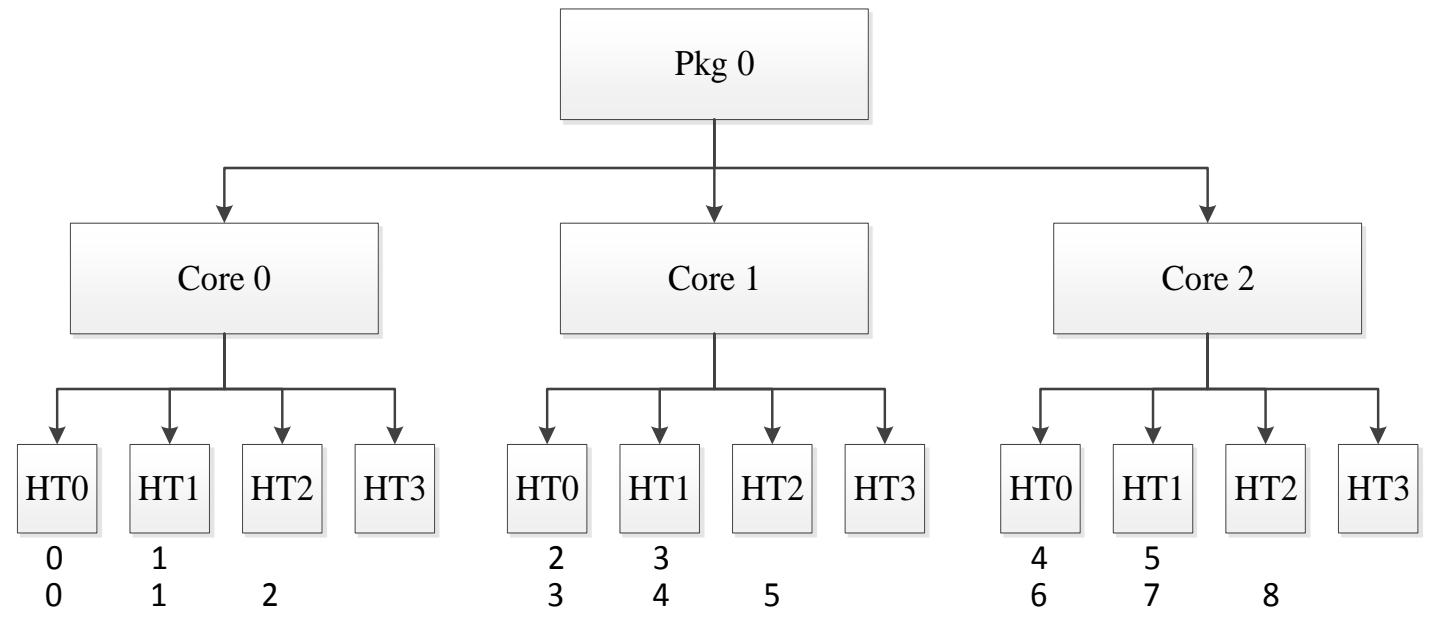

Figure 4.47 Allocation with balanced affinity type

(Line 1 has 6 threads, line 2 has 9 threads) 


\subsubsection{Heisenberg Chain}

Table 4.38 Speedup of Chain $(N)$ with $N=26,24$ and 22 on an Intel Xeon Phi

\begin{tabular}{|c|c|c|c|c|c|c|c|c|c|}
\hline Thread & & $=2$ & & & $N=24$ & & & $N=22$ & \\
\hline 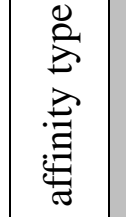 & 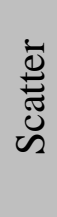 & $\begin{array}{l}\tilde{\tilde{E}} \\
\tilde{\Xi} \\
\tilde{\Xi}\end{array}$ & 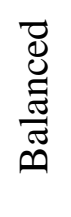 & 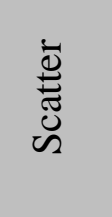 & 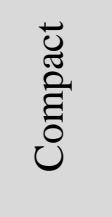 & 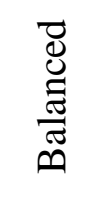 & 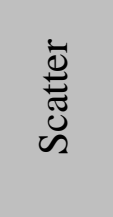 & 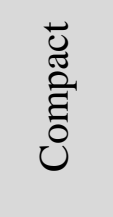 & 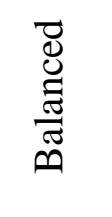 \\
\hline 1 & - & - & - & 1.00 & 1.00 & 1.00 & 1.00 & 1.00 & 1.00 \\
\hline 10 & - & - & - & 4.34 & 3.11 & 4.10 & 4.24 & 3.11 & 3.47 \\
\hline 20 & - & - & - & 7.23 & 5.94 & 6.86 & 7.29 & 5.70 & 5.82 \\
\hline 30 & - & - & - & 9.98 & 8.63 & 9.56 & 10.09 & 8.12 & 8.00 \\
\hline 40 & - & - & - & 13.27 & 11.28 & 12.37 & 13.79 & 10.47 & 10.67 \\
\hline 50 & - & - & - & 16.08 & 13.93 & 15.55 & 16.57 & 12.54 & 12.91 \\
\hline 60 & - & - & - & 18.90 & 16.14 & 18.11 & 18.92 & 14.56 & 14.76 \\
\hline 70 & - & - & - & 20.72 & 18.51 & 20.15 & 20.44 & 16.57 & 16.11 \\
\hline 80 & - & - & - & 23.82 & 21.12 & 22.65 & 22.97 & 19.17 & 18.92 \\
\hline 90 & - & - & - & 25.50 & 22.90 & 24.34 & 24.23 & 20.96 & 19.69 \\
\hline 100 & - & - & - & 27.39 & 25.02 & 25.73 & 26.17 & 23.48 & 21.34 \\
\hline 110 & - & - & - & 28.66 & 26.89 & 27.56 & 28.02 & 25.61 & 22.44 \\
\hline 120 & - & - & - & 29.96 & 28.58 & 29.25 & 29.34 & 27.38 & 23.70 \\
\hline 130 & - & - & - & 30.24 & 30.26 & 30.28 & 29.77 & 28.86 & 24.50 \\
\hline 140 & - & - & - & 31.29 & 31.89 & 31.78 & 30.56 & 30.53 & 25.88 \\
\hline 150 & - & - & - & 33.21 & 33.59 & 33.37 & 25.61 & 26.15 & 6.80 \\
\hline 160 & - & - & - & 33.57 & 35.62 & 35.09 & 26.06 & 26.36 & 6.15 \\
\hline 170 & - & - & - & 33.94 & 35.48 & 35.38 & 26.07 & 25.81 & 5.43 \\
\hline 180 & - & - & - & 35.99 & 38.57 & 37.66 & 25.46 & 25.70 & 4.76 \\
\hline 190 & - & - & - & 36.89 & 40.44 & 38.63 & 25.35 & 25.50 & 4.79 \\
\hline 200 & - & - & - & 37.67 & 41.53 & 40.36 & 24.80 & 24.85 & 3.84 \\
\hline 210 & - & - & - & 39.35 & 42.67 & 41.72 & 24.45 & 24.18 & 3.54 \\
\hline 220 & - & - & - & 40.63 & 43.18 & 41.90 & 23.99 & 23.38 & 3.75 \\
\hline 230 & - & - & - & 43.06 & 44.89 & 44.96 & 23.73 & 22.52 & 3.36 \\
\hline 240 & - & - & - & 43.36 & 45.93 & 45.99 & 22.33 & 21.63 & 3.08 \\
\hline
\end{tabular}


Table 4.39 Speedup of Chain $(N)$ with $N=20,18$ and 16 on an Intel Xeon Phi

\begin{tabular}{|c|c|c|c|c|c|c|c|c|c|}
\hline Thread & & $N=20$ & & & $=18$ & & & $=16$ & \\
\hline 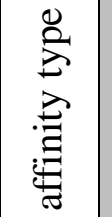 & 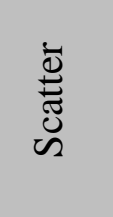 & 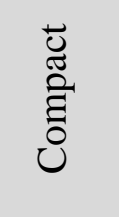 & 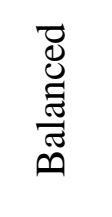 & $\underset{\mathscr{J}}{\stackrel{\Xi}{\Xi}}$ & 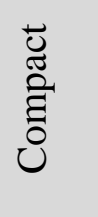 & 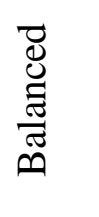 & $\begin{array}{l}\bar{\Xi} \\
\text { ज्ञ } \\
\mathscr{U}\end{array}$ & 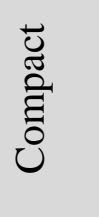 & 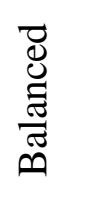 \\
\hline 1 & 1.00 & 1.00 & 1.00 & 1.00 & 1.00 & 1.00 & 1.00 & 1.00 & 1.00 \\
\hline 10 & 4.61 & 2.98 & 13.78 & 3.87 & 2.66 & 3.52 & 2.36 & 1.76 & 2.17 \\
\hline 20 & 7.46 & 5.29 & 15.94 & 4.97 & 3.48 & 4.07 & 2.34 & 1.88 & 2.02 \\
\hline 30 & 9.87 & 7.90 & 20.13 & 7.00 & 4.23 & 5.14 & 2.07 & 1.76 & 1.66 \\
\hline 40 & 10.87 & 9.04 & 16.79 & 6.73 & 4.20 & 4.29 & 1.75 & 1.62 & 1.28 \\
\hline 50 & 11.74 & 9.81 & 13.39 & 6.18 & 4.07 & 3.42 & 1.44 & 1.48 & 0.98 \\
\hline 60 & 12.27 & 10.25 & 10.48 & 5.51 & 3.91 & 2.68 & 1.21 & 1.36 & 0.76 \\
\hline 70 & 12.21 & 10.53 & 6.90 & 4.89 & 3.73 & 1.76 & 1.02 & 1.25 & 0.61 \\
\hline 80 & 12.48 & 10.88 & 6.37 & 4.47 & 3.52 & 1.63 & 0.92 & 1.16 & 0.55 \\
\hline 90 & 14.73 & 12.03 & 5.83 & 4.15 & 3.33 & 1.49 & 0.84 & 1.07 & 0.50 \\
\hline 100 & 13.95 & 11.61 & 5.42 & 3.88 & 3.19 & 1.38 & 0.77 & 0.99 & 0.46 \\
\hline 110 & 13.38 & 11.27 & 4.87 & 3.61 & 3.03 & 1.24 & 0.71 & 0.93 & 0.41 \\
\hline 120 & 12.82 & 10.83 & 4.62 & 3.32 & 2.84 & 1.18 & 0.66 & 0.88 & 0.38 \\
\hline 130 & 11.90 & 10.42 & 4.16 & 3.05 & 2.69 & 1.06 & 0.61 & 0.82 & 0.35 \\
\hline 140 & 11.28 & 10.02 & 3.85 & 2.88 & 2.57 & 0.98 & 0.57 & 0.76 & 0.32 \\
\hline 150 & 10.74 & 9.66 & 3.64 & 2.73 & 2.49 & 0.93 & 0.54 & 0.73 & 0.30 \\
\hline 160 & 10.29 & 9.31 & 3.41 & 2.58 & 2.35 & 0.87 & 0.51 & 0.68 & 0.27 \\
\hline 170 & 9.87 & 8.97 & 3.21 & 2.46 & 2.24 & 0.82 & 0.48 & 0.64 & 0.26 \\
\hline 180 & 9.49 & 8.64 & 3.04 & 2.34 & 2.15 & 0.78 & 0.47 & 0.61 & 0.24 \\
\hline 190 & 8.73 & 8.34 & 3.01 & 2.20 & 2.06 & 0.77 & 0.44 & 0.58 & 0.23 \\
\hline 200 & 8.44 & 8.04 & 2.85 & 2.11 & 1.98 & 0.73 & 0.42 & 0.55 & 0.22 \\
\hline 210 & 8.14 & 7.74 & 2.68 & 2.03 & 1.90 & 0.69 & 0.40 & 0.52 & 0.20 \\
\hline 220 & 7.90 & 7.49 & 2.52 & 1.95 & 1.84 & 0.64 & 0.38 & 0.50 & 0.19 \\
\hline 230 & 7.68 & 7.22 & 2.36 & 1.88 & 1.76 & 0.60 & 0.37 & 0.47 & 0.18 \\
\hline 240 & 7.36 & 6.95 & 2.27 & 1.81 & 1.70 & 0.58 & 0.35 & 0.44 & 0.17 \\
\hline
\end{tabular}

The Table 4.38 and Table 4.39 summarize 18 tables: from Table 6.10 to Table 6.27 


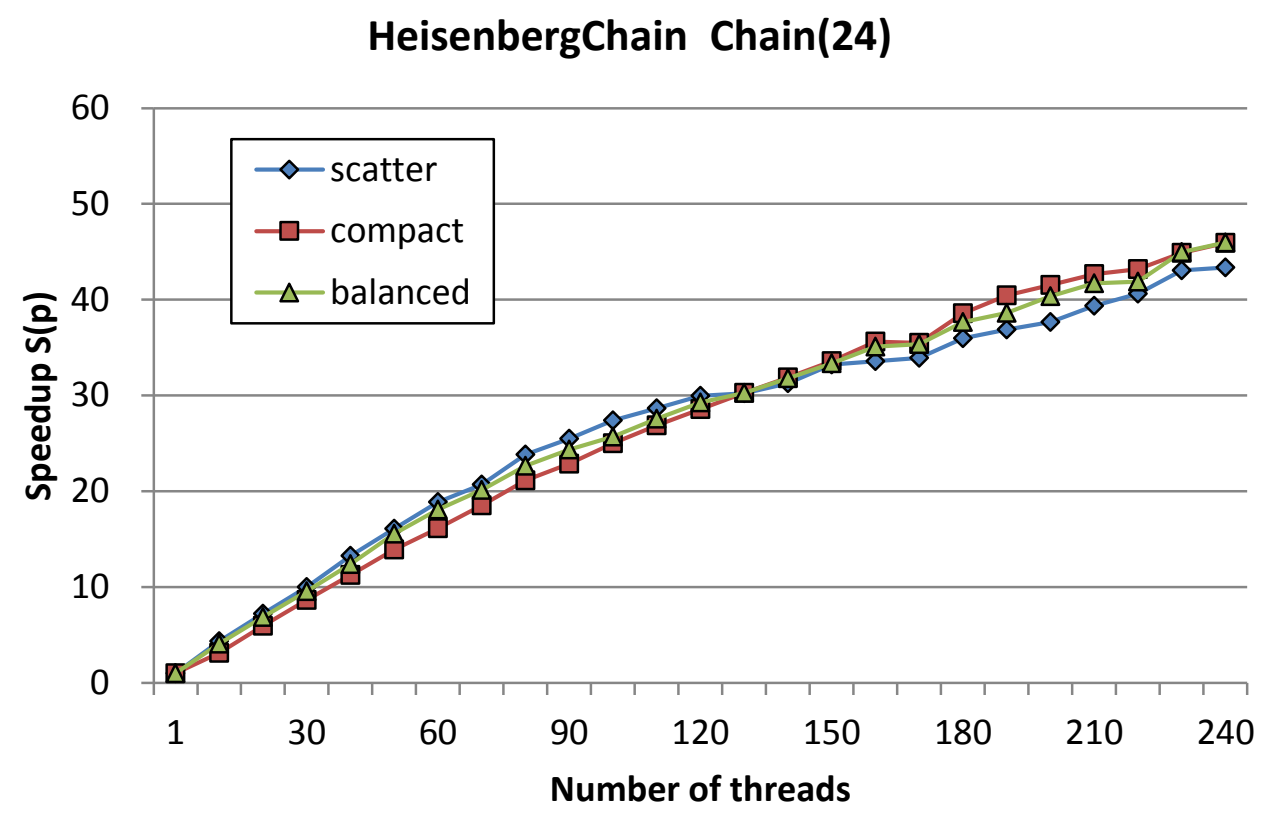

Figure 4.48 Parallel speedup of Chain(24) model on an Intel Xeon Phi with KMP_THREAD_AFFINITY set to scatter, compact and balanced

\section{HeisenbergChain Chain(22)}

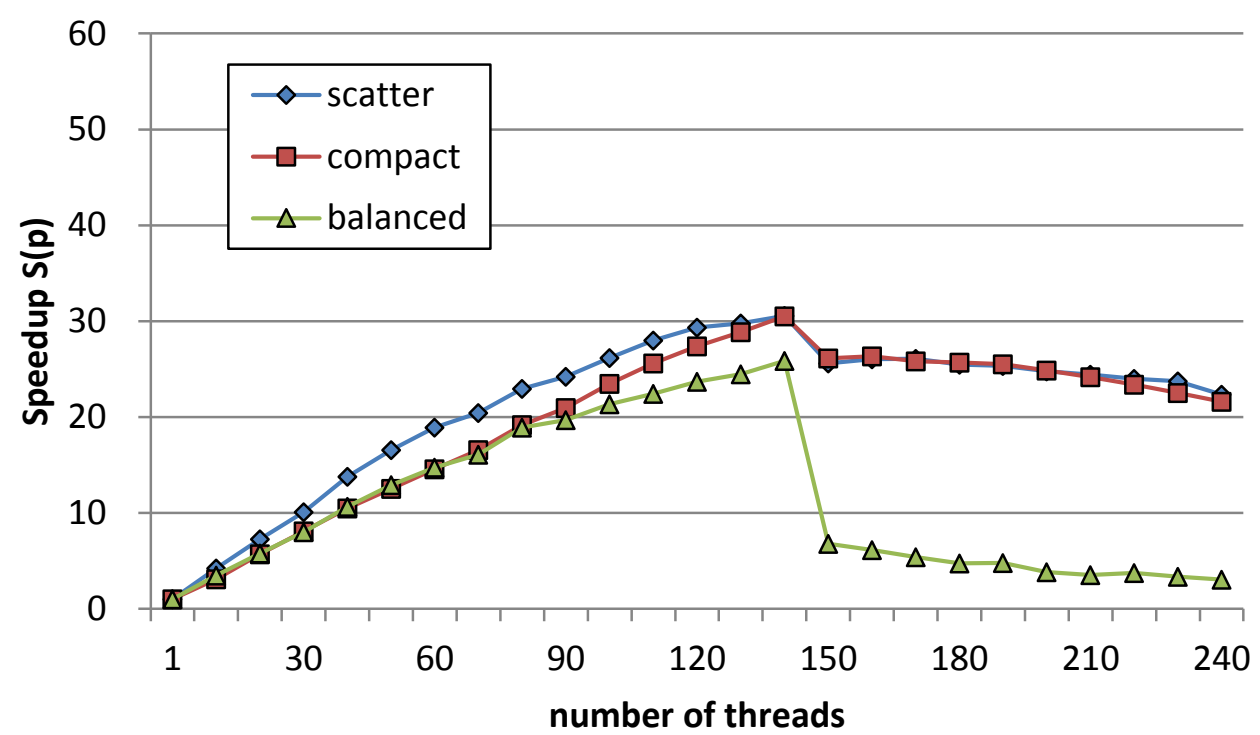

Figure 4.49 Parallel speedup of Chain(22) model on an Intel Xeon Phi with KMP_THREAD_AFFINITY set to scatter, compact and balanced 


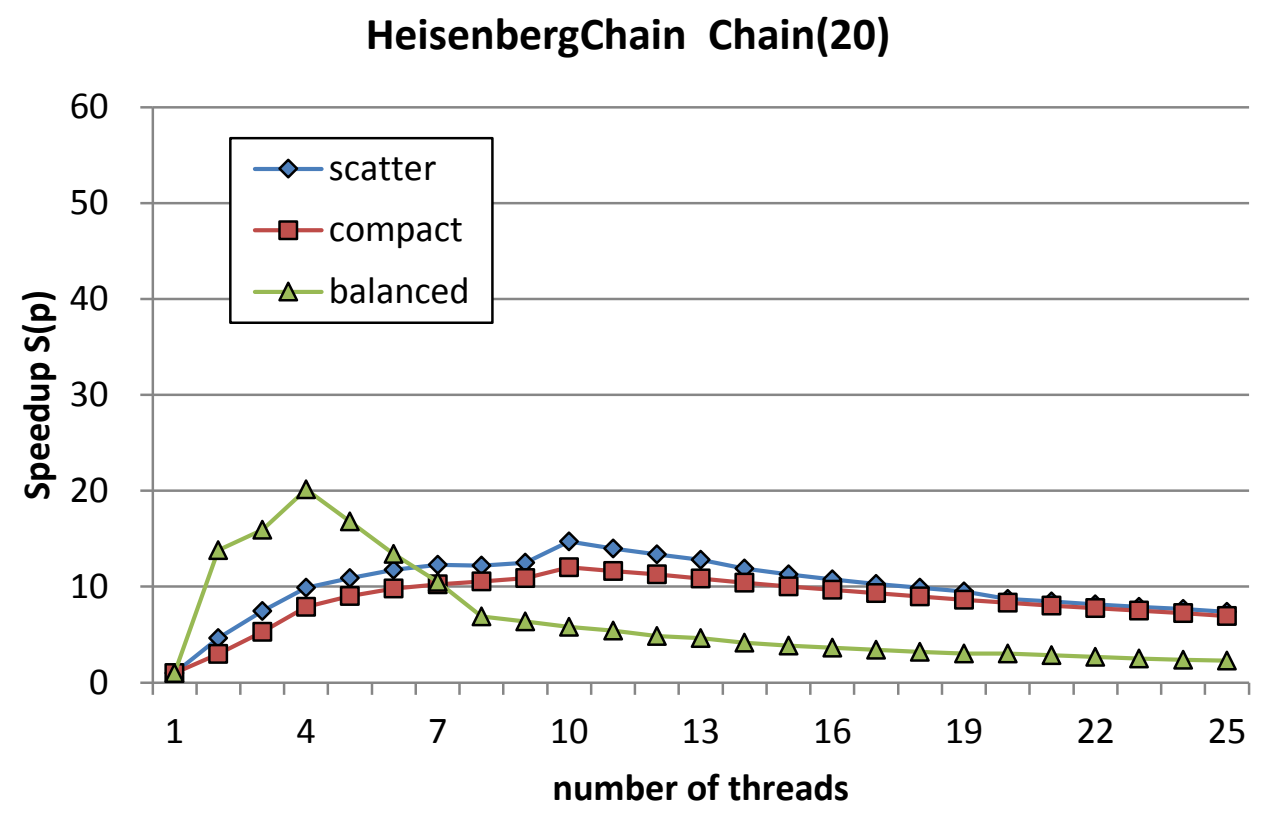

Figure 4.50 Parallel speedup of Chain(24) model on an Intel Xeon Phi with KMP_THREAD_AFFINITY set to scatter, compact and balanced

\section{HeisenbergChain Chain(18)}

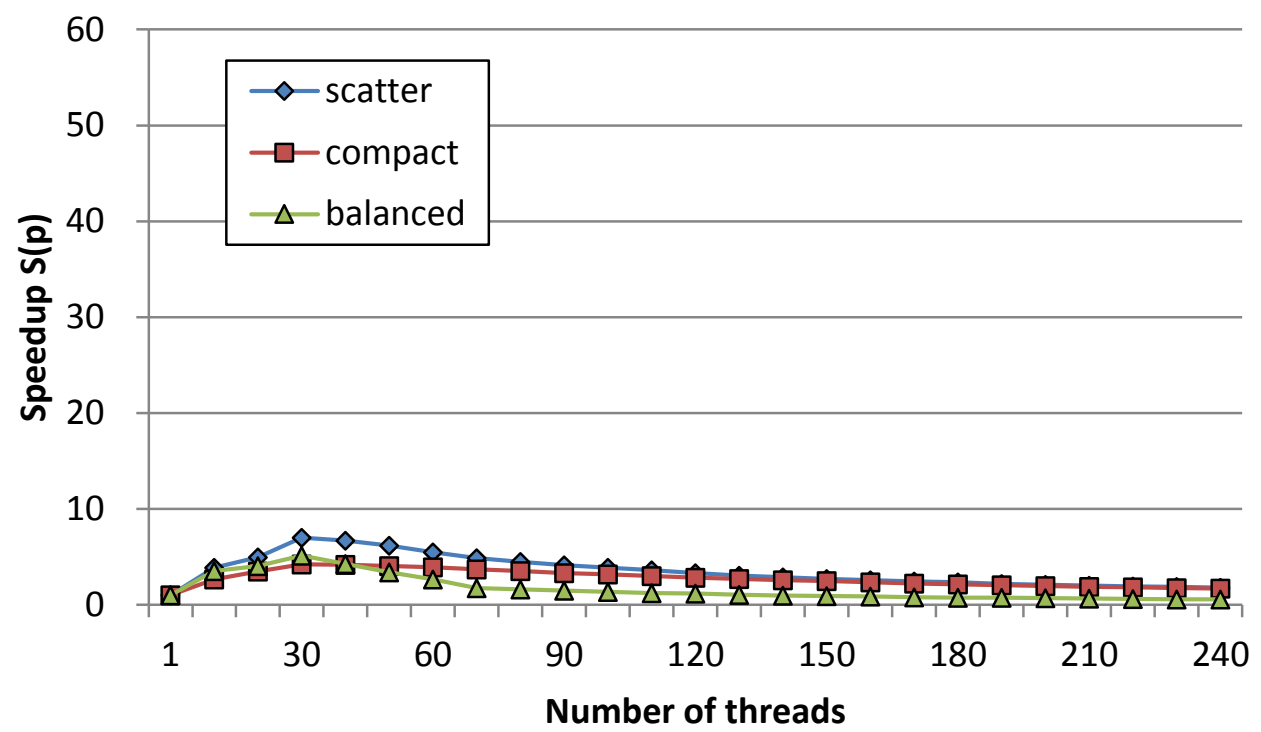

Figure 4.51 Parallel speedup of Chain(18) model on an Intel Xeon Phi with KMP_THREAD_AFFINITY set to scatter, compact and balanced 


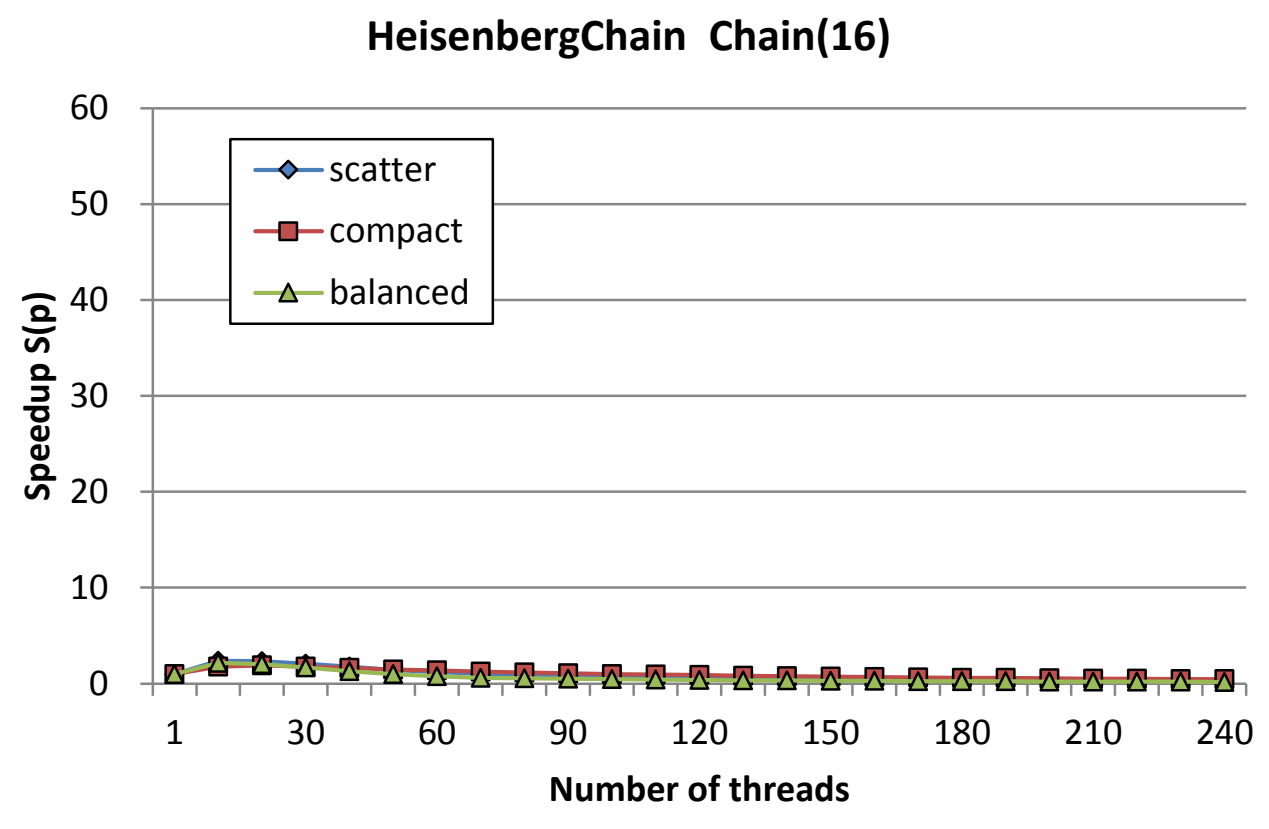

Figure 4.52 Parallel speedup of Chain(16) model on an Intel Xeon Phi with KMP_THREAD_AFFINITY set to scatter, compact and balanced

According the Figure 4.48, Figure 4.49, Figure 4.50, Figure 4.51 and Figure 4.52 as well as Table 4.38 and Table 4.39 , we can find the largest speedup of the Chain model for $N=24$. It is approximate 46 times faster than the execution time using a single thread.

When size $N$ is equal to 24 or larger than 24 , the speedup graph appears to be a linear which looks like monotonically increasing function, that shows, the number of speedup is growing when the number of thread is increasing. Before the number of thread increase to 130 , speedup of Scatter is better than the other two. After thread number reaches to 140, Compact and Balanced type is better than Scatter. 


\subsubsection{Heisenberg Ladder}

Table 4.40 Speedup of Ladde2(N) with $N=26,24$ and 22 on an Intel Xeon Phi

\begin{tabular}{|c|c|c|c|c|c|c|c|c|c|}
\hline Thread & & $=2$ & & & $N=24$ & & & $N=22$ & \\
\hline 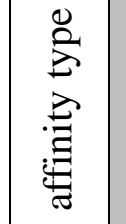 & 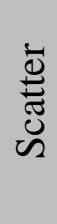 & $\begin{array}{l}\overline{\tilde{E}} \\
\stackrel{\tilde{O}}{\tilde{0}} \\
\tilde{0}\end{array}$ & 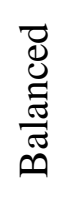 & 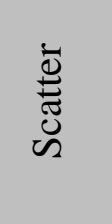 & 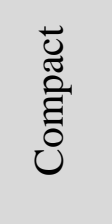 & 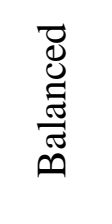 & 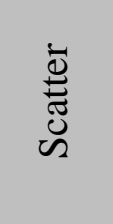 & 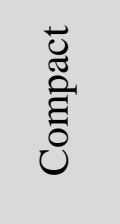 & 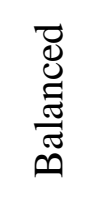 \\
\hline 1 & - & - & - & 1.00 & 1.00 & 1.00 & 1.00 & 1.00 & 1.00 \\
\hline 10 & - & - & - & 4.25 & 3.37 & 4.25 & 4.05 & 3.26 & 4.03 \\
\hline 20 & - & - & - & 7.43 & 6.30 & 7.10 & 6.96 & 5.89 & 7.24 \\
\hline 30 & - & - & - & 9.77 & 9.24 & 10.41 & 10.20 & 8.44 & 10.49 \\
\hline 40 & - & - & - & 13.09 & 12.00 & 12.70 & 13.35 & 10.91 & 13.10 \\
\hline 50 & - & - & - & 15.50 & 14.77 & 15.34 & 15.94 & 12.99 & 16.13 \\
\hline 60 & - & - & - & 18.06 & 17.20 & 18.07 & 18.41 & 15.26 & 18.68 \\
\hline 70 & - & - & - & 20.25 & 19.88 & 20.07 & 20.41 & 17.46 & 20.36 \\
\hline 80 & - & - & - & 22.88 & 22.55 & 22.86 & 23.19 & 20.09 & 23.08 \\
\hline 90 & - & - & - & 25.16 & 24.59 & 25.12 & 23.85 & 22.07 & 24.32 \\
\hline 100 & - & - & - & 26.52 & 26.77 & 27.59 & 26.16 & 24.47 & 26.56 \\
\hline 110 & - & - & - & 28.47 & 28.91 & 29.36 & 28.40 & 26.87 & 28.30 \\
\hline 120 & - & - & - & 30.19 & 30.82 & 31.27 & 29.71 & 28.88 & 29.37 \\
\hline 130 & - & - & - & 31.80 & 32.67 & 32.39 & 30.52 & 30.39 & 30.18 \\
\hline 140 & - & - & - & 33.81 & 34.69 & 34.58 & 31.83 & 32.49 & 32.04 \\
\hline 150 & - & - & - & 35.00 & 36.66 & 35.44 & 27.96 & 28.44 & 8.45 \\
\hline 160 & - & - & - & 35.86 & 38.83 & 37.76 & 28.31 & 28.84 & 7.61 \\
\hline 170 & - & - & - & 35.78 & 39.01 & 38.15 & 28.15 & 28.44 & 6.72 \\
\hline 180 & - & - & - & 37.93 & 42.01 & 40.76 & 27.82 & 28.32 & 5.88 \\
\hline 190 & - & - & - & 38.11 & 44.10 & 41.19 & 27.39 & 28.18 & 5.94 \\
\hline 200 & - & - & - & 40.22 & 45.36 & 42.99 & 26.97 & 27.42 & 4.72 \\
\hline 210 & - & - & - & 42.18 & 46.68 & 45.16 & 26.71 & 26.73 & 4.35 \\
\hline 220 & - & - & - & 42.70 & 47.50 & 45.51 & 26.30 & 25.90 & 4.63 \\
\hline 230 & - & - & - & 46.51 & 49.37 & 49.46 & 26.03 & 25.06 & 4.15 \\
\hline 240 & - & - & - & 47.29 & 50.86 & 50.86 & 25.50 & 24.20 & 3.78 \\
\hline
\end{tabular}


Table 4.41 Speedup of Ladder2 $(N)$ with $N=20,18$ and 16 on an Intel Xeon Phi

\begin{tabular}{|c|c|c|c|c|c|c|c|c|c|}
\hline Thread & & $N=20$ & & & $=18$ & & & $=16$ & \\
\hline 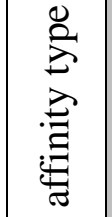 & 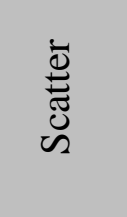 & $\begin{array}{l}\check{\tilde{U}} \\
\stackrel{\tilde{\Xi}}{0} \\
\tilde{0}\end{array}$ & 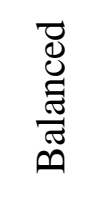 & 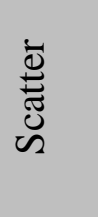 & $\begin{array}{l}\overleftarrow{\tilde{E}} \\
\stackrel{\tilde{E}}{0} \\
\tilde{0}\end{array}$ & 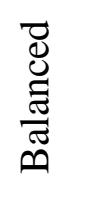 & $\begin{array}{l}\dot{\bar{\Xi}} \\
\stackrel{\Xi}{\tilde{U}} \\
\tilde{\omega}\end{array}$ & $\begin{array}{l}\bar{\circlearrowright} \\
\tilde{\tilde{\Xi}} \\
\tilde{\Xi} \\
\tilde{O}\end{array}$ & 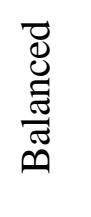 \\
\hline 1 & 1.00 & 1.00 & 1.00 & 1.00 & 1.00 & 1.00 & 1.00 & 1.00 & 1.00 \\
\hline 10 & 4.43 & 3.11 & 4.51 & 4.01 & 2.92 & 3.61 & 2.65 & 1.93 & 2.43 \\
\hline 20 & 7.63 & 5.55 & 7.45 & 5.22 & 3.98 & 4.52 & 2.65 & 2.07 & 2.38 \\
\hline 30 & 10.18 & 8.26 & 10.06 & 7.45 & 4.82 & 6.02 & 2.40 & 1.98 & 2.01 \\
\hline 40 & 11.45 & 9.71 & 8.74 & 6.88 & 4.90 & 5.32 & 2.06 & 1.83 & 1.62 \\
\hline 50 & 12.57 & 10.64 & 8.81 & 6.03 & 4.73 & 4.54 & 1.72 & 1.65 & 1.29 \\
\hline 60 & 13.32 & 11.35 & 8.48 & 5.11 & 4.59 & 3.73 & 1.43 & 1.52 & 1.02 \\
\hline 70 & 13.56 & 11.81 & 4.68 & 4.56 & 4.32 & 2.41 & 1.28 & 1.39 & 0.82 \\
\hline 80 & 13.90 & 12.23 & 4.09 & 4.18 & 4.10 & 2.23 & 1.15 & 1.28 & 0.74 \\
\hline 90 & 13.51 & 12.06 & 3.18 & 3.84 & 3.83 & 2.07 & 1.04 & 1.18 & 0.66 \\
\hline 100 & 13.34 & 11.90 & 2.74 & 3.53 & 3.61 & 1.93 & 0.95 & 1.10 & 0.60 \\
\hline 110 & 13.06 & 11.69 & 2.70 & 3.27 & 3.38 & 1.78 & 0.88 & 1.02 & 0.54 \\
\hline 120 & 12.65 & 11.50 & 2.66 & 3.03 & 3.16 & 1.67 & 0.82 & 0.94 & 0.50 \\
\hline 130 & 13.78 & 12.19 & 2.37 & 2.78 & 2.97 & 1.50 & 0.75 & 0.87 & 0.46 \\
\hline 140 & 13.24 & 11.72 & 2.72 & 2.61 & 2.80 & 1.40 & 0.70 & 0.81 & 0.42 \\
\hline 150 & 12.65 & 11.37 & 2.30 & 2.47 & 2.63 & 1.31 & 0.67 & 0.75 & 0.39 \\
\hline 160 & 12.11 & 10.96 & 2.03 & 2.34 & 2.48 & 1.21 & 0.63 & 0.70 & 0.36 \\
\hline 170 & 11.57 & 10.60 & 1.98 & 2.22 & 2.31 & 1.14 & 0.60 & 0.66 & 0.33 \\
\hline 180 & 11.29 & 10.22 & 1.95 & 2.11 & 2.17 & 1.08 & 0.57 & 0.61 & 0.31 \\
\hline 190 & 10.68 & 9.88 & 2.08 & 1.99 & 2.03 & 1.07 & 0.54 & 0.57 & 0.30 \\
\hline 200 & 10.26 & 9.55 & 2.07 & 1.90 & 1.91 & 1.02 & 0.51 & 0.54 & 0.28 \\
\hline 210 & 9.97 & 9.20 & 1.85 & 1.83 & 1.78 & 0.97 & 0.49 & 0.50 & 0.27 \\
\hline 220 & 9.68 & 8.90 & 1.69 & 1.75 & 1.68 & 0.91 & 0.47 & 0.47 & 0.25 \\
\hline 230 & 9.33 & 8.59 & 1.64 & 1.69 & 1.58 & 0.86 & 0.45 & 0.43 & 0.23 \\
\hline 240 & 9.05 & 8.30 & 1.60 & 1.62 & 1.50 & 0.82 & 0.44 & 0.41 & 0.22 \\
\hline
\end{tabular}

The Table 4.40 and Table 4.41 summarize 18 tables: from Table 6.28 to Table 6.45 in section 6.2 


\section{Heisenberg Ladder2(24)}

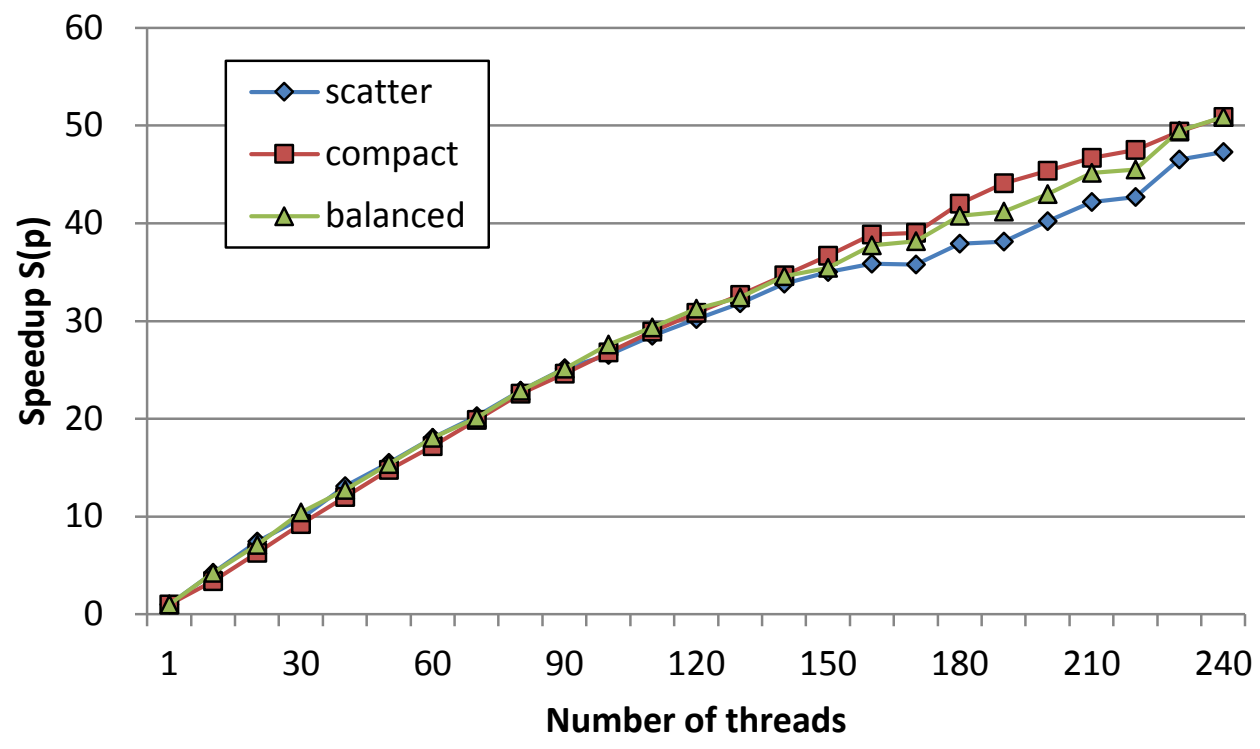

Figure 4.53 Parallel speedup of Ladder2(24) model on an Intel Xeon Phi with KMP_THREAD_AFFINITY set to scatter, compact and balanced

\section{Heisenberg Ladder2(22)}

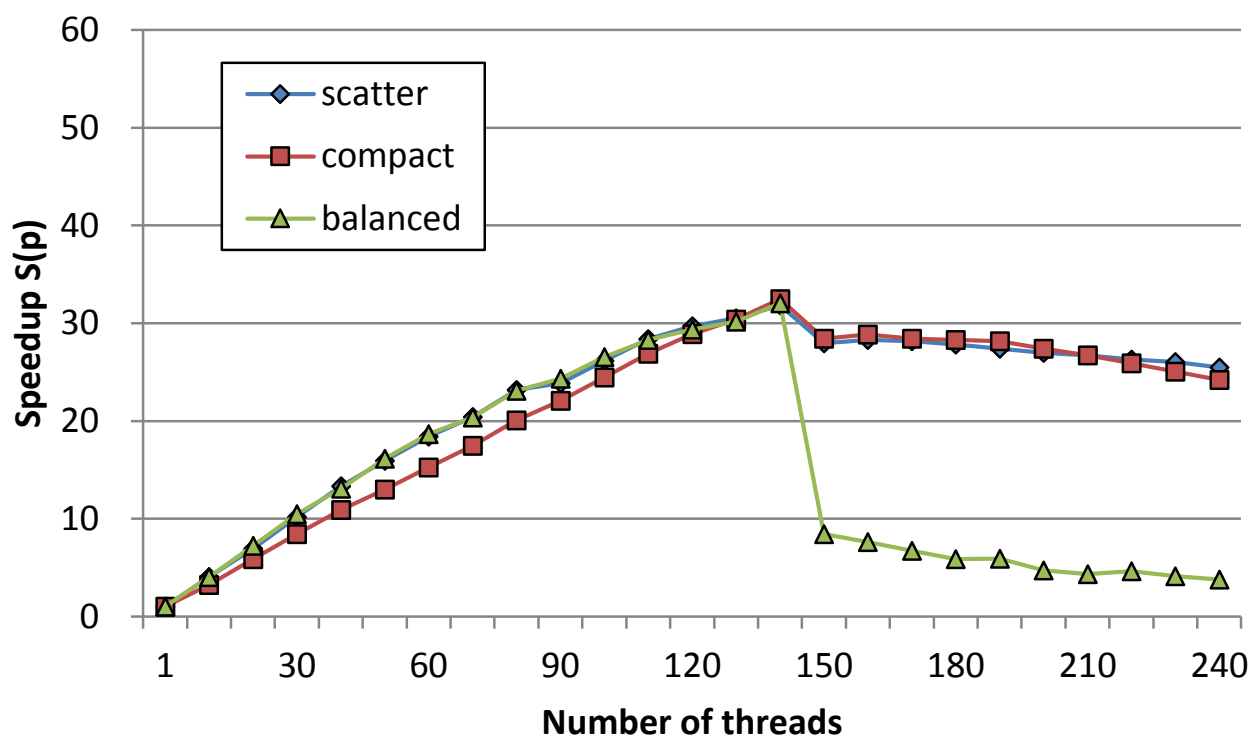

Figure 4.54 Parallel speedup of Ladder2(22) model on an Intel Xeon Phi with KMP_THREAD_AFFINITY set to scatter, compact and balanced 


\section{Heisenberg Ladder2(20)}

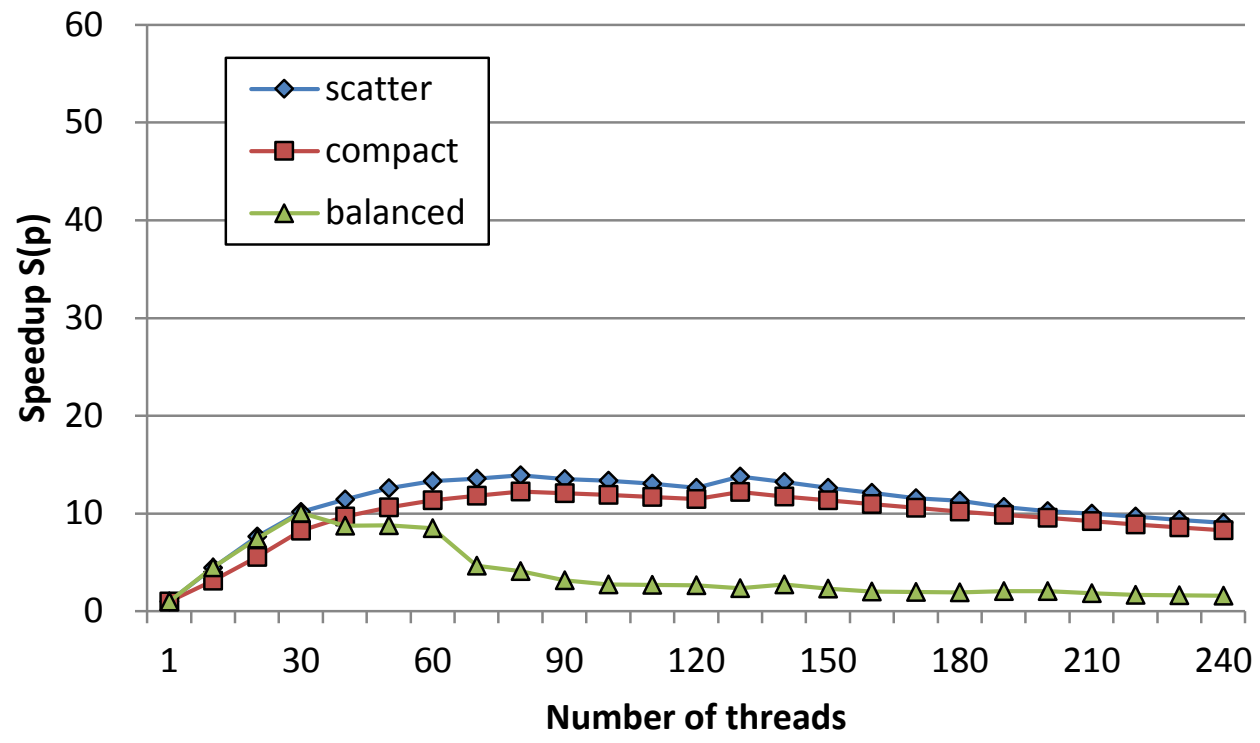

Figure 4.55 Parallel speedup of Ladder2(20) model on an Intel Xeon Phi with KMP_THREAD_AFFINITY set to scatter, compact and balanced

\section{Heisenberg Ladder2(18)}

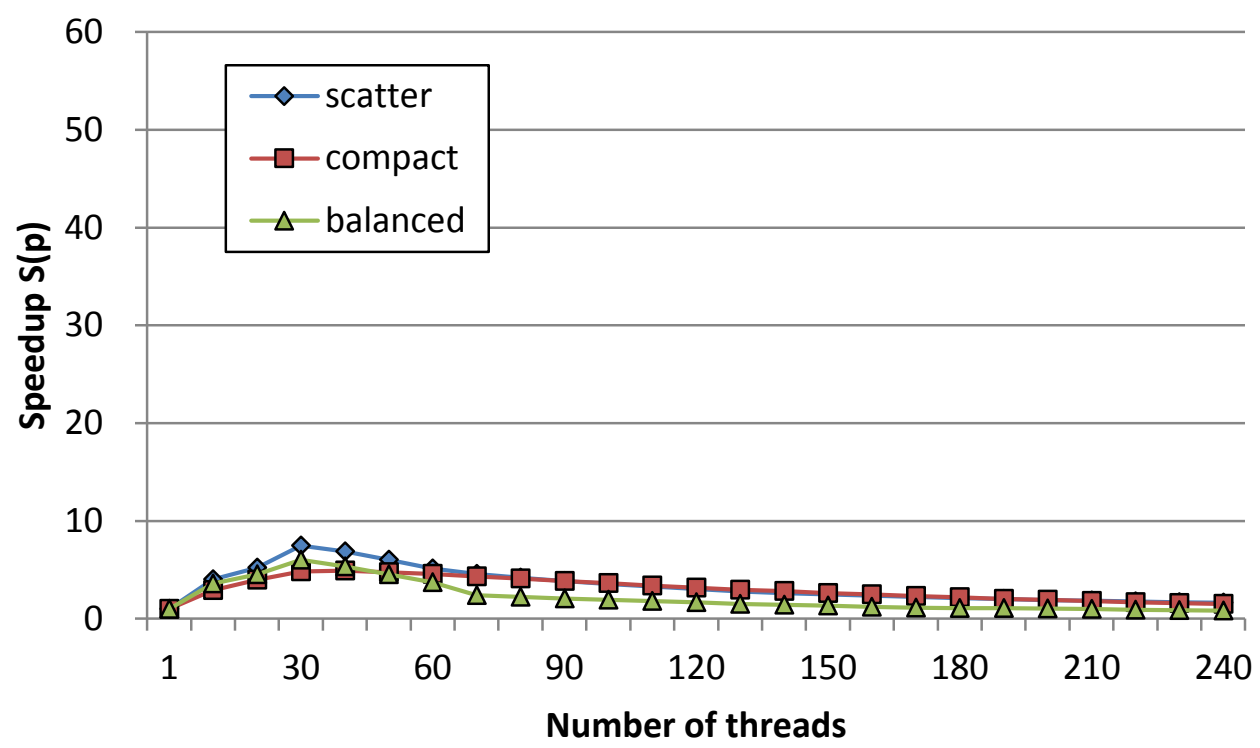

Figure 4.56 Parallel speedup of Ladder2(18) mode on an Intel Xeon Phi with KMP_THREAD_AFFINITY set to scatter, compact and balanced 


\section{Heisenberg Ladder(16)}

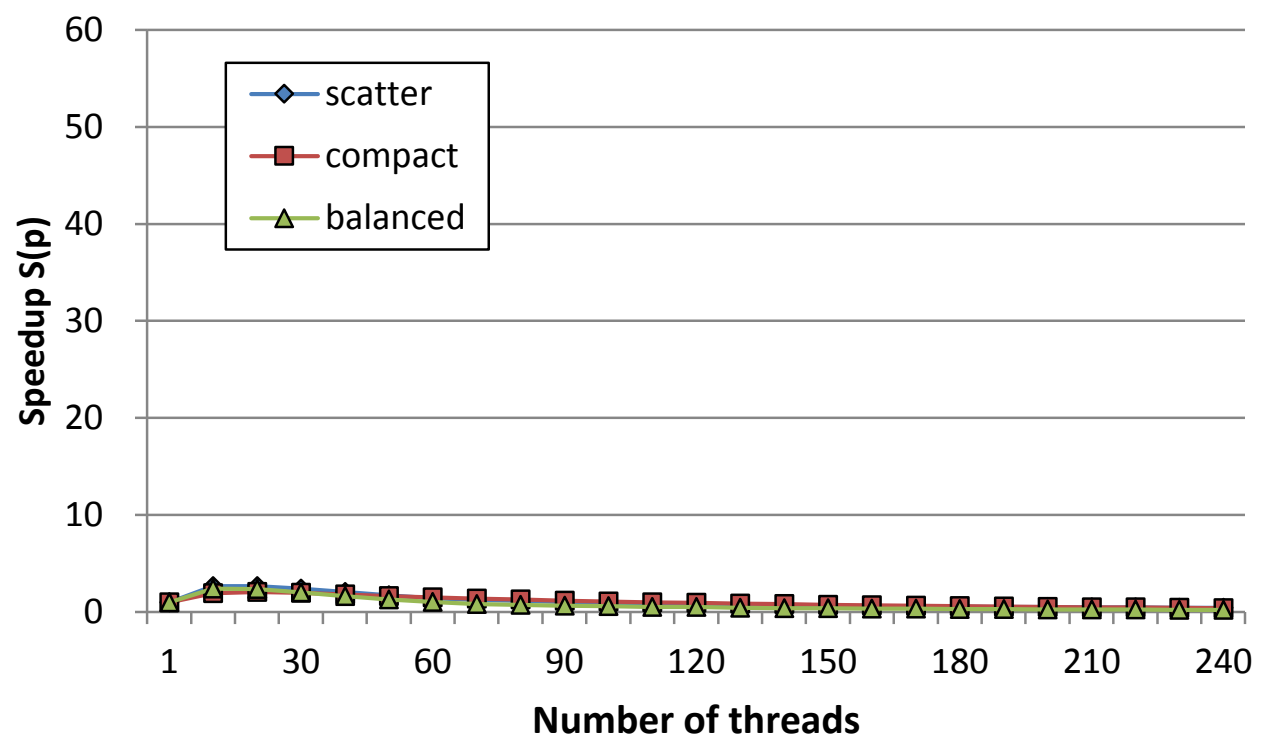

Figure 4.57 Parallel speedup of Ladder2(16) model on an Intel Xeon Phi with KMP_THREAD_AFFINITY set to scatter, compact and balanced

See above the Figure 4.53, Figure 4.54, Figure 4.55, Figure 4.56 and Figure 4.57, review Table 4.40 and Table 4.41, we can find out the largest number of speedup of Ladder2 model is happened when $N=24$, it is 50.86 times faster than the execution time using single thread.

When $N \geq 24$, the speedup graph appears a linear which looks like monotonically increasing function, that shows, the number of speedup is growing when the number of thread is increasing.

Before the number of thread increase to 130, speedup of Scatter is better than the other two. After thread number reaches to 140, Compact and Balanced type is better than Scatter. 


\subsubsection{Heisenberg Ladder 215C}

Table 4.42 Speedup of Ladder 215C $(N)$ with $N=26,24$ and 22 on Xeon Phi

\begin{tabular}{|c|c|c|c|c|c|c|c|c|c|}
\hline Thread & & $N=26$ & & & $N=24$ & & & $N=22$ & \\
\hline 氖 & 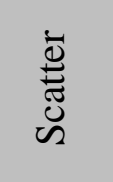 & $\begin{array}{l}\check{\tilde{\Xi}} \\
\stackrel{\Xi}{0} \\
\tilde{\Xi} \\
\tilde{O}\end{array}$ & 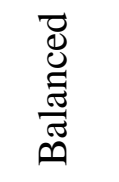 & 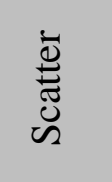 & $\begin{array}{l}\check{\tilde{U}} \\
\stackrel{\tilde{Z}}{0} \\
\tilde{0}\end{array}$ & 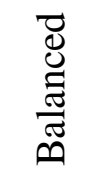 & $\underset{\mathscr{E}}{\stackrel{\bar{\Xi}}{E}}$ & $\begin{array}{l}\check{U} \\
\tilde{\Xi} \\
\tilde{\Xi} \\
\tilde{0}\end{array}$ & 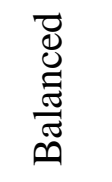 \\
\hline 1 & 1.00 & 1.00 & 0 & 1.00 & 1.00 & 1.00 & 1.00 & 1.00 & 1.00 \\
\hline 10 & 4.16 & 3.44 & 4.07 & 4.18 & 3.38 & 4.34 & 4.40 & 3.26 & 4.22 \\
\hline 20 & 7.11 & 6.54 & 7.21 & 7.29 & 6.29 & 7.30 & 7.21 & 5.89 & 7.38 \\
\hline 30 & 9.92 & 9.50 & .29 & 9.92 & 9.21 & 9.97 & 9.99 & 8.43 & 0.27 \\
\hline 40 & 13.26 & 12.61 & .27 & 12.68 & 12.00 & 2.97 & 12.97 & 10.90 & 13.27 \\
\hline 50 & 15.62 & 15.65 & 15.91 & 15.51 & 14.77 & 15.71 & 16.04 & 13.00 & 15.99 \\
\hline 60 & 18.39 & 18.43 & 43 & 18.30 & 17.20 & 18.12 & 18.87 & 5.26 & 17.85 \\
\hline 70 & 20.67 & 21.40 & 20 & 20.57 & 19.88 & 20.32 & 20.88 & 7.47 & 19.84 \\
\hline 80 & 23.36 & 24.35 & 23.49 & 22.85 & 22.55 & 23.27 & 22.81 & 20.10 & 22.54 \\
\hline 90 & 25.35 & 26.75 & 25.56 & 25.28 & 24.58 & 24.88 & 24.30 & 22.04 & 24.29 \\
\hline 100 & 26.55 & 29.28 & 28.18 & 27.45 & 26.76 & 27.04 & 26.40 & 24.46 & 26.44 \\
\hline 110 & 29.57 & 31.88 & 31.09 & 29.44 & 28.91 & 29.05 & 28.27 & 26.81 & 28.14 \\
\hline 120 & 98 & 34.11 & 32.88 & 4 & 5 & 5 & 29.76 & 28.79 & 29.50 \\
\hline 130 & 54 & 36.39 & 3 & 31.62 & 32.71 & 6 & 30 & 38 & 30.42 \\
\hline 140 & 34.81 & 38.91 & 36.43 & 33.88 & 34.71 & 34.19 & 32.17 & 32.45 & 32.03 \\
\hline 150 & 35.18 & 41.21 & 38.21 & 35.19 & 36.65 & 36.01 & 28.34 & 28.59 & 8.45 \\
\hline 160 & 37.89 & 43.81 & 40.49 & 36.14 & 38.88 & 38.39 & 28.09 & 29.00 & 7.61 \\
\hline 170 & 39.14 & 45.69 & 55 & 36.66 & 38.99 & 38.59 & 27.93 & 28.66 & 6.69 \\
\hline 180 & 40.43 & 47.68 & 43.27 & 37.94 & 42.06 & 4 & 27.94 & 20.05 & 5.87 \\
\hline 190 & 41.52 & 50.27 & 33 & 37 & 7 & 1 & 27 & DJ & 5.92 \\
\hline 200 & 42.12 & 51.82 & 47.37 & 39.98 & 45.40 & 43.13 & 27.12 & 27.96 & 4.73 \\
\hline 210 & 44.30 & 53.65 & 49.12 & 42.49 & 46.72 & 45.27 & 26.82 & 27.38 & 4.36 \\
\hline 220 & 46.42 & 55.11 & 50.42 & 43.88 & 47.59 & 45.55 & 26.55 & 26.66 & 4.64 \\
\hline 230 & 50.18 & 56.83 & 56.79 & 46.66 & 49.39 & 49.51 & 26.25 & 25.91 & 4.14 \\
\hline 240 & 50.81 & 58.37 & 58.21 & 47.27 & 50.89 & 50.92 & 25.68 & 25.23 & 3.79 \\
\hline
\end{tabular}


Table 4.43 Speedup of Ladder $215 \mathrm{C}(N)$ with $N=20,18$ and 16 on an Intel Xeon Phi

\begin{tabular}{|c|c|c|c|c|c|c|c|c|c|}
\hline \multirow{2}{*}{ 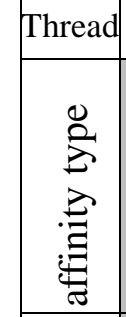 } & \multicolumn{3}{|c|}{$N=20$} & \multicolumn{3}{|c|}{$N=18$} & \multicolumn{3}{|c|}{$N=16$} \\
\hline & 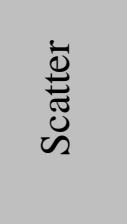 & $\begin{array}{l}\check{\tilde{U}} \\
\stackrel{\tilde{\Xi}}{0} \\
\tilde{0}\end{array}$ & 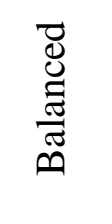 & 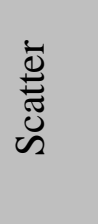 & $\begin{array}{l}\overleftarrow{U} \\
\tilde{\Xi} \\
\tilde{\Xi} \\
\tilde{0}\end{array}$ & 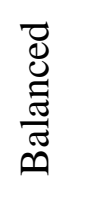 & 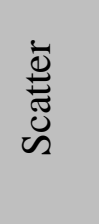 & 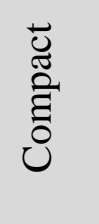 & 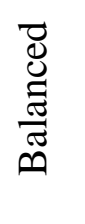 \\
\hline 1 & 1.00 & 1.00 & 1.00 & 1.00 & 1.00 & 1.00 & 1.00 & 1.00 & 1.00 \\
\hline 10 & 4.69 & 3.14 & 4.59 & 3.98 & 2.95 & 3.66 & 2.71 & 1.97 & 2.41 \\
\hline 20 & 7.53 & 5.60 & 7.53 & 5.24 & 4.02 & 4.46 & 2.74 & 2.12 & 2.33 \\
\hline 30 & 9.91 & 8.33 & 10.03 & 7.57 & 4.87 & 5.76 & 2.47 & 2.02 & 1.91 \\
\hline 40 & 11.45 & 9.78 & 8.74 & 7.06 & 5.01 & 5.03 & 2.12 & 1.88 & 1.52 \\
\hline 50 & 12.34 & 10.71 & 8.71 & 6.21 & 4.82 & 4.11 & 1.78 & 1.71 & 1.20 \\
\hline 60 & 12.90 & 11.43 & 8.22 & 5.26 & 4.73 & 3.25 & 1.49 & 1.57 & 0.93 \\
\hline 70 & 12.99 & 11.89 & 4.64 & 4.64 & 4.50 & 2.13 & 1.31 & 1.45 & 0.75 \\
\hline 80 & 13.08 & 12.35 & 3.96 & 4.24 & 4.27 & 1.99 & 1.19 & 1.34 & 0.67 \\
\hline 90 & 12.55 & 12.25 & 3.09 & 3.89 & 4.06 & 1.85 & 1.07 & 1.22 & 0.60 \\
\hline 100 & 12.16 & 12.10 & 2.67 & 3.58 & 3.84 & 1.69 & 0.98 & 1.14 & 0.55 \\
\hline 110 & 11.77 & 11.87 & 2.62 & 3.32 & 3.63 & 1.55 & 0.91 & 1.05 & 0.49 \\
\hline 120 & 11.38 & 11.62 & 2.58 & 3.08 & 3.45 & 1.46 & 0.84 & 0.98 & 0.46 \\
\hline 130 & 12.08 & 12.36 & 2.27 & 2.84 & 3.27 & 1.34 & 0.78 & 0.91 & 0.42 \\
\hline 140 & 11.47 & 11.93 & 2.58 & 2.65 & 3.11 & 1.24 & 0.73 & 0.86 & 0.38 \\
\hline 150 & 10.82 & 11.58 & 2.18 & 2.51 & 2.97 & 1.16 & 0.69 & 0.81 & 0.36 \\
\hline 160 & 10.32 & 11.19 & 1.94 & 2.37 & 2.84 & 1.08 & 0.65 & 0.76 & 0.33 \\
\hline 170 & 9.90 & 10.84 & 1.88 & 2.26 & 2.71 & 1.02 & 0.62 & 0.71 & 0.31 \\
\hline 180 & 9.72 & 10.45 & 1.84 & 2.15 & 2.59 & 0.98 & 0.59 & 0.67 & 0.29 \\
\hline 190 & 9.15 & 10.12 & 1.98 & 2.02 & 2.49 & 0.96 & 0.55 & 0.63 & 0.27 \\
\hline 200 & 8.84 & 9.81 & 1.93 & 1.93 & 2.38 & 0.92 & 0.53 & 0.60 & 0.26 \\
\hline 210 & 8.50 & 9.47 & 1.72 & 1.85 & 2.29 & 0.86 & 0.51 & 0.56 & 0.24 \\
\hline 220 & 8.22 & 9.16 & 1.58 & 1.78 & 2.20 & 0.81 & 0.49 & 0.53 & 0.23 \\
\hline 230 & 7.96 & 8.85 & 1.54 & 1.71 & 2.12 & 0.76 & 0.47 & 0.50 & 0.22 \\
\hline 240 & 7.68 & 8.57 & 1.51 & 1.64 & 2.03 & 0.73 & 0.45 & 0.47 & 0.21 \\
\hline
\end{tabular}

The Table 4.42 and Table 4.43 summarize 18 tables: from Table 6.46 to Table 6.63 in section 6.2 


\section{Heisenberg Ladder215C(26)}

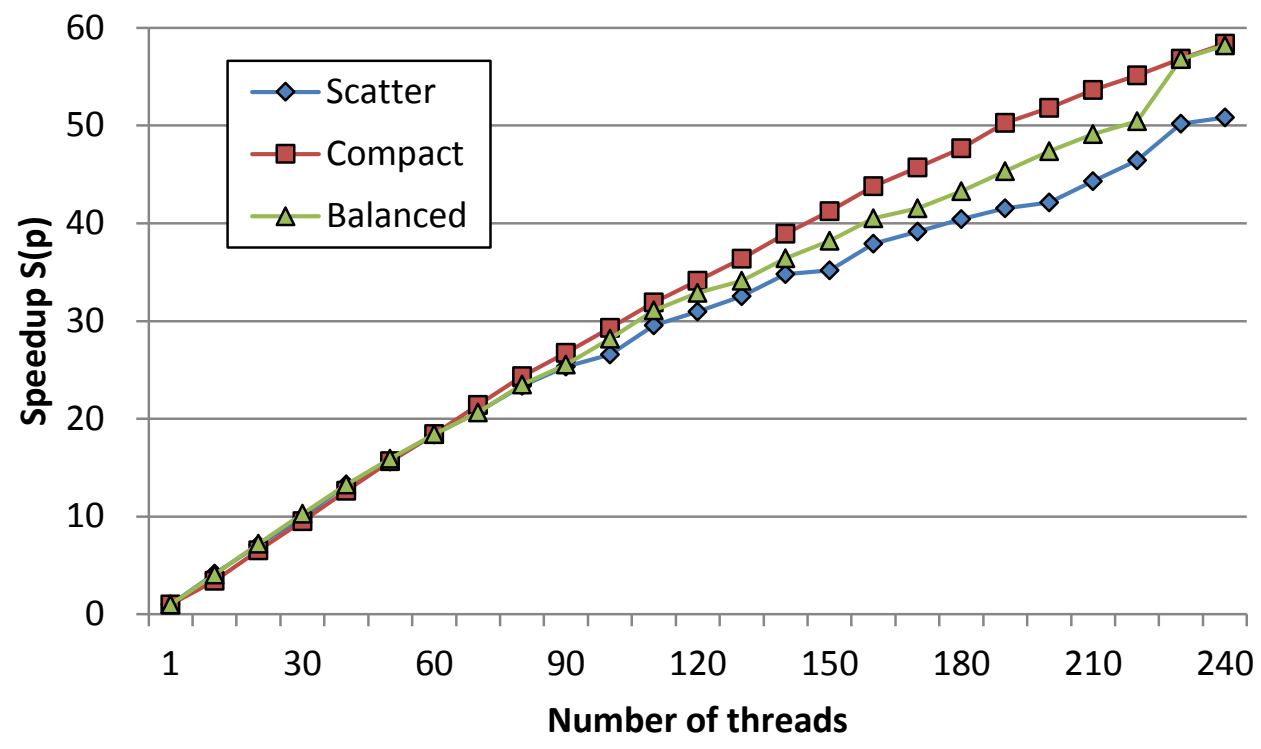

Figure 4.58 Parallel speedup of Ladder215C(26) model on an Intel Xeon Phi with KMP_THREAD_AFFINITY set to scatter, compact and balanced

\section{Heisenberg Ladder215C(24)}

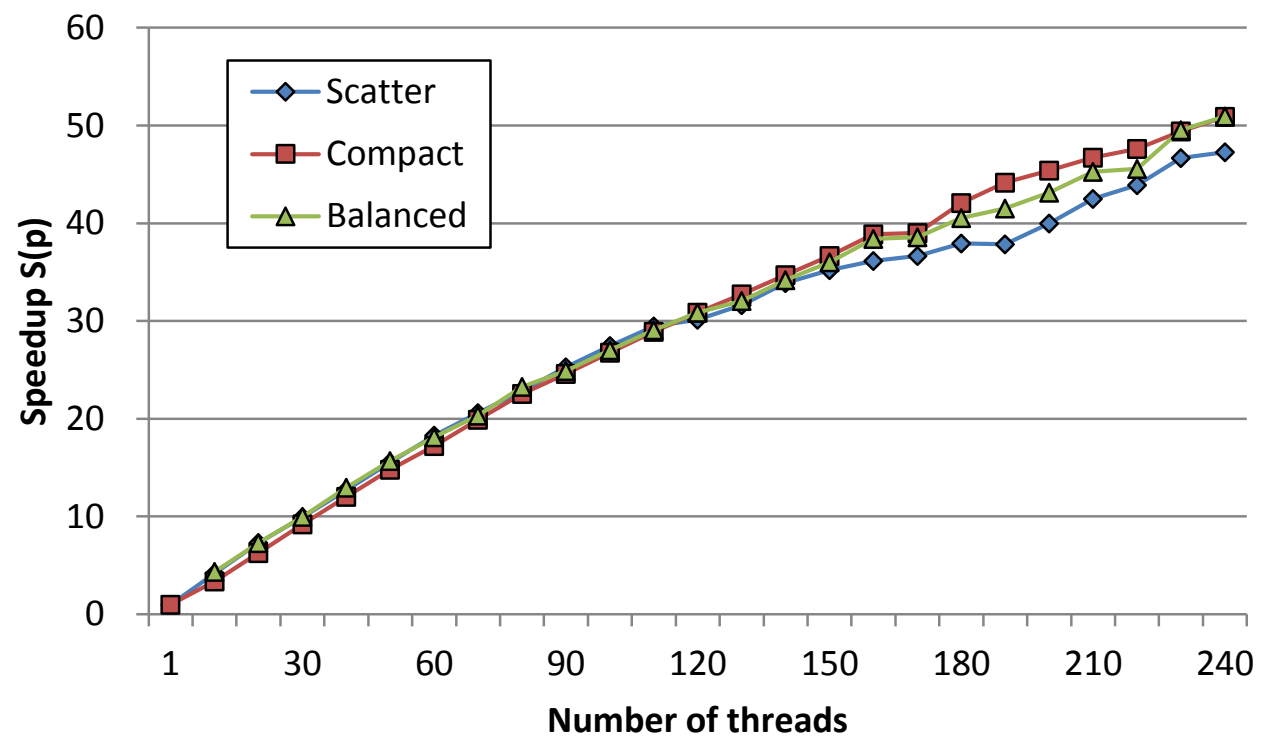

Figure 4.59 Parallel speedup of Ladder215C(24) model on an Intel Xeon Phi with KMP_THREAD_AFFINITY set to scatter, compact and balanced 


\section{Heisenberg Ladder215C(22)}

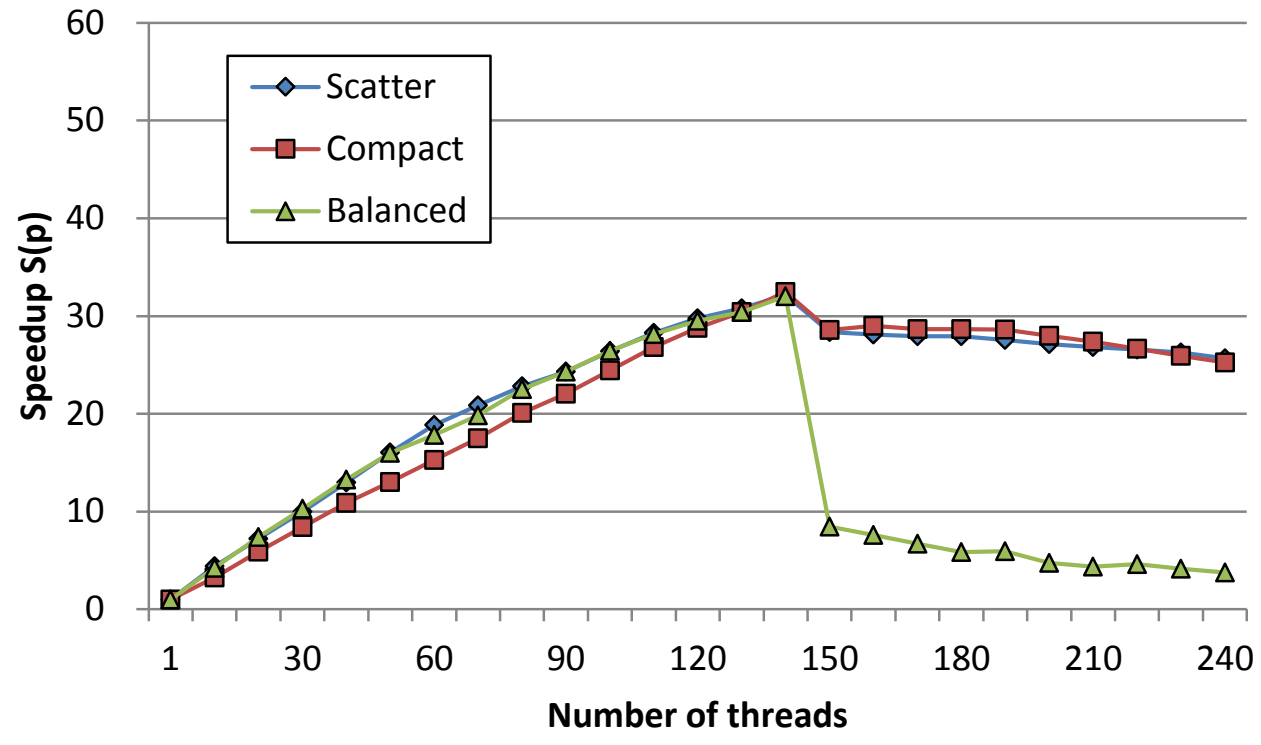

Figure 4.60 Parallel speedup of Ladder215C(22) model on an Intel Xeon Phi with KMP_THREAD_AFFINITY set to scatter, compact and balanced

\section{Heisenberg Ladder215C(20)}

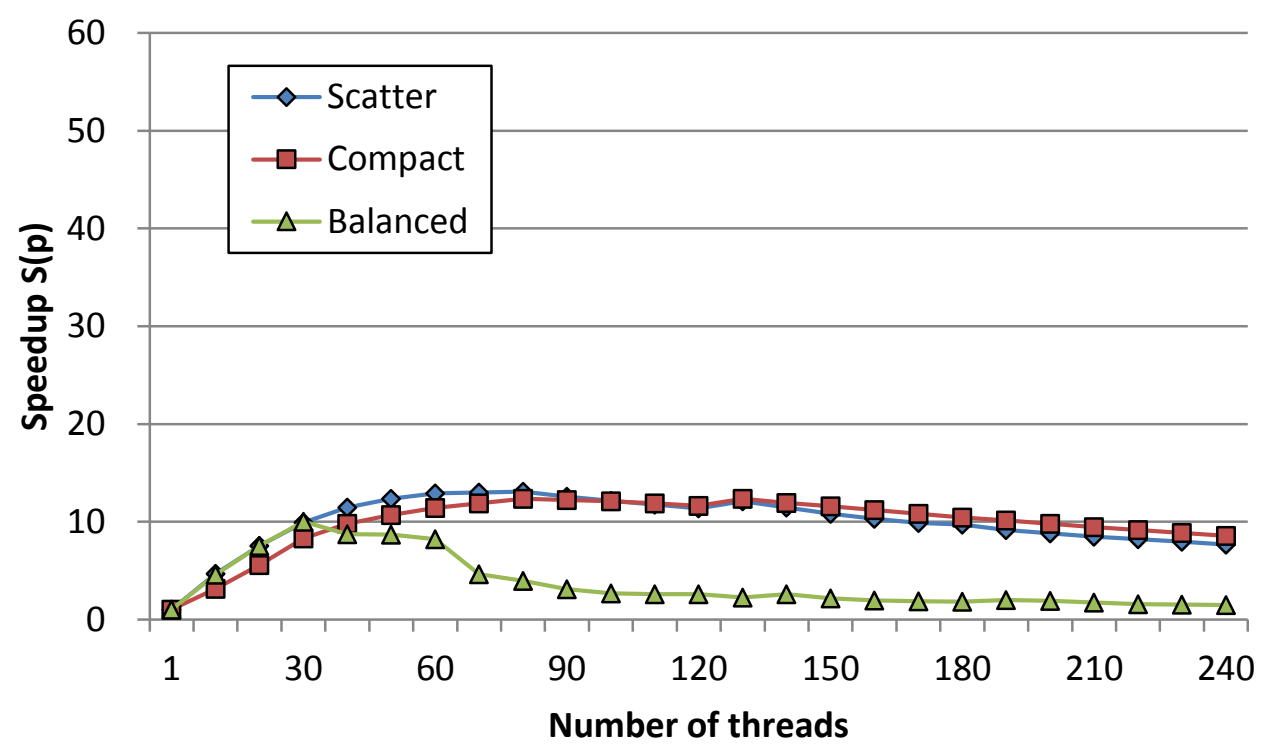

Figure 4.61 Parallel speedup of Ladder215C(20) model on an Intel Xeon Phi with KMP_THREAD_AFFINITY set to scatter, compact and balanced 


\section{Heisenberg Ladder215C(18)}

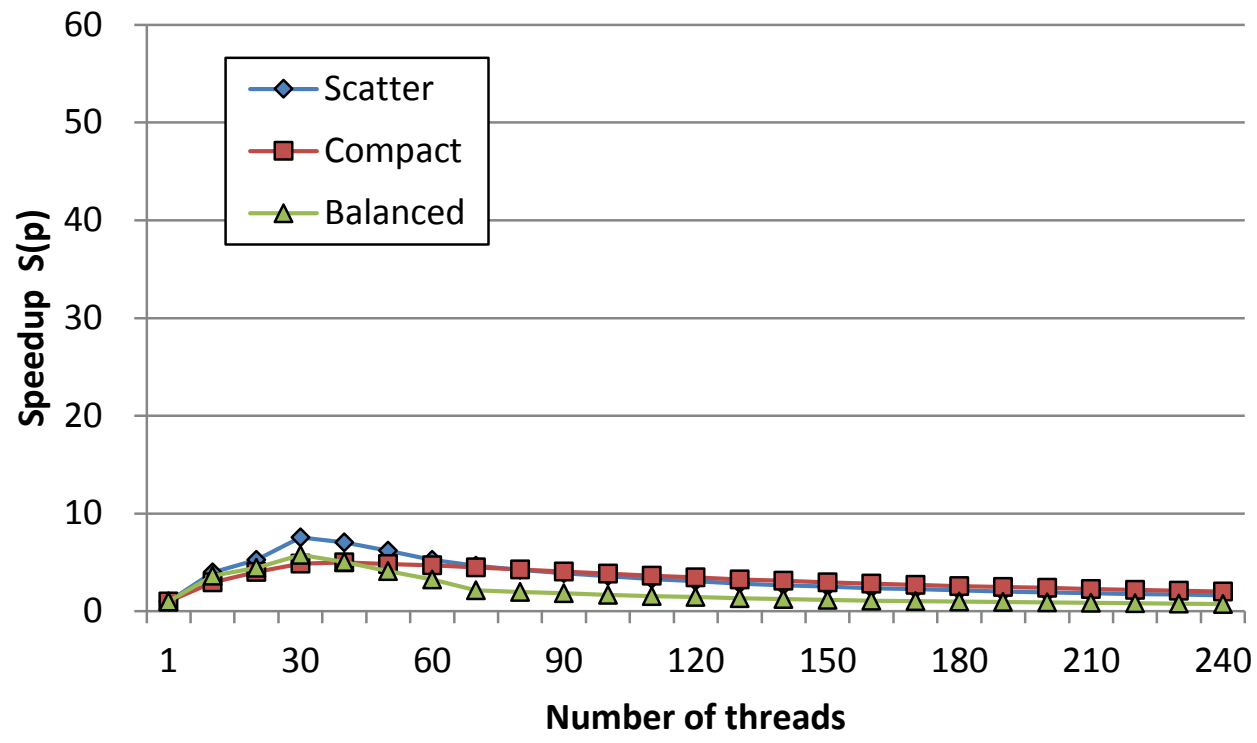

Figure 4.62 Parallel speedup of Ladder215C(18) model on an Intel Xeon Phi with KMP_THREAD_AFFINITY set to scatter, compact and balanced

\section{Heisenberg Ladder215C(16)}

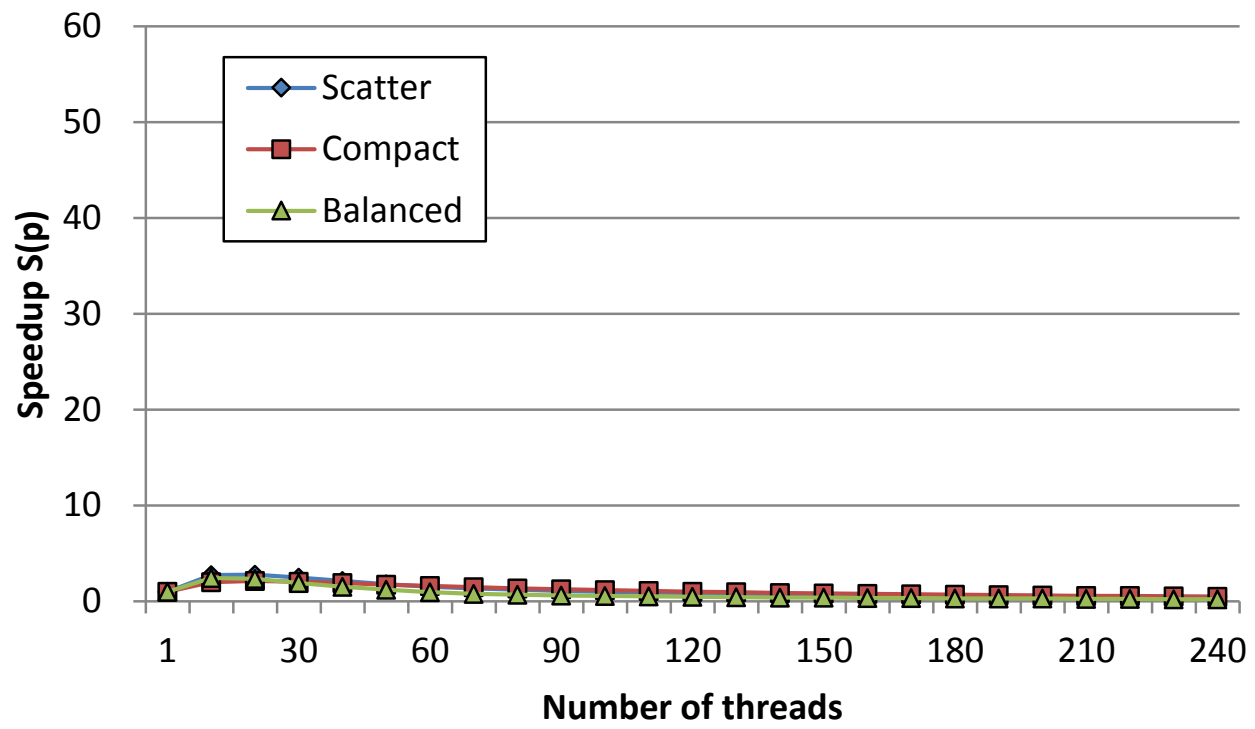

Figure 4.63 Parallel speedup of Ladder215C(16) model on an Intel Xeon Phi with KMP_THREAD_AFFINITY set to scatter, compact and balanced 
See above the Figure 4.58, Figure 4.59, Figure 4.60, Figure 4.61, Figure 4.62 and Figure 4.63, review Table 4.42 and Table 4.43, we can find out the largest number of speedup of Ladder215C model is happened when $N=26$, it is 58.37 times faster than the execution time using single thread. This set of data with $N=26$ is the fastest one of all. The maximum speedups for $N=24$ and 26 range from 50 to 60 . I can also be seen that for the larger systems compact core binding gives better speedups.

The speedups of scatter, compact and balanced are very similar when $N=$ 24 and 26, if $N$ is less than 20, the coprocessor is inefficient, and these speedup numbers of Ladder215C are similar to Ladder2 model. 


\subsubsection{Heisenberg Ladder 215S}

Table 4.44 Speedup of Ladder $215 \mathrm{~S}(N)$ with $N=26,24$ and 22 on an Intel Xeon Phi

\begin{tabular}{|c|c|c|c|c|c|c|c|c|c|}
\hline \multirow{2}{*}{ 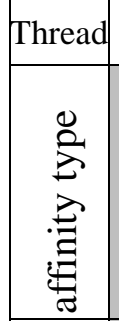 } & \multicolumn{3}{|c|}{$N=26$} & \multicolumn{3}{|c|}{$N=24$} & \multicolumn{3}{|c|}{$N=22$} \\
\hline & 离 & 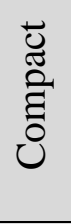 & 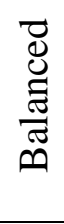 & 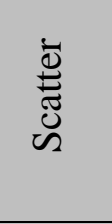 & $\begin{array}{l}\overline{\tilde{U}} \\
\stackrel{0}{0} \\
\tilde{0}\end{array}$ & 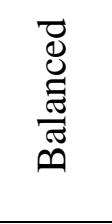 & 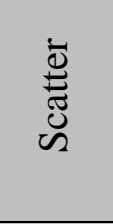 & $\begin{array}{l}\overline{\tilde{U}} \\
\stackrel{0}{0} \\
\tilde{0}\end{array}$ & 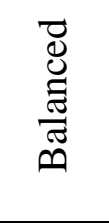 \\
\hline 1 & - & - & - & 1.00 & 1.00 & 1.00 & 1.00 & 1.00 & 1.00 \\
\hline 10 & - & - & - & 4.13 & 3.38 & 4.29 & 4.23 & 3.26 & 4.31 \\
\hline 20 & - & - & - & 7.46 & 6.28 & 7.23 & 6.88 & 5.90 & 7.22 \\
\hline 30 & - & - & - & 10.16 & 9.22 & 9.79 & 10.04 & 8.45 & 9.93 \\
\hline 40 & - & - & - & 13.06 & 12.00 & 12.87 & 13.12 & 10.92 & 13.25 \\
\hline 50 & - & - & - & 15.80 & 14.77 & 15.34 & 15.98 & 13.02 & 16.02 \\
\hline 60 & - & - & - & 18.08 & 17.47 & 18.17 & 18.65 & 15.28 & 18.32 \\
\hline 70 & - & - & - & 20.48 & 19.89 & 20.55 & 20.51 & 17.50 & 19.99 \\
\hline 80 & - & - & - & 23.32 & 22.56 & 23.28 & 23.20 & 20.12 & 23.48 \\
\hline 90 & - & - & - & 24.93 & 24.79 & 25.22 & 24.67 & 22.07 & 24.44 \\
\hline 100 & - & - & - & 27.93 & 26.77 & 27.89 & 26.51 & 24.50 & 26.49 \\
\hline 110 & - & - & - & 30.12 & 28.93 & 29.43 & 28.23 & 26.85 & 27.85 \\
\hline 120 & - & - & - & 30.26 & 30.84 & 31.15 & 29.81 & 28.82 & 29.41 \\
\hline 130 & - & - & - & 31.81 & 32.73 & 32.36 & 30.91 & 30.36 & 30.41 \\
\hline 140 & - & - & - & 33.91 & 34.72 & 34.11 & 32.47 & 32.40 & 32.12 \\
\hline 150 & - & - & - & 34.99 & 36.68 & 36.37 & 28.07 & 28.60 & 8.44 \\
\hline 160 & - & - & - & 36.54 & 39.01 & 38.31 & 28.08 & 29.14 & 7.64 \\
\hline 170 & - & - & - & 36.87 & 39.03 & 38.41 & 27.32 & 28.83 & 6.74 \\
\hline 180 & - & - & - & 38.44 & 42.06 & 40.55 & 26.91 & 28.94 & 5.90 \\
\hline 190 & - & - & - & 39.34 & 44.15 & 41.13 & 26.38 & 29.15 & 5.94 \\
\hline 200 & - & - & - & 40.47 & 45.38 & 43.25 & 25.65 & 28.65 & 4.77 \\
\hline 210 & - & - & - & 43.17 & 46.71 & 45.33 & 25.13 & 28.32 & 4.39 \\
\hline 220 & - & - & - & 44.03 & 47.56 & 45.62 & 24.74 & 27.61 & 4.66 \\
\hline 230 & - & - & - & 46.54 & 49.37 & 49.48 & 24.22 & 27.05 & 4.17 \\
\hline 240 & - & - & - & 47.44 & 50.91 & 50.94 & 23.58 & 26.50 & 3.82 \\
\hline
\end{tabular}


Table 4.45 Speedup of Ladder $215 \mathrm{~S}(N)$ with $N=20,18$ and 16 on an Intel Xeon Phi

\begin{tabular}{|c|c|c|c|c|c|c|c|c|c|}
\hline \multirow{2}{*}{ 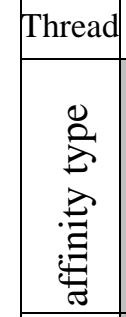 } & \multicolumn{3}{|c|}{$N=20$} & \multicolumn{3}{|c|}{$N=18$} & \multicolumn{3}{|c|}{$N=16$} \\
\hline & 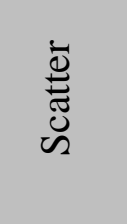 & 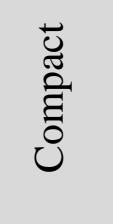 & 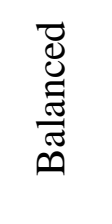 & 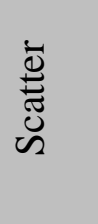 & $\begin{array}{l}\overleftarrow{\tilde{E}} \\
\stackrel{\tilde{E}}{0} \\
\tilde{0}\end{array}$ & 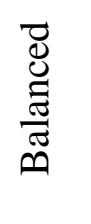 & 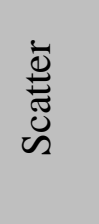 & 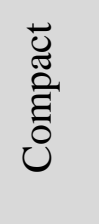 & 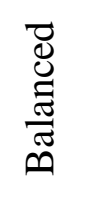 \\
\hline 1 & 1.00 & 1.00 & 1.00 & 1.00 & 1.00 & 1.00 & 1.00 & 1.00 & 1.00 \\
\hline 10 & 4.70 & 3.13 & 4.18 & 4.09 & 2.93 & 3.66 & 2.66 & 1.95 & 2.42 \\
\hline 20 & 7.57 & 5.58 & 7.35 & 5.40 & 3.98 & 4.49 & 2.67 & 2.10 & 2.35 \\
\hline 30 & 10.02 & 8.30 & 10.17 & 7.91 & 4.82 & 5.94 & 2.47 & 2.01 & 1.94 \\
\hline 40 & 11.47 & 9.77 & 8.83 & 7.71 & 4.90 & 5.25 & 2.23 & 1.86 & 1.54 \\
\hline 50 & 12.56 & 10.68 & 8.80 & 7.19 & 4.72 & 4.45 & 1.95 & 1.70 & 1.20 \\
\hline 60 & 13.22 & 11.34 & 8.35 & 6.50 & 4.55 & 3.65 & 1.69 & 1.56 & 0.94 \\
\hline 70 & 13.52 & 11.74 & 4.65 & 5.83 & 4.30 & 2.32 & 1.51 & 1.43 & 0.75 \\
\hline 80 & 13.90 & 12.08 & 3.98 & 5.39 & 4.04 & 2.15 & 1.39 & 1.33 & 0.67 \\
\hline 90 & 13.53 & 11.84 & 3.13 & 4.92 & 3.81 & 1.99 & 1.25 & 1.21 & 0.61 \\
\hline 100 & 13.39 & 11.64 & 2.70 & 4.53 & 3.61 & 1.85 & 1.16 & 1.12 & 0.54 \\
\hline 110 & 13.12 & 11.40 & 2.64 & 4.31 & 3.37 & 1.70 & 1.03 & 1.04 & 0.49 \\
\hline 120 & 12.74 & 11.12 & 2.60 & 3.99 & 3.15 & 1.60 & 0.96 & 0.97 & 0.45 \\
\hline 130 & 13.71 & 11.58 & 2.30 & 3.69 & 2.95 & 1.46 & 0.90 & 0.90 & 0.41 \\
\hline 140 & 13.09 & 11.07 & 2.66 & 3.48 & 2.78 & 1.35 & 0.85 & 0.84 & 0.38 \\
\hline 150 & 12.56 & 10.55 & 2.24 & 3.31 & 2.60 & 1.27 & 0.80 & 0.78 & 0.36 \\
\hline 160 & 12.01 & 10.01 & 1.99 & 3.15 & 2.45 & 1.17 & 0.76 & 0.73 & 0.33 \\
\hline 170 & 11.58 & 9.51 & 1.94 & 3.01 & 2.28 & 1.11 & 0.73 & 0.69 & 0.31 \\
\hline 180 & 11.24 & 8.99 & 1.89 & 2.95 & 2.14 & 1.06 & 0.69 & 0.65 & 0.29 \\
\hline 190 & 10.62 & 8.53 & 2.04 & 2.75 & 2.00 & 1.05 & 0.65 & 0.61 & 0.27 \\
\hline 200 & 10.32 & 8.04 & 2.01 & 2.63 & 1.88 & 1.01 & 0.63 & 0.57 & 0.26 \\
\hline 210 & 9.94 & 7.54 & 1.79 & 2.54 & 1.75 & 0.96 & 0.60 & 0.53 & 0.25 \\
\hline 220 & 9.65 & 7.14 & 1.64 & 2.45 & 1.65 & 0.90 & 0.58 & 0.50 & 0.23 \\
\hline 230 & 9.36 & 6.70 & 1.60 & 2.36 & 1.54 & 0.85 & 0.56 & 0.46 & 0.22 \\
\hline 240 & 9.01 & 6.40 & 1.56 & 2.27 & 1.47 & 0.81 & 0.54 & 0.44 & 0.21 \\
\hline
\end{tabular}

The Table 4.44 and Table 4.45 summarize 18 tables: from Table 6.64 to Table 6.81 in section 6.2 
Heisenberg Ladder215S(24)

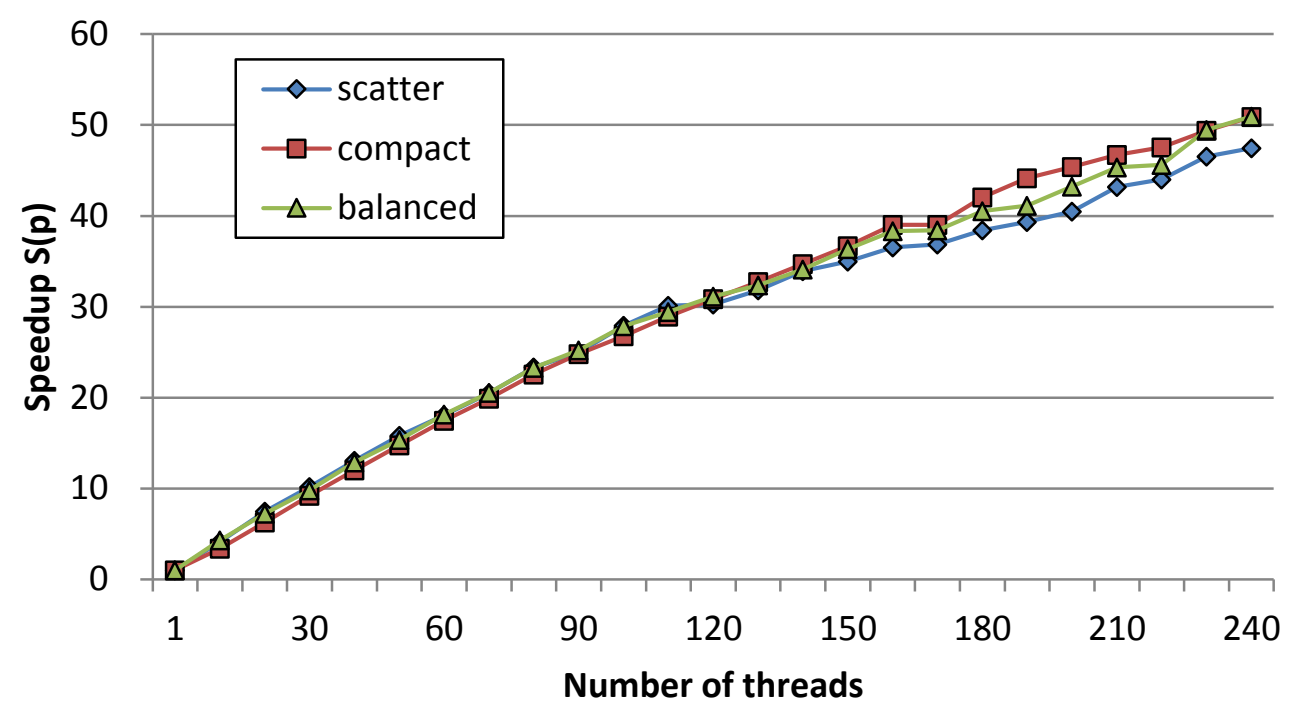

Figure 4.64 Parallel speedup of Ladder215S(24) model on an Intel Xeon Phi with KMP_THREAD_AFFINITY set to scatter, compact and balanced Heisenberg Ladder215S(22)

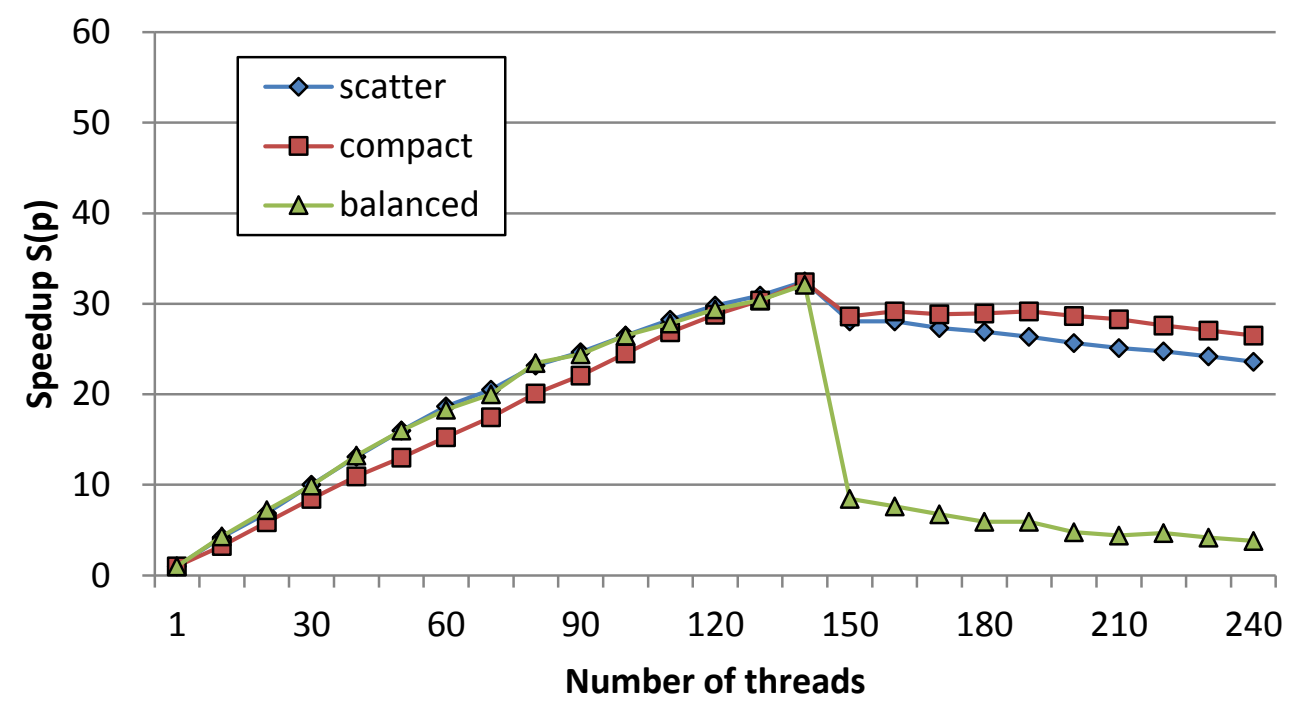

Figure 4.65 Parallel speedup of Ladder215S(22) model on an Intel Xeon Phi with KMP_THREAD_AFFINITY set to scatter, compact and balanced 
Heisenberg Ladder215S(20)

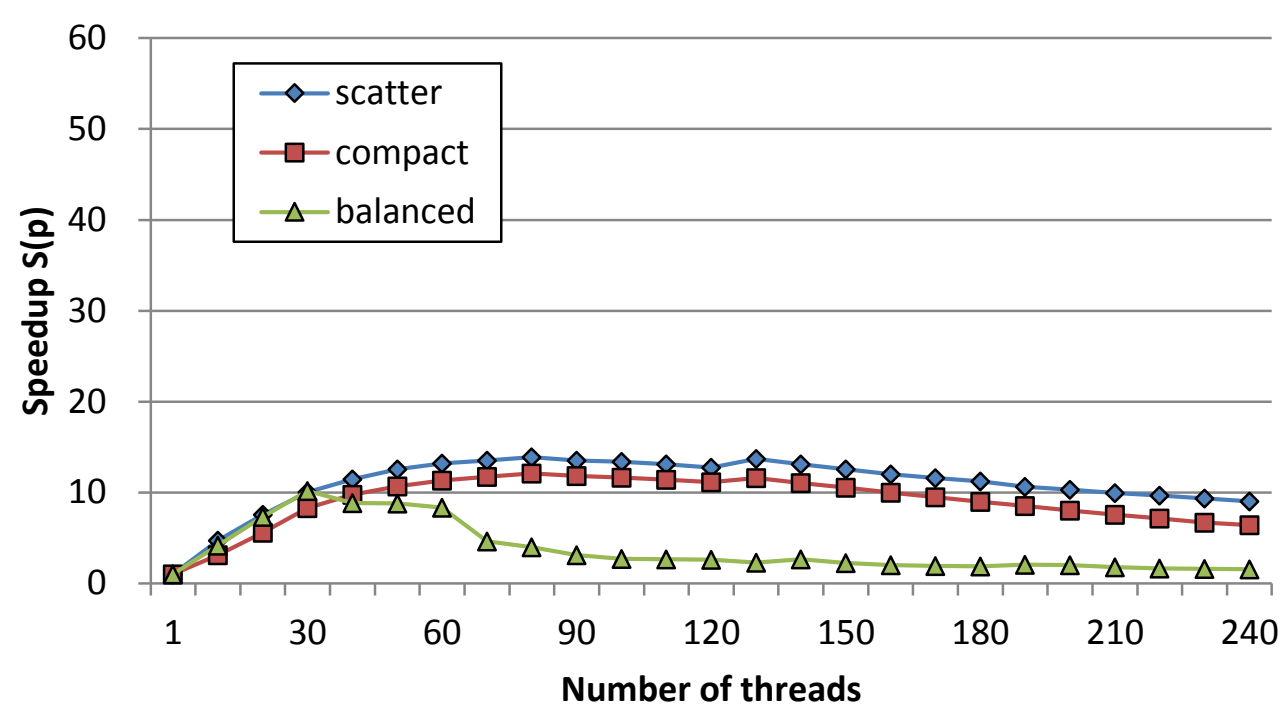

Figure 4.66 Parallel speedup of Ladder215S(20) model on an Intel Xeon Phi with KMP_THREAD_AFFINITY set to scatter, compact and balanced Heisenberg Ladder215S(18)

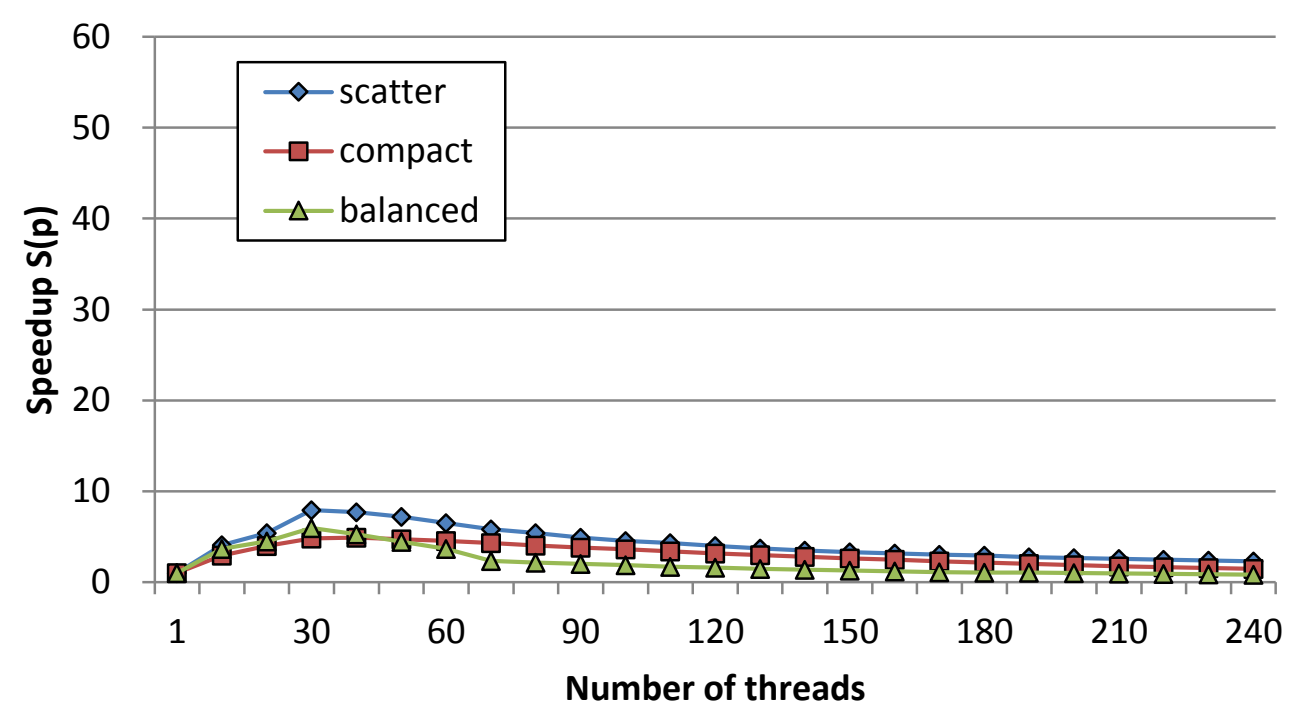

Figure 4.67 Parallel speedup of Ladder215S(18) model on an Intel Xeon Phi with KMP_THREAD_AFFINITY set to scatter, compact and balanced 
Heisenberg Ladder215S(16)

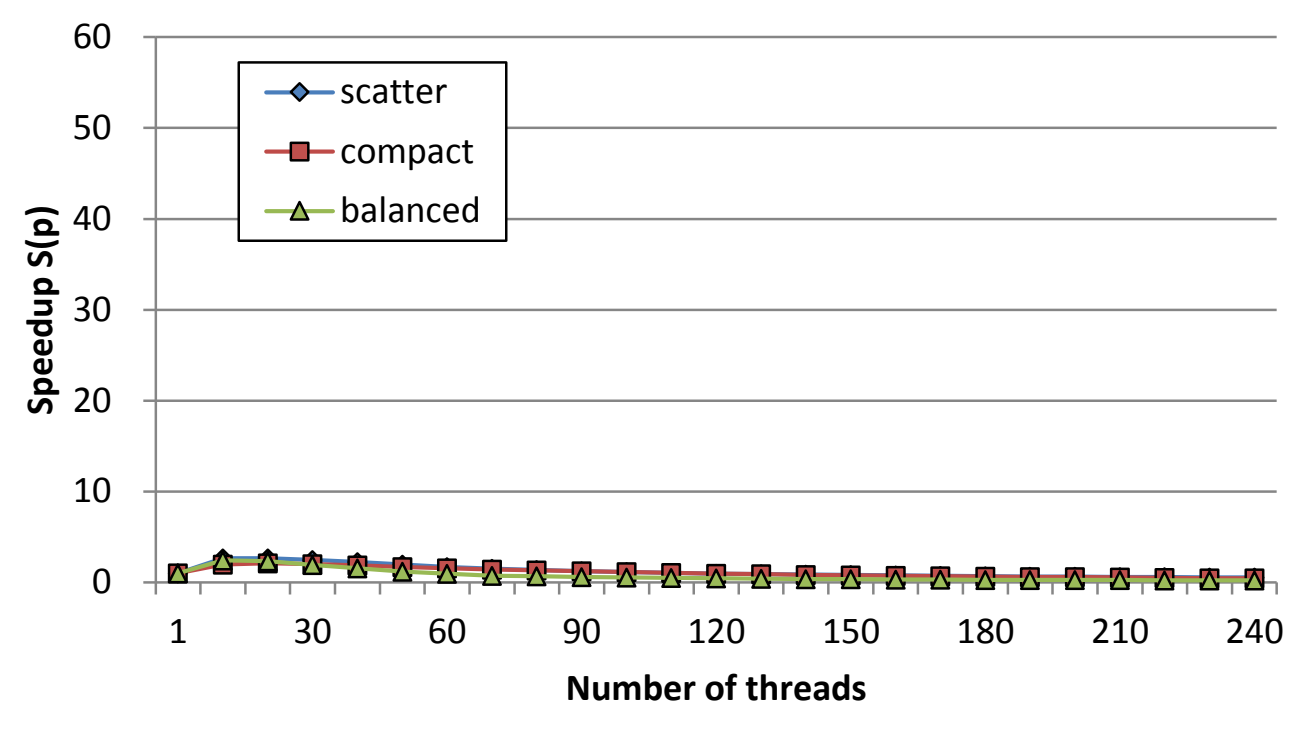

Figure 4.68 Parallel speedup of Ladder215S(16) model on an Intel Xeon Phi with KMP_THREAD_AFFINITY set to scatter, compact and balanced

The speedup of Ladder215S model is almost same as the one of Ladder215C model, I do not have data about speedup of the Ladder215S with $N=26$ because of the Xeon Phi machine had some problem, but I can predict the speedup of Ladder215S model has almost same as Ladder215C, because the speedup of Ladder215S with size $N=24,22$, 20, 18 and 16 are rather similar with speedup of Ladder215C models. Actually, we can observe all two-leg Heisenberg Ladder models (Ladder2, Ladder215C and Ladder215S) has almost same speedup. 


\subsubsection{Heisenberg Ladder 315C}

Table 4.46 Speedup of Ladder $315 \mathrm{C}(N)$ with $N=24$ and 18 on an Intel Xeon Phi

\begin{tabular}{|c|c|c|c|c|c|c|}
\hline Thread & \multicolumn{3}{|c|}{$N=24$} & \multicolumn{3}{|c|}{$N=18$} \\
\hline $\begin{array}{c}\text { affinity } \\
\text { type }\end{array}$ & Scatter & Compact & Balanced & Scatter & Compact & Balanced \\
\hline 1 & 1.00 & 1.00 & 1.00 & 1.00 & 1.00 & 1.00 \\
\hline 10 & 4.12 & 3.27 & 4.13 & 4.27 & 3.02 & 3.72 \\
\hline 20 & 7.18 & 6.37 & 7.16 & 5.67 & 4.19 & 4.73 \\
\hline 30 & 10.08 & 9.32 & 9.76 & 6.01 & 4.59 & 4.66 \\
\hline 40 & 12.58 & 12.14 & 12.52 & 7.58 & 5.18 & 5.17 \\
\hline 50 & 15.77 & 15.09 & 15.52 & 6.68 & 5.10 & 4.22 \\
\hline 60 & 17.88 & 17.52 & 18.03 & 5.71 & 4.86 & 3.36 \\
\hline 70 & 20.09 & 20.05 & 20.49 & 5.06 & 4.62 & 2.17 \\
\hline 80 & 22.69 & 22.65 & 23.15 & 4.57 & 4.41 & 2.01 \\
\hline 90 & 24.63 & 25.03 & 25.68 & 4.20 & 4.17 & 1.86 \\
\hline 100 & 27.42 & 27.38 & 27.85 & 3.89 & 3.91 & 1.73 \\
\hline 110 & 29.17 & 29.35 & 29.98 & 3.62 & 3.63 & 1.58 \\
\hline 120 & 30.41 & 31.47 & 31.59 & 3.34 & 3.44 & 1.49 \\
\hline 130 & 31.45 & 33.43 & 32.72 & 3.08 & 3.22 & 1.37 \\
\hline 140 & 33.67 & 35.28 & 34.68 & 2.89 & 3.02 & 1.30 \\
\hline 150 & 35.43 & 37.22 & 36.50 & 2.74 & 2.83 & 1.20 \\
\hline 160 & 36.14 & 39.48 & 37.97 & 2.60 & 2.66 & 1.11 \\
\hline 170 & 37.15 & 39.98 & 38.71 & 2.47 & 2.48 & 1.06 \\
\hline 180 & 38.58 & 43.18 & 41.29 & 2.36 & 2.33 & 1.01 \\
\hline 190 & 39.52 & 44.87 & 41.64 & 2.22 & 2.18 & 1.00 \\
\hline 200 & 41.79 & 46.54 & 43.70 & 2.12 & 2.05 & 0.95 \\
\hline 210 & 43.69 & 48.02 & 46.01 & 2.04 & 1.91 & 0.91 \\
\hline 220 & 44.69 & 48.85 & 46.51 & 1.97 & 1.84 & 0.84 \\
\hline 230 & 47.87 & 50.89 & 50.77 & 1.94 & 1.69 & 0.80 \\
\hline 240 & 48.36 & 52.40 & 52.31 & 1.81 & 1.60 & 0.75 \\
\hline
\end{tabular}

The Table 4.46 summarize 18 tables: from Table 6.82 to Table 6.87 in section 6.2 


\section{Heisenberg Ladder315C(24)}

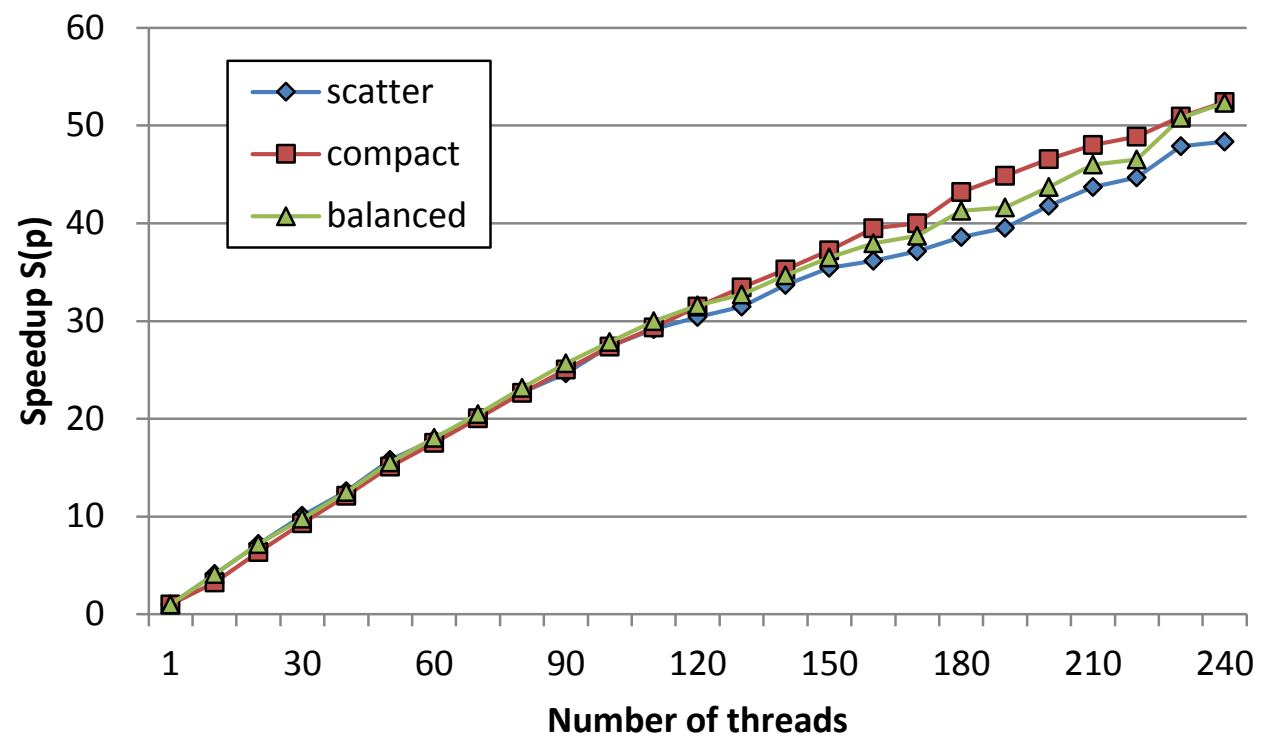

Figure 4.69 Parallel speedup of Ladder315C(24) model on an Intel Xeon Phi with KMP_THREAD_AFFINITY set to scatter, compact and balanced

\section{Heisenberg Ladder315C(18)}

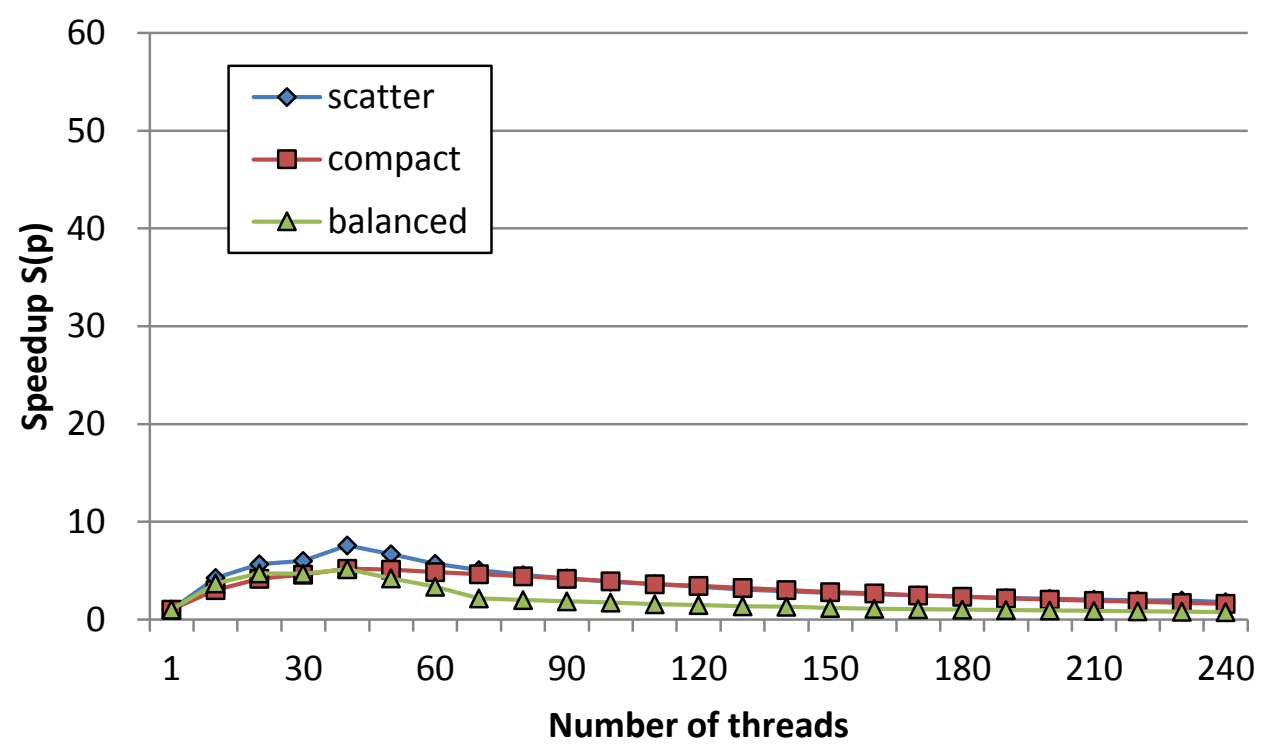

Figure 4.70 Parallel speedup of Ladder315C(18) model on an Intel Xeon Phi with KMP_THREAD_AFFINITY set to scatter, compact and balanced 


\subsubsection{Heisenberg Ladder 315S}

Table 4.47 Speedup of Ladder $315 \mathrm{~S}(N)$ with $N=24$ and 18 on an Intel Xeon Phi

\begin{tabular}{|c|c|c|c|c|c|c|}
\hline Thread & \multicolumn{3}{|c|}{$N=24$} & \multicolumn{3}{|c|}{$N=18$} \\
\hline $\begin{array}{l}\text { affinity } \\
\text { type }\end{array}$ & Scatter & Compact & Balanced & Scatter & Compact & Balanced \\
\hline 1 & 1.00 & 1.00 & 1.00 & 1.00 & 1.00 & 1.00 \\
\hline 10 & 4.15 & 3.32 & 4.15 & 4.24 & 3.02 & 3.98 \\
\hline 20 & 7.05 & 6.37 & 7.24 & 5.58 & 4.29 & 5.12 \\
\hline 30 & 9.78 & 9.38 & 10.02 & 6.15 & 4.62 & 4.95 \\
\hline 40 & 12.40 & 12.13 & 12.41 & 8.35 & 5.28 & 5.51 \\
\hline 50 & 15.75 & 15.09 & 15.84 & 8.12 & 5.14 & 4.50 \\
\hline 60 & 17.83 & 17.51 & 18.07 & 7.45 & 5.05 & 3.60 \\
\hline 70 & 19.93 & 20.06 & 20.06 & 6.55 & 4.71 & 2.31 \\
\hline 80 & 22.18 & 22.75 & 23.31 & 6.27 & 4.45 & 2.15 \\
\hline 90 & 24.97 & 25.02 & 25.31 & 5.56 & 4.32 & 1.95 \\
\hline 100 & 27.22 & 27.39 & 27.86 & 5.36 & 4.00 & 1.81 \\
\hline 110 & 29.36 & 29.36 & 29.52 & 5.01 & 3.81 & 1.66 \\
\hline 120 & 30.41 & 31.46 & 31.49 & 4.58 & 3.66 & 1.56 \\
\hline 130 & 31.23 & 33.42 & 32.76 & 4.15 & 3.50 & 1.45 \\
\hline 140 & 33.40 & 35.27 & 34.60 & 4.01 & 3.23 & 1.33 \\
\hline 150 & 35.09 & 37.24 & 36.86 & 3.73 & 3.05 & 1.23 \\
\hline 160 & 34.94 & 39.48 & 38.84 & 3.59 & 2.86 & 1.15 \\
\hline 170 & 37.25 & 39.79 & 38.79 & 3.40 & 2.69 & 1.09 \\
\hline 180 & 38.86 & 43.18 & 41.19 & 3.30 & 2.61 & 1.03 \\
\hline 190 & 40.48 & 44.91 & 41.82 & 3.05 & 2.56 & 1.01 \\
\hline 200 & 41.59 & 46.54 & 44.01 & 2.94 & 2.41 & 0.97 \\
\hline 210 & 43.76 & 48.39 & 46.18 & 2.97 & 2.17 & 0.91 \\
\hline 220 & 45.02 & 48.82 & 46.91 & 2.74 & 2.11 & 0.85 \\
\hline 230 & 47.67 & 50.90 & 50.87 & 2.69 & 2.00 & 0.84 \\
\hline 240 & 48.34 & 52.71 & 52.31 & 2.54 & 2.03 & 0.77 \\
\hline
\end{tabular}

The Table 4.47 summarize 18 tables: from Table 6.88 to Table 6.93 in section 6.2 


\section{Heisenberg Ladder315S(24)}

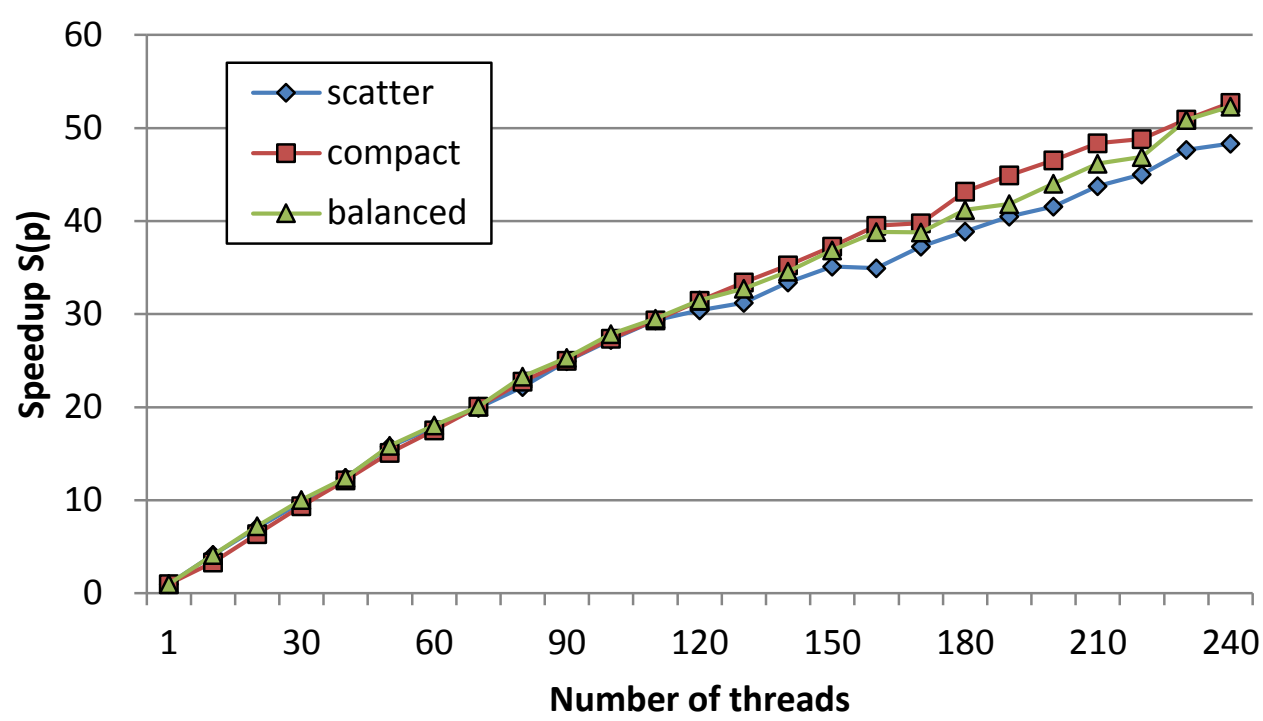

Figure 4.71 Parallel speedup of Ladder315S(24) model on an Intel Xeon Phi with KMP_THREAD_AFFINITY set to scatter, compact and balanced

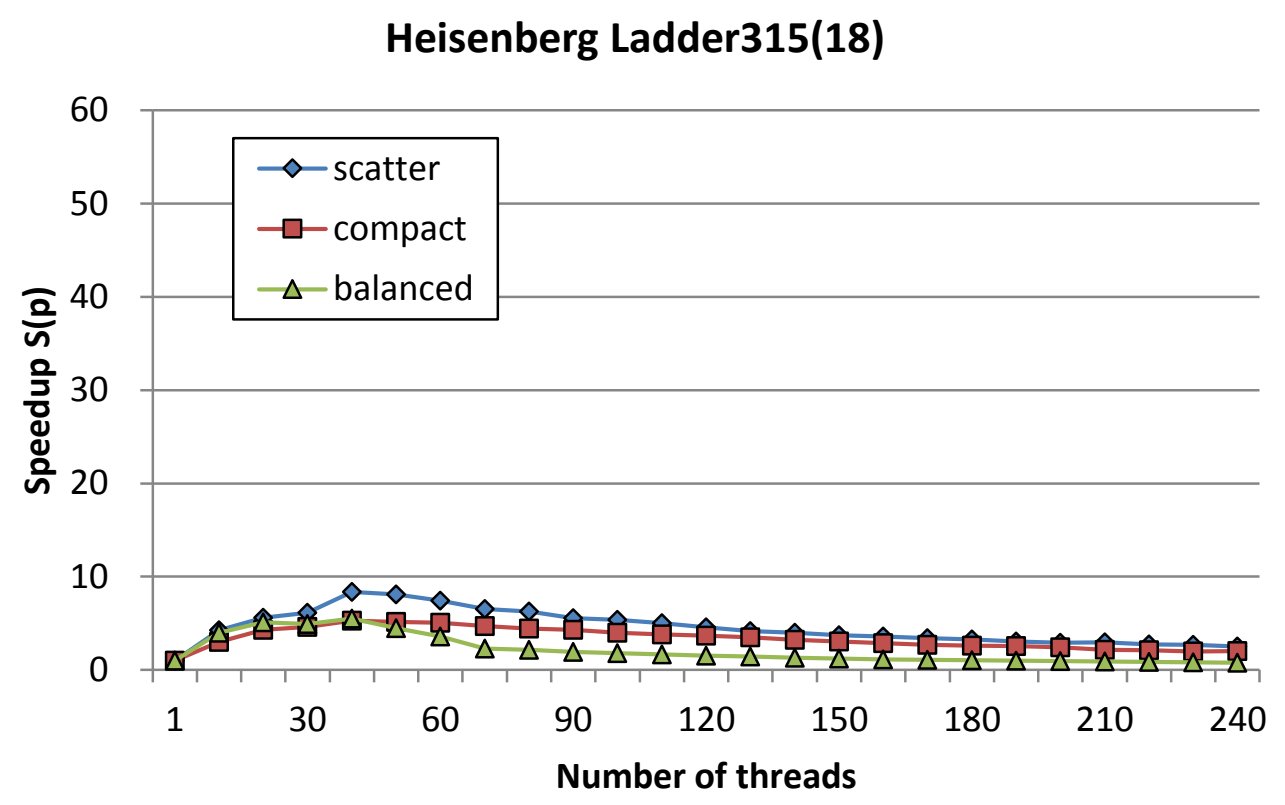

Figure 4.72 Parallel speedup of Ladder315S(24) model on an Intel Xeon Phi with KMP_THREAD_AFFINITY set to scatter, compact and balanced 
The dimension of three-leg Heisenberg Ladder models require that size is divided exactly by 6 , I tested speedup of three-leg Ladder models with $N=24$ and 18 . In Table 4.46, Figure 4.69 and Figure 4.70 show the speedup of the Ladder315C and Ladder315S are very similar, or even almost same, as before the speedups rise linearly when $N=24$, but speedup of three-leg Ladder models is a bit better than the ones of two-leg Ladder models. In Table 4.47, Figure 4.71 and Figure 4.72 the speedup with $N=18$ in three-leg Ladder models is low as before. 


\subsubsection{Time and speedup of different models on Xeon Phi}

In Figure 4.73, Figure 4.74 and Figure 4.75, I show the parallel speedup obtained with the variants RMM-DIIS (RMM-DIIS $V_{\max }$ and $V_{\max }$ ) method on the Intel Xeon Phi coprocessor for the dimerized two-leg Heisenberg ladder215C model for various system size $N$, I tested three settings of the environment variable KMP_THREAD_AFFINITY scatter, compact and balanced. This variable affects the binding of threads to processor cores for OpenMP and the MKL on the coprocessor. If set to scatter the runtime system distributed the threads over as many cores as possible whereas compact uses as few cores as possible, balanced uses cores as groups. The speedups of compact and balanced are very similar when $N=24$ and 26, if $N$ is less than 24 , the speedups of balanced are much lower than speedups of scatter and compact specially in the system with small $N$, almost all speedups of balanced are the lowest one among three types. The figures show that for small system with $N=$ 16,18 and 20, the coprocessor is inefficient with maximum speedups far below what be expected form a 60 core device, even some time speedup is less than 1 . For $N=22$ a maximum speedup of 30 is reached at 130 threads, the speedups declined, especially in bal anced type, the speedup from 30 drop to 8 (see Figure 4.60 in page 103), whereas for $N=24$ and 26 the speedup rises continuously until $p=240$ threads. The maximum speedups for $N=24$ and 26 range from 50 to 60 . I can also be seen that for the larger systems compact core binding gives better speedups. 


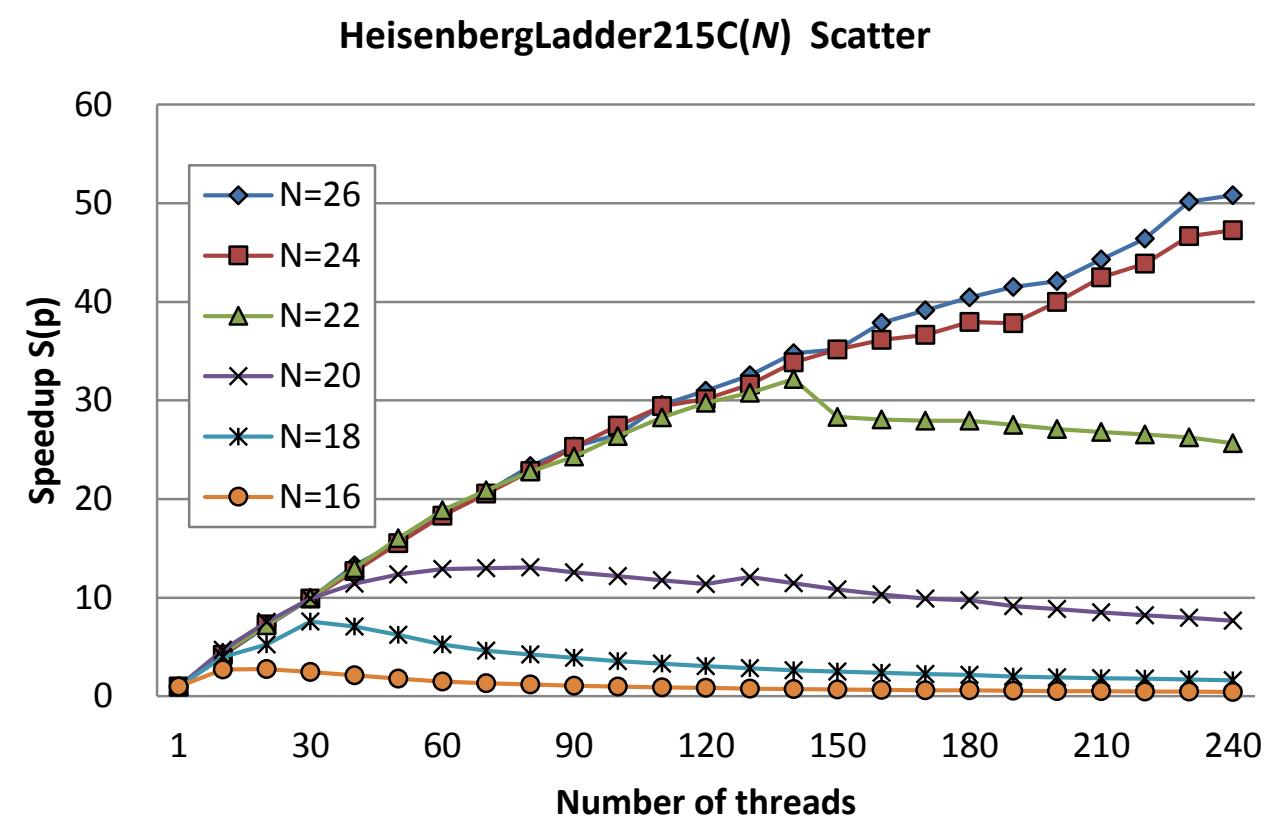

Figure 4.73 Parallel speedup of Ladder215C $(N)$ with $N=16,18,20,22,24$ and 26 on an Intel Xeon Phi coprocessor with KMP_THREAD_AFFINITY is Scatter

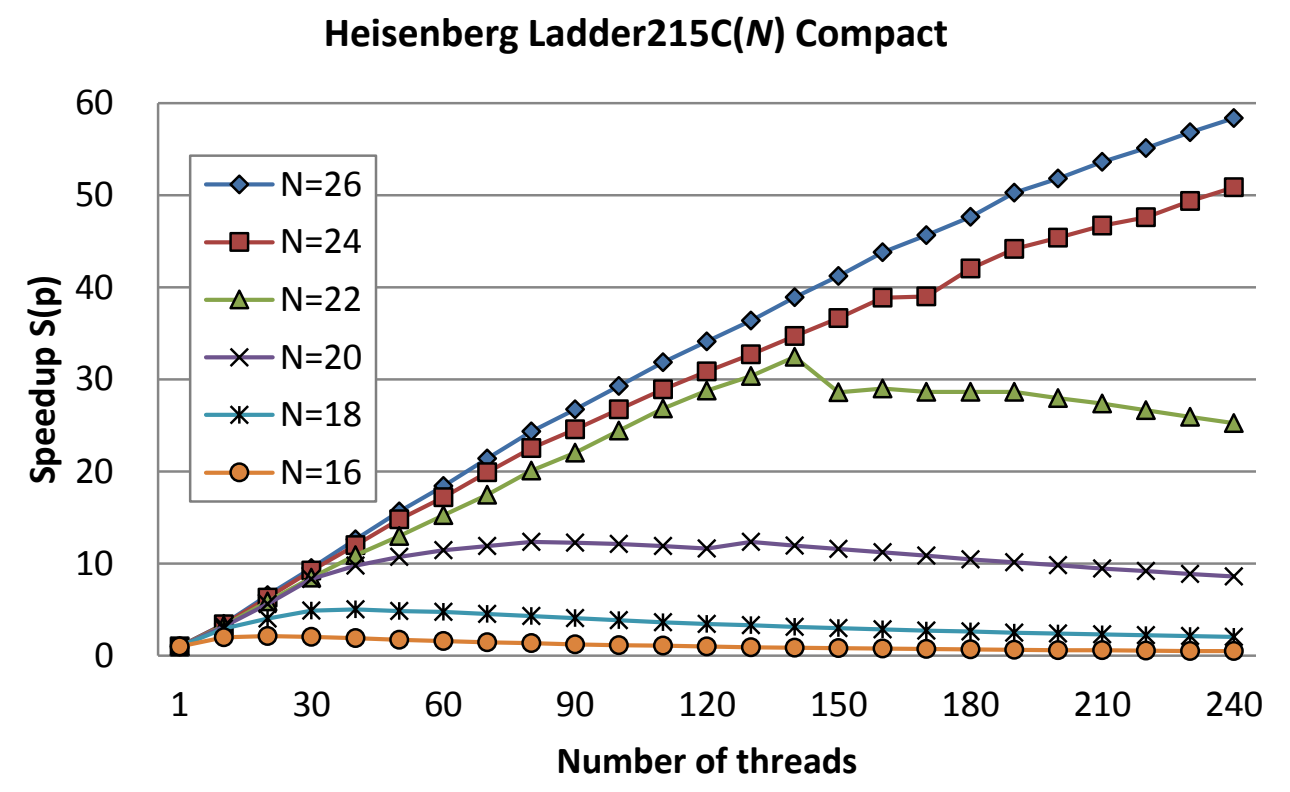

Figure 4.74 Parallel speedup of Ladder215C $(N)$ with $N=16,18,20,22,24$ and 26 on an Intel Xeon Phi coprocessor with KMP_THREAD_AFFINITY is compact 


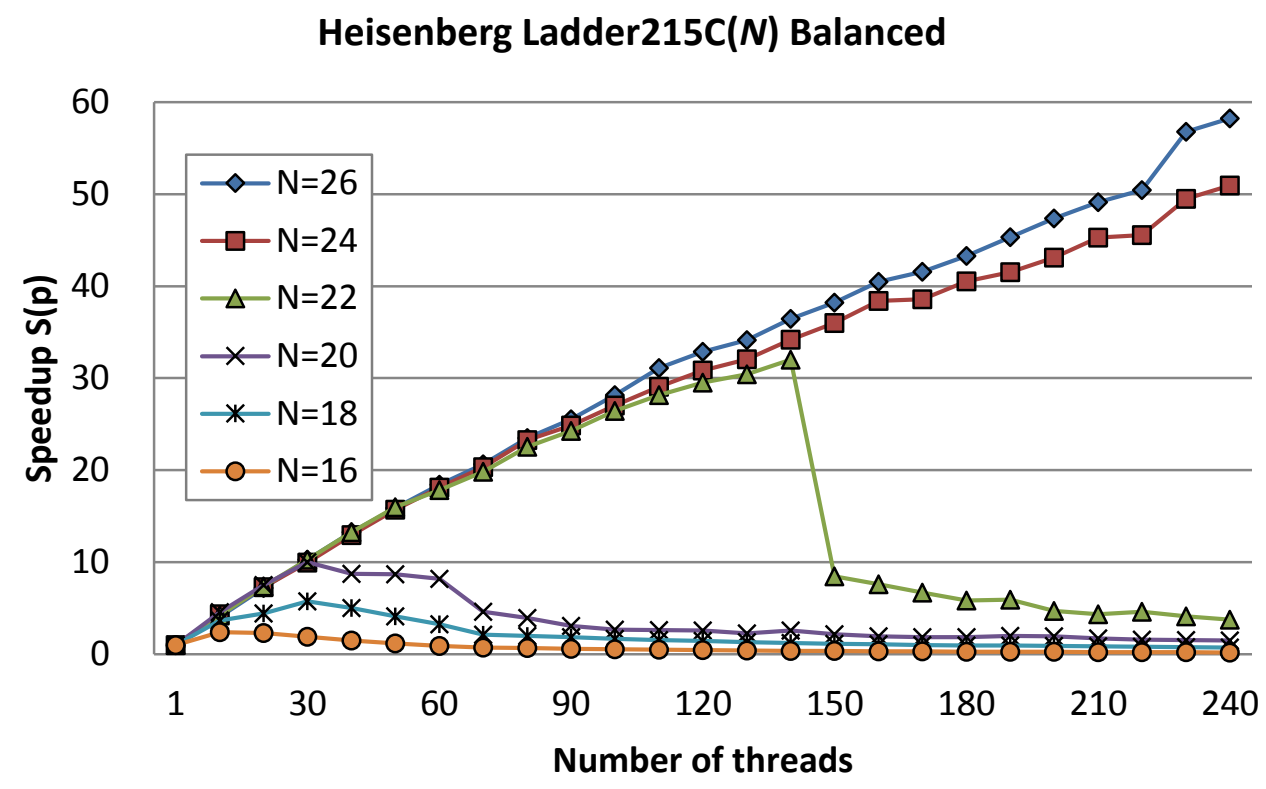

Figure 4.75 Parallel speedup of Ladder215C $(N)$ with $N=16,18,20,22,24$ and 26 on an Intel Xeon Phi coprocessor with KMP_THREAD_AFFINITY is Balanced

In Table 4.48, Table 4.49, Table 4.50, Table 4.51, Table 4.52and Table 4.53 we compare the parallel speedups obtained for all our benchmark models with $N=24$ and 18 on the Xeon Phi coprocessor for different number of threads. As in Figure 4.73, Figure 4.74 and Figure 4.75, I have considered three types of thread binding. For thread numbers $p$ up to sixty, the speedups are similar for all models and correspond to a parallel efficiency of about $33 \%$. As the number of threads $p$ goes beyond 60 (i.e. more than one thread per core), the speedup continues to increase but differences between the models appear. This is best seen at $p=240$. The highest speedup is obtained by the three-leg dimerized ladder systems. All two-leg ladder modes have slightly lower speedup follow by the chain system. The data show that the speedup is determined by the number of legs of the model, all two leg ladders have (nearly) the same speedups independent of parameters. The same is true for the three-leg models. This indicates that the speedup is determined 
by the sparsity of matrix. Execution time of Chain system is the fastest one of all, although its speedup is the lowest of all. Three-leg dimerized ladder systems has the best speedup, however the execution time of it is more than two-leg dimerized ladder system and Chain system. The reason for why three-leg dimerized ladder systems has good speedup but slower is there are more non-zero elements in three-leg dimerized ladder system, hence it need more time to calculate. The number of non-zero entries per row is 13,19 , and 21 for the chain, two-leg and three-leg models, respectively. 
Table 4.48 Comparison of time and speedup of Chain, Ladder and Ladder 215C with $N$ $=24$ on an Intel Xeon Phi with KMP_THREAD_AFFINITY set to scatter

\begin{tabular}{|c|c|c|c|c|c|c|}
\hline \multicolumn{7}{|c|}{ Scatter $N=24$ (time in seconds) } \\
\hline model & \multicolumn{2}{|c|}{ Chain $(N)$} & \multicolumn{2}{|c|}{$\operatorname{Ladder}(N)$} & \multicolumn{2}{|c|}{ Ladder $215 \mathrm{C}(N)$} \\
\hline threads & time & speedup & time & speedup & time & speedup \\
\hline 1 & 1797.89 & 1.00 & 2354.22 & 1.00 & 2354.32 & 1.00 \\
\hline 10 & 414.00 & 4.34 & 553.43 & 4.25 & 563.25 & 4.18 \\
\hline 20 & 248.57 & 7.23 & 316.80 & 7.43 & 322.80 & 7.29 \\
\hline 30 & 180.10 & 9.98 & 241.08 & 9.77 & 237.32 & 9.92 \\
\hline 40 & 135.48 & 13.27 & 179.88 & 13.09 & 185.61 & 12.68 \\
\hline 50 & 111.78 & 16.08 & 151.88 & 15.50 & 151.79 & 15.51 \\
\hline 60 & 95.14 & 18.90 & 130.34 & 18.06 & 128.63 & 18.30 \\
\hline 70 & 86.75 & 20.72 & 116.26 & 20.25 & 114.44 & 20.57 \\
\hline 80 & 75.47 & 23.82 & 102.89 & 22.88 & 103.02 & 22.85 \\
\hline 90 & 70.51 & 25.50 & 93.56 & 25.16 & 93.12 & 25.28 \\
\hline 100 & 65.64 & 27.39 & 88.78 & 26.52 & 85.77 & 27.45 \\
\hline 110 & 62.73 & 28.66 & 82.68 & 28.47 & 79.96 & 29.44 \\
\hline 120 & 60.01 & 29.96 & 77.99 & 30.19 & 78.13 & 30.14 \\
\hline 130 & 59.45 & 30.24 & 74.04 & 31.80 & 74.45 & 31.62 \\
\hline 140 & 57.45 & 31.29 & 69.64 & 33.81 & 69.50 & 33.88 \\
\hline 150 & 54.14 & 33.21 & 67.26 & 35.00 & 66.90 & 35.19 \\
\hline 160 & 53.56 & 33.57 & 65.66 & 35.86 & 65.15 & 36.14 \\
\hline 170 & 52.98 & 33.94 & 65.79 & 35.78 & 64.22 & 36.66 \\
\hline 180 & 49.96 & 35.99 & 62.07 & 37.93 & 62.05 & 37.94 \\
\hline 190 & 48.74 & 36.89 & 61.78 & 38.11 & 62.22 & 37.84 \\
\hline 200 & 47.73 & 37.67 & 58.54 & 40.22 & 58.89 & 39.98 \\
\hline 210 & 45.69 & 39.35 & 55.81 & 42.18 & 55.40 & 42.49 \\
\hline 220 & 44.26 & 40.63 & 55.13 & 42.70 & 53.65 & 43.88 \\
\hline 230 & 41.75 & 43.06 & 50.61 & 46.51 & 50.45 & 46.66 \\
\hline 240 & 41.46 & 43.36 & 49.78 & 47.29 & 49.80 & 47.27 \\
\hline
\end{tabular}


Table 4.49 Comparison of time and speedup of Ladder215S, Ladder315C and Ladder315S with $N=24$ on an Intel Xeon Phi coprocessor with KMP_THREAD_AFFINITY set to scatter

\begin{tabular}{|c|c|c|c|c|c|c|}
\hline \multicolumn{7}{|c|}{ Scatter $N=24$ (time in seconds) } \\
\hline \multirow{2}{*}{$\begin{array}{l}\text { model } \\
\text { threads }\end{array}$} & \multicolumn{2}{|c|}{ Ladder $215 \mathrm{~S}(N)$} & \multicolumn{2}{|c|}{ Ladder $315 \mathrm{C}(N)$} & \multicolumn{2}{|c|}{ Ladder $315 \mathrm{~S}(N)$} \\
\hline & time & speedup & time & speedup & time & speedup \\
\hline 1 & 2355.34 & 1.00 & 2568.94 & 1.00 & 2568.89 & 1.00 \\
\hline 10 & 570.38 & 4.13 & 623.26 & 4.12 & 619.72 & 4.15 \\
\hline 20 & 315.66 & 7.46 & 357.64 & 7.18 & 364.62 & 7.05 \\
\hline 30 & 231.80 & 10.16 & 254.79 & 10.08 & 262.78 & 9.78 \\
\hline 40 & 180.33 & 13.06 & 204.19 & 12.58 & 207.23 & 12.40 \\
\hline 50 & 149.11 & 15.80 & 162.92 & 15.77 & 163.13 & 15.75 \\
\hline 60 & 130.28 & 18.08 & 143.68 & 17.88 & 144.07 & 17.83 \\
\hline 70 & 115.02 & 20.48 & 127.89 & 20.09 & 128.89 & 19.93 \\
\hline 80 & 101.02 & 23.32 & 113.24 & 22.69 & 115.80 & 22.18 \\
\hline 90 & 94.48 & 24.93 & 104.28 & 24.63 & 102.88 & 24.97 \\
\hline 100 & 84.32 & 27.93 & 93.69 & 27.42 & 94.36 & 27.22 \\
\hline 110 & 78.20 & 30.12 & 88.07 & 29.17 & 87.50 & 29.36 \\
\hline 120 & 77.85 & 30.26 & 84.48 & 30.41 & 84.49 & 30.41 \\
\hline 130 & 74.05 & 31.81 & 81.67 & 31.45 & 82.26 & 31.23 \\
\hline 140 & 69.46 & 33.91 & 76.29 & 33.67 & 76.90 & 33.40 \\
\hline 150 & 67.32 & 34.99 & 72.51 & 35.43 & 73.20 & 35.09 \\
\hline 160 & 64.46 & 36.54 & 71.09 & 36.14 & 73.52 & 34.94 \\
\hline 170 & 63.88 & 36.87 & 69.15 & 37.15 & 68.97 & 37.25 \\
\hline 180 & 61.28 & 38.44 & 66.59 & 38.58 & 66.11 & 38.86 \\
\hline 190 & 59.87 & 39.34 & 65.00 & 39.52 & 63.46 & 40.48 \\
\hline 200 & 58.20 & 40.47 & 61.48 & 41.79 & 61.77 & 41.59 \\
\hline 210 & 54.57 & 43.17 & 58.80 & 43.69 & 58.70 & 43.76 \\
\hline 220 & 53.50 & 44.03 & 57.48 & 44.69 & 57.06 & 45.02 \\
\hline 230 & 50.60 & 46.54 & 53.66 & 47.87 & 53.89 & 47.67 \\
\hline 240 & 49.65 & 47.44 & 53.12 & 48.36 & 53.14 & 48.34 \\
\hline
\end{tabular}


Table 4.50 Comparison of time and speedup of Chain, Ladder and Ladder 215C with $N$ $=24$ on an Intel Xeon Phi coprocessor with KMP_THREAD_AFFINITY set to compact

\begin{tabular}{|r|r|r|r|r|r|r|}
\hline \multicolumn{7}{|c|}{ Compact $N=24$ (time in seconds) } \\
\hline model & \multicolumn{2}{|c|}{ Chain $(N)$} & \multicolumn{2}{|c|}{ Ladder $(N)$} & \multicolumn{2}{c|}{ Ladder $215 \mathrm{C}(N)$} \\
\hline threads & \multicolumn{1}{c|}{ time } & \multicolumn{1}{c|}{ speedup } & \multicolumn{1}{c|}{ time } & \multicolumn{1}{c|}{ speedup } & \multicolumn{1}{c|}{ time } & \multicolumn{1}{c|}{ speedup } \\
\hline 1 & 1797.89 & 1.00 & 2354.22 & 1.00 & 2354.32 & 1.00 \\
\hline 10 & 577.55 & 3.11 & 698.39 & 3.37 & 696.48 & 3.38 \\
\hline 20 & 302.90 & 5.94 & 373.96 & 6.30 & 374.54 & 6.29 \\
\hline 30 & 208.23 & 8.63 & 254.70 & 9.24 & 255.51 & 9.21 \\
\hline 40 & 159.40 & 11.28 & 196.21 & 12.00 & 196.13 & 12.00 \\
\hline 50 & 129.06 & 13.93 & 159.40 & 14.77 & 159.40 & 14.77 \\
\hline 60 & 111.40 & 16.14 & 136.87 & 17.20 & 136.85 & 17.20 \\
\hline 70 & 97.12 & 18.51 & 118.39 & 19.88 & 118.40 & 19.88 \\
\hline 80 & 85.11 & 21.12 & 104.39 & 22.55 & 104.41 & 22.55 \\
\hline 90 & 78.52 & 22.90 & 95.73 & 24.59 & 95.78 & 24.58 \\
\hline 100 & 71.86 & 25.02 & 87.95 & 26.77 & 87.98 & 26.76 \\
\hline 110 & 66.86 & 26.89 & 81.45 & 28.91 & 81.43 & 28.91 \\
\hline 120 & 62.91 & 28.58 & 76.40 & 30.82 & 76.31 & 30.85 \\
\hline 130 & 59.42 & 30.26 & 72.05 & 32.67 & 71.96 & 32.71 \\
\hline 140 & 56.38 & 31.89 & 67.87 & 34.69 & 67.82 & 34.71 \\
\hline 150 & 53.53 & 33.59 & 64.22 & 36.66 & 64.23 & 36.65 \\
\hline 160 & 50.48 & 35.62 & 60.63 & 38.83 & 60.56 & 38.88 \\
\hline 170 & 50.67 & 35.48 & 60.35 & 39.01 & 60.38 & 38.99 \\
\hline 180 & 46.61 & 38.57 & 56.04 & 42.01 & 55.98 & 42.06 \\
\hline 190 & 44.45 & 40.44 & 53.38 & 44.10 & 53.31 & 44.17 \\
\hline 200 & 43.29 & 41.53 & 51.90 & 45.36 & 51.85 & 45.40 \\
\hline 210 & 42.13 & 42.67 & 50.44 & 46.68 & 50.40 & 46.72 \\
\hline 220 & 41.63 & 43.18 & 49.56 & 47.50 & 49.47 & 47.59 \\
\hline 230 & 40.05 & 44.89 & 47.69 & 49.37 & 47.67 & 49.39 \\
\hline 240 & 39.14 & 45.93 & 46.29 & 50.86 & 46.26 & 50.89 \\
\hline
\end{tabular}


Table 4.51 Comparison of time and speedup of Ladder215S, Ladder315C and Ladder315S with $N=24$ on an Intel Xeon Phi coprocessor with KMP_THREAD_AFFINITY set to compact

\begin{tabular}{|c|c|c|c|c|c|c|}
\hline \multicolumn{7}{|c|}{ Compact $N=24$ (time in seconds) } \\
\hline \multirow{2}{*}{$\frac{\text { model }}{\text { threads }}$} & \multicolumn{2}{|c|}{ Ladder $215 \mathrm{~S}(N)$} & \multicolumn{2}{|c|}{ Ladder $315 \mathrm{C}(N)$} & \multicolumn{2}{|c|}{ Ladder $315 \mathrm{~S}(N)$} \\
\hline & time & speedup & time & speedup & time & speedup \\
\hline 1 & 2355.34 & 1.00 & 2568.94 & 1.00 & 2568.89 & 1.00 \\
\hline 10 & 696.70 & 3.38 & 785.06 & 3.27 & 774.36 & 3.32 \\
\hline 20 & 374.76 & 6.28 & 403.20 & 6.37 & 403.12 & 6.37 \\
\hline 30 & 255.56 & 9.22 & 275.59 & 9.32 & 273.91 & 9.38 \\
\hline 40 & 196.31 & 12.00 & 211.64 & 12.14 & 211.72 & 12.13 \\
\hline 50 & 159.52 & 14.77 & 170.30 & 15.09 & 170.23 & 15.09 \\
\hline 60 & 134.78 & 17.47 & 146.65 & 17.52 & 146.67 & 17.51 \\
\hline 70 & 118.39 & 19.89 & 128.09 & 20.05 & 128.06 & 20.06 \\
\hline 80 & 104.40 & 22.56 & 113.39 & 22.65 & 112.93 & 22.75 \\
\hline 90 & 95.01 & 24.79 & 102.64 & 25.03 & 102.69 & 25.02 \\
\hline 100 & 87.97 & 26.77 & 93.81 & 27.38 & 93.79 & 27.39 \\
\hline 110 & 81.43 & 28.93 & 87.52 & 29.35 & 87.51 & 29.36 \\
\hline 120 & 76.37 & 30.84 & 81.64 & 31.47 & 81.66 & 31.46 \\
\hline 130 & 71.97 & 32.73 & 76.84 & 33.43 & 76.87 & 33.42 \\
\hline 140 & 67.84 & 34.72 & 72.82 & 35.28 & 72.83 & 35.27 \\
\hline 150 & 64.21 & 36.68 & 69.01 & 37.22 & 68.97 & 37.24 \\
\hline 160 & 60.38 & 39.01 & 65.07 & 39.48 & 65.06 & 39.48 \\
\hline 170 & 60.35 & 39.03 & 64.26 & 39.98 & 64.55 & 39.79 \\
\hline 180 & 56.01 & 42.06 & 59.49 & 43.18 & 59.49 & 43.18 \\
\hline 190 & 53.35 & 44.15 & 57.25 & 44.87 & 57.20 & 44.91 \\
\hline 200 & 51.90 & 45.38 & 55.20 & 46.54 & 55.20 & 46.54 \\
\hline 210 & 50.43 & 46.71 & 53.49 & 48.02 & 53.09 & 48.39 \\
\hline 220 & 49.53 & 47.56 & 52.59 & 48.85 & 52.62 & 48.82 \\
\hline 230 & 47.71 & 49.37 & 50.48 & 50.89 & 50.47 & 50.90 \\
\hline 240 & 46.26 & 50.91 & 49.02 & 52.40 & 48.74 & 52.71 \\
\hline
\end{tabular}


Table 4.52 Comparison of time and speedup of Chain, Ladder and Ladder 215C with $N$ $=24$ on an Intel Xeon Phi coprocessor with KMP_THREAD_AFFINITY set to balanced

\begin{tabular}{|c|c|c|c|c|c|c|}
\hline \multicolumn{7}{|c|}{ Balanced $N=24$ (time in seconds) } \\
\hline \multirow{2}{*}{$\begin{array}{c}\text { model } \\
\text { threads }\end{array}$} & \multicolumn{2}{|c|}{$\operatorname{Chain}(N)$} & \multicolumn{2}{|c|}{ Ladder $(N)$} & \multicolumn{2}{|c|}{ Ladder $215 \mathrm{C}(N)$} \\
\hline & time & speedup & time & speedup & time & speedup \\
\hline 1 & 1797.89 & 1.00 & 2354.22 & 1.00 & 2354.32 & 1.00 \\
\hline 10 & 438.57 & 4.10 & 554.35 & 4.25 & 541.91 & 4.34 \\
\hline 20 & 262.23 & 6.86 & 331.76 & 7.10 & 322.58 & 7.30 \\
\hline 30 & 187.99 & 9.56 & 226.18 & 10.41 & 236.08 & 9.97 \\
\hline 40 & 145.30 & 12.37 & 185.39 & 12.70 & 181.50 & 12.97 \\
\hline 50 & 115.59 & 15.55 & 153.42 & 15.34 & 149.90 & 15.71 \\
\hline 60 & 99.26 & 18.11 & 130.32 & 18.07 & 129.96 & 18.12 \\
\hline 70 & 89.24 & 20.15 & 117.29 & 20.07 & 115.86 & 20.32 \\
\hline 80 & 79.38 & 22.65 & 102.97 & 22.86 & 101.16 & 23.27 \\
\hline 90 & 73.86 & 24.34 & 93.70 & 25.12 & 94.63 & 24.88 \\
\hline 100 & 69.89 & 25.73 & 85.32 & 27.59 & 87.06 & 27.04 \\
\hline 110 & 65.23 & 27.56 & 80.18 & 29.36 & 81.03 & 29.05 \\
\hline 120 & 61.47 & 29.25 & 75.29 & 31.27 & 76.30 & 30.85 \\
\hline 130 & 59.37 & 30.28 & 72.67 & 32.39 & 73.43 & 32.06 \\
\hline 140 & 56.58 & 31.78 & 68.08 & 34.58 & 68.85 & 34.19 \\
\hline 150 & 53.88 & 33.37 & 66.43 & 35.44 & 65.37 & 36.01 \\
\hline 160 & 51.24 & 35.09 & 62.35 & 37.76 & 61.32 & 38.39 \\
\hline 170 & 50.82 & 35.38 & 61.71 & 38.15 & 61.01 & 38.59 \\
\hline 180 & 47.74 & 37.66 & 57.76 & 40.76 & 58.07 & 40.54 \\
\hline 190 & 46.54 & 38.63 & 57.16 & 41.19 & 56.72 & 41.51 \\
\hline 200 & 44.55 & 40.36 & 54.76 & 42.99 & 54.59 & 43.13 \\
\hline 210 & 43.09 & 41.72 & 52.13 & 45.16 & 52.01 & 45.27 \\
\hline 220 & 42.91 & 41.90 & 51.74 & 45.51 & 51.69 & 45.55 \\
\hline 230 & 39.99 & 44.96 & 47.60 & 49.46 & 47.55 & 49.51 \\
\hline 240 & 39.10 & 45.99 & 46.29 & 50.86 & 46.24 & 50.92 \\
\hline
\end{tabular}


Table 4.53 Comparison of time and speedup of Ladder215S, Ladder315C and Ladder315S with $N=24$ on an Intel Xeon Phi coprocessor with KMP_THREAD_AFFINITY set to balanced

\begin{tabular}{|c|c|c|c|c|c|c|}
\hline \multicolumn{7}{|c|}{ Balanced $N=24$ (time in seconds) } \\
\hline \multirow{2}{*}{$\frac{\text { model }}{\text { threads }}$} & \multicolumn{2}{|c|}{ Ladder $215 \mathrm{~S}(N)$} & \multicolumn{2}{|c|}{ Ladder $315 \mathrm{C}(N)$} & \multicolumn{2}{|c|}{ Ladder $315 \mathrm{~S}(N)$} \\
\hline & time & speedup & time & speedup & time & speedup \\
\hline 1 & 2355.34 & 1.00 & 2568.94 & 1.00 & 2568.89 & 1.00 \\
\hline 10 & 549.57 & 4.29 & 622.22 & 4.13 & 619.44 & 4.15 \\
\hline 20 & 325.76 & 7.23 & 358.71 & 7.16 & 354.97 & 7.24 \\
\hline 30 & 240.50 & 9.79 & 263.17 & 9.76 & 256.50 & 10.02 \\
\hline 40 & 183.06 & 12.87 & 205.25 & 12.52 & 206.93 & 12.41 \\
\hline 50 & 153.58 & 15.34 & 165.51 & 15.52 & 162.17 & 15.84 \\
\hline 60 & 129.63 & 18.17 & 142.44 & 18.03 & 142.15 & 18.07 \\
\hline 70 & 114.59 & 20.55 & 125.40 & 20.49 & 128.03 & 20.06 \\
\hline 80 & 101.17 & 23.28 & 110.97 & 23.15 & 110.19 & 23.31 \\
\hline 90 & 93.39 & 25.22 & 100.03 & 25.68 & 101.48 & 25.31 \\
\hline 100 & 84.45 & 27.89 & 92.25 & 27.85 & 92.21 & 27.86 \\
\hline 110 & 80.03 & 29.43 & 85.70 & 29.98 & 87.02 & 29.52 \\
\hline 120 & 75.62 & 31.15 & 81.33 & 31.59 & 81.57 & 31.49 \\
\hline 130 & 72.78 & 32.36 & 78.51 & 32.72 & 78.42 & 32.76 \\
\hline 140 & 69.06 & 34.11 & 74.08 & 34.68 & 74.25 & 34.60 \\
\hline 150 & 64.76 & 36.37 & 70.38 & 36.50 & 69.69 & 36.86 \\
\hline 160 & 61.47 & 38.31 & 67.66 & 37.97 & 66.15 & 38.84 \\
\hline 170 & 61.33 & 38.41 & 66.37 & 38.71 & 66.22 & 38.79 \\
\hline 180 & 58.09 & 40.55 & 62.21 & 41.29 & 62.36 & 41.19 \\
\hline 190 & 57.26 & 41.13 & 61.70 & 41.64 & 61.43 & 41.82 \\
\hline 200 & 54.46 & 43.25 & 58.78 & 43.70 & 58.37 & 44.01 \\
\hline 210 & 51.96 & 45.33 & 55.83 & 46.01 & 55.63 & 46.18 \\
\hline 220 & 51.63 & 45.62 & 55.23 & 46.51 & 54.76 & 46.91 \\
\hline 230 & 47.60 & 49.48 & 50.60 & 50.77 & 50.50 & 50.87 \\
\hline 240 & 46.24 & 50.94 & 49.11 & 52.31 & 49.11 & 52.31 \\
\hline
\end{tabular}


Table 4.54 Comparison of time and speedup of Chain, Ladder and Ladder 215C with $N$ $=18$ on an Intel Xeon Phi coprocessor with KMP_THREAD_AFFINITY set to scatter

\begin{tabular}{|c|c|c|c|c|c|c|}
\hline \multicolumn{7}{|c|}{ Scatter $N=18$ (time in seconds) } \\
\hline \multirow{2}{*}{$\begin{array}{c}\text { model } \\
\text { threads }\end{array}$} & \multicolumn{2}{|c|}{ Chain $(N)$} & \multicolumn{2}{|c|}{ Ladder $(N)$} & \multicolumn{2}{|c|}{ Ladder $215 \mathrm{C}(N)$} \\
\hline & time & speedup & time & speedup & time & speedup \\
\hline 1 & 27.67 & 1.00 & 32.94 & 1.00 & 33.01 & 1.00 \\
\hline 10 & 7.15 & 3.87 & 8.21 & 4.01 & 8.29 & 3.98 \\
\hline 20 & 5.56 & 4.97 & 6.31 & 5.22 & 6.30 & 5.24 \\
\hline 30 & 3.95 & 7.00 & 4.42 & 7.45 & 4.36 & 7.57 \\
\hline 40 & 4.11 & 6.73 & 4.79 & 6.88 & 4.68 & 7.06 \\
\hline 50 & 4.48 & 6.18 & 5.47 & 6.03 & 5.32 & 6.21 \\
\hline 60 & 5.02 & 5.51 & 6.44 & 5.11 & 6.28 & 5.26 \\
\hline 70 & 5.66 & 4.89 & 7.23 & 4.56 & 7.11 & 4.64 \\
\hline 80 & 6.19 & 4.47 & 7.88 & 4.18 & 7.79 & 4.24 \\
\hline 90 & 6.67 & 4.15 & 8.59 & 3.84 & 8.49 & 3.89 \\
\hline 100 & 7.13 & 3.88 & 9.33 & 3.53 & 9.21 & 3.58 \\
\hline 110 & 7.66 & 3.61 & 10.07 & 3.27 & 9.95 & 3.32 \\
\hline 120 & 8.35 & 3.32 & 10.87 & 3.03 & 10.73 & 3.08 \\
\hline 130 & 9.09 & 3.05 & 11.87 & 2.78 & 11.64 & 2.84 \\
\hline 140 & 9.59 & 2.88 & 12.64 & 2.61 & 12.44 & 2.65 \\
\hline 150 & 10.14 & 2.73 & 13.34 & 2.47 & 13.15 & 2.51 \\
\hline 160 & 10.72 & 2.58 & 14.10 & 2.34 & 13.90 & 2.37 \\
\hline 170 & 11.26 & 2.46 & 14.86 & 2.22 & 14.63 & 2.26 \\
\hline 180 & 11.80 & 2.34 & 15.58 & 2.11 & 15.33 & 2.15 \\
\hline 190 & 12.60 & 2.20 & 16.55 & 1.99 & 16.33 & 2.02 \\
\hline 200 & 13.11 & 2.11 & 17.30 & 1.90 & 17.09 & 1.93 \\
\hline 210 & 13.66 & 2.03 & 18.03 & 1.83 & 17.81 & 1.85 \\
\hline 220 & 14.22 & 1.95 & 18.78 & 1.75 & 18.54 & 1.78 \\
\hline 230 & 14.71 & 1.88 & 19.52 & 1.69 & 19.29 & 1.71 \\
\hline 240 & 15.29 & 1.81 & 20.35 & 1.62 & 20.10 & 1.64 \\
\hline
\end{tabular}


Table 4.55 Comparison of time and speedup of Ladder215S, Ladder315C and Ladder315S with $N=18$ on an Intel Xeon Phi coprocessor with KMP_THREAD_AFFINITY set to scatter

\begin{tabular}{|r|r|r|r|r|r|r|}
\hline \multicolumn{2}{|c|}{ Scatter $N=18$ (time in seconds) } \\
\hline model & \multicolumn{1}{|c|}{ Ladder $215 \mathrm{~S}(N)$} & \multicolumn{2}{|l|}{ Ladder $315 \mathrm{C}(N)$} & \multicolumn{2}{c|}{ Ladder $315 \mathrm{~S}(N)$} \\
\hline threads & \multicolumn{1}{c|}{ time } & \multicolumn{1}{c|}{ speedup } & \multicolumn{1}{c|}{ time } & \multicolumn{1}{c|}{ speedup } & \multicolumn{1}{c|}{ time } & \multicolumn{1}{c|}{ speedup } \\
\hline 1 & 32.93 & 1.00 & 35.73 & 1.00 & 35.78 & 1.00 \\
\hline 10 & 8.06 & 4.09 & 8.38 & 4.27 & 8.43 & 4.24 \\
\hline 20 & 6.10 & 5.40 & 6.30 & 5.67 & 6.41 & 5.58 \\
\hline 30 & 4.16 & 7.91 & 5.95 & 6.01 & 5.82 & 6.15 \\
\hline 40 & 4.27 & 7.71 & 4.72 & 7.58 & 4.28 & 8.35 \\
\hline 50 & 4.58 & 7.19 & 5.35 & 6.68 & 4.40 & 8.12 \\
\hline 60 & 5.07 & 6.50 & 6.26 & 5.71 & 4.80 & 7.45 \\
\hline 70 & 5.65 & 5.83 & 7.06 & 5.06 & 5.46 & 6.55 \\
\hline 80 & 6.12 & 5.39 & 7.82 & 4.57 & 5.71 & 6.27 \\
\hline 90 & 6.69 & 4.92 & 8.50 & 4.20 & 6.44 & 5.56 \\
\hline 100 & 7.27 & 4.53 & 9.18 & 3.89 & 6.68 & 5.36 \\
\hline 110 & 7.64 & 4.31 & 9.88 & 3.62 & 7.14 & 5.01 \\
\hline 120 & 8.26 & 3.99 & 10.68 & 3.34 & 7.82 & 4.58 \\
\hline 130 & 8.92 & 3.69 & 11.59 & 3.08 & 8.61 & 4.15 \\
\hline 140 & 9.47 & 3.48 & 12.36 & 2.89 & 8.92 & 4.01 \\
\hline 150 & 9.94 & 3.31 & 13.06 & 2.74 & 9.60 & 3.73 \\
\hline 160 & 10.47 & 3.15 & 13.76 & 2.60 & 9.95 & 3.59 \\
\hline 170 & 10.94 & 3.01 & 14.48 & 2.47 & 10.51 & 3.40 \\
\hline 180 & 11.17 & 2.95 & 15.14 & 2.36 & 10.84 & 3.30 \\
\hline 190 & 11.99 & 2.75 & 16.13 & 2.22 & 11.73 & 3.05 \\
\hline 200 & 12.50 & 2.63 & 16.84 & 2.12 & 12.17 & 2.94 \\
\hline 210 & 12.97 & 2.54 & 17.53 & 2.04 & 12.04 & 2.97 \\
\hline 220 & 13.46 & 2.45 & 18.12 & 1.97 & 13.07 & 2.74 \\
\hline 230 & 13.95 & 2.36 & 18.37 & 1.94 & 13.31 & 2.69 \\
\hline 240 & 14.53 & 2.27 & 19.76 & 1.81 & 14.09 & 2.54 \\
\hline
\end{tabular}


Table 4.56 Comparison of time and speedup of Chain, Ladder and Ladder 215C with $N$ $=18$ on an Intel Xeon Phi coprocessor with KMP_THREAD_AFFINITY set to compact

\begin{tabular}{|r|r|r|r|r|r|r|}
\hline \multicolumn{7}{|c|}{ Compact $N=18$ (time in seconds) } \\
\hline model & \multicolumn{2}{|c|}{ Chain $(N)$} & \multicolumn{2}{|c|}{ Ladder $(N)$} & \multicolumn{2}{c|}{ Ladder $215 \mathrm{C}(N)$} \\
\hline threads & \multicolumn{1}{c|}{ time } & \multicolumn{1}{c|}{ speedup } & \multicolumn{1}{c|}{ time } & \multicolumn{1}{c|}{ speedup } & \multicolumn{1}{c|}{ time } & \multicolumn{1}{c|}{ speedup } \\
\hline 1 & 26.60 & 1.00 & 32.94 & 1.00 & 33.01 & 1.00 \\
\hline 10 & 10.02 & 2.66 & 11.27 & 2.92 & 11.20 & 2.95 \\
\hline 20 & 7.64 & 3.48 & 8.29 & 3.98 & 8.21 & 4.02 \\
\hline 30 & 6.28 & 4.23 & 6.84 & 4.82 & 6.78 & 4.87 \\
\hline 40 & 6.33 & 4.20 & 6.72 & 4.90 & 6.59 & 5.01 \\
\hline 50 & 6.53 & 4.07 & 6.96 & 4.73 & 6.85 & 4.82 \\
\hline 60 & 6.80 & 3.91 & 7.18 & 4.59 & 6.98 & 4.73 \\
\hline 70 & 7.13 & 3.73 & 7.63 & 4.32 & 7.33 & 4.50 \\
\hline 80 & 7.55 & 3.52 & 8.03 & 4.10 & 7.73 & 4.27 \\
\hline 90 & 8.00 & 3.33 & 8.61 & 3.83 & 8.14 & 4.06 \\
\hline 100 & 8.34 & 3.19 & 9.12 & 3.61 & 8.60 & 3.84 \\
\hline 110 & 8.77 & 3.03 & 9.76 & 3.38 & 9.10 & 3.63 \\
\hline 120 & 9.37 & 2.84 & 10.43 & 3.16 & 9.57 & 3.45 \\
\hline 130 & 9.88 & 2.69 & 11.11 & 2.97 & 10.09 & 3.27 \\
\hline 140 & 10.35 & 2.57 & 11.76 & 2.80 & 10.61 & 3.11 \\
\hline 150 & 10.70 & 2.49 & 12.54 & 2.63 & 11.11 & 2.97 \\
\hline 160 & 11.34 & 2.35 & 13.30 & 2.48 & 11.64 & 2.84 \\
\hline 170 & 11.86 & 2.24 & 14.25 & 2.31 & 12.17 & 2.71 \\
\hline 180 & 12.39 & 2.15 & 15.20 & 2.17 & 12.74 & 2.59 \\
\hline 190 & 12.89 & 2.06 & 16.22 & 2.03 & 13.28 & 2.49 \\
\hline 200 & 13.42 & 1.98 & 17.26 & 1.91 & 13.85 & 2.38 \\
\hline 210 & 13.98 & 1.90 & 18.46 & 1.78 & 14.42 & 2.29 \\
\hline 220 & 14.44 & 1.84 & 19.58 & 1.68 & 14.98 & 2.20 \\
\hline 230 & 15.08 & 1.76 & 20.89 & 1.58 & 15.58 & 2.12 \\
\hline 240 & 15.68 & 1.70 & 21.97 & 1.50 & 16.25 & 2.03 \\
\hline
\end{tabular}


Table 4.57 Comparison of time and speedup of Ladder215S, Ladder315C and Ladder315S with $N=18$ on an Intel Xeon Phi coprocessor with KMP_THREAD_AFFINITY set to compact

\begin{tabular}{|c|c|c|c|c|c|c|}
\hline \multicolumn{7}{|c|}{ Compact $N=18$ (time in seconds) } \\
\hline \multirow{2}{*}{$\frac{\text { model }}{\text { threads }}$} & \multicolumn{2}{|c|}{ Ladder $215 \mathrm{~S}(N)$} & \multicolumn{2}{|c|}{ Ladder $315 \mathrm{C}(N)$} & \multicolumn{2}{|c|}{ Ladder $315 \mathrm{~S}(N)$} \\
\hline & time & speedup & time & speedup & time & Speedup \\
\hline 1 & 32.93 & 1.00 & 35.73 & 1.00 & 35.78 & 1.00 \\
\hline 10 & 11.26 & 2.93 & 11.84 & 3.02 & 11.85 & 3.02 \\
\hline 20 & 8.27 & 3.98 & 8.54 & 4.19 & 8.34 & 4.29 \\
\hline 30 & 6.84 & 4.82 & 7.78 & 4.59 & 7.75 & 4.62 \\
\hline 40 & 6.72 & 4.90 & 6.90 & 5.18 & 6.77 & 5.28 \\
\hline 50 & 6.98 & 4.72 & 7.01 & 5.10 & 6.96 & 5.14 \\
\hline 60 & 7.24 & 4.55 & 7.35 & 4.86 & 7.09 & 5.05 \\
\hline 70 & 7.66 & 4.30 & 7.73 & 4.62 & 7.60 & 4.71 \\
\hline 80 & 8.15 & 4.04 & 8.10 & 4.41 & 8.04 & 4.45 \\
\hline 90 & 8.64 & 3.81 & 8.58 & 4.17 & 8.29 & 4.32 \\
\hline 100 & 9.13 & 3.61 & 9.14 & 3.91 & 8.94 & 4.00 \\
\hline 110 & 9.76 & 3.37 & 9.85 & 3.63 & 9.38 & 3.81 \\
\hline 120 & 10.45 & 3.15 & 10.40 & 3.44 & 9.78 & 3.66 \\
\hline 130 & 11.15 & 2.95 & 11.11 & 3.22 & 10.21 & 3.50 \\
\hline 140 & 11.83 & 2.78 & 11.83 & 3.02 & 11.09 & 3.23 \\
\hline 150 & 12.64 & 2.60 & 12.63 & 2.83 & 11.75 & 3.05 \\
\hline 160 & 13.43 & 2.45 & 13.42 & 2.66 & 12.50 & 2.86 \\
\hline 170 & 14.41 & 2.28 & 14.38 & 2.48 & 13.28 & 2.69 \\
\hline 180 & 15.39 & 2.14 & 15.32 & 2.33 & 13.73 & 2.61 \\
\hline 190 & 16.47 & 2.00 & 16.36 & 2.18 & 13.99 & 2.56 \\
\hline 200 & 17.52 & 1.88 & 17.41 & 2.05 & 14.85 & 2.41 \\
\hline 210 & 18.81 & 1.75 & 18.68 & 1.91 & 16.51 & 2.17 \\
\hline 220 & 19.97 & 1.65 & 19.39 & 1.84 & 16.95 & 2.11 \\
\hline 230 & 21.32 & 1.54 & 21.17 & 1.69 & 17.91 & 2.00 \\
\hline 240 & 22.47 & 1.47 & 22.30 & 1.60 & 17.61 & 2.03 \\
\hline
\end{tabular}


Table 4.58 Comparison of time and speedup of Chain, Ladder and Ladder 215C with $N$ $=18$ on an Intel Xeon Phi coprocessor with KMP_THREAD_AFFINITY set to balanced

\begin{tabular}{|c|c|c|c|c|c|c|}
\hline \multicolumn{7}{|c|}{ Balanced $N=18$ (time in seconds) } \\
\hline \multirow{2}{*}{$\begin{array}{l}\text { model } \\
\text { threads }\end{array}$} & \multicolumn{2}{|c|}{ Chain $(N)$} & \multicolumn{2}{|c|}{ Ladder $(N)$} & \multicolumn{2}{|c|}{ Ladder $215 \mathrm{C}(N)$} \\
\hline & time(sec.) & speedup & time(sec.) & speedup & time(sec.) & speedup \\
\hline 1 & 27.67 & 1.00 & 32.94 & 1.00 & 33.01 & 1.00 \\
\hline 10 & 7.86 & 3.52 & 9.13 & 3.61 & 9.02 & 3.66 \\
\hline 20 & 6.79 & 4.07 & 7.28 & 4.52 & 7.41 & 4.46 \\
\hline 30 & 5.38 & 5.14 & 5.47 & 6.02 & 5.73 & 5.76 \\
\hline 40 & 6.45 & 4.29 & 6.19 & 5.32 & 6.56 & 5.03 \\
\hline 50 & 8.09 & 3.42 & 7.25 & 4.54 & 8.03 & 4.11 \\
\hline 60 & 10.33 & 2.68 & 8.83 & 3.73 & 10.15 & 3.25 \\
\hline 70 & 15.69 & 1.76 & 13.69 & 2.41 & 15.46 & 2.13 \\
\hline 80 & 16.99 & 1.63 & 14.79 & 2.23 & 16.58 & 1.99 \\
\hline 90 & 18.59 & 1.49 & 15.94 & 2.07 & 17.87 & 1.85 \\
\hline 100 & 19.98 & 1.38 & 17.03 & 1.93 & 19.47 & 1.69 \\
\hline 110 & 22.23 & 1.24 & 18.50 & 1.78 & 21.30 & 1.55 \\
\hline 120 & 23.45 & 1.18 & 19.77 & 1.67 & 22.54 & 1.46 \\
\hline 130 & 26.04 & 1.06 & 21.92 & 1.50 & 24.60 & 1.34 \\
\hline 140 & 28.14 & 0.98 & 23.55 & 1.40 & 26.58 & 1.24 \\
\hline 150 & 29.77 & 0.93 & 25.15 & 1.31 & 28.41 & 1.16 \\
\hline 160 & 31.78 & 0.87 & 27.32 & 1.21 & 30.53 & 1.08 \\
\hline 170 & 33.71 & 0.82 & 29.00 & 1.14 & 32.36 & 1.02 \\
\hline 180 & 35.58 & 0.78 & 30.44 & 1.08 & 33.81 & 0.98 \\
\hline 190 & 35.97 & 0.77 & 30.82 & 1.07 & 34.35 & 0.96 \\
\hline 200 & 38.04 & 0.73 & 32.16 & 1.02 & 35.90 & 0.92 \\
\hline 210 & 40.38 & 0.69 & 33.98 & 0.97 & 38.23 & 0.86 \\
\hline 220 & 42.95 & 0.64 & 36.03 & 0.91 & 40.91 & 0.81 \\
\hline 230 & 45.91 & 0.60 & 38.29 & 0.86 & 43.22 & 0.76 \\
\hline 240 & 47.75 & 0.58 & 40.16 & 0.82 & 45.41 & 0.73 \\
\hline
\end{tabular}


Table 4.59 Comparison of time and speedup of Ladder215S, Ladder315C and Ladder315S with $N=18$ on an Intel Xeon Phi coprocessor with KMP_THREAD_AFFINITY set to balanced

\begin{tabular}{|c|c|c|c|c|c|c|}
\hline \multicolumn{7}{|c|}{ Balanced $N=18$ (time in seconds) } \\
\hline \multirow{2}{*}{$\begin{array}{c}\text { model } \\
\text { threads }\end{array}$} & \multicolumn{2}{|c|}{ Ladder $215 \mathrm{~S}(N)$} & \multicolumn{2}{|c|}{ Ladder $315 \mathrm{C}(N)$} & \multicolumn{2}{|c|}{ Ladder $315 \mathrm{~S}(N)$} \\
\hline & time & speedup & time & speedup & time & speedup \\
\hline 1 & 32.93 & 1.00 & 35.73 & 1.00 & 35.78 & 1.00 \\
\hline 10 & 9.00 & 3.66 & 9.61 & 3.72 & 9.00 & 3.98 \\
\hline 20 & 7.33 & 4.49 & 7.56 & 4.73 & 6.99 & 5.12 \\
\hline 30 & 5.55 & 5.94 & 7.66 & 4.66 & 7.23 & 4.95 \\
\hline 40 & 6.28 & 5.25 & 6.92 & 5.17 & 6.50 & 5.51 \\
\hline 50 & 7.40 & 4.45 & 8.46 & 4.22 & 7.95 & 4.50 \\
\hline 60 & 9.02 & 3.65 & 10.64 & 3.36 & 9.93 & 3.60 \\
\hline 70 & 14.19 & 2.32 & 16.50 & 2.17 & 15.49 & 2.31 \\
\hline 80 & 15.35 & 2.15 & 17.80 & 2.01 & 16.67 & 2.15 \\
\hline 90 & 16.51 & 1.99 & 19.17 & 1.86 & 18.33 & 1.95 \\
\hline 100 & 17.76 & 1.85 & 20.67 & 1.73 & 19.73 & 1.81 \\
\hline 110 & 19.43 & 1.70 & 22.64 & 1.58 & 21.57 & 1.66 \\
\hline 120 & 20.59 & 1.60 & 24.03 & 1.49 & 22.96 & 1.56 \\
\hline 130 & 22.60 & 1.46 & 26.17 & 1.37 & 24.76 & 1.45 \\
\hline 140 & 24.46 & 1.35 & 27.42 & 1.30 & 26.98 & 1.33 \\
\hline 150 & 25.93 & 1.27 & 29.72 & 1.20 & 28.98 & 1.23 \\
\hline 160 & 28.12 & 1.17 & 32.11 & 1.11 & 31.11 & 1.15 \\
\hline 170 & 29.69 & 1.11 & 33.76 & 1.06 & 32.97 & 1.09 \\
\hline 180 & 31.10 & 1.06 & 35.41 & 1.01 & 34.59 & 1.03 \\
\hline 190 & 31.45 & 1.05 & 35.80 & 1.00 & 35.34 & 1.01 \\
\hline 200 & 32.67 & 1.01 & 37.69 & 0.95 & 37.01 & 0.97 \\
\hline 210 & 34.48 & 0.96 & 39.47 & 0.91 & 39.41 & 0.91 \\
\hline 220 & 36.43 & 0.90 & 42.79 & 0.84 & 42.09 & 0.85 \\
\hline 230 & 38.92 & 0.85 & 44.66 & 0.80 & 42.72 & 0.84 \\
\hline 240 & 40.70 & 0.81 & 47.60 & 0.75 & 46.35 & 0.77 \\
\hline
\end{tabular}




\subsubsection{Comparison of Speedup between Xeon Phi and Odin}

To sum up the above arguments, Compact type with 240 threads when $N=24$ can get a better speedup on Intel Xeon Phi, with the same size $N=24$, set 8 threads on Odin can obtain a good speedup.

Table 4.60 the execution speed of the same problem on Odin and the Xeon Phi coprocessor is computed. See that the Xeon Phi is around 3.6 times faster than Odin for all 6 models. Efficiency $=$ Speedup/amount of cores, on the Xeon Phi, the efficiency of Ladder models is up to 0.88 , it is very close to 1 , therefore, when $N=24$, Xeon Phi has a rather good speedup. A good speedup can be got when Odin set 8 threads, I picked the data form the Table 4.35, (time in seconds)

Table 4.36 and Table 4.37 where threads $p=8, N=24$. The efficiency of each core is very low, just around 0.16 , in this case, speed of Odin is disappointing.

Table 4.60 Comparison of time and speedup between Xeon Phi and Odin with $N=24$

\begin{tabular}{|c|c|c|c|c|c|c|}
\hline$N=24$ & \multicolumn{2}{|c|}{ Xeon Phi Compact (240 threads) } & \multicolumn{3}{|c|}{ Odin (8 threads) } \\
\hline models name & time(sec.) & speedup & efficiency & time(sec.) & speedup & efficiency \\
\hline Chain & 39.14 & 45.93 & 0.77 & 146.09 & 1.75 & 0.15 \\
\hline Ladder2 & 46.29 & 50.86 & 0.85 & 169.46 & 1.81 & 0.15 \\
\hline Ladder215C & 46.26 & 50.89 & 0.85 & 166.61 & 1.89 & 0.16 \\
\hline Ladder215S & 46.26 & 50.91 & 0.85 & 170.62 & 1.86 & 0.16 \\
\hline Ladder315C & 49.02 & 52.40 & 0.87 & 180.08 & 1.95 & 0.16 \\
\hline Ladder315S & 48.74 & 52.71 & 0.88 & 177.01 & 2.04 & 0.17 \\
\hline
\end{tabular}


Table 4.61 Comparison of time and speedup between Xeon Phi and Odin with $N=18$

\begin{tabular}{|c|r|c|c|c|c|c|}
\hline \multirow{2}{*}{$N=18$} & \multicolumn{3}{|c|}{ Xeon Phi Compact (40 threads) } & \multicolumn{3}{c|}{ Odin (8 threads) } \\
\hline models name & time(sec.) & speedup & efficiency & time(sec.) & speedup & efficiency \\
\hline Chain & 6.33 & 4.20 & 0.07 & 0.74 & 4.04 & 0.34 \\
\hline Ladder2 & 6.72 & 4.90 & 0.08 & 0.79 & 4.48 & 0.37 \\
\hline Ladder215C & 6.59 & 5.01 & 0.08 & 0.79 & 4.44 & 0.37 \\
\hline Ladder215S & 6.72 & 4.90 & 0.08 & 0.73 & 4.67 & 0.39 \\
\hline Ladder315C & 6.90 & 5.18 & 0.09 & 0.91 & 4.31 & 0.36 \\
\hline Ladder315S & 6.77 & 5.28 & 0.09 & 1.00 & 3.93 & 0.33 \\
\hline
\end{tabular}

Table 4.61 compared time and speedup between Intel Xeon and Odin with $N=18$. Compact affinity type with 40 threads can get the best speedup on Xeon Phi, so I pick this set of data as the object of comparison; In Odin, I also use the data with number of threads $p=8$. Odin and Xeon Phi have a similar speedup in this size, but the result of Odin is faster than Xeon Phi. I have to admit that is Xeon Phi do not fit on a calculation with small size $\mathrm{N}$, the smaller the slower. Hence, the calculations with small size is better executed on Odin than on the Xeon Phi. 
Table 4.62 Comparison Ladder 215C $(N)$ with $N=16,18,20,22,24,26$ on two machines

\begin{tabular}{|c|r|r|r|r|}
\hline Ladder 215C $(N)$ & \multicolumn{2}{|c|}{ Xeon Phi Compact } & \multicolumn{2}{c|}{ Odin } \\
\hline$N$ & Time (sec.) & \multicolumn{1}{|c|}{ Speedup } & Time (sec.) & \multicolumn{1}{c|}{ Speedup } \\
\hline 16 & 17.73 & 0.47 & 0.32 & 2.54 \\
\hline 18 & 16.25 & 2.03 & 0.79 & 4.44 \\
\hline 20 & 15.75 & 8.57 & 6.42 & 2.47 \\
\hline 22 & 21.31 & 25.23 & 33.75 & 2.10 \\
\hline 24 & 46.26 & 50.89 & 166.61 & 1.89 \\
\hline 26 & 95.00 & 94.98 & 601.43 & 2.41 \\
\hline
\end{tabular}

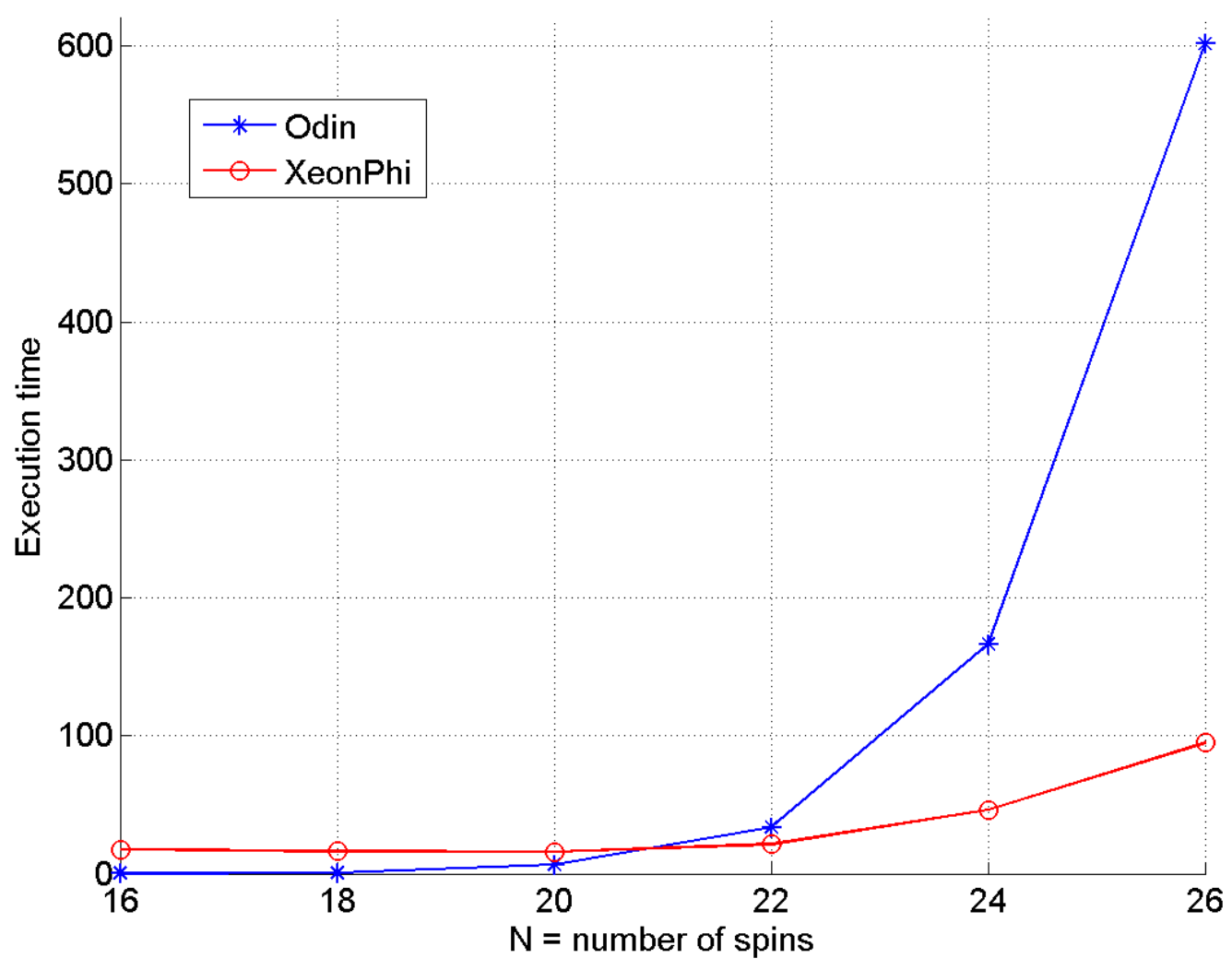

Figure 4.76 Comparison of time between Odin and Xeon Phi on Ladder215C(24) 
Table 4.62 shows the execution times and parallel speedups obtained on odin and the Intel Xeon Phi machine for the Ladder 215C model. The execution times are also shown graphically by Figure 4.76 . The data show clearly that for smaller $N$, the conventional multi-core architecture of odin yields much better results. For larger $N$ 's however the situation changes and the Intel Xeon Phi becomes the faster machine.

These results can be understood by the architectural differences between the two machines. Modern multi-core CPU's (like those in odin) have an out-of-order core architecture that has a superior performance of a single core than the in-order architecture of the Xeon-Phi cores. For smaller system sizes where memory access speed is not the limiting factor, the better performance of the individual cores gives the multi-core CPU an advantage. For larger system sizes, however, the execution speed of the program becomes more and more limited by memory access time and the out-oforder core architecture loses its advantage. The Xeon-Phi however has not only a large number of cores on the chip but also provides a large memory bandwidth of $320 \mathrm{~GB} / \mathrm{s}$. This large memory bandwidth explains the better performance of the Xeon Phi coprocessor in case of the larger $N$ 's. 


\section{Summary and Conclusions}

The work described in this thesis has two goals. The first goal is to study the performance and possible advantages of the Block-Davidson and RMM-DIIS algorithms for the exact diagonalization of quantum spin systems. In order to achieve this, programs were developed for each of these algorithms and applied to typical spin1/2 Heisenberg models.

The second goal of this thesis is to test whether the new Intel Many-Integrated-Core technology provides a suitable architecture to carry out exact diagonalization studies. To this end, the RMM-DIIS code developed in the first part of the thesis was tested extensively on an Intel Xeon Phi coprocessor as well as a conventional multi-core machine.

\subsection{Block Davidson and RMM-DIIS Algorithms}

The results presented in chapter 4 show that the Block Davidson method as well as the RMM-DIIS algorithms can be used for the determination of the lowest eigenpairs of the Hamilton matrices describing typical quantum spin systems. Repeated tests with different initial trial vectors did not indicate any specific convergence problems for either method.

As expected, the RMM-DIIS method reaches the same level of convergence in less

computing time than the Block Davidson method due to the reduced need for 
reorthogonalization. As shown by the results in Sec. 4.3, the difference is sometimes rather large. The reason for this is the extreme size of the matrices which makes the reorthogonalization computationally very costly.

In electronic structure calculations it has been found Ref. [3] that one has to be careful with the initialization of the RMM-DIIS algorithm. The reason is that this algorithm will find the eigenvectors closest to the initial trial vectors and there is no guarantee that the states found will be the lowest vectors. In addition to this, there are two possibilities to perform the intermediate Ritz projections. One method $\left(V_{\max }\right)$ uses the full iterative subspace obtained for all trial states in the Ritz step. The other method $\left(V_{\min }\right)$ uses only the latest iteration of each trial vector in the Ritz step.

In total, I have developed and tested three different versions of the RMM-DIIS algorithm. The first version $\left(\mathrm{BKDV}-V_{\min }\right)$ starts the calculations with the BlockDavidson algorithm and then switches to the $V_{\min }$ version of RMM-DIIS. The second variant $\left(V_{\max }\right)$ uses exclusively the $V_{\max }$ version of the algorithm. The third variant $\left(V_{\max }-V_{\min }\right)$ starts the algorithm with $V_{\max }$ and then switches to the Vmin version of the algorithm.

Surprisingly, the tests indicated no initialization problems for any of the three variants of the RMM-DIIS method. The most efficient method was the third variant $V_{\text {max }}-V_{\text {min }}$. It is not clear why in our tests, the lowest eigenvectors were found even for the second and third variant which use the RMM-DIIS method exclusively. Possible reasons are the fact that compared to typical electronic structure calculations, only very few eigenvectors were sought. It might also play a role that the electronic structure calculations in Ref. [3] are self-consistent calculations in which the Hamilton matrix 
depends on the eigenvectors. This dependency might compound the initialization problem.

A severe practical problem of exact diagonalization studies is the extreme size of even single vectors in the problem. This usually imposes severe limits on the maximum size of the system. In this thesis, this problem was not directly addressed. The RMM-DIIS algorithm would nevertheless be a promising candidate for a memory saving scheme. The reason is that between the Ritz steps, this algorithm iterates only individual eigenstates. It is therefore conceivable to design a program that stores the vectors on different nodes and communicates them during the Ritz step.

\subsection{Parallel Speedup on Intel Xeon Phi}

In order to test the performance of the Intel Many-Integrated Core technology for the diagonalization of quantum spin systems, I ran the RMM-DIIS algorithm on an Intel Xeon Phi coprocessor and a convention multi-core machine (Odin).

The maximum parallel speedups obtained on the conventional 12-core machine was less than 5. This was not unexpected due to the large size of the matrices and vectors. In addition to this, operations on sparse matrices often access the memory in a non-ideal manner.

On the Intel Xeon Phi, the maximum parallel speedup and the performance in general depends strongly on the size of the problem. As shown in Sec. 4.4, the coprocessor is 
not working efficiently at all for the smaller systems $(N=16-20)$. For the large systems $(N=24,26)$ however, the Xeon Phi coprocessor achieves excellent speedups in the range 50 - 60. The speedups vary slightly between the different models. The controlling factor appears to be the number of elements per row in the matrix. The more elements there are (i.e. the less sparse the matrix is) the better the performance.

The speedup of the Xeon Phi coprocessor has to be seen in relation to the performance of one of its cores. While the device has 60 cores, a single core is much less powerful than a core on a typical multi-core machine like odin. Nevertheless, for the larger systems, the Xeon Phi coprocessor outperforms odin by a factor of three to four.

The results obtained in this thesis indicate that the Xeon Phi coprocessor is an excellent architecture for exact diagonalization problems. Most likely it is its large memory bandwidth that is responsible for this. One might ask, however, why the speedup of the Xeon Phi coprocessor is below 60 although each of the coprocessor cores can manage four hardware threads Ref.[12]. Most likely this is due to the fact that the program remains limited by memory access speed. In addition to this, the four hardware threads still must share some of the cores physical units

\subsection{Comparison with other exact diagonalization studies}

In this work only relatively small systems with $N \leq 26$ have been considered. This is in contrast to recent applications of the exact diagonalization method which uses system sizes $N$ in the range from 28 to 40 Ref. [2][15][24][25]. There are two reasons for this discrepancy. 
On the one hand, the calculations in this work have focused on the case where not only the lowest eigenvalue is sought but several of the lowest eigenvalues. This increases the memory requirements and reduces the size of the attainable systems. By lowering the number of eigenvalues from eight to four and by using some memory saving strategies it might well be possible to treat systems with $N=28$ with the program developed in this thesis.

On the other hand, system sizes of $N=30$ and above can usually only be treated if all symmetries of the quantum models are exploited. This is a cumbersome and time consuming task. The goal of this thesis was however not to reach the largest possible system sizes but to study the properties of the Block Davidson and RMM-DIIS method in general. For this reason, only the relatively simple conservation of $S^{Z}$ was used in this work. By using more symmetries it should be possible to extend the size of the systems considerably.

A conclusion from this work is that for exact diagonalization studies of quantum spin systems that require more than the lowest eigenvalue, the application of the RMM-DIIS method and the Block Davidson method is possible. In particular, the RMM-DIIS method might lead to shorter computing times due to the reduced need for orthogonalization of the trial states. In addition to this, the benchmark calculations show that the Xeon Phi co-processor is an interesting architecture for studies with system sizes above $N=24$. 


\section{Appendix A}

\subsection{Speedup data on Odin}

Table 6.1 Time and speedup of Chain $(N)$ model with $N=26,24$ and 22 on Odin

\begin{tabular}{|c|c|c|c|c|c|c|c|}
\hline \multicolumn{8}{|c|}{ Heisenberg chain $(N)$ (recorded time in seconds) } \\
\hline Thread & Data 1 & Data 2 & Data 3 & Data 4 & Data 5 & Average & Speedup \\
\hline$N=26$ & $8582 \mathrm{MB}$ & $5963 \mathrm{MB}$ & & & & & \\
\hline 1 & 1208.36 & 1173.69 & 1083.89 & 1170.62 & 1212.05 & 1169.72 & 1.00 \\
\hline 2 & 701.42 & 693.72 & 705.53 & 693.85 & 714.45 & 701.79 & 1.67 \\
\hline 4 & 576.42 & 573.71 & 589.59 & 578.34 & 586.83 & 580.98 & 2.01 \\
\hline 6 & 525.60 & 541.38 & 553.69 & 542.88 & 542.50 & 541.21 & 2.16 \\
\hline 8 & 513.80 & 547.78 & 557.58 & 542.54 & 540.81 & 540.50 & 2.16 \\
\hline 10 & 526.09 & 544.83 & 554.14 & 540.99 & 540.78 & 541.37 & 2.16 \\
\hline 12 & 502.99 & 539.58 & 546.44 & 542.71 & 548.79 & 536.10 & 2.18 \\
\hline$N=24$ & $2865 \mathrm{MB}$ & $2184 \mathrm{MB}$ & & & & & \\
\hline 1 & 254.78 & 254.81 & 259.26 & 256.69 & 254.76 & 256.06 & 1.00 \\
\hline 2 & 181.44 & 181.91 & 178.47 & 180.68 & 183.19 & 181.14 & 1.41 \\
\hline 4 & 152.10 & 153.49 & 149.31 & 150.31 & 155.22 & 152.09 & 1.68 \\
\hline 6 & 148.49 & 148.00 & 145.21 & 144.35 & 152.75 & 147.76 & 1.73 \\
\hline 8 & 147.23 & 148.51 & 143.31 & 143.19 & 148.19 & 146.09 & 1.75 \\
\hline 10 & 151.60 & 153.08 & 147.87 & 148.21 & 152.80 & 150.71 & 1.70 \\
\hline 12 & 155.19 & 155.10 & 149.78 & 150.28 & 155.88 & 153.24 & 1.67 \\
\hline$N=22$ & $1411 \mathrm{MB}$ & $1234 \mathrm{MB}$ & & & & & \\
\hline 1 & 61.45 & 60.78 & 59.88 & 59.42 & 59.22 & 60.15 & 1.00 \\
\hline 2 & 41.38 & 37.97 & 39.97 & 36.33 & 36.00 & 38.33 & 1.57 \\
\hline 4 & 32.24 & 29.81 & 30.30 & 28.24 & 29.23 & 29.97 & 2.01 \\
\hline 6 & 27.09 & 26.44 & 28.09 & 25.48 & 26.94 & 26.81 & 2.24 \\
\hline 8 & 26.45 & 25.07 & 27.17 & 27.10 & 26.10 & 26.38 & 2.28 \\
\hline 10 & 27.69 & 26.28 & 27.49 & 27.44 & 26.80 & 27.14 & 2.22 \\
\hline 12 & 26.63 & 26.67 & 27.54 & 25.14 & 26.78 & 26.55 & 2.27 \\
\hline
\end{tabular}


Table 6.2 Time and speedup of Chain $(N)$ model with $N=20,18$ and16 on Odin

\begin{tabular}{|c|c|c|c|c|c|c|c|}
\hline \multicolumn{8}{|c|}{ Heisenberg Chain $(N)$ (recorded time in seconds) } \\
\hline Thread & Data 1 & Data 2 & Data 3 & Data 4 & Data 5 & Average & Speedup \\
\hline$N=20$ & 1039MB & 992MB & & & & & \\
\hline 1 & 13.36 & 13.16 & 15.84 & 13.18 & 13.21 & 13.75 & 1.00 \\
\hline 2 & 8.02 & 8.15 & 8.19 & 8.21 & 8.10 & 8.13 & 1.69 \\
\hline 4 & 5.97 & 6.21 & 5.90 & 6.16 & 6.19 & 6.09 & 2.26 \\
\hline 6 & 5.49 & 5.50 & 5.57 & 5.81 & 5.34 & 5.54 & 2.48 \\
\hline 8 & 5.29 & 5.58 & 5.57 & 5.66 & 5.62 & 5.55 & 2.48 \\
\hline 10 & 5.27 & 5.45 & 5.66 & 5.57 & 5.45 & 5.48 & 2.51 \\
\hline 12 & 5.53 & 5.60 & 5.66 & 5.71 & 5.61 & 5.62 & 2.45 \\
\hline$N=18$ & $982 \mathrm{MB}$ & $963 \mathrm{MB}$ & & & & & \\
\hline 1 & 3.02 & 2.95 & 3.04 & 2.95 & 2.95 & 2.98 & 1.00 \\
\hline 2 & 1.70 & 1.52 & 1.58 & 1.87 & 1.58 & 1.65 & 1.81 \\
\hline 4 & 1.00 & 0.90 & 1.06 & 0.90 & 0.89 & 0.95 & 3.13 \\
\hline 6 & 0.71 & 0.70 & 0.93 & 0.76 & 0.73 & 0.77 & 3.89 \\
\hline 8 & 0.74 & 0.69 & 0.73 & 0.77 & 0.77 & 0.74 & 4.04 \\
\hline 10 & 0.75 & 0.75 & 0.76 & 0.79 & 0.80 & 0.77 & 3.87 \\
\hline 12 & 0.91 & 0.97 & 0.96 & 0.92 & 0.99 & 0.95 & 3.14 \\
\hline$N=16$ & $952 \mathrm{MB}$ & & & & & & \\
\hline 1 & 0.71 & 0.70 & 0.70 & 0.70 & 0.70 & 0.70 & 1.00 \\
\hline 2 & 0.46 & 0.42 & 0.40 & 0.40 & 0.40 & 0.41 & 1.70 \\
\hline 4 & 0.31 & 0.30 & 0.30 & 0.30 & 0.30 & 0.30 & 2.33 \\
\hline 6 & 0.33 & 0.33 & 0.30 & 0.29 & 0.30 & 0.31 & 2.25 \\
\hline 8 & 0.30 & 0.33 & 0.30 & 0.31 & 0.33 & 0.31 & 2.24 \\
\hline 10 & 0.42 & 0.42 & 0.42 & 0.36 & 0.40 & 0.40 & 1.73 \\
\hline 12 & 0.45 & 0.44 & 0.45 & 0.46 & 0.45 & 0.45 & 1.56 \\
\hline
\end{tabular}


Table 6.3 Time and speedup of Ladder2( $N)$ model with $N=26,24$ and 22, on Odin

\begin{tabular}{|c|c|c|c|c|c|c|c|}
\hline \multicolumn{8}{|c|}{ Heisenberg Ladder2 $(N)$ (recorded time in seconds) } \\
\hline Thread & Data 1 & Data 2 & Data 3 & Data 4 & Data 5 & Average & Speedup \\
\hline$N=26$ & 9354MB & $6768 \mathrm{MB}$ & & & & & \\
\hline 1 & 1491.41 & 1485.34 & 1498.03 & 1484.01 & 1484.85 & 1488.73 & 1.00 \\
\hline 2 & 845.32 & 844.28 & 865.33 & 845.96 & 839.35 & 848.05 & 1.76 \\
\hline 4 & 656.97 & 686.26 & 660.47 & 688.01 & 675.54 & 673.45 & 2.21 \\
\hline 6 & 630.07 & 623.82 & 639.71 & 616.73 & 631.19 & 628.30 & 2.37 \\
\hline 8 & 589.68 & 622.94 & 633.13 & 602.35 & 620.53 & 613.73 & 2.43 \\
\hline 10 & 584.50 & 604.59 & 594.01 & 628.19 & 617.31 & 605.72 & 2.46 \\
\hline 12 & 630.43 & 601.42 & 633.51 & 623.66 & 602.78 & 618.36 & 2.41 \\
\hline$N=24$ & $3058 \mathrm{MB}$ & $2378 \mathrm{MB}$ & & & & & \\
\hline 1 & 307.87 & 308.99 & 304.96 & 304.91 & 308.64 & 307.08 & 1.00 \\
\hline 2 & 219.38 & 219.40 & 218.63 & 218.61 & 217.77 & 218.76 & 1.40 \\
\hline 4 & 184.31 & 179.66 & 181.38 & 180.51 & 180.11 & 181.19 & 1.69 \\
\hline 6 & 172.98 & 160.76 & 173.67 & 172.16 & 173.65 & 170.64 & 1.80 \\
\hline 8 & 173.15 & 157.28 & 173.01 & 171.08 & 172.78 & 169.46 & 1.81 \\
\hline 10 & 175.18 & 174.32 & 175.57 & 174.94 & 174.81 & 174.97 & 1.76 \\
\hline 12 & 179.92 & 175.82 & 179.30 & 177.91 & 175.87 & 177.76 & 1.73 \\
\hline$N=22$ & $1458 \mathrm{MB}$ & $1280 \mathrm{MB}$ & & & & & \\
\hline 1 & 70.01 & 70.08 & 70.03 & 70.06 & 74.29 & 70.89 & 1.00 \\
\hline 2 & 43.79 & 44.73 & 45.37 & 43.73 & 41.74 & 43.87 & 1.62 \\
\hline 4 & 34.96 & 35.37 & 35.97 & 34.97 & 32.98 & 34.85 & 2.03 \\
\hline 6 & 32.88 & 33.25 & 33.36 & 33.11 & 30.63 & 32.65 & 2.17 \\
\hline 8 & 32.40 & 33.23 & 32.70 & 32.64 & 30.04 & 32.20 & 2.20 \\
\hline 10 & 33.23 & 33.41 & 31.03 & 33.45 & 29.81 & 32.19 & 2.20 \\
\hline 12 & 34.19 & 33.90 & 30.33 & 34.20 & 29.54 & 32.43 & 2.19 \\
\hline
\end{tabular}


Table 6.4 Time and speedup of Ladder2(N) model with $N=20,18$ and16 on Odin

\begin{tabular}{|c|c|c|c|c|c|c|c|}
\hline \multicolumn{8}{|c|}{ Heisenberg Ladder2 $(N)$ (recorded time in seconds) } \\
\hline Thread & Data 1 & Data 2 & Data 3 & Data 4 & Data 5 & Average & Speedup \\
\hline$N=20$ & $1050 \mathrm{MB}$ & $1003 \mathrm{MB}$ & & & & & \\
\hline 1 & 15.52 & 15.53 & 15.25 & 15.49 & 15.66 & 15.49 & 1.00 \\
\hline 2 & 9.66 & 9.74 & 9.72 & 9.55 & 9.49 & 9.63 & 1.61 \\
\hline 4 & 7.62 & 7.50 & 7.51 & 7.49 & 7.32 & 7.49 & 2.07 \\
\hline 6 & 6.73 & 6.68 & 6.58 & 6.81 & 6.40 & 6.64 & 2.33 \\
\hline 8 & 6.47 & 6.72 & 6.64 & 6.57 & 6.31 & 6.54 & 2.37 \\
\hline 10 & 6.64 & 6.61 & 6.86 & 6.64 & 6.53 & 6.66 & 2.33 \\
\hline 12 & 6.93 & 7.00 & 6.73 & 6.95 & 6.93 & 6.91 & 2.24 \\
\hline$N=18$ & $984 \mathrm{MB}$ & $981 \mathrm{MB}$ & & & & & \\
\hline 1 & 3.49 & 3.54 & 3.54 & 3.53 & 3.55 & 3.53 & 1.00 \\
\hline 2 & 1.84 & 1.85 & 1.86 & 1.85 & 1.86 & 1.85 & 1.91 \\
\hline 4 & 1.13 & 1.14 & 1.13 & 1.20 & 1.17 & 1.15 & 3.07 \\
\hline 6 & 0.91 & 0.84 & 0.87 & 0.86 & 0.85 & 0.87 & 4.08 \\
\hline 8 & 0.73 & 0.78 & 0.88 & 0.75 & 0.80 & 0.79 & 4.48 \\
\hline 10 & 0.87 & 0.79 & 0.95 & 0.89 & 0.82 & 0.86 & 4.09 \\
\hline 12 & 0.98 & 0.98 & 1.03 & 1.05 & 1.05 & 1.02 & 3.47 \\
\hline$N=16$ & $951 \mathrm{MB}$ & $948 \mathrm{MB}$ & & & & & \\
\hline 1 & 0.82 & 0.82 & 0.82 & 0.82 & 0.82 & 0.82 & 1.00 \\
\hline 2 & 0.46 & 0.46 & 0.46 & 0.51 & 0.46 & 0.47 & 1.74 \\
\hline 4 & 0.33 & 0.33 & 0.34 & 0.34 & 0.33 & 0.33 & 2.46 \\
\hline 6 & 0.33 & 0.32 & 0.34 & 0.35 & 0.32 & 0.33 & 2.46 \\
\hline 8 & 0.33 & 0.32 & 0.33 & 0.32 & 0.32 & 0.32 & 2.53 \\
\hline 10 & 0.37 & 0.36 & 0.38 & 0.34 & 0.44 & 0.38 & 2.16 \\
\hline 12 & 0.48 & 0.46 & 0.48 & 0.46 & 0.46 & 0.47 & 1.75 \\
\hline
\end{tabular}


Table 6.5 Time and speedup of Ladder $215 \mathrm{C}(N)$ with $N=26,24$ and 22 on Odin

\begin{tabular}{|c|c|c|c|c|c|c|c|}
\hline \multicolumn{8}{|c|}{ Heisenberg Ladder215C $(N)$ (recorded time in seconds) } \\
\hline Thread & Data 1 & Data 2 & Data 3 & Data 4 & Data 5 & Average & Speedup \\
\hline$N=26$ & $9354 \mathrm{MB}$ & $6768 \mathrm{MB}$ & & & & & \\
\hline 1 & 1358.32 & 1523.56 & 1501.96 & 1504.02 & 1365.02 & 1450.58 & 1.00 \\
\hline 2 & 861.17 & 867.98 & 852.24 & 867.11 & 852.99 & 860.30 & 1.69 \\
\hline 4 & 662.47 & 662.25 & 695.12 & 673.30 & 692.11 & 677.05 & 2.14 \\
\hline 6 & 627.53 & 609.93 & 616.93 & 624.01 & 642.56 & 624.19 & 2.32 \\
\hline 8 & 615.06 & 591.68 & 607.90 & 604.46 & 588.06 & 601.43 & 2.41 \\
\hline 10 & 600.64 & 580.73 & 625.91 & 593.96 & 626.13 & 605.47 & 2.40 \\
\hline 12 & 624.96 & 577.95 & 631.38 & 581.71 & 612.63 & 605.73 & 2.39 \\
\hline$N=24$ & $3058 \mathrm{MB}$ & $2378 \mathrm{MB}$ & & & & & \\
\hline 1 & 312.11 & 312.37 & 314.66 & 315.11 & 317.42 & 314.33 & 1.00 \\
\hline 2 & 215.57 & 229.89 & 214.73 & 213.01 & 213.20 & 217.28 & 1.45 \\
\hline 4 & 179.66 & 191.82 & 176.63 & 177.41 & 174.74 & 180.05 & 1.75 \\
\hline 6 & 168.85 & 175.67 & 167.34 & 167.96 & 165.85 & 169.13 & 1.86 \\
\hline 8 & 168.77 & 169.02 & 165.11 & 165.79 & 164.36 & 166.61 & 1.89 \\
\hline 10 & 169.74 & 169.39 & 167.10 & 167.05 & 166.54 & 167.96 & 1.87 \\
\hline 12 & 173.86 & 169.03 & 171.77 & 169.47 & 168.07 & 170.44 & 1.84 \\
\hline$N=22$ & $1458 \mathrm{MB}$ & $1280 \mathrm{MB}$ & & & & & \\
\hline 1 & 69.98 & 71.05 & 71.03 & 70.91 & 71.64 & 70.92 & 1.00 \\
\hline 2 & 44.55 & 45.27 & 45.11 & 45.36 & 45.22 & 45.10 & 1.57 \\
\hline 4 & 36.08 & 35.83 & 36.66 & 39.05 & 36.36 & 36.80 & 1.93 \\
\hline 6 & 33.61 & 34.14 & 34.56 & 34.57 & 34.16 & 34.20 & 2.07 \\
\hline 8 & 32.93 & 33.59 & 34.17 & 33.78 & 34.29 & 33.75 & 2.10 \\
\hline 10 & 33.58 & 33.27 & 34.62 & 34.72 & 34.90 & 34.22 & 2.07 \\
\hline 12 & 33.93 & 33.27 & 35.10 & 35.02 & 35.36 & 34.54 & 2.05 \\
\hline
\end{tabular}


Table 6.6 Time and speedup of Ladder $215 \mathrm{C}(N)$ with $N=20,18$ and16 on Odin

\begin{tabular}{|c|c|c|c|c|c|c|c|}
\hline \multicolumn{8}{|c|}{ Heisenberg Ladder215C $(N)$ (recorded time in seconds) } \\
\hline Thread & Data 1 & Data 2 & Data 3 & Data 4 & Data 5 & Average & Speedup \\
\hline$N=20$ & $1050 \mathrm{MB}$ & $1003 \mathrm{MB}$ & & & & & \\
\hline 1 & 16.44 & 16.51 & 15.49 & 15.49 & 15.48 & 15.88 & 1.00 \\
\hline 2 & 9.30 & 9.30 & 9.65 & 9.72 & 9.72 & 9.53 & 1.67 \\
\hline 4 & 7.18 & 7.04 & 7.47 & 7.61 & 7.55 & 7.37 & 2.16 \\
\hline 6 & 6.29 & 6.33 & 6.66 & 6.66 & 6.69 & 6.53 & 2.43 \\
\hline 8 & 6.20 & 6.22 & 6.63 & 6.57 & 6.47 & 6.42 & 2.47 \\
\hline 10 & 6.16 & 6.14 & 6.58 & 6.59 & 6.64 & 6.42 & 2.47 \\
\hline 12 & 6.30 & 6.19 & 6.74 & 6.81 & 6.87 & 6.58 & 2.41 \\
\hline$N=18$ & $984 \mathrm{MB}$ & $981 \mathrm{MB}$ & & & & & \\
\hline 1 & 3.49 & 3.52 & 3.53 & 3.52 & 3.53 & 3.52 & 1.00 \\
\hline 2 & 2.08 & 1.85 & 1.86 & 2.14 & 1.85 & 1.96 & 1.80 \\
\hline 4 & 1.16 & 1.21 & 1.14 & 1.10 & 1.13 & 1.15 & 3.07 \\
\hline 6 & 0.97 & 1.00 & 1.12 & 0.89 & 1.09 & 1.01 & 3.47 \\
\hline 8 & 0.74 & 0.86 & 0.81 & 0.78 & 0.77 & 0.79 & 4.44 \\
\hline 10 & 0.78 & 0.93 & 0.83 & 0.94 & 0.86 & 0.87 & 4.06 \\
\hline 12 & 1.00 & 0.97 & 1.05 & 0.99 & 1.06 & 1.01 & 3.47 \\
\hline$N=16$ & $951 \mathrm{MB}$ & $948 \mathrm{MB}$ & & & & & \\
\hline 1 & 0.80 & 0.82 & 0.82 & 0.82 & 0.80 & 0.81 & 1.00 \\
\hline 2 & 0.47 & 0.51 & 0.48 & 0.46 & 0.45 & 0.47 & 1.72 \\
\hline 4 & 0.32 & 0.33 & 0.31 & 0.33 & 0.32 & 0.32 & 2.51 \\
\hline 6 & 0.33 & 0.33 & 0.32 & 0.33 & 0.32 & 0.33 & 2.49 \\
\hline 8 & 0.31 & 0.33 & 0.29 & 0.34 & 0.32 & 0.32 & 2.54 \\
\hline 10 & 0.36 & 0.44 & 0.43 & 0.41 & 0.35 & 0.40 & 2.04 \\
\hline 12 & 0.47 & 0.46 & 0.46 & 0.46 & 0.46 & 0.46 & 1.76 \\
\hline
\end{tabular}


Table 6.7 Time and speedup of Ladder $215 \mathrm{~S}(N)$ with $N=26,24$ and 22 on Odin

\begin{tabular}{|c|c|c|c|c|c|c|c|}
\hline \multicolumn{8}{|c|}{ Heisenberg Ladder215S $(N)$ (recorded time in seconds) } \\
\hline Thread & Data 1 & Data 2 & Data 3 & Data 4 & Data 5 & Average & Speedup \\
\hline$N=26$ & $9354 \mathrm{MB}$ & $6768 \mathrm{MB}$ & & & & & \\
\hline 1 & 1470.10 & 1534.82 & 1405.18 & 1514.15 & 1508.64 & 1486.58 & 1.00 \\
\hline 2 & 841.47 & 869.74 & 882.98 & 888.47 & 849.31 & 866.39 & 1.72 \\
\hline 4 & 684.45 & 690.01 & 731.44 & 680.91 & 676.13 & 692.59 & 2.15 \\
\hline 6 & 614.11 & 619.52 & 640.68 & 624.63 & 631.91 & 626.17 & 2.37 \\
\hline 8 & 589.24 & 596.88 & 621.57 & 601.81 & 613.16 & 604.53 & 2.46 \\
\hline 10 & 589.81 & 603.36 & 624.11 & 589.38 & 613.08 & 603.95 & 2.46 \\
\hline 12 & 623.06 & 608.92 & 659.49 & 578.15 & 646.98 & 623.32 & 2.38 \\
\hline$N=24$ & $3058 \mathrm{MB}$ & $2378 \mathrm{MB}$ & & & & & \\
\hline 1 & 306.74 & 327.60 & 304.39 & 305.01 & 343.28 & 317.40 & 1.00 \\
\hline 2 & 218.43 & 206.97 & 218.86 & 220.24 & 205.02 & 213.90 & 1.48 \\
\hline 4 & 180.30 & 171.00 & 181.41 & 182.58 & 164.67 & 175.99 & 1.80 \\
\hline 6 & 171.07 & 171.52 & 174.43 & 174.36 & 154.13 & 169.10 & 1.88 \\
\hline 8 & 179.25 & 173.43 & 172.67 & 172.47 & 155.28 & 170.62 & 1.86 \\
\hline 10 & 198.52 & 176.30 & 176.43 & 176.24 & 155.31 & 176.56 & 1.80 \\
\hline 12 & 185.35 & 181.10 & 179.57 & 177.88 & 157.37 & 176.25 & 1.80 \\
\hline$N=22$ & $1458 \mathrm{MB}$ & $1280 \mathrm{MB}$ & & & & & \\
\hline 1 & 70.02 & 71.07 & 70.45 & 70.02 & 69.39 & 70.19 & 1.00 \\
\hline 2 & 44.57 & 45.35 & 45.54 & 44.08 & 44.92 & 44.89 & 1.56 \\
\hline 4 & 35.97 & 35.49 & 36.84 & 35.62 & 35.63 & 35.91 & 1.95 \\
\hline 6 & 33.34 & 31.87 & 33.97 & 33.36 & 33.20 & 33.15 & 2.12 \\
\hline 8 & 32.77 & 33.73 & 34.00 & 32.60 & 33.36 & 33.29 & 2.11 \\
\hline 10 & 33.44 & 34.47 & 34.88 & 34.27 & 33.50 & 34.11 & 2.06 \\
\hline 12 & 34.24 & 33.76 & 35.96 & 34.82 & 34.13 & 34.58 & 2.03 \\
\hline
\end{tabular}


Table 6.8 Time and speedup of Ladder $215 \mathrm{~S}(N)$ with $N=20,18$ and 16 on Odin

\begin{tabular}{|c|c|c|c|c|c|c|c|}
\hline \multicolumn{8}{|c|}{ Heisenberg Ladder215S $(N)$ (recorded time in seconds) } \\
\hline Thread & Data 1 & Data 2 & Data 3 & Data 4 & Data 5 & Average & Speedup \\
\hline$N=20$ & $1050 \mathrm{MB}$ & $1003 \mathrm{MB}$ & & & & & \\
\hline 1 & 15.39 & 15.37 & 15.36 & 15.45 & 15.44 & 15.40 & 1.00 \\
\hline 2 & 9.62 & 9.64 & 9.66 & 9.76 & 9.61 & 9.66 & 1.59 \\
\hline 4 & 7.49 & 7.52 & 7.49 & 7.52 & 7.64 & 7.53 & 2.05 \\
\hline 6 & 6.75 & 6.95 & 6.74 & 6.79 & 6.75 & 6.80 & 2.27 \\
\hline 8 & 6.96 & 6.72 & 6.77 & 6.82 & 6.40 & 6.73 & 2.29 \\
\hline 10 & 6.86 & 6.87 & 6.94 & 6.83 & 6.77 & 6.86 & 2.25 \\
\hline 12 & 7.13 & 7.00 & 7.09 & 6.85 & 6.83 & 6.98 & 2.21 \\
\hline$N=18$ & $984 \mathrm{MB}$ & $981 \mathrm{MB}$ & & & & & \\
\hline 1 & 3.45 & 3.56 & 3.27 & 3.33 & 3.46 & 3.41 & 1.00 \\
\hline 2 & 1.80 & 2.35 & 2.10 & 1.81 & 1.68 & 1.95 & 1.75 \\
\hline 4 & 1.15 & 1.04 & 0.96 & 1.03 & 1.04 & 1.05 & 3.27 \\
\hline 6 & 0.87 & 0.79 & 0.98 & 0.80 & 0.75 & 0.84 & 4.08 \\
\hline 8 & 0.72 & 0.65 & 0.83 & 0.71 & 0.75 & 0.73 & 4.67 \\
\hline 10 & 0.83 & 0.73 & 0.70 & 0.77 & 0.78 & 0.76 & 4.48 \\
\hline 12 & 0.98 & 0.98 & 0.91 & 1.03 & 0.93 & 0.96 & 3.54 \\
\hline$N=16$ & $951 \mathrm{MB}$ & $948 \mathrm{MB}$ & & & & & \\
\hline 1 & 0.71 & 0.71 & 0.72 & 0.64 & 0.65 & 0.69 & 1.00 \\
\hline 2 & 0.45 & 0.42 & 0.41 & 0.48 & 0.38 & 0.43 & 1.60 \\
\hline 4 & 0.37 & 0.30 & 0.32 & 0.30 & 0.23 & 0.30 & 2.25 \\
\hline 6 & 0.37 & 0.29 & 0.31 & 0.31 & 0.26 & 0.31 & 2.23 \\
\hline 8 & 0.34 & 0.30 & 0.30 & 0.30 & 0.25 & 0.30 & 2.29 \\
\hline 10 & 0.37 & 0.41 & 0.38 & 0.47 & 0.34 & 0.39 & 1.74 \\
\hline 12 & 0.39 & 0.48 & 0.47 & 0.50 & 0.42 & 0.45 & 1.52 \\
\hline
\end{tabular}


Table 6.9 Time and speedup of Ladder315C $(N)$ and $315 \mathrm{~S}(N)$ model with $N=24$ and 18 on Odin

\begin{tabular}{|c|c|c|c|c|c|c|c|}
\hline \multicolumn{8}{|c|}{ Heisenberg Ladder315C $(N)$ (recorded time in seconds) } \\
\hline Thread & Data 1 & Data 2 & Data 3 & Data 4 & Data 5 & Average & Speedup \\
\hline$N=24$ & $3123 \mathrm{MB}$ & 2442MB & & & & & \\
\hline 1 & 351.96 & 351.86 & 352.01 & 351.70 & 351.60 & 351.83 & 1.00 \\
\hline 2 & 249.88 & 247.97 & 248.97 & 249.33 & 250.35 & 249.30 & 1.41 \\
\hline 4 & 198.04 & 198.05 & 199.62 & 199.23 & 198.70 & 198.73 & 1.77 \\
\hline 6 & 188.00 & 187.12 & 187.62 & 185.86 & 187.19 & 187.16 & 1.88 \\
\hline 8 & 179.09 & 179.01 & 182.60 & 180.49 & 179.24 & 180.08 & 1.95 \\
\hline 10 & 183.33 & 183.73 & 183.54 & 183.47 & 183.22 & 183.46 & 1.92 \\
\hline 12 & 187.43 & 187.45 & 187.66 & 187.97 & 188.19 & 187.74 & 1.87 \\
\hline$N=18$ & $985 \mathrm{MB}$ & $982 \mathrm{MB}$ & & & & & \\
\hline 1 & 3.92 & 3.92 & 3.91 & 3.91 & 3.92 & 3.92 & 1.00 \\
\hline 2 & 2.08 & 2.33 & 2.48 & 2.05 & 2.05 & 2.20 & 1.78 \\
\hline 4 & 1.26 & 1.17 & 1.15 & 1.34 & 1.26 & 1.23 & 3.17 \\
\hline 6 & 1.01 & 0.91 & 1.10 & 0.93 & 0.95 & 0.98 & 3.99 \\
\hline 8 & 0.93 & 0.82 & 1.03 & 0.92 & 0.84 & 0.91 & 4.31 \\
\hline 10 & 1.02 & 0.86 & 1.09 & 1.04 & 0.87 & 0.98 & 4.01 \\
\hline 12 & 1.02 & 1.00 & 1.02 & 1.00 & 1.05 & 1.02 & 3.84 \\
\hline \multicolumn{8}{|c|}{ Heisenberg Ladder315S $(N)$ (recorded time in seconds) } \\
\hline$N=24$ & $3123 \mathrm{MB}$ & $2442 \mathrm{MB}$ & & & & & \\
\hline 1 & 374.54 & 352.03 & 352.03 & 363.48 & 363.26 & 361.07 & 1.00 \\
\hline 2 & 231.54 & 249.75 & 252.14 & 252.05 & 250.75 & 247.25 & 1.46 \\
\hline 4 & 179.94 & 197.71 & 198.49 & 196.26 & 183.03 & 191.09 & 1.89 \\
\hline 6 & 168.42 & 186.61 & 186.71 & 172.95 & 186.93 & 180.32 & 2.00 \\
\hline 8 & 165.48 & 181.62 & 178.58 & 178.36 & 181.00 & 177.01 & 2.04 \\
\hline 10 & 178.46 & 183.07 & 182.85 & 183.06 & 181.26 & 181.74 & 1.99 \\
\hline 12 & 184.60 & 185.16 & 187.84 & 187.90 & 185.65 & 186.23 & 1.94 \\
\hline$N=18$ & $985 \mathrm{MB}$ & $982 \mathrm{MB}$ & & & & & \\
\hline 1 & 3.91 & 3.92 & 3.93 & 3.92 & 3.92 & 3.92 & 1.00 \\
\hline 2 & 2.35 & 2.08 & 2.48 & 2.53 & 2.53 & 2.39 & 1.64 \\
\hline 4 & 1.16 & 1.31 & 1.38 & 1.37 & 1.22 & 1.29 & 3.04 \\
\hline 6 & 0.91 & 1.01 & 0.95 & 1.16 & 1.13 & 1.03 & 3.80 \\
\hline 8 & 0.84 & 1.09 & 0.89 & 1.13 & 1.04 & 1.00 & 3.93 \\
\hline 10 & 0.85 & 0.92 & 0.96 & 0.91 & 1.14 & 0.96 & 4.10 \\
\hline 12 & 1.06 & 1.08 & 1.08 & 1.03 & 1.12 & 1.07 & 3.65 \\
\hline
\end{tabular}




\subsection{Speedup data on Xeon Phi}

\subsubsection{Heisenberg Chain on Xeon Phi}

Table 6.10 Time and speedup of Chain $(N)$ model with $N=26$ on an Intel Xeon Phi coprocessor with KMP_THREAD_AFFINITY set to scatter

\begin{tabular}{|r|c|c|c|c|c|c|c|}
\hline \multicolumn{6}{|c|}{ Scatter Heisenberg Chain(26) (recorded time in seconds) } \\
\hline Thread & Data 1 & Data 2 & Data 3 & Data 4 & Data 5 & Average & Speedup \\
\hline 1 & - & - & - & - & - & - & - \\
\hline 10 & - & - & - & - & - & - & - \\
\hline 20 & - & - & - & - & - & - & - \\
\hline 30 & - & - & - & - & - & - & - \\
\hline 40 & - & - & - & - & - & - & - \\
\hline 50 & - & - & - & - & - & - & - \\
\hline 60 & - & - & - & - & - & - & - \\
\hline 70 & - & - & - & - & - & - & - \\
\hline 80 & - & - & - & - & - & - & - \\
\hline 90 & - & - & - & - & - & - & - \\
\hline 100 & - & - & - & - & - & - & - \\
\hline 110 & - & - & - & - & - & - & - \\
\hline 120 & - & - & - & - & - & - & - \\
\hline 130 & - & - & - & - & - & - & - \\
\hline 140 & - & - & - & - & - & - & - \\
\hline 150 & - & - & - & - & - & - & - \\
\hline 160 & - & - & - & - & - & - & - \\
\hline 170 & - & - & - & - & - & - & - \\
\hline 180 & - & - & - & - & - & - & - \\
\hline 190 & - & - & - & - & - & - & - \\
\hline 200 & - & - & - & - & - & - & - \\
\hline 210 & - & - & - & - & - & - & - \\
\hline 220 & - & - & - & - & - & - & - \\
\hline 230 & - & - & - & - & - & - & - \\
\hline 240 & - & - & - & - & - & - & - \\
\hline
\end{tabular}


Table 6.11 Time and speedup of Chain $(N)$ model with $N=24$ on an Intel Xeon Phi coprocessor with KMP_THREAD_AFFINITY set to scatter

\begin{tabular}{|c|c|c|c|c|c|c|c|}
\hline \multicolumn{8}{|c|}{ Scatter Heisenberg Chain(24) (recorded time in seconds) } \\
\hline Thread & Data 1 & Data 2 & Data 3 & Data 4 & Data 5 & Average & Speedup \\
\hline 1 & 1797.55 & 1798.52 & 1797.94 & 1797.59 & 1797.87 & 1797.89 & 1.00 \\
\hline 10 & 408.80 & 399.77 & 384.35 & 448.46 & 428.65 & 414.00 & 4.34 \\
\hline 20 & 236.42 & 252.82 & 231.32 & 260.13 & 262.17 & 248.57 & 7.23 \\
\hline 30 & 170.63 & 192.00 & 178.05 & 181.27 & 178.54 & 180.10 & 9.98 \\
\hline 40 & 138.15 & 139.45 & 133.77 & 134.82 & 131.20 & 135.48 & 13.27 \\
\hline 50 & 109.58 & 113.72 & 113.82 & 109.84 & 111.93 & 111.78 & 16.08 \\
\hline 60 & 97.33 & 96.03 & 90.53 & 96.13 & 95.72 & 95.14 & 18.90 \\
\hline 70 & 88.95 & 86.39 & 82.71 & 89.39 & 86.34 & 86.75 & 20.72 \\
\hline 80 & 76.29 & 75.00 & 74.04 & 74.72 & 77.29 & 75.47 & 23.82 \\
\hline 90 & 69.56 & 68.96 & 71.00 & 73.05 & 70.00 & 70.51 & 25.50 \\
\hline 100 & 66.41 & 63.45 & 68.11 & 67.01 & 63.22 & 65.64 & 27.39 \\
\hline 110 & 62.47 & 62.51 & 65.99 & 60.10 & 62.60 & 62.73 & 28.66 \\
\hline 120 & 60.72 & 59.27 & 59.91 & 60.06 & 60.07 & 60.01 & 29.96 \\
\hline 130 & 59.27 & 58.18 & 61.14 & 57.11 & 61.54 & 59.45 & 30.24 \\
\hline 140 & 56.53 & 57.06 & 54.90 & 58.87 & 59.90 & 57.45 & 31.29 \\
\hline 150 & 53.15 & 52.62 & 53.68 & 56.79 & 54.43 & 54.14 & 33.21 \\
\hline 160 & 51.47 & 52.55 & 50.36 & 55.08 & 58.34 & 53.56 & 33.57 \\
\hline 170 & 52.61 & 51.83 & 51.53 & 52.29 & 56.63 & 52.98 & 33.94 \\
\hline 180 & 48.93 & 48.64 & 50.17 & 48.99 & 53.07 & 49.96 & 35.99 \\
\hline 190 & 48.79 & 47.53 & 50.04 & 47.84 & 49.52 & 48.74 & 36.89 \\
\hline 200 & 48.00 & 46.30 & 48.64 & 46.73 & 48.98 & 47.73 & 37.67 \\
\hline 210 & 44.96 & 44.95 & 45.93 & 45.09 & 47.52 & 45.69 & 39.35 \\
\hline 220 & 43.63 & 43.12 & 43.07 & 44.67 & 46.79 & 44.26 & 40.63 \\
\hline 230 & 41.86 & 41.68 & 41.74 & 41.57 & 41.90 & 41.75 & 43.06 \\
\hline 240 & 41.40 & 41.43 & 41.52 & 41.52 & 41.43 & 41.46 & 43.36 \\
\hline
\end{tabular}


Table 6.12 Time and speedup of Chain $(N)$ model with $N=22$ on an Intel Xeon Phi coprocessor with KMP_THREAD_AFFINITY set to scatter

\begin{tabular}{|c|c|c|c|c|c|c|c|}
\hline \multicolumn{8}{|c|}{ Scatter Heisenberg Chain(22) (recorded time in seconds) } \\
\hline Thread & Data 1 & Data 2 & Data 3 & Data 4 & Data 5 & Average & Speedup \\
\hline 1 & 433.11 & 434.21 & 434.14 & 434.11 & 434.01 & 433.92 & 1.00 \\
\hline 10 & 111.12 & 95.31 & 94.57 & 99.04 & 111.58 & 102.32 & 4.24 \\
\hline 20 & 60.25 & 60.50 & 59.53 & 55.35 & 61.86 & $\mathbf{5 9 . 5 0}$ & 7.29 \\
\hline 30 & 42.34 & 47.07 & 42.29 & 40.78 & 42.61 & 43.02 & 10.09 \\
\hline 40 & 31.92 & 31.26 & 31.48 & 31.38 & 31.34 & 31.47 & 13.79 \\
\hline 50 & 26.28 & 26.51 & 26.03 & 26.09 & 26.00 & 26.18 & 16.57 \\
\hline 60 & 22.94 & 23.12 & 22.63 & 23.00 & 22.99 & 22.94 & 18.92 \\
\hline 70 & 21.45 & 21.14 & 21.10 & 21.26 & 21.20 & 21.23 & 20.44 \\
\hline 80 & 20.23 & 18.12 & 18.71 & 18.73 & 18.67 & 18.89 & 22.97 \\
\hline 90 & 18.46 & 18.21 & 17.58 & 17.66 & 17.64 & 17.91 & 24.23 \\
\hline 100 & 16.73 & 16.66 & 16.65 & 16.59 & 16.27 & 16.58 & 26.17 \\
\hline 110 & 15.59 & 15.56 & 15.51 & 15.52 & 15.25 & 15.49 & 28.02 \\
\hline 120 & 14.78 & 14.66 & 14.91 & 14.70 & 14.90 & 14.79 & 29.34 \\
\hline 130 & 14.90 & 14.43 & 14.55 & 14.38 & 14.61 & 14.58 & 29.77 \\
\hline 140 & 14.99 & 14.33 & 13.97 & 13.82 & 13.89 & 14.20 & 30.56 \\
\hline 150 & 17.25 & 16.71 & 17.40 & 16.63 & 16.71 & 16.94 & 25.61 \\
\hline 160 & 17.09 & 16.65 & 16.67 & 16.53 & 16.32 & 16.65 & 26.06 \\
\hline 170 & 16.99 & 16.55 & 17.15 & 16.26 & 16.27 & 16.65 & 26.07 \\
\hline 180 & 17.21 & 17.18 & 17.51 & 16.57 & 16.73 & 17.04 & 25.46 \\
\hline 190 & 16.95 & 16.86 & 18.06 & 16.73 & 16.99 & 17.12 & 25.35 \\
\hline 200 & 17.28 & 17.28 & 18.64 & 17.04 & 17.22 & 17.49 & 24.80 \\
\hline 210 & 17.53 & 17.37 & 19.08 & 17.27 & 17.49 & 17.75 & 24.45 \\
\hline 220 & 17.86 & 17.64 & 19.61 & 17.50 & 17.83 & 18.09 & 23.99 \\
\hline 230 & 18.06 & 17.78 & 19.95 & 17.60 & 18.03 & 18.28 & 23.73 \\
\hline 240 & 21.00 & 18.38 & 20.82 & 18.22 & 18.73 & 19.43 & 22.33 \\
\hline
\end{tabular}


Table 6.13 Time and speedup of Chain $(N)$ model with $N=20$ on an Intel Xeon Phi coprocessor with KMP_THREAD_AFFINITY set to scatter

\begin{tabular}{|c|c|c|c|c|c|c|c|}
\hline \multicolumn{8}{|c|}{ Scatter Heisenberg Chain(20) (recorded time in seconds) } \\
\hline Thread & Data 1 & Data 2 & Data 3 & Data 4 & Data 5 & Average & Speedup \\
\hline 1 & 108.24 & 108.40 & 108.28 & 108.27 & 108.29 & 108.30 & 1.00 \\
\hline 10 & 26.13 & 22.82 & 22.84 & 22.82 & 22.80 & 23.49 & 4.61 \\
\hline 20 & 14.89 & 14.69 & 14.73 & 14.16 & 14.13 & 14.52 & 7.46 \\
\hline 30 & 10.74 & 11.11 & 10.82 & 11.09 & 11.08 & 10.97 & 9.87 \\
\hline 40 & 9.99 & 9.96 & 10.07 & 9.93 & 9.88 & 9.97 & 10.87 \\
\hline 50 & 9.24 & 9.18 & 9.31 & 9.21 & 9.19 & 9.23 & 11.74 \\
\hline 60 & 8.87 & 8.85 & 8.71 & 8.88 & 8.83 & 8.83 & 12.27 \\
\hline 70 & 8.88 & 8.83 & 8.88 & 8.92 & 8.84 & 8.87 & 12.21 \\
\hline 80 & 8.66 & 8.62 & 8.76 & 8.74 & 8.61 & 8.68 & 12.48 \\
\hline 90 & 7.26 & 7.24 & 7.54 & 7.46 & 7.26 & 7.35 & 14.73 \\
\hline 100 & 7.75 & 7.57 & 7.92 & 7.78 & 7.78 & 7.76 & 13.95 \\
\hline 110 & 8.11 & 7.93 & 8.12 & 8.16 & 8.17 & 8.10 & 13.38 \\
\hline 120 & 8.47 & 8.39 & 8.43 & 8.46 & 8.48 & 8.45 & 12.82 \\
\hline 130 & 9.09 & 9.07 & 9.08 & 9.14 & 9.12 & 9.10 & 11.90 \\
\hline 140 & 9.52 & 9.57 & 9.64 & 9.73 & 9.56 & 9.60 & 11.28 \\
\hline 150 & 10.01 & 10.05 & 10.04 & 10.22 & 10.08 & 10.08 & 10.74 \\
\hline 160 & 10.49 & 10.44 & 10.46 & 10.57 & 10.68 & 10.53 & 10.29 \\
\hline 170 & 10.96 & 10.88 & 11.00 & 11.00 & 11.00 & 10.97 & 9.87 \\
\hline 180 & 11.36 & 11.45 & 11.46 & 11.40 & 11.40 & 11.41 & 9.49 \\
\hline 190 & 12.37 & 12.38 & 12.38 & 12.45 & 12.42 & 12.40 & 8.73 \\
\hline 200 & 12.80 & 12.84 & 12.84 & 12.86 & 12.82 & 12.83 & 8.44 \\
\hline 210 & 13.27 & 13.23 & 13.35 & 13.34 & 13.30 & 13.30 & 8.14 \\
\hline 220 & 13.67 & 13.66 & 13.72 & 13.74 & 13.73 & 13.70 & 7.90 \\
\hline 230 & 14.06 & 14.06 & 14.13 & 14.14 & 14.10 & 14.10 & 7.68 \\
\hline 240 & 14.86 & 14.54 & 14.77 & 14.77 & 14.68 & 14.72 & 7.36 \\
\hline
\end{tabular}


Table 6.14 Time and speedup of Chain $(N)$ model with $N=18$ on an Intel Xeon Phi coprocessor with KMP_THREAD_AFFINITY set to scatter

\begin{tabular}{|c|c|c|c|c|c|c|c|}
\hline \multicolumn{8}{|c|}{ Scatter Heisenberg Chain(18) (recorded time in seconds) } \\
\hline Thread & Data 1 & Data 2 & Data 3 & Data 4 & Data 5 & Average & Speedup \\
\hline 1 & 28.00 & 27.91 & 27.94 & 27.93 & 26.60 & 27.67 & 1.00 \\
\hline 10 & 7.08 & 7.55 & 7.04 & 7.04 & 7.05 & 7.15 & 3.87 \\
\hline 20 & 5.58 & 5.74 & 5.55 & 5.41 & 5.54 & 5.56 & 4.97 \\
\hline 30 & 3.87 & 4.04 & 3.94 & 3.97 & 3.95 & 3.95 & 7.00 \\
\hline 40 & 4.06 & 4.13 & 4.10 & 4.14 & 4.13 & 4.11 & 6.73 \\
\hline 50 & 4.46 & 4.43 & 4.46 & 4.53 & 4.51 & 4.48 & 6.18 \\
\hline 60 & 5.02 & 4.99 & 5.00 & 5.03 & 5.05 & 5.02 & 5.51 \\
\hline 70 & 5.65 & 5.63 & 5.66 & 5.70 & 5.68 & 5.66 & 4.89 \\
\hline 80 & 6.22 & 6.17 & 6.16 & 6.19 & 6.19 & 6.19 & 4.47 \\
\hline 90 & 6.54 & 6.68 & 6.70 & 6.74 & 6.70 & 6.67 & 4.15 \\
\hline 100 & 7.18 & 6.96 & 7.15 & 7.19 & 7.20 & 7.13 & 3.88 \\
\hline 110 & 7.65 & 7.64 & 7.65 & 7.68 & 7.70 & 7.66 & 3.61 \\
\hline 120 & 8.34 & 8.31 & 8.33 & 8.37 & 8.39 & 8.35 & 3.32 \\
\hline 130 & 9.11 & 9.06 & 9.06 & 9.09 & 9.11 & 9.09 & 3.05 \\
\hline 140 & 9.66 & 9.60 & 9.61 & 9.41 & 9.68 & 9.59 & 2.88 \\
\hline 150 & 10.15 & 10.12 & 10.14 & 10.14 & 10.15 & 10.14 & 2.73 \\
\hline 160 & 10.74 & 10.70 & 10.72 & 10.72 & 10.72 & 10.72 & 2.58 \\
\hline 170 & 11.31 & 11.24 & 11.22 & 11.26 & 11.25 & 11.26 & 2.46 \\
\hline 180 & 11.82 & 11.77 & 11.75 & 11.85 & 11.81 & 11.80 & 2.34 \\
\hline 190 & 12.65 & 12.57 & 12.56 & 12.59 & 12.61 & 12.60 & 2.20 \\
\hline 200 & 13.17 & 13.10 & 13.09 & 13.06 & 13.12 & 13.11 & 2.11 \\
\hline 210 & 13.74 & 13.65 & 13.62 & 13.63 & 13.67 & 13.66 & 2.03 \\
\hline 220 & 14.30 & 14.21 & 14.18 & 14.18 & 14.22 & 14.22 & 1.95 \\
\hline 230 & 14.80 & 14.71 & 14.67 & 14.66 & 14.71 & 14.71 & 1.88 \\
\hline 240 & 15.63 & 15.25 & 15.17 & 15.17 & 15.23 & 15.29 & 1.81 \\
\hline
\end{tabular}


Table 6.15 Time and speedup of Chain $(N)$ model with $N=16$ on an Intel Xeon Phi coprocessor with KMP_THREAD_AFFINITY set to scatter

\begin{tabular}{|c|c|c|c|c|c|c|c|}
\hline Thread & Data 1 & Data 2 & Data 3 & Data 4 & Data 5 & Average & Speedup \\
\hline 1 & 7.07 & 6.99 & 7.01 & 7.02 & 7.03 & 7.02 & 1.00 \\
\hline 10 & 3.07 & 2.93 & 3.01 & 2.94 & 2.94 & 2.98 & 2.36 \\
\hline 20 & 2.98 & 3.01 & 3.00 & 3.00 & 3.00 & 3.00 & 2.34 \\
\hline 30 & 3.38 & 3.38 & 3.42 & 3.40 & 3.38 & 3.39 & 2.07 \\
\hline 40 & 4.02 & 4.02 & 4.00 & 4.03 & 4.05 & 4.02 & 1.75 \\
\hline 50 & 4.88 & 4.89 & 4.89 & 4.90 & 4.90 & 4.89 & 1.44 \\
\hline 60 & 5.96 & 5.51 & 6.32 & 5.67 & 5.68 & 5.83 & 1.21 \\
\hline 70 & 6.78 & 6.76 & 7.24 & 6.79 & 6.81 & 6.87 & 1.02 \\
\hline 80 & 7.49 & 7.51 & 7.96 & 7.52 & 7.52 & 7.60 & 0.92 \\
\hline 90 & 8.33 & 8.32 & 8.87 & 8.35 & 7.96 & 8.36 & 0.84 \\
\hline 100 & 8.83 & 9.04 & 9.60 & 9.06 & 9.06 & 9.12 & 0.77 \\
\hline 110 & 9.83 & 9.82 & 9.83 & 9.84 & 9.87 & 9.84 & 0.71 \\
\hline 120 & 10.64 & 10.62 & 10.62 & 10.66 & 10.67 & 10.64 & 0.66 \\
\hline 130 & 11.55 & 11.53 & 11.53 & 11.55 & 11.04 & 11.44 & 0.61 \\
\hline 140 & 12.02 & 12.28 & 12.29 & 12.30 & 12.32 & 12.24 & 0.57 \\
\hline 150 & 13.04 & 13.01 & 12.72 & 13.05 & 13.05 & 12.97 & 0.54 \\
\hline 160 & 13.78 & 13.76 & 13.76 & 13.80 & 13.79 & 13.78 & 0.51 \\
\hline 170 & 14.50 & 14.51 & 14.49 & 14.53 & 14.54 & 14.51 & 0.48 \\
\hline 180 & 15.21 & 14.55 & 14.88 & 14.93 & 15.26 & 14.96 & 0.47 \\
\hline 190 & 16.17 & 16.15 & 16.13 & 16.20 & 15.13 & 15.96 & 0.44 \\
\hline 200 & 16.89 & 16.89 & 16.50 & 16.93 & 16.93 & 16.83 & 0.42 \\
\hline 210 & 17.64 & 17.65 & 17.63 & 17.68 & 17.69 & 17.66 & 0.40 \\
\hline 220 & 18.40 & 18.01 & 18.41 & 18.44 & 18.44 & 18.34 & 0.38 \\
\hline 230 & 19.12 & 19.14 & 19.14 & 19.16 & 19.16 & 19.15 & 0.37 \\
\hline 240 & 20.35 & 19.88 & 19.88 & 19.90 & 19.91 & 19.98 & 0.35 \\
\hline
\end{tabular}


Table 6.16 Time and speedup of Chain $(N)$ model with $N=26$ on an Intel Xeon Phi coprocessor with KMP_THREAD_AFFINITY set to compact

\begin{tabular}{|c|c|c|c|c|c|c|c|}
\hline \multicolumn{8}{|c|}{ Compact Heisenberg Chain(26) (recorded time in seconds) } \\
\hline Thread & Data 1 & Data 2 & Data 3 & Data 4 & Data 5 & Average & Speedup \\
\hline 1 & - & - & - & - & - & - & - \\
\hline 10 & - & - & - & - & - & - & - \\
\hline 20 & - & - & - & - & - & - & - \\
\hline 30 & - & - & - & - & - & - & - \\
\hline 40 & - & - & - & - & - & - & - \\
\hline 50 & - & - & - & - & - & - & - \\
\hline 60 & - & - & - & - & - & - & - \\
\hline 70 & - & - & - & - & - & - & - \\
\hline 80 & - & - & - & - & - & - & - \\
\hline 90 & - & - & - & - & - & - & - \\
\hline 100 & - & - & - & - & - & - & - \\
\hline 110 & - & - & - & - & - & - & - \\
\hline 120 & - & - & - & - & - & - & - \\
\hline 130 & - & - & - & - & - & - & - \\
\hline 140 & - & - & - & - & - & - & - \\
\hline 150 & - & - & - & - & - & - & - \\
\hline 160 & - & - & - & - & - & - & - \\
\hline 170 & - & - & - & - & - & - & - \\
\hline 180 & - & - & - & - & - & - & - \\
\hline 190 & - & - & - & - & - & - & - \\
\hline 200 & - & - & - & - & - & - & - \\
\hline 210 & - & - & - & - & - & - & - \\
\hline 220 & - & - & - & - & - & - & - \\
\hline 230 & - & - & - & - & - & - & - \\
\hline 240 & - & - & - & - & - & - & - \\
\hline
\end{tabular}


Table 6.17 Time and speedup of Chain $(N)$ model with $N=24$ on an Intel Xeon Phi coprocessor with KMP_THREAD_AFFINITY set to compact

\begin{tabular}{|c|c|c|c|c|c|c|c|}
\hline \multicolumn{8}{|c|}{ Compact Heisenberg Chain(24) (recorded time in seconds) } \\
\hline Thread & Data 1 & Data 2 & Data 3 & Data 4 & Data 5 & Average & Speedup \\
\hline 1 & 1797.55 & 1798.52 & 1797.94 & 1797.59 & 1797.87 & $\mathbf{1 7 9 7 . 8 9}$ & 1.00 \\
\hline 10 & 575.70 & 587.43 & 577.94 & 573.34 & 573.34 & $\mathbf{5 7 7 . 5 5}$ & 3.11 \\
\hline 20 & 304.72 & 302.29 & 302.37 & 302.55 & 302.55 & 302.90 & 5.94 \\
\hline 30 & 211.64 & 207.18 & 207.83 & 207.27 & 207.27 & 208.23 & 8.63 \\
\hline 40 & 159.52 & 159.40 & 159.21 & 159.44 & 159.44 & 159.40 & 11.28 \\
\hline 50 & 129.09 & 129.10 & 128.99 & 129.05 & 129.05 & 129.06 & 13.93 \\
\hline 60 & 111.45 & 111.44 & 111.39 & 111.37 & 111.37 & 111.40 & 16.14 \\
\hline 70 & 97.13 & 97.15 & 97.14 & 97.08 & 97.08 & 97.12 & 18.51 \\
\hline 80 & 85.09 & 85.12 & 85.07 & 85.14 & 85.14 & 85.11 & 21.12 \\
\hline 90 & 78.55 & 78.55 & 78.53 & 78.49 & 78.49 & 78.52 & 22.90 \\
\hline 100 & 71.88 & 71.91 & 71.82 & 71.85 & 71.85 & 71.86 & 25.02 \\
\hline 110 & 66.87 & 66.91 & 66.82 & 66.84 & 66.84 & 66.86 & 26.89 \\
\hline 120 & 62.93 & 62.97 & 62.92 & 62.87 & 62.87 & 62.91 & 28.58 \\
\hline 130 & 59.44 & 59.53 & 59.36 & 59.39 & 59.39 & 59.42 & 30.26 \\
\hline 140 & 56.40 & 56.47 & 56.38 & 56.33 & 56.33 & 56.38 & 31.89 \\
\hline 150 & 53.52 & 53.61 & 53.46 & 53.54 & 53.54 & 53.53 & 33.59 \\
\hline 160 & 50.49 & 50.55 & 50.44 & 50.46 & 50.46 & 50.48 & 35.62 \\
\hline 170 & 50.83 & 50.80 & 50.62 & 50.56 & 50.56 & 50.67 & 35.48 \\
\hline 180 & 46.65 & 46.74 & 46.58 & 46.54 & 46.54 & 46.61 & 38.57 \\
\hline 190 & 44.46 & 44.55 & 44.45 & 44.41 & 44.41 & 44.45 & 40.44 \\
\hline 200 & 43.31 & 43.41 & 43.26 & 43.23 & 43.23 & 43.29 & 41.53 \\
\hline 210 & 42.15 & 42.26 & 42.09 & 42.08 & 42.08 & 42.13 & 42.67 \\
\hline 220 & 41.64 & 41.76 & 41.61 & 41.57 & 41.57 & 41.63 & 43.18 \\
\hline 230 & 40.08 & 40.18 & 39.98 & 40.00 & 40.00 & 40.05 & 44.89 \\
\hline 240 & 39.19 & 39.22 & 39.15 & 39.08 & 39.08 & 39.14 & 45.93 \\
\hline
\end{tabular}


Table 6.18 Time and speedup of Chain $(N)$ model with $N=22$ on an Intel Xeon Phi coprocessor with KMP_THREAD_AFFINITY set to compact

\begin{tabular}{|c|c|c|c|c|c|c|c|}
\hline \multicolumn{8}{|c|}{ Compact Heisenberg Chain(22) (recorded time in seconds) } \\
\hline Thread & Data 1 & Data 2 & Data 3 & Data 4 & Data 5 & Average & Speedup \\
\hline 1 & 433.11 & 434.21 & 434.14 & 434.11 & 434.01 & 433.92 & 1.00 \\
\hline 10 & 139.61 & 139.40 & 139.32 & 140.05 & 139.36 & 139.55 & 3.11 \\
\hline 20 & 76.15 & 76.15 & 76.07 & 76.19 & 76.00 & 76.11 & 5.70 \\
\hline 30 & 53.50 & 53.51 & 53.46 & 53.48 & 53.34 & 53.46 & 8.12 \\
\hline 40 & 41.50 & 41.44 & 41.47 & 41.41 & 41.43 & 41.45 & 10.47 \\
\hline 50 & 34.65 & 34.58 & 34.57 & 34.60 & 34.59 & 34.60 & 12.54 \\
\hline 60 & 29.85 & 29.80 & 29.83 & 29.80 & 29.75 & 29.81 & 14.56 \\
\hline 70 & 26.22 & 26.17 & 26.22 & 26.25 & 26.11 & 26.19 & 16.57 \\
\hline 80 & 22.64 & 22.61 & 22.71 & 22.65 & 22.58 & 22.64 & 19.17 \\
\hline 90 & 20.70 & 20.74 & 20.80 & 20.68 & 20.61 & 20.71 & 20.96 \\
\hline 100 & 18.46 & 18.52 & 18.55 & 18.46 & 18.43 & 18.48 & 23.48 \\
\hline 110 & 16.93 & 16.95 & 17.07 & 16.90 & 16.88 & 16.94 & 25.61 \\
\hline 120 & 15.78 & 15.86 & 15.97 & 15.86 & 15.77 & 15.85 & 27.38 \\
\hline 130 & 15.01 & 15.04 & 15.16 & 15.01 & 14.95 & 15.04 & 28.86 \\
\hline 140 & 14.17 & 14.24 & 14.34 & 14.17 & 14.12 & 14.21 & 30.53 \\
\hline 150 & 16.53 & 16.54 & 16.76 & 16.60 & 16.54 & 16.59 & 26.15 \\
\hline 160 & 16.39 & 16.32 & 16.67 & 16.53 & 16.40 & 16.46 & 26.36 \\
\hline 170 & 16.71 & 16.63 & 17.05 & 16.93 & 16.74 & 16.81 & 25.81 \\
\hline 180 & 16.82 & 16.61 & 17.14 & 17.02 & 16.84 & 16.88 & 25.70 \\
\hline 190 & 16.97 & 16.60 & 17.32 & 17.31 & 16.87 & 17.01 & 25.50 \\
\hline 200 & 17.46 & 16.84 & 17.84 & 17.86 & 17.29 & 17.46 & 24.85 \\
\hline 210 & 18.00 & 17.14 & 18.35 & 18.38 & 17.85 & 17.94 & 24.18 \\
\hline 220 & 18.61 & 17.56 & 19.05 & 19.19 & 18.38 & 18.56 & 23.38 \\
\hline 230 & 19.32 & 18.10 & 19.83 & 19.97 & 19.09 & 19.26 & 22.52 \\
\hline 240 & 20.19 & 18.63 & 20.76 & 20.85 & 19.90 & 20.06 & 21.63 \\
\hline
\end{tabular}


Table 6.19 Time and speedup of Chain $(N)$ model with $N=20$ on an Intel Xeon Phi coprocessor with KMP_THREAD_AFFINITY set to compact

\begin{tabular}{|c|c|c|c|c|c|c|c|}
\hline \multicolumn{8}{|c|}{ Compact Heisenberg Chain(20) (recorded time in seconds) } \\
\hline Thread & Data 1 & Data 2 & Data 3 & Data 4 & Data 5 & Average & Speedup \\
\hline 1 & 108.24 & 108.40 & 108.28 & 108.27 & 108.29 & 108.30 & 1.00 \\
\hline 10 & 36.33 & 36.35 & 36.35 & 36.25 & 36.25 & 36.31 & 2.98 \\
\hline 20 & 20.51 & 20.51 & 20.51 & 20.41 & 20.40 & 20.47 & 5.29 \\
\hline 30 & 13.71 & 13.72 & 13.74 & 13.69 & 13.66 & 13.71 & 7.90 \\
\hline 40 & 12.00 & 12.01 & 12.01 & 11.96 & 11.94 & 11.98 & 9.04 \\
\hline 50 & 11.05 & 11.05 & 11.06 & 11.01 & 11.01 & 11.04 & 9.81 \\
\hline 60 & 10.59 & 10.57 & 10.57 & 10.55 & 10.54 & 10.56 & 10.25 \\
\hline 70 & 10.31 & 10.28 & 10.33 & 10.27 & 10.24 & 10.28 & 10.53 \\
\hline 80 & 9.97 & 9.96 & 9.98 & 9.92 & 9.93 & 9.95 & 10.88 \\
\hline 90 & 9.00 & 8.97 & 9.02 & 9.01 & 9.01 & 9.00 & 12.03 \\
\hline 100 & 9.32 & 9.33 & 9.32 & 9.34 & 9.33 & 9.33 & 11.61 \\
\hline 110 & 9.59 & 9.57 & 9.63 & 9.62 & 9.63 & 9.61 & 11.27 \\
\hline 120 & 9.99 & 10.00 & 9.99 & 10.00 & 10.00 & 10.00 & 10.83 \\
\hline 130 & 10.40 & 10.38 & 10.39 & 10.38 & 10.40 & 10.39 & 10.42 \\
\hline 140 & 10.83 & 10.81 & 10.83 & 10.80 & 10.79 & 10.81 & 10.02 \\
\hline 150 & 11.24 & 11.21 & 11.21 & 11.19 & 11.21 & 11.21 & 9.66 \\
\hline 160 & 11.64 & 11.63 & 11.64 & 11.61 & 11.63 & 11.63 & 9.31 \\
\hline 170 & 12.04 & 12.08 & 12.08 & 12.10 & 12.07 & 12.07 & 8.97 \\
\hline 180 & 12.50 & 12.53 & 12.54 & 12.56 & 12.52 & 12.53 & 8.64 \\
\hline 190 & 12.98 & 12.99 & 12.98 & 13.01 & 12.99 & 12.99 & 8.34 \\
\hline 200 & 13.46 & 13.48 & 13.48 & 13.47 & 13.45 & 13.47 & 8.04 \\
\hline 210 & 13.99 & 13.99 & 13.98 & 13.98 & 13.99 & 13.99 & 7.74 \\
\hline 220 & 14.47 & 14.46 & 14.46 & 14.47 & 14.46 & 14.47 & 7.49 \\
\hline 230 & 15.00 & 14.99 & 15.00 & 14.99 & 15.00 & 15.00 & 7.22 \\
\hline 240 & 15.81 & 15.51 & 15.54 & 15.52 & 15.50 & 15.58 & 6.95 \\
\hline
\end{tabular}


Table 6.20 Time and speedup of Chain $(N)$ model with $N=18$ on an Intel Xeon Phi coprocessor with KMP_THREAD_AFFINITY set to compact

\begin{tabular}{|r|r|r|r|r|r|r|r|}
\hline \multicolumn{7}{|c|}{ Compact Heisenberg Chain(18) (recorded time in seconds) } \\
\hline Thread & \multicolumn{1}{|c|}{ Data 1 } & Data 2 & \multicolumn{1}{c|}{ Data 3 } & Data 4 & Data 5 & \multicolumn{1}{c|}{ Average } & Speedup \\
\hline 1 & 28.00 & 27.91 & 27.94 & 27.93 & 26.60 & $\mathbf{2 6 . 6 0}$ & 1.00 \\
\hline 10 & 10.02 & 10.04 & 10.02 & 10.01 & 9.99 & $\mathbf{1 0 . 0 2}$ & 2.66 \\
\hline 20 & 7.65 & 7.65 & 7.66 & 7.63 & 7.63 & $\mathbf{7 . 6 4}$ & 3.48 \\
\hline 30 & 6.12 & 6.33 & 6.32 & 6.32 & 6.30 & $\mathbf{6 . 2 8}$ & 4.23 \\
\hline 40 & 6.33 & 6.30 & 6.34 & 6.32 & 6.35 & $\mathbf{6 . 3 3}$ & 4.20 \\
\hline 50 & 6.50 & 6.54 & 6.56 & 6.53 & 6.53 & $\mathbf{6 . 5 3}$ & 4.07 \\
\hline 60 & 6.80 & 6.82 & 6.79 & 6.81 & 6.80 & $\mathbf{6 . 8 0}$ & 3.91 \\
\hline 70 & 7.16 & 7.17 & 7.18 & 6.94 & 7.17 & $\mathbf{7 . 1 3}$ & 3.73 \\
\hline 80 & 7.55 & 7.55 & 7.52 & 7.56 & 7.57 & $\mathbf{7 . 5 5}$ & 3.52 \\
\hline 90 & 8.02 & 8.00 & 7.95 & 8.00 & 8.02 & $\mathbf{8 . 0 0}$ & 3.33 \\
\hline 100 & 8.38 & 8.38 & 8.38 & 8.41 & 8.15 & $\mathbf{8 . 3 4}$ & 3.19 \\
\hline 110 & 8.80 & 8.82 & 8.55 & 8.83 & 8.85 & $\mathbf{8 . 7 7}$ & 3.03 \\
\hline 120 & 9.37 & 9.36 & 9.34 & 9.39 & 9.40 & $\mathbf{9 . 3 7}$ & 2.84 \\
\hline 130 & 9.88 & 9.87 & 9.89 & 9.88 & 9.90 & $\mathbf{9 . 8 8}$ & 2.69 \\
\hline 140 & 10.34 & 10.35 & 10.35 & 10.36 & 10.35 & $\mathbf{1 0 . 3 5}$ & 2.57 \\
\hline 150 & 10.80 & 10.23 & 10.78 & 10.85 & 10.84 & $\mathbf{1 0 . 7 0}$ & 2.49 \\
\hline 160 & 11.33 & 11.34 & 11.32 & 11.34 & 11.35 & $\mathbf{1 1 . 3 4}$ & 2.35 \\
\hline 170 & 11.87 & 11.84 & 11.86 & 11.87 & 11.87 & $\mathbf{1 1 . 8 6}$ & 2.24 \\
\hline 180 & 12.43 & 12.36 & 12.40 & 12.39 & 12.39 & $\mathbf{1 2 . 3 9}$ & 2.15 \\
\hline 190 & 12.89 & 12.88 & 12.91 & 12.87 & 12.89 & $\mathbf{1 2 . 8 9}$ & 2.06 \\
\hline 200 & 13.44 & 13.40 & 13.41 & 13.40 & 13.43 & $\mathbf{1 3 . 4 2}$ & 1.98 \\
\hline 210 & 13.97 & 13.99 & 14.00 & 13.95 & 13.97 & $\mathbf{1 3 . 9 8}$ & 1.90 \\
\hline 220 & 14.51 & 14.48 & 14.18 & 14.51 & 14.53 & $\mathbf{1 4 . 4 4}$ & 1.84 \\
\hline 230 & 15.07 & 15.08 & 15.10 & 15.07 & 15.08 & $\mathbf{1 5 . 0 8}$ & 1.76 \\
\hline 240 & 15.94 & 15.62 & 15.60 & 15.60 & 15.65 & $\mathbf{1 5 . 6 8}$ & 1.70 \\
\hline
\end{tabular}


Table 6.21 Time and speedup of Chain $(N)$ model with $N=16$ on an Intel Xeon Phi coprocessor with KMP_THREAD_AFFINITY set to compact

\begin{tabular}{|c|c|c|c|c|c|c|c|}
\hline \multicolumn{8}{|c|}{ Compact Heisenberg Chain(16) (recorded time in seconds) } \\
\hline Thread & Data 1 & Data 2 & Data 3 & Data 4 & Data 5 & Average & Speedup \\
\hline 1 & 7.07 & 6.99 & 7.01 & 7.02 & 7.03 & 7.02 & 1.00 \\
\hline 10 & 4.01 & 3.99 & 3.90 & 4.04 & 3.99 & 3.98 & 1.76 \\
\hline 20 & 3.79 & 3.65 & 3.77 & 3.77 & 3.72 & 3.74 & 1.88 \\
\hline 30 & 4.00 & 3.97 & 4.00 & 4.00 & 3.97 & 3.99 & 1.76 \\
\hline 40 & 4.34 & 4.32 & 4.32 & 4.35 & 4.32 & 4.33 & 1.62 \\
\hline 50 & 4.71 & 4.69 & 4.74 & 4.81 & 4.78 & 4.75 & 1.48 \\
\hline 60 & 5.12 & 5.16 & 5.14 & 5.23 & 5.22 & 5.17 & 1.36 \\
\hline 70 & 5.39 & 5.52 & 5.53 & 5.81 & 5.77 & 5.61 & 1.25 \\
\hline 80 & 5.83 & 5.98 & 5.99 & 6.32 & 6.27 & 6.08 & 1.16 \\
\hline 90 & 6.43 & 6.44 & 6.26 & 6.84 & 6.83 & 6.56 & 1.07 \\
\hline 100 & 6.90 & 6.88 & 6.91 & 7.32 & 7.30 & 7.06 & 0.99 \\
\hline 110 & 7.39 & 7.35 & 7.39 & 7.87 & 7.86 & 7.57 & 0.93 \\
\hline 120 & 7.66 & 7.86 & 7.47 & 8.43 & 8.41 & 7.97 & 0.88 \\
\hline 130 & 8.38 & 8.37 & 7.74 & 9.12 & 9.04 & 8.53 & 0.82 \\
\hline 140 & 8.88 & 8.88 & 8.87 & 9.71 & 9.67 & 9.20 & 0.76 \\
\hline 150 & 9.42 & 9.39 & 9.39 & 9.89 & 10.35 & 9.69 & 0.73 \\
\hline 160 & 9.92 & 9.90 & 9.90 & 11.04 & 11.05 & 10.36 & 0.68 \\
\hline 170 & 10.41 & 10.41 & 10.40 & 11.80 & 11.79 & 10.96 & 0.64 \\
\hline 180 & 10.96 & 10.96 & 10.97 & 12.51 & 12.51 & 11.58 & 0.61 \\
\hline 190 & 11.51 & 11.48 & 11.50 & 13.34 & 13.04 & 12.17 & 0.58 \\
\hline 200 & 11.49 & 11.72 & 12.01 & 14.13 & 14.14 & 12.70 & 0.55 \\
\hline 210 & 12.60 & 12.60 & 12.56 & 15.05 & 15.05 & 13.57 & 0.52 \\
\hline 220 & 13.12 & 13.10 & 13.09 & 15.96 & 15.24 & 14.10 & 0.50 \\
\hline 230 & 13.61 & 13.69 & 13.65 & 17.10 & 17.06 & 15.02 & 0.47 \\
\hline 240 & 14.54 & 14.19 & 14.44 & 18.32 & 17.98 & 15.90 & 0.44 \\
\hline
\end{tabular}


Table 6.22 Time and speedup of Chain $(N)$ model with $N=26$ on an Intel Xeon Phi coprocessor with KMP_THREAD_AFFINITY set to ba lanced

\begin{tabular}{|c|c|c|c|c|c|c|c|}
\hline Balan & ced Hei & berg $\mathrm{Ch}$ & 26) (recc & ded time & second & & \\
\hline Thread & Data 1 & Data 2 & Data 3 & Data 4 & Data 5 & Average & Speedup \\
\hline 1 & - & - & - & - & - & - & - \\
\hline 10 & - & - & - & - & - & - & - \\
\hline 20 & - & - & - & - & - & - & - \\
\hline 30 & - & - & - & - & - & - & - \\
\hline 40 & - & - & - & - & - & - & - \\
\hline 50 & - & - & - & - & - & - & - \\
\hline 60 & - & - & - & - & - & - & - \\
\hline 70 & - & - & - & - & - & - & - \\
\hline 80 & - & - & - & - & - & - & - \\
\hline 90 & - & - & - & - & - & - & - \\
\hline 100 & - & - & - & - & - & - & - \\
\hline 110 & - & - & - & - & - & - & - \\
\hline 120 & - & - & - & - & - & - & - \\
\hline 130 & - & - & - & - & - & - & - \\
\hline 140 & - & - & - & - & - & - & - \\
\hline 150 & - & - & - & - & - & - & - \\
\hline 160 & - & - & - & - & - & - & - \\
\hline 170 & - & - & - & - & - & - & - \\
\hline 180 & - & - & - & - & - & - & - \\
\hline 190 & - & - & - & - & - & - & - \\
\hline 200 & - & - & - & - & - & - & - \\
\hline 210 & - & - & - & - & - & - & - \\
\hline 220 & - & - & - & - & - & - & - \\
\hline 230 & - & - & - & - & - & - & - \\
\hline 240 & - & - & - & - & - & - & - \\
\hline
\end{tabular}


Table 6.23 Time and speedup of Chain $(N)$ model with $N=24$ on an Intel Xeon Phi coprocessor with KMP_THREAD_AFFINITY set to balanced

\begin{tabular}{|c|c|c|c|c|c|c|c|}
\hline Thread & Data 1 & Data 2 & Data 3 & Data 4 & Data 5 & Average & Speedup \\
\hline 1 & 1797.55 & 1798.52 & 1797.94 & 1797.59 & 1797.87 & 1797.89 & 1.00 \\
\hline 10 & 574.70 & 445.37 & 377.58 & 408.04 & 387.16 & 438.57 & 4.10 \\
\hline 20 & 302.79 & 238.96 & 254.07 & 259.27 & 256.06 & 262.23 & 6.86 \\
\hline 30 & 207.42 & 190.01 & 183.26 & 178.95 & 180.32 & 187.99 & 9.56 \\
\hline 40 & 159.36 & 144.71 & 140.65 & 140.03 & 141.76 & 145.30 & 12.37 \\
\hline 50 & 129.12 & 109.01 & 112.21 & 114.96 & 112.64 & 115.59 & 15.55 \\
\hline 60 & 111.44 & 94.13 & 95.71 & 97.17 & 97.84 & 99.26 & 18.11 \\
\hline 70 & 97.07 & 87.01 & 85.61 & 87.73 & 88.77 & 89.24 & 20.15 \\
\hline 80 & 85.05 & 75.88 & 78.55 & 81.46 & 75.97 & 79.38 & 22.65 \\
\hline 90 & 78.51 & 75.99 & 75.46 & 69.21 & 70.14 & 73.86 & 24.34 \\
\hline 100 & 71.80 & 70.11 & 70.04 & 70.79 & 66.69 & 69.89 & 25.73 \\
\hline 110 & 66.85 & 65.09 & 64.30 & 64.77 & 65.13 & 65.23 & 27.56 \\
\hline 120 & 62.89 & 61.15 & 60.63 & 61.38 & 61.28 & 61.47 & 29.25 \\
\hline 130 & 59.37 & 58.98 & 59.04 & 59.89 & 59.55 & 59.37 & 30.28 \\
\hline 140 & 56.33 & 55.92 & 56.53 & 56.90 & 57.22 & 56.58 & 31.78 \\
\hline 150 & 53.54 & 53.95 & 52.81 & 54.06 & 55.04 & 53.88 & 33.37 \\
\hline 160 & 50.35 & 50.75 & 51.49 & 51.71 & 51.89 & 51.24 & 35.09 \\
\hline 170 & 50.67 & 49.92 & 51.32 & 51.13 & 51.04 & 50.82 & 35.38 \\
\hline 180 & 46.54 & 48.32 & 47.98 & 47.95 & 47.93 & 47.74 & 37.66 \\
\hline 190 & 44.33 & 45.66 & 47.68 & 47.66 & 47.36 & 46.54 & 38.63 \\
\hline 200 & 43.25 & 44.73 & 45.13 & 44.61 & 45.01 & 44.55 & 40.36 \\
\hline 210 & 42.07 & 43.57 & 43.30 & 43.86 & 42.66 & 43.09 & 41.72 \\
\hline 220 & 41.72 & 43.14 & 43.48 & 43.17 & 43.06 & 42.91 & 41.90 \\
\hline 230 & 40.00 & 39.86 & 40.07 & 40.11 & 39.88 & 39.99 & 44.96 \\
\hline 240 & 39.11 & 39.12 & 39.04 & 39.20 & 39.01 & 39.10 & 45.99 \\
\hline
\end{tabular}


Table 6.24 Time and speedup of Chain $(N)$ model with $N=22$ on an Intel Xeon Phi coprocessor with KMP_THREAD_AFFINITY set to bal anced

\begin{tabular}{|c|c|c|c|c|c|c|c|}
\hline \multicolumn{8}{|c|}{ Balanced Heisenberg Chain(22) (recorded time in seconds) } \\
\hline Thread & Data 1 & Data 2 & Data 3 & Data 4 & Data 5 & Average & Speedup \\
\hline 1 & 433.11 & 434.21 & 434.14 & 434.11 & 434.01 & 433.92 & 1.00 \\
\hline 10 & 124.48 & 121.27 & 131.55 & 127.16 & 119.93 & 124.88 & 3.47 \\
\hline 20 & 68.84 & 75.78 & 78.13 & 76.67 & 73.68 & 74.62 & 5.82 \\
\hline 30 & 54.59 & 53.60 & 55.13 & 54.42 & 53.51 & 54.25 & 8.00 \\
\hline 40 & 41.42 & 39.47 & 41.24 & 40.88 & 40.26 & 40.65 & 10.67 \\
\hline 50 & 34.28 & 33.11 & 33.34 & 33.75 & 33.61 & 33.62 & 12.91 \\
\hline 60 & 29.46 & 28.81 & 29.55 & 29.78 & 29.41 & 29.40 & 14.76 \\
\hline 70 & 26.44 & 27.75 & 27.55 & 26.56 & 26.40 & 26.94 & 16.11 \\
\hline 80 & 21.04 & 24.07 & 23.12 & 23.30 & 23.15 & 22.94 & 18.92 \\
\hline 90 & 21.69 & 23.27 & 21.65 & 21.88 & 21.70 & 22.04 & 19.69 \\
\hline 100 & 20.04 & 21.56 & 20.94 & 19.23 & 19.90 & 20.33 & 21.34 \\
\hline 110 & 18.58 & 19.86 & 19.32 & 19.42 & 19.50 & 19.34 & 22.44 \\
\hline 120 & 18.34 & 18.48 & 18.00 & 18.28 & 18.46 & 18.31 & 23.70 \\
\hline 130 & 17.82 & 17.84 & 17.55 & 17.59 & 17.75 & 17.71 & 24.50 \\
\hline 140 & 16.63 & 16.72 & 16.81 & 16.79 & 16.89 & 16.77 & 25.88 \\
\hline 150 & 63.99 & 63.97 & 63.89 & 63.66 & 63.57 & 63.82 & 6.80 \\
\hline 160 & 70.50 & 70.35 & 70.98 & 70.86 & 69.90 & 70.52 & 6.15 \\
\hline 170 & 79.78 & 80.28 & 80.09 & 79.96 & 79.65 & 79.95 & 5.43 \\
\hline 180 & 91.59 & 90.93 & 90.74 & 91.24 & 91.67 & 91.23 & 4.76 \\
\hline 190 & 91.01 & 91.31 & 89.98 & 90.08 & 90.64 & 90.60 & 4.79 \\
\hline 200 & 113.74 & 113.06 & 112.10 & 112.97 & 112.55 & 112.88 & 3.84 \\
\hline 210 & 122.83 & 122.59 & 122.96 & 122.95 & 122.36 & 122.74 & 3.54 \\
\hline 220 & 115.38 & 115.92 & 115.48 & 115.85 & 115.30 & 115.59 & 3.75 \\
\hline 230 & 129.02 & 128.81 & 129.01 & 129.39 & 129.74 & 129.19 & 3.36 \\
\hline 240 & 141.84 & 140.37 & 141.19 & 140.81 & 140.96 & 141.03 & 3.08 \\
\hline
\end{tabular}


Table 6.25 Time and speedup of Chain $(N)$ model with $N=20$ on an Intel Xeon Phi coprocessor with KMP_THREAD_AFFINITY set to balanced

\begin{tabular}{|c|c|c|c|c|c|c|c|}
\hline \multicolumn{8}{|c|}{ Balanced Heisenberg Chain(20) (recorded time in seconds) } \\
\hline Thread & Data 1 & Data 2 & Data 3 & Data 4 & Data 5 & Average & Speedup \\
\hline 1 & 108.24 & 108.40 & 108.28 & 108.27 & 108.29 & 108.30 & 1.00 \\
\hline 10 & 7.81 & 7.81 & 7.83 & 7.98 & 7.86 & 7.86 & 13.78 \\
\hline 20 & 6.74 & 6.73 & 6.73 & 6.91 & 6.86 & 6.79 & 15.94 \\
\hline 30 & 5.40 & 5.40 & 5.28 & 5.46 & 5.37 & 5.38 & 20.13 \\
\hline 40 & 6.44 & 6.45 & 6.44 & 6.53 & 6.41 & 6.45 & 16.79 \\
\hline 50 & 8.06 & 8.12 & 8.04 & 8.12 & 8.08 & 8.09 & 13.39 \\
\hline 60 & 10.34 & 10.35 & 10.14 & 10.44 & 10.40 & 10.33 & 10.48 \\
\hline 70 & 15.64 & 15.61 & 15.67 & 15.83 & 15.72 & 15.69 & 6.90 \\
\hline 80 & 16.90 & 16.90 & 16.84 & 17.00 & 17.32 & 16.99 & 6.37 \\
\hline 90 & 18.53 & 18.80 & 18.54 & 18.46 & 18.60 & 18.59 & 5.83 \\
\hline 100 & 20.20 & 19.91 & 19.99 & 20.27 & 19.55 & 19.98 & 5.42 \\
\hline 110 & 22.20 & 22.23 & 22.19 & 22.31 & 22.23 & 22.23 & 4.87 \\
\hline 120 & 23.48 & 23.47 & 22.91 & 23.76 & 23.65 & 23.45 & 4.62 \\
\hline 130 & 25.94 & 26.12 & 26.14 & 25.92 & 26.07 & 26.04 & 4.16 \\
\hline 140 & 28.33 & 27.94 & 28.24 & 28.07 & 28.09 & 28.14 & 3.85 \\
\hline 150 & 29.86 & 29.43 & 30.04 & 29.86 & 29.64 & 29.77 & 3.64 \\
\hline 160 & 32.00 & 31.94 & 31.96 & 31.23 & 31.76 & 31.78 & 3.41 \\
\hline 170 & 33.57 & 33.56 & 33.59 & 33.81 & 34.01 & 33.71 & 3.21 \\
\hline 180 & 35.60 & 35.61 & 35.74 & 35.62 & 35.34 & 35.58 & 3.04 \\
\hline 190 & 36.16 & 36.07 & 36.26 & 36.05 & 35.33 & 35.97 & 3.01 \\
\hline 200 & 37.96 & 38.21 & 38.02 & 37.81 & 38.22 & 38.04 & 2.85 \\
\hline 210 & 40.27 & 40.53 & 40.44 & 40.25 & 40.41 & 40.38 & 2.68 \\
\hline 220 & 43.28 & 43.09 & 42.61 & 42.69 & 43.10 & 42.95 & 2.52 \\
\hline 230 & 46.24 & 45.78 & 45.67 & 45.85 & 46.00 & 45.91 & 2.36 \\
\hline 240 & 48.43 & 46.76 & 47.66 & 47.64 & 48.23 & 47.75 & 2.27 \\
\hline
\end{tabular}


Table 6.26 Time and speedup of Chain $(N)$ model with $N=18$ on an Intel Xeon Phi coprocessor with KMP_THREAD_AFFINITY set to balanced

\begin{tabular}{|c|c|c|c|c|c|c|c|}
\hline \multicolumn{8}{|c|}{ Balanced Heisenberg Chain(18) (recorded time in seconds) } \\
\hline Thread & Data 1 & Data 2 & Data 3 & Data 4 & Data 5 & Average & Speedup \\
\hline 1 & 28.00 & 27.91 & 27.94 & 27.93 & 26.60 & 27.67 & 1.00 \\
\hline 10 & 7.81 & 7.81 & 7.83 & 7.98 & 7.86 & 7.86 & 3.52 \\
\hline 20 & 6.74 & 6.73 & 6.73 & 6.91 & 6.86 & 6.79 & 4.07 \\
\hline 30 & 5.40 & 5.40 & 5.28 & 5.46 & 5.37 & 5.38 & 5.14 \\
\hline 40 & 6.44 & 6.45 & 6.44 & 6.53 & 6.41 & 6.45 & 4.29 \\
\hline 50 & 8.06 & 8.12 & 8.04 & 8.12 & 8.08 & 8.09 & 3.42 \\
\hline 60 & 10.34 & 10.35 & 10.14 & 10.44 & 10.40 & 10.33 & 2.68 \\
\hline 70 & 15.64 & 15.61 & 15.67 & 15.83 & 15.72 & 15.69 & 1.76 \\
\hline 80 & 16.90 & 16.90 & 16.84 & 17.00 & 17.32 & 16.99 & 1.63 \\
\hline 90 & 18.53 & 18.80 & 18.54 & 18.46 & 18.60 & 18.59 & 1.49 \\
\hline 100 & 20.20 & 19.91 & 19.99 & 20.27 & 19.55 & 19.98 & 1.38 \\
\hline 110 & 22.20 & 22.23 & 22.19 & 22.31 & 22.23 & 22.23 & 1.24 \\
\hline 120 & 23.48 & 23.47 & 22.91 & 23.76 & 23.65 & 23.45 & 1.18 \\
\hline 130 & 25.94 & 26.12 & 26.14 & 25.92 & 26.07 & 26.04 & 1.06 \\
\hline 140 & 28.33 & 27.94 & 28.24 & 28.07 & 28.09 & 28.14 & 0.98 \\
\hline 150 & 29.86 & 29.43 & 30.04 & 29.86 & 29.64 & 29.77 & 0.93 \\
\hline 160 & 32.00 & 31.94 & 31.96 & 31.23 & 31.76 & 31.78 & 0.87 \\
\hline 170 & 33.57 & 33.56 & 33.59 & 33.81 & 34.01 & 33.71 & 0.82 \\
\hline 180 & 35.60 & 35.61 & 35.74 & 35.62 & 35.34 & 35.58 & 0.78 \\
\hline 190 & 36.16 & 36.07 & 36.26 & 36.05 & 35.33 & 35.97 & 0.77 \\
\hline 200 & 37.96 & 38.21 & 38.02 & 37.81 & 38.22 & 38.04 & 0.73 \\
\hline 210 & 40.27 & 40.53 & 40.44 & 40.25 & 40.41 & 40.38 & 0.69 \\
\hline 220 & 43.28 & 43.09 & 42.61 & 42.69 & 43.10 & 42.95 & 0.64 \\
\hline 230 & 46.24 & 45.78 & 45.67 & 45.85 & 46.00 & 45.91 & 0.60 \\
\hline 240 & 48.43 & 46.76 & 47.66 & 47.64 & 48.23 & 47.75 & 0.58 \\
\hline
\end{tabular}


Table 6.27 Time and speedup of Chain $(N)$ model with $N=16$ on an Intel Xeon Phi coprocessor with KMP_THREAD_AFFINITY set to balanced

\begin{tabular}{|c|c|c|c|c|c|c|c|}
\hline \multicolumn{8}{|c|}{ Balanced Heisenberg Chain(16) (recorded time in seconds) } \\
\hline Thread & Data 1 & Data 2 & Data 3 & Data 4 & Data 5 & Average & Speedup \\
\hline 1 & 7.07 & 6.99 & 7.01 & 7.02 & 7.03 & 7.02 & 1.00 \\
\hline 10 & 3.22 & 3.28 & 3.23 & 3.26 & 3.23 & 3.24 & 2.17 \\
\hline 20 & 3.44 & 3.50 & 3.46 & 3.48 & 3.54 & 3.48 & 2.02 \\
\hline 30 & 4.27 & 4.05 & 4.27 & 4.29 & 4.30 & 4.24 & 1.66 \\
\hline 40 & 5.45 & 5.46 & 5.48 & 5.51 & 5.54 & 5.49 & 1.28 \\
\hline 50 & 7.08 & 7.11 & 7.15 & 7.13 & 7.20 & 7.13 & 0.98 \\
\hline 60 & 9.31 & 9.35 & 9.16 & 9.33 & 9.32 & 9.29 & 0.76 \\
\hline 70 & 11.60 & 11.29 & 11.66 & 11.65 & 11.65 & 11.57 & 0.61 \\
\hline 80 & 12.76 & 12.83 & 12.85 & 12.76 & 12.48 & 12.73 & 0.55 \\
\hline 90 & 14.07 & 14.09 & 14.21 & 14.31 & 13.83 & 14.10 & 0.50 \\
\hline 100 & 15.07 & 15.58 & 15.58 & 15.14 & 15.55 & 15.38 & 0.46 \\
\hline 110 & 17.11 & 17.27 & 17.26 & 17.28 & 17.18 & 17.22 & 0.41 \\
\hline 120 & 18.51 & 18.71 & 18.54 & 18.64 & 18.53 & 18.59 & 0.38 \\
\hline 130 & 20.19 & 20.31 & 20.30 & 20.38 & 20.24 & 20.28 & 0.35 \\
\hline 140 & 22.01 & 21.51 & 22.03 & 22.06 & 22.07 & 21.94 & 0.32 \\
\hline 150 & 23.58 & 23.79 & 23.64 & 23.93 & 23.73 & 23.73 & 0.30 \\
\hline 160 & 25.97 & 25.84 & 25.71 & 25.91 & 25.90 & 25.87 & 0.27 \\
\hline 170 & 27.83 & 27.59 & 27.53 & 27.63 & 27.00 & 27.52 & 0.26 \\
\hline 180 & 29.43 & 29.56 & 29.52 & 29.62 & 29.60 & 29.55 & 0.24 \\
\hline 190 & 30.58 & 30.53 & 30.51 & 29.91 & 30.44 & 30.39 & 0.23 \\
\hline 200 & 31.69 & 31.64 & 32.56 & 32.52 & 31.09 & 31.90 & 0.22 \\
\hline 210 & 34.74 & 34.85 & 34.84 & 34.78 & 34.87 & 34.81 & 0.20 \\
\hline 220 & 36.91 & 36.92 & 36.85 & 36.93 & 37.04 & 36.93 & 0.19 \\
\hline 230 & 39.03 & 38.24 & 37.36 & 38.57 & 37.52 & 38.14 & 0.18 \\
\hline 240 & 41.14 & 38.31 & 39.89 & 40.90 & 40.84 & 40.22 & 0.17 \\
\hline
\end{tabular}




\subsubsection{Heisenberg Ladder on Xeon Phi}

Table 6.28 Time and speedup of Ladder2( $N)$ model with $N=26$ on an Intel Xeon Phi coprocessor with KMP_THREAD_AFFINITY set to scatter

\begin{tabular}{|c|c|c|c|c|c|c|c|}
\hline \multicolumn{8}{|c|}{ Scatter Heisenberg Ladder2(26) (recorded time in seconds) } \\
\hline Thread & Data 1 & Data 2 & Data 3 & Data 4 & Data 5 & Average & Speedup \\
\hline 1 & - & - & - & - & - & - & - \\
\hline 10 & - & - & - & - & - & - & - \\
\hline 20 & - & - & - & - & - & - & - \\
\hline 30 & - & - & - & - & - & - & - \\
\hline 40 & - & - & - & - & - & - & - \\
\hline 50 & - & - & - & - & - & - & - \\
\hline 60 & - & - & - & - & - & - & - \\
\hline 70 & - & - & - & - & - & - & - \\
\hline 80 & - & - & - & - & - & - & - \\
\hline 90 & - & - & - & - & - & - & - \\
\hline 100 & - & - & - & - & - & - & - \\
\hline 110 & - & - & - & - & - & - & - \\
\hline 120 & - & - & - & - & - & - & - \\
\hline 130 & - & - & - & - & - & - & - \\
\hline 140 & - & - & - & - & - & - & - \\
\hline 150 & - & - & - & - & - & - & - \\
\hline 160 & - & - & - & - & - & - & - \\
\hline 170 & - & - & - & - & - & - & - \\
\hline 180 & - & - & - & - & - & - & - \\
\hline 190 & - & - & - & - & - & - & - \\
\hline 200 & - & - & - & - & - & - & - \\
\hline 210 & - & - & - & - & - & - & - \\
\hline 220 & - & - & - & - & - & - & - \\
\hline 230 & - & - & - & - & - & - & - \\
\hline 240 & - & - & - & - & - & - & - \\
\hline
\end{tabular}


Table 6.29 Time and speedup of Ladder2 $(N)$ model with $N=24$ on an Intel Xeon Phi coprocessor with KMP_THREAD_AFFINITY set to scatter

\begin{tabular}{|c|c|c|c|c|c|c|c|}
\hline \multicolumn{8}{|c|}{ Scatter Heisenberg Ladder2(24) (recorded time in seconds) } \\
\hline Thread & Data 1 & Data 2 & Data 3 & Data 4 & Data 5 & Average & Speedup \\
\hline 1 & 2352.88 & 2354.23 & 2354.63 & 2355.27 & 2354.12 & 2354.22 & 1.00 \\
\hline 10 & 554.40 & 579.01 & 542.70 & 553.20 & 537.86 & 553.43 & 4.25 \\
\hline 20 & 322.49 & 317.95 & 299.73 & 317.82 & 326.01 & 316.80 & 7.43 \\
\hline 30 & 246.32 & 239.08 & 229.18 & 235.76 & 255.04 & 241.08 & 9.77 \\
\hline 40 & 185.69 & 175.71 & 182.13 & 174.23 & 181.62 & 179.88 & 13.09 \\
\hline 50 & 152.52 & 157.67 & 146.06 & 152.02 & 151.11 & 151.88 & 15.50 \\
\hline 60 & 130.45 & 130.59 & 129.63 & 132.26 & 128.77 & 130.34 & 18.06 \\
\hline 70 & 117.04 & 118.71 & 115.49 & 115.66 & 114.41 & 116.26 & 20.25 \\
\hline 80 & 100.40 & 103.29 & 101.93 & 101.65 & 107.20 & 102.89 & 22.88 \\
\hline 90 & 90.80 & 91.14 & 93.64 & 98.43 & 93.78 & 93.56 & 25.16 \\
\hline 100 & 90.77 & 85.09 & 89.40 & 90.78 & 87.89 & 88.78 & 26.52 \\
\hline 110 & 82.38 & 82.19 & 80.04 & 83.35 & 85.44 & 82.68 & 28.47 \\
\hline 120 & 76.77 & 77.19 & 78.30 & 77.85 & 79.82 & 77.99 & 30.19 \\
\hline 130 & 72.68 & 73.57 & 75.77 & 74.29 & 73.87 & 74.04 & 31.80 \\
\hline 140 & 69.30 & 69.72 & 70.44 & 69.04 & 69.69 & 69.64 & 33.81 \\
\hline 150 & 66.61 & 70.65 & 67.66 & 65.16 & 66.21 & 67.26 & 35.00 \\
\hline 160 & 62.66 & 67.48 & 64.46 & 68.40 & 65.31 & 65.66 & 35.86 \\
\hline 170 & 65.80 & 64.93 & 64.54 & 64.79 & 68.89 & 65.79 & 35.78 \\
\hline 180 & 64.00 & 61.06 & 60.90 & 61.11 & 63.32 & 62.07 & 37.93 \\
\hline 190 & 61.38 & 59.62 & 62.10 & 62.43 & 63.34 & 61.78 & 38.11 \\
\hline 200 & 59.89 & 58.08 & 57.75 & 58.61 & 58.36 & 58.54 & 40.22 \\
\hline 210 & 56.13 & 56.55 & 55.10 & 55.25 & 56.03 & 55.81 & 42.18 \\
\hline 220 & 54.24 & 57.53 & 53.36 & 53.27 & 57.27 & 55.13 & 42.70 \\
\hline 230 & 50.48 & 51.12 & 50.55 & 50.40 & 50.52 & 50.61 & 46.51 \\
\hline 240 & 49.70 & 49.89 & 49.83 & 49.66 & 49.85 & 49.78 & 47.29 \\
\hline
\end{tabular}


Table 6.30 Time and speedup of Ladder $2(N)$ model with $N=22$ on an Intel Xeon Phi coprocessor with KMP_THREAD_AFFINITY set to scatter

\begin{tabular}{|c|c|c|c|c|c|c|c|}
\hline \multicolumn{8}{|c|}{ Scatter Heisenberg Ladder2(22) (recorded time in seconds) } \\
\hline Thread & Data 1 & Data 2 & Data 3 & Data 4 & Data 5 & Average & Speedup \\
\hline 1 & 534.20 & 539.41 & 539.06 & 540.01 & 539.19 & 538.37 & 1.00 \\
\hline 10 & 127.81 & 135.78 & 135.87 & 139.20 & 125.51 & 132.83 & 4.05 \\
\hline 20 & 79.92 & 77.81 & 77.73 & 72.80 & 78.24 & 77.30 & 6.96 \\
\hline 30 & 55.09 & 49.34 & 54.87 & 49.48 & 55.10 & 52.78 & 10.20 \\
\hline 40 & 41.26 & 40.86 & 40.57 & 37.98 & 40.91 & 40.32 & 13.35 \\
\hline 50 & 33.77 & 33.78 & 34.39 & 33.40 & 33.57 & 33.78 & 15.94 \\
\hline 60 & 29.44 & 29.14 & 29.38 & 29.59 & 28.69 & 29.25 & 18.41 \\
\hline 70 & 26.23 & 26.72 & 26.06 & 26.50 & 26.37 & 26.38 & 20.41 \\
\hline 80 & 22.86 & 23.33 & 22.91 & 23.24 & 23.73 & 23.21 & 23.19 \\
\hline 90 & 23.84 & 23.19 & 22.53 & 21.70 & 21.61 & 22.58 & 23.85 \\
\hline 100 & 21.86 & 20.57 & 20.25 & 20.37 & 19.83 & 20.58 & 26.16 \\
\hline 110 & 19.69 & 19.06 & 18.45 & 19.15 & 18.45 & 18.96 & 28.40 \\
\hline 120 & 18.06 & 17.96 & 18.29 & 18.02 & 18.28 & 18.12 & 29.71 \\
\hline 130 & 18.19 & 17.33 & 17.61 & 17.46 & 17.59 & 17.64 & 30.52 \\
\hline 140 & 17.26 & 16.71 & 16.70 & 17.23 & 16.69 & 16.92 & 31.83 \\
\hline 150 & 19.86 & 19.02 & 18.98 & 19.53 & 18.90 & 19.26 & 27.96 \\
\hline 160 & 19.00 & 19.27 & 18.78 & 19.31 & 18.73 & 19.02 & 28.31 \\
\hline 170 & 19.27 & 19.34 & 18.87 & 19.26 & 18.87 & 19.12 & 28.15 \\
\hline 180 & 19.28 & 19.43 & 19.27 & 19.30 & 19.50 & 19.36 & 27.82 \\
\hline 190 & 19.56 & 19.51 & 20.02 & 19.55 & 19.65 & 19.66 & 27.39 \\
\hline 200 & 19.89 & 19.93 & 20.20 & 19.86 & 19.95 & 19.97 & 26.97 \\
\hline 210 & 20.07 & 20.09 & 20.41 & 20.07 & 20.13 & 20.16 & 26.71 \\
\hline 220 & 20.39 & 20.43 & 20.73 & 20.41 & 20.39 & 20.47 & 26.30 \\
\hline 230 & 20.68 & 20.65 & 20.71 & 20.67 & 20.69 & 20.68 & 26.03 \\
\hline 240 & 21.31 & 21.07 & 21.11 & 21.03 & 21.03 & 21.11 & 25.50 \\
\hline
\end{tabular}


Table 6.31 Time and speedup of Ladder2 $(N)$ model with $N=20$ on an Intel Xeon Phi coprocessor with KMP_THREAD_AFFINITY set to scatter

\begin{tabular}{|c|c|c|c|c|c|c|c|}
\hline \multicolumn{8}{|c|}{ Scatter Heisenberg Ladder2(20) (recorded time in seconds) } \\
\hline Thread & Data 1 & Data 2 & Data 3 & Data 4 & Data 5 & Average & Speedup \\
\hline 1 & 133.70 & 133.85 & 133.96 & 134.29 & 134.28 & 134.02 & 1.00 \\
\hline 10 & 29.42 & 32.05 & 30.37 & 28.52 & 30.84 & 30.24 & 4.43 \\
\hline 20 & 18.72 & 17.56 & 17.25 & 17.17 & 17.17 & 17.58 & 7.63 \\
\hline 30 & 13.22 & 13.14 & 12.94 & 13.66 & 12.87 & 13.17 & 10.18 \\
\hline 40 & 11.85 & 11.51 & 11.80 & 11.61 & 11.76 & 11.71 & 11.45 \\
\hline 50 & 10.76 & 10.76 & 10.74 & 10.43 & 10.63 & 10.66 & 12.57 \\
\hline 60 & 10.05 & 10.13 & 10.09 & 10.05 & 10.02 & 10.06 & 13.32 \\
\hline 70 & 9.90 & 9.85 & 10.03 & 9.83 & 9.81 & 9.88 & 13.56 \\
\hline 80 & 9.64 & 9.62 & 9.82 & 9.55 & 9.58 & 9.64 & 13.90 \\
\hline 90 & 9.92 & 9.92 & 10.05 & 9.85 & 9.86 & 9.92 & 13.51 \\
\hline 100 & 10.04 & 10.03 & 10.12 & 9.96 & 10.09 & 10.05 & 13.34 \\
\hline 110 & 10.30 & 10.24 & 10.29 & 10.21 & 10.25 & 10.26 & 13.06 \\
\hline 120 & 10.62 & 10.63 & 10.60 & 10.56 & 10.55 & 10.59 & 12.65 \\
\hline 130 & 9.66 & 9.68 & 9.72 & 9.80 & 9.76 & 9.73 & 13.78 \\
\hline 140 & 10.05 & 10.14 & 10.32 & 10.05 & 10.07 & 10.13 & 13.24 \\
\hline 150 & 10.68 & 10.59 & 10.65 & 10.52 & 10.53 & 10.59 & 12.65 \\
\hline 160 & 11.13 & 10.98 & 11.16 & 10.96 & 11.12 & 11.07 & 12.11 \\
\hline 170 & 11.63 & 11.48 & 11.67 & 11.54 & 11.60 & 11.58 & 11.57 \\
\hline 180 & 11.92 & 11.81 & 11.82 & 11.92 & 11.87 & 11.87 & 11.29 \\
\hline 190 & 12.53 & 12.57 & 12.55 & 12.53 & 12.56 & 12.55 & 10.68 \\
\hline 200 & 13.03 & 13.09 & 13.08 & 13.06 & 13.05 & 13.06 & 10.26 \\
\hline 210 & 13.42 & 13.46 & 13.43 & 13.45 & 13.43 & 13.44 & 9.97 \\
\hline 220 & 13.83 & 13.87 & 13.86 & 13.84 & 13.83 & 13.84 & 9.68 \\
\hline 230 & 14.35 & 14.38 & 14.40 & 14.38 & 14.32 & 14.37 & 9.33 \\
\hline 240 & 15.08 & 14.72 & 14.79 & 14.78 & 14.69 & 14.81 & 9.05 \\
\hline
\end{tabular}


Table 6.32 Time and speedup of Ladder2 $(N)$ model with $N=18$ on an Intel Xeon Phi coprocessor with KMP_THREAD_AFFINITY set to scatter

\begin{tabular}{|c|c|c|c|c|c|c|c|}
\hline \multicolumn{8}{|c|}{ Scatter Heisenberg Ladder2(18) (recorded time in seconds) } \\
\hline Thread & Data 1 & Data 2 & Data 3 & Data 4 & Data 5 & Average & Speedup \\
\hline 1 & 32.89 & 32.85 & 33.03 & 33.01 & 32.93 & 32.94 & 1.00 \\
\hline 10 & 8.12 & 8.83 & 8.09 & 8.02 & 8.00 & 8.21 & 4.01 \\
\hline 20 & 6.23 & 6.38 & 6.18 & 6.41 & 6.37 & 6.31 & 5.22 \\
\hline 30 & 4.38 & 4.38 & 4.34 & 4.58 & 4.43 & 4.42 & 7.45 \\
\hline 40 & 4.75 & 4.71 & 4.72 & 5.02 & 4.75 & 4.79 & 6.88 \\
\hline 50 & 5.39 & 5.40 & 5.34 & 5.78 & 5.42 & 5.47 & 6.03 \\
\hline 60 & 6.40 & 6.34 & 6.33 & 6.79 & 6.36 & 6.44 & 5.11 \\
\hline 70 & 7.21 & 7.22 & 7.16 & 7.32 & 7.24 & 7.23 & 4.56 \\
\hline 80 & 7.89 & 7.88 & 7.84 & 7.90 & 7.90 & 7.88 & 4.18 \\
\hline 90 & 8.57 & 8.62 & 8.54 & 8.59 & 8.61 & 8.59 & 3.84 \\
\hline 100 & 9.31 & 9.32 & 9.30 & 9.36 & 9.36 & 9.33 & 3.53 \\
\hline 110 & 10.07 & 10.07 & 10.01 & 10.09 & 10.10 & 10.07 & 3.27 \\
\hline 120 & 10.89 & 10.87 & 10.83 & 10.87 & 10.89 & 10.87 & 3.03 \\
\hline 130 & 11.89 & 11.88 & 11.80 & 11.89 & 11.87 & 11.87 & 2.78 \\
\hline 140 & 12.64 & 12.65 & 12.58 & 12.66 & 12.66 & 12.64 & 2.61 \\
\hline 150 & 13.34 & 13.34 & 13.29 & 13.39 & 13.35 & 13.34 & 2.47 \\
\hline 160 & 14.10 & 14.10 & 14.09 & 14.11 & 14.11 & 14.10 & 2.34 \\
\hline 170 & 14.87 & 14.85 & 14.82 & 14.87 & 14.88 & 14.86 & 2.22 \\
\hline 180 & 15.59 & 15.57 & 15.50 & 15.59 & 15.65 & 15.58 & 2.11 \\
\hline 190 & 16.56 & 16.54 & 16.49 & 16.56 & 16.56 & 16.55 & 1.99 \\
\hline 200 & 17.33 & 17.32 & 17.25 & 17.30 & 17.32 & 17.30 & 1.90 \\
\hline 210 & 18.05 & 18.04 & 17.98 & 18.04 & 18.05 & 18.03 & 1.83 \\
\hline 220 & 18.78 & 18.79 & 18.74 & 18.79 & 18.79 & 18.78 & 1.75 \\
\hline 230 & 19.53 & 19.53 & 19.47 & 19.52 & 19.53 & 19.52 & 1.69 \\
\hline 240 & 20.65 & 20.29 & 20.22 & 20.29 & 20.30 & 20.35 & 1.62 \\
\hline
\end{tabular}


Table 6.33 Time and speedup of Ladder2 $(N)$ model with $N=16$ on an Intel Xeon Phi coprocessor with KMP_THREAD_AFFINITY set to scatter

\begin{tabular}{|c|c|c|c|c|c|c|c|}
\hline \multicolumn{8}{|c|}{ Scatter Heisenberg Ladder2(16) (recorded time in seconds) } \\
\hline Thread & Data 1 & Data 2 & Data 3 & Data 4 & Data 5 & Average & Speedup \\
\hline 1 & 8.28 & 8.20 & 8.22 & 8.24 & 8.25 & 8.24 & 1.00 \\
\hline 10 & 3.11 & 3.10 & 3.10 & 3.11 & 3.13 & 3.11 & 2.65 \\
\hline 20 & 3.10 & 3.10 & 3.10 & 3.11 & 3.11 & 3.10 & 2.65 \\
\hline 30 & 3.44 & 3.43 & 3.44 & 3.45 & 3.44 & 3.44 & 2.40 \\
\hline 40 & 3.98 & 3.99 & 3.99 & 3.99 & 4.00 & 3.99 & 2.06 \\
\hline 50 & 4.77 & 4.78 & 4.77 & 4.80 & 4.85 & 4.79 & 1.72 \\
\hline 60 & 5.74 & 5.73 & 5.74 & 5.75 & 5.76 & 5.74 & 1.43 \\
\hline 70 & 6.44 & 6.48 & 6.46 & 6.48 & 6.46 & 6.46 & 1.28 \\
\hline 80 & 7.14 & 7.14 & 7.17 & 7.14 & 7.16 & 7.15 & 1.15 \\
\hline 90 & 7.91 & 7.92 & 7.92 & 7.93 & 7.95 & 7.93 & 1.04 \\
\hline 100 & 8.66 & 8.66 & 8.67 & 8.67 & 8.68 & 8.67 & 0.95 \\
\hline 110 & 9.38 & 9.37 & 9.38 & 9.39 & 9.41 & 9.39 & 0.88 \\
\hline 120 & 10.07 & 10.07 & 10.09 & 10.09 & 10.10 & 10.08 & 0.82 \\
\hline 130 & 10.92 & 10.91 & 10.92 & 10.94 & 10.94 & 10.92 & 0.75 \\
\hline 140 & 11.68 & 11.68 & 11.70 & 11.70 & 11.70 & 11.69 & 0.70 \\
\hline 150 & 12.38 & 12.38 & 12.37 & 12.39 & 12.39 & 12.38 & 0.67 \\
\hline 160 & 13.05 & 13.05 & 13.06 & 13.07 & 13.07 & 13.06 & 0.63 \\
\hline 170 & 13.76 & 13.76 & 13.78 & 13.78 & 13.78 & 13.77 & 0.60 \\
\hline 180 & 14.44 & 14.44 & 14.44 & 14.45 & 14.46 & 14.45 & 0.57 \\
\hline 190 & 15.34 & 15.31 & 15.32 & 15.35 & 15.34 & 15.33 & 0.54 \\
\hline 200 & 16.04 & 16.03 & 16.04 & 16.04 & 16.05 & 16.04 & 0.51 \\
\hline 210 & 16.74 & 16.72 & 16.73 & 16.71 & 16.73 & 16.72 & 0.49 \\
\hline 220 & 17.45 & 17.43 & 17.45 & 17.43 & 17.45 & 17.44 & 0.47 \\
\hline 230 & 18.14 & 18.12 & 18.12 & 18.12 & 18.13 & 18.13 & 0.45 \\
\hline 240 & 19.16 & 18.81 & 18.81 & 18.81 & 18.85 & 18.89 & 0.44 \\
\hline
\end{tabular}


Table 6.34 Time and speedup of Ladder2(N) model with $N=26$ on an Intel Xeon Phi coprocessor with KMP_THREAD_AFFINITY set to compact

\begin{tabular}{|c|c|c|c|c|c|c|c|}
\hline Compa & ct Heise & erg Lad & (26) (rec & rded tim & n secon & & \\
\hline Thread & Data 1 & Data 2 & Data 3 & Data 4 & Data 5 & Average & Speedup \\
\hline 1 & - & - & - & - & - & - & - \\
\hline 10 & - & - & - & - & - & - & - \\
\hline 20 & - & - & - & - & - & - & - \\
\hline 30 & - & - & - & - & - & - & - \\
\hline 40 & - & - & - & - & - & - & - \\
\hline 50 & - & - & - & - & - & - & - \\
\hline 60 & - & - & - & - & - & - & - \\
\hline 70 & - & - & - & - & - & - & - \\
\hline 80 & - & - & - & - & - & - & - \\
\hline 90 & - & - & - & - & - & - & - \\
\hline 100 & - & - & - & - & - & - & - \\
\hline 110 & - & - & - & - & - & - & - \\
\hline 120 & - & - & - & - & - & - & - \\
\hline 130 & - & - & - & - & - & - & - \\
\hline 140 & - & - & - & - & - & - & - \\
\hline 150 & - & - & - & - & - & - & - \\
\hline 160 & - & - & - & - & - & - & - \\
\hline 170 & - & - & - & - & - & - & - \\
\hline 180 & - & - & - & - & - & - & - \\
\hline 190 & - & - & - & - & - & - & - \\
\hline 200 & - & - & - & - & - & - & - \\
\hline 210 & - & - & - & - & - & - & - \\
\hline 220 & - & - & - & - & - & - & - \\
\hline 230 & - & - & - & - & - & - & - \\
\hline 240 & - & - & - & - & - & - & - \\
\hline
\end{tabular}


Table 6.35 Time and speedup of Ladder2 $(N)$ model with $N=24$ on an Intel Xeon Phi coprocessor with KMP_THREAD_AFFINITY set to compact

\begin{tabular}{|c|c|c|c|c|c|c|c|}
\hline \multicolumn{8}{|c|}{ Compact Heisenberg Ladder2(24) (recorded time in seconds) } \\
\hline Thread & Data 1 & Data 2 & Data 3 & Data 4 & Data 5 & Average & Speedup \\
\hline 1 & 2352.88 & 2354.23 & 2354.63 & 2355.27 & 2354.12 & 2354.22 & 1.00 \\
\hline 10 & 698.26 & 697.62 & 698.07 & 700.38 & 697.64 & 698.39 & 3.37 \\
\hline 20 & 373.91 & 373.94 & 373.94 & 373.97 & 374.04 & 373.96 & 6.30 \\
\hline 30 & 254.73 & 254.64 & 254.70 & 254.66 & 254.75 & 254.70 & 9.24 \\
\hline 40 & 196.22 & 196.15 & 196.22 & 196.18 & 196.28 & 196.21 & 12.00 \\
\hline 50 & 159.29 & 159.26 & 159.92 & 159.24 & 159.28 & 159.40 & 14.77 \\
\hline 60 & 136.88 & 136.83 & 136.87 & 136.88 & 136.92 & 136.87 & 17.20 \\
\hline 70 & 118.42 & 118.34 & 118.39 & 118.41 & 118.40 & 118.39 & 19.88 \\
\hline 80 & 104.34 & 104.47 & 104.38 & 104.37 & 104.37 & 104.39 & 22.55 \\
\hline 90 & 95.73 & 95.73 & 95.73 & 95.74 & 95.72 & 95.73 & 24.59 \\
\hline 100 & 87.98 & 87.96 & 87.94 & 87.94 & 87.95 & 87.95 & 26.77 \\
\hline 110 & 81.42 & 81.46 & 81.45 & 81.43 & 81.48 & 81.45 & 28.91 \\
\hline 120 & 76.40 & 76.39 & 76.38 & 76.41 & 76.39 & 76.40 & 30.82 \\
\hline 130 & 72.02 & 72.02 & 72.05 & 72.13 & 72.04 & 72.05 & 32.67 \\
\hline 140 & 67.88 & 67.89 & 67.87 & 67.86 & 67.87 & 67.87 & 34.69 \\
\hline 150 & 64.23 & 64.19 & 64.21 & 64.24 & 64.25 & 64.22 & 36.66 \\
\hline 160 & 60.64 & 60.62 & 60.60 & 60.64 & 60.63 & 60.63 & 38.83 \\
\hline 170 & 60.39 & 60.31 & 60.48 & 60.29 & 60.30 & 60.35 & 39.01 \\
\hline 180 & 56.07 & 56.01 & 56.04 & 56.05 & 56.05 & 56.04 & 42.01 \\
\hline 190 & 53.34 & 53.39 & 53.40 & 53.39 & 53.38 & 53.38 & 44.10 \\
\hline 200 & 51.88 & 51.91 & 51.91 & 51.90 & 51.92 & 51.90 & 45.36 \\
\hline 210 & 50.43 & 50.46 & 50.45 & 50.42 & 50.44 & 50.44 & 46.68 \\
\hline 220 & 49.55 & 49.58 & 49.57 & 49.57 & 49.55 & 49.56 & 47.50 \\
\hline 230 & 47.69 & 47.72 & 47.69 & 47.67 & 47.66 & 47.69 & 49.37 \\
\hline 240 & 46.36 & 46.31 & 46.26 & 46.29 & 46.22 & 46.29 & 50.86 \\
\hline
\end{tabular}


Table 6.36 Time and speedup of Ladder2 $(N)$ model with $N=22$ on an Intel Xeon Phi coprocessor with KMP_THREAD_AFFINITY set to compact

\begin{tabular}{|c|c|c|c|c|c|c|c|}
\hline Thread & Data 1 & Data 2 & Data 3 & Data 4 & Data 5 & Average & Speedup \\
\hline 1 & 534.20 & 539.41 & 539.06 & 540.01 & 539.19 & 538.37 & 1.00 \\
\hline 10 & 165.25 & 165.26 & 165.31 & 165.28 & 165.32 & 165.29 & 3.26 \\
\hline 20 & 91.37 & 91.39 & 91.42 & 91.37 & 91.38 & 91.38 & 5.89 \\
\hline 30 & 63.76 & 63.80 & 63.78 & 63.80 & 63.81 & 63.79 & 8.44 \\
\hline 40 & 49.35 & 49.37 & 49.36 & 49.38 & 49.36 & 49.37 & 10.91 \\
\hline 50 & 41.46 & 41.46 & 41.47 & 41.43 & 41.43 & 41.45 & 12.99 \\
\hline 60 & 35.26 & 35.28 & 35.27 & 35.30 & 35.26 & 35.27 & 15.26 \\
\hline 70 & 30.83 & 30.84 & 30.86 & 30.83 & 30.83 & 30.84 & 17.46 \\
\hline 80 & 26.81 & 26.80 & 26.80 & 26.81 & 26.77 & 26.80 & 20.09 \\
\hline 90 & 24.41 & 24.40 & 24.39 & 24.38 & 24.39 & 24.40 & 22.07 \\
\hline 100 & 22.00 & 22.04 & 21.99 & 22.00 & 21.97 & 22.00 & 24.47 \\
\hline 110 & 20.04 & 20.01 & 20.07 & 20.03 & 20.03 & 20.04 & 26.87 \\
\hline 120 & 18.65 & 18.65 & 18.64 & 18.64 & 18.62 & 18.64 & 28.88 \\
\hline 130 & 17.74 & 17.71 & 17.72 & 17.71 & 17.69 & 17.71 & 30.39 \\
\hline 140 & 16.60 & 16.56 & 16.57 & 16.57 & 16.56 & 16.57 & 32.49 \\
\hline 150 & 18.96 & 18.92 & 18.92 & 18.93 & 18.92 & 18.93 & 28.44 \\
\hline 160 & 18.70 & 18.64 & 18.67 & 18.66 & 18.66 & 18.66 & 28.84 \\
\hline 170 & 18.95 & 18.93 & 18.92 & 18.93 & 18.92 & 18.93 & 28.44 \\
\hline 180 & 19.02 & 19.00 & 19.01 & 19.01 & 19.01 & 19.01 & 28.32 \\
\hline 190 & 19.16 & 19.09 & 19.11 & 19.11 & 19.07 & 19.11 & 28.18 \\
\hline 200 & 19.65 & 19.64 & 19.66 & 19.63 & 19.58 & 19.63 & 27.42 \\
\hline 210 & 20.15 & 20.13 & 20.15 & 20.13 & 20.13 & 20.14 & 26.73 \\
\hline 220 & 20.81 & 20.76 & 20.76 & 20.82 & 20.79 & 20.79 & 25.90 \\
\hline 230 & 21.52 & 21.48 & 21.46 & 21.51 & 21.45 & 21.48 & 25.06 \\
\hline 240 & 22.46 & 22.18 & 22.17 & 22.20 & 22.22 & 22.25 & 24.20 \\
\hline
\end{tabular}


Table 6.37 Time and speedup of Ladder2 $(N)$ model with $N=20$ on an Intel Xeon Phi coprocessor with KMP_THREAD_AFFINITY set to compact

\begin{tabular}{|c|c|c|c|c|c|c|c|}
\hline \multicolumn{8}{|c|}{ Compact Heisenberg Ladder2(20) (recorded time in seconds) } \\
\hline Thread & Data 1 & Data 2 & Data 3 & Data 4 & Data 5 & Average & Speedup \\
\hline 1 & 133.70 & 133.85 & 133.96 & 134.29 & 134.28 & 134.02 & 1.00 \\
\hline 10 & 42.97 & 43.35 & 43.00 & 42.97 & 42.97 & 43.05 & 3.11 \\
\hline 20 & 24.23 & 24.21 & 24.21 & 24.06 & 24.07 & 24.16 & 5.55 \\
\hline 30 & 16.26 & 16.22 & 16.22 & 16.21 & 16.21 & 16.22 & 8.26 \\
\hline 40 & 13.83 & 13.81 & 13.83 & 13.77 & 13.77 & 13.80 & 9.71 \\
\hline 50 & 12.60 & 12.59 & 12.62 & 12.58 & 12.57 & 12.59 & 10.64 \\
\hline 60 & 11.82 & 11.82 & 11.81 & 11.77 & 11.78 & 11.80 & 11.35 \\
\hline 70 & 11.37 & 11.34 & 11.36 & 11.36 & 11.33 & 11.35 & 11.81 \\
\hline 80 & 10.97 & 10.97 & 10.97 & 10.94 & 10.94 & 10.96 & 12.23 \\
\hline 90 & 11.12 & 11.12 & 11.11 & 11.09 & 11.12 & 11.11 & 12.06 \\
\hline 100 & 11.29 & 11.26 & 11.26 & 11.25 & 11.26 & 11.26 & 11.90 \\
\hline 110 & 11.50 & 11.46 & 11.45 & 11.47 & 11.46 & 11.47 & 11.69 \\
\hline 120 & 11.66 & 11.68 & 11.66 & 11.66 & 11.64 & 11.66 & 11.50 \\
\hline 130 & 10.97 & 11.00 & 11.00 & 11.01 & 11.01 & 11.00 & 12.19 \\
\hline 140 & 11.45 & 11.42 & 11.42 & 11.45 & 11.43 & 11.44 & 11.72 \\
\hline 150 & 11.75 & 11.78 & 11.80 & 11.80 & 11.81 & 11.79 & 11.37 \\
\hline 160 & 12.22 & 12.20 & 12.24 & 12.24 & 12.22 & 12.22 & 10.96 \\
\hline 170 & 12.61 & 12.66 & 12.65 & 12.67 & 12.65 & 12.65 & 10.60 \\
\hline 180 & 13.11 & 13.06 & 13.11 & 13.16 & 13.15 & 13.12 & 10.22 \\
\hline 190 & 13.58 & 13.55 & 13.52 & 13.56 & 13.60 & 13.56 & 9.88 \\
\hline 200 & 14.08 & 14.03 & 14.02 & 14.03 & 14.02 & 14.04 & 9.55 \\
\hline 210 & 14.60 & 14.55 & 14.54 & 14.55 & 14.57 & 14.56 & 9.20 \\
\hline 220 & 15.10 & 15.06 & 15.03 & 15.03 & 15.10 & 15.06 & 8.90 \\
\hline 230 & 15.65 & 15.59 & 15.58 & 15.58 & 15.62 & 15.61 & 8.59 \\
\hline 240 & 16.45 & 16.06 & 16.03 & 16.06 & 16.10 & 16.14 & 8.30 \\
\hline
\end{tabular}


Table 6.38 Time and speedup of Ladder $2(N)$ model with $N=18$ on an Intel Xeon Phi coprocessor with KMP_THREAD_AFFINITY set to compact

\begin{tabular}{|c|c|c|c|c|c|c|c|}
\hline Thread & Data 1 & Data 2 & Data 3 & Data 4 & Data 5 & Average & Speedup \\
\hline 1 & 32.89 & 32.85 & 33.03 & 33.01 & 32.93 & 32.94 & 1.00 \\
\hline 10 & 11.31 & 11.27 & 11.29 & 11.25 & 11.24 & 11.27 & 2.92 \\
\hline 20 & 8.35 & 8.29 & 8.29 & 8.26 & 8.24 & 8.29 & 3.98 \\
\hline 30 & 6.88 & 6.87 & 6.89 & 6.78 & 6.78 & 6.84 & 4.82 \\
\hline 40 & 6.75 & 6.71 & 6.73 & 6.69 & 6.74 & 6.72 & 4.90 \\
\hline 50 & 6.99 & 6.96 & 6.97 & 6.94 & 6.94 & 6.96 & 4.73 \\
\hline 60 & 7.19 & 7.18 & 7.20 & 7.16 & 7.19 & 7.18 & 4.59 \\
\hline 70 & 7.67 & 7.61 & 7.65 & 7.61 & 7.61 & 7.63 & 4.32 \\
\hline 80 & 8.05 & 8.05 & 8.04 & 8.02 & 8.01 & 8.03 & 4.10 \\
\hline 90 & 8.62 & 8.59 & 8.62 & 8.60 & 8.60 & 8.61 & 3.83 \\
\hline 100 & 9.14 & 9.12 & 9.14 & 9.11 & 9.10 & 9.12 & 3.61 \\
\hline 110 & 9.80 & 9.75 & 9.79 & 9.73 & 9.73 & 9.76 & 3.38 \\
\hline 120 & 10.47 & 10.42 & 10.42 & 10.43 & 10.41 & 10.43 & 3.16 \\
\hline 130 & 11.14 & 11.10 & 11.12 & 11.09 & 11.09 & 11.11 & 2.97 \\
\hline 140 & 11.77 & 11.73 & 11.77 & 11.77 & 11.78 & 11.76 & 2.80 \\
\hline 150 & 12.57 & 12.51 & 12.53 & 12.55 & 12.53 & 12.54 & 2.63 \\
\hline 160 & 13.32 & 13.26 & 13.32 & 13.29 & 13.30 & 13.30 & 2.48 \\
\hline 170 & 14.27 & 14.21 & 14.27 & 14.27 & 14.26 & 14.25 & 2.31 \\
\hline 180 & 15.25 & 15.14 & 15.18 & 15.20 & 15.21 & 15.20 & 2.17 \\
\hline 190 & 16.29 & 16.15 & 16.24 & 16.20 & 16.22 & 16.22 & 2.03 \\
\hline 200 & 17.32 & 17.17 & 17.23 & 17.31 & 17.28 & 17.26 & 1.91 \\
\hline 210 & 18.54 & 18.40 & 18.38 & 18.50 & 18.50 & 18.46 & 1.78 \\
\hline 220 & 19.64 & 19.55 & 19.51 & 19.60 & 19.59 & 19.58 & 1.68 \\
\hline 230 & 20.95 & 20.83 & 20.82 & 20.92 & 20.94 & 20.89 & 1.58 \\
\hline 240 & 22.29 & 21.82 & 21.83 & 21.95 & 21.96 & 21.97 & 1.50 \\
\hline
\end{tabular}


Table 6.39 Time and speedup of Ladder2 $(N)$ model with $N=16$ on an Intel Xeon Phi coprocessor with KMP_THREAD_AFFINITY set to compact

\begin{tabular}{|c|c|c|c|c|c|c|c|}
\hline Thread & Data 1 & Data 2 & Data 3 & Data 4 & Data 5 & Average & Speedup \\
\hline 1 & 8.28 & 8.20 & 8.22 & 8.24 & 8.25 & 8.24 & 1.00 \\
\hline 10 & 4.25 & 4.25 & 4.27 & 4.28 & 4.29 & 4.27 & 1.93 \\
\hline 20 & 3.98 & 3.98 & 3.96 & 3.98 & 3.98 & 3.98 & 2.07 \\
\hline 30 & 4.15 & 4.13 & 4.15 & 4.18 & 4.17 & 4.16 & 1.98 \\
\hline 40 & 4.50 & 4.47 & 4.48 & 4.51 & 4.51 & 4.49 & 1.83 \\
\hline 50 & 4.98 & 4.99 & 4.98 & 4.99 & 5.01 & 4.99 & 1.65 \\
\hline 60 & 5.40 & 5.39 & 5.41 & 5.44 & 5.41 & 5.41 & 1.52 \\
\hline 70 & 5.90 & 5.90 & 5.93 & 5.91 & 5.91 & 5.91 & 1.39 \\
\hline 80 & 6.42 & 6.42 & 6.41 & 6.46 & 6.41 & 6.42 & 1.28 \\
\hline 90 & 6.95 & 6.96 & 6.97 & 6.97 & 6.98 & 6.96 & 1.18 \\
\hline 100 & 7.49 & 7.47 & 7.50 & 7.50 & 7.50 & 7.49 & 1.10 \\
\hline 110 & 8.11 & 8.09 & 8.12 & 8.12 & 8.14 & 8.12 & 1.02 \\
\hline 120 & 8.80 & 8.78 & 8.80 & 8.80 & 8.79 & 8.79 & 0.94 \\
\hline 130 & 9.48 & 9.47 & 9.51 & 9.48 & 9.50 & 9.49 & 0.87 \\
\hline 140 & 10.17 & 10.16 & 10.14 & 10.17 & 10.18 & 10.16 & 0.81 \\
\hline 150 & 10.93 & 10.93 & 10.93 & 10.96 & 10.96 & 10.94 & 0.75 \\
\hline 160 & 11.72 & 11.68 & 11.69 & 11.70 & 11.71 & 11.70 & 0.70 \\
\hline 170 & 12.56 & 12.58 & 12.58 & 12.59 & 12.59 & 12.58 & 0.66 \\
\hline 180 & 13.40 & 13.40 & 13.39 & 13.42 & 13.43 & 13.41 & 0.61 \\
\hline 190 & 14.45 & 14.43 & 14.44 & 14.44 & 14.45 & 14.44 & 0.57 \\
\hline 200 & 15.37 & 15.32 & 15.32 & 15.31 & 15.34 & 15.33 & 0.54 \\
\hline 210 & 16.61 & 16.57 & 16.58 & 16.56 & 16.59 & 16.58 & 0.50 \\
\hline 220 & 17.69 & 17.66 & 17.67 & 17.70 & 17.68 & 17.68 & 0.47 \\
\hline 230 & 18.98 & 18.95 & 18.91 & 18.95 & 18.95 & 18.95 & 0.43 \\
\hline 240 & 20.36 & 20.03 & 20.01 & 20.02 & 20.03 & 20.09 & 0.41 \\
\hline
\end{tabular}


Table 6.40 Time and speedup of Ladder2(N) model with $N=26$ on an Intel Xeon Phi coprocessor with KMP_THREAD_AFFINITY set to ba lanced

\begin{tabular}{|c|c|c|c|c|c|c|c|}
\hline Thread & Data 1 & Data 2 & Data 3 & Data 4 & Data 5 & Average & Speedup \\
\hline 1 & - & - & - & - & - & - & - \\
\hline 10 & - & - & - & - & - & - & - \\
\hline 20 & - & - & - & - & - & - & - \\
\hline 30 & - & - & - & - & - & - & - \\
\hline 40 & - & - & - & - & - & - & - \\
\hline 50 & - & - & - & - & - & - & - \\
\hline 60 & - & - & - & - & - & - & - \\
\hline 70 & - & - & - & - & - & - & - \\
\hline 80 & - & - & - & - & - & - & - \\
\hline 90 & - & - & - & - & - & - & - \\
\hline 100 & - & - & - & - & - & - & - \\
\hline 110 & - & - & - & - & - & - & - \\
\hline 120 & - & - & - & - & - & - & - \\
\hline 130 & - & - & - & - & - & - & - \\
\hline 140 & - & - & - & - & - & - & - \\
\hline 150 & - & - & - & - & - & - & - \\
\hline 160 & - & - & - & - & - & - & - \\
\hline 170 & - & - & - & - & - & - & - \\
\hline 180 & - & - & - & - & - & - & - \\
\hline 190 & - & - & - & - & - & - & - \\
\hline 200 & - & - & - & - & - & - & - \\
\hline 210 & - & - & - & - & - & - & - \\
\hline 220 & - & - & - & - & - & - & - \\
\hline 230 & - & - & - & - & - & - & - \\
\hline 240 & - & - & - & - & - & - & - \\
\hline
\end{tabular}


Table 6.41 Time and speedup of Ladder2 $(N)$ model with $N=24$ on an Intel Xeon Phi coprocessor with KMP_THREAD_AFFINITY set to balanced

\begin{tabular}{|c|c|c|c|c|c|c|c|}
\hline \multicolumn{8}{|c|}{ Balanced Heisenberg Ladder2(24) (recorded time in seconds) } \\
\hline Thread & Data 1 & Data 2 & Data 3 & Data 4 & Data 5 & Average & Speedup \\
\hline 1 & 2352.88 & 2354.23 & 2354.63 & 2355.27 & 2354.12 & 2354.22 & 1.00 \\
\hline 10 & 570.37 & 531.96 & 529.47 & 565.59 & 574.36 & 554.35 & 4.25 \\
\hline 20 & 352.77 & 323.24 & 333.92 & 344.63 & 304.25 & 331.76 & 7.10 \\
\hline 30 & 230.39 & 216.88 & 221.68 & 233.30 & 228.63 & 226.18 & 10.41 \\
\hline 40 & 190.24 & 185.76 & 182.14 & 184.46 & 184.36 & 185.39 & 12.70 \\
\hline 50 & 160.14 & 151.48 & 152.97 & 151.61 & 150.93 & 153.42 & 15.34 \\
\hline 60 & 134.38 & 129.75 & 128.54 & 129.22 & 129.69 & 130.32 & 18.07 \\
\hline 70 & 115.42 & 120.86 & 116.21 & 117.65 & 116.32 & 117.29 & 20.07 \\
\hline 80 & 106.82 & 102.17 & 101.95 & 104.03 & 99.90 & 102.97 & 22.86 \\
\hline 90 & 93.92 & 92.17 & 90.67 & 96.93 & 94.80 & 93.70 & 25.12 \\
\hline 100 & 85.45 & 83.18 & 87.40 & 84.60 & 85.96 & 85.32 & 27.59 \\
\hline 110 & 79.51 & 76.49 & 80.94 & 83.73 & 80.23 & 80.18 & 29.36 \\
\hline 120 & 75.10 & 77.33 & 74.66 & 74.36 & 75.01 & 75.29 & 31.27 \\
\hline 130 & 72.89 & 73.69 & 72.82 & 71.64 & 72.35 & 72.67 & 32.39 \\
\hline 140 & 68.46 & 69.39 & 66.88 & 67.17 & 68.52 & 68.08 & 34.58 \\
\hline 150 & 67.02 & 65.78 & 66.50 & 66.86 & 65.98 & 66.43 & 35.44 \\
\hline 160 & 62.68 & 61.53 & 62.15 & 61.51 & 63.89 & 62.35 & 37.76 \\
\hline 170 & 62.09 & 60.91 & 60.94 & 61.50 & 63.14 & 61.71 & 38.15 \\
\hline 180 & 58.39 & 57.59 & 57.45 & 57.67 & 57.69 & 57.76 & 40.76 \\
\hline 190 & 57.24 & 57.20 & 57.08 & 57.08 & 57.20 & 57.16 & 41.19 \\
\hline 200 & 54.97 & 54.78 & 55.01 & 54.78 & 54.27 & 54.76 & 42.99 \\
\hline 210 & 51.82 & 52.03 & 52.50 & 52.35 & 51.95 & 52.13 & 45.16 \\
\hline 220 & 51.17 & 51.74 & 51.94 & 51.92 & 51.92 & 51.74 & 45.51 \\
\hline 230 & 47.61 & 47.60 & 47.58 & 47.58 & 47.65 & 47.60 & 49.46 \\
\hline 240 & 46.34 & 46.31 & 46.24 & 46.28 & 46.26 & 46.29 & 50.86 \\
\hline
\end{tabular}


Table 6.42 Time and speedup of Ladder $2(N)$ model with $N=22$ on an Intel Xeon Phi coprocessor with KMP_THREAD_AFFINITY set to balanced

\begin{tabular}{|c|c|c|c|c|c|c|c|}
\hline Thread & Data 1 & Data 2 & Data 3 & Data 4 & Data 5 & Average & Speedup \\
\hline 1 & 534.20 & 539.41 & 539.06 & 540.01 & 539.19 & 538.37 & 1.00 \\
\hline 10 & 130.34 & 141.66 & 141.54 & 133.68 & 120.60 & 133.56 & 4.03 \\
\hline 20 & 78.49 & 72.05 & 72.49 & 78.92 & 69.75 & 74.34 & 7.24 \\
\hline 30 & 54.29 & 50.96 & 54.91 & 48.22 & 48.23 & 51.32 & 10.49 \\
\hline 40 & 43.25 & 39.89 & 40.50 & 41.55 & 40.26 & 41.09 & 13.10 \\
\hline 50 & 33.79 & 33.28 & 33.20 & 33.61 & 32.96 & 33.37 & 16.13 \\
\hline 60 & 29.20 & 28.58 & 29.11 & 28.65 & 28.54 & 28.82 & 18.68 \\
\hline 70 & 26.11 & 26.32 & 26.49 & 26.10 & 27.22 & 26.45 & 20.36 \\
\hline 80 & 23.12 & 23.18 & 23.16 & 23.03 & 24.16 & 23.33 & 23.08 \\
\hline 90 & 22.40 & 21.56 & 21.53 & 23.57 & 21.63 & 22.14 & 24.32 \\
\hline 100 & 20.66 & 20.68 & 20.27 & 19.89 & 19.85 & 20.27 & 26.56 \\
\hline 110 & 18.45 & 19.37 & 19.44 & 19.37 & 18.49 & 19.02 & 28.30 \\
\hline 120 & 18.36 & 18.50 & 18.34 & 18.22 & 18.24 & 18.33 & 29.37 \\
\hline 130 & 17.90 & 17.87 & 17.59 & 17.90 & 17.94 & 17.84 & 30.18 \\
\hline 140 & 16.94 & 16.63 & 16.54 & 16.71 & 17.20 & 16.80 & 32.04 \\
\hline 150 & 63.69 & 63.25 & 63.93 & 63.86 & 63.79 & 63.70 & 8.45 \\
\hline 160 & 70.80 & 70.72 & 70.69 & 70.96 & 70.36 & 70.71 & 7.61 \\
\hline 170 & 80.37 & 80.53 & 80.21 & 79.86 & 79.61 & 80.12 & 6.72 \\
\hline 180 & 91.94 & 91.29 & 91.90 & 92.08 & 90.96 & 91.63 & 5.88 \\
\hline 190 & 90.70 & 90.60 & 90.29 & 90.98 & 90.79 & 90.67 & 5.94 \\
\hline 200 & 114.24 & 113.82 & 114.13 & 113.57 & 114.10 & 113.97 & 4.72 \\
\hline 210 & 123.48 & 123.83 & 124.46 & 123.50 & 123.35 & 123.72 & 4.35 \\
\hline 220 & 115.42 & 116.56 & 116.27 & 116.94 & 116.01 & 116.24 & 4.63 \\
\hline 230 & 47.61 & 47.60 & 47.58 & 47.58 & 47.65 & 47.60 & 49.46 \\
\hline 240 & 46.34 & 46.31 & 46.24 & 46.28 & 46.26 & 46.29 & 50.86 \\
\hline
\end{tabular}


Table 6.43 Time and speedup of Ladder $2(N)$ model with $N=20$ on an Intel Xeon Phi coprocessor with KMP_THREAD_AFFINITY set to balanced

\begin{tabular}{|c|c|c|c|c|c|c|c|}
\hline \multicolumn{8}{|c|}{ Balanced Heisenberg Ladder2(20) (recorded time in seconds) } \\
\hline Thread & Data 1 & Data 2 & Data 3 & Data 4 & Data 5 & Average & Speedup \\
\hline 1 & 133.70 & 133.85 & 133.96 & 134.29 & 134.28 & 134.02 & 1.00 \\
\hline 10 & 28.71 & 29.31 & 29.58 & 30.70 & 30.27 & 29.71 & 4.51 \\
\hline 20 & 17.21 & 17.64 & 17.97 & 18.74 & 18.45 & 18.00 & 7.45 \\
\hline 30 & 12.94 & 13.11 & 13.23 & 13.73 & 13.58 & 13.32 & 10.06 \\
\hline 40 & 15.45 & 15.41 & 15.34 & 15.08 & 15.40 & 15.34 & 8.74 \\
\hline 50 & 15.20 & 15.29 & 15.09 & 15.23 & 15.26 & 15.21 & 8.81 \\
\hline 60 & 15.78 & 15.81 & 15.83 & 15.83 & 15.81 & 15.81 & 8.48 \\
\hline 70 & 28.62 & 28.48 & 28.56 & 28.75 & 28.76 & 28.63 & 4.68 \\
\hline 80 & 32.61 & 32.98 & 32.74 & 33.16 & 32.45 & 32.79 & 4.09 \\
\hline 90 & 41.97 & 42.60 & 41.95 & 42.19 & 41.75 & 42.09 & 3.18 \\
\hline 100 & 48.82 & 48.56 & 49.13 & 49.07 & 49.11 & 48.94 & 2.74 \\
\hline 110 & 49.98 & 49.20 & 50.08 & 49.52 & 49.58 & 49.67 & 2.70 \\
\hline 120 & 50.43 & 50.58 & 50.35 & 50.25 & 50.30 & 50.38 & 2.66 \\
\hline 130 & 56.80 & 56.92 & 56.39 & 56.06 & 56.54 & 56.54 & 2.37 \\
\hline 140 & 49.36 & 49.12 & 49.30 & 48.86 & 49.42 & 49.21 & 2.72 \\
\hline 150 & 57.91 & 58.27 & 57.92 & 58.41 & 58.55 & 58.21 & 2.30 \\
\hline 160 & 66.40 & 66.00 & 65.59 & 66.06 & 66.38 & 66.08 & 2.03 \\
\hline 170 & 67.88 & 67.66 & 66.98 & 67.83 & 67.52 & 67.57 & 1.98 \\
\hline 180 & 68.78 & 69.21 & 68.62 & 68.75 & 68.62 & 68.80 & 1.95 \\
\hline 190 & 64.16 & 64.64 & 64.48 & 64.32 & 64.25 & 64.37 & 2.08 \\
\hline 200 & 64.55 & 64.49 & 65.00 & 65.12 & 64.58 & 64.75 & 2.07 \\
\hline 210 & 72.44 & 72.47 & 73.15 & 72.57 & 72.43 & 72.61 & 1.85 \\
\hline 220 & 79.47 & 79.55 & 79.31 & 79.55 & 79.61 & 79.50 & 1.69 \\
\hline 230 & 81.54 & 82.35 & 81.55 & 81.97 & 81.54 & 81.79 & 1.64 \\
\hline 240 & 83.52 & 83.44 & 83.83 & 83.04 & 83.88 & 83.54 & 1.60 \\
\hline
\end{tabular}


Table 6.44 Time and speedup of Ladder $2(N)$ model with $N=18$ on an Intel Xeon Phi coprocessor with KMP_THREAD_AFFINITY set to ba lanced

\begin{tabular}{|c|c|c|c|c|c|c|c|}
\hline \multicolumn{8}{|c|}{ Balanced Heisenberg Ladder2(18) (recorded time in seconds) } \\
\hline Thread & Data 1 & Data 2 & Data 3 & Data 4 & Data 5 & Average & Speedup \\
\hline 1 & 32.89 & 32.85 & 33.03 & 33.01 & 32.93 & 32.94 & 1.00 \\
\hline 10 & 9.03 & 9.11 & 9.50 & 8.71 & 9.29 & 9.13 & 3.61 \\
\hline 20 & 7.21 & 7.21 & 7.48 & 7.30 & 7.21 & 7.28 & 4.52 \\
\hline 30 & 5.49 & 5.42 & 5.53 & 5.52 & 5.39 & 5.47 & 6.02 \\
\hline 40 & 6.27 & 6.12 & 6.22 & 6.24 & 6.07 & 6.19 & 5.32 \\
\hline 50 & 7.15 & 7.31 & 7.25 & 7.27 & 7.29 & 7.25 & 4.54 \\
\hline 60 & 8.79 & 8.83 & 8.89 & 8.78 & 8.88 & 8.83 & 3.73 \\
\hline 70 & 13.56 & 13.84 & 13.63 & 13.69 & 13.73 & 13.69 & 2.41 \\
\hline 80 & 14.57 & 14.84 & 14.75 & 14.85 & 14.94 & 14.79 & 2.23 \\
\hline 90 & 15.79 & 15.99 & 15.98 & 15.95 & 15.99 & 15.94 & 2.07 \\
\hline 100 & 16.93 & 17.05 & 17.17 & 16.92 & 17.09 & 17.03 & 1.93 \\
\hline 110 & 18.60 & 18.44 & 18.39 & 18.53 & 18.54 & 18.50 & 1.78 \\
\hline 120 & 19.64 & 19.94 & 19.61 & 19.90 & 19.76 & 19.77 & 1.67 \\
\hline 130 & 22.05 & 21.93 & 22.05 & 21.81 & 21.76 & 21.92 & 1.50 \\
\hline 140 & 23.49 & 23.38 & 23.53 & 23.72 & 23.63 & 23.55 & 1.40 \\
\hline 150 & 25.23 & 25.11 & 25.05 & 25.16 & 25.18 & 25.15 & 1.31 \\
\hline 160 & 27.09 & 27.28 & 27.35 & 27.57 & 27.31 & 27.32 & 1.21 \\
\hline 170 & 28.89 & 28.82 & 29.24 & 29.18 & 28.87 & 29.00 & 1.14 \\
\hline 180 & 30.46 & 30.26 & 30.70 & 30.26 & 30.54 & 30.44 & 1.08 \\
\hline 190 & 30.74 & 30.95 & 30.94 & 30.71 & 30.79 & 30.82 & 1.07 \\
\hline 200 & 32.12 & 32.31 & 32.09 & 32.25 & 32.04 & 32.16 & 1.02 \\
\hline 210 & 34.24 & 34.12 & 33.77 & 34.00 & 33.75 & 33.98 & 0.97 \\
\hline 220 & 35.89 & 35.81 & 36.01 & 36.16 & 36.27 & 36.03 & 0.91 \\
\hline 230 & 38.37 & 38.34 & 38.09 & 38.44 & 38.18 & 38.29 & 0.86 \\
\hline 240 & 40.35 & 39.98 & 40.31 & 40.00 & 40.14 & 40.16 & 0.82 \\
\hline
\end{tabular}


Table 6.45 Time and speedup of Ladder2 $(N)$ model with $N=16$ on an Intel Xeon Phi coprocessor with KMP_THREAD_AFFINITY set to ba lanced

\begin{tabular}{|c|c|c|c|c|c|c|c|}
\hline \multicolumn{8}{|c|}{ Balanced Heisenberg Ladder2(16) (recorded time in seconds) } \\
\hline Thread & Data 1 & Data 2 & Data 3 & Data 4 & Data 5 & Average & Speedup \\
\hline 1 & 8.28 & 8.20 & 8.22 & 8.24 & 8.25 & 8.24 & 1.00 \\
\hline 10 & 3.37 & 3.39 & 3.45 & 3.37 & 3.36 & 3.39 & 2.43 \\
\hline 20 & 3.44 & 3.45 & 3.51 & 3.45 & 3.45 & 3.46 & 2.38 \\
\hline 30 & 4.09 & 4.10 & 4.12 & 4.10 & 4.12 & 4.11 & 2.01 \\
\hline 40 & 5.07 & 5.04 & 5.12 & 5.06 & 5.10 & 5.08 & 1.62 \\
\hline 50 & 6.42 & 6.39 & 6.36 & 6.40 & 6.41 & 6.40 & 1.29 \\
\hline 60 & 8.05 & 8.07 & 8.00 & 8.09 & 8.04 & 8.05 & 1.02 \\
\hline 70 & 10.05 & 10.05 & 10.02 & 10.00 & 10.07 & 10.04 & 0.82 \\
\hline 80 & 11.10 & 11.11 & 11.08 & 11.12 & 11.11 & 11.10 & 0.74 \\
\hline 90 & 12.38 & 12.46 & 12.42 & 12.42 & 12.40 & 12.41 & 0.66 \\
\hline 100 & 13.70 & 13.71 & 13.62 & 13.74 & 13.76 & 13.71 & 0.60 \\
\hline 110 & 15.25 & 15.28 & 15.20 & 15.26 & 15.28 & 15.25 & 0.54 \\
\hline 120 & 16.39 & 16.33 & 16.37 & 16.44 & 16.34 & 16.37 & 0.50 \\
\hline 130 & 18.03 & 18.09 & 18.03 & 18.10 & 18.07 & 18.06 & 0.46 \\
\hline 140 & 19.68 & 19.74 & 19.91 & 19.81 & 19.86 & 19.80 & 0.42 \\
\hline 150 & 21.22 & 21.24 & 21.20 & 21.19 & 21.21 & 21.21 & 0.39 \\
\hline 160 & 23.15 & 23.10 & 23.11 & 23.16 & 23.12 & 23.13 & 0.36 \\
\hline 170 & 24.69 & 24.57 & 24.74 & 24.81 & 24.78 & 24.72 & 0.33 \\
\hline 180 & 26.29 & 26.23 & 26.32 & 26.19 & 26.16 & 26.24 & 0.31 \\
\hline 190 & 27.82 & 27.97 & 27.85 & 27.85 & 27.84 & 27.86 & 0.30 \\
\hline 200 & 29.51 & 29.59 & 29.71 & 29.67 & 29.74 & 29.65 & 0.28 \\
\hline 210 & 30.59 & 30.51 & 30.40 & 30.51 & 30.58 & 30.52 & 0.27 \\
\hline 220 & 33.14 & 32.98 & 33.16 & 33.05 & 33.02 & 33.07 & 0.25 \\
\hline 230 & 35.46 & 35.41 & 35.14 & 35.17 & 35.11 & 35.26 & 0.23 \\
\hline 240 & 37.11 & 36.77 & 36.59 & 36.70 & 36.62 & 36.76 & 0.22 \\
\hline
\end{tabular}




\subsubsection{Heisenberg Ladder 215C on Xeon Phi}

Table 6.46 Time and speedup of Ladder $215 \mathrm{C}(N)$ model with $N=26$ on an Intel Xeon

Phi coprocessor with KMP_THREAD_AFFINITY set to scatter

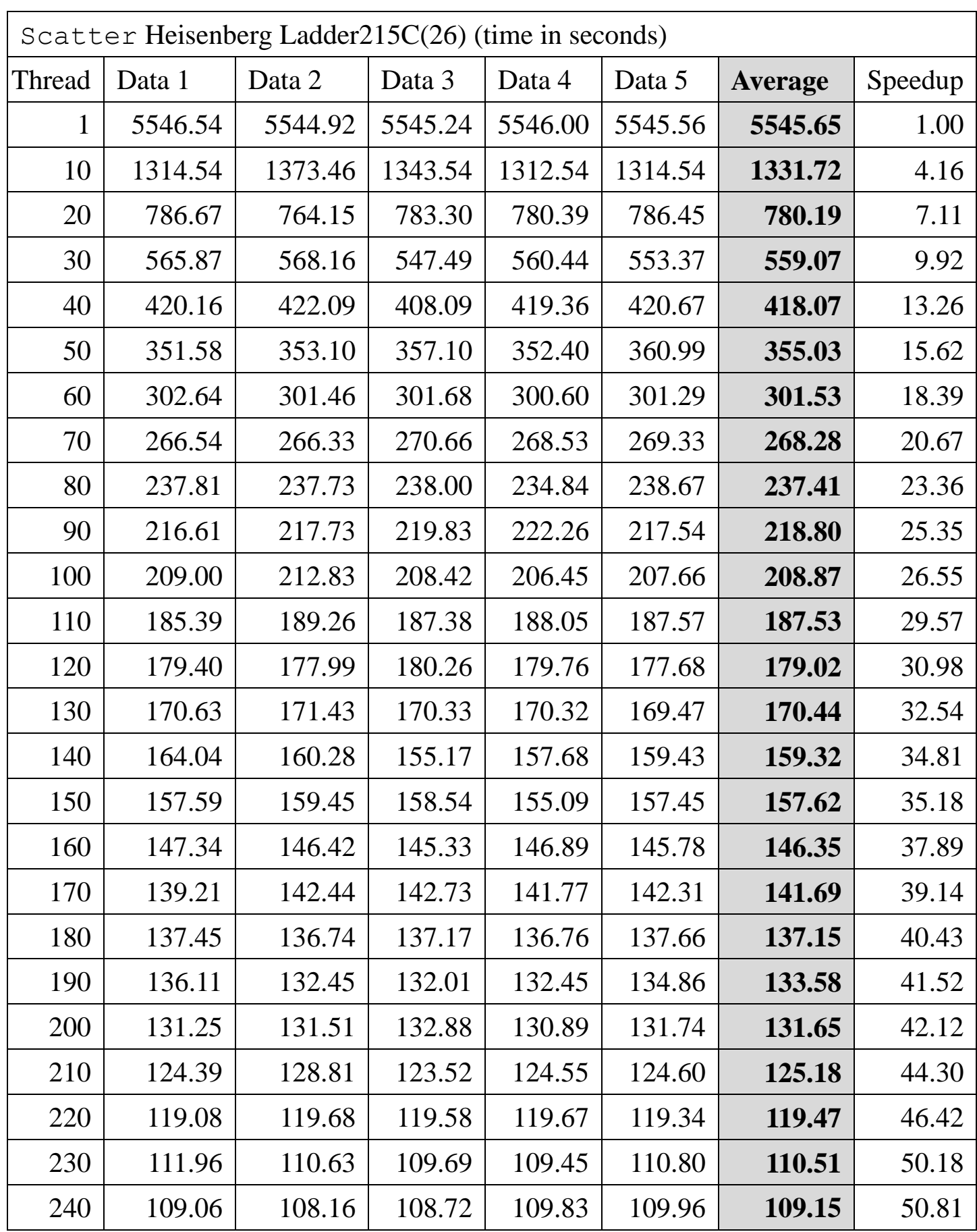


Table 6.47 Time and speedup of Ladder $215 \mathrm{C}(N)$ model with $N=24$ on an Intel Xeon Phi coprocessor with KMP_THREAD_AFFINITY set to scatter

\begin{tabular}{|c|c|c|c|c|c|c|c|}
\hline \multicolumn{8}{|c|}{ Scatter Heisenberg Ladder215C(24) (time in seconds) } \\
\hline Thread & Data 1 & Data 2 & Data 3 & Data 4 & Data 5 & Average & Speedup \\
\hline 1 & 2351.55 & 2355.30 & 2354.26 & 2355.33 & 2355.14 & 2354.32 & 1.00 \\
\hline 10 & 569.02 & 533.47 & 556.68 & 604.12 & 552.95 & 563.25 & 4.18 \\
\hline 20 & 311.97 & 322.75 & 321.53 & 326.19 & 331.58 & 322.80 & 7.29 \\
\hline 30 & 247.05 & 224.24 & 240.73 & 245.90 & 228.66 & 237.32 & 9.92 \\
\hline 40 & 186.12 & 183.99 & 185.63 & 185.66 & 186.67 & 185.61 & 12.68 \\
\hline 50 & 153.67 & 150.16 & 151.67 & 153.88 & 149.60 & 151.79 & 15.51 \\
\hline 60 & 128.68 & 126.90 & 129.58 & 129.32 & 128.69 & 128.63 & 18.30 \\
\hline 70 & 114.06 & 112.09 & 115.44 & 115.11 & 115.49 & 114.44 & 20.57 \\
\hline 80 & 102.05 & 105.42 & 101.52 & 104.50 & 101.58 & 103.02 & 22.85 \\
\hline 90 & 94.41 & 94.03 & 92.26 & 92.65 & 92.23 & 93.12 & 25.28 \\
\hline 100 & 89.06 & 88.90 & 83.84 & 83.50 & 83.54 & 85.77 & 27.45 \\
\hline 110 & 82.15 & 75.95 & 76.56 & 82.51 & 82.65 & 79.96 & 29.44 \\
\hline 120 & 77.56 & 77.75 & 78.34 & 78.33 & 78.64 & 78.13 & 30.14 \\
\hline 130 & 74.62 & 74.25 & 74.61 & 74.03 & 74.73 & 74.45 & 31.62 \\
\hline 140 & 69.36 & 70.14 & 68.83 & 69.71 & 69.45 & 69.50 & 33.88 \\
\hline 150 & 67.02 & 66.63 & 68.00 & 66.20 & 66.65 & 66.90 & 35.19 \\
\hline 160 & 62.89 & 62.40 & 67.17 & 66.71 & 66.57 & 65.15 & 36.14 \\
\hline 170 & 61.65 & 66.98 & 61.03 & 64.91 & 66.55 & 64.22 & 36.66 \\
\hline 180 & 64.54 & 62.09 & 61.62 & 60.27 & 61.72 & 62.05 & 37.94 \\
\hline 190 & 64.06 & 64.84 & 63.51 & 59.01 & 59.68 & 62.22 & 37.84 \\
\hline 200 & 60.33 & 60.95 & 57.56 & 58.71 & 56.89 & 58.89 & 39.98 \\
\hline 210 & 55.91 & 55.04 & 55.61 & 54.39 & 56.08 & 55.40 & 42.49 \\
\hline 220 & 52.24 & 53.38 & 52.69 & 53.23 & 56.71 & 53.65 & 43.88 \\
\hline 230 & 50.30 & 50.44 & 50.26 & 50.49 & 50.80 & 50.45 & 46.66 \\
\hline 240 & 50.01 & 49.58 & 49.80 & 49.59 & 50.04 & 49.80 & 47.27 \\
\hline
\end{tabular}


Table 6.48 Time and speedup of Ladder $215 \mathrm{C}(N)$ model with $N=22$ on an Intel Xeon Phi coprocessor with KMP_THREAD_AFFINITY set to scatter

\begin{tabular}{|c|c|c|c|c|c|c|c|}
\hline \multicolumn{8}{|c|}{ Scatter Heisenberg Ladder215C(22) (time in seconds) } \\
\hline Thread & Data 1 & Data 2 & Data 3 & Data 4 & Data 5 & Average & Speedup \\
\hline 1 & 537.74 & 537.83 & 534.50 & 539.50 & 539.20 & 537.75 & 1.00 \\
\hline 10 & 120.58 & 127.88 & 122.16 & 120.74 & 120.13 & 122.30 & 4.40 \\
\hline 20 & 72.74 & 77.01 & 77.08 & 68.84 & 77.39 & 74.61 & 7.21 \\
\hline 30 & 51.73 & 53.79 & 53.48 & 55.20 & 54.85 & 53.81 & 9.99 \\
\hline 40 & 41.84 & 41.53 & 41.21 & 41.11 & 41.65 & 41.47 & 12.97 \\
\hline 50 & 33.60 & 32.90 & 33.89 & 33.43 & 33.76 & 33.52 & 16.04 \\
\hline 60 & 28.43 & 28.63 & 28.61 & 28.39 & 28.43 & 28.50 & 18.87 \\
\hline 70 & 25.70 & 25.77 & 25.80 & 25.76 & 25.76 & 25.76 & 20.88 \\
\hline 80 & 24.06 & 22.77 & 24.18 & 22.80 & 24.08 & 23.58 & 22.81 \\
\hline 90 & 22.34 & 21.85 & 22.57 & 21.44 & 22.47 & 22.13 & 24.30 \\
\hline 100 & 20.47 & 20.51 & 20.66 & 19.71 & 20.50 & 20.37 & 26.40 \\
\hline 110 & 18.85 & 18.94 & 19.12 & 19.11 & 19.09 & 19.02 & 28.27 \\
\hline 120 & 17.94 & 17.96 & 18.20 & 18.12 & 18.12 & 18.07 & 29.76 \\
\hline 130 & 17.37 & 17.39 & 17.57 & 17.51 & 17.50 & 17.47 & 30.78 \\
\hline 140 & 17.09 & 16.54 & 16.68 & 16.63 & 16.63 & 16.72 & 32.17 \\
\hline 150 & 19.38 & 18.86 & 18.92 & 18.86 & 18.86 & 18.97 & 28.34 \\
\hline 160 & 19.20 & 19.26 & 19.88 & 18.73 & 18.65 & 19.14 & 28.09 \\
\hline 170 & 19.04 & 19.28 & 19.14 & 19.46 & 19.36 & 19.25 & 27.93 \\
\hline 180 & 19.07 & 19.22 & 19.30 & 19.29 & 19.34 & 19.24 & 27.94 \\
\hline 190 & 19.31 & 19.37 & 19.39 & 20.07 & 19.48 & 19.52 & 27.54 \\
\hline 200 & 19.67 & 19.77 & 19.73 & 20.18 & 19.78 & 19.83 & 27.12 \\
\hline 210 & 19.99 & 20.02 & 19.97 & 20.23 & 20.04 & 20.05 & 26.82 \\
\hline 220 & 20.20 & 20.24 & 20.28 & 20.29 & 20.26 & 20.26 & 26.55 \\
\hline 230 & 20.47 & 20.47 & 20.48 & 20.48 & 20.51 & 20.48 & 26.25 \\
\hline 240 & 21.09 & 20.92 & 20.86 & 20.95 & 20.87 & 20.94 & 25.68 \\
\hline
\end{tabular}


Table 6.49 Time and speedup of Ladder $215 \mathrm{C}(N)$ model with $N=20$ on an Intel Xeon Phi coprocessor with KMP_THREAD_AFFINITY set to scatter

\begin{tabular}{|c|c|c|c|c|c|c|c|}
\hline \multicolumn{8}{|c|}{ Scatter Heisenberg Ladder215C(20) (time in seconds) } \\
\hline Thread & Data 1 & Data 2 & Data 3 & Data 4 & Data 5 & Average & Speedup \\
\hline 1 & 135.34 & 136.35 & 134.47 & 134.58 & 134.60 & 135.07 & 1.00 \\
\hline 10 & 28.84 & 28.79 & 28.77 & 28.53 & 28.92 & 28.77 & 4.69 \\
\hline 20 & 17.32 & 18.62 & 17.89 & 17.20 & 18.71 & 17.95 & 7.53 \\
\hline 30 & 12.99 & 13.72 & 13.84 & 13.72 & 13.87 & 13.63 & 9.91 \\
\hline 40 & 11.49 & 11.95 & 11.88 & 11.86 & 11.83 & 11.80 & 11.45 \\
\hline 50 & 10.96 & 10.97 & 10.95 & 10.89 & 10.96 & 10.95 & 12.34 \\
\hline 60 & 10.52 & 10.48 & 10.45 & 10.46 & 10.44 & 10.47 & 12.90 \\
\hline 70 & 10.40 & 10.37 & 10.37 & 10.34 & 10.50 & 10.40 & 12.99 \\
\hline 80 & 10.27 & 10.23 & 10.60 & 10.20 & 10.32 & 10.32 & 13.08 \\
\hline 90 & 10.64 & 10.60 & 11.23 & 10.72 & 10.63 & 10.76 & 12.55 \\
\hline 100 & 10.96 & 11.02 & 11.51 & 10.98 & 11.03 & 11.10 & 12.16 \\
\hline 110 & 11.41 & 11.36 & 11.89 & 11.30 & 11.40 & 11.47 & 11.77 \\
\hline 120 & 11.78 & 11.73 & 12.37 & 11.73 & 11.73 & 11.87 & 11.38 \\
\hline 130 & 10.98 & 10.96 & 11.97 & 11.03 & 10.96 & 11.18 & 12.08 \\
\hline 140 & 11.50 & 11.60 & 12.48 & 11.74 & 11.56 & 11.77 & 11.47 \\
\hline 150 & 12.18 & 12.41 & 13.23 & 12.37 & 12.22 & 12.48 & 10.82 \\
\hline 160 & 12.82 & 13.01 & 13.74 & 12.84 & 13.01 & 13.08 & 10.32 \\
\hline 170 & 13.69 & 13.54 & 14.08 & 13.42 & 13.52 & 13.65 & 9.90 \\
\hline 180 & 13.96 & 13.85 & 13.85 & 13.92 & 13.92 & 13.90 & 9.72 \\
\hline 190 & 14.81 & 14.81 & 14.72 & 14.72 & 14.75 & 14.76 & 9.15 \\
\hline 200 & 15.28 & 15.27 & 15.30 & 15.28 & 15.31 & 15.29 & 8.84 \\
\hline 210 & 15.89 & 15.88 & 15.91 & 15.91 & 15.90 & 15.90 & 8.50 \\
\hline 220 & 16.40 & 16.45 & 16.44 & 16.41 & 16.43 & 16.43 & 8.22 \\
\hline 230 & 16.94 & 17.02 & 16.95 & 16.94 & 16.98 & 16.97 & 7.96 \\
\hline 240 & 17.82 & 17.51 & 17.53 & 17.54 & 17.55 & 17.59 & 7.68 \\
\hline
\end{tabular}


Table 6.50 Time and speedup of Ladder $215 \mathrm{C}(N)$ model with $N=18$ on an Intel Xeon Phi coprocessor with KMP_THREAD_AFFINITY set to scatter

\begin{tabular}{|c|c|c|c|c|c|c|c|}
\hline \multicolumn{8}{|c|}{ Scatter Heisenberg Ladder215C(18) (time in seconds) } \\
\hline Thread & Data 1 & Data 2 & Data 3 & Data 4 & Data 5 & Average & Speedup \\
\hline 1 & 32.99 & 32.94 & 33.02 & 32.97 & 33.13 & 33.01 & 1.00 \\
\hline 10 & 8.00 & 8.09 & 8.71 & 8.72 & 7.92 & 8.29 & 3.98 \\
\hline 20 & 6.17 & 6.29 & 6.30 & 6.30 & 6.45 & 6.30 & 5.24 \\
\hline 30 & 4.35 & 4.29 & 4.38 & 4.40 & 4.37 & 4.36 & 7.57 \\
\hline 40 & 4.67 & 4.64 & 4.64 & 4.75 & 4.68 & 4.68 & 7.06 \\
\hline 50 & 5.29 & 5.30 & 5.30 & 5.40 & 5.30 & 5.32 & 6.21 \\
\hline 60 & 6.22 & 6.26 & 6.24 & 6.37 & 6.30 & 6.28 & 5.26 \\
\hline 70 & 7.05 & 7.04 & 7.08 & 7.28 & 7.10 & 7.11 & 4.64 \\
\hline 80 & 7.76 & 7.74 & 7.75 & 7.90 & 7.78 & 7.79 & 4.24 \\
\hline 90 & 8.44 & 8.45 & 8.47 & 8.63 & 8.49 & 8.49 & 3.89 \\
\hline 100 & 9.16 & 9.17 & 9.19 & 9.32 & 9.21 & 9.21 & 3.58 \\
\hline 110 & 9.94 & 9.90 & 9.91 & 10.07 & 9.94 & 9.95 & 3.32 \\
\hline 120 & 10.73 & 10.69 & 10.72 & 10.76 & 10.73 & 10.73 & 3.08 \\
\hline 130 & 11.61 & 11.61 & 11.68 & 11.67 & 11.64 & 11.64 & 2.84 \\
\hline 140 & 12.47 & 12.41 & 12.43 & 12.45 & 12.45 & 12.44 & 2.65 \\
\hline 150 & 13.13 & 13.14 & 13.15 & 13.18 & 13.17 & 13.15 & 2.51 \\
\hline 160 & 13.88 & 13.88 & 13.89 & 13.92 & 13.94 & 13.90 & 2.37 \\
\hline 170 & 14.59 & 14.60 & 14.63 & 14.64 & 14.68 & 14.63 & 2.26 \\
\hline 180 & 15.31 & 15.32 & 15.35 & 15.35 & 15.34 & 15.33 & 2.15 \\
\hline 190 & 16.34 & 16.31 & 16.34 & 16.32 & 16.34 & 16.33 & 2.02 \\
\hline 200 & 17.08 & 17.08 & 17.10 & 17.08 & 17.10 & 17.09 & 1.93 \\
\hline 210 & 17.80 & 17.81 & 17.83 & 17.80 & 17.81 & 17.81 & 1.85 \\
\hline 220 & 18.53 & 18.55 & 18.56 & 18.51 & 18.54 & 18.54 & 1.78 \\
\hline 230 & 19.28 & 19.29 & 19.31 & 19.27 & 19.30 & 19.29 & 1.71 \\
\hline 240 & 20.34 & 20.01 & 20.04 & 20.04 & 20.05 & 20.10 & 1.64 \\
\hline
\end{tabular}


Table 6.51 Time and speedup of Ladder $215 \mathrm{C}(N)$ model with $N=16$ on an Intel Xeon Phi coprocessor with KMP_THREAD_AFFINITY set to scatter

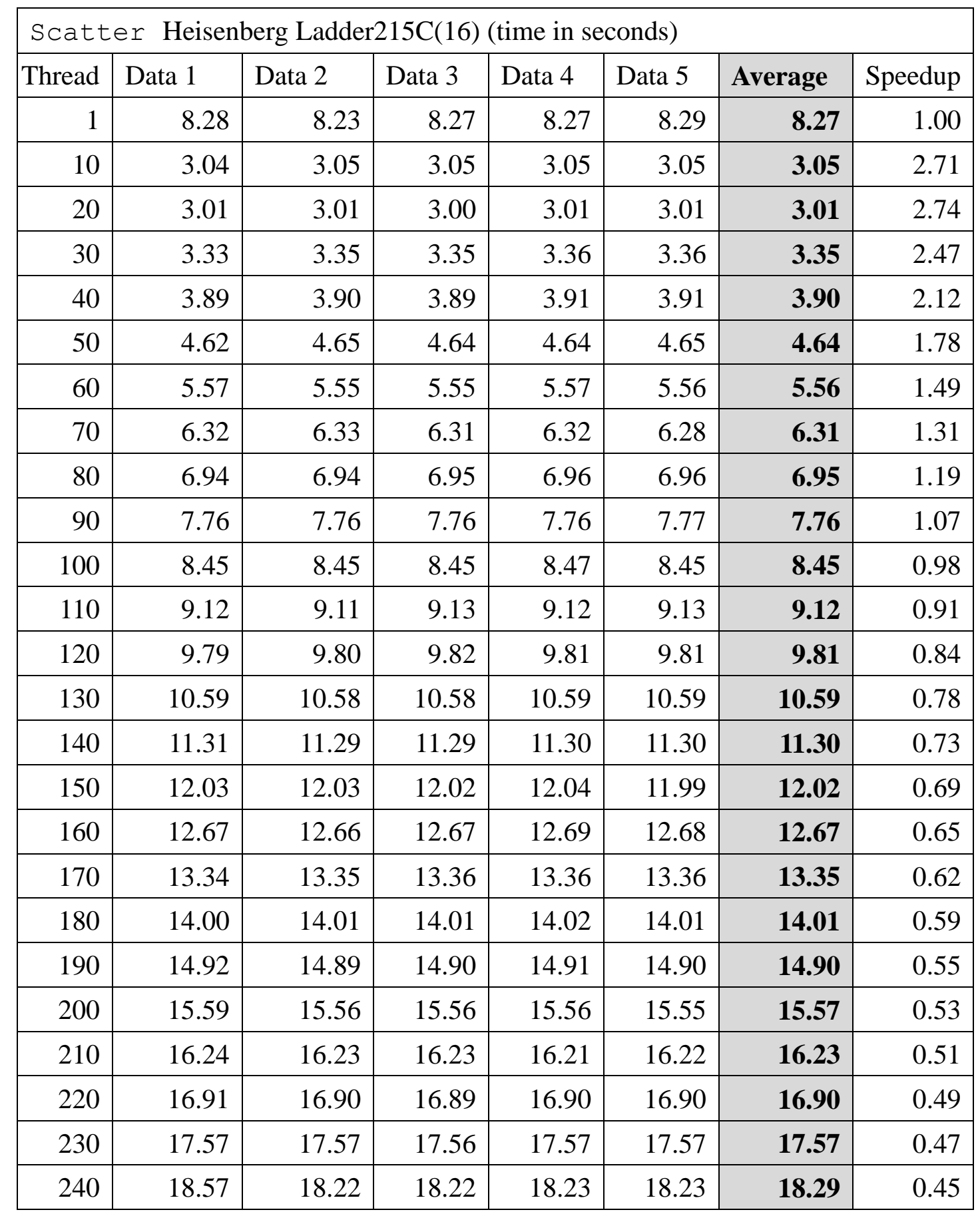


Table 6.52 Time and speedup of Ladder $215 \mathrm{C}(N)$ model with $N=26$ on an Intel Xeon Phi coprocessor with KMP_THREAD_AFFINITY set to compact

\begin{tabular}{|c|c|c|c|c|c|c|c|}
\hline \multicolumn{8}{|c|}{ Compact Heisenberg Ladder215C(26) (time in seconds) } \\
\hline Thread & Data 1 & Data 2 & Data 3 & Data 4 & Data 5 & Average & Speedup \\
\hline 1 & 5546.54 & 5544.92 & 5545.24 & 5546.00 & 5545.56 & 5545.65 & 5546.54 \\
\hline 10 & 1591.48 & 1615.95 & 1620.90 & 1617.41 & 1616.34 & 1612.42 & 1591.48 \\
\hline 20 & 848.94 & 848.35 & 848.39 & 848.67 & 848.66 & 848.60 & 848.94 \\
\hline 30 & 590.84 & 573.56 & 585.34 & 583.45 & 584.33 & 583.50 & 590.84 \\
\hline 40 & 439.94 & 439.65 & 439.77 & 439.53 & 439.55 & 439.69 & 439.94 \\
\hline 50 & 359.70 & 352.95 & 353.01 & 353.02 & 353.00 & 354.33 & 359.70 \\
\hline 60 & 300.94 & 301.04 & 301.11 & 301.02 & 300.67 & 300.96 & 300.94 \\
\hline 70 & 259.29 & 259.05 & 259.11 & 259.24 & 259.11 & 259.16 & 259.29 \\
\hline 80 & 227.82 & 227.68 & 227.81 & 227.63 & 227.90 & 227.77 & 227.82 \\
\hline 90 & 207.29 & 207.41 & 207.36 & 207.34 & 207.29 & 207.34 & 207.29 \\
\hline 100 & 189.46 & 189.35 & 189.40 & 189.30 & 189.33 & 189.37 & 189.46 \\
\hline 110 & 173.97 & 173.86 & 174.00 & 174.02 & 173.92 & 173.95 & 173.97 \\
\hline 120 & 162.69 & 162.73 & 162.37 & 162.44 & 162.56 & 162.56 & 162.69 \\
\hline 130 & 152.31 & 152.42 & 152.33 & 152.55 & 152.35 & 152.39 & 152.31 \\
\hline 140 & 142.54 & 142.55 & 142.49 & 142.47 & 142.54 & 142.52 & 142.54 \\
\hline 150 & 134.42 & 134.45 & 134.77 & 134.61 & 134.54 & 134.56 & 134.42 \\
\hline 160 & 126.73 & 126.51 & 126.53 & 126.64 & 126.55 & 126.59 & 126.73 \\
\hline 170 & 121.15 & 121.12 & 121.06 & 122.36 & 121.25 & 121.39 & 121.15 \\
\hline 180 & 116.28 & 116.38 & 116.23 & 116.27 & 116.44 & 116.32 & 116.28 \\
\hline 190 & 110.40 & 110.32 & 110.29 & 110.28 & 110.24 & 110.31 & 110.40 \\
\hline 200 & 107.14 & 107.00 & 106.99 & 106.96 & 106.97 & 107.01 & 107.14 \\
\hline 210 & 103.46 & 103.34 & 103.59 & 103.44 & 103.01 & 103.37 & 103.46 \\
\hline 220 & 100.68 & 100.54 & 100.60 & 100.67 & 100.69 & 100.64 & 100.68 \\
\hline 230 & 97.74 & 97.66 & 97.62 & 97.33 & 97.56 & 97.58 & 97.74 \\
\hline 240 & 94.98 & 95.57 & 94.79 & 94.80 & 94.88 & 95.00 & 94.98 \\
\hline
\end{tabular}


Table 6.53 Time and speedup of Ladder $215 \mathrm{C}(N)$ model with $N=24$ on an Intel Xeon Phi coprocessor with KMP_THREAD_AFFINITY set to compact

\begin{tabular}{|c|c|c|c|c|c|c|c|}
\hline \multicolumn{8}{|c|}{ Compact Heisenberg Ladder215C(24) (time in seconds) } \\
\hline Thread & Data 1 & Data 2 & Data 3 & Data 4 & Data 5 & Average & Speedup \\
\hline 1 & 2351.55 & 2355.30 & 2354.26 & 2355.33 & 2355.14 & 2354.32 & 1.00 \\
\hline 10 & 696.64 & 696.05 & 696.79 & 696.64 & 696.26 & 696.48 & 3.38 \\
\hline 20 & 374.57 & 374.48 & 374.59 & 374.60 & 374.46 & 374.54 & 6.29 \\
\hline 30 & 254.88 & 254.92 & 257.87 & 254.99 & 254.91 & 255.51 & 9.21 \\
\hline 40 & 196.22 & 196.08 & 196.12 & 196.16 & 196.07 & 196.13 & 12.00 \\
\hline 50 & 159.36 & 159.39 & 159.45 & 159.42 & 159.36 & 159.40 & 14.77 \\
\hline 60 & 136.88 & 136.84 & 136.86 & 136.86 & 136.81 & 136.85 & 17.20 \\
\hline 70 & 118.40 & 118.40 & 118.40 & 118.41 & 118.40 & 118.40 & 19.88 \\
\hline 80 & 104.43 & 104.44 & 104.45 & 104.36 & 104.35 & 104.41 & 22.55 \\
\hline 90 & 95.80 & 95.77 & 95.79 & 95.78 & 95.74 & 95.78 & 24.58 \\
\hline 100 & 87.99 & 87.97 & 88.00 & 87.98 & 87.98 & 87.98 & 26.76 \\
\hline 110 & 81.53 & 81.40 & 81.40 & 81.39 & 81.42 & 81.43 & 28.91 \\
\hline 120 & 76.29 & 76.29 & 76.32 & 76.32 & 76.33 & 76.31 & 30.85 \\
\hline 130 & 71.96 & 71.93 & 71.93 & 71.95 & 72.05 & 71.96 & 32.71 \\
\hline 140 & 67.81 & 67.79 & 67.84 & 67.82 & 67.84 & 67.82 & 34.71 \\
\hline 150 & 64.22 & 64.22 & 64.22 & 64.20 & 64.29 & 64.23 & 36.65 \\
\hline 160 & 60.59 & 60.59 & 60.51 & 60.56 & 60.54 & 60.56 & 38.88 \\
\hline 170 & 60.52 & 60.33 & 60.33 & 60.37 & 60.35 & 60.38 & 38.99 \\
\hline 180 & 55.96 & 55.99 & 55.95 & 55.98 & 55.99 & 55.98 & 42.06 \\
\hline 190 & 53.28 & 53.34 & 53.33 & 53.31 & 53.27 & 53.31 & 44.17 \\
\hline 200 & 51.82 & 51.87 & 51.86 & 51.85 & 51.87 & 51.85 & 45.40 \\
\hline 210 & 50.40 & 50.40 & 50.38 & 50.40 & 50.40 & 50.40 & 46.72 \\
\hline 220] & 49.47 & 49.46 & 49.46 & 49.47 & 49.48 & 49.47 & 47.59 \\
\hline 230 & 47.68 & 47.67 & 47.66 & 47.67 & 47.65 & 47.67 & 49.39 \\
\hline 240 & 46.40 & 46.20 & 46.21 & 46.26 & 46.23 & 46.26 & 50.89 \\
\hline
\end{tabular}


Table 6.54 Time and speedup of Ladder $215 \mathrm{C}(N)$ model with $N=22$ on an Intel Xeon Phi coprocessor with KMP_THREAD_AFFINITY set to compact

\begin{tabular}{|c|c|c|c|c|c|c|c|}
\hline Comp & Heisen & g Ladde & $5 C(22)$ & me in se & nds) & & \\
\hline Thread & Data 1 & Data 2 & Data 3 & Data 4 & Data 5 & Average & Speedup \\
\hline 1 & 537.74 & 537.83 & 534.50 & 539.50 & 539.20 & 537.75 & 1.00 \\
\hline 10 & 165.07 & 165.08 & 165.11 & 165.09 & 165.10 & 165.09 & 3.26 \\
\hline 20 & 91.31 & 91.30 & 91.35 & 91.35 & 91.30 & 91.32 & 5.89 \\
\hline 30 & 63.78 & 63.80 & 63.78 & 63.76 & 63.75 & 63.77 & 8.43 \\
\hline 40 & 49.33 & 49.34 & 49.37 & 49.35 & 49.35 & 49.35 & 10.90 \\
\hline 50 & 41.38 & 41.35 & 41.36 & 41.36 & 41.38 & 41.37 & 13.00 \\
\hline 60 & 35.26 & 35.23 & 35.22 & 35.24 & 35.22 & 35.23 & 15.26 \\
\hline 70 & 30.79 & 30.77 & 30.77 & 30.76 & 30.77 & 30.77 & 17.47 \\
\hline 80 & 26.79 & 26.75 & 26.77 & 26.74 & 26.74 & 26.76 & 20.10 \\
\hline 90 & 24.42 & 24.41 & 24.37 & 24.37 & 24.41 & 24.39 & 22.04 \\
\hline 100 & 22.01 & 21.99 & 22.00 & 21.98 & 21.97 & 21.99 & 24.46 \\
\hline 110 & 20.08 & 20.05 & 20.03 & 20.05 & 20.07 & 20.06 & 26.81 \\
\hline 120 & 18.72 & 18.66 & 18.68 & 18.69 & 18.66 & 18.68 & 28.79 \\
\hline 130 & 17.71 & 17.70 & 17.70 & 17.69 & 17.69 & 17.70 & 30.38 \\
\hline 140 & 16.58 & 16.56 & 16.58 & 16.58 & 16.56 & 16.57 & 32.45 \\
\hline 150 & 18.82 & 18.79 & 18.82 & 18.83 & 18.78 & 18.81 & 28.59 \\
\hline 160 & 18.57 & 18.53 & 18.54 & 18.55 & 18.53 & 18.54 & 29.00 \\
\hline 170 & 18.78 & 18.77 & 18.77 & 18.76 & 18.74 & 18.76 & 28.66 \\
\hline 180 & 18.78 & 18.76 & 18.77 & 18.77 & 18.77 & 18.77 & 28.65 \\
\hline 190 & 18.79 & 18.76 & 18.79 & 18.80 & 18.77 & 18.78 & 28.63 \\
\hline 200 & 19.25 & 19.23 & 19.24 & 19.21 & 19.23 & 19.23 & 27.96 \\
\hline 210 & 19.66 & 19.63 & 19.62 & 19.64 & 19.67 & 19.64 & 27.38 \\
\hline 220 & 20.17 & 20.18 & 20.13 & 20.18 & 20.19 & 20.17 & 26.66 \\
\hline 230 & 20.75 & 20.75 & 20.75 & 20.76 & 20.75 & 20.75 & 25.91 \\
\hline 240 & 21.58 & 21.25 & 21.24 & 21.25 & 21.24 & 21.31 & 25.23 \\
\hline
\end{tabular}


Table 6.55 Time and speedup of Ladder $215 \mathrm{C}(N)$ model with $N=20$ on an Intel Xeon Phi coprocessor with KMP_THREAD_AFFINITY set to compact

\begin{tabular}{|c|c|c|c|c|c|c|c|}
\hline Thread & Data 1 & Data 2 & Data 3 & Data 4 & Data 5 & Average & Speedup \\
\hline 1 & 135.34 & 136.35 & 134.47 & 134.58 & 134.60 & 135.07 & 1.00 \\
\hline 10 & 42.95 & 42.91 & 42.91 & 43.00 & 43.01 & 42.96 & 3.14 \\
\hline 20 & 24.18 & 24.15 & 24.13 & 24.11 & 24.12 & 24.14 & 5.60 \\
\hline 30 & 16.27 & 16.19 & 16.20 & 16.22 & 16.22 & 16.22 & 8.33 \\
\hline 40 & 13.84 & 13.79 & 13.80 & 13.81 & 13.80 & 13.81 & 9.78 \\
\hline 50 & 12.63 & 12.60 & 12.61 & 12.59 & 12.63 & 12.61 & 10.71 \\
\hline 60 & 11.84 & 11.82 & 11.79 & 11.80 & 11.83 & 11.82 & 11.43 \\
\hline 70 & 11.35 & 11.37 & 11.36 & 11.36 & 11.34 & 11.36 & 11.89 \\
\hline 80 & 10.95 & 10.94 & 10.93 & 10.93 & 10.94 & 10.94 & 12.35 \\
\hline 90 & 11.05 & 11.00 & 11.03 & 11.02 & 11.03 & 11.03 & 12.25 \\
\hline 100 & 11.18 & 11.16 & 11.17 & 11.16 & 11.16 & 11.16 & 12.10 \\
\hline 110 & 11.42 & 11.35 & 11.37 & 11.36 & 11.38 & 11.37 & 11.87 \\
\hline 120 & 11.63 & 11.62 & 11.63 & 11.62 & 11.60 & 11.62 & 11.62 \\
\hline 130 & 10.91 & 10.89 & 10.88 & 10.96 & 10.98 & 10.92 & 12.36 \\
\hline 140 & 11.27 & 11.29 & 11.27 & 11.41 & 11.38 & 11.32 & 11.93 \\
\hline 150 & 11.66 & 11.63 & 11.64 & 11.70 & 11.71 & 11.67 & 11.58 \\
\hline 160 & 12.04 & 12.04 & 12.05 & 12.12 & 12.09 & 12.07 & 11.19 \\
\hline 170 & 12.45 & 12.44 & 12.45 & 12.48 & 12.47 & 12.46 & 10.84 \\
\hline 180 & 12.90 & 12.89 & 12.89 & 12.98 & 12.94 & 12.92 & 10.45 \\
\hline 190 & 13.41 & 13.28 & 13.29 & 13.38 & 13.35 & 13.34 & 10.12 \\
\hline 200 & 13.80 & 13.75 & 13.75 & 13.75 & 13.79 & 13.77 & 9.81 \\
\hline 210 & 14.32 & 14.22 & 14.23 & 14.26 & 14.28 & 14.26 & 9.47 \\
\hline 220 & 14.80 & 14.70 & 14.70 & 14.71 & 14.83 & 14.75 & 9.16 \\
\hline 230 & 15.36 & 15.26 & 15.22 & 15.20 & 15.29 & 15.26 & 8.85 \\
\hline 240 & 16.09 & 15.66 & 15.65 & 15.65 & 15.73 & 15.75 & 8.57 \\
\hline
\end{tabular}


Table 6.56 Time and speedup of Ladder $215 \mathrm{C}(N)$ model with $N=18$ on an Intel Xeon Phi coprocessor with KMP_THREAD_AFFINITY set to compact

\begin{tabular}{|c|c|c|c|c|c|c|c|}
\hline \multicolumn{8}{|c|}{ Compact Heisenberg Ladder215C(18) (time in seconds) } \\
\hline Thread & Data 1 & Data 2 & Data 3 & Data 4 & Data 5 & Average & Speedup \\
\hline 1 & 32.99 & 32.94 & 33.02 & 32.97 & 33.13 & 33.01 & 1.00 \\
\hline 10 & 11.21 & 11.21 & 11.23 & 11.17 & 11.18 & 11.20 & 2.95 \\
\hline 20 & 8.24 & 8.21 & 8.23 & 8.19 & 8.19 & 8.21 & 4.02 \\
\hline 30 & 6.83 & 6.80 & 6.83 & 6.72 & 6.74 & 6.78 & 4.87 \\
\hline 40 & 6.61 & 6.58 & 6.61 & 6.58 & 6.56 & 6.59 & 5.01 \\
\hline 50 & 6.90 & 6.88 & 6.89 & 6.79 & 6.79 & 6.85 & 4.82 \\
\hline 60 & 7.00 & 6.98 & 6.99 & 6.99 & 6.97 & 6.98 & 4.73 \\
\hline 70 & 7.34 & 7.34 & 7.34 & 7.30 & 7.32 & 7.33 & 4.50 \\
\hline 80 & 7.75 & 7.74 & 7.73 & 7.72 & 7.71 & 7.73 & 4.27 \\
\hline 90 & 8.15 & 8.15 & 8.15 & 8.13 & 8.12 & 8.14 & 4.06 \\
\hline 100 & 8.61 & 8.61 & 8.64 & 8.58 & 8.55 & 8.60 & 3.84 \\
\hline 110 & 9.13 & 9.10 & 9.08 & 9.11 & 9.10 & 9.10 & 3.63 \\
\hline 120 & 9.57 & 9.58 & 9.58 & 9.58 & 9.56 & 9.57 & 3.45 \\
\hline 130 & 10.09 & 10.07 & 10.09 & 10.09 & 10.08 & 10.09 & 3.27 \\
\hline 140 & 10.62 & 10.60 & 10.62 & 10.61 & 10.61 & 10.61 & 3.11 \\
\hline 150 & 11.11 & 11.10 & 11.11 & 11.12 & 11.10 & 11.11 & 2.97 \\
\hline 160 & 11.64 & 11.62 & 11.63 & 11.66 & 11.64 & 11.64 & 2.84 \\
\hline 170 & 12.17 & 12.18 & 12.15 & 12.17 & 12.19 & 12.17 & 2.71 \\
\hline 180 & 12.77 & 12.72 & 12.74 & 12.74 & 12.72 & 12.74 & 2.59 \\
\hline 190 & 13.31 & 13.25 & 13.28 & 13.26 & 13.29 & 13.28 & 2.49 \\
\hline 200 & 13.85 & 13.85 & 13.87 & 13.84 & 13.82 & 13.85 & 2.38 \\
\hline 210 & 14.42 & 14.41 & 14.42 & 14.41 & 14.43 & 14.42 & 2.29 \\
\hline 220 & 14.97 & 14.98 & 14.97 & 14.98 & 14.99 & 14.98 & 2.20 \\
\hline 230 & 15.59 & 15.57 & 15.58 & 15.57 & 15.59 & 15.58 & 2.12 \\
\hline 240 & 16.49 & 16.17 & 16.18 & 16.17 & 16.22 & 16.25 & 2.03 \\
\hline
\end{tabular}


Table 6.57 Time and speedup of Ladder $215 \mathrm{C}(N)$ model with $N=16$ on an Intel Xeon Phi coprocessor with KMP_THREAD_AFFINITY set to compact

\begin{tabular}{|c|c|c|c|c|c|c|c|}
\hline Thread & Data 1 & Data 2 & Data 3 & Data 4 & Data 5 & Average & Speedup \\
\hline 1 & 8.28 & 8.23 & 8.27 & 8.27 & 8.29 & 8.27 & 1.00 \\
\hline 10 & 4.17 & 4.15 & 4.16 & 4.28 & 4.24 & 4.20 & 1.97 \\
\hline 20 & 3.87 & 3.86 & 3.86 & 3.98 & 3.95 & 3.90 & 2.12 \\
\hline 30 & 4.04 & 4.07 & 4.04 & 4.17 & 4.13 & 4.09 & 2.02 \\
\hline 40 & 4.38 & 4.36 & 4.37 & 4.46 & 4.43 & 4.40 & 1.88 \\
\hline 50 & 4.81 & 4.82 & 4.82 & 4.87 & 4.86 & 4.84 & 1.71 \\
\hline 60 & 5.25 & 5.23 & 5.21 & 5.31 & 5.28 & 5.25 & 1.57 \\
\hline 70 & 5.70 & 5.70 & 5.68 & 5.78 & 5.72 & 5.72 & 1.45 \\
\hline 80 & 6.16 & 6.13 & 6.14 & 6.24 & 6.20 & 6.18 & 1.34 \\
\hline 90 & 6.75 & 6.74 & 6.76 & 6.79 & 6.79 & 6.77 & 1.22 \\
\hline 100 & 7.25 & 7.23 & 7.23 & 7.31 & 7.31 & 7.27 & 1.14 \\
\hline 110 & 7.84 & 7.83 & 7.84 & 7.87 & 7.85 & 7.84 & 1.05 \\
\hline 120 & 8.42 & 8.39 & 8.39 & 8.46 & 8.44 & 8.42 & 0.98 \\
\hline 130 & 9.03 & 9.01 & 9.01 & 9.09 & 9.07 & 9.04 & 0.91 \\
\hline 140 & 9.62 & 9.57 & 9.59 & 9.70 & 9.69 & 9.64 & 0.86 \\
\hline 150 & 10.24 & 10.19 & 10.21 & 10.31 & 10.29 & 10.25 & 0.81 \\
\hline 160 & 10.90 & 10.85 & 10.85 & 11.00 & 10.97 & 10.91 & 0.76 \\
\hline 170 & 11.61 & 11.56 & 11.57 & 11.69 & 11.66 & 11.62 & 0.71 \\
\hline 180 & 12.32 & 12.26 & 12.27 & 12.45 & 12.35 & 12.33 & 0.67 \\
\hline 190 & 13.06 & 13.00 & 13.02 & 13.20 & 13.13 & 13.08 & 0.63 \\
\hline 200 & 13.86 & 13.78 & 13.79 & 13.99 & 13.91 & 13.87 & 0.60 \\
\hline 210 & 14.83 & 14.74 & 14.75 & 14.95 & 14.85 & 14.83 & 0.56 \\
\hline 220 & 15.63 & 15.48 & 15.48 & 15.76 & 15.70 & 15.61 & 0.53 \\
\hline 230 & 16.66 & 16.53 & 16.54 & 16.80 & 16.76 & 16.66 & 0.50 \\
\hline 240 & 17.92 & 17.45 & 17.44 & 18.17 & 17.69 & 17.73 & 0.47 \\
\hline
\end{tabular}


Table 6.58 Time and speedup of Ladder $215 \mathrm{C}(N)$ model with $N=26$ on an Intel Xeon Phi coprocessor with KMP_THREAD_AFFINITY set to balanced

\begin{tabular}{|c|c|c|c|c|c|c|c|}
\hline Thread & Data 1 & Data 2 & Data 3 & Data 4 & Data 5 & Average & Speedup \\
\hline 1 & 5546.54 & 5544.92 & 5545.24 & 5546.00 & 5545.56 & 5545.65 & 1.00 \\
\hline 10 & 1355.22 & 1366.78 & 1366.92 & 1361.38 & 1366.33 & 1363.33 & 4.07 \\
\hline 20 & 733.29 & 783.94 & 785.89 & 760.13 & 784.44 & 769.54 & 7.21 \\
\hline 30 & 534.78 & 536.15 & 536.44 & 546.62 & 540.03 & 538.81 & 10.29 \\
\hline 40 & 407.68 & 426.61 & 417.82 & 417.13 & 420.44 & 417.94 & 13.27 \\
\hline 50 & 345.18 & 351.43 & 348.59 & 349.43 & 348.13 & 348.55 & 15.91 \\
\hline 60 & 300.32 & 300.42 & 301.99 & 300.92 & 301.23 & 300.98 & 18.43 \\
\hline 70 & 266.42 & 269.05 & 270.08 & 268.44 & 269.23 & 268.64 & 20.64 \\
\hline 80 & 236.94 & 234.77 & 236.32 & 234.40 & 237.89 & 236.06 & 23.49 \\
\hline 90 & 216.77 & 218.17 & 216.58 & 217.00 & 216.49 & 217.00 & 25.56 \\
\hline 100 & 194.73 & 198.56 & 196.94 & 197.33 & 196.34 & 196.78 & 28.18 \\
\hline 110 & 177.39 & 178.04 & 179.52 & 178.55 & 178.36 & 178.37 & 31.09 \\
\hline 120 & 168.43 & 168.10 & 170.22 & 168.50 & 168.18 & 168.69 & 32.88 \\
\hline 130 & 161.37 & 164.04 & 163.84 & 161.45 & 161.66 & 162.47 & 34.13 \\
\hline 140 & 151.37 & 151.88 & 154.45 & 151.32 & 152.03 & 152.21 & 36.43 \\
\hline 150 & 145.00 & 144.37 & 145.62 & 145.22 & 145.55 & 145.15 & 38.21 \\
\hline 160 & 138.49 & 138.14 & 136.85 & 134.97 & 136.34 & 136.96 & 40.49 \\
\hline 170 & 133.04 & 132.66 & 133.86 & 134.47 & 133.36 & 133.48 & 41.55 \\
\hline 180 & 127.21 & 128.96 & 128.81 & 127.76 & 128.00 & 128.15 & 43.27 \\
\hline 190 & 120.98 & 122.09 & 123.21 & 122.44 & 122.99 & 122.34 & 45.33 \\
\hline 200 & 116.68 & 117.17 & 116.97 & 117.67 & 116.83 & 117.07 & 47.37 \\
\hline 210 & 113.22 & 112.88 & 111.69 & 113.32 & 113.43 & 112.91 & 49.12 \\
\hline 220 & 110.07 & 110.34 & 110.78 & 109.27 & 109.45 & 109.98 & 50.42 \\
\hline 230 & 97.60 & 97.52 & 97.83 & 97.46 & 97.89 & 97.66 & 56.79 \\
\hline 240 & 95.49 & 95.50 & 94.96 & 94.55 & 95.84 & 95.27 & 58.21 \\
\hline
\end{tabular}


Table 6.59 Time and speedup of Ladder $215 \mathrm{C}(N)$ model with $N=24$ on an Intel Xeon Phi coprocessor with KMP_THREAD_AFFINITY set to balanced

\begin{tabular}{|c|c|c|c|c|c|c|c|}
\hline Thread & Data 1 & Data 2 & Data 3 & Data 4 & Data 5 & Average & Speedup \\
\hline 1 & 2351.55 & 2355.30 & 2354.26 & 2355.33 & 2355.14 & 2354.32 & 1.00 \\
\hline 10 & 537.24 & 529.52 & 529.76 & 549.94 & 563.11 & 541.91 & 4.34 \\
\hline 20 & 333.32 & 327.72 & 310.97 & 301.85 & 339.03 & 322.58 & 7.30 \\
\hline 30 & 242.93 & 233.59 & 225.93 & 237.99 & 239.96 & 236.08 & 9.97 \\
\hline 40 & 176.10 & 180.54 & 181.38 & 186.06 & 183.42 & 181.50 & 12.97 \\
\hline 50 & 152.09 & 153.17 & 145.13 & 145.77 & 153.33 & 149.90 & 15.71 \\
\hline 60 & 128.39 & 130.69 & 129.76 & 129.97 & 130.98 & 129.96 & 18.12 \\
\hline 70 & 114.72 & 116.04 & 116.52 & 118.53 & 113.49 & 115.86 & 20.32 \\
\hline 80 & 102.94 & 97.58 & 106.08 & 99.15 & 100.05 & 101.16 & 23.27 \\
\hline 90 & 95.64 & 96.18 & 92.26 & 96.71 & 92.37 & 94.63 & 24.88 \\
\hline 100 & 85.61 & 85.58 & 88.32 & 88.18 & 87.59 & 87.06 & 27.04 \\
\hline 110 & 80.90 & 80.16 & 81.74 & 81.25 & 81.11 & 81.03 & 29.05 \\
\hline 120 & 76.35 & 75.05 & 78.04 & 75.77 & 76.31 & 76.30 & 30.85 \\
\hline 130 & 75.38 & 72.23 & 74.20 & 71.48 & 73.86 & 73.43 & 32.06 \\
\hline 140 & 70.20 & 69.03 & 68.77 & 68.37 & 67.91 & 68.85 & 34.19 \\
\hline 150 & 65.23 & 65.65 & 65.74 & 64.29 & 65.94 & 65.37 & 36.01 \\
\hline 160 & 61.91 & 60.72 & 61.30 & 60.88 & 61.82 & 61.32 & 38.39 \\
\hline 170 & 61.54 & 61.84 & 59.33 & 61.22 & 61.11 & 61.01 & 38.59 \\
\hline 180 & 57.56 & 57.97 & 58.26 & 58.38 & 58.17 & 58.07 & 40.54 \\
\hline 190 & 57.18 & 56.40 & 56.54 & 56.96 & 56.54 & 56.72 & 41.51 \\
\hline 200 & 54.77 & 55.38 & 54.08 & 54.95 & 53.77 & 54.59 & 43.13 \\
\hline 210 & 51.89 & 52.10 & 52.22 & 51.95 & 51.87 & 52.01 & 45.27 \\
\hline 220] & 51.34 & 52.11 & 52.33 & 51.61 & 51.06 & 51.69 & 45.55 \\
\hline 230 & 47.56 & 47.68 & 47.46 & 47.55 & 47.52 & 47.55 & 49.51 \\
\hline 240 & 46.30 & 46.18 & 46.25 & 46.21 & 46.24 & 46.24 & 50.92 \\
\hline
\end{tabular}


Table 6.60 Time and speedup of Ladder $215 \mathrm{C}(N)$ model with $N=22$ on an Intel Xeon Phi coprocessor with KMP_THREAD_AFFINITY set to balanced

\begin{tabular}{|c|c|c|c|c|c|c|c|}
\hline Thread & Data 1 & Data 2 & Data 3 & Data 4 & Data 5 & Average & Speedup \\
\hline 1 & 537.74 & 537.83 & 534.50 & 539.50 & 539.20 & 537.75 & 1.00 \\
\hline 10 & 119.87 & 124.44 & 132.22 & 123.49 & 136.63 & 127.33 & 4.22 \\
\hline 20 & 70.99 & 72.28 & 74.65 & 74.18 & 72.36 & 72.90 & 7.38 \\
\hline 30 & 49.90 & 51.60 & 53.15 & 52.06 & 55.15 & 52.37 & 10.27 \\
\hline 40 & 39.89 & 40.85 & 40.17 & 40.40 & 41.31 & 40.52 & 13.27 \\
\hline 50 & 33.65 & 33.39 & 34.16 & 32.61 & 34.34 & 33.63 & 15.99 \\
\hline 60 & 31.04 & 31.49 & 29.29 & 29.04 & 29.79 & 30.13 & 17.85 \\
\hline 70 & 26.49 & 26.26 & 27.88 & 28.38 & 26.51 & 27.10 & 19.84 \\
\hline 80 & 23.16 & 24.76 & 23.13 & 24.80 & 23.45 & 23.86 & 22.54 \\
\hline 90 & 21.68 & 22.94 & 21.55 & 22.96 & 21.57 & 22.14 & 24.29 \\
\hline 100 & 19.89 & 20.81 & 19.97 & 20.21 & 20.81 & 20.34 & 26.44 \\
\hline 110 & 19.67 & 19.42 & 18.54 & 18.50 & 19.40 & 19.11 & 28.14 \\
\hline 120 & 18.18 & 18.22 & 18.44 & 18.19 & 18.10 & 18.23 & 29.50 \\
\hline 130 & 17.60 & 17.82 & 17.52 & 17.79 & 17.66 & 17.68 & 30.42 \\
\hline 140 & 17.06 & 16.53 & 16.77 & 16.94 & 16.64 & 16.79 & 32.03 \\
\hline 150 & 63.28 & 63.66 & 63.72 & 63.87 & 63.62 & 63.63 & 8.45 \\
\hline 160 & 70.41 & 70.75 & 71.16 & 70.47 & 70.71 & 70.70 & 7.61 \\
\hline 170 & 80.32 & 80.41 & 80.48 & 80.50 & 80.35 & 80.41 & 6.69 \\
\hline 180 & 90.98 & 91.84 & 91.91 & 91.72 & 91.72 & 91.63 & 5.87 \\
\hline 190 & 90.99 & 90.64 & 90.74 & 91.18 & 90.64 & 90.84 & 5.92 \\
\hline 200 & 113.68 & 114.41 & 113.43 & 113.72 & 113.40 & 113.73 & 4.73 \\
\hline 210 & 122.18 & 123.51 & 123.20 & 124.56 & 123.25 & 123.34 & 4.36 \\
\hline 220 & 116.07 & 115.84 & 116.35 & 115.99 & 115.40 & 115.93 & 4.64 \\
\hline 230 & 129.81 & 129.72 & 130.59 & 129.37 & 130.24 & 129.95 & 4.14 \\
\hline 240 & 142.34 & 142.49 & 141.47 & 141.47 & 142.28 & 142.01 & 3.79 \\
\hline
\end{tabular}


Table 6.61 Time and speedup of Ladder $215 \mathrm{C}(N)$ model with $N=20$ on an Intel Xeon Phi coprocessor with KMP_THREAD_AFFINITY set to balanced

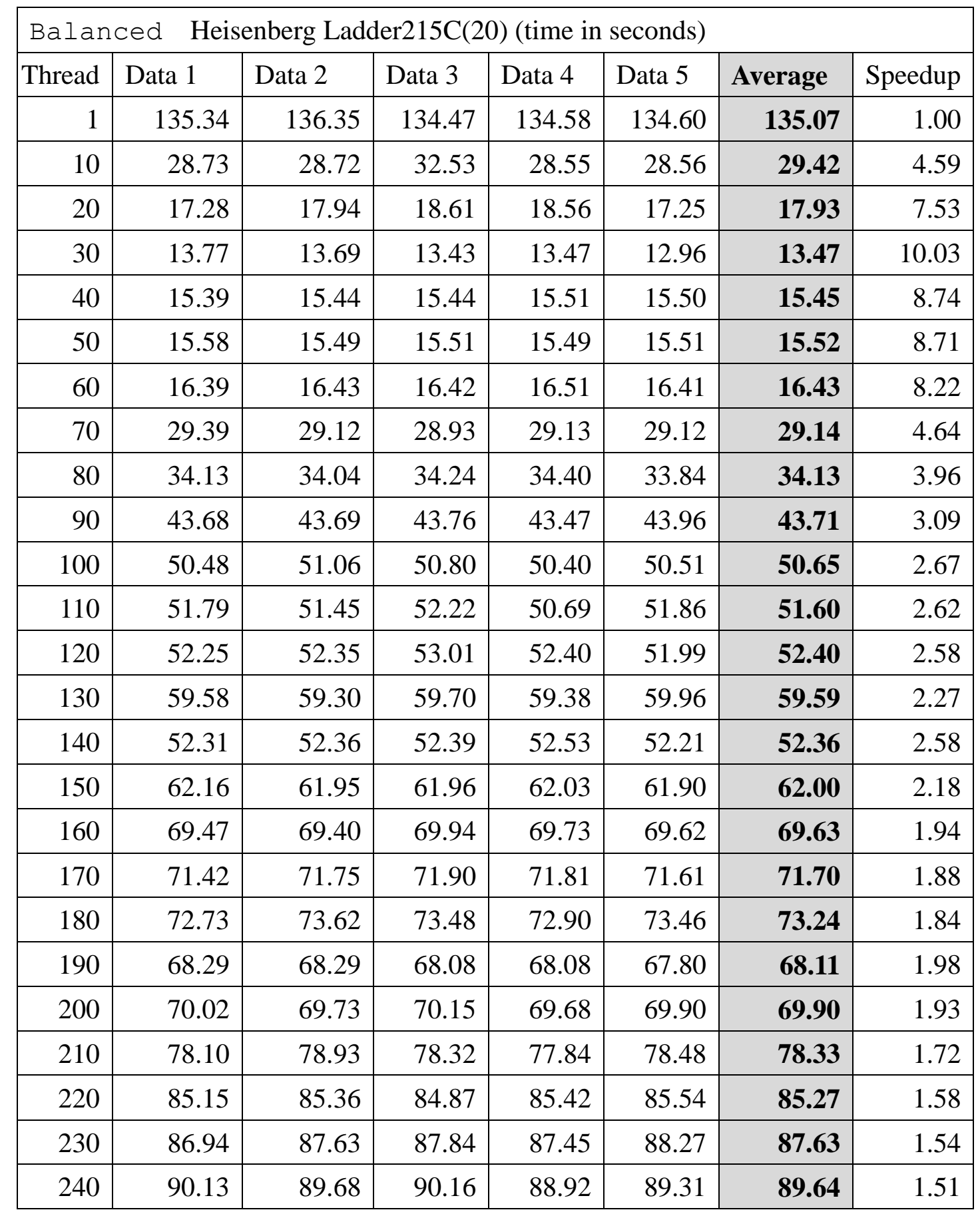


Table 6.62 Time and speedup of Ladder $215 \mathrm{C}(N)$ model with $N=18$ on an Intel Xeon Phi coprocessor with KMP_THREAD_AFFINITY set to balanced

\begin{tabular}{|c|c|c|c|c|c|c|c|}
\hline Thread & Data 1 & Data 2 & Data 3 & Data 4 & Data 5 & Average & Speedup \\
\hline 1 & 32.99 & 32.94 & 33.02 & 32.97 & 33.13 & 33.01 & 1.00 \\
\hline 10 & 8.85 & 9.58 & 8.84 & 8.83 & 8.99 & 9.02 & 3.66 \\
\hline 20 & 7.50 & 7.46 & 7.46 & 7.34 & 7.26 & 7.41 & 4.46 \\
\hline 30 & 5.74 & 5.76 & 5.80 & 5.66 & 5.71 & 5.73 & 5.76 \\
\hline 40 & 6.53 & 6.56 & 6.57 & 6.55 & 6.60 & 6.56 & 5.03 \\
\hline 50 & 7.98 & 8.01 & 8.05 & 8.08 & 8.03 & 8.03 & 4.11 \\
\hline 60 & 10.26 & 10.04 & 10.08 & 10.15 & 10.22 & 10.15 & 3.25 \\
\hline 70 & 15.17 & 15.57 & 15.57 & 15.44 & 15.55 & 15.46 & 2.13 \\
\hline 80 & 16.67 & 16.55 & 16.50 & 16.61 & 16.59 & 16.58 & 1.99 \\
\hline 90 & 17.73 & 17.80 & 18.00 & 17.82 & 18.01 & 17.87 & 1.85 \\
\hline 100 & 19.55 & 19.45 & 19.36 & 19.33 & 19.68 & 19.47 & 1.69 \\
\hline 110 & 21.33 & 21.36 & 21.20 & 21.31 & 21.32 & 21.30 & 1.55 \\
\hline 120 & 22.54 & 22.61 & 22.28 & 22.54 & 22.75 & 22.54 & 1.46 \\
\hline 130 & 24.46 & 24.48 & 24.68 & 24.70 & 24.67 & 24.60 & 1.34 \\
\hline 140 & 26.48 & 26.63 & 26.53 & 26.65 & 26.60 & 26.58 & 1.24 \\
\hline 150 & 28.53 & 28.27 & 28.26 & 28.39 & 28.62 & 28.41 & 1.16 \\
\hline 160 & 30.55 & 30.69 & 30.37 & 30.61 & 30.41 & 30.53 & 1.08 \\
\hline 170 & 32.18 & 32.50 & 32.20 & 32.48 & 32.44 & 32.36 & 1.02 \\
\hline 180 & 33.71 & 33.94 & 33.84 & 33.81 & 33.74 & 33.81 & 0.98 \\
\hline 190 & 34.48 & 34.40 & 34.33 & 34.33 & 34.23 & 34.35 & 0.96 \\
\hline 200 & 35.81 & 35.91 & 35.67 & 36.18 & 35.93 & 35.90 & 0.92 \\
\hline 210 & 38.19 & 38.29 & 38.34 & 38.19 & 38.14 & 38.23 & 0.86 \\
\hline 220 & 40.69 & 41.19 & 40.59 & 40.98 & 41.12 & 40.91 & 0.81 \\
\hline 230 & 43.37 & 43.27 & 43.20 & 43.14 & 43.11 & 43.22 & 0.76 \\
\hline 240 & 45.59 & 45.30 & 45.36 & 45.44 & 45.36 & 45.41 & 0.73 \\
\hline
\end{tabular}


Table 6.63 Time and speedup of Ladder $215 \mathrm{C}(N)$ model with $N=16$ on an Intel Xeon Phi coprocessor with KMP_THREAD_AFFINITY set to balanced

\begin{tabular}{|c|c|c|c|c|c|c|c|}
\hline Thread & Data 1 & Data 2 & Data 3 & Data 4 & Data 5 & Average & Speedup \\
\hline 1 & 8.28 & 8.23 & 8.27 & 8.27 & 8.29 & 8.27 & 1.00 \\
\hline 10 & 3.37 & 3.43 & 3.53 & 3.36 & 3.46 & 3.43 & 2.41 \\
\hline 20 & 3.52 & 3.56 & 3.57 & 3.52 & 3.56 & 3.55 & 2.33 \\
\hline 30 & 4.27 & 4.28 & 4.28 & 4.58 & 4.27 & 4.34 & 1.91 \\
\hline 40 & 5.36 & 5.38 & 5.35 & 5.73 & 5.40 & 5.44 & 1.52 \\
\hline 50 & 6.86 & 6.86 & 6.88 & 6.92 & 6.87 & 6.88 & 1.20 \\
\hline 60 & 8.98 & 8.88 & 8.86 & 8.88 & 8.87 & 8.89 & 0.93 \\
\hline 70 & 11.01 & 11.02 & 11.12 & 11.12 & 11.20 & 11.09 & 0.75 \\
\hline 80 & 12.34 & 12.24 & 12.40 & 12.22 & 12.37 & 12.31 & 0.67 \\
\hline 90 & 13.60 & 13.68 & 13.77 & 13.65 & 13.72 & 13.68 & 0.60 \\
\hline 100 & 15.05 & 14.99 & 15.04 & 15.14 & 15.61 & 15.17 & 0.55 \\
\hline 110 & 16.68 & 16.86 & 16.67 & 16.61 & 17.43 & 16.85 & 0.49 \\
\hline 120 & 18.01 & 17.98 & 18.04 & 17.98 & 18.03 & 18.01 & 0.46 \\
\hline 130 & 19.80 & 19.85 & 19.87 & 19.70 & 19.75 & 19.79 & 0.42 \\
\hline 140 & 21.51 & 21.50 & 21.57 & 21.68 & 21.63 & 21.58 & 0.38 \\
\hline 150 & 23.15 & 23.03 & 23.19 & 23.07 & 23.33 & 23.15 & 0.36 \\
\hline 160 & 25.09 & 25.02 & 25.10 & 25.23 & 25.41 & 25.17 & 0.33 \\
\hline 170 & 26.83 & 26.69 & 26.89 & 26.83 & 26.93 & 26.83 & 0.31 \\
\hline 180 & 28.57 & 28.54 & 28.52 & 28.64 & 28.60 & 28.57 & 0.29 \\
\hline 190 & 30.11 & 30.13 & 30.20 & 30.17 & 30.16 & 30.15 & 0.27 \\
\hline 200 & 31.25 & 31.28 & 31.20 & 31.16 & 31.24 & 31.23 & 0.26 \\
\hline 210 & 33.76 & 33.78 & 33.63 & 33.80 & 33.78 & 33.75 & 0.24 \\
\hline 220 & 36.01 & 36.24 & 36.23 & 36.05 & 36.14 & 36.14 & 0.23 \\
\hline 230 & 38.14 & 38.00 & 38.10 & 38.11 & 38.11 & 38.09 & 0.22 \\
\hline 240 & 40.23 & 39.83 & 39.93 & 39.83 & 39.92 & 39.95 & 0.21 \\
\hline
\end{tabular}




\subsubsection{Heisenberg Ladder 215S on Xeon Phi}

Table 6.64 Time and speedup of Ladder $215 \mathrm{~S}(N)$ model with $N=26$ on an Intel Xeon

Phi coprocessor with KMP_THREAD_AFFINITY set to scatter

\begin{tabular}{|c|c|c|c|c|c|c|c|}
\hline \multicolumn{8}{|c|}{ Scatter Heisenberg Ladder215S(26) (time in seconds) } \\
\hline Thread & Data 1 & Data 2 & Data 3 & Data 4 & Data 5 & Average & Speedup \\
\hline 1 & - & - & - & - & - & - & - \\
\hline 10 & - & - & - & - & - & - & - \\
\hline 20 & - & - & - & - & - & - & - \\
\hline 30 & - & - & - & - & - & - & - \\
\hline 40 & - & - & - & - & - & - & - \\
\hline 50 & - & - & - & - & - & - & - \\
\hline 60 & - & - & - & - & - & - & - \\
\hline 70 & - & - & - & - & - & - & - \\
\hline 80 & - & - & - & - & - & - & - \\
\hline 90 & - & - & - & - & - & - & - \\
\hline 100 & - & - & - & - & - & - & - \\
\hline 110 & - & - & - & - & - & - & - \\
\hline 120 & - & - & - & - & - & - & - \\
\hline 130 & - & - & - & - & - & - & - \\
\hline 140 & - & - & - & - & - & - & - \\
\hline 150 & - & - & - & - & - & - & - \\
\hline 160 & - & - & - & - & - & - & - \\
\hline 170 & - & - & - & - & - & - & - \\
\hline 180 & - & - & - & - & - & - & - \\
\hline 190 & - & - & - & - & - & - & - \\
\hline 200 & - & - & - & - & - & - & - \\
\hline 210 & - & - & - & - & - & - & - \\
\hline 220 & - & - & - & - & - & - & - \\
\hline 230 & - & - & - & - & - & - & - \\
\hline 240 & - & - & - & - & - & - & - \\
\hline
\end{tabular}


Table 6.65 Time and speedup of Ladder $215 \mathrm{~S}(N)$ model with $N=24$ on an Intel Xeon Phi coprocessor with KMP_THREAD_AFFINITY set to scatter

\begin{tabular}{|c|c|c|c|c|c|c|c|}
\hline \multicolumn{8}{|c|}{ Scatter Heisenberg Ladder215S(24) (time in seconds) } \\
\hline Thread & Data 1 & Data 2 & Data 3 & Data 4 & Data 5 & Average & Speedup \\
\hline 1 & 2354.56 & 2355.89 & 2356.18 & 2355.27 & 2354.79 & 2355.34 & 1.00 \\
\hline 10 & 554.12 & 595.99 & 570.68 & 558.85 & 572.24 & 570.38 & 4.13 \\
\hline 20 & 308.48 & 325.02 & 325.96 & 317.64 & 301.21 & 315.66 & 7.46 \\
\hline 30 & 236.02 & 233.41 & 235.03 & 240.10 & 214.45 & 231.80 & 10.16 \\
\hline 40 & 175.22 & 183.40 & 183.80 & 179.79 & 179.45 & 180.33 & 13.06 \\
\hline 50 & 148.45 & 148.29 & 149.98 & 148.17 & 150.67 & 149.11 & 15.80 \\
\hline 60 & 130.43 & 129.77 & 129.99 & 131.34 & 129.88 & 130.28 & 18.08 \\
\hline 70 & 114.94 & 114.38 & 115.27 & 114.81 & 115.73 & 115.02 & 20.48 \\
\hline 80 & 100.23 & 102.51 & 99.64 & 101.05 & 101.68 & 101.02 & 23.32 \\
\hline 90 & 93.27 & 92.66 & 97.70 & 93.19 & 95.57 & 94.48 & 24.93 \\
\hline 100 & 82.71 & 83.02 & 82.50 & 88.32 & 85.07 & 84.32 & 27.93 \\
\hline 110 & 78.38 & 77.63 & 82.14 & 81.34 & 71.50 & 78.20 & 30.12 \\
\hline 120 & 78.01 & 77.96 & 77.63 & 78.13 & 77.51 & 77.85 & 30.26 \\
\hline 130 & 73.96 & 74.96 & 73.87 & 74.31 & 73.14 & 74.05 & 31.81 \\
\hline 140 & 70.14 & 69.13 & 69.96 & 69.04 & 69.00 & 69.46 & 33.91 \\
\hline 150 & 68.10 & 68.70 & 66.76 & 66.11 & 66.94 & 67.32 & 34.99 \\
\hline 160 & 65.98 & 65.49 & 62.91 & 64.61 & 63.29 & 64.46 & 36.54 \\
\hline 170 & 60.96 & 63.88 & 67.24 & 64.57 & 62.74 & 63.88 & 36.87 \\
\hline 180 & 62.01 & 61.07 & 61.73 & 60.64 & 60.94 & 61.28 & 38.44 \\
\hline 190 & 59.57 & 60.19 & 60.26 & 59.29 & 60.01 & 59.87 & 39.34 \\
\hline 200 & 58.33 & 58.37 & 57.84 & 58.29 & 58.16 & 58.20 & 40.47 \\
\hline 210 & 54.51 & 54.59 & 54.53 & 54.51 & 54.69 & 54.57 & 43.17 \\
\hline 220 & 53.32 & 53.51 & 53.54 & 53.30 & 53.81 & 53.50 & 44.03 \\
\hline 230 & 50.51 & 50.63 & 50.64 & 50.50 & 50.74 & 50.60 & 46.54 \\
\hline 240 & 49.62 & 49.58 & 49.65 & 49.71 & 49.67 & 49.65 & 47.44 \\
\hline
\end{tabular}


Table 6.66 Time and speedup of Ladder $215 \mathrm{~S}(N)$ model with $N=22$ on an Intel Xeon Phi coprocessor with KMP_THREAD_AFFINITY set to scatter

\begin{tabular}{|c|c|c|c|c|c|c|c|}
\hline \multicolumn{8}{|c|}{ Scatter Heisenberg Ladder215S(22) (time in seconds) } \\
\hline Thread & Data 1 & Data 2 & Data 3 & Data 4 & Data 5 & Average & Speedup \\
\hline 1 & 534.98 & 539.45 & 539.39 & 539.47 & 539.60 & 538.58 & 1.00 \\
\hline 10 & 127.11 & 133.64 & 132.48 & 123.07 & 119.89 & 127.24 & 4.23 \\
\hline 20 & 79.67 & 79.76 & 76.60 & 82.03 & 73.36 & 78.29 & 6.88 \\
\hline 30 & 48.19 & 55.14 & 53.75 & 56.84 & 54.30 & 53.64 & 10.04 \\
\hline 40 & 40.99 & 40.78 & 40.95 & 42.57 & 39.91 & 41.04 & 13.12 \\
\hline 50 & 33.59 & 33.82 & 33.47 & 34.45 & 33.16 & 33.70 & 15.98 \\
\hline 60 & 28.71 & 29.40 & 28.64 & 29.14 & 28.47 & 28.87 & 18.65 \\
\hline 70 & 26.17 & 27.49 & 25.78 & 26.12 & 25.75 & 26.26 & 20.51 \\
\hline 80 & 22.80 & 24.32 & 22.67 & 22.97 & 23.31 & 23.21 & 23.20 \\
\hline 90 & 21.37 & 22.56 & 21.29 & 21.41 & 22.53 & 21.83 & 24.67 \\
\hline 100 & 20.67 & 20.69 & 20.60 & 19.91 & 19.72 & 20.32 & 26.51 \\
\hline 110 & 19.01 & 19.05 & 18.93 & 19.14 & 19.25 & 19.08 & 28.23 \\
\hline 120 & 17.04 & 17.99 & 18.36 & 18.72 & 18.21 & 18.07 & 29.81 \\
\hline 130 & 17.38 & 17.43 & 17.55 & 17.28 & 17.48 & 17.43 & 30.91 \\
\hline 140 & 16.53 & 16.61 & 16.71 & 16.44 & 16.64 & 16.59 & 32.47 \\
\hline 150 & 18.98 & 19.03 & 19.10 & 19.48 & 19.34 & 19.19 & 28.07 \\
\hline 160 & 18.33 & 19.29 & 19.10 & 19.53 & 19.65 & 19.18 & 28.08 \\
\hline 170 & 19.35 & 19.83 & 19.89 & 19.63 & 19.87 & 19.71 & 27.32 \\
\hline 180 & 20.16 & 20.05 & 20.04 & 19.90 & 19.92 & 20.01 & 26.91 \\
\hline 190 & 20.43 & 20.56 & 20.48 & 20.29 & 20.33 & 20.42 & 26.38 \\
\hline 200 & 20.90 & 21.33 & 20.95 & 20.93 & 20.86 & 20.99 & 25.65 \\
\hline 210 & 21.28 & 21.64 & 21.38 & 21.32 & 21.54 & 21.43 & 25.13 \\
\hline 220 & 21.70 & 21.86 & 21.75 & 21.80 & 21.74 & 21.77 & 24.74 \\
\hline 230 & 22.17 & 22.29 & 22.28 & 22.22 & 22.24 & 22.24 & 24.22 \\
\hline 240 & 22.95 & 22.78 & 22.85 & 22.81 & 22.80 & 22.84 & 23.58 \\
\hline
\end{tabular}


Table 6.67 Time and speedup of Ladder $215 \mathrm{~S}(N)$ model with $N=20$ on an Intel Xeon Phi coprocessor with KMP_THREAD_AFFINITY set to scatter

\begin{tabular}{|c|c|c|c|c|c|c|c|}
\hline Thread & Data 1 & Data 2 & Data 3 & Data 4 & Data 5 & Average & Speedup \\
\hline 1 & 134.13 & 134.28 & 134.34 & 134.30 & 134.31 & 134.27 & 1.00 \\
\hline 10 & 28.71 & 28.65 & 28.64 & 28.49 & 28.44 & 28.59 & 4.70 \\
\hline 20 & 17.27 & 17.21 & 17.25 & 18.45 & 18.50 & 17.73 & 7.57 \\
\hline 30 & 13.06 & 12.94 & 13.49 & 13.75 & 13.73 & 13.39 & 10.02 \\
\hline 40 & 11.83 & 11.84 & 11.41 & 11.73 & 11.74 & 11.71 & 11.47 \\
\hline 50 & 10.81 & 10.81 & 10.51 & 10.80 & 10.50 & 10.69 & 12.56 \\
\hline 60 & 10.16 & 10.14 & 10.16 & 10.18 & 10.15 & 10.16 & 13.22 \\
\hline 70 & 9.95 & 9.97 & 9.92 & 9.90 & 9.91 & 9.93 & 13.52 \\
\hline 80 & 9.65 & 9.81 & 9.62 & 9.65 & 9.59 & 9.66 & 13.90 \\
\hline 90 & 9.92 & 10.01 & 9.92 & 9.89 & 9.88 & 9.92 & 13.53 \\
\hline 100 & 10.00 & 10.07 & 9.99 & 10.12 & 9.98 & 10.03 & 13.39 \\
\hline 110 & 10.26 & 10.25 & 10.18 & 10.29 & 10.18 & 10.23 & 13.12 \\
\hline 120 & 10.55 & 10.54 & 10.54 & 10.53 & 10.51 & 10.54 & 12.74 \\
\hline 130 & 9.80 & 9.66 & 9.77 & 9.87 & 9.86 & 9.79 & 13.71 \\
\hline 140 & 10.16 & 10.24 & 10.37 & 10.23 & 10.27 & 10.26 & 13.09 \\
\hline 150 & 10.63 & 10.70 & 10.67 & 10.71 & 10.72 & 10.69 & 12.56 \\
\hline 160 & 11.04 & 11.26 & 11.14 & 11.32 & 11.14 & 11.18 & 12.01 \\
\hline 170 & 11.50 & 11.73 & 11.52 & 11.71 & 11.54 & 11.60 & 11.58 \\
\hline 180 & 11.93 & 11.94 & 11.88 & 12.02 & 11.99 & 11.95 & 11.24 \\
\hline 190 & 12.61 & 12.60 & 12.70 & 12.66 & 12.66 & 12.65 & 10.62 \\
\hline 200 & 12.98 & 13.00 & 13.01 & 13.04 & 13.04 & 13.02 & 10.32 \\
\hline 210 & 13.51 & 13.45 & 13.48 & 13.50 & 13.57 & 13.50 & 9.94 \\
\hline 220 & 13.89 & 13.89 & 13.91 & 13.90 & 13.99 & 13.92 & 9.65 \\
\hline 230 & 14.36 & 14.28 & 14.31 & 14.34 & 14.46 & 14.35 & 9.36 \\
\hline 240 & 15.14 & 14.74 & 14.81 & 14.83 & 14.95 & 14.89 & 9.01 \\
\hline
\end{tabular}


Table 6.68 Time and speedup of Ladder $215 \mathrm{~S}(N)$ model with $N=18$ on an Intel Xeon Phi coprocessor with KMP_THREAD_AFFINITY set to scatter

\begin{tabular}{|c|c|c|c|c|c|c|c|}
\hline Thread & Data 1 & Data 2 & Data 3 & Data 4 & Data 5 & Average & Speedup \\
\hline 1 & 32.96 & 32.91 & 32.93 & 32.92 & 32.94 & 32.93 & 1.00 \\
\hline 10 & 7.91 & 7.88 & 7.84 & 8.75 & 7.91 & 8.06 & 4.09 \\
\hline 20 & 6.02 & 5.97 & 5.93 & 6.39 & 6.19 & 6.10 & 5.40 \\
\hline 30 & 4.08 & 4.07 & 4.06 & 4.40 & 4.20 & 4.16 & 7.91 \\
\hline 40 & 4.20 & 4.18 & 4.17 & 4.55 & 4.26 & 4.27 & 7.71 \\
\hline 50 & 4.48 & 4.48 & 4.45 & 4.94 & 4.54 & 4.58 & 7.19 \\
\hline 60 & 4.95 & 4.97 & 4.93 & 5.50 & 5.01 & 5.07 & 6.50 \\
\hline 70 & 5.50 & 5.50 & 5.52 & 6.19 & 5.56 & 5.65 & 5.83 \\
\hline 80 & 5.95 & 5.89 & 5.93 & 6.69 & 6.12 & 6.12 & 5.39 \\
\hline 90 & 6.52 & 6.44 & 6.50 & 7.38 & 6.61 & 6.69 & 4.92 \\
\hline 100 & 7.05 & 7.11 & 7.03 & 8.06 & 7.08 & 7.27 & 4.53 \\
\hline 110 & 7.43 & 7.41 & 7.43 & 8.46 & 7.48 & 7.64 & 4.31 \\
\hline 120 & 8.02 & 8.01 & 8.04 & 9.12 & 8.09 & 8.26 & 3.99 \\
\hline 130 & 8.66 & 8.62 & 8.73 & 9.91 & 8.69 & 8.92 & 3.69 \\
\hline 140 & 9.18 & 9.16 & 9.19 & 10.53 & 9.27 & 9.47 & 3.48 \\
\hline 150 & 9.64 & 9.62 & 9.66 & 11.05 & 9.73 & 9.94 & 3.31 \\
\hline 160 & 10.20 & 10.12 & 10.16 & 11.66 & 10.22 & 10.47 & 3.15 \\
\hline 170 & 10.64 & 10.60 & 10.65 & 12.13 & 10.69 & 10.94 & 3.01 \\
\hline 180 & 11.14 & 11.13 & 11.11 & 11.30 & 11.17 & 11.17 & 2.95 \\
\hline 190 & 12.01 & 11.93 & 11.97 & 12.04 & 12.04 & 11.99 & 2.75 \\
\hline 200 & 12.53 & 12.45 & 12.49 & 12.50 & 12.54 & 12.50 & 2.63 \\
\hline 210 & 13.00 & 12.92 & 12.96 & 12.97 & 13.01 & 12.97 & 2.54 \\
\hline 220 & 13.49 & 13.40 & 13.46 & 13.45 & 13.50 & 13.46 & 2.45 \\
\hline 230 & 13.98 & 13.89 & 13.94 & 13.94 & 13.99 & 13.95 & 2.36 \\
\hline 240 & 14.82 & 14.41 & 14.44 & 14.45 & 14.50 & 14.53 & 2.27 \\
\hline
\end{tabular}


Table 6.69 Time and speedup of Ladder $215 \mathrm{~S}(N)$ model with $N=16$ on an Intel Xeon Phi coprocessor with KMP_THREAD_AFFINITY set to scatter

\begin{tabular}{|c|c|c|c|c|c|c|c|}
\hline \multicolumn{8}{|c|}{ Scatter Heisenberg Ladder215S(16) (time in seconds) } \\
\hline Thread & Data 1 & Data 2 & Data 3 & Data 4 & Data 5 & Average & Speedup \\
\hline 1 & 8.30 & 8.25 & 8.26 & 8.31 & 8.28 & 8.28 & 1.00 \\
\hline 10 & 3.08 & 3.18 & 2.98 & 3.26 & 3.09 & 3.12 & 2.66 \\
\hline 20 & 3.07 & 3.09 & 3.08 & 3.22 & 3.05 & 3.10 & 2.67 \\
\hline 30 & 3.34 & 3.30 & 3.30 & 3.52 & 3.31 & 3.35 & 2.47 \\
\hline 40 & 3.70 & 3.70 & 3.73 & 3.72 & 3.71 & 3.71 & 2.23 \\
\hline 50 & 4.23 & 4.23 & 4.24 & 4.25 & 4.27 & 4.24 & 1.95 \\
\hline 60 & 4.89 & 4.88 & 4.87 & 4.90 & 4.92 & 4.89 & 1.69 \\
\hline 70 & 5.48 & 5.50 & 5.47 & 5.53 & 5.50 & 5.50 & 1.51 \\
\hline 80 & 5.85 & 6.02 & 6.02 & 5.87 & 6.03 & 5.96 & 1.39 \\
\hline 90 & 6.61 & 6.60 & 6.62 & 6.62 & 6.61 & 6.61 & 1.25 \\
\hline 100 & 7.18 & 7.18 & 7.02 & 7.20 & 7.20 & 7.16 & 1.16 \\
\hline 110 & 7.84 & 8.57 & 7.86 & 7.88 & 7.88 & 8.01 & 1.03 \\
\hline 120 & 8.48 & 9.00 & 8.48 & 8.50 & 8.50 & 8.59 & 0.96 \\
\hline 130 & 9.23 & 9.23 & 9.22 & 9.23 & 9.23 & 9.23 & 0.90 \\
\hline 140 & 9.77 & 9.78 & 9.77 & 9.80 & 9.80 & 9.79 & 0.85 \\
\hline 150 & 10.31 & 10.31 & 10.30 & 10.32 & 10.32 & 10.31 & 0.80 \\
\hline 160 & 10.84 & 10.85 & 10.84 & 10.86 & 10.86 & 10.85 & 0.76 \\
\hline 170 & 11.37 & 11.38 & 11.38 & 11.41 & 11.38 & 11.39 & 0.73 \\
\hline 180 & 11.94 & 11.94 & 11.93 & 11.94 & 11.95 & 11.94 & 0.69 \\
\hline 190 & 12.70 & 12.66 & 12.65 & 12.68 & 12.68 & 12.67 & 0.65 \\
\hline 200 & 13.24 & 13.22 & 13.21 & 13.21 & 13.23 & 13.22 & 0.63 \\
\hline 210 & 13.55 & 13.77 & 13.78 & 13.78 & 13.78 & 13.73 & 0.60 \\
\hline 220 & 14.34 & 14.31 & 14.00 & 14.31 & 14.35 & 14.26 & 0.58 \\
\hline 230 & 14.85 & 14.84 & 14.83 & 14.50 & 14.85 & 14.78 & 0.56 \\
\hline 240 & 15.72 & 15.37 & 15.07 & 15.38 & 15.38 & 15.38 & 0.54 \\
\hline
\end{tabular}


Table 6.70 Time and speedup of Ladder $215 \mathrm{~S}(N)$ model with $N=26$ on an Intel Xeon Phi coprocessor with KMP_THREAD_AFFINITY set to compact

\begin{tabular}{|r|c|c|c|c|c|c|c|}
\hline \multicolumn{7}{|c|}{ Compact Heisenberg Ladder215S(26) (time in seconds) } \\
\hline Thread & Data 1 & Data 2 & Data 3 & Data 4 & Data 5 & Average & Speedup \\
\hline 1 & - & - & - & - & - & - & - \\
\hline 10 & - & - & - & - & - & - & - \\
\hline 20 & - & - & - & - & - & - & - \\
\hline 30 & - & - & - & - & - & - & - \\
\hline 40 & - & - & - & - & - & - & - \\
\hline 50 & - & - & - & - & - & - & - \\
\hline 60 & - & - & - & - & - & - & - \\
\hline 70 & - & - & - & - & - & - & - \\
\hline 80 & - & - & - & - & - & - & - \\
\hline 90 & - & - & - & - & - & - & - \\
\hline 100 & - & - & - & - & - & - & - \\
\hline 110 & - & - & - & - & - & - & - \\
\hline 120 & - & - & - & - & - & - & - \\
\hline 130 & - & - & - & - & - & - & - \\
\hline 140 & - & - & - & - & - & - & - \\
\hline 150 & - & - & - & - & - & - & - \\
\hline 160 & - & - & - & - & - & - & - \\
\hline 170 & - & - & - & - & - & - & - \\
\hline 180 & - & - & - & - & - & - & - \\
\hline 190 & - & - & - & - & - & - & - \\
\hline 200 & - & - & - & - & - & - & - \\
\hline 210 & - & - & - & - & - & - & - \\
\hline 220 & - & - & - & - & - & - & - \\
\hline 230 & - & - & - & - & - & - & - \\
\hline 240 & - & - & - & - & - & - & - \\
\hline
\end{tabular}


Table 6.71 Time and speedup of Ladder $215 \mathrm{~S}(N)$ model with $N=24$ on an Intel Xeon

Phi coprocessor with KMP_THREAD_AFFINITY set to compact

\begin{tabular}{|c|c|c|c|c|c|c|c|}
\hline Compa & $t$ Heiser & g Ladd & $5 \mathrm{~S}(24)$ & me in $\mathrm{s}$ & onds) & & \\
\hline Thread & Data 1 & Data 2 & Data 3 & Data 4 & Data 5 & Average & Speedup \\
\hline 1 & 2354.56 & 2355.89 & 2356.18 & 2355.27 & 2354.79 & 2355.34 & 1.00 \\
\hline 10 & 696.75 & 696.73 & 696.99 & 696.89 & 696.15 & 696.70 & 3.38 \\
\hline 20 & 374.73 & 374.63 & 374.72 & 374.78 & 374.93 & 374.76 & 6.28 \\
\hline 30 & 255.16 & 257.06 & 255.28 & 255.18 & 255.14 & 255.56 & 9.22 \\
\hline 40 & 196.31 & 196.27 & 196.29 & 196.29 & 196.41 & 196.31 & 12.00 \\
\hline 50 & 159.43 & 159.43 & 159.48 & 159.81 & 159.45 & 159.52 & 14.77 \\
\hline 60 & 136.81 & 136.78 & 136.81 & 131.77 & 131.75 & 134.78 & 17.47 \\
\hline 70 & 118.38 & 118.37 & 118.41 & 118.38 & 118.41 & 118.39 & 19.89 \\
\hline 80 & 104.40 & 104.39 & 104.40 & 104.39 & 104.41 & 104.40 & 22.56 \\
\hline 90 & 95.73 & 95.72 & 95.71 & 92.18 & 95.71 & 95.01 & 24.79 \\
\hline 100 & 87.98 & 87.97 & 87.96 & 87.98 & 87.97 & 87.97 & 26.77 \\
\hline 110 & 81.41 & 81.44 & 81.42 & 81.44 & 81.43 & 81.43 & 28.93 \\
\hline 120 & 76.35 & 76.40 & 76.38 & 76.35 & 76.36 & 76.37 & 30.84 \\
\hline 130 & 71.99 & 71.96 & 71.96 & 71.96 & 71.98 & 71.97 & 32.73 \\
\hline 140 & 67.85 & 67.83 & 67.85 & 67.83 & 67.84 & 67.84 & 34.72 \\
\hline 150 & 64.29 & 64.19 & 64.17 & 64.18 & 64.20 & 64.21 & 36.68 \\
\hline 160 & 60.61 & 59.49 & 60.60 & 60.60 & 60.60 & 60.38 & 39.01 \\
\hline 170 & 60.25 & 60.37 & 60.37 & 60.37 & 60.38 & 60.35 & 39.03 \\
\hline 180 & 55.97 & 56.01 & 56.00 & 56.03 & 56.02 & 56.01 & 42.06 \\
\hline 190 & 53.35 & 53.36 & 53.36 & 53.35 & 53.34 & 53.35 & 44.15 \\
\hline 200 & 51.89 & 51.90 & 51.90 & 51.92 & 51.91 & 51.90 & 45.38 \\
\hline 210 & 50.44 & 50.41 & 50.43 & 50.43 & 50.42 & 50.43 & 46.71 \\
\hline 220 & 49.49 & 49.52 & 49.55 & 49.55 & 49.53 & 49.53 & 47.56 \\
\hline 230 & 47.70 & 47.70 & 47.71 & 47.73 & 47.72 & 47.71 & 49.37 \\
\hline 240 & 46.30 & 46.25 & 46.26 & 46.27 & 46.25 & 46.26 & 50.91 \\
\hline
\end{tabular}


Table 6.72 Time and speedup of Ladder $215 \mathrm{~S}(N)$ model with $N=22$ on an Intel Xeon Phi coprocessor with KMP_THREAD_AFFINITY set to compact

\begin{tabular}{|c|c|c|c|c|c|c|c|}
\hline Thread & Data 1 & Data 2 & Data 3 & Data 4 & Data 5 & Average & Speedup \\
\hline 1 & 534.98 & 539.45 & 539.39 & 539.47 & 539.60 & 538.58 & 1.00 \\
\hline 10 & 165.16 & 165.15 & 165.12 & 165.11 & 165.17 & 165.14 & 3.26 \\
\hline 20 & 91.34 & 91.37 & 91.29 & 91.28 & 91.29 & 91.32 & 5.90 \\
\hline 30 & 63.74 & 63.73 & 63.75 & 63.76 & 63.74 & 63.74 & 8.45 \\
\hline 40 & 49.34 & 49.33 & 49.32 & 49.33 & 49.31 & 49.33 & 10.92 \\
\hline 50 & 41.36 & 41.35 & 41.40 & 41.36 & 41.38 & 41.37 & 13.02 \\
\hline 60 & 35.25 & 35.31 & 35.22 & 35.23 & 35.25 & 35.25 & 15.28 \\
\hline 70 & 30.79 & 30.78 & 30.78 & 30.77 & 30.78 & 30.78 & 17.50 \\
\hline 80 & 26.77 & 26.78 & 26.76 & 26.78 & 26.75 & 26.77 & 20.12 \\
\hline 90 & 24.44 & 24.40 & 24.40 & 24.41 & 24.39 & 24.41 & 22.07 \\
\hline 100 & 22.00 & 21.97 & 22.00 & 21.97 & 21.98 & 21.99 & 24.50 \\
\hline 110 & 20.07 & 20.05 & 20.06 & 20.07 & 20.05 & 20.06 & 26.85 \\
\hline 120 & 18.71 & 18.68 & 18.69 & 18.66 & 18.69 & 18.69 & 28.82 \\
\hline 130 & 17.74 & 17.74 & 17.75 & 17.72 & 17.74 & 17.74 & 30.36 \\
\hline 140 & 16.61 & 16.63 & 16.61 & 16.62 & 16.63 & 16.62 & 32.40 \\
\hline 150 & 18.82 & 18.85 & 18.83 & 18.82 & 18.82 & 18.83 & 28.60 \\
\hline 160 & 18.49 & 18.47 & 18.47 & 18.47 & 18.50 & 18.48 & 29.14 \\
\hline 170 & 18.71 & 18.67 & 18.67 & 18.67 & 18.69 & 18.68 & 28.83 \\
\hline 180 & 18.63 & 18.62 & 18.62 & 18.59 & 18.60 & 18.61 & 28.94 \\
\hline 190 & 18.51 & 18.46 & 18.50 & 18.47 & 18.47 & 18.48 & 29.15 \\
\hline 200 & 18.79 & 18.78 & 18.82 & 18.77 & 18.82 & 18.80 & 28.65 \\
\hline 210 & 19.05 & 19.01 & 19.03 & 18.99 & 19.02 & 19.02 & 28.32 \\
\hline 220 & 19.50 & 19.52 & 19.50 & 19.50 & 19.50 & 19.50 & 27.61 \\
\hline 230 & 19.93 & 19.90 & 19.90 & 19.91 & 19.90 & 19.91 & 27.05 \\
\hline 240 & 20.48 & 20.31 & 20.23 & 20.29 & 20.31 & 20.32 & 26.50 \\
\hline
\end{tabular}


Table 6.73 Time and speedup of Ladder $215 \mathrm{~S}(N)$ model with $N=20$ on an Intel Xeon Phi coprocessor with KMP_THREAD_AFFINITY set to compact

\begin{tabular}{|c|c|c|c|c|c|c|c|}
\hline \multicolumn{8}{|c|}{ Compact Heisenberg Ladder215S(20) (time in seconds) } \\
\hline Thread & Data 1 & Data 2 & Data 3 & Data 4 & Data 5 & Average & Speedup \\
\hline 1 & 134.13 & 134.28 & 134.34 & 134.30 & 134.31 & 134.27 & 1.00 \\
\hline 10 & 42.83 & 42.80 & 42.78 & 42.88 & 42.89 & 42.83 & 3.13 \\
\hline 20 & 24.12 & 24.05 & 24.06 & 24.03 & 24.03 & 24.06 & 5.58 \\
\hline 30 & 16.20 & 16.14 & 16.12 & 16.19 & 16.21 & 16.17 & 8.30 \\
\hline 40 & 13.77 & 13.72 & 13.71 & 13.75 & 13.76 & 13.74 & 9.77 \\
\hline 50 & 12.58 & 12.54 & 12.55 & 12.59 & 12.58 & 12.57 & 10.68 \\
\hline 60 & 11.87 & 11.82 & 11.80 & 11.86 & 11.85 & 11.84 & 11.34 \\
\hline 70 & 11.45 & 11.43 & 11.39 & 11.42 & 11.47 & 11.43 & 11.74 \\
\hline 80 & 11.17 & 11.11 & 11.08 & 11.11 & 11.11 & 11.12 & 12.08 \\
\hline 90 & 11.39 & 11.33 & 11.31 & 11.33 & 11.34 & 11.34 & 11.84 \\
\hline 100 & 11.57 & 11.53 & 11.52 & 11.53 & 11.53 & 11.54 & 11.64 \\
\hline 110 & 11.86 & 11.75 & 11.74 & 11.79 & 11.78 & 11.78 & 11.40 \\
\hline 120 & 12.11 & 12.04 & 12.03 & 12.09 & 12.09 & 12.07 & 11.12 \\
\hline 130 & 11.56 & 11.54 & 11.55 & 11.64 & 11.66 & 11.59 & 11.58 \\
\hline 140 & 12.11 & 12.09 & 12.10 & 12.18 & 12.18 & 12.13 & 11.07 \\
\hline 150 & 12.72 & 12.69 & 12.70 & 12.76 & 12.76 & 12.73 & 10.55 \\
\hline 160 & 13.38 & 13.38 & 13.37 & 13.48 & 13.46 & 13.41 & 10.01 \\
\hline 170 & 14.12 & 14.10 & 14.06 & 14.18 & 14.16 & 14.12 & 9.51 \\
\hline 180 & 14.99 & 14.89 & 14.91 & 14.96 & 14.95 & 14.94 & 8.99 \\
\hline 190 & 15.80 & 15.73 & 15.71 & 15.74 & 15.71 & 15.74 & 8.53 \\
\hline 200 & 16.76 & 16.66 & 16.67 & 16.67 & 16.73 & 16.70 & 8.04 \\
\hline 210 & 17.88 & 17.77 & 17.79 & 17.77 & 17.81 & 17.80 & 7.54 \\
\hline 220 & 18.92 & 18.79 & 18.81 & 18.61 & 18.85 & 18.80 & 7.14 \\
\hline 230 & 20.14 & 20.00 & 20.00 & 20.00 & 20.06 & 20.04 & 6.70 \\
\hline 240 & 21.34 & 20.90 & 20.90 & 20.89 & 20.94 & 20.99 & 6.40 \\
\hline
\end{tabular}


Table 6.74 Time and speedup of Ladder $215 \mathrm{~S}(N)$ model with $N=18$ on an Intel Xeon Phi coprocessor with KMP_THREAD_AFFINITY set to compact

\begin{tabular}{|c|c|c|c|c|c|c|c|}
\hline \multicolumn{8}{|c|}{ Compact Heisenberg Ladder215S(18) (time in seconds) } \\
\hline Thread & Data 1 & Data 2 & Data 3 & Data 4 & Data 5 & Average & Speedup \\
\hline 1 & 32.96 & 32.91 & 32.93 & 32.92 & 32.94 & 32.93 & 1.00 \\
\hline 10 & 11.28 & 11.25 & 11.26 & 11.26 & 11.24 & 11.26 & 2.93 \\
\hline 20 & 8.30 & 8.29 & 8.28 & 8.26 & 8.24 & 8.27 & 3.98 \\
\hline 30 & 6.84 & 6.84 & 6.86 & 6.82 & 6.83 & 6.84 & 4.82 \\
\hline 40 & 6.70 & 6.70 & 6.72 & 6.74 & 6.72 & 6.72 & 4.90 \\
\hline 50 & 6.99 & 6.98 & 7.00 & 6.97 & 6.98 & 6.98 & 4.72 \\
\hline 60 & 7.22 & 7.22 & 7.21 & 7.27 & 7.25 & 7.24 & 4.55 \\
\hline 70 & 7.68 & 7.63 & 7.66 & 7.66 & 7.69 & 7.66 & 4.30 \\
\hline 80 & 8.14 & 8.12 & 8.14 & 8.18 & 8.16 & 8.15 & 4.04 \\
\hline 90 & 8.63 & 8.59 & 8.62 & 8.68 & 8.69 & 8.64 & 3.81 \\
\hline 100 & 9.14 & 9.13 & 9.12 & 9.14 & 9.12 & 9.13 & 3.61 \\
\hline 110 & 9.73 & 9.75 & 9.74 & 9.79 & 9.79 & 9.76 & 3.37 \\
\hline 120 & 10.44 & 10.42 & 10.44 & 10.48 & 10.47 & 10.45 & 3.15 \\
\hline 130 & 11.14 & 11.13 & 11.13 & 11.17 & 11.17 & 11.15 & 2.95 \\
\hline 140 & 11.83 & 11.79 & 11.84 & 11.85 & 11.84 & 11.83 & 2.78 \\
\hline 150 & 12.64 & 12.63 & 12.64 & 12.67 & 12.64 & 12.64 & 2.60 \\
\hline 160 & 13.41 & 13.42 & 13.42 & 13.45 & 13.46 & 13.43 & 2.45 \\
\hline 170 & 14.40 & 14.40 & 14.43 & 14.46 & 14.37 & 14.41 & 2.28 \\
\hline 180 & 15.43 & 15.38 & 15.38 & 15.43 & 15.34 & 15.39 & 2.14 \\
\hline 190 & 16.51 & 16.47 & 16.50 & 16.50 & 16.40 & 16.47 & 2.00 \\
\hline 200 & 17.58 & 17.48 & 17.53 & 17.55 & 17.44 & 17.52 & 1.88 \\
\hline 210 & 18.85 & 18.80 & 18.85 & 18.82 & 18.72 & 18.81 & 1.75 \\
\hline 220 & 20.08 & 19.96 & 19.98 & 19.96 & 19.88 & 19.97 & 1.65 \\
\hline 230 & 21.34 & 21.34 & 21.36 & 21.31 & 21.24 & 21.32 & 1.54 \\
\hline 240 & 22.73 & 22.40 & 22.43 & 22.43 & 22.34 & 22.47 & 1.47 \\
\hline
\end{tabular}


Table 6.75 Time and speedup of Ladder $215 \mathrm{~S}(N)$ model with $N=16$ on an Intel Xeon Phi coprocessor with KMP_THREAD_AFFINITY set to compact

\begin{tabular}{|c|c|c|c|c|c|c|c|}
\hline Thread & Data 1 & Data 2 & Data 3 & Data 4 & Data 5 & Average & Speedup \\
\hline 1 & 8.30 & 8.25 & 8.26 & 8.31 & 8.28 & 8.28 & 1.00 \\
\hline 10 & 4.22 & 4.23 & 4.25 & 4.29 & 4.29 & 4.26 & 1.95 \\
\hline 20 & 3.92 & 3.93 & 3.95 & 4.01 & 3.95 & 3.95 & 2.10 \\
\hline 30 & 4.10 & 4.11 & 4.12 & 4.15 & 4.14 & 4.13 & 2.01 \\
\hline 40 & 4.44 & 4.43 & 4.45 & 4.50 & 4.47 & 4.46 & 1.86 \\
\hline 50 & 4.80 & 4.86 & 4.88 & 4.91 & 4.90 & 4.87 & 1.70 \\
\hline 60 & 5.29 & 5.29 & 5.32 & 5.35 & 5.33 & 5.32 & 1.56 \\
\hline 70 & 5.79 & 5.77 & 5.77 & 5.77 & 5.82 & 5.78 & 1.43 \\
\hline 80 & 6.22 & 6.24 & 6.23 & 6.27 & 6.27 & 6.25 & 1.33 \\
\hline 90 & 6.83 & 6.84 & 6.80 & 6.84 & 6.85 & 6.83 & 1.21 \\
\hline 100 & 7.36 & 7.37 & 7.37 & 7.40 & 7.39 & 7.38 & 1.12 \\
\hline 110 & 7.98 & 7.95 & 7.96 & 7.97 & 7.97 & 7.96 & 1.04 \\
\hline 120 & 8.56 & 8.55 & 8.57 & 8.59 & 8.57 & 8.57 & 0.97 \\
\hline 130 & 9.24 & 9.22 & 9.23 & 9.21 & 9.25 & 9.23 & 0.90 \\
\hline 140 & 9.89 & 9.88 & 9.89 & 9.89 & 9.70 & 9.85 & 0.84 \\
\hline 150 & 10.61 & 10.60 & 10.62 & 10.57 & 10.60 & 10.60 & 0.78 \\
\hline 160 & 11.28 & 11.27 & 11.30 & 11.28 & 11.28 & 11.28 & 0.73 \\
\hline 170 & 12.08 & 12.06 & 12.08 & 12.07 & 12.07 & 12.07 & 0.69 \\
\hline 180 & 12.83 & 12.80 & 12.84 & 12.79 & 12.81 & 12.81 & 0.65 \\
\hline 190 & 13.76 & 13.75 & 13.76 & 13.73 & 13.10 & 13.62 & 0.61 \\
\hline 200 & 14.01 & 14.65 & 14.32 & 14.60 & 14.64 & 14.44 & 0.57 \\
\hline 210 & 15.75 & 15.72 & 15.74 & 15.32 & 15.61 & 15.63 & 0.53 \\
\hline 220 & 16.74 & 16.74 & 16.74 & 16.61 & 16.68 & 16.70 & 0.50 \\
\hline 230 & 17.92 & 17.91 & 17.91 & 17.84 & 17.86 & 17.89 & 0.46 \\
\hline 240 & 19.27 & 18.94 & 18.95 & 18.86 & 18.91 & 18.99 & 0.44 \\
\hline
\end{tabular}


Table 6.76 Time and speedup of Ladder $215 \mathrm{~S}(N)$ model with $N=26$ on an Intel Xeon Phi coprocessor with KMP_THREAD_AFFINITY set to balanced

\begin{tabular}{|c|c|c|c|c|c|c|c|}
\hline Balan & ced $\mathrm{He}$ & berg Lac & $215 \mathrm{~S}(2$ & (time in & conds) & & \\
\hline Thread & Data 1 & Data 2 & Data 3 & Data 4 & Data 5 & Average & Speedup \\
\hline 1 & - & - & - & - & - & - & - \\
\hline 10 & - & - & - & - & - & - & - \\
\hline 20 & - & - & - & - & - & - & - \\
\hline 30 & - & - & - & - & - & - & - \\
\hline 40 & - & - & - & - & - & - & - \\
\hline 50 & - & - & - & - & - & - & - \\
\hline 60 & - & - & - & - & - & - & - \\
\hline 70 & - & - & - & - & - & - & - \\
\hline 80 & - & - & - & - & - & - & - \\
\hline 90 & - & - & - & - & - & - & - \\
\hline 100 & - & - & - & - & - & - & - \\
\hline 110 & - & - & - & - & - & - & - \\
\hline 120 & - & - & - & - & - & - & - \\
\hline 130 & - & - & - & - & - & - & - \\
\hline 140 & - & - & - & - & - & - & - \\
\hline 150 & - & - & - & - & - & - & - \\
\hline 160 & - & - & - & - & - & - & - \\
\hline 170 & - & - & - & - & - & - & - \\
\hline 180 & - & - & - & - & - & - & - \\
\hline 190 & - & - & - & - & - & - & - \\
\hline 200 & - & - & - & - & - & - & - \\
\hline 210 & - & - & - & - & - & - & - \\
\hline 220 & - & - & - & - & - & - & - \\
\hline 230 & - & - & - & - & - & - & - \\
\hline 240 & - & - & - & - & - & - & - \\
\hline
\end{tabular}


Table 6.77 Time and speedup of Ladder $215 \mathrm{~S}(N)$ model with $N=24$ on an Intel Xeon Phi coprocessor with KMP_THREAD_AFFINITY set to balanced

\begin{tabular}{|c|c|c|c|c|c|c|c|}
\hline Thread & Data 1 & Data 2 & Data 3 & Data 4 & Data 5 & Average & Speedup \\
\hline 1 & 2354.56 & 2355.89 & 2356.18 & 2355.27 & 2354.79 & 2355.34 & 1.00 \\
\hline 10 & 544.79 & 547.65 & 536.39 & 555.49 & 563.53 & 549.57 & 4.29 \\
\hline 20 & 337.22 & 326.28 & 327.61 & 305.80 & 331.90 & 325.76 & 7.23 \\
\hline 30 & 234.18 & 242.23 & 245.66 & 240.92 & 239.49 & 240.50 & 9.79 \\
\hline 40 & 177.17 & 178.09 & 185.46 & 186.77 & 187.82 & 183.06 & 12.87 \\
\hline 50 & 152.39 & 152.27 & 153.14 & 152.21 & 157.87 & 153.58 & 15.34 \\
\hline 60 & 129.28 & 129.17 & 129.64 & 130.35 & 129.71 & 129.63 & 18.17 \\
\hline 70 & 112.29 & 113.49 & 116.21 & 116.36 & 114.60 & 114.59 & 20.55 \\
\hline 80 & 95.16 & 101.43 & 102.31 & 101.09 & 105.85 & 101.17 & 23.28 \\
\hline 90 & 91.01 & 95.79 & 95.52 & 91.45 & 93.19 & 93.39 & 25.22 \\
\hline 100 & 81.47 & 88.15 & 83.16 & 81.98 & 87.50 & 84.45 & 27.89 \\
\hline 110 & 79.69 & 79.85 & 80.87 & 79.77 & 79.95 & 80.03 & 29.43 \\
\hline 120 & 76.19 & 76.03 & 75.43 & 75.75 & 74.72 & 75.62 & 31.15 \\
\hline 130 & 72.98 & 75.75 & 73.22 & 67.41 & 74.54 & 72.78 & 32.36 \\
\hline 140 & 68.48 & 69.32 & 69.10 & 67.82 & 70.57 & 69.06 & 34.11 \\
\hline 150 & 65.84 & 63.31 & 66.80 & 63.43 & 64.40 & 64.76 & 36.37 \\
\hline 160 & 60.63 & 61.10 & 61.72 & 61.98 & 61.95 & 61.47 & 38.31 \\
\hline 170 & 62.35 & 61.52 & 61.94 & 59.67 & 61.16 & 61.33 & 38.41 \\
\hline 180 & 58.46 & 58.24 & 58.05 & 57.99 & 57.69 & 58.09 & 40.55 \\
\hline 190 & 56.87 & 57.18 & 58.12 & 56.59 & 57.55 & 57.26 & 41.13 \\
\hline 200 & 54.27 & 54.54 & 54.99 & 53.93 & 54.59 & 54.46 & 43.25 \\
\hline 210 & 51.57 & 52.45 & 51.98 & 52.37 & 51.47 & 51.96 & 45.33 \\
\hline 220 & 51.63 & 51.64 & 51.67 & 51.25 & 51.98 & 51.63 & 45.62 \\
\hline 230 & 47.50 & 47.66 & 47.56 & 47.51 & 47.79 & 47.60 & 49.48 \\
\hline 240 & 46.29 & 46.23 & 46.22 & 46.25 & 46.21 & 46.24 & 50.94 \\
\hline
\end{tabular}


Table 6.78 Time and speedup of Ladder $215 \mathrm{~S}(N)$ model with $N=22$ on an Intel Xeon Phi coprocessor with KMP_THREAD_AFFINITY set to bal anced

\begin{tabular}{|c|c|c|c|c|c|c|c|}
\hline Thread & Data 1 & Data 2 & Data 3 & Data 4 & Data 5 & Average & Speedup \\
\hline 1 & 534.98 & 539.45 & 539.39 & 539.47 & 539.60 & 538.58 & 1.00 \\
\hline 10 & 124.48 & 121.27 & 131.55 & 127.16 & 119.93 & 124.88 & 4.31 \\
\hline 20 & 68.84 & 75.78 & 78.13 & 76.67 & 73.68 & 74.62 & 7.22 \\
\hline 30 & 54.59 & 53.60 & 55.13 & 54.42 & 53.51 & 54.25 & 9.93 \\
\hline 40 & 41.42 & 39.47 & 41.24 & 40.88 & 40.26 & 40.65 & 13.25 \\
\hline 50 & 34.28 & 33.11 & 33.34 & 33.75 & 33.61 & 33.62 & 16.02 \\
\hline 60 & 29.46 & 28.81 & 29.55 & 29.78 & 29.41 & 29.40 & 18.32 \\
\hline 70 & 26.44 & 27.75 & 27.55 & 26.56 & 26.40 & 26.94 & 19.99 \\
\hline 80 & 21.04 & 24.07 & 23.12 & 23.30 & 23.15 & 22.94 & 23.48 \\
\hline 90 & 21.69 & 23.27 & 21.65 & 21.88 & 21.70 & 22.04 & 24.44 \\
\hline 100 & 20.04 & 21.56 & 20.94 & 19.23 & 19.90 & 20.33 & 26.49 \\
\hline 110 & 18.58 & 19.86 & 19.32 & 19.42 & 19.50 & 19.34 & 27.85 \\
\hline 120 & 18.34 & 18.48 & 18.00 & 18.28 & 18.46 & 18.31 & 29.41 \\
\hline 130 & 17.82 & 17.84 & 17.55 & 17.59 & 17.75 & 17.71 & 30.41 \\
\hline 140 & 16.63 & 16.72 & 16.81 & 16.79 & 16.89 & 16.77 & 32.12 \\
\hline 150 & 63.99 & 63.97 & 63.89 & 63.66 & 63.57 & 63.82 & 8.44 \\
\hline 160 & 70.50 & 70.35 & 70.98 & 70.86 & 69.90 & 70.52 & 7.64 \\
\hline 170 & 79.78 & 80.28 & 80.09 & 79.96 & 79.65 & 79.95 & 6.74 \\
\hline 180 & 91.59 & 90.93 & 90.74 & 91.24 & 91.67 & 91.23 & 5.90 \\
\hline 190 & 91.01 & 91.31 & 89.98 & 90.08 & 90.64 & 90.60 & 5.94 \\
\hline 200 & 113.74 & 113.06 & 112.10 & 112.97 & 112.55 & 112.88 & 4.77 \\
\hline 210 & 122.83 & 122.59 & 122.96 & 122.95 & 122.36 & 122.74 & 4.39 \\
\hline 220 & 115.38 & 115.92 & 115.48 & 115.85 & 115.30 & 115.59 & 4.66 \\
\hline 230 & 129.02 & 128.81 & 129.01 & 129.39 & 129.74 & 129.19 & 4.17 \\
\hline 240 & 141.84 & 140.37 & 141.19 & 140.81 & 140.96 & 141.03 & 3.82 \\
\hline
\end{tabular}


Table 6.79 Time and speedup of Ladder $215 \mathrm{~S}(N)$ model with $N=20$ on an Intel Xeon Phi coprocessor with KMP_THREAD_AFFINITY set to balanced

\begin{tabular}{|c|c|c|c|c|c|c|c|}
\hline Thread & Data 1 & Data 2 & Data 3 & Data 4 & Data 5 & Average & Speedup \\
\hline 1 & 134.13 & 134.28 & 134.34 & 134.30 & 134.31 & 134.27 & 1.00 \\
\hline 10 & 33.62 & 33.52 & 31.11 & 30.65 & 31.62 & 32.10 & 4.18 \\
\hline 20 & 18.69 & 18.68 & 18.05 & 18.54 & 17.42 & 18.28 & 7.35 \\
\hline 30 & 13.67 & 13.13 & 13.25 & 12.87 & 13.09 & 13.20 & 10.17 \\
\hline 40 & 14.99 & 15.29 & 15.31 & 15.27 & 15.17 & 15.21 & 8.83 \\
\hline 50 & 15.23 & 15.27 & 15.22 & 15.30 & 15.27 & 15.26 & 8.80 \\
\hline 60 & 16.12 & 16.07 & 16.07 & 16.04 & 16.08 & 16.08 & 8.35 \\
\hline 70 & 29.06 & 29.12 & 28.63 & 28.56 & 29.17 & 28.91 & 4.65 \\
\hline 80 & 33.37 & 34.14 & 33.77 & 33.57 & 33.79 & 33.73 & 3.98 \\
\hline 90 & 43.54 & 42.66 & 42.75 & 42.89 & 42.68 & 42.90 & 3.13 \\
\hline 100 & 49.92 & 50.04 & 50.30 & 49.16 & 49.46 & 49.78 & 2.70 \\
\hline 110 & 50.64 & 51.02 & 50.91 & 50.63 & 51.28 & 50.89 & 2.64 \\
\hline 120 & 51.61 & 51.82 & 51.67 & 51.35 & 51.45 & 51.58 & 2.60 \\
\hline 130 & 58.18 & 58.78 & 57.71 & 58.41 & 58.52 & 58.32 & 2.30 \\
\hline 140 & 50.32 & 50.78 & 50.21 & 49.67 & 51.03 & 50.40 & 2.66 \\
\hline 150 & 59.73 & 59.87 & 59.67 & 59.86 & 60.01 & 59.83 & 2.24 \\
\hline 160 & 67.27 & 67.14 & 67.70 & 67.38 & 67.72 & 67.44 & 1.99 \\
\hline 170 & 69.03 & 69.23 & 69.29 & 69.30 & 69.08 & 69.19 & 1.94 \\
\hline 180 & 70.23 & 70.73 & 71.02 & 70.96 & 71.46 & 70.88 & 1.89 \\
\hline 190 & 65.19 & 65.88 & 65.69 & 65.80 & 65.99 & 65.71 & 2.04 \\
\hline 200 & 66.38 & 66.65 & 66.48 & 67.37 & 66.71 & 66.72 & 2.01 \\
\hline 210 & 75.60 & 74.88 & 74.89 & 75.03 & 75.52 & 75.18 & 1.79 \\
\hline 220 & 81.43 & 81.53 & 82.35 & 81.62 & 81.72 & 81.73 & 1.64 \\
\hline 230 & 84.52 & 83.82 & 84.47 & 83.75 & 83.69 & 84.05 & 1.60 \\
\hline 240 & 86.32 & 85.91 & 86.19 & 85.81 & 85.78 & 86.00 & 1.56 \\
\hline
\end{tabular}


Table 6.80 Time and speedup of Ladder $215 \mathrm{~S}(N)$ model with $N=18$ on an Intel Xeon Phi coprocessor with KMP_THREAD_AFFINITY set to balanced

\begin{tabular}{|c|c|c|c|c|c|c|c|}
\hline \multicolumn{8}{|c|}{ Balanced Heisenberg Ladder215S(18) (time in seconds) } \\
\hline Thread & Data 1 & Data 2 & Data 3 & Data 4 & Data 5 & Average & Speedup \\
\hline 1 & 32.96 & 32.91 & 32.93 & 32.92 & 32.94 & 32.93 & 1.00 \\
\hline 10 & 9.24 & 9.49 & 8.83 & 8.74 & 8.71 & 9.00 & 3.66 \\
\hline 20 & 7.48 & 7.37 & 7.25 & 7.19 & 7.36 & 7.33 & 4.49 \\
\hline 30 & 5.62 & 5.59 & 5.46 & 5.51 & 5.55 & 5.55 & 5.94 \\
\hline 40 & 6.25 & 6.36 & 6.25 & 6.27 & 6.26 & 6.28 & 5.25 \\
\hline 50 & 7.38 & 7.40 & 7.35 & 7.41 & 7.46 & 7.40 & 4.45 \\
\hline 60 & 8.95 & 9.06 & 8.98 & 9.13 & 9.00 & 9.02 & 3.65 \\
\hline 70 & 14.49 & 14.03 & 14.12 & 14.15 & 14.16 & 14.19 & 2.32 \\
\hline 80 & 15.22 & 15.33 & 15.38 & 15.31 & 15.53 & 15.35 & 2.15 \\
\hline 90 & 16.70 & 16.60 & 16.41 & 16.46 & 16.39 & 16.51 & 1.99 \\
\hline 100 & 17.63 & 17.75 & 17.76 & 17.82 & 17.86 & 17.76 & 1.85 \\
\hline 110 & 19.50 & 19.28 & 19.32 & 19.71 & 19.34 & 19.43 & 1.70 \\
\hline 120 & 20.66 & 20.45 & 20.55 & 20.70 & 20.57 & 20.59 & 1.60 \\
\hline 130 & 22.51 & 22.67 & 22.74 & 22.59 & 22.51 & 22.60 & 1.46 \\
\hline 140 & 24.23 & 24.48 & 24.54 & 24.46 & 24.58 & 24.46 & 1.35 \\
\hline 150 & 25.76 & 25.80 & 25.77 & 25.85 & 26.49 & 25.93 & 1.27 \\
\hline 160 & 28.28 & 27.99 & 27.91 & 27.90 & 28.54 & 28.12 & 1.17 \\
\hline 170 & 29.95 & 29.63 & 29.63 & 29.65 & 29.60 & 29.69 & 1.11 \\
\hline 180 & 31.09 & 31.05 & 31.14 & 31.03 & 31.20 & 31.10 & 1.06 \\
\hline 190 & 31.45 & 31.52 & 31.18 & 31.67 & 31.44 & 31.45 & 1.05 \\
\hline 200 & 32.89 & 32.55 & 32.66 & 32.58 & 32.65 & 32.67 & 1.01 \\
\hline 210 & 34.25 & 34.81 & 34.54 & 34.40 & 34.39 & 34.48 & 0.96 \\
\hline 220 & 36.45 & 36.45 & 36.36 & 36.38 & 36.50 & 36.43 & 0.90 \\
\hline 230 & 39.17 & 38.92 & 38.98 & 38.82 & 38.71 & 38.92 & 0.85 \\
\hline 240 & 40.83 & 40.76 & 40.39 & 40.65 & 40.87 & 40.70 & 0.81 \\
\hline
\end{tabular}


Table 6.81 Time and speedup of Ladder $215 \mathrm{~S}(N)$ model with $N=16$ on an Intel Xeon Phi coprocessor with KMP_THREAD_AFFINITY set to balanced

\begin{tabular}{|c|c|c|c|c|c|c|c|}
\hline \multicolumn{8}{|c|}{ Balanced Heisenberg Ladder215S(16) (time in seconds) } \\
\hline Thread & Data 1 & Data 2 & Data 3 & Data 4 & Data 5 & Average & Speedup \\
\hline 1 & 8.30 & 8.25 & 8.26 & 8.31 & 8.28 & 8.28 & 1.00 \\
\hline 10 & 3.46 & 3.37 & 3.38 & 3.37 & 3.49 & 3.42 & 2.42 \\
\hline 20 & 3.49 & 3.49 & 3.51 & 3.51 & 3.59 & 3.52 & 2.35 \\
\hline 30 & 4.24 & 4.24 & 4.24 & 4.30 & 4.30 & 4.26 & 1.94 \\
\hline 40 & 5.36 & 5.39 & 5.36 & 5.45 & 5.39 & 5.39 & 1.54 \\
\hline 50 & 6.88 & 6.96 & 6.72 & 6.96 & 6.89 & 6.88 & 1.20 \\
\hline 60 & 8.92 & 8.75 & 8.93 & 8.95 & 8.71 & 8.85 & 0.94 \\
\hline 70 & 11.08 & 11.16 & 11.09 & 11.13 & 10.89 & 11.07 & 0.75 \\
\hline 80 & 12.42 & 12.27 & 12.26 & 12.45 & 12.34 & 12.35 & 0.67 \\
\hline 90 & 13.70 & 13.65 & 13.62 & 13.69 & 13.70 & 13.67 & 0.61 \\
\hline 100 & 15.13 & 15.16 & 15.22 & 15.07 & 15.76 & 15.27 & 0.54 \\
\hline 110 & 17.03 & 16.87 & 17.30 & 16.86 & 16.84 & 16.98 & 0.49 \\
\hline 120 & 18.23 & 18.22 & 18.26 & 18.22 & 18.22 & 18.23 & 0.45 \\
\hline 130 & 20.12 & 19.99 & 20.07 & 20.00 & 20.10 & 20.06 & 0.41 \\
\hline 140 & 21.83 & 21.91 & 21.88 & 21.94 & 21.83 & 21.88 & 0.38 \\
\hline 150 & 23.32 & 22.79 & 23.43 & 23.31 & 22.83 & 23.14 & 0.36 \\
\hline 160 & 25.50 & 25.35 & 25.42 & 24.91 & 25.44 & 25.32 & 0.33 \\
\hline 170 & 27.10 & 27.19 & 27.11 & 27.19 & 27.10 & 27.14 & 0.31 \\
\hline 180 & 28.96 & 28.95 & 29.15 & 28.95 & 29.02 & 29.00 & 0.29 \\
\hline 190 & 30.27 & 30.28 & 30.27 & 30.28 & 29.66 & 30.15 & 0.27 \\
\hline 200 & 31.76 & 31.77 & 31.86 & 31.84 & 31.72 & 31.79 & 0.26 \\
\hline 210 & 34.02 & 30.79 & 34.16 & 34.18 & 34.19 & 33.47 & 0.25 \\
\hline 220 & 36.29 & 36.21 & 36.28 & 35.59 & 36.50 & 36.18 & 0.23 \\
\hline 230 & 38.45 & 38.40 & 38.42 & 38.40 & 37.61 & 38.26 & 0.22 \\
\hline 240 & 40.34 & 40.01 & 39.43 & 40.23 & 40.11 & 40.03 & 0.21 \\
\hline
\end{tabular}




\subsubsection{Heisenberg Ladder 315C on Xeon Phi}

Table 6.82 Time and speedup of Ladder $315 \mathrm{C}(N)$ model with $N=24$ on an Intel Xeon Phi coprocessor with KMP_THREAD_AFFINITY set to scatter

\begin{tabular}{|c|c|c|c|c|c|c|c|}
\hline \multicolumn{8}{|c|}{ Scatter Heisenberg Ladder215S(24) (time in seconds) } \\
\hline Thread & Data 1 & Data 2 & Data 3 & Data 4 & Data 5 & Average & Speedup \\
\hline 1 & 2566.78 & 2569.76 & 2570.14 & 2568.15 & 2569.88 & 2568.94 & 1.00 \\
\hline 10 & 614.93 & 640.37 & 625.17 & 624.50 & 611.34 & 623.26 & 4.12 \\
\hline 20 & 350.78 & 352.20 & 376.72 & 358.77 & 349.76 & 357.64 & 7.18 \\
\hline 30 & 252.83 & 228.58 & 259.25 & 270.46 & 262.84 & 254.79 & 10.08 \\
\hline 40 & 206.77 & 195.42 & 205.11 & 206.07 & 207.56 & 204.19 & 12.58 \\
\hline 50 & 162.46 & 166.52 & 164.73 & 159.60 & 161.28 & 162.92 & 15.77 \\
\hline 60 & 144.32 & 146.04 & 142.27 & 142.51 & 143.28 & 143.68 & 17.88 \\
\hline 70 & 127.30 & 127.16 & 131.05 & 126.79 & 127.17 & 127.89 & 20.09 \\
\hline 80 & 117.72 & 112.35 & 112.89 & 109.83 & 113.40 & 113.24 & 22.69 \\
\hline 90 & 104.20 & 101.01 & 101.67 & 104.51 & 110.03 & 104.28 & 24.63 \\
\hline 100 & 96.68 & 91.72 & 90.98 & 92.65 & 96.41 & 93.69 & 27.42 \\
\hline 110 & 85.32 & 89.89 & 90.45 & 86.10 & 88.58 & 88.07 & 29.17 \\
\hline 120 & 83.32 & 83.66 & 84.93 & 84.97 & 85.50 & 84.48 & 30.41 \\
\hline 130 & 84.45 & 81.31 & 81.43 & 80.70 & 80.46 & 81.67 & 31.45 \\
\hline 140 & 76.70 & 77.09 & 76.78 & 75.99 & 74.88 & 76.29 & 33.67 \\
\hline 150 & 74.31 & 72.08 & 72.25 & 71.31 & 72.58 & 72.51 & 35.43 \\
\hline 160 & 71.78 & 75.82 & 67.85 & 68.42 & 71.56 & 71.09 & 36.14 \\
\hline 170 & 69.87 & 69.90 & 70.30 & 69.52 & 66.16 & 69.15 & 37.15 \\
\hline 180 & 65.68 & 66.69 & 65.82 & 66.02 & 68.75 & 66.59 & 38.58 \\
\hline 190 & 65.22 & 64.83 & 65.25 & 65.24 & 64.45 & 65.00 & 39.52 \\
\hline 200 & 61.12 & 60.50 & 61.53 & 61.88 & 62.35 & 61.48 & 41.79 \\
\hline 210 & 58.41 & 58.56 & 59.01 & 58.95 & 59.08 & 58.80 & 43.69 \\
\hline 220 & 56.98 & 57.09 & 57.26 & 57.59 & 58.49 & 57.48 & 44.69 \\
\hline 230 & 53.54 & 53.67 & 53.76 & 53.61 & 53.71 & 53.66 & 47.87 \\
\hline 240 & 53.15 & 53.11 & 52.92 & 53.08 & 53.32 & 53.12 & 48.36 \\
\hline
\end{tabular}


Table 6.83 Time and speedup of Ladder $315 \mathrm{C}(N)$ model with $N=18$ on an Intel Xeon Phi coprocessor with KMP_THREAD_AFFINITY set to scatter

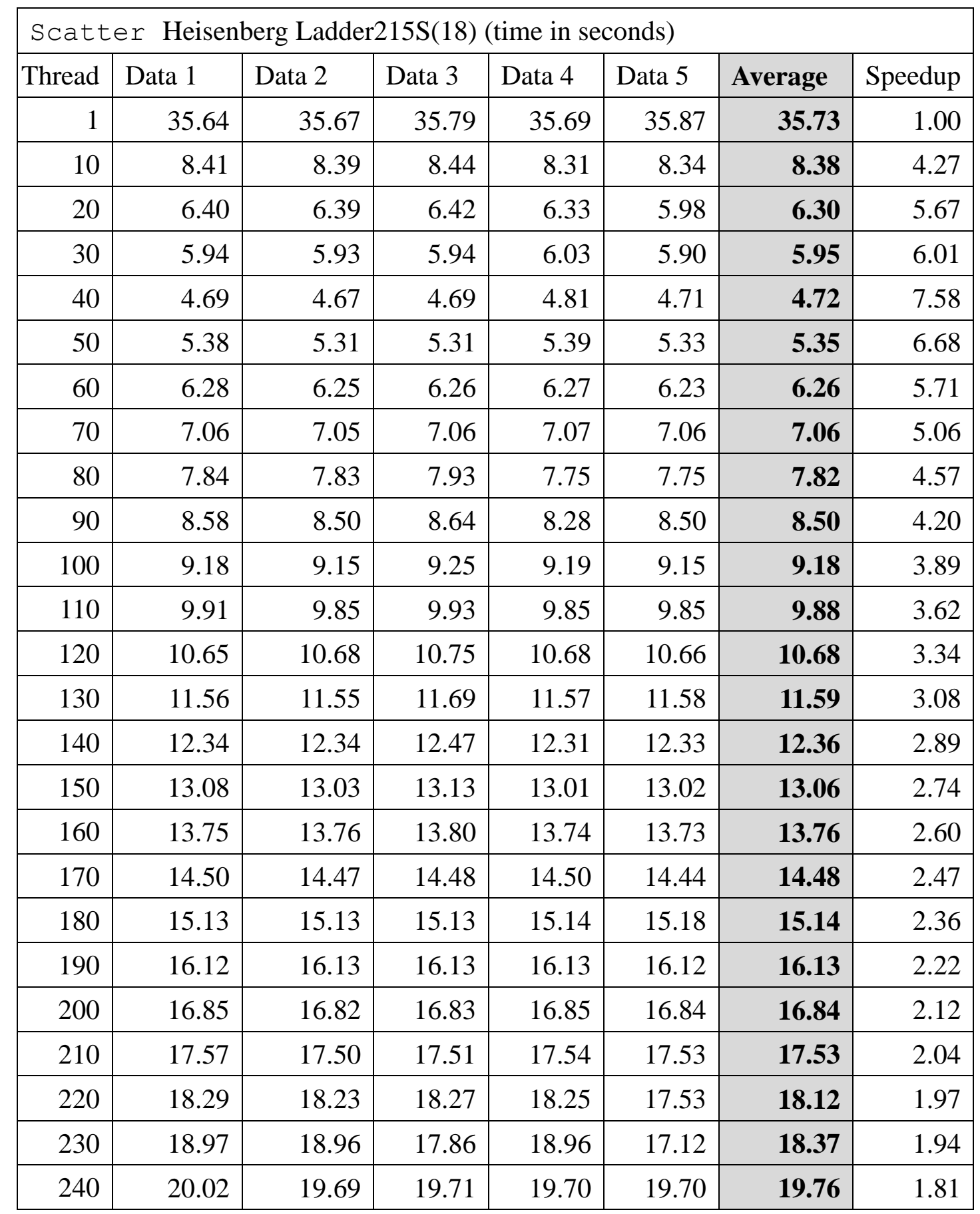


Table 6.84 Time and speedup of Ladder $315 \mathrm{C}(N)$ model with $N=24$ on an Intel Xeon Phi coprocessor with KMP_THREAD_AFFINITY set to compact

\begin{tabular}{|c|c|c|c|c|c|c|c|}
\hline Thread & Data 1 & Data 2 & Data 3 & Data 4 & Data 5 & Average & Speedup \\
\hline 1 & 2566.78 & 2569.76 & 2570.14 & 2568.15 & 2569.88 & 2568.94 & 1.00 \\
\hline 10 & 784.03 & 784.53 & 784.71 & 785.20 & 786.81 & 785.06 & 3.27 \\
\hline 20 & 403.16 & 403.20 & 403.24 & 403.22 & 403.18 & 403.20 & 6.37 \\
\hline 30 & 277.42 & 277.70 & 274.23 & 274.24 & 274.38 & 275.59 & 9.32 \\
\hline 40 & 211.65 & 211.68 & 211.66 & 211.59 & 211.62 & 211.64 & 12.14 \\
\hline 50 & 170.29 & 170.31 & 170.29 & 170.30 & 170.30 & 170.30 & 15.09 \\
\hline 60 & 146.63 & 146.68 & 146.64 & 146.63 & 146.65 & 146.65 & 17.52 \\
\hline 70 & 128.17 & 128.04 & 128.11 & 128.08 & 128.07 & 128.09 & 20.05 \\
\hline 80 & 113.35 & 113.41 & 113.40 & 113.41 & 113.40 & 113.39 & 22.65 \\
\hline 90 & 102.64 & 102.63 & 102.64 & 102.66 & 102.64 & 102.64 & 25.03 \\
\hline 100 & 93.82 & 93.80 & 93.81 & 93.81 & 93.81 & 93.81 & 27.38 \\
\hline 110 & 87.54 & 87.52 & 87.53 & 87.53 & 87.51 & 87.52 & 29.35 \\
\hline 120 & 81.63 & 81.64 & 81.65 & 81.65 & 81.63 & 81.64 & 31.47 \\
\hline 130 & 76.88 & 76.85 & 76.83 & 76.84 & 76.80 & 76.84 & 33.43 \\
\hline 140 & 72.83 & 72.83 & 72.83 & 72.82 & 72.81 & 72.82 & 35.28 \\
\hline 150 & 69.02 & 69.01 & 69.02 & 69.04 & 68.98 & 69.01 & 37.22 \\
\hline 160 & 65.04 & 65.08 & 65.10 & 65.07 & 65.06 & 65.07 & 39.48 \\
\hline 170 & 64.30 & 64.18 & 64.21 & 64.31 & 64.28 & 64.26 & 39.98 \\
\hline 180 & 59.47 & 59.49 & 59.51 & 59.50 & 59.48 & 59.49 & 43.18 \\
\hline 190 & 57.20 & 57.28 & 57.26 & 57.23 & 57.27 & 57.25 & 44.87 \\
\hline 200 & 55.16 & 55.20 & 55.25 & 55.18 & 55.20 & 55.20 & 46.54 \\
\hline 210 & 53.44 & 53.49 & 53.50 & 53.51 & 53.53 & 53.49 & 48.02 \\
\hline 220 & 52.56 & 52.57 & 52.59 & 52.61 & 52.60 & 52.59 & 48.85 \\
\hline 230 & 50.44 & 50.48 & 50.52 & 50.44 & 50.53 & 50.48 & 50.89 \\
\hline 240 & 49.07 & 49.02 & 48.99 & 49.04 & 49.00 & 49.02 & 52.40 \\
\hline
\end{tabular}


Table 6.85 Time and speedup of Ladder $315 \mathrm{C}(N)$ model with $N=18$ on an Intel Xeon Phi coprocessor with KMP_THREAD_AFFINITY set to compact

\begin{tabular}{|c|c|c|c|c|c|c|c|}
\hline \multicolumn{8}{|c|}{ Compact Heisenberg Ladder315C(18) (time in seconds) } \\
\hline Thread & Data 1 & Data 2 & Data 3 & Data 4 & Data 5 & Average & Speedup \\
\hline 1 & 35.64 & 35.67 & 35.79 & 35.69 & 35.87 & 35.73 & 1.00 \\
\hline 10 & 11.88 & 11.90 & 11.86 & 11.79 & 11.79 & 11.84 & 3.02 \\
\hline 20 & 8.57 & 8.59 & 8.57 & 8.47 & 8.48 & 8.54 & 4.19 \\
\hline 30 & 7.84 & 7.81 & 7.81 & 7.73 & 7.73 & 7.78 & 4.59 \\
\hline 40 & 6.94 & 6.95 & 6.93 & 6.84 & 6.85 & 6.90 & 5.18 \\
\hline 50 & 7.05 & 7.04 & 7.00 & 6.98 & 6.96 & 7.01 & 5.10 \\
\hline 60 & 7.36 & 7.37 & 7.35 & 7.32 & 7.33 & 7.35 & 4.86 \\
\hline 70 & 7.79 & 7.75 & 7.76 & 7.67 & 7.68 & 7.73 & 4.62 \\
\hline 80 & 8.21 & 7.77 & 8.18 & 8.15 & 8.17 & 8.10 & 4.41 \\
\hline 90 & 8.74 & 7.96 & 8.73 & 8.73 & 8.72 & 8.58 & 4.17 \\
\hline 100 & 9.23 & 8.76 & 9.22 & 9.23 & 9.25 & 9.14 & 3.91 \\
\hline 110 & 9.86 & 9.85 & 9.85 & 9.85 & 9.85 & 9.85 & 3.63 \\
\hline 120 & 10.45 & 10.46 & 10.44 & 10.23 & 10.43 & 10.40 & 3.44 \\
\hline 130 & 11.11 & 11.12 & 11.10 & 11.11 & 11.11 & 11.11 & 3.22 \\
\hline 140 & 11.83 & 11.83 & 11.83 & 11.82 & 11.82 & 11.83 & 3.02 \\
\hline 150 & 12.65 & 12.64 & 12.61 & 12.63 & 12.61 & 12.63 & 2.83 \\
\hline 160 & 13.42 & 13.43 & 13.43 & 13.40 & 13.41 & 13.42 & 2.66 \\
\hline 170 & 14.40 & 14.39 & 14.38 & 14.36 & 14.38 & 14.38 & 2.48 \\
\hline 180 & 15.38 & 15.33 & 15.30 & 15.30 & 15.31 & 15.32 & 2.33 \\
\hline 190 & 16.37 & 16.37 & 16.37 & 16.32 & 16.38 & 16.36 & 2.18 \\
\hline 200 & 17.44 & 17.43 & 17.39 & 17.39 & 17.42 & 17.41 & 2.05 \\
\hline 210 & 18.68 & 18.67 & 18.68 & 18.66 & 18.72 & 18.68 & 1.91 \\
\hline 220 & 17.57 & 19.84 & 19.84 & 19.84 & 19.86 & 19.39 & 1.84 \\
\hline 230 & 21.17 & 21.11 & 21.20 & 21.19 & 21.18 & 21.17 & 1.69 \\
\hline 240 & 22.58 & 22.14 & 22.26 & 22.27 & 22.26 & 22.30 & 1.60 \\
\hline
\end{tabular}


Table 6.86 Time and speedup of Ladder $315 \mathrm{C}(N)$ model with $N=24$ on an Intel Xeon Phi coprocessor with KMP_THREAD_AFFINITY set to bal anced

\begin{tabular}{|c|c|c|c|c|c|c|c|}
\hline Thread & Data 1 & Data 2 & Data 3 & Data 4 & Data 5 & Average & Speedup \\
\hline 1 & 2566.78 & 2569.76 & 2570.14 & 2568.15 & 2569.88 & 2568.94 & 1.00 \\
\hline 10 & 596.15 & 633.40 & 636.07 & 615.49 & 630.02 & 622.22 & 4.13 \\
\hline 20 & 350.59 & 357.93 & 365.96 & 369.76 & 349.31 & 358.71 & 7.16 \\
\hline 30 & 252.62 & 269.55 & 259.16 & 258.60 & 275.90 & 263.17 & 9.76 \\
\hline 40 & 207.07 & 208.64 & 209.85 & 200.92 & 199.76 & 205.25 & 12.52 \\
\hline 50 & 168.33 & 157.19 & 170.30 & 165.52 & 166.21 & 165.51 & 15.52 \\
\hline 60 & 139.29 & 139.73 & 147.62 & 144.22 & 141.36 & 142.44 & 18.03 \\
\hline 70 & 124.46 & 123.70 & 125.36 & 127.71 & 125.76 & 125.40 & 20.49 \\
\hline 80 & 105.77 & 109.38 & 117.61 & 110.90 & 111.20 & 110.97 & 23.15 \\
\hline 90 & 99.18 & 99.15 & 100.23 & 100.79 & 100.80 & 100.03 & 25.68 \\
\hline 100 & 90.78 & 89.29 & 90.61 & 96.26 & 94.30 & 92.25 & 27.85 \\
\hline 110 & 86.28 & 87.58 & 85.01 & 84.92 & 84.69 & 85.70 & 29.98 \\
\hline 120 & 80.59 & 81.46 & 82.53 & 80.90 & 81.17 & 81.33 & 31.59 \\
\hline 130 & 77.65 & 78.81 & 78.58 & 79.70 & 77.82 & 78.51 & 32.72 \\
\hline 140 & 73.57 & 74.46 & 74.70 & 73.17 & 74.48 & 74.08 & 34.68 \\
\hline 150 & 70.27 & 70.38 & 69.11 & 71.53 & 70.61 & 70.38 & 36.50 \\
\hline 160 & 67.43 & 67.70 & 67.06 & 68.21 & 67.89 & 67.66 & 37.97 \\
\hline 170 & 66.25 & 66.22 & 66.09 & 66.81 & 66.48 & 66.37 & 38.71 \\
\hline 180 & 62.67 & 61.79 & 62.09 & 62.58 & 61.94 & 62.21 & 41.29 \\
\hline 190 & 61.83 & 61.90 & 61.49 & 61.46 & 61.82 & 61.70 & 41.64 \\
\hline 200 & 58.06 & 59.20 & 58.59 & 59.16 & 58.90 & 58.78 & 43.70 \\
\hline 210 & 55.98 & 55.54 & 55.65 & 55.96 & 56.02 & 55.83 & 46.01 \\
\hline 220 & 54.98 & 55.57 & 55.36 & 55.05 & 55.18 & 55.23 & 46.51 \\
\hline 230 & 50.70 & 50.42 & 50.75 & 50.66 & 50.47 & 50.60 & 50.77 \\
\hline 240 & 49.19 & 49.08 & 49.10 & 49.08 & 49.09 & 49.11 & 52.31 \\
\hline
\end{tabular}


Table 6.87 Time and speedup of Ladder $315 \mathrm{C}(N)$ model with $N=18$ on an Intel Xeon Phi coprocessor with KMP_THREAD_AFFINITY set to bal anced

\begin{tabular}{|c|c|c|c|c|c|c|c|}
\hline Thread & Data 1 & Data 2 & Data 3 & Data 4 & Data 5 & Average & Speedup \\
\hline 1 & 35.64 & 35.67 & 35.79 & 35.69 & 35.87 & 35.73 & 1.00 \\
\hline 10 & 10.01 & 9.20 & 9.46 & 10.08 & 9.31 & 9.61 & 3.72 \\
\hline 20 & 7.66 & 7.54 & 7.20 & 7.77 & 7.60 & 7.56 & 4.73 \\
\hline 30 & 7.68 & 7.56 & 7.75 & 7.71 & 7.60 & 7.66 & 4.66 \\
\hline 40 & 6.81 & 6.93 & 6.91 & 7.02 & 6.91 & 6.92 & 5.17 \\
\hline 50 & 8.47 & 8.41 & 8.49 & 8.41 & 8.52 & 8.46 & 4.22 \\
\hline 60 & 10.64 & 10.59 & 10.57 & 10.74 & 10.68 & 10.64 & 3.36 \\
\hline 70 & 16.45 & 16.50 & 16.61 & 16.44 & 16.49 & 16.50 & 2.17 \\
\hline 80 & 17.78 & 17.86 & 17.71 & 17.83 & 17.80 & 17.80 & 2.01 \\
\hline 90 & 19.10 & 18.95 & 19.37 & 19.20 & 19.22 & 19.17 & 1.86 \\
\hline 100 & 20.62 & 20.56 & 20.58 & 20.90 & 20.71 & 20.67 & 1.73 \\
\hline 110 & 22.63 & 22.35 & 22.61 & 22.77 & 22.81 & 22.64 & 1.58 \\
\hline 120 & 23.95 & 24.07 & 24.02 & 24.31 & 23.81 & 24.03 & 1.49 \\
\hline 130 & 26.26 & 26.14 & 26.27 & 26.14 & 26.04 & 26.17 & 1.37 \\
\hline 140 & 27.80 & 27.96 & 28.26 & 28.05 & 25.03 & 27.42 & 1.30 \\
\hline 150 & 29.69 & 29.91 & 30.16 & 28.85 & 29.97 & 29.72 & 1.20 \\
\hline 160 & 32.34 & 31.74 & 32.14 & 32.10 & 32.22 & 32.11 & 1.11 \\
\hline 170 & 33.70 & 33.39 & 33.88 & 33.92 & 33.89 & 33.76 & 1.06 \\
\hline 180 & 35.37 & 35.44 & 35.52 & 35.25 & 35.48 & 35.41 & 1.01 \\
\hline 190 & 35.84 & 35.72 & 35.63 & 35.87 & 35.92 & 35.80 & 1.00 \\
\hline 200 & 38.06 & 37.57 & 37.53 & 37.53 & 37.77 & 37.69 & 0.95 \\
\hline 210 & 40.38 & 40.43 & 40.46 & 40.43 & 35.67 & 39.47 & 0.91 \\
\hline 220 & 42.90 & 42.84 & 42.62 & 42.83 & 42.76 & 42.79 & 0.84 \\
\hline 230 & 45.27 & 41.10 & 45.77 & 45.64 & 45.49 & 44.66 & 0.80 \\
\hline 240 & 47.92 & 47.36 & 47.63 & 47.48 & 47.63 & 47.60 & 0.75 \\
\hline
\end{tabular}




\subsubsection{Heisenberg Ladder 315S on Xeon Phi}

Table 6.88 Time and speedup of Ladder $315 \mathrm{~S}(N)$ model with $N=24$ on an Intel Xeon

Phi coprocessor with KMP_THREAD_AFFINITY set to scatter

\begin{tabular}{|c|c|c|c|c|c|c|c|}
\hline \multicolumn{8}{|c|}{ Scatter Heisenberg Ladder315S(24) (time in seconds) } \\
\hline Thread & Data 1 & Data 2 & Data 3 & Data 4 & Data 5 & Average & Speedup \\
\hline 1 & 2565.74 & 2569.90 & 2569.13 & 2569.56 & 2570.11 & 2568.89 & 1.00 \\
\hline 10 & 634.26 & 639.13 & 607.93 & 620.80 & 596.48 & 619.72 & 4.15 \\
\hline 20 & 376.11 & 385.95 & 366.75 & 355.90 & 338.40 & 364.62 & 7.05 \\
\hline 30 & 262.39 & 257.53 & 262.53 & 267.41 & 264.03 & 262.78 & 9.78 \\
\hline 40 & 202.56 & 208.22 & 208.06 & 208.26 & 209.03 & 207.23 & 12.40 \\
\hline 50 & 156.74 & 166.11 & 166.98 & 164.72 & 161.10 & 163.13 & 15.75 \\
\hline 60 & 140.80 & 150.12 & 142.74 & 142.53 & 144.15 & 144.07 & 17.83 \\
\hline 70 & 127.88 & 133.37 & 127.35 & 127.86 & 127.99 & 128.89 & 19.93 \\
\hline 80 & 119.86 & 117.38 & 113.98 & 116.31 & 111.49 & 115.80 & 22.18 \\
\hline 90 & 97.99 & 107.04 & 102.54 & 104.67 & 102.16 & 102.88 & 24.97 \\
\hline 100 & 97.07 & 92.93 & 91.55 & 95.22 & 95.03 & 94.36 & 27.22 \\
\hline 110 & 86.42 & 89.37 & 85.07 & 87.30 & 89.35 & 87.50 & 29.36 \\
\hline 120 & 84.68 & 83.71 & 84.22 & 83.72 & 86.11 & 84.49 & 30.41 \\
\hline 130 & 81.39 & 81.86 & 80.09 & 81.00 & 86.99 & 82.26 & 31.23 \\
\hline 140 & 76.46 & 84.02 & 69.73 & 77.06 & 77.25 & 76.90 & 33.40 \\
\hline 150 & 71.35 & 72.13 & 77.31 & 72.55 & 72.66 & 73.20 & 35.09 \\
\hline 160 & 68.38 & 78.77 & 78.80 & 68.86 & 72.77 & 73.52 & 34.94 \\
\hline 170 & 67.46 & 66.58 & 70.87 & 70.59 & 69.36 & 68.97 & 37.25 \\
\hline 180 & 66.24 & 66.92 & 65.38 & 66.26 & 65.76 & 66.11 & 38.86 \\
\hline 190 & 65.13 & 57.42 & 65.01 & 64.55 & 65.22 & 63.46 & 40.48 \\
\hline 200 & 62.26 & 62.58 & 61.17 & 61.49 & 61.37 & 61.77 & 41.59 \\
\hline 210 & 58.69 & 58.95 & 58.70 & 58.16 & 59.03 & 58.70 & 43.76 \\
\hline 220 & 57.74 & 57.46 & 57.49 & 57.55 & 55.04 & 57.06 & 45.02 \\
\hline 230 & 53.87 & 53.95 & 53.88 & 53.97 & 53.79 & 53.89 & 47.67 \\
\hline 240 & 53.21 & 53.00 & 53.23 & 53.08 & 53.19 & 53.14 & 48.34 \\
\hline
\end{tabular}


Table 6.89 Time and speedup of Ladder $315 \mathrm{~S}(N)$ model with $N=18$ on an Intel Xeon Phi coprocessor with KMP_THREAD_AFFINITY set to scatter

\begin{tabular}{|c|c|c|c|c|c|c|c|}
\hline \multicolumn{8}{|c|}{ Scatter Heisenberg Ladder315S(18) (time in seconds) } \\
\hline Thread & Data 1 & Data 2 & Data 3 & Data 4 & Data 5 & Average & Speedup \\
\hline 1 & 35.80 & 35.71 & 35.81 & 35.72 & 35.88 & 35.78 & 1.00 \\
\hline 10 & 8.30 & 8.49 & 8.74 & 8.34 & 8.29 & 8.43 & 4.24 \\
\hline 20 & 6.38 & 6.62 & 6.44 & 6.31 & 6.30 & 6.41 & 5.58 \\
\hline 30 & 5.78 & 5.92 & 5.81 & 5.74 & 5.86 & 5.82 & 6.15 \\
\hline 40 & 4.24 & 4.37 & 4.25 & 4.25 & 4.31 & 4.28 & 8.35 \\
\hline 50 & 4.49 & 4.29 & 4.26 & 4.50 & 4.49 & 4.40 & 8.12 \\
\hline 60 & 4.73 & 4.93 & 4.91 & 4.55 & 4.88 & 4.80 & 7.45 \\
\hline 70 & 5.46 & 5.40 & 5.48 & 5.50 & 5.49 & 5.46 & 6.55 \\
\hline 80 & 5.10 & 5.89 & 5.84 & 5.85 & 5.85 & 5.71 & 6.27 \\
\hline 90 & 6.42 & 6.49 & 6.41 & 6.44 & 6.43 & 6.44 & 5.56 \\
\hline 100 & 6.84 & 6.88 & 6.20 & 6.60 & 6.86 & 6.68 & 5.36 \\
\hline 110 & 7.03 & 6.77 & 7.29 & 7.31 & 7.30 & 7.14 & 5.01 \\
\hline 120 & 7.73 & 7.87 & 7.87 & 7.74 & 7.89 & 7.82 & 4.58 \\
\hline 130 & 8.60 & 8.60 & 8.62 & 8.62 & 8.63 & 8.61 & 4.15 \\
\hline 140 & 9.20 & 8.29 & 8.97 & 8.99 & 9.15 & 8.92 & 4.01 \\
\hline 150 & 9.60 & 9.60 & 9.60 & 9.60 & 9.60 & 9.60 & 3.73 \\
\hline 160 & 10.05 & 9.48 & 10.08 & 10.09 & 10.07 & 9.95 & 3.59 \\
\hline 170 & 10.51 & 10.54 & 10.51 & 10.50 & 10.51 & 10.51 & 3.40 \\
\hline 180 & 10.34 & 10.96 & 10.95 & 10.95 & 11.02 & 10.84 & 3.30 \\
\hline 190 & 11.73 & 11.74 & 11.74 & 11.70 & 11.73 & 11.73 & 3.05 \\
\hline 200 & 12.17 & 12.17 & 12.17 & 12.15 & 12.18 & 12.17 & 2.94 \\
\hline 210 & 9.72 & 12.62 & 12.63 & 12.62 & 12.62 & 12.04 & 2.97 \\
\hline 220 & 13.07 & 13.06 & 13.08 & 13.05 & 13.07 & 13.07 & 2.74 \\
\hline 230 & 13.51 & 13.52 & 13.52 & 13.51 & 12.48 & 13.31 & 2.69 \\
\hline 240 & 14.36 & 14.02 & 14.01 & 14.04 & 14.02 & 14.09 & 2.54 \\
\hline
\end{tabular}


Table 6.90 Time and speedup of Ladder $315 \mathrm{~S}(N)$ model with $N=24$ on an Intel Xeon

Phi coprocessor with KMP_THREAD_AFFINITY set to compact

\begin{tabular}{|c|c|c|c|c|c|c|c|}
\hline \multicolumn{8}{|c|}{ Compact Heisenberg Ladder315S(24) (time in seconds) } \\
\hline Thread & Data 1 & Data 2 & Data 3 & Data 4 & Data 5 & Average & Speedup \\
\hline 1 & 2565.74 & 2569.90 & 2569.13 & 2569.56 & 2570.11 & 2568.89 & 1.00 \\
\hline 10 & 763.46 & 762.06 & 769.37 & 790.99 & 785.94 & 774.36 & 3.32 \\
\hline 20 & 402.93 & 402.86 & 402.86 & 403.52 & 403.42 & 403.12 & 6.37 \\
\hline 30 & 273.55 & 273.65 & 273.61 & 274.46 & 274.27 & 273.91 & 9.38 \\
\hline 40 & 211.63 & 211.75 & 211.66 & 211.73 & 211.85 & 211.72 & 12.13 \\
\hline 50 & 170.08 & 170.13 & 170.07 & 170.47 & 170.42 & 170.23 & 15.09 \\
\hline 60 & 146.72 & 146.67 & 146.68 & 146.64 & 146.66 & 146.67 & 17.51 \\
\hline 70 & 127.98 & 128.06 & 127.97 & 128.13 & 128.14 & 128.06 & 20.06 \\
\hline 80 & 113.28 & 113.31 & 113.30 & 111.31 & 113.44 & 112.93 & 22.75 \\
\hline 90 & 102.57 & 102.59 & 102.62 & 102.82 & 102.86 & 102.69 & 25.02 \\
\hline 100 & 93.74 & 93.77 & 93.79 & 93.83 & 93.83 & 93.79 & 27.39 \\
\hline 110 & 87.44 & 87.49 & 87.46 & 87.58 & 87.57 & 87.51 & 29.36 \\
\hline 120 & 81.66 & 81.66 & 81.60 & 81.67 & 81.71 & 81.66 & 31.46 \\
\hline 130 & 76.83 & 76.85 & 76.84 & 76.91 & 76.91 & 76.87 & 33.42 \\
\hline 140 & 72.82 & 72.81 & 72.82 & 72.86 & 72.85 & 72.83 & 35.27 \\
\hline 150 & 68.91 & 68.99 & 68.97 & 69.02 & 68.99 & 68.97 & 37.24 \\
\hline 160 & 65.07 & 65.02 & 65.05 & 65.09 & 65.07 & 65.06 & 39.48 \\
\hline 170 & 64.67 & 64.54 & 64.47 & 64.50 & 64.59 & 64.55 & 39.79 \\
\hline 180 & 59.46 & 59.49 & 59.49 & 59.52 & 59.48 & 59.49 & 43.18 \\
\hline 190 & 57.15 & 57.19 & 57.17 & 57.23 & 57.28 & 57.20 & 44.91 \\
\hline 200 & 55.15 & 55.21 & 55.18 & 55.21 & 55.24 & 55.20 & 46.54 \\
\hline 210 & 53.46 & 51.50 & 53.42 & 53.52 & 53.56 & 53.09 & 48.39 \\
\hline 220 & 52.57 & 52.62 & 52.62 & 52.60 & 52.67 & 52.62 & 48.82 \\
\hline 230 & 50.43 & 50.44 & 50.42 & 50.51 & 50.54 & 50.47 & 50.90 \\
\hline 240 & 49.38 & 48.98 & 48.95 & 47.29 & 49.08 & 48.74 & 52.71 \\
\hline
\end{tabular}


Table 6.91 Time and speedup of Ladder $315 \mathrm{~S}(N)$ model with $N=18$ on an Intel Xeon Phi coprocessor with KMP_THREAD_AFFINITY set to compact

\begin{tabular}{|c|c|c|c|c|c|c|c|}
\hline \multicolumn{8}{|c|}{ Compact Heisenberg Ladder315S(18) (time in seconds) } \\
\hline Thread & Data 1 & Data 2 & Data 3 & Data 4 & Data 5 & Average & Speedup \\
\hline 1 & 35.80 & 35.71 & 35.81 & 35.72 & 35.88 & 35.78 & 1.00 \\
\hline 10 & 11.87 & 11.89 & 11.87 & 11.82 & 11.81 & 11.85 & 3.02 \\
\hline 20 & 8.53 & 7.63 & 8.55 & 8.49 & 8.48 & 8.34 & 4.29 \\
\hline 30 & 7.78 & 7.77 & 7.77 & 7.70 & 7.71 & 7.75 & 4.62 \\
\hline 40 & 6.89 & 6.53 & 6.77 & 6.85 & 6.83 & 6.77 & 5.28 \\
\hline 50 & 7.00 & 7.00 & 6.95 & 6.92 & 6.94 & 6.96 & 5.14 \\
\hline 60 & 7.29 & 7.30 & 7.26 & 6.37 & 7.24 & 7.09 & 5.05 \\
\hline 70 & 7.66 & 7.65 & 7.61 & 7.46 & 7.61 & 7.60 & 4.71 \\
\hline 80 & 8.05 & 8.03 & 8.03 & 8.05 & 8.03 & 8.04 & 4.45 \\
\hline 90 & 8.50 & 8.46 & 8.47 & 7.58 & 8.43 & 8.29 & 4.32 \\
\hline 100 & 8.95 & 8.96 & 8.91 & 8.92 & 8.94 & 8.94 & 4.00 \\
\hline 110 & 9.13 & 9.48 & 9.44 & 9.42 & 9.43 & 9.38 & 3.81 \\
\hline 120 & 9.98 & 9.07 & 9.94 & 9.95 & 9.96 & 9.78 & 3.66 \\
\hline 130 & 10.58 & 10.58 & 8.82 & 10.53 & 10.54 & 10.21 & 3.50 \\
\hline 140 & 11.15 & 10.94 & 11.11 & 11.13 & 11.11 & 11.09 & 3.23 \\
\hline 150 & 11.82 & 11.81 & 11.55 & 11.79 & 11.77 & 11.75 & 3.05 \\
\hline 160 & 12.50 & 12.51 & 12.50 & 12.48 & 12.51 & 12.50 & 2.86 \\
\hline 170 & 13.31 & 13.30 & 13.27 & 13.28 & 13.25 & 13.28 & 2.69 \\
\hline 180 & 13.98 & 14.01 & 12.66 & 14.01 & 13.98 & 13.73 & 2.61 \\
\hline 190 & 14.82 & 14.29 & 13.43 & 12.59 & 14.84 & 13.99 & 2.56 \\
\hline 200 & 12.05 & 15.54 & 15.57 & 15.58 & 15.54 & 14.85 & 2.41 \\
\hline 210 & 16.53 & 16.53 & 16.52 & 16.50 & 16.49 & 16.51 & 2.17 \\
\hline 220 & 17.43 & 17.41 & 17.08 & 17.42 & 15.43 & 16.95 & 2.11 \\
\hline 230 & 15.66 & 18.47 & 18.49 & 18.47 & 18.46 & 17.91 & 2.00 \\
\hline 240 & 19.72 & 14.22 & 17.17 & 19.41 & 17.55 & 17.61 & 2.03 \\
\hline
\end{tabular}


Table 6.92 Time and speedup of Ladder $315 \mathrm{~S}(N)$ model with $N=24$ on an Intel Xeon Phi coprocessor with KMP_THREAD_AFFINITY set to balanced

\begin{tabular}{|c|c|c|c|c|c|c|c|}
\hline Thread & Data 1 & Data 2 & Data 3 & Data 4 & Data 5 & Average & Speedup \\
\hline 1 & 2565.74 & 2569.90 & 2569.13 & 2569.56 & 2570.11 & 2568.89 & 1.00 \\
\hline 10 & 633.32 & 615.96 & 601.85 & 628.27 & 617.80 & 619.44 & 4.15 \\
\hline 20 & 358.79 & 353.39 & 358.15 & 342.32 & 362.17 & 354.97 & 7.24 \\
\hline 30 & 255.15 & 263.30 & 244.04 & 257.84 & 262.17 & 256.50 & 10.02 \\
\hline 40 & 207.99 & 207.69 & 206.36 & 206.84 & 205.77 & 206.93 & 12.41 \\
\hline 50 & 163.20 & 165.28 & 166.02 & 160.07 & 156.31 & 162.17 & 15.84 \\
\hline 60 & 140.84 & 141.97 & 142.81 & 142.21 & 142.94 & 142.15 & 18.07 \\
\hline 70 & 124.50 & 126.09 & 128.79 & 134.83 & 125.94 & 128.03 & 20.06 \\
\hline 80 & 107.91 & 108.08 & 113.08 & 112.78 & 109.10 & 110.19 & 23.31 \\
\hline 90 & 101.81 & 92.49 & 104.68 & 106.45 & 101.98 & 101.48 & 25.31 \\
\hline 100 & 92.60 & 91.05 & 90.78 & 93.13 & 93.47 & 92.21 & 27.86 \\
\hline 110 & 87.55 & 87.07 & 87.02 & 86.50 & 86.97 & 87.02 & 29.52 \\
\hline 120 & 81.90 & 81.04 & 80.99 & 82.28 & 81.65 & 81.57 & 31.49 \\
\hline 130 & 76.94 & 78.16 & 79.32 & 79.00 & 78.67 & 78.42 & 32.76 \\
\hline 140 & 75.09 & 74.35 & 74.49 & 73.73 & 73.62 & 74.25 & 34.60 \\
\hline 150 & 70.59 & 70.00 & 69.48 & 69.29 & 69.08 & 69.69 & 36.86 \\
\hline 160 & 68.61 & 65.82 & 65.27 & 65.21 & 65.82 & 66.15 & 38.84 \\
\hline 170 & 67.51 & 65.78 & 66.26 & 64.86 & 66.69 & 66.22 & 38.79 \\
\hline 180 & 62.95 & 62.34 & 61.35 & 62.88 & 62.29 & 62.36 & 41.19 \\
\hline 190 & 61.48 & 61.67 & 61.24 & 61.03 & 61.74 & 61.43 & 41.82 \\
\hline 200 & 58.00 & 57.88 & 59.11 & 58.99 & 57.88 & 58.37 & 44.01 \\
\hline 210 & 55.71 & 55.56 & 55.66 & 55.32 & 55.90 & 55.63 & 46.18 \\
\hline 220 & 55.08 & 55.23 & 52.48 & 55.46 & 55.57 & 54.76 & 46.91 \\
\hline 230 & 50.52 & 50.43 & 50.56 & 50.45 & 50.52 & 50.50 & 50.87 \\
\hline 240 & 49.07 & 49.19 & 49.14 & 49.06 & 49.12 & 49.11 & 52.31 \\
\hline
\end{tabular}


Table 6.93 Time and speedup of Ladder $315 \mathrm{~S}(N)$ model with $N=18$ on an Intel Xeon Phi coprocessor with KMP_THREAD_AFFINITY set to bal anced

\begin{tabular}{|c|c|c|c|c|c|c|c|}
\hline Thread & Data 1 & Data 2 & Data 3 & Data 4 & Data 5 & Average & Speedup \\
\hline 1 & 35.80 & 35.71 & 35.81 & 35.72 & 35.88 & 35.78 & 1.00 \\
\hline 10 & 9.13 & 8.38 & 9.15 & 9.99 & 8.34 & 9.00 & 3.98 \\
\hline 20 & 6.93 & 6.61 & 7.27 & 6.55 & 7.60 & 6.99 & 5.12 \\
\hline 30 & 7.43 & 7.59 & 5.94 & 7.69 & 7.50 & 7.23 & 4.95 \\
\hline 40 & 5.61 & 6.71 & 6.62 & 6.84 & 6.70 & 6.50 & 5.51 \\
\hline 50 & 8.10 & 7.65 & 8.06 & 7.82 & 8.14 & 7.95 & 4.50 \\
\hline 60 & 9.50 & 10.28 & 10.08 & 10.41 & 9.39 & 9.93 & 3.60 \\
\hline 70 & 15.53 & 14.72 & 15.35 & 15.91 & 15.92 & 15.49 & 2.31 \\
\hline 80 & 16.99 & 17.09 & 16.73 & 14.77 & 17.75 & 16.67 & 2.15 \\
\hline 90 & 18.21 & 18.13 & 18.21 & 18.36 & 18.74 & 18.33 & 1.95 \\
\hline 100 & 19.60 & 19.58 & 19.64 & 19.96 & 19.87 & 19.73 & 1.81 \\
\hline 110 & 21.49 & 21.76 & 21.52 & 21.52 & 21.56 & 21.57 & 1.66 \\
\hline 120 & 22.95 & 23.02 & 22.80 & 22.82 & 23.20 & 22.96 & 1.56 \\
\hline 130 & 24.40 & 23.68 & 25.08 & 25.22 & 25.40 & 24.76 & 1.45 \\
\hline 140 & 27.04 & 27.14 & 26.36 & 27.09 & 27.26 & 26.98 & 1.33 \\
\hline 150 & 29.03 & 28.53 & 28.97 & 29.12 & 29.25 & 28.98 & 1.23 \\
\hline 160 & 30.88 & 31.17 & 31.44 & 31.13 & 30.96 & 31.11 & 1.15 \\
\hline 170 & 33.06 & 32.75 & 32.92 & 33.04 & 33.07 & 32.97 & 1.09 \\
\hline 180 & 34.65 & 34.59 & 34.53 & 34.58 & 34.62 & 34.59 & 1.03 \\
\hline 190 & 35.31 & 35.44 & 35.51 & 35.24 & 35.20 & 35.34 & 1.01 \\
\hline 200 & 37.24 & 36.95 & 36.97 & 36.85 & 37.03 & 37.01 & 0.97 \\
\hline 210 & 39.42 & 38.84 & 39.55 & 39.64 & 39.62 & 39.41 & 0.91 \\
\hline 220 & 42.13 & 41.90 & 42.03 & 42.11 & 42.25 & 42.09 & 0.85 \\
\hline 230 & 34.27 & 44.88 & 45.04 & 44.75 & 44.66 & 42.72 & 0.84 \\
\hline 240 & 44.74 & 46.87 & 46.80 & 46.61 & 46.71 & 46.35 & 0.77 \\
\hline
\end{tabular}




\section{Reference}

[1] A. Honecker, A comparative study of the magnetization process of two-dimensional antiferromagnets, J. Phys.: Condens. Matter 11, 4697-4713 (1999).

[2] Andreas Lüscher and Andreas M. Läuchli, Exact diagonalization study of the antiferromagnetic spin-1/2 Heisenberg model on the square lattice in a magnetic field, Phys. Rev. B 79, 195102 (2009).

[3] B: G. Kresse and J. Furthmüller, Phys. Rev. B 54, 11169 (1996).

[4] Balanced Affinity Type, Intel® Fortran Compiler XE 13.1 User and Reference Guides, https://software.intel.com/sites/products/documentation/doclib/stdxe/2013/composerxe/ compiler/fortran-lin/GUID-8FCD3720-6F73-429C-AE65-7144ED0B991A.htm retrieved November $12^{\text {th }}, 2013$.

[5] Barnes, T., Dagotto, E., Riera, J., Swanson, E.S.: Excitation spectrum of Heisenberg spin ladders. Phys. Rev. B 47, 3196-3203 (1993).

[6] Crouzeix, M., Phillipe, B., Sadkane, M.: The Davidson method. SIAM J. Sci. Comput. 15(1), 62-76 (1994).

[7] David L. Harrar II, "On the Davidson and Jacobi-Davidson Methods for Large-Scale Eigenvalue Problems", Research Report, Centre for Mathematics and Applications, Australian National University.

[8] Davidson, E.R.: The iterative calculation of a few lowest eigenvalues and corresponding eigenvectors of large symmetric matrices. Comput. Phys. 17, 87-94 (1975).

[9] Fehske, H. Schneider, R. and Weiße, A. (eds.), Computational Many-Particle Physics, Lect. Notes Phys. 739 (Springer-Verlag Berlin Heidelberg 2008).

[10] Gene H.G.: Matrix Computations, 3rd edition. Johns Hopkins University Press; 471-489 (1996). 
http://maths-intranet.anu.edu.au/research.reports/mrr/99/047/MRR99-047.pdf

[11] J. B. Parkinson and J. C. Bonner, Spin chain in a field: Crossover from quantum to classical behavior, Phys. Rev. B 32, 4703-4724 (1985).

[12] J. Jeffers and J. Reinders, Intel Xeon Phi Coprocessor High Performance Programming (Morgan Kaufman, NewYork, 2013).

[13] J. Oitmaa and D. D. Betts, The ground state of two quantum models of magnetism, Can. J. Phys. 56, 897 (1978).

[14] Jill C. Bonner and Michael E. Fisher, Linear Magnetic Chains with Anisotropic Coupling, Phys. Rev. 135, A640-A658 (1964).

[15] Johannes Richter, Jörg Schulenburg, and Andreas Honecker, Quantum Magnetism in Two Dimensions: From Semi-classical Néel Order to Magnetic Disorder, Lect. Notes Phys. 645, 85-153 (2004).

[16] Kresse, G., Furthmüller, J.: Efficiency of ab-initio total energy calculations for metals and semiconductors using a plane-wave basis set. Comput. Mater. Sci. 6, 15-50 (1996).

[17] Liu, B.: Numerical Algorithms in Chemistry: Algebraic method, vol. LBL-8158, chap. The simultaneous expansion for the solution of several of the lowest eigenvalues and corresponding eigenvectors of large real-symmetric matrices, pp. 49-53. Lawrence Berkeley Lab., CA (1978).

[18] Mamoru Usami and Sei-ichiro Suga, Critical properties of $S=1 / 2$ Heisenberg ladders in magnetic fields, Phys. Rev. B 58, 14401-14406 (1998).

[19] Martín-Delgado, M.A., Shankar, R., Sierra, G.: Phase transitions in staggered spin ladders. Phys. Rev. Lett. 77, 3443-3446 (1996).

[20] Murray, C.W., Racine, S.C., Davidson, E.R.: Improved algorithms for the lowest few eigenvalues and associated eigenvectors of large matrices. J. Comput. Phys. 103, 382389 (1992).

[21] N. Flocke, Exact diagonalization study on spin-1/2 ladders as a function of two coupling 
parameters using the symmetric group approach, Phys. Rev. B 56, 13673-13676 (1997).

[22] Parkinson, J.B., Farnell, D.J.J.: An Introduction to Quantum Spin Systems, Lect. Notes Phys., 816. Springer-Verlag, Berlin Heidelberg (2010).

[23] Pulay, P.: Convergence acceleration of iterative sequences. The case of SCF iteration. Chem. Phys. Lett. 73, 393-398 (1980).

[24] S. Nemati, S. Batebi, and S. Mahdavifar, Concurrence and entanglement entropy in a dimerised spin-1/2 two-leg ladder, Eur. Phys. J. B 83, 329-335 (2011).

[25] Sandra J. Gibson, R. Meyer and Gennady Y. Chitov, Numerical study of critical properties and hidden orders in dimerized spin ladders, Phys. Rev. B 83, 104423 (2011).

[26] Sparse Matrix Wikipedia, http://en.wikipedia.org/wiki/Sparse_matrix Retrieved Novermber $16^{\text {th }}, 2013$.

[27] Steven R. White, Density matrix formulation fro quantum renormalization groups, Phys. Rev. Lett. 69, 2863-2866 (1992).

[28] Thijssen, J.: Computational Physics, $2^{\text {nd }}$ edition. Cambridge University Press, Cambrige (2010).

[29] Totsuka, K., Suzuki, M.: The spin-1/2 Heisenberg spin ladder with bond alternation. J. Phys: Condens. Matter 7, 6079-6096 (1995).

[30] Weiße, A., Fehske, H.: Computational Many-Particle Physics, Lect. Notes Phys., vol. 739, chap. Exact Diagonalization Techniques, pp. 529-544. Springer-Verlag, Berlin Heidelberg (2008).

[31] Wood, D.M., Zunger, A.: A new method for diagonalising large matrices. J. Phys. A: Math. Gen. 18, 1343-1359 (1985). 